\title{
Attenuation of Shock Waves in Copper and Stainless Steel
}

LA- - 10753-T

William Bryan Harvey

$\mathrm{DE} 86 \quad 014879$

\section{BISCLAIMER}

This report was prepared as an account of work sponsored 'y an agency of the L inited States finvernment. Neither the United States Government nor any agenc: thereot, nur any of their employees. makes any warrany, express or implied, or assumes any legal liability as responsi. hility for the atcuracy. completeness, or usefulness af any information, apparatus, product. of process disclosed, or represents that its use would nos infringe provaly owned rights. Reforence herein to any specilic commercial producl. precess, ar service by trade nance Irademark. manufacturer. of wherwise does not necessarily constitute or umply ts endotsetuen, recommendation, wr favoring hy the United States fovernment or any ageney thereot. The views and opinions of authors expressed hereer do not necessarly state or reflec: those of the Iniled Siater Guvernment or any agency theroor. 


\begin{abstract}
By using shock pins, data were gathered on the trajectories of shock waves in stainless steel (SS-304L) and oxygen-free-high-conductivity copper (OFHC-Cu). Shock pressures were generated in these materials by impacting the appropriate target with thin $(\sim 1.5 \mathrm{~mm})$ flying plates. The flying plates in these experiments were accelerated to high velocities $(\sim 4 \mathrm{~km} / \mathrm{s})$ by high explosives. Six experiments were conducted, three using SS-304L as the target material and three experiments using OFHC-Cu as the target material. Peak shock pressures generated in the steel experiments were approximately 109, 130, and $147 \mathrm{GPa}$ and in the copper experiments, the peak shock pressures were approximately 111,132 , and $143 \mathrm{GPa}$. In each experiment, an attenuation of the shock wave by a following release wave was clearly observed.
\end{abstract}

Ar extensive effort using two characteristic codes (described in this work) to theoretically calculate the attenuation of the shock waves was made. The efficacy 
of several different constitutive equations to successfully model the "yperiments was stulied hy comparing the calculated shock trijectories to the experimmetat datit. Based on such conparions, the conchusion an be drawn that OtHC-Cu enters a melt phase at about 130 GPa on the principal Hugnusot. There was nosign

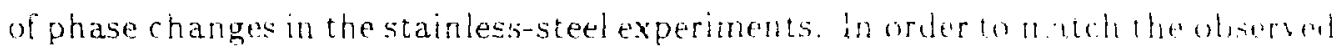

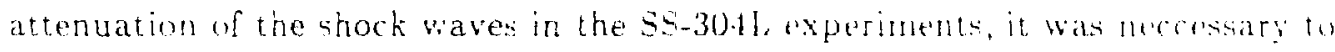
include strength effects ir the alculations. It was foumd that the values for the parameters in the strength equations were dependent on the equation of state used in the modteling of the experiments. 


\section{Table of fontents}

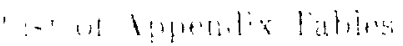

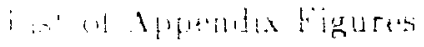

xini

i.hither

! $1911011 ! 1 \cdots 10 \div$

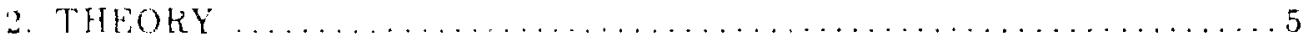

2A. Basic Assumptinns . . . . . . . . . . . . . . . . . . . .

2k. The Rankine-Hugoniot Equations $\ldots \ldots \ldots \ldots \ldots \ldots \ldots \ldots \ldots \ldots \ldots \ldots \ldots$

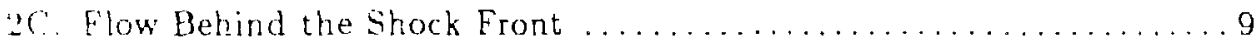

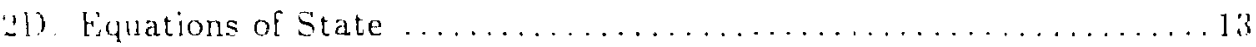

$2 \mathrm{~L}$. The Release Wave and the Gruneisen Parameter .............25

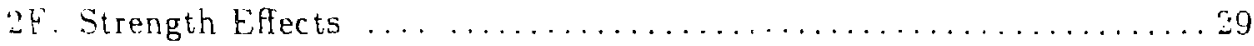

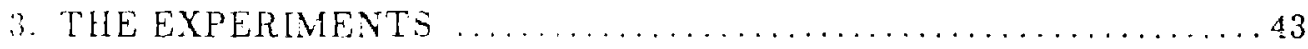

3A. Expermental Method and Design $\ldots \ldots \ldots \ldots \ldots \ldots \ldots \ldots \ldots \ldots$

3B. The Flying Plate Experiments $\ldots \ldots \ldots \ldots \ldots \ldots \ldots \ldots \ldots \ldots \ldots \ldots \ldots \ldots \ldots \ldots \ldots$

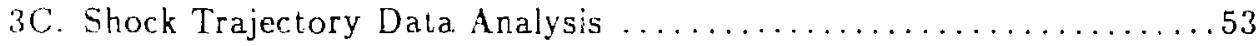

3D. The Shock-Attenuation Experiments .................. 59 


\section{Table of Contents Continued}

Chapter

4. COMPLTER MODELIVG OF THE EXPERIMENTS .........76

1. Preliminaries . . . . . . . . . . . . . . . . . . .

4B. Hydrodynamic and Simple Strength Modeling ............. 85

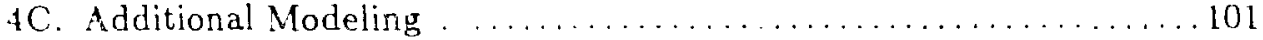

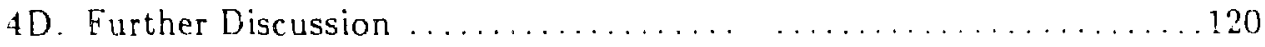

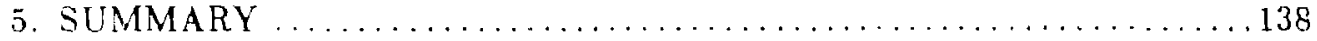

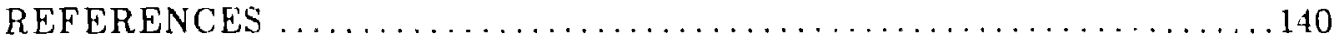

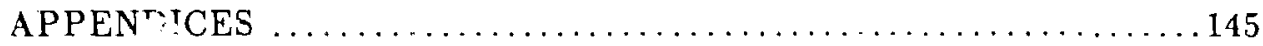

A. Characteristic Codes for One-Dimensional Flow ............ 145

B. An Analytical Solution for Shock Attenuation ................ 159

C. Melting on the Principal Hugoniot $\ldots \ldots \ldots \ldots \ldots \ldots \ldots \ldots \ldots \ldots$

D. The Gruneisen Parametn and the Cold Curve ............. 174

E. Initial Conditions in the Flyer Plate ................... 179

F. Equation of State Parameters and Sound Speeds ............ 183

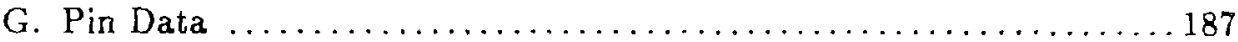

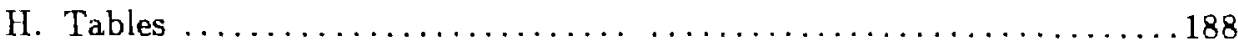

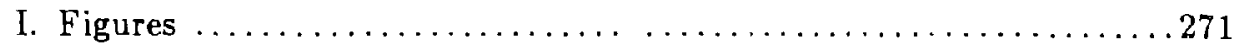




\section{List of Appendix Tables}

intite

page

B. Mran Bestiption of the Experiments

$\because$. Rs-2301: Parameters for the Fit

t $\quad a_{1} \cdot a_{r} x+a_{y} y \cdot a_{z} z+a_{r} r^{2}$.

2t. RS-2301: Parameters for the Fit

: $a_{1,}+a_{y} y+a_{z} z+a_{r} r^{2}$

3a. RS-2307: Parameters for the Fit

$$
t \quad a_{1}+a_{x} x+a_{y} y+a_{z} z
$$

3b. RS-2307: Parameters for the Fit

$$
t=a_{0}+a_{y} y+a_{z} z
$$

4a. RS-2310: Parameters for the Fit

$$
+\cdots a_{0}+a_{x} x+a_{y} y+a_{z} z+a_{1} r+a_{2} r^{2}
$$

4b. RS-2310: Parameters for the Fit

$$
t \therefore a_{0}+a_{y} y+a_{z} z+a_{1} r+a_{2} r^{2}
$$

5. Measured Flyer Plate Velocities and Predicted Shock Speeds . . . . . . 192

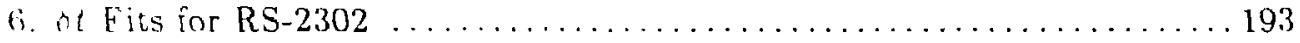

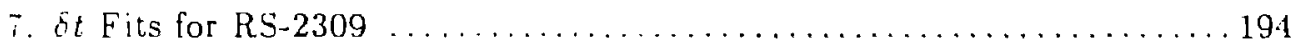

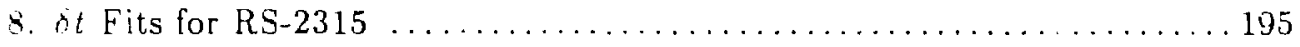

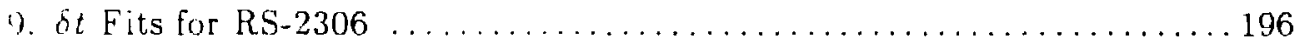

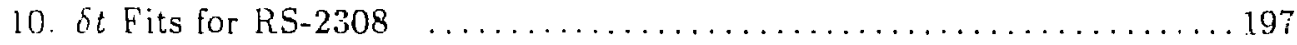

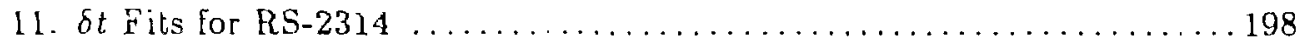

12. Probable Overtake Region ............ . . . . . . . . . . . 199

13. RS-2302: Data Analysis and Code Parameters for Figure $70 \ldots \ldots 200$

14. Variables on a Right-Going Characteristic ................

15. RS-2302: Data Analysis and Code Parameters for Figure $71 \ldots \ldots 2$

16. RS-2302: Data Analysis and Code Parameters fo- Figure $72 \ldots \ldots .203$

17. RS-2302: Data Analysis and Code Parameters for Figure 73 . . . . 204 
List of Appendix Tables Continued

Table page

18. RS-2315: Data Analysis and Code Parameters for Figure 74 205

19. RS-2315: Data Analysis and Code Parameters for Figure 75 ....... 206

20. RS-2315: Data Analysis and Code Parameters for Figure 76 207

21. RS-2315: Data. Analysis and Code Parameters for Figure 77 . ......208

22. RS-2309: Data Analysis and Code Parameters for Figure 78 ........209

23. RS-2309: Data Analysis and Code Parameters for Figure $79 \ldots \ldots \ldots 210$

24. RS-2309: Data Analysis and Code Parameters for Figure $80 \ldots \ldots \ldots 211$

25. RS-2309: Data Analysis and Code Parameters for Figure $81 \ldots \ldots \ldots 212$

26. RS-2302: Data Analysis and Code Parameters for Figure $85 \ldots \ldots \ldots 213$

27. RS-2:02: Data Analysis and Code Parameters for Figure $88 \ldots \ldots \ldots 214$

28. RS-2315: Data Analysis and Code Parameters for Figure $90 \ldots \ldots \ldots 215$

29. RS-2309: Data Analysis and Code Parameters for Figure 91 .......216

30. RS-2302: Data Analysis and Code Parameters for Figure $94 \ldots \ldots \ldots 217$

31. RS-2315: Data Analysis and Code Parameters for Figure $96 \ldots \ldots \ldots 218$

32. RS-2309: Data Analysis and Code Parameters for Figure 98 ........219

33. RS-2302: Data Analysis and Code Parameters for Figure $91 \ldots \ldots \ldots 220$

34. RS-2306: Data Analysis and Code Parameters for Figure 102 .......221

35. RS-2306: Data Analysis and Code Parameters for Figure $104 \ldots \ldots .222$

36. RS-2506: Data Analysis and Code Parameters for Figure 105 .......223

37. RS-2306: Data Analysis and Code Parameters for Figure $106 \ldots \ldots \ldots 224$

38. RS-2306: Data Analysis and Code Parameters for Figure $107 \ldots \ldots \ldots 225$

39. RS-2306: Data Analysis and Code Parameters for Figure $108 \ldots \ldots \ldots 226$

40. RS-2314: Data Anaiysis and Code Parameters for Figure 109 .......227

41. RS-2314: Data Analysis and Code Parameters for Figure $110 \ldots \ldots \ldots 228$

42. RS-2314: Data Analysis and Code Parameters for Figure 111 .......229 
List of Appendix Tables Continued

liable

page

43. Rs-2314: Data Analysis and Code Parameters for Figure $112 \ldots 230$

44. RS-2328: Data Analysis and Code Parameters for Figuce $115 \ldots \ldots .231$

45. RS-2328: Data Analysis and Code Parameters for Figure $117 \ldots \ldots 232$

16. RS-2308: Data Analysis and Cocie Parameters for Figure $119 \ldots \ldots 233$

47. RS-2308: Data Analysis and Code Parameters for Figure $121 \ldots 234$

48. RS-2302: Data Analysis and Code Parameters for Figure 123 . . . . .235

19. RS-2302: Data Analysis and Code Parameters for Figure 124 . . . 236

50. R5-2302: Data Analysis and Code Parameters for Figure $125 \ldots \ldots 237$

5i. RS-2315: Data Analysis and Code Parameters for Figure 129 .....238

52. RS-2309: Data Analysis and Code Parameters for Figure $131 \ldots 239$

53. RS-2302: Data Analysis and Code Parameters for Figure $133 \ldots \ldots 240$

54. RS-2315: Data Analysis and Code Parameters for Figure 135 . . ..241

55. RS-2309: Data Analysis and Code Parameters for Figure $137 \ldots \ldots 242$

56. RS-2302: Data Analysis and Code Parameters for Figure 139 .....243

57. RS-2315: Data Analysis and Code Parameters foi Figure $141 \ldots 244$

58. RS-2309: Data Analysis and Code Parameters for Figure $143 \ldots \ldots 245$

59. RS-2302: Datia Analysis and Code Parameters for Figure 145 . . . . .246

60. RS-2315: Data Analysis and Code Parameters for Figure $147 \ldots \ldots 247$

61. RS-2309: Data Analysis and Code Parameters for Figure 149 . . . . 248

62. RS-2302: Data Analysis and Code Farameters for Figure $151 \ldots 249$

63. RS-2315: Data Analysis and Code Parameters for Figure $153 \ldots \ldots .250$

64. RS-2309: Data Analysis and Code Parameters for Figure 155 . . . . 251

65. RS-2306: Data Analysis and Code Parameters for Figure $157 \ldots \ldots 252$

66. RS-2314: Data Analysis and Code Parameters for Figure $159 \ldots \ldots 253$

67. RS-2308: Data Analysis and Code Parameters for Figure $161 \ldots 254$ 


\section{List of Appendix Tables Continued}

Table

page

68. Experimental and Calculated Sound Speeds for Tantalum . ........255

69. Hugoniot Pressure and Residual Temperalure .................256

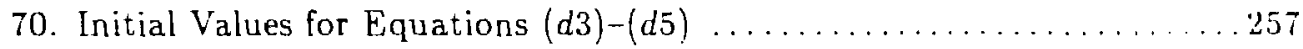

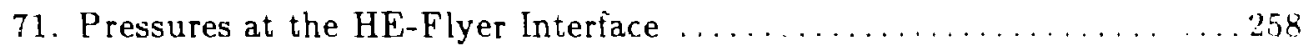

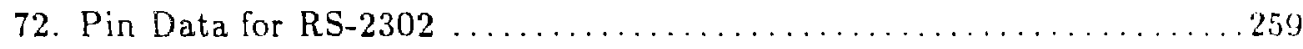

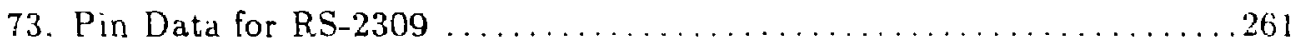

74. Pin Data for RS-2315 ................................... 263

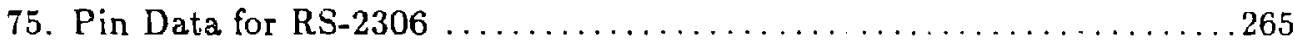

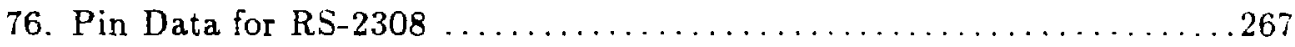

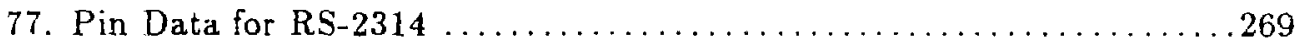


List of Appendix Figures

$r: g u r e$

page

1. I thagram showing the wave interaction for a flyer plate

hulling as serni-infinite target............................. 27

$\because$ Schematic diagram for a ypical experiment..................272

3. Iverage times for a given radius in the plane $x=0$

for the three flying wate experiments......................... 273

1 Observed times, $t_{i}$, plotted against pin positon, $x_{\mathfrak{n}}$,

in the target plate for experiment RS-2302.

5. An example of the adjusted times, $\delta t_{i}$, plotted against

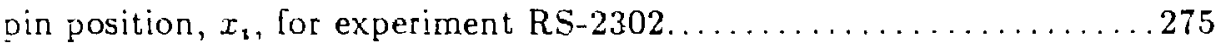

6 A plot of $\delta t$ versus $x$ in which no aitempt has been made to account for the non-planarity of the shock wave for experiment RS-2302 . . . . 276

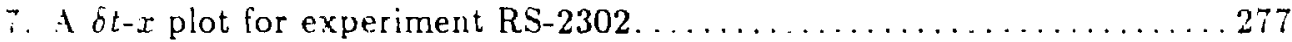

8 . A plot of shuck velocity, $U_{\mathrm{s}}$, versus position, $x$, in the target plate taken from the fit to the data shown in figure $z$ for experiment RS-2302...278

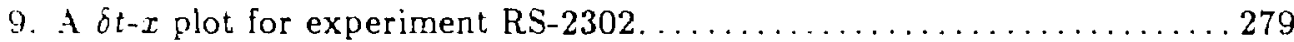

10. A $U_{s}$ versus $\mathcal{I}$ plot for $\mathrm{RS}-2302$ tak $\mathrm{n}$ from the fit to the

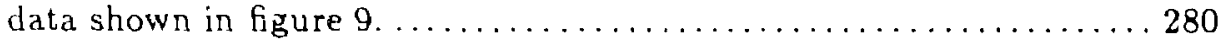

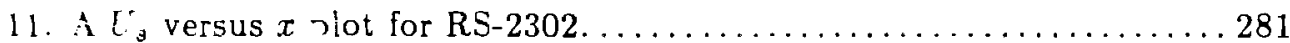

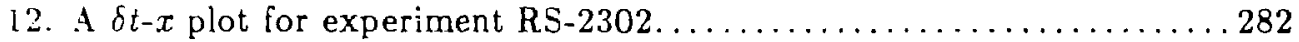

13. A $U_{s}$ versus $x$ plot for $\mathrm{KS}$-2302 taken from the fit to the

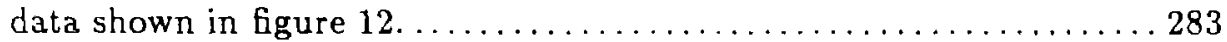

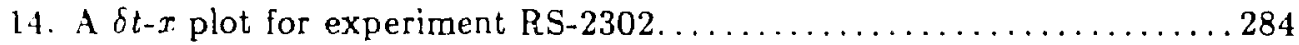

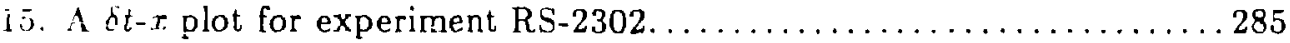

16. A $U_{s}$ versus $x$ piot for RS-2302 taken from the fit to the

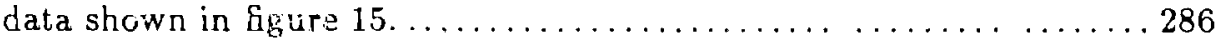

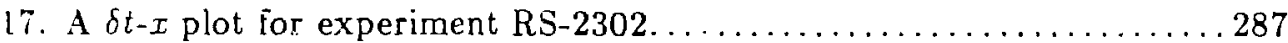


List of Appendix Figures Continued

Figure

18. A $U_{s}$ versus $x$ plot for RS-2302 taken from the fit to the data shown in figure $17 \ldots \ldots \ldots \ldots \ldots \ldots \ldots \ldots \ldots \ldots$

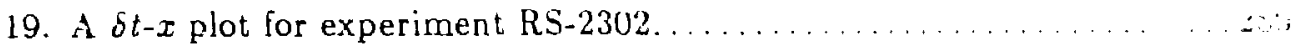

20. A $U_{3}$ versus $x$ plot for RS-2302 taken from the fit to the

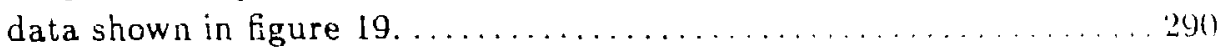

21. A $U_{3}$ versus $x$ plot taken from a fit to the data of $\mathrm{RS}-2302 \ldots \ldots \ldots 29$

22. A $U_{s}$ versus $x$ plot taken from a fit to the data of RS-2302 using the same functional fitting forms as those used for figure $21 \ldots \ldots \ldots 292$

23. A $\delta t-x$ plot for experiment RS-2302 ....................... 293

24. A $U_{s}$ versus $x$ plot for RS-2302 taken from the fit to the

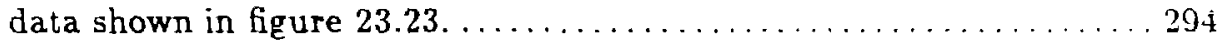

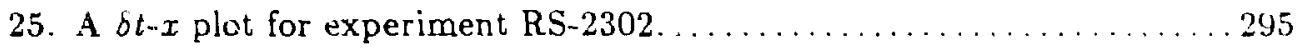

26. A $U_{s}$ versus $x$ plot for RS-2302 taken from the fit to the

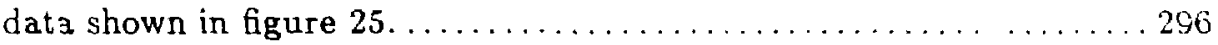

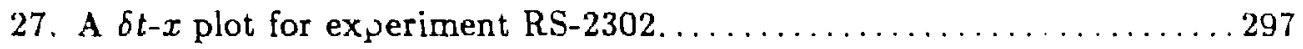

28. A $U_{s}$ versus $x$ plot for RS-2302 taken from the fit to the

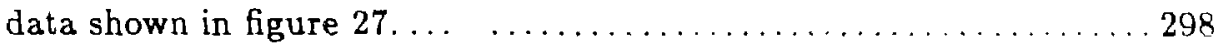

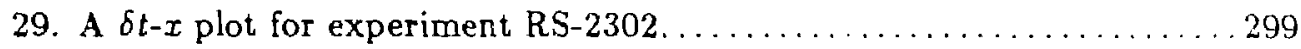

30. A $U_{a}$ versus $x$ plot for RS-2302 taken from the tit to the

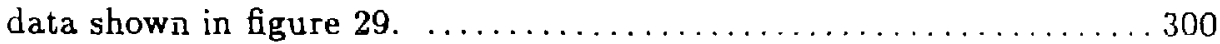

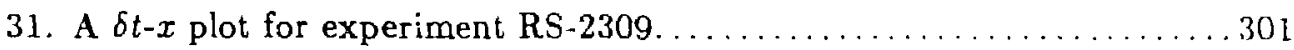

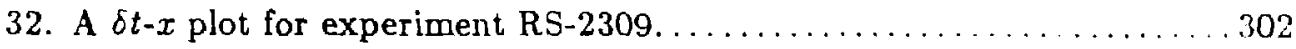

33. A $U_{s}$ versus $x$ plot for RS-2309 taken from the fit to the

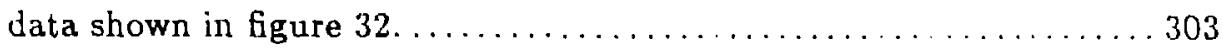

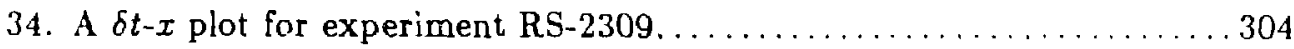

35. A $U_{8}$ versus $x$ plot for RS-2309 taken from the fit to the

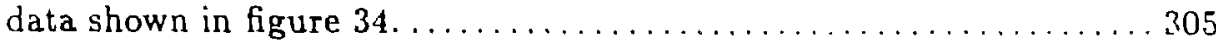




\section{List of Appendix Figures Continued}

Figure

page

36. A $U$ y versus $x$ plot taken from a tit to the data of RS-2309 using the same functional fitting for $\pi$ as those used for figure $35 \ldots \ldots \ldots . . .306$

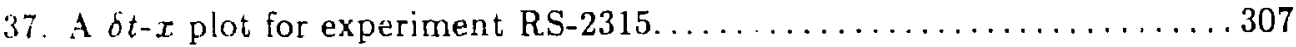

38. A $U$, versus $I$ plot for RS-2315 taken from the fit to the

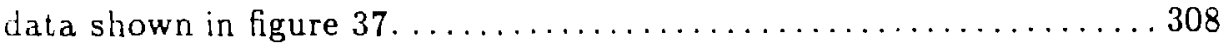

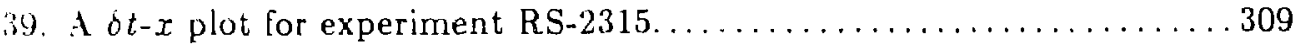

40. $A U_{a}$ versus $x$ plot for RS-2315 taken from the fit to the

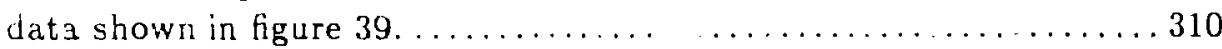

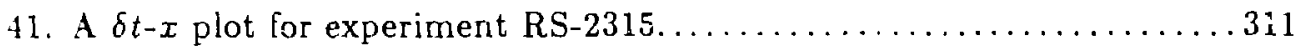

42. A $U_{s}$ versus $x$ plot for RS-2315 taken from the fit to the

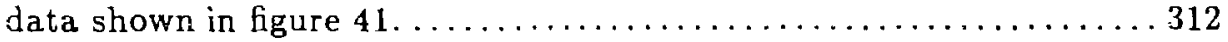

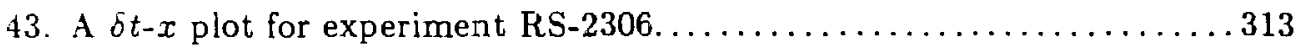

14. A $U_{3}$ versis $x$ plot for RS-2306 taker from the fit to the

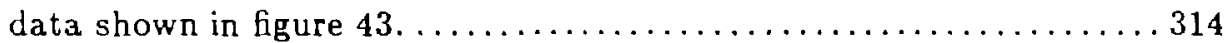

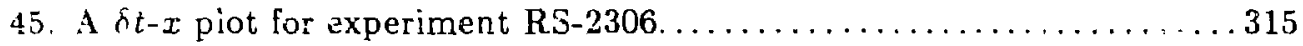

46. A $U_{y}$ versus $x$ plot for RS-2306 taken from the fit to the

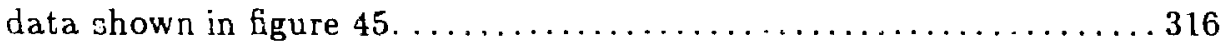

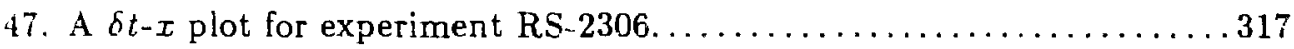

48. A $U_{s}$ versus $x$ plot for RS-2306 taken from the fit to the

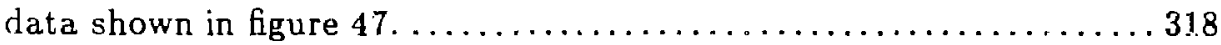

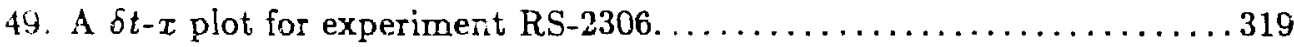

50. A $U_{\mathrm{a}}$ versus $x$ plot for RS-2306 taken from the fit to the

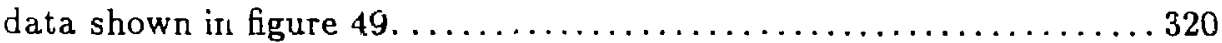

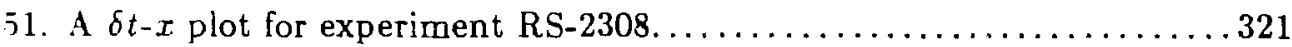

52. A $U_{a}$ versus $x$ plot for RS-230s taken from the fit to the

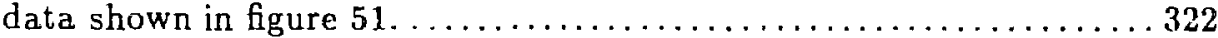

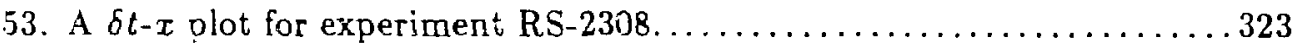


List of Appendix Figures Continued

Figure

54. A $U_{3}$ versus $x$ plot for RS-2308 taken from the fit to the data shown in figure 53.

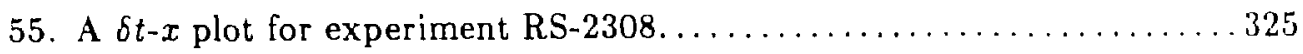

56. A $U_{s}$ versus $x$ plot for RS-2308 taken from the fit to the data shown in figure 55 .

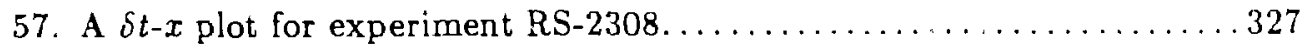

58. A $U_{s}$ versus $x$ plot for RS-2308 taken from the fit to the data shown in figure 57 .

59. A $\delta t-x$ plot for experiment RS-2308.

60. A $U_{s}$ versus $x$ plot for RS-2308 taken from the fit to the

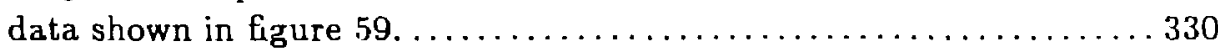

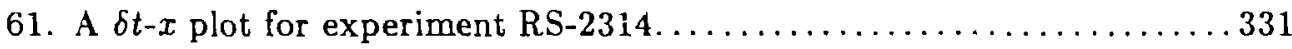

62. A $U_{s}$ versus $x$ plot for RS-2314 taken from the fit to the

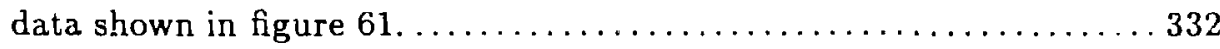

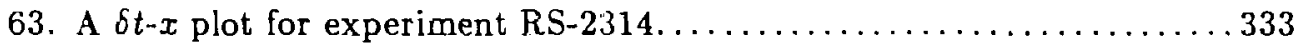

64. A $U_{s}$ versus $x$ plot for RS-2314 taken from the fit to the

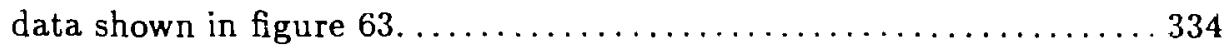

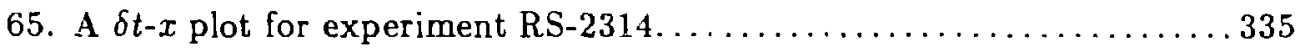

66. A $U_{s}$ versus $x$ plot for RS-2314 taken from the fit to the

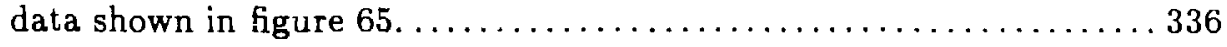

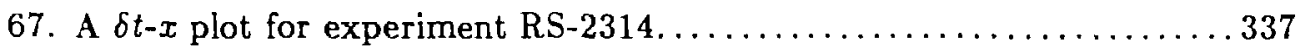

68. A $U_{a}$ versus $x$ plot for RS-2314 taken from the fit to the

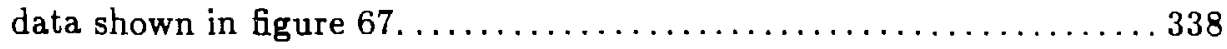

69. Detailed diagram of the different regions that occur when a simple-centered release wave attenuates a shock. .

70. Comparison of the $\delta t$ data for RS-2302 and calculational results from the code SHKSIM.

71. Comparison of the $\delta t$ data for RS-2302 and calculational results from the code SHKSIM. 


\section{List of Appendix Figures Continued}

ligure

page

72. Comparison of the $\delta t$ data for RS-2302 and calculational results from the code SHKSLM.

73. Comparicon of the $\delta t$ data for RS-2302 and calculational results from the code SHKSIM

7 . Comparison of the $\delta t$ data for RS-2315 and calculational results from the code SHKSIM.

75. Comparison of the $\delta t$ data for KS-2315 and calculational results from the code SHKSIM.

76. Comparison of the $\delta t$ data for RS-2315 and calculational results from the code ShKSIM..................... 346

77. Comparison of the $\delta t$ data for RS-2315 and calculationai results from the code SHKSIM.

78 . Comparison of the $\delta t$ data for RS-2309 and calculational results from the code SHKSLM.

79. Comparison of the $\delta t$ data for RS-2309 and calculational results from the code SHKSlYí. . . . . . . . . . . . . . . . . . . . . 349

80. Comparison of the $\delta t$ data for RS-2309 and calculational results frorn the code SHKSIM.

81. Comparison of the $\delta t$ data for RS-2309 and calculational results from the code SHKSIM.

82. A comparison of the shock velocity from the code calculation and the shock velocity curve from a fit to the data for $\mathrm{KS}-2302 \ldots \ldots \ldots \ldots \ldots 2$

83. A comparison of the shock velocity from the code calculation and the shock velocity curve from a fit to the dat.a for RS-2315 .......... 353

84. A comparison of the shock velocity from the code calculation and the shock velocity curve from a fit to the data for RS-2309. . . . . . . . 354

85. Comparison of the $\delta t$ data fur RS-2302 and calculational results from the code SHKSJB.

86. A comparison of the shock velocity from the code calculation and the shock velocity curve from a fit to the data for $\mathrm{RS}-2302$. 


\section{List of Appendix Figures Continued}

Figure

87. A ccmparison of the shock velocity from the code calculation and the shock velocity curve from a fit to the data for RS-2302 . . . . . . . 357

88. Comparison of the $\delta t$ data for RS-2302 and calculational results from the code SHKSJB.

89. A comparison of the shock velocity from the code calculation and the shock velucity curve from a fit to the data for RS-2302 . . . . . . . 359

90. Comparison of the $\delta t$ data for RS-2315 and calculational results from the code SHKSJB.

91. Comparison of the $\delta t$ data for RS-2309 and calculational results from the code SHKSJB.

92. A comparison of the shock velocity from the code calculation and the shock velocity curve from a fit to the data for RS-2315 . . . . . . 362

93. A comparison of the shock velocity from the code calculation and the shock velocity curve from a fit to the data for RS-2309. ......... 363

94. Comparison of the $\delta t$ data for RS-2302 and calculational results from tine code SHKSJB.

95. A comparison of the shock velocity from the ccde calculation and the shock velocity curve from a fit to the data for RS-2302 . . . . . . . 365

96. Comparison of the $\delta t$ data for RS-2315 and calculational results from the code SHKSJB.

97. A comparison of the shock velocity from the code calculation and the shock velocity curve from a fit to the data for RS-2315 . . . . . . . 367

98. Comparison of the $\delta t$ data for RS-2309 and calculational results from the code SHKSJB.

99. A comparison of the shock velocity from the code calculation and the shock velocity curve from a fit to the data for RS-2309. ......... 369

100. Comparison of the $\delta t$ data for RS-2302 and calculational results from the code SHKSJB.

101. A comparison of the shock velucity from the code calculation and the shock velocity curve from a fit to the data for RS-2302 . . . . . . . 371 
list of Appendix Figures Continued

Figure:

page

102. Compariscn of the $\delta t$ data for RS-2306 and calculational results from the code SHKSJB.

103. A comparison of the shock velocity from the code calculation and the whock velocity curve from a fit to the data for RS-2306. . . . . . . 373

104. Comparison of the $\delta t$ data tor RS-2306 and calculational results from the code SHKSJB.

105. Comparison of the $\delta t$ data for RS-230ti and calculational results from the code SHKSJB.

106. Comparison of the $\delta t$ data for RS-2306 and calculational results from the code SHKSJB.

107. Comparison of the $\delta t$ data for RS-2306 and calculational results from the code SHKSJB.

108. Comparison of the $\delta t$ data for RS-2306 and calculational results from the code SHKSJB. . . . . . . . . . . . . . . 378

109. Comparison of the $\delta t$ data for RS-2314 and calculational results from the code SHKSJB.

110. Comparison of the $\delta t$ data for RS-2314 and calculational results from the code SHKSJB.

111. Comparison of the $\delta t$ data for RS-2314 and calculaioional results from the code SHKSJB.

112. Comparison of the $\delta t$ data for RS-2314 and calculational results from the code SHKSJB.

113. A comparison of the shock velocity from the code calculation and the shock velocity curve from a fit to the data for $\mathrm{RS}-2314 \ldots \ldots \ldots \ldots \ldots 38$

114. A comparison of the shock velocity from the code calculation and the shock velocity curve from a fit to the data for RS-2314 . . . . . . 384

115. Comparison of the $\delta t$ data for RS-2308 and calculational results from the code SHKSJB.

116. A comparison of the shock velocity from the code calculation and the shock velocity curve from a fit to the data for RS-2308. 386 
List of Appendix Figures Continued

Figure

frage

i17. Comparison of the $\varepsilon t$ data for RS-2308 and calculational results from the code SHKSJB.

118. A comparison of the shock velocity from the code calculation and the shock velocity curve from a fit to the data for RS-2308 ......... 388

119. Comparison of the $\delta t$ data for RS-2308 and calculational results from the code SHKSJB.

120. A comparison of the shock velocity from the code calculation and the shock velocity curve from a fit to the data for RS-2308 . . . . . . . . 390

121. Comparison of the $\delta t$ data for RS-2308 and calculational results from the code SHKSJB.

122. A comparison of the shock velocity from the code calculation and the

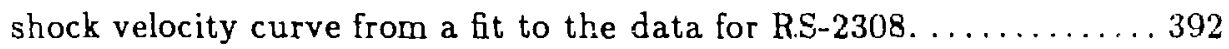

123. Comparison of the $\delta t$ data for R.S-2302 and calculational results from the code SHKSJB.

124. Comparison of the $\delta t$ data for RS-2302 and calculational results from the code SHKSJB.

125. Comparison of the $\delta t$ data for RS-2302 and calculational results from the code SHKSJB.

126. A comparison of the shock velocity from the code calculation and the shock velocity curve from a fit to the data for RS-2302 . . . . . . 396

127. A comparison of the shock velocity from the code calculation and the shock velocity curve from a fit to the data for RS-2302 . . . . . . . . 397

128. A comparison of the shock velocity from the code calculation and the shock velocity curve from a fit to the data for RS-2302 . . . . . . . . 398

129. Comparison of the $\delta t$ data for RS-2315 and calculational results from the code SHKSJB.

130. A comparison of the shock velocity from the code calculation and the shock relocity curve from a fit to the data for RS-2315, ........ 400

131. Comparison of the $\delta t$ data for RS-2309 and calculational results from the code SHKSJB. 
i.: of tppendi Figures Contmued

(:115\%

13:. A romparison of the shock veiocity from the code calculation and the whock velocity curve from at fit to the data for RS-2309. .......... 40?

$13:$ Comparison of the $\delta t$ data for RS-2302 and calculational results from the code SHKSJB.

13 A crmparison of the shock velocity from the code calculation and the shock velucity curve from a fit to the data for RS-2302. ...........4

13. Comparison of the $\delta t$ data for RS-2315 and calculational results from the code SHKSJB.

136. A comparison of the shock velocity from the code calculation and the shock velocity curve from a fit to the data for RS-2315.......... 406

137. Comparison of the $\delta t$ data for RS-2309 and calculational results from the code SHKSJB. 407

138. A comparison of the shock velucity from the code calculation and the shock velocity curve frum a fit to the data for RS-2309. . . . . . . 408

139. Comparison of the $\delta t$ data for $R S-2302$ and calculational results from the code SHKSJB.

110. A comparison of the shock velocity from the code calculation and the shock velocity curve irum a fit to the data for RS-2302 ......... 110

$1+1$. Comparison of the $\delta t$ data for RS-2315 and calculational results from the code SHKSJP.

142. A comparison of the shock velocity from the code calculation and the shock velocity curve from a fit to the data for RS-2315 ......... 412

143. Comparison of the $\delta t$ data for RS-2309 and calculational : esults frcm the code SHKSJB.

144. A comparison of the shock velocity from the code calculation and the shock velocity curve from a fit to the data for RS-2309.

115. Comparison of the $\delta t$ data ior RS-2302 and calculational results from the code SHKSJB.

1.46. A comparison of the shock velocity from the code calculation and the shock velocity curve from a fit to the data for RS-2 202 . 
List of Appendix Figures Continued

Figure

147. Comparison of the $\delta t$ data for RS-2315 and calculational results from the code SHKSJB.

148. A comparison of the shock velocity from the code calculiation and the shock velocity curve from a fit to the data for $\mathrm{RS}-2315 \ldots \ldots \ldots \ldots$

149. Comparison of the $\delta t$ data for RS-2309 and calculational results from the code SHKSJB.

150. A comparison of the shock velocity from the code calculation and the shock velocity curve from a fit to the data for RS-2309.

151. Comparison of the $\delta t$ data for RS-2302 and calculational results from the code SHKSJB.

152. A comparison of the shock velocity from the code calculation and the shock velocity curve from a fit to the data for RS-2302.

153. Comparison of the $\delta t$ data for RS-2315 and calculational results from the code SHKSJB.

154. A comparison of the shock velocity from the code calculation and the shock velocity curve from a fit to the data for RS-2315 . . . . . . . . 424

155. Comparison of the $\delta t$ data for RS-2309 and calculational results from the code SHKSJB.

156. A comparison of the shock velocity from the code calculation and the shock velocity curve from a fit to the data for RS-2309. . . . . . . 426

157. Comparison of the $\delta t$ data for RS-2306 and calculational results from the code SHKSJB.

158. A comparison of the shock velocity from the code calculation and the shock velocity curve from a fit to the data for RS-2306.

159. Comparison of the $\delta t$ data for RS-2314 and calculational results from the code SHKSJB.

160. A comparison of the shock velocity from the code calculation and the shock velocity curve from a fit to the data for RS-2314 . . . . . 430

161. Comparison of the $\delta t$ data for RS-2308 and calculational results from the code SHKSJB. 
Lint of Appendix Figures Conlinued

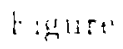

page

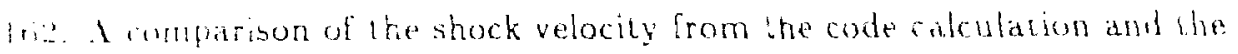
who velocity curve from a fit to the data for RS-2308. ...........132

ini comparison of numerical results from SHKSIM and an analytical colution for shock attenuation

thit Comparison of numerical results from SHKSJB and an analytical solution for shock attenuation.

l(ii). Comparison of numerica! results from SHKSJB and an analytical solution for shock attenuation.

166. Comparison of the shock velocities from SHJSJB and an analytical solution plotted as a function of shock position.

167. Temperature along the principal Hugoniot for copper taken from the integration of equations $(d 3)-(d 5)$.

Iris. Temperature along the principal Hugoniot for stainless-steel taken from the integration of equations $(d 3)-(d 5)$

169. The Gruneisen parameter for copper plotted as a function of $\eta$ from different formulaz.

170. Pressure as a function of $\eta$ alon zero-degree isotherms for copper.

171. The Gruneisen parameter for stainless-steel plotted as a function of $\eta$ from different formulas.

172. Pressure as a function of $\eta$ along zero-degree isotherms for stainless-steel.

173. The Hugoniot for stainless-steel in the $P-\rho$ plane as calculated from the Mie-Gruneisen equation of state.

174. The Hugoniot for stainless-steel in the $P-\rho$ plane as calculated from the Rice equation of state.

175. The Hugoniot for stainless-steel in the $P$ - $\rho$ plane as calculated from the Tillotson equation of state.

176. The Hugoniot for stainless-steel in the $P$ - $\rho$ plane as calculated from the Gray equation of state. . 


\section{List of Appendix Figures Continued}

Figure

177. The Hugoniot for copne $\vdots$ ine P-p plane as calculated from ne Mie-Gruneisen equation of state. . . . . . . . 4.17

178. The Hugoniot for copper in the $P$-p plane as calculated from the Rice equation of state...............4

179. The Hugoniot for copper in the $P$ - $\rho$ plane as caiculated from the Tillotson equation of state..............49

180. The Hugoniot for copper in the $P$ - $\rho$ plane as calculated from the Gray equation of state . . . . . . . . . . 450

181. The Hugoniot for stainless-steel in the $E-P$ plane as calculated from the Mie-Gruneisen equation of state.

182. The Hugoniot for stainless-steel in the $E-P$ plane as calculated from the Rice equation of state.

183. The Hugoniot for stainless-steel in the $E-P$ plane as calculated from the Tillotson equation of state.

184. The Hugoniot for stainless-steel in the $E$ - $P$ plane as calculated from the Giay equation of state.

185. The Hugoniot fo ${ }^{-}$copper in the $E-P$ plane as calculated from the Mie-Gruneisen equation of state.

186. 'The Hugoniot for copper in the $E-P$ plane as calculated from the Rice equation of state............. 456

187. The Hugoniot for copper in the $E$ - $P$ plane as calculated from the Tillotson equation of st te.

188. The Hugoniot for copper in the $E-P$ plane as calculated from the Gray equation of stale.

189. The sound speeds for stainless-steel along the Hugoniot as calculated from the various equations of state.

190. The sound speeds for copper along the Hugoniot as calculated from the various equations of state

191. The sound speeds for stainless-steel along the 150-GPa isentrope as calculated from the various equations of state. 
List of Appendix Figures Continued

bigure

192. The sound speeds for copper along the 150-GPa isentrope as calculated from the various equations of state. . . . . . . . 162

193. The sound speeds for stainless-steel along the 80-GPa isentrope as calculated from the various equations of state.............463

19.4. The sound speeds for copper along the 80-GPa isentrope as calculated from the various equations of state............464 


\section{INTRODUCTION}

The physics of matter subjected to high pressure is an area of research that is of interest to many different branches of engincering, chemistry, and physics. High pressure experimental and theoretical research cover a very wide range of pressures from just a few tenths of a gigapascal (GPa): in ihe study of pressure vessels, to pressures of nearly a huncired gigapascals in experimental work using high pressure anvils, to the extremely high pressures in the theoretical study of the death of a star ${ }^{1,2}$ The present work is both an experimental and calculational investigation of the behavior of materials subjected to moderately high pressures (on the order of $100 \mathrm{GPa}$ ).

In the experimental work, these pressures were gererated by strong shock waves and data wer: gathered on peak shock strengths and attenuation of these shock waves. Specifically, these experiments were designed to gather data relevant to the equations of state for oxygen-free-high-conductivity (OFHC) copper and a commercial grade of stainless steel. The stainless steel used in these experiments was SS-304L. The calculational effort centered on modeling the experiments and comparing the calculational results with the data to draw conclusions about the constitutive equations used in the modeling of the experiments.

$\dagger$ The unit of pressure in this work is the gigapascal (GPa). One pascal is equal to one newton per square meter. 
Experiments involving pressures above ambient conditions thate a long history. A brief but interesting historical account of high pressure experiments is given by Bridgman. ${ }^{3}$ The experiments referenced by Bridgman were all static experiments with maximum pressures ranging from 2 to .6 GPa. In this century Bridgman did some of the most signi.icant work in high pressure experiments studying a wide range of physical phenomena. He routinely used pressures in the range of $1-2 \mathrm{GPa}$ and achieved pressures as high as '0 GPa in the study of the compressibilities of solids. These experiments laid an important foundation for later work.

Expirimental methods using shock waves to produce high pressures began to receive much attention with the advent of World War II. In this country much of this work was started at Los Alamos by Goranson and others. ${ }^{4,5}$ The mathematical framework needed for the understanding of shock waves was laid by Rankine, Hugoniot, Hadamard, and others. ${ }^{5}$ Following World War II, high pressure physics research continued with much emphasis placed on dynamic experiments using shock waves. In the 1950's the upper limit on the pressures that could be achieved in static experiments was around $10 \mathrm{GPa}$. The attractiveness of experiments using high explosives to generate shock waves was that a pressure regime nearly an order of magnitude greater than that in static experiments was open to researchers. An excellent review of the experimental shock wave work through 1979 is given by Davison and Graham. ${ }^{7}$ Before 1960 , the majority of the experimental shock-wave 


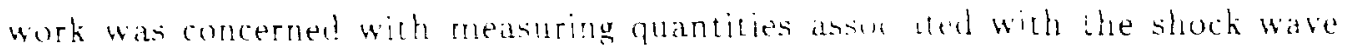
such as the speed of the shock wave, and the velocity imparted to the material by the shock wave. The need to buter understand the behavior of material shoched to high pressures led investigators to measure other quantities beside the shock speed and particle velicity, such ar sound speeds in the high pressure state and the decay of the stock wave. Among the earliest work along this line was that of Altshuler and others." Many others have performed experiments measuring similar quantities but using diflerent techniques.

It was work along such lines that provided the impetus and inspiration for the present experimental work. By measuring the attenuation of a shock wave ir a material, data are gathered on the sound speed and hence the compressibility of the material in a high pressure state. When such data are used in conjunction with calculin: ional modeling of the experiments, inferences may be drawn about the equation of state and other aspects of material response. The present experimenta! data and calculational modeling indicate that material rigidity or strength is an important ingredient in describing the material response of stainless-steel to shock pressures of at least $150 \mathrm{GPa}$ and probably higher. For copper, however, the present experiments suggest a purely bulk equation of state is adequate for modeling the material response of copper at shock pressures of $110 \mathrm{GPa}$. There is some indication that the copper may well he undergoing a phase transformation 
to a liquid state in the experiments performed at still higher pressures.

Shock waves and fluid dynamics are intimately connected and chapter 2 lays much of the theoretical fuundation needed for the understanding of shock waves and the interaction of shock waves with the flow equations of fluid dynamics. In chapter 3 the experiments are described in some detail. The manner in which the raw data are analyzed is also described in chapter 3 . The calculational modeling of the shock-attenuation experiments is discussed at length in chapter 4 . The implicat ons provided by the comparisons of the calculational results with the experimental data for the physical models is also covered in chapter 4 . Additional details about the codes used for the calculations and other aspects of the physics involved in the modeling of these experiments are given in the appendices. 


\section{THEORY}

\section{A. Basic Assumptions}

In working with materials that are dynamically shocked, four assumptions are made concerning the nature and structure of the shock wave and the state of the material behind the shock front. Before proceeding with any analysis, an elaboration of these assumptions is necessary.

The foremost assumption in the present investigation is that the shock front is infinitesimally thin, which implies that the shock process is discontinuous. In reality, the shock front has a finite thickness, but experimental estimates of the thickness of shock fionts have very small values. Experiments using laser-induced shocks in aluminum ${ }^{9}$ have shown the thickness of the shock front to be less than $0.7 \mu \mathrm{m}$ at a shock pressure of $200 \mathrm{GPa}$. The very thin nature of the shock front supports the treatment of the shock wave as a discontinuity.

Anolher important assumption concerns the state of the material once it has been shocked. It is assumed that the material is in local thermodynamic equilibrium and may be described by a suitable equilibrium constitutive equation or equation of state. The basis for this assumption is partially drawn from the fact that pressure-volume data taken by shock-wave measurements extrapolates fairly well to lower pressure data taken statically. ${ }^{10}$ 
The last two assumptions are concerned with the nature of the flow behind the shock front. T e first assumption is that the flow is adiabatic and this implies that heat conduction is being ignored. The whole process of shocking a material and then releasing down to ambient pressures occurs in less than $10 \mu \mathrm{s}$ in these experiments. This is just too short a time scale for heat conduction to have any significant effect. Lising even very high estimates of the thermal conductivity, such as $2 \times 10^{9}$ ergs (cm-s-K), the energy flow per unit area due to heat conduction is still at least three orders of magnitude lower than that associated with the flow equations given in section $2 \mathrm{C}$. The second assumption pertaining to the flow is that viscosity effects may be safely ignored. The extremely small thickness of a shock front implies that the coefficient of viscosity is on the same order of magnitude as that for a fluid under normal conditions. ${ }^{11}$ This in turn suggests that viscous effects may be neglected. The neglect oi these effects will insure that the system of partial differential equations governing the flow will be a first-order system.

\section{B. The Rankine-Hugoniot Equations}

The Rankine-Hugoniot equations ${ }^{10,12,13}$ relate kinematic and thermodynamic variables between an initial state and a final staie of a material that has been shocked. Consider a planar shock traversing a material that is initially at rest. The shock wave will travel at a wave velocity, $U_{8}$, with respect to the unshocked 
material. In the shocked state, the material acquires a particle velocity, $u_{p}$. Across the sılock front conservation of mass, momentum, aid energy must hold. These conservation laws lead to the Rankine-Hugoniot equations. The Rankine-Hugoniot equations are

$$
\begin{gathered}
\rho_{o} U_{s}=\rho\left(U_{s}-u_{p}\right), \\
P-P_{o}=\rho_{o} U_{s} u_{p},
\end{gathered}
$$

and

$$
E-E_{o}=\frac{1}{2}\left(P+P_{o}\right)\left(\frac{1}{\rho_{o}}-\frac{1}{\rho}\right) .
$$

In these equations, $\rho$ is the density, $P$ is the pressure, and $E$ is the specific internal energy. The zero subscripted variables refer to the material in the initial or unshocked state, while the unscripted variables refer to the material in the shocked state. If strength effects are important such that the material can support a deviatoric stress, then the component of the total stress that results in a force normal to the shock front replaces the pressure in equations (2.2) and (2.3).

Experimentally it is found that many materials obey the following empirical relation between shock velocity and particle velocity $10,14,15$ :

$$
U_{s}=c_{o}+s u_{p}
$$

The parameters $c_{o}$ and $s$ are constants characteristic of the material being shocked. These parameters also depend on the initial conditions, but in general they are 
insensitive to slight changes of the initial conditions. When equation (2.4) is used in conjunction with the Rankine-Hugoniot equations, a rclatively simple expression can be written for the principal Hugoniot. The principal Hugoniot is the locus of all points in the $P$ - $\rho$ plane that can be reached by a single shock from the initial state $\Gamma_{1}, \rho_{0}$. With the use of equation (2.4), the expression for the pressure on the principal Hugoniot is

$$
l^{\prime} \quad l_{1}: \rho_{1} c_{0}^{2} \eta(1-s \eta)^{2},
$$

where

$$
\eta=1-\frac{\rho_{o}}{\rho}
$$

Similarly the particle velocity and specific internal energy can also be expressed in terms of the density:

$$
u_{p}=c_{0} \eta(1-s \eta)^{-1}
$$

and

$$
E=E_{0}+\frac{\eta P_{o}}{\rho_{o}}+\frac{1}{2} c_{o}^{2} \eta^{2}(1-s \eta)^{-2} .
$$

Frequently, the reference energy $E_{o}$ is chosen to be zero, and the inital pressure term $P_{0}$ is often ignored because it is anywhere from three to six orders of magnitude less than the pressures of interest in shock experiments. 


\section{C. Flow Behind the Shock Front}

After the shock front has passed a region of the material, this portion of the material is in a compressed state at high pressure or stress. Further flow occurs as the shocked region releases down to ambient pressure. This flow is always continuous provided the material is normal in the sense of the BetheWeyl conditions. ${ }^{16}$ There is the possibility of supporting a rarefaction shock if the material undergoes a marked phase change such that at a lower pressure the material can support a faster longitudinal or bulk sound speed than at a higher pressure; however, in this case the material is not normal in the sense of tire BetheWeyl conditions. If the material does not exhibit this behavior, the whole release process will be continuous. Discontinuities may exist in the spatial gradients of the flow variables, but the flow variables such as pressure, density, energy and particle velocity will be spatially continuous behind the shock front.

The derivation of the flow equations proceeds from the consideration of a control volume and the conservation of mass, momentum, and energy. In the absence of body forces and dissipative mechanisms, the flow equations are

$$
\begin{gathered}
\frac{\partial \rho}{\partial t}+\frac{\partial\left(\rho u_{i}\right)}{\partial x_{i}}=0 \\
\frac{\partial u_{i}}{\partial t}+u_{j} \frac{\partial u_{i}}{\partial x_{j}}+\frac{1}{\rho} \frac{\partial P}{\partial x_{i}}=0
\end{gathered}
$$

and

$$
\frac{\partial E}{\partial t}+u_{i} \frac{\partial E}{\partial x_{i}}+\frac{P}{\rho} \frac{\partial u_{i}}{\partial x_{i}}=0 .
$$


The indices :un from 1 to 3 , and the summation convention is used for repeated indices. The term $u_{3}$ is the material velocity in the $i^{\text {th }}$ direction. For a material that can support a deviatoric stress, equation (2.9) remains the same, but equations (2.10) and (2.11) become

$$
\frac{\partial u_{i}}{\partial t}+u_{j} \frac{\partial u_{i}}{\partial x_{j}}+\frac{1}{\rho} \frac{\partial \sigma_{j i}}{\partial x_{j}}=0
$$

and

$$
\frac{\partial E}{\partial t}+u_{i} \frac{\partial E}{\partial x_{i}}+\frac{\sigma_{i j}}{\rho} \frac{\partial u_{j}}{\partial x_{i}}=0
$$

The term $\sigma_{i j}$ is a component of the stress tensor. The sign convention has been chosen such that the sum of the principal stresses is positive in compression.

In the present investigation, the flow is essentially one-dimensional with no flow in the lateral directions. The stress tensor, when reduced to its principal values, will have one component that can be considered as a force per unit area normal to the shock front and two components that are tangential to the shock. From symmetry arguments, the tangential components must be equal. This greatly simplifies the flow equations. For plane flow in one dimension, the governing system of partial differential equations is

$$
\begin{gathered}
\frac{\partial \rho}{\partial t}+\frac{\partial(\rho u)}{\partial x}=0 \\
\frac{\partial u}{\partial t}+u \frac{\partial u}{\partial x}+\frac{1}{\rho} \frac{\partial P}{\partial x}=0,
\end{gathered}
$$


and

$$
\frac{\partial E}{\partial t}+u \frac{\partial E}{\partial I}+\frac{\mu}{\rho} \frac{\partial u}{\partial x}=0 .
$$

If the material can support a stress, then $P$ must be replaced in equations (2.15) and (2.16) by $\sigma_{n}$, the normal component of the stress tersor.

Equations (2.14)-(2.16) form a system of partial differential equations that are functions of iwo independent variables, $x$ and $t$. Once an equation of siate has been specified then finite differencing techniques can be applied to this system of equations to numerically solve for configurations later in time. A slightly different approach is to apply the method of characteristics ${ }^{17,18}$ to equations (2.14) (2.16). The method of characteristics essentially transforms the system from three partial differential equations to a system of six first-order, ordinary differential equations.

In the method of characteristics, sol. . to the flow are evolved along certain characteristc directions in the $x-t$ plane. For equations (2.14)-(2.16), three characteristic directions are defined and they are

$$
\begin{gathered}
\frac{d x}{d t}=u, \\
\frac{d x}{d t}=u+c,
\end{gathered}
$$

and

$$
\frac{d x}{d t}=u-c
$$


Along each characteristic direction, a compatibility equation holds that specifies the manuer in which the dependent variables must change. For the three characteristic directions that have been defined, the compatiblity equations are, respectively,

$$
\begin{gathered}
d E=\frac{P}{\rho^{2}} d \rho, \\
d P--\rho c d u,
\end{gathered}
$$

and

$$
d P=\rho c d u
$$

The quantity $c$ in these equations can be identified as a bulk sound speed, and is defined by the following equation:

$$
c^{2}=\left(\frac{\partial P}{\partial \rho}\right)_{E}+\frac{P}{\rho^{2}}\left(\frac{\partial P}{\partial E}\right)_{\rho} .
$$

Initial conditions or boundary conditions must be specified along a line in the $x-t$ plane which is nowhere tangent to a characteristic direction in the domain of the problem. Numerical differencing techniques can then be applied to the system of equations (2.17)-(2.22).

If strength effects are to be modeled, whe method of characteristics can still be used. The characteristic directions remain the same, but the compatibilty equations are changed. Modeling of strength effects are explained in more detail 
in section $2 \mathrm{~F}$. In appendix $\mathrm{A}$, two computer programs using the method of characteristics to solve for the flow behind the shock front, as well as the trajectory of the shock front, are described.

There are many existing hyrodynamic codes that have been written for the problem of compressible fluid flow in one dimension. By and large the majority of such hydrodynamic codes use finite differencing techniques applied to the Lagrangian form ${ }^{19}$ of equations $(2.14)-(2.16)$. Generally speaking, these codes use artificial viscosity techniques to foliow a shock front. Artificial viscosity techniques do not solve the Rankine-Hugonict equations in order to follow a shock. By using the method of characteristics, the Rankine-Hugoniot equations are solved exactly to relate the conditions at the shock front to the continuous flow immediately behind the shock front. It was for this reason that the computer programs described in appendix A were developed.

\section{D. Equations of State}

In any quantitative modeling of shock wave attenuation, it will be necessary to have an equation of state giving the pressure as a function of the specific internal energy and density. The equation of state is important because wave speeds in the rarefaction region are determined by it. The basis on which an equation of state 
may be constructed, as well as sonte specific equations of state, will be discussed in this section.

Kunwledge of the qualitative nature of the structure of metals forms a basis from which an equation of state may be constructed. A simple approach is to consider the metal as a crystal lattice with the valence electrons forming an electron gas. In this approach, the interaction between lattice points and the free electrons is not explicitly taken in to account. The total sperific internal energy can then be written as the sum of three terms ${ }^{20}$ :

$$
E=E_{\mathrm{c}}+E_{\mathrm{t}}+E_{e}
$$

The first term, $F_{i:}$, arises from the potential of the crystal and is assumed to be a function of specific volume or density only. The zero-point vibrational energy of the lattice may be included in this first term, as well as any other contribution to the internal energy which can be assumed to be a function of volume only. The second term, $E_{t}$, is the contribution to the specific internal energy from thermal vibrations of the lattice. The last term, $E_{e}$, is the thermal contribution of the electron gas to the specific internal energy.

The assumption that the total internal energy can be written as the sum of three terms leads to a similar equation for the pressure. From thermodynamic theory, the contribution to the total pressure from the first term in equation (2.24) 
is

$$
I_{c}-\frac{d E_{c}}{d v}
$$

In this equation $v$ is the specific volume, and the Lnta derivative of $E_{6}$, is taken since $E_{c}$ is assumed to be a funtion of specitic volume only. Eyuation (2.25) is frequently called the cold compression curve since this is the pressure the anderial would exhibit along a zero-degree isotherm. If the temperature is not too great, then the electronic term in equation (2.24) may be neglected; but even if it is not neglected, the total pressure may still be written as

$$
P=P_{\varepsilon}(v)+P_{\mathrm{t}}(v, T)
$$

In equation (2.26) $T$ is the absolute temperature, and $F_{\llcorner}$represents all the thermal contributions to the pressure.

An interesting form for the equation of state can be derived by taking the partial derivative of equation (2.26) with respect to the temperature holding tne specific volume constant:

$$
\left(\frac{\partial P}{\partial T}\right)_{v}=\left(\frac{\partial P_{t}}{\partial T}\right)_{v}
$$

The left-hand side of equation (2.27) can be rewritten using the thermodynamic definition of the Gruneisen parameter and the definition of the specific heat at constant volume. The thermodynamic definition of the Gruneisen parameter ${ }^{2}$ is

$$
\gamma=\frac{1}{\rho}\left(\frac{\partial P}{\partial E}\right)_{\nu} .
$$


The defining equation for the specific heat at constant volume is

$$
c_{v}=T\left(\frac{\partial S}{\partial T}\right)_{v}=\left(\frac{\partial E}{\partial T}\right)_{v}
$$

where $S$ is the specific entropy. Use of these equations suggests that the equation for the total pressure may be written in the following manner:

$$
P=P_{c}-\int \gamma \rho c_{v} d T
$$

If the Gruneisen parameter is assumed to be independent of temperature, then $\gamma \rho$ can be taken outside the integral in equation (2.30), and the equation becomes

$$
P=P_{\mathrm{c}}+\gamma \rho \int c_{v} d T
$$

Now the integral in equation (2.31) is nothing more than the difference between the total specific internal energy and the energy on the cold compression curve. Replacing the integral by this difference, the equation of state becomes

$$
P=P_{c}+\gamma \rho\left(E-E_{c}\right)
$$

Equation (2.32) is the form for the Mie-Gruneisen equation of state. ${ }^{10,13,21}$ To arrive at equation (2.32) it was necessary to assume that $\gamma$ was independent of temperature. For a metal this implies that electronic effects are neglected, and that the metal remains in a single phase.

If $E$ is known for a locus of states in the $P-p$ or $P-v$ plane, then equation (2.32) leads to a first-order differential equation for $E_{c}$. One such locus of states 
that is known is the principal Hugonius. Ii the material obeys a linear $U_{s}-u_{p}$ relation, then equation (2.5) gives the pressure on the principal Ilugoniot and equation (2.8) gives the specific internal enerey. Assuming that $F_{n}$ and $P_{n}$, can be neglected, the differential equation for $E_{\text {, is }}$

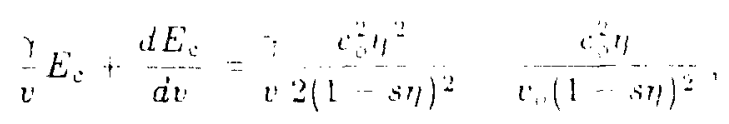

$v$ : vre $n$ is given by equation $(2,6)$. The functional ifpendence of $\gamma$ on the specitic volume must still be known before equation (2.33) an be integrated to find $E_{1}$; however, it is not necessary to find $f_{i}$ to arrive at an equation of state. I'sing equation (2.33), equation (2.32) can b= written as

$$
P \quad{ }_{v}^{\gamma} E+\frac{c^{2} \eta}{v_{v}(1-s \eta)^{2}}-\frac{\gamma}{2 v(1-s \eta)^{2}} .
$$

The functional dependence of $y$ on $v$ or $\rho$ is stıll not specified in equation (2.34). The derivation of equation (2.34) is based on the following assumptions: the material obeys a linear $U_{s}-u_{p}$ relation given by equation (2.4), electronic effects may be neglected, and the Gruneisen parameter depends only upon density or specific volume and is thus independent of temperature or specific internal encrgy.

A slightly different way of deriving equation (2.32) uses a more explicit treatment of a crystal as an assembly of harmonic oscillators. The Helmholtz free energy for an assembly of harmonic oscillators ${ }^{22,23}$ is given by

$$
A=A_{o}+\frac{k T}{m} \sum_{i} \ln \left(1-\exp \left(-\frac{h \nu_{i}}{k T}\right)\right) .
$$


In this equation, $k$ is Botlzmann's constant, $h$ is Planck's constant, $m$ is the mass, and $\nu_{i}$ is the frequency for the $i^{\text {th }}$ norma! mode of vibration. The term $A_{0}$ is dependent on the specific volume only and is identical to the term $E_{E}$ in equation (2.24). The sum is over all normal modes. The pressure is given by

$$
P=-\left(\frac{\partial A}{\partial v}\right)_{T}=-\frac{d A_{0}}{d v}-\frac{1}{m v} \sum_{i} \frac{h \nu_{i}}{\exp \left(\frac{h \nu_{i}}{k T}\right)-1} \frac{v}{\nu_{i}}\left(\frac{\partial \nu_{i}}{\partial v}\right)_{T}
$$

When the frequencies are independent of temperature, the specific internal energy is

$$
E=A_{o}+\frac{1}{m} \sum_{i} \frac{h \nu_{i}}{\exp \left(\frac{h \nu_{i}}{k T}\right)-1}
$$

Now make the assumption that all frequency changes vary in the same marner, and define the Gruneisen parameter by

$$
\gamma=-\frac{v}{\nu_{i}} \frac{d \nu_{i}}{d v}
$$

Using equations (2.37) and (2.38), equation (2.36) may be written as

$$
P=-\frac{d A_{\circ}}{d v}+\frac{\gamma}{v}\left(E-A_{\circ}\right)
$$

This equation is in the same form as equation (2.32).

In deriving equation (2.32), one assumed that the Gruneisen parameter was independent of temperature. In deriving equation (2.39), one assumed that the normal frequencies were independent of temperature. A second assumption, that 
the normal frequencies all varied in exactly the same manner with respect to changes in density or specific volume, was also needed. The thermodynamic definition of the Gruneisen paraneter, erfualion (2.25), and the delinition given by equation (2.38) are identical under these assumptions.

An interesting point to consider is the definition of the Gruneisen parameter given by equation (2.38) and the maximium frequency postulated in Debye theory. There are many different expressions relating the maximum or cutoff frequency in Debye theory to the Gruneisen parameter and to bulk properties. ${ }^{24}$ Some of these theories are discussed in greater detail in appendix D. A relatively simple formula for the Gruneisen parameter can be found when it is assumed that the normal frequencies of a material are only linearly dependent on the density. To arrive at this formula replace the specific volume by the density in equation (2.38). Then take the derivatives of both sides with respect to the density. Assuming that the second derivative of the frequencies with respect to the density is zero, leads to the following differential equation for the Gruneisen parameter:

$$
\frac{d(\gamma \rho)}{d \rho}+\left(\frac{\gamma \rho}{\rho}\right)^{2}=0 .
$$

The solution to equation $(2.40)$ is

$$
\gamma \rho=\frac{\gamma_{0} \rho_{o}}{\left(1+\gamma_{o} \eta\right)}
$$


This specific form for the Gruneisen parameter may now be substituted into equation (2.34) to give the following for the equation of state:

$$
P=\frac{\gamma_{0} \rho_{0}}{1+\gamma_{0} \eta}\left[E-\frac{\left(c_{0} \eta\right)^{2}}{2(1-s \eta)^{2}}\right]+\frac{\rho_{0} c_{0}^{2} \eta}{(1-s \eta)^{2}}
$$

When equation (2.42) is used for the equation of state, the sound speed is independent of the specific internal energy. Equation (2.42) was developed and used by Rice ${ }^{25}$ in his analysis of some Hugoniot data for $\mathrm{NaCl}$ that he had taker Equation (2.42) will be referred to as the Rice equation of state.

W' hen the compression of the material is not too great, the functional dependence of the Gruneisen parameter on the density as given by equation (2.40) may be approximated by the equation

$$
\gamma=\frac{\gamma_{0} \rho_{0}}{\rho}
$$

It turns out that equation $(2.43)$ is a reasonable representation of the functional dependence of the Gruneisen parameter on the density as determined from experiments on porous alumirum. ${ }^{26}$ Equation (2.43) is frequently used in high-pressure research. Another aspect of equation (2.43) is that it simplifies the equation of state. The term that is linear in the internal energy no longer involves the density. With equation (2.43) inserted into equation (2.34), the equation of state becomes

$$
P=\gamma_{o} \rho_{o} E+\rho_{o} c_{o}^{2} \eta \frac{1-\frac{1}{2} \gamma_{o} \eta}{(1-s \eta)^{2}}
$$


Equations (2.42) and (2.44) are two equations of state that are used in examining the present experiments. Both these equations of state are based on a linear $U_{3}-u_{p}$ relation and reference the principal Hugoniot. The analytical simplicity of these equations makes them particularly useful in hydrodynamic computations. The deficiencies in these equations of state are fairly obvious. They do not model phase changes, and the Gruneisen parameter as defined in equation (2.28) is assumed to be a function of density or specific volume only. More complicated equations of state can be used. In additon to equations (2.42) and (2.44) two other equations of state are used to model the experiments. They are the Tillotson equation of state and the Gray equation of state.

The Tillotson equation of state ${ }^{27}$ was constructed to be a general analytical equation of state valid for a wide pressure range from 0 to a maximum pressure of approximately $10^{5} \mathrm{GPa}$. It is thought that approximately $10^{3} \mathrm{GPa}$ is the minimum pressure where the Thomas-Fermi or Thomas-Fermi-Dirac equation of state 28,29 is applicable; thus the pressure range of $10^{3}$ to $10^{5} \mathrm{GPa}$ for the Tillotson equation of state overlaps a region where the Thomas-Fermi equation of state is believed to be valid. Tillotson constructed his equation of state to extrapolate smoothly from the pressure region where Hugoniot data has been taken to the Thomas-Fermi region.

The form for the Tillotson equation of state is very similar to the Mie- 
Gruneisen form; specifically, the form is

$$
P=\gamma \rho E+f(\rho)
$$

The major difference between the Tillotson and the Mie-Gruneisen equation of state is that the parameter $\gamma$ in! equation (2.45) is now a function of both density and the specific internal energy. In the Tillotson equation of state, the parameter $y$ is given by the equation

$$
\gamma=\alpha+\frac{\left(\gamma_{\circ}-\alpha\right) B}{E(1-\eta)^{2}+B} .
$$

In this equation $B$ is an adjustable parameter, $E$ is the specific internal energy, and $\gamma_{0}$ is the Gruneisen parameter in the ambient state. In the limit of high internal energies, equation (2.46) gives a limiting value of $\alpha$ for $\gamma$. For the Tillotson equation of state, the value of $\alpha$ for most materials is one-half.

The last equation of state used in the modeling of these experiments is the the Gray eos ${ }^{30,31}$ (equation of state). The Gray eos is a multiphase equation of state that takes into account solid, liquid, and gas phases of a material. The contribution of an electron gas in a metal to the specific internal energy is also taken into account. The Gray eos proceeds from the assumption that a suitable equation of state can always be written in the form

$$
P=P_{c}+\frac{\Gamma}{v}\left(E-E_{c}\right)
$$


where the terms $P_{\mathfrak{c}}$ and $E_{\mathrm{c}}$ refer to quantities on the zero-degree isotherm. At this point the Gray equation of state is quite similar to the Mie-Gruneisen form (equation (2.32)). The difference between the two equations is that $\Gamma$ in equation (2.47) is dependent on the internal energy or temperature as well as the density, while the parameter $y$ in equation $(2.32)$ is assumed to be a function of density only.

In order to determine the behavior of the cold curve for the Gray equation of state, equation (2.47) is abandoned and equation (2.32) is used to relate the principal Hugoniot to the cold curve; that is $P_{H}$, the pressure on the principal Hugoniot, and $E_{H}$, the specific internal energy on the principal Hugoniot, replace $P$ and $E$ in equation (2.32). For a material that follows a linear $U_{s}-u_{p}$ relation, equation (2.33) is the differential equation for $E_{c}$. To solve for $E_{c}$ it is assumed in the Gray eos that $y$ has a linear dependence on $\eta$ :

$$
\gamma=\gamma_{o}-a \eta
$$

The parameters $\gamma_{o}$ and $a$ are material constants. Instead of using an exact soluiion to equation (2.33), the Gray eos uses an approximate solution for $E_{c}$ whose principal term is

$$
E_{c}=\frac{c_{o}^{2} \eta^{2}}{2(1-s \eta)}\left[1+\frac{s \eta}{3}+\frac{s^{2} \eta^{2}}{6}\left(1-\frac{\gamma_{o}}{s}\right)\right]
$$

It is apparent there is a slight inconsistency in the derivation of the Gray equation 
of state. Temperature effects are included in writing equation (2.47) but then are ignored in deriving equation (2.49).

Referencing the principal Hugoniot to the cold curve by use of equations (2.32) and (2.48) enables one to rewrite equation (2.47) in the following manner:

$$
\begin{gathered}
P=P_{H}\left(1-\frac{1}{2}\left(\gamma_{c}-a \eta\right) 1-\frac{\eta}{\eta}\right)+\rho E\left(\gamma_{0}-a \eta\right) \\
+\rho\left(l-\eta_{H}-a \eta\right)\left(E-F_{c}\right) .
\end{gathered}
$$

When the equation of state is written in this form, it is clear that the first two terms of equation (2.50) represent a Mie-Gruneisen form for the equation of state in which the Gruneisen paramster is given by equation (2.48). The nonlinear effects of the specific internal energy or temperature are included in the third term and this term may be viewed as a correction term to a Mie-Gruneisen equation of state.

In the Gray equation of state it is necessary to calculate a temperature in order to calculate the correction term in equation (2.50). The temperature is calculated from the specific internal energy. For a material in the solid phase in this eos, the relation of the temperature to the specific internal energy is given by

$$
E=E_{c}+c_{\nu} T+\frac{1}{2} g T^{2}
$$

The second term, $c_{\imath} T$, in equation (2.51) corresponds to $E_{t}$ in equation (2.24) and the third term of equation (2.51) to $E_{e}$ in equation (2.24). The specific heat 
at constant volume, $c_{v}$, refers to the lattice only and is assumed to be constant at the classica! Dulong and Petit value as long as the material remains in the solid phase. The term, $\frac{1}{2} g T$, is the electronic specific heat. Equations similar to equation (2.51) which take into account the latent heat of phase tranformations are used when the material is in a liquid or dense gas phase to calculate the temperature. The correction term of the Gray eos for a material in the solid phase can be found from the equation

$$
\left(\Gamma-\gamma_{o}+a \eta\right)\left(E-E_{c}\right)=\frac{1}{2} g T^{2}\left(\gamma_{e}-\gamma_{0}+a \eta\right) .
$$

The parameter $\gamma_{e}$ is an electronic Gruneisen parameter and is usually assumed to be equal to two-thirds for most metals in the Gray eos. Equation (2.52) completes the specification of the Gray eos for a material in the solid phase.

\section{E. The Release Wave and the Gruneisen Parameter}

For one-dimensional planar motion, an interesting relation can be derived between the slope of the principal Hugoniot, the bulk or longitudinal wave speed of the head of the rarefaction fan, and the Gruneisen parameter. This relation is based on the assumption that the initial part of the release wave is an isentropic process. If strength effects are present, this assumption implies that the initial release is an elastic process. For simplification, strength effects will be ignored. 
From thermodynamics, one has that changes in the specific internal energy are governed by the equation

$$
\text { di. T.LS }+\frac{\Gamma}{\rho^{2}} d \rho
$$

where sis rhe specific entropy. Similarly, changes in internal energy along the principal Hugoniot are given by

$$
\text { dL } \quad{ }_{2 \rho}^{n} d l^{\prime}+\frac{P}{2 \rho^{2}} d \rho
$$

Substituing the right-hand side of equation (2.53) for $d E$ in equation (2.54) leads to the equation

$$
T d S+\frac{P}{\rho^{2}} d \rho=\frac{\eta}{2 \rho_{\rho}} d P+\frac{P}{2 \rho^{2}} d \rho
$$

If the specific entropy is expressed in terms of $P$ and $\rho$, then

$$
T d S=\frac{1}{\gamma \rho} d P+T\left(\frac{\partial S}{\partial \rho}\right)_{P} d \rho
$$

but

$$
T\left(\frac{\partial S}{\partial \rho}\right)_{P}=-\frac{1}{\gamma \rho}\left(\frac{\partial P}{\partial \rho}\right)_{S}=-\frac{c^{2}}{\gamma \rho}
$$

The thermodynamic definition of the Gruneisen parameter (equation (2.28)) was used in deriving equations (2.56) ard (2.57). With the use of equations (2.56) and (2.57), equation $(2.55)$ can be written as

$$
\frac{1}{\gamma \rho} d P-\frac{c^{2}}{\gamma \rho} d \rho=\frac{\eta}{2 \rho_{o}} d P+\frac{P}{2 \rho^{2}} d \rho .
$$


Equation (2.58) holds along the principal Hugoniot in the $P-\rho$ plane. Dividing by $d \rho$ and rearranging terms, equation (2.58) becomes ${ }^{32}$

$$
c^{2}=\left(1-\frac{\eta \gamma \rho}{2 \rho_{0}}\right)\left(\frac{\partial P}{\partial \rho}\right)_{H}+\frac{D_{H}}{2 \rho^{2}} \gamma \rho
$$

In equation (2.59), the subscript $U$ refers to quantities on the principal Hugoniot or to derivatives taken along the Hugoniot. Equation (2.59) gives the square of the bulk sound speed along the principal Hugoniot; or, phrased in another manner, the slope of any isentrope intersecting the principal Hugoniot is given by equation (2.59).

A more illustrative representation of equation $(2.59)$ is

$$
\left(\frac{\partial P}{\partial \rho}\right)_{S}=\left(\frac{\partial P}{\partial \rho}\right)_{H}-\frac{\gamma \rho}{2}\left[\left(\frac{\partial P}{\partial \rho}\right)_{H} \frac{\eta}{\rho_{o}}-\frac{P_{H}}{\rho^{2}}\right]
$$

Since the Gruneisen parameter is a positive quantity, the term inside the brackets in equation (2.60) determines whether the slope of the Hugoniot is greater or less than the slope of the isentrope in the $P$ - $\rho$ plane. To $d$-termine the sign of the quantity in the brackets, consider the intersection point between the principal Hugoniot and an isentrope. For any increase in density, the change in specific internal energy along the principal Hugoniot must be given by equation (2.53). Along the isentrope the change in specific energy is also given by equation (2.53) but the specific entropy term is identically zero. The change in specific internal 
energy along the principal Hugoniot must also be given by equation (2.54). This inplies the following must hold:

$$
\frac{\eta}{2 \rho_{0}} d P+\frac{P}{2 \rho^{2}} d \rho \geq \frac{P}{\rho^{2}} d \rho
$$

or

$$
\frac{\eta}{\rho_{o}}\left(\frac{\partial P}{\partial \rho}\right)_{H} \geq \frac{P_{H}}{\rho^{2}}
$$

From this it an be seen that the slope of the principal Hugoniot is greater than the slope of the intersecting isentrope. The equality sign in equation (2.61) holds at the foot of the Hugoniot, and thus the isentrope that goes through the point $\rho_{\circ}$ will have the aame slope as the principal Hugoniot at this point. The influence of the Gruneisen parameter on the speed of the head of any release wave is readily apparent in equation $(2.60)$. For a given shock state on the principal Hugoniot the larger the Gruneisen parameter is, the smaller the sound speed becomes.

It is interesting to apply equation (2.59) to a material which has a linear $U_{a^{-}}$ $u_{p}$ relation. For such a material the pressure on the principal Hugoniot is given by equation (2.5). The derivative of the pressure with respect to the density along the Hugoniot is

$$
\left(\frac{\partial P}{\partial \rho}\right)_{H}=\left(\frac{\rho_{o} c_{o}}{\rho}\right)^{2} \frac{1+s \eta}{(1-s \eta)^{3}}
$$

This leads to the following equation for the sound speed along the Hugoniot:

$$
c^{2}=\left(\frac{\partial P}{\partial \rho}\right)_{S}=\left(\frac{\rho_{o} c_{o}}{\rho}\right)^{2} \frac{\left(1+s \eta-s \eta^{2} \gamma \rho / \rho_{o}\right)}{(1-s \eta)^{3}} .
$$


No special assumptions had to be made concering the Gruneisen parameter in deriving equation (2.64). It is interesting to compare this equation with the bulk sound speed predictel by a Mie-Gruneisen equation of state. For this equation of state the Gruneisen parameter is assumed to be independent of temperature or specific internal energy. Using equation (2.23) to calculate the sound speed from equation (2.34), one has that the slope of the isentrope is given by

$$
\begin{aligned}
c^{2} & =\left(\frac{\partial F}{\partial \rho}\right)_{S}=\left(\frac{\rho_{o} c_{0}}{\rho}\right)^{2} \frac{1+s \eta-s \eta^{2} \gamma \rho / \rho_{0}}{(1-s \eta)^{3}} \\
& +\left[E-\frac{c_{o}^{2} \eta^{2}}{2(1-s \eta)^{2}}\right]\left[\frac{d(\gamma \rho)}{d \rho}+\left(\frac{\gamma \rho}{\rho}\right)^{2}\right] .
\end{aligned}
$$

The first term on the right-hand side of equation (2.65) is identical to equation (2.63). As long as

$$
\frac{d(\gamma \rho)}{d \rho}+\left(\frac{\gamma \rho}{\rho}\right)^{2}>0
$$

then the effect of shock heating is to increase the sound speed on the release isentrope for a given density. If $\gamma$ is given by equation (2.41), then the slope on a release isentrope is independent of shock heating.

\section{F. Strength Effects}

In the previous sections the problem of shock compression and subsequent release to ambient pressure has been approached with the explicit assumption 
that $a$ hydrolynamic description is adequate. This can only be justified aposteriori. If a hydrodynamic description is not appropriate, then the effects of material rigidity or strength must be taken into account. This opens a virtual Pandora's box of possible constitutive equations and maierial response functions. $\dagger$ These ditficulties are further complicated by material symmetry. To eliminate this last complication, the discussion will be restricted to the idealized case of isotropic materials. A natural division of material response functions are those materials which are viscid (stresses are rate-dependent) and those that are inviscid (stresses are rate-independent). When deaiing with a viscid material, the set of equations governing any flow process is a system of second-order partial differential equations. Flow for an inviscid material is described by a system of first-order partial differential equations. The assumptions that a material is isotropic and inviscid will limit the scope of possible constitutive equations but a great variety of material models is still possible. As in the hydrodynamic case, the assumption that a material is isotropic and inviscid can only be justified aposteriori. The first order of business, in view of the complexities involved, is to develop a clear picture of the simıpler isotropic and rate independent materials.

† The nomenclature (stress, strain, plasticity, viscid materials, etc.) that is used in this section as well as the scope of possible constitutive relations and material response functions can be found in books or articles on continuum mechanics. Some good sources are listed in references 33-36. 
In dealing with strength, the obvious first step is to write the flow equations using the stress tensor instead of the pressure as was done in section 20 . Materiat isutropy was assumed in the argunent in that section concerning the normal and tangential components of the stress tensor for one-dimensional flow. It must be emphasized that the primary aifference between the hydrodynamic system and the system in which strength is important is the introduction of non-zero deviatoric stresses. Alt lough a stress lensor must now be used, the concept of pressure can still be retained by defining a pressure in terms of the first invariant of the stress tensor as

$$
P=\frac{1}{3} I_{\sigma}=\frac{1}{3}\left(\sigma_{n}+2 \sigma_{t}\right),
$$

where $I_{\sigma}$ is the first invariant of the stress tensor. For the presen $i$ case of onedimensional flow, $\sigma_{n}$ is the component parallel to the flow and $\sigma_{t}$ is the component perpendicular to the flow. The sign convention for the stress tensor is the same as that in section $2 \mathrm{C}$. With the use of equation (2.67), the deviatoric stresses can be defined as

$$
\tau_{n}=\sigma_{n}-P
$$

and

$$
r_{t}=\sigma_{t} \cdot P \text {. }
$$

From these definitions it is seen that the following equation holds for the deviatoric 
stresses:

$$
\tau_{n}+2 \tau_{t}=0
$$

With the deviatoric stresses given by equations (2.68) and (2.69), the set of partial differential equations governing the flow behind the shock front can be written as

$$
\begin{gathered}
\frac{\partial \rho}{\partial t}+\frac{\partial(\rho u)}{\partial x}=0 \\
\frac{\partial u}{\partial t}+u \frac{\partial u}{\partial x}+\frac{1}{\rho} \frac{\partial\left(P+\tau_{n}\right)}{\partial x}=0
\end{gathered}
$$

and

$$
\frac{\partial E}{\partial t}+u \frac{\partial E}{\partial x}+\frac{P+\tau_{n}}{\rho} \frac{\partial u}{\partial x}=0
$$

It is now assumed that the pressure defined in equaiion (2.67) and used in equations (2.72) and (2.73) is given by an equation of state similar to one of those in section $2 \mathrm{D}$. Once a constitutive equation is specified for the deviatoric stress $\tau_{n}$, the system of partial differential equations governing the flow can be solved. The remainder of this section will be devoted to the description of a suitable model for the deviatoric stress.

The mechanical behavior of solids and metals in particular can be qualitatively classified in one of two broad categories, elastic behavior and plastic behavior. ${ }^{37}$ The simplest descriptions of each phenomenom are fundamentally different, but each can be described by rather simple examples. A perfectly elastic material is deformed according to Hooke's law when a force or stress is applied 
to the material. When the force or stress is relieved, the material returns to its original shape. For a material that is perfectly plastic there is no deformation until the stress field reaches a certain magnitude. The material then flows undergoing a deformation. When the stress is relieved from a plastic material, the flow ceases and the material retains a permanent deformation. All metals exhibit to some extent the features of both elasticity and plasticity. The constitutive equation for $\tau_{n}$ should reflect some type of elastic-plastic behavior.

Elasticity and plasticity have been studied from the point of view of solid-state physics and from a phenomenological viewpoint. Historically, the phenomenological approach is the oldest of the two approaches and the more pervasive in the formulation of elastic-plastic models. An important factor in the phenomenological description of an elastic-plastic material is the concept of a yield strength or yield surface. Examples of a yield surface or yield criteria are the Tresca and the von Mises yield conditions. ${ }^{38}$ A yield condition is the mathematical expression, written in terms of the stress tensor, of the experimental observation that a material can only support a certain maximum stress field before it flows plastically or fails catastrophically. The Tresca yield condition is written in terms of the principal stress components of the stress tensor as

$$
\sigma_{1}-\sigma_{3}=Y
$$

where $\sigma_{1}$ is the maximum vaiue of the principal stress components and $\sigma_{3}$ is the 
minimum value. The von Mises yield condition is written in terms of the stress deviators as

$$
\frac{3}{2} \tau_{i j} \tau_{i j}=Y^{2}
$$

In equations (2.74) and (2.75) the parameter, $Y$, is the yield strength in simple tension. For a problem in which deformation only occurs in one dimension, these yield conditions both give

$$
\left|\tau_{n}\right|=\frac{2}{3} Y
$$

Equations (2.74) and (2.75) represent the conditions that the stress tensor must meet when the material fails. These are limiting values of the stress tensor or the deviatoric stress tensor; thus, the deviatoric stress of the material will always be limited in absolute magnitude and equation (2.76) can be written as

$$
\left|\tau_{n}\right| \leq \frac{2}{3} Y
$$

In general, the yield conditions exp:essed in equations (2.74) and (2.75) resulted from experimental studies performed at much lower static pressures than the peak pressures in these experiments. Even though this is true, it will be assumed that equation (2.77) can be extrapolated to high pressure regions as long as the material remains in the solid phase. The yield strength may be considered tu be a function of thermodynamic variables such as density or strain, internal energy or temperature, or it may depend on the stress-strain history such as in 
work hardening. It may also depend on a dynamic property such as strain rate, but it is simplest to treat $Y$ in equation (2.77) as a constant.

The yield condition of equation (2.77) can be considered as the criterion for separating the material response into two regions. The first region occurs when the inequality sign holds in equation (2.77). For this region the material has some sort of elastic response. The problem is to incorporate some elastic model in with the bulk properties that are assumed to dominate. Depending on the sign of the velocity gradient and whether $\tau_{n}$ is at an upper or lower yield limit, the material may also respond elastically when the equality sign in equation (2.77) holds. When the material is in the plastic state, the equality sign in equation (2.77) holds.

A physical quantity that is used in the modeling of solids is the quantity called strain. Depending upon the circumstance and what one wants to model, there are many different definitions of strain. Fortunately, the case of deformation in one dimension makes the definition of strain relatively easy. The definition that is appropriate for this type of deformation is the natural or logarithmic strain. As in the case of the stress tensor, the stiain tensor will be resolved into one normal component and two tangential components. The tangential components will be equal from symmetry arguments. The normal component, $\varepsilon_{n}$, is given by the 
equation

$$
\varepsilon_{n}=\ln \left(\frac{\rho_{c}}{\rho}\right)
$$

and the tangential components by the equation

$$
\varepsilon_{t}=0
$$

One approach to the description of an elastic-plastic material is to asssume that each of the total strain components may be considered to be the sum of an elastic term and a plastic term. This implies the following equations:

$$
\varepsilon_{n}=e_{n}+\lambda_{n}=\ln \left(\frac{\rho_{o}}{\rho}\right)
$$

and

$$
\varepsilon_{t}=e_{t}+\lambda_{t}=0
$$

The quantity $e$ denotes the elastic strains and $\lambda$ denotes the plastic strains. It is also assumed that the plastic strains do not produce a volume change and ihis assumption results in the equations

$$
\lambda_{n}+2 \lambda_{t}=0
$$

and

$$
e_{n}+2 e_{t}=\ln \left(\frac{\rho_{o}}{\rho}\right)
$$

From these equations it is possible to define strain deviators. It is clear from equation (2.82) that the plastic strain deviator is identical to the plastic strain. 
This is not true for the elastic strain deviator. $\xi$, and the components of the elastic strain deviator are given by the equations

$$
\xi_{n}=e_{n}-\frac{1}{3} \ln \left(\frac{\rho_{o}}{\rho}\right)
$$

and

$$
\xi_{t}=e_{t}-\frac{1}{3} \ln \left(\frac{\rho_{o}}{\rho}\right)
$$

The deviators are needed since the constitutive equations for $\tau_{n}$ in simple elasticity theory are given in terms of the normal component of the elastic strain deviator.

The strength model may be summarized up to this point as dividing the response of a material to a deformation into two broad categories, an elastic response and a plastic one. These two types of material response are separated by a yield condition. For one-dimensional deformation, the constitutive equation for $\tau_{n}$ in the plastic region must satisfy the equality constraint of equation (2.77). When $\tau_{n}$ satisfies the inequality in equation (2.76), the material is responding elastically but equation (2.77) no longer provides a constitutive equation for $\tau_{n}$. Appealing to simple elasticity theory, ${ }^{39}$ a constitutive equation for $\tau_{n}$ in terms of the elastic strain deviator is

$$
T_{n}=-2 \mu \xi_{n} .
$$

Another constitutive equation that has been given for $\tau_{n}$ in terms of $\xi_{n}$ is, ${ }^{40}$

$$
\tau_{n}=-2 \mu \frac{\rho}{\rho_{o}} \xi_{n} .
$$


The minussign has been added to these equations to agree with the sign convention of section $2 \mathrm{C}$ that stress is positive in compression. The term $\mu$ is the shear or rigidity modulus and may be assumed to be a constant.

Equations (2.86) and (2.87) are constitutive equations from elasticity theory that have been applied to one-dimensional deformations. In particular they have been applied to small deformations and quasi-static problems. For the present problem, equations (2.86) and (2.87) will not be used in the forms given but will be generalized to give the deviatoric stress rates. This is similar to hypoelasticity. The geners.'izations for the deviatoric stress rate in the elastic region are

$$
\frac{d \tau_{n}}{d t}=-2 \mu \frac{d \xi_{n}}{d t}
$$

and

$$
\frac{d \tau_{n}}{d t}=-2 \mu \frac{\rho}{\rho_{o}} \frac{d \xi_{n}}{d t}
$$

Now equation (2.88) can be derived from equation (2.86) when the rigidity modulus is constant but equation (2.89) certainly cannot be directly derived from equation (2.87). When the material is flowing plastically, the deviatoric stress rate is

$$
\frac{d \tau_{r_{n}}}{d t}=0
$$

assuming that the yield strength is constant. Equations (2.88) and (2.89) may be written in terms of the density. Recognizing that the plastic strain rates are iden- 
tically zero in the elastic regime, equation (2.84) may be expressed as a function of the total strain and the plastic strain and then differentiated to arrive at

$$
\frac{d \xi_{n}}{d t}=-\frac{2}{3 \rho} \frac{d \rho}{d t}
$$

Equation (2.88) then becomes

$$
\frac{d i_{n}}{d t}=\frac{4}{3} \frac{\mu}{\rho} \frac{d \rho}{d t}
$$

and equation (2.89) may be rewritten as

$$
\frac{d \tau_{n}}{d t}=\frac{4}{3} \frac{\mu}{\rho_{0}} \frac{d \rho}{d t}
$$

Equations (2.77), (2.90), and (2.92) or (2.93), allow a complete description of $\tau_{n}$ in a region where the flow is continuous.

An interesting sidelight to these strength models is the prediction of a Hugoniot elastic limit. ${ }^{41}$ At the Hugoniot elastic limit the normal component of the deviatoric stress will satisfy equation (2.76) but the jump conditions across the shock can be found from the equations governing the flow of an elastic material. Considering the yield strength and the rigidity modulus to be constant and the initial state to be at normal density with zero stress, the equations across the shock front can be approximated as

$$
u_{\mathrm{p}}=U_{\mathrm{a}} \frac{w}{1+w},
$$




$$
\begin{gathered}
\rho-p_{u}(1+w), \\
E=\frac{1}{2} U_{s}^{2}\left(\frac{w}{1+w}\right)^{2},
\end{gathered}
$$

and

$$
P=\rho_{0} U_{g}^{2}\left(\frac{w}{1+w}\right)-\frac{2}{3} Y
$$

The parameter $w$ in equations (2.94) through (2.97) is given by,

$$
w 1=\frac{Y}{2 \mu} .
$$

A quick perusal of these equations reveals that the density at the Hugoniot elastic limit is not fixed by the shock speed but rather by the strength parameters which result from the constitutive equation (2.92) or (2.93). The normal stress at the Hugoniot elastic limit is given by

$$
\sigma_{n}=P+\frac{2}{3} Y
$$

To determine the stress at the Hugoniot elastic limit, the pressure in equation (2.97) must be known. Because equations (2.94)-(2.97) are not sufficient to solve for all the unknowns, the specification of the equation of state is the necessary fifth equation that allows the determination of all relevant quantities at the Hugoniot elastic limit.

The phenomenological strength models related in this section are mainly introduced to describe the effects that non-zero deviatoric stresses may have on 
wave propagation in a very high pressure regime. They are not intended to be detailed descriptions of either plastcicity or elasticity. The strength models are being added in a rather ad hoc fashion to the equations of state of section $2 \mathrm{D}$ in an attempt to furnish a more complete description of material behavior than is possible in a purely hydrodynamic model. The fact that the equations of state reference the principal Hugoniot and the principal Hugoniot is really a measurement of a normal stress component casts some doubt on the accuracy of the equations of state. However, it should be noted that most principal Hugoniot data have been taken at states well beyond the Hugoniot elastic limit and in these pressure states it is expected that the stress deviator should be just a small percentage of the total stress. This last statement is only true if it is assumed that the yield strength does not increase by orders of magnitude with increasing pressure. In any case, one would expect that bulk properties must dominate in determining the principal Hugoniot and the inclusion of strength models should not greatly alter the pressure calculation along the principal Hugoniot at these very high pressures.

The situation is much different when one considers the possible effects that a strength model has on the speed of a release wave. If the initial release from a state on the principal Hugoniot is elastic then the longitudinal sound speed, $c_{\ell}$, is given by the equation

$$
c_{\ell}^{2}=c_{\beta}^{2}+\frac{4}{3} \frac{\mu}{\rho}+\frac{\tau_{n}}{\rho^{2}}\left(\frac{\partial P}{\partial E}\right)_{\rho} .
$$


The term $c_{\beta}$ in equation $(2.100)$ is the bulk sound speed and is given by the righthand side of equation (2.23). Equation (2.100) is valid when the stress rate is giveth by equation (2.92). For the stress rate given by equation $(2.93), c_{\ell}$ is given by

$$
c_{P}^{\frac{2}{2}}=c_{Q}^{2}+\frac{4 \mu}{3 \rho_{0}}+\frac{T_{\mathrm{r}}}{\rho^{2}}\left(\frac{\partial P}{\partial E}\right) .
$$

The bulk sound speed increases as the density increases; thus, the difference between the longitutinal and hulk somnd speed will decrease when the rigidity modulus is treated as a constart. At zero or ambient pressure the bulk sound speed may be twenty to thirty percent smaller than the longitudinal sound speed, and although this difference decreases in these particular strengih models with increasing pressure, the ditference in the sound speeds is still appieciable.

A last remark must be made on the propagation of waves in the plastic region. In this region the stress rate is given by equation (2.90). This leads to the following equation for the sound speed in the plastic state:

$$
c_{\ell}^{2}=c_{\beta}^{2}+\frac{\tau_{n}}{\rho^{2}}\left(\frac{\partial P}{\partial E}\right)_{\rho} .
$$

Since the normal component of the deviatoric stress is limited in magnitude by equation (2.77) and the isentropic bulk modulus is usually very much greater than the yield strength, it is seen that wave speeds in the plastic region are nearly the same as wave speeds calculated in models that do not include strength effects. 


\section{THE EXPEKIMENTS}

\section{A. Experimental Method and Design}

There is more than one method by which one may measure the speed of a release wave in a materal, but the method chosen for these experiments was the "overtaking-relaxation" method. ${ }^{8}$ This method involves producing a strong shock wave of constant velocity in a target materia! and then introducing a rarefaction or release wave into the target that overtakes the shock and attenuates or "relaxes" :t. By observing the point at which the attenuation first occurs, one can determine the speed of the wave if the spatial and temporal origins of the wave are known.

The actual experimental technique entails accelerating a thin flyer plate to some velocity and letting it collide with a thicker target plate. If the velocity of the flyer plate is sufficiently high, a strong shock wave is produced that travels forward into the target plate and another strong shock wave is also produced that travels backward into the flyer plate. When the shock wave in the flyer plate reaches the back surface of the flyer, a release wave is created that travels forward through the flyer and into the target plate, where it eventually overtakes and then attenuates the shock wave in the target. A schematic of this process in a $t$ (time) versus $x$ (position) diagram is shown in figure 1 (appendix I). A flyer plate of thickness, $h$, traveling at velocity, $u_{o}$, impacts a target plate at $t=0, x=0$. This creaies the forward-traveling shock wave with velocity $U_{s}$ shown in the target plate. When 
the backward-traveling shock wave in the flyer plate reaches the back surface of the flyer plate, a release wave is created at $t=t_{1}, x=x_{1}$. The head or lead characteristic of the release wave is shown ovetaking the shock wave in the target plate at $t=t_{3}, I=x_{3}$. Experimentally, the quantities that were measured were the trajectory of the flyer plate just before it collided with the target plate and the trajectory of the shock wave in the target plate. It must be mentioned that figure 1 is for the idealized rase in which there are no strength effects in the high pressure state. Strength effects are often assumed to be negligible even when the material remains in the solid phase upon shock loading.

The trajectory of the flyer plate and the trajectory of the shock wave in the target plate were measured by means of electrical contactor pins. ${ }^{42}$ These pins are coaxial in nature with an inner conductor and an outer jacket or sleeve that serves as a ground. When the sleeve is shorted to the inner conductor, an electrical signal is produced. In the present experiments the inner conductor was set back approximately $0.025 \mathrm{~mm}$ with respect to the sleeve. To measure the trajectory of the flyer, holes were drilled through the target plate and the ends of the contactor pins protruded through these holes. A thin nickel cap covered the end of each pin to prevent pre-shorting from ionized gases or ions ejected from the surface of the flyer plate prior to mechanical contact of the flyer plate with the pins. To measure the shock trajectory in the target plate, flat-bottomed holes were drilled in the 
target and an uncapped pin was butted against the bottom of each hole.

Figure 2 is a layout drawing of the experiments. The explosive driver system in each experiment was comprised of a detonator, a P/i20 HE (High Explosive) lens, and a cylindrical slab of $\mathrm{HE}$. The slab of $\mathrm{HE}$ had a nominal dianeter of $304 \mathrm{~mm}$ and a nominal thickness if $50.8 \mathrm{~mm}$. The purpose of the $\mathrm{P} / 120 \mathrm{HE}$ lens was to initiate a planar detonation in the cylindrical slab of $\mathrm{HE}$. The detonation products from the slab of HE then drove the flyer plate across a 25.4-rnm gap where the flyer impacted the target plate. A machined ring of mild steel was used to holi the HE slab. This steel ring had an inside iip on which the HE slab rested. The lip wis $6.35-m m$ thick and provided a standoff between the slab and the flyer plate. The flyer plate also had a thin sheet $(\sim 0.38 \mathrm{~mm})$ of polyethylene attached to the back surface of the flyer plate. The purpose of the polyethylers sheet and the $6.35-\mathrm{mm}$ standofi was to reduce the peak shock pressure in the flyer plate and to provide a smoother acceleration of the flyer plate. The mild steel rirg was bolied to the flyer plate and to an aluminum standoff ring. The thickr ess of the aluminum standoff ring in all experiments was $25.4 \pm 0.014 \mathrm{~mm}$. This whole arrangement then bolted to an end plate in which the target plate fitted. By using these machin':d pieces, careful alignment of the HE, flyer plate, and target piate was achieved. The experiments were made such that the expolsive driving system, flyer plate, and the impact surface of the target plate were all in an evacuated 
region. The evacuation greatly reduced the amount of mass from gases or air between the flyer plate and target plate. Typical pressures in the canristers at time of firing were 500 millitorr.

'lo control the peak shock pressure in the target plate, the velocity of the flyer plate had to be controlled. The velocity of the liyer could be controlled by changing the amount of $\mathrm{HE}$, the type of $\mathrm{HE}$, the thickness of the flyer plate, or the length of run between the flyer and target. In the present experiments, it was decided that the velocity of the flyer plate could be most easily controlled by changing the type of high explosive used for the cylindrical HE slab. Three different types of $\mathrm{HE}$ were used in these experiments, and are listed in table 1 (appendix H). The explosives used were a TNT explosive, a RDX-based explosive, and a HMX-basrd explosive. ${ }^{43}$ The Comp B explosive listed in table 1 is the RDXbased explosive and PBX-9404 is the HMX-based explosive. Properties that can be used to characterize these explosives are the initial density and the detonation or Chapman-Jouget (CJ) pressure. ${ }^{44}$ The PBX-9404 has the greistest initial density and CJ pressure while the TNT has the lowest initial density and lowest CJ pressure of the three explosives used in these exp'srimerts. Before any experiment was conducted involving the measurement of shock attenuation in a target plate, a preliminary experiment was conducted to characterize the kinematics of the flyer plate for a given explosive ćriving system. 


\section{B. The Flying Plate Experiments}

The purpuse of the flyer experiments was threefold. First, the timing and velocity of the flyer plate for a particular explosive driving system had to be determined. Second, the planarity of the flyer plate and the shock wave produced by the collision of the flyer with the target plate had to be examined. Third, if the system were sufficiently planar, then a region free from edge perturbations had to be found.

A basic experimental problem using explosively driven flyer plates for onedimensional shock experiments is the lack of planarity of the system. Two perturbations are prevalent, tilt of the flying plate and bowing of the flying plate. The amount of tilt can be minimized by careful alignment of the system. The present experiments were designed to be axially symmeteric about a line drawn from the center of the detonator through the center of the flyer plate and target plate. The bowing of the flyer plate appears to be radially dependent with respect to this axis of symmetry. To check or these perturbations the data from each flying plate experiment were fit to the following equations:

$$
\begin{aligned}
& t=a_{\circ}+a_{x} x+a_{y} y+a_{z} z+a_{r} r \\
& i=a_{o}+a_{x} x+a_{y} y+a_{z} z+a_{r} r^{2} .
\end{aligned}
$$

If the radial dependence of the fit was found to be negligible, the data were then 
fit to the equation

$$
t=a_{o}+a_{x} x+a_{y} y+a_{z} z
$$

The coordinate system was chosen such that the $x$-axis coincided with the axis of symmetry; further, the impact surface of the flyer plate and target was chosen as the plane $x=0$. For this coordinate system, $r$ is defined by

$$
r^{2}=y^{2}+z^{2}
$$

In each flying plate experiment, the target or witness plate was made of 6061 aluminum. Although no attempt was made to measure shock wave attenuation in the aluminum witness plates, pins were placed in the witness plates to check on the shape of the shock wave produced by the collision of the flyer plate with the witness plate. At least three planes or levels of pins were used in each flying plate experiment. One group of pins was placed to monitor the shape of the flyer plate just before collision with the target plate. A second group was set flush with the impact surface (the plane $x=0$ ) and a third group was placed in the witness plate to monitor the shock wave. The level of pins in the impact surface served a ciual roie in each flyer experiment; first, this level was used in determining the shape and velocity of the flyer plate and then was used along with the last level of pins in deteriming the shape and velocity of the shock wave. When using the pirs in the $x=0$ plare in this manner, the assumption is implicitly made that the 
shock wave is created the instant the flyer plate makes contact with the witness or target plate.

By their very nature pin data are Eulerian measurements and not Lagrangian measurements, in that they provide a time record for a particular point in space and do not follow a material point in the flyer plate or witness plate. When pin data are fit to equations $(3.1),(3.2)$, or $(3.3)$, the pin measurements are being used in the most propitious manner. Specifically, the pin measurements are being used for interpolation to determine isochrones for the shock front or the front surface of the Hyer plate. Although the material velocity of any particular point in the surface of the flyer plate is not deterimined, a velocity for the surface can be found from the fitted data. The magnitude of a velocity in a direction normal to the surface for equation ( 31 1) iw given by the equation

$$
u=\left[a^{2}+\left(a_{y}+a_{r} y r^{-\frac{1}{2}}\right)^{2}+\left(a_{z}+a_{r} z r^{-\frac{1}{2}}\right)^{2}\right]^{-\frac{1}{2}} .
$$

For equations (3.2) and (3.3) the equations for velocity are, respectively,

$$
u=\left[a_{x}^{2}+\left(a_{y}+2 a_{r} y\right)^{2}+\left(a_{z}+2 a_{r} z\right)^{2}\right]^{-\frac{1}{2}},
$$

and

$$
u=\left[a_{x}^{2}+a_{y}^{2}+a_{z}^{2}\right]^{-\frac{1}{2}} .
$$

It was found that in all the present flying plate experiments that the parameter $a_{x}$ completely dominates all other parameters in equations (3.5)-(3.7) in determining the speed of the flyer plate. 
An interesting point to consider is the comparison of the shape of the shock front to the shape of the flyer plate. This comparison can be made by fitting the pin data from the $x=0$ plane independently of the pin data from the witness plate. The fitting equations should reflecc the fact that within a given level of pins, the $x$-values are nominally constant. A suitable set of such equations are

$$
\begin{aligned}
& t=a_{0}+a_{y} y+a_{z} z+a_{r} r \\
& t=a_{0}+a_{y} y+a_{z} z+a_{r} r^{2}
\end{aligned}
$$

and

$$
t=a_{c}+a_{y} y+a_{z} z
$$

If the only component of material velocity of the flyer plate is in the $x$ direction and is independent of material position in the flyer, then the shock front should show the same tilt and bowing as the flyer plate. The shapes of the fronts are compared in tables $2 b, 3 b$, and $4 b$.

The first flying plate experiment was RS-2301. The high explosive used to drive the flyer was TNT. A total of one hundred and fifty-six contactor pins were used in this experiment. Fifty-four capped pins were placed flush with the front surface of the target or witness plate. Another fifty-four uncapped pins were butted against the free surface of the witness plate. The aluminum witness plate had an average thickness of $\mathbf{1 5 . 2 2} \mathrm{mm}$. Of the remaining forty-eight pins, twentyfour capped pins were placed approximately 1-mm away from the impact surface 
and the other twenty-four pins were placed approxinately 2-mm away from the same surface. The pins were thus grouped into four planes, nominally at $x=-2$, $x=-1, x=0$, and $x=15.22 \mathrm{~mm}$. Within each plane the pins were further placed in circular arrays. These arrays were concentric about the $x$-axis. For the $x=0$ plane the innermost array had a radius of $30 \mathrm{~mm}$ and the array with the greatest radius had a radius of $95 \mathrm{~mm}$. For the plane at $x=-2 \mathrm{~mm}$ and the plane at $x=-1 \mathrm{~mm}$ the arrays extended from a radius of $50 \mathrm{~mm}$ to a radius of $95 \mathrm{~mm}$. The pins in the $15.22-\mathrm{mm}$ plane covered an area with an inner radius of $2 \mathrm{~mm}$ and an outer radius of $110 \mathrm{~mm}$. Three pins were equally spaced (angular separation of $120^{\circ}$ ) in the 110 -mm radius array. The next circular array was at $105 \mathrm{~mm}$ with three pins placed in this array. Unfortunately the signals from these pins failed to be recorded and no time data for pins with a radius greater than $100 \mathrm{~mm}$ were recorded.

For the second experiment in the series of fying plate experiments only three levels or planes of pins were used. For experiment RS-2307, the planes were $-1.5,0$, and $5 \mathrm{~mm}$. A total of one hundred and fourteen piris were used in this experiment. Thirty-eight pins were placed in each level. Within each plane the pins were positioned in concentric arrays about the $t r$-axis as was done in experiment RS-2301. In each plane the area covered by the pins was an annulus with an inner radius of $20 \mathrm{~mm}$ and an outer radius of $110 \mathrm{~mm}$. The pin array for 
the last flying plate experiment, RS-2310, in the series was the same as experiment RS-2307 except that the first level or plane of pins was nominally set at $x=-1.2$ mm.

In fitting the data to any of the equations such as $(3.1),(3.2)$, or $(3.3)$, it was assumed that positioning errors were negligible. The method of least squares was then used to determine the coefficients of the equations. This entailed minimizing $R$ with respect to the parameters $a_{\circ}, a_{x}$, etc. $R$ is defined by the equation

$$
R=\sum_{i=1}^{N}\left[t_{i}-f\left(x_{i}, y_{i}, z_{i}\right)\right]^{2}
$$

In equation (3.11), $N$ is the number of data points and $f$ can be any of the functions represented in equations (3.1)-(3.3) or (3.8)-(3.10). The standard deviation, $\sigma$, of the fit was then taken to be

$$
\sigma=\left(\frac{R}{N-N_{a}}\right)^{\frac{1}{2}}
$$

where $N_{\mathrm{a}}$ is the number of coefficients or parameters in the equation used for the fit.

The results from the flying plate experimenis are presented in tables 2,3 , and 4. Each table lists the values of the coefficients for that equation that best fit the data for that particular experiment. The error bars for earh parameter were calculated from the standard error matrix using a $90 \%$ confidence level. 
Experiment RS-2301 was fitted best by equation (3.2). Experiment RS-2307 was best fit by equation (3.3). For experiment RS-2310 it was found that the fitting equation needed both the linear and quadrartic term in the radius to give the best representation of the data. Figure 3 plots average times for each circular array in the $x=0$ plane against the radius for each of the three flying plate experiments.

\section{C. Shock Trajectory Data Analysis}

As stated in section $3 \mathrm{~A}$, the principal measurement in each shock-wave attenuation experiment was the trajectory of the shock wave in the target plate. The trajectory data consisted of a set of discrete points. The analyses of these data proceeded from certain assumptions concerning the state of the flyer plate and the nature of the shock wave introduced into the target plate. For the data reduction discussed in this section it was assumed that the flyer plate had uniform properties; that is, all material points in the flyer plate had the same particle velocity, density, and specific internal energy. This assumption then implied that a shock wav $=$ of constant velocity would be introduced into the target plate and would remain at a constant velocity until it was overtaken by the release wave. These assurpitions form the premise on which the data analysis of each shockwave experiment was based. Further discussion and quantitative refinement of these idealized assumptions are given in chapter 4. 
The placement of the shock pins in the target plate was made on the basis of the flyer plate experiment for that particular explosive driving system. In general each shock-wave attenuation experiment had from fifteen to twenty levels of shock pins. These levels were spaced appoximately 1 -mm apart in the $x$ direction. Every experiment had from four to six shock pins in each level. Further details about the positions of the shock pins for each experiment are given in appendix G.

The main difficulty in analyzing these shock wave experiments is determining the point at which the attenuation of the shock wave first begins. An example of shock trajectory data is plotted in figure 4. The measured arrival time, $t$, of the shock wave at a depth, $x$, into the target plate is plotted against $x$ in this figure. The data are from experiment RS-2302. As can be seen in this plot, the amount of attenuation does not appear to be great. To better show that attentuation is indeed present, a plot of " $\delta i^{n}$ versus $x$ is given in figure 5 . The curvature of the data that is attributable to a decrease in the velocity of the shock wave is readily apparent in this plot.

The term " $\delta t "$ is a calculated quantity which is based on a fit to the experimental data. The first step in calculating $\delta t$ is to fit the first few levels of pin data to the equation

$$
t=a_{o}+a_{x} x
$$

On the basis of this fit a calculated time, $t c_{i}$, is found for every shock pin. Once 
this has been done, an unitial value of $\delta t_{i}$ for each shock pin is then found from the equation

$$
\delta t_{i}=t_{i}-t c_{i}
$$

where $t_{i}$ is "he observed or measured time for the $i^{\text {th }}$ shock pin.

The next, step in arriving at a final answer for $\delta t$ is the calculation of a shock velocity at the position of each shock pin. For the first few levels of pin data that are fi $_{\mathrm{i}}$ so equation (3.13), the shock velocity is simply

$$
U_{s}=\frac{1}{a_{x}}
$$

For the remainder of the pin data the calculation of a shock velocity is a little more involved.

All shock pin date not explicitjy fit to equation (3.13) can be considered to be in the attenuation region where the shock wave is decaying. The $\delta t_{i}$ data in this attenuation region may be fit to a polynomial or a spline function of the independent variable $x$. It was decided that a suitable function for fitting the data would be a spline function with two break points. The choice of the position of the first of these break points is discissed in section 3D and in chapter 4 . From strictly a statisticai point of view the position of the break points is somewhat arbitrary but is clear that the first break point, $x_{1}$, should always be assumed to lie between the last level of pin data fit to equation (3.13) and the next level of pins. 
This first break point, $x_{1}$ corresponds to $x_{3}$ in figure 1 . The only requirement for the second beak point, $x_{2}$, is that it should be greater than or equal to the first break point. A combination of both cubic and quadratic polynomials were used in fitting the $\delta t$ data. A physical basis for the choice of cubic or quadratic polynomials and the position of $x_{2}$ is also discussed in jection $3 \mathrm{D}$.

When the data are analyzed in this manner the data are divided into three regions or zones. In the first zone $\left(x_{1} \leq x_{1}\right)$, the appropriate data are fitted to equation (3.13). On the basis of this fit a $t c_{i}$ is calculated for each datum. Then a $\delta t_{i}$ is found from equation (3.14). In the second zone $\left(x_{1}<x_{i} \leq x_{2}\right)$, the $\delta t_{i}$ data are fit to the equation

$$
\begin{gathered}
\delta t=b_{0}+b_{1}\left(x-x_{1}\right)+b_{2}\left(x-x_{1}\right)^{2} \\
+b_{3}\left(x-x_{1}\right)^{3} .
\end{gathered}
$$

If a quadratic polynomial is used in this zone then $b_{3}$ is identically set to zero. For the third zone $\left(x_{2}<x_{i}\right)$, the $\delta t_{i}$ data are fit to the equation

$$
\begin{gathered}
\delta t=c_{0}+c_{1}\left(x-x_{2}\right)+c_{2}\left(x-x_{2}\right)^{2} \\
+c_{3}\left(x-x_{2}\right)^{3} .
\end{gathered}
$$

As in the second zone, if a quadratic fit is used then the coefficient for the cubic term is set to zero. 
The coefficients in equations (3.16) and (3.17) are not independent and are subject to constraints. The first constraint is that $\delta t$ should be continuous in $x$. Since the first zone $\left(x \leq x_{1}\right)$ is the region where $\delta t$ is nominally zero, the continuity of $\delta t$ implies that $b_{0}$ must be zero in equation (3.16). The sccond and last constraint is that once the shock wave is formed the velocity of the shock wave is continuous in $x$. This second constraint is equivalent to insisting that the first derivative of $\delta t$ with respect to $x$ is rontinuous. This implies that $b$ : in equation (3.16) must also be zero. These continuity conditions on $b t$ and its first derivative imply that at the point $x=x_{2}$ the following relations must hold:

$$
c_{0}=b_{2}\left(x_{2}-x_{1}\right)^{2}+b_{3}\left(x_{2}-x_{1}\right)^{3},
$$

and

$$
c_{1}=2 b_{2}\left(x_{2}-x_{1}\right)+3 b_{3}\left(x_{2}-x_{1}\right)^{3} .
$$

It is possible to obtain a velocity for the shock wave from these fits to the data in a straightforward manner. In the first region the shock velocity is simply given by (3.15). If in the second and third zones the representation for $\delta t$ is

$$
\delta t=f(x)
$$

then the shock wave velocity at any point in the attenuation region is given by

$$
U_{s}=-\left(\frac{d f(x)}{d x}+a_{x}\right)^{-1}
$$


In equation (3.21) the parameter $a_{r}$ is the same parameter that is in equation $(3.15)$

After a shock velocity has been found for each pin, an average va!ve for $x$ is calculated for each group of pins that are in the same level. The measured time for each pin is then adjusted to the average $x$ value for that particular pin leva!. Letting $x_{2}$ be the average value of $x$ for a group of pins in a given level, the new adjusted tine, $t_{1}$, is given by

$$
i_{1}=t_{1}+x_{1} x_{1}
$$

The shock velocity, $U_{i}$, is calculated from either equation (3.15) or equation (3.21) depending on whether the datum is in the shork-attenuation region or not.

The $i$, are not the final adjustments that are made to the measured times. Equation (3.20) shows that within each pin !evel all measured times in that pin ievel have been adjusted to an average value of $x$ for that group of pins. Within each group or level of pins there is still much scatter in the data. Much of this scatter can be attributed to tilt or bowing or both. To account for these perturbations each level of data is fit to either equations (3.8) or (3.10). If a given level of data does not have enough pins to fit to these equations, then that level of pin data is simply ignored. Based on these fits, average values for these perturbation parameters are found. The final adjusted times. $\tilde{t}_{i}$, are then calculated from

$$
\bar{t}_{i}=\bar{t}_{i}-a_{y} y_{i}-a_{z} z_{i}-a_{r} r_{i}
$$


These new adjusted times are an attempt to rake into account the non-plararitj' of the shock. The $\tilde{t}_{i}$ are then fit to equation $(3.13)$ and the $\delta t_{i}$ data are recalculated from equation (3.14) using $\tilde{t}_{i}$ instead of $t_{1}$. The plot of st in figure 5 was made using this analysis technique. Figure 6 is a $\delta$ plot using the raw times $t_{t}$ instead of the adjusted times, $\tilde{t}_{i}$. It is very apparent there is much more scatter in the data when no attempt is made to take into account the perturbations to the planarity of the shock wave.

\section{D. The Shock-Attenuation Experiments}

The analysis procedure described in section $3 \mathrm{C}$ does not give any quantitative means for the determination of the initial ovcrtaking point. Section $3 \mathrm{C}$ merely describes the handling of tha data when the initial overtaking point, $x_{1}$, and the second break point, $x_{2}$, are given. It is possible to include $x_{1}$ and $x_{2}$ in the equations of seciion $3 \mathrm{C}$ as unknowns that are to be determined by a minimization of some objective function such as the sum of the squares of the residuals. The problem with such a technique is that it leads to a highly nonlinear fitting routine. Another problem is a progiam such as this is dependent to some extent on the continuity of the objective function with respect to the unknown parameters. In the present case the objective function is not continuous with respect to $r_{1}$ and $x_{2}$. Besides these problems, it is not clear whether the scatter in the data wouid allow 
an accurate determination of these break points. This question will be examined in some detail in the present section.

It is desirable to have some quantitative guide for judging or choosing where the point $x_{1}$ might lie. The starting point for determining where this point mi, be is the consideration of the assumptions stated in section $3 \mathrm{C}$ for the analysis of the data. Specifically, the assumption that the flyer plate has uniform properties is needed here. When the additional assumption is made that the flyer plate is at normal density and zero pressure just before it collides with the target plate, one can derive a rather simple formula for the point $x_{1}$. For a Hyer and target plate made of the same material, the equation for $x_{1}$ is

$$
x_{1}=h \frac{c+U_{s}-u_{p}}{c-U_{s}+u_{p}} .
$$

In this equation, $h$ is the thickness of the flyer plate and $c$ is the sound speed of the lead characteristic in the release wave. The shock speed in equation (3.24) does not have to be measured from the shock-wave trajectory data. In each experiment the trajectcry of the fyer plate is also measured and the flyer plate velocity may be calculated from this data. The shock speed in the target may then be calculated on the basis of the flyer plate velocity. By making reasonable estimates about the maximum and minim um values for $c$ in equation (3.24), quantitative estimates for the minimum and maximum values of the overtaking point may be made. 
A maximum value for $c$ in equation (3.24) can be found by considering the nature of the shock process. The shock represents an irreversible process, while the release wave is assumed to be isentropic. This implies that in the $P$ - $\rho$ plane any isentrope that intersects the principal Hugoniot must have a slope less than the slope of the Hugoniot at the point of intersection. For a material whose principal Hugoniot is adequately described by a linear $U_{a^{-}} u_{\mathrm{p}}$ relation, equation (2.59) or equation (2.60) can be used to find a maximum value for $c$.

A rrinimum value for $c$ can also be found from equation (2.59) or (2.60). In appendix $D_{\text {it }}$ is shown that in the absence of any phase changes, $\gamma$ will decrease as the density increases along the principal Hugoniot. A reasonable estimate for the minimum value of $c$ can thus be found by replacing $\gamma$ by $\gamma_{0}$ in equation (2.60).

In each shock-wave attenuation experiment the pin data may be divided into two separate sets. One set of pin measurements pertains to the fiyer plate while the second set is the $t-x$ data for the shock wave in the target plate. The analysis of this first set of pin data is the same as the anlysis of the data of the flying plate experiments in section 3B. The pin data for the flyer plate in each experiment are fit to equation (3.1). From this fit the velocity of the flyer plate can be calculated. Based solely on the caiculated flyer plate velocity, the initial shock speed and the minimum and maximum values of $\nu_{1}$ in the target plate can be predicted. Table 5 gives the measured flyer plaie velocity for each shock-atteruztion experiment, the 
initial snock speed as predicted from the flyer plate velocity, and the minimum and maximum values of $x_{1}$ using equations (2.59) anc่ $(2.60)$ to calculate maximum and minimum sound speeds. From this table it is very noticeable that the values for $r_{1}$ are smaller in the copper experiments than in the stainless-steel experiments. This occurs because of the higher shock speed in stainless steel as opposed to copper for comparable pressures.

The $s t$ plots do result from a fitting procedure as explained in section $3 \mathrm{C}$. The division of the shock-wave data into three zones $\left(x_{i} \leq x_{1}, x_{1}<x_{i} \leq x_{2}, x_{2}<x_{i}\right)$ permits a sharp demarcation of the data into two differen regions. The first zone is the region where the shock is assumed to be moving at a constant velocity. Th: a second region is composed of the second and third $z$, nes where it is assumed that the shock wave is being attenuate.. by the releas: wave. The separation of this second region inis two zones by the break point, $x_{2}$, is partly a convenience for fitting the a djusted $\delta t$ data by equations (3.16) . nd (3.17). The fitting or smoothing functions are of course quadratic or cubic polynumials.

Obviously, the choice of where $x_{1}$ lies has an effect on the calculation of $\delta t$. Consider for the moment when the true posiuion of $x_{1}$ is $x^{*}$. If $x_{1}$ is now chosen such that $x_{1}$ is very much greater than $x^{*}$, then one would eypect the calculation of the initial shock velocity would be too low and the values of $\delta t$ for the zones where $x_{i}>x_{1}$ would alsc be too low. If the assumption that an initially flat- 
topped shock is introduced into the target is correct, it would be expected that the shock velocity calculated when $x_{1}$ is much smaller than $x^{*}$ would be very close to the value of the shock velocity when all the pins with $x_{1} * x^{*}$ are used to calculate it. The values for $\delta t$ in the attenuation region would also be expected to be very similar in these two cases.

The position of the second break point, $x_{2}$, and the option of cubic or quadratic polynomials for the fitting or smoothing functions ir. the second and third zones have a negligible effect on the calculation of $\delta t$; however, they do have a pronounced effect on the calculation of shock velocities in these zones. The choices of the position of $x_{2}$ and the option of the fitting functions are thus not strictly a matter of convenience, but can be made from an examination of the shock velocity plots.

For experiment RS-2302, table 5 suggests that the earliest ouservation of the overtaking point should occur between the 7 and 8 -mm pin levels. Figure 7 is a $\delta t-x$ plot for this experiment in which the first break point is at $7.8 \mathrm{~mm}$ and the second break point is at $8.5 \mathrm{~mm}$. The dashed line in the figure is the result of fitting equations (3.16) and (3.17) to the $\delta t$ data in the attenuation region. In tinis particular plet the quadratic forms for equations (3.16) and (3.17) were used. The shock velocity as calculated from these fits is shown in figure 8 . Referring to figure 7 , it is fairly safe $: \leq 4 y$ that those pins at the $11-\mathrm{r} m$ m leve! and beyond ar: 
definitely in the region where the shock wave is decaying. Following the general shape of the data in the attenuation region, it is probably safe to conclude that the pins at the $10-\mathrm{mm}$ levr! are also in the attenuation region. What is dfficult to conclude is whether the pins in the 8 -and $9-\mathrm{mm}$ levels should be in the attenuation region.

As previously stated, the general effect of changing the degree of the fitting functions and the position of the point $x_{2}$ can dramatically change the shape of the shock velocity curve while having a negligible effect on the $\delta t-x$ plot. Figure 9 plots $\delta t$ in which the first and second break points are the same as in figure 7 but cubic polynomials have been used in zones two and three. The $\delta t$ data have changed imperceptily, but the velocity graph (figure 10) based on these new fits has changed considerably. The shock velocity in the first zone is the same as that calculated for the first zone in figure 8 . The difference is the shock velocity in the attenuation region. Once the attenuation of the shock wave has begun no positive acceleration of the shock wave is expected. Thus the large increase in the shoik velocity seen in the second zone is physically unreasorable. If a simple substitution is made in zone two of a quadratic polynomial instead of a cubic polynomial, one then gets figure 11 for the shock velocity. This simple change of the fitting function for zone two has eliminated the acceleration of the shock wave and pioduced a smooth velocity curve as can be seen in t'is plot. 
The maiil problem with using a cubic polynomial in either zone two or three is the possibility of the $\delta t$ data naving too inuch scatter and the cubic polynomial following the scatter in the data. This is what happened in figure 10. In the same figure, the large amount of data in zone three has produced a reasonatly smooth portion of the velocity curve even though a cubic polynomial has been used for this zone. 'The suitability of using a quadratic or cubic polynomial is thus dependent not only on the scatter in the data but on the amount of data in the zone as well. An example of this is seen in figures 12 and 13 . In these figures, $x_{1}$ is the same as in figure 9 and cubic polynomials were used in she attenuation region but the second break point has been moved from 8.5 to $11.5 \mathrm{~mm}$. A comparison of the velocity ${ }^{\prime}$ 'ts in figures 10 and 13 shows that by simply changing the position of the second break point a much smoother velocity curve has been achieved.

When $x_{1}$ is varied: the interval between two levels of pir data, there is essentially no rhange in the $\delta t$ data and no change in the calculated shock velocity of zone one. The variation of $x_{1}$ only produces a change in the $\delta t$ data and the initial shock velocity when the number of pin levels in the first zone changes. This effect can be seen when figure 7 is compared with figure 14. The $\delta t$ data for figure 14 is the same as in figure 7 even though $x_{1}$ has been moved from 7.8 to $7.1 \mathrm{~mm}$. When the first break point is moved to $4.5 \mathrm{~mm}$, the $5 t$ points do indeed $\mathrm{c}^{1}$ iange as a comparison of figure 14 with figure 15 shows. The changes in the data occur 
because the calculated value for the shock velocity in the first zone has changed. When only the first four pin levels as opposed to the first seven pin levels are used in zone one, a lower initial shock velocity is calculated for experiment RS-2302, and this in turn produces the noticeable differences in the $\delta t$ plots of figures 14 and 15. The shock velocity plot for $x_{1}$ at $4.5 \mathrm{~mm}$ and $x_{2}$ at $8.5 \mathrm{~mm}$ using quadratic polynomials in the attenuation region is shown in figure 16. Just as in figure 10 , zone two in this plot shows a positive acceleration of the shock wave.

The maximum value of $x_{1}$ given in table 5 for experiment RS-2302 implies that the pins in the $10 \mathrm{~mm}$ level may belong to the unperturbed region instead of the region where the shock wave is decaying. A qualitative examination of figure 7 suggested this was not the case. When $x_{1}$ is taken to be at $10.2 \mathrm{~mm}, x_{2}$ at 10.6 nm, and quadratic polynomials are used in zones two and three, one arrives at ñgure 17. The shock velocity plot for this fit is shown in figure 18. A comparison of figlre 17 with figure 7 reveals that the $\delta t$ data have not changed very much, but the shock velocity in the attenuation region has changed dramatically. There is a large and extremely rapid drop in the shock velocity in zone two and the discontinuity in the slope of the shock velocity at the second break point has become mure pronounced. A shift of the first break point from 10.2 to 9.2 leads to similar results shown in figures 19 and 20. Attempts at smonthing the velocity plots can be made by moving $x_{2}$ and going to a cubic fitting function for zone 
three. With $x_{1}$ at $10.2 \mathrm{~mm}, x_{2}$ at 12.5 , and a change from a quadratic to a cubic polynomial in zone three one gets the shock velocity curve shown in figure 21. The large and rapid decrease in shock velocity in zone wwo is still present and a positive acceleration of the shock wave has now appeared in zone three. A similar result for $x_{1}$ at $9.2 \mathrm{~mm}$ is seen in figure 22 . When $x_{2}$ is left at $12.5 \mathrm{~mm}$ and the fitting functions are lefo as a quadratic polynomial in the second zone and a cubic polynomial in the third zone but $x_{1}$ is moved to $8.2 \mathrm{~mm}$, one obtains figures 23 and 24 . The $\delta t$ plot for $x_{1}$ at $8.2 \mathrm{~mm}$ is not dissimilar from the $\delta t$ plots with $x_{1}$ at 10.2 and $9.2 \mathrm{~mm}$, but the behavior of the shock velocity shows a much smoother curve. The question is whether one expects a smooth decay or attenuation of the shock. Just on the basis of the $\delta t$ plots alone one might not be able to conclude rnuch about the position of $x_{1}$, but when the velocity plots are also considered the situation is considerably altered. Assuming that the sharp decreases in the shock velocity seen iri figures 18 and 20 are not the expected behavior, one may conclude the 9 and $10-\mathrm{mm}$ pin levels are in the attenuation region in experiment iis̀-2302.

The initia! shock velocities calculated for zone one and the standard deviations for some of the fits to the data for experiment RS-2302 are listed in table 6 . The column headed by the term "spline" refers to the polynomials that were used in the attenuation region. An entry in this column such as " $2-3^{n}$ means that a 
quadratic polynomial was used in $z o n e$ two and a cubic polynimial in zone three. The term $\sigma_{1}$ is the standard deviation of the fit in the first zone or unperturbed region and $\sigma_{2}$ is the standard deviation in the attenuation region. The term $\sigma$ is the standard deviation of the overall fit.

Although the table does not contain as much information as the $\delta t$ and shock velocity plots, it does show some quantitative information that is difficult to discern from the plots. Even though the fits with the first break point less than $7 \mathrm{~mm}$ in experiment RS-2302 may be rejected on the arguments of the maximum speed of the release wave, they are included in the table as illustrating how the shock speed calculation in the unperturbed region is changing as more pin levels are added to the first zone. The statistics of the fits with $x_{1}$ tetween 7.0 and $9.0 \mathrm{~mm}$ show there is essentially no statistical difference among these fits and one cannot distinguish the position of $x_{1}$ on the basis of these fits alone. The additional tools for the acceptance or rejection of the first break point are physical arguments on the region where $x_{1}$ might lie and the expected manner in which the shock wave will be attenuated. It shouls be noted that the smallest standard deviation for the data of experiment RS-2302 is around 7.1 ns and this must be considered as the inherent scatter of the data for this experiment.

The fact that the variation of $x_{1}$ in tine interval between successive pin levels does not change the shock velocity in zone one or tie $\delta t$ plots indicates that an 
adequate choice for the initial analysis of the pin data may be made by choosing $x_{1}$ to lie halfway between two successive pin levels. To arrive at velocity plots, one can make the rather arbitrary choice that $x_{2}$ will be $1 \mathrm{~mm}$ away from $x_{1}$. Since this choice will mean that the second zone will have a paucity of daia, one an insist on a quadratic polynomial in zone swo to prevent the scatter in the data from having an undue influence on the shock velocity plot. The choice of a quadratic or cubic polynomial for zone three may not have the same influence as in zone two but again a quadratic polynomial may be chosen as being less susceptible to strange accelerations of the shock wave in zone three if there is a good deal of scatter in the data for this zone. An illustration of using such a procedure for experiment RS-2302 is shown in figures 25 through 30 . The $\delta t$ plots and their respective velocity plots show essentially the same behavior as that determined previously. The remainder of the shock-wave experiments will be analyzed in this simplified roanner.

The next experiment after RS-2302 using stainless steel as the target plate was experiment RS-230s. This shot produced the greatest shock speeds measured in any of the shock-attenuation experiments. Table 7 lists data for some of the fits to the data of experiment RS-2309. The first of these fits had $x_{1}$ at $5.5 \mathrm{~mm}$ and in successive fits the first break point was stepped in 1-mm increments out to a maximum value of $9.5 \mathrm{~m} \cdot \mathrm{n}$. Only quadratic polynomials were used in these 
fits. Just a cursory examination of this table reveals an increase ir. the overall standard deviation for the fit with $x_{1}$ at $8.5 \mathrm{~mm}$. Figure 31 shows the reason for this increase: the 8 -rum pin level should not be included in zone one, but rather it should be inciuded with the pin data in the attenuation region. Figure 32 shows the $\delta t-x$ plot for the fit with $x_{1}$ at $7.5 \mathrm{~mm}$ and figure 33 is the shock velocity plot for this fit. The deacceleration of the shock wave in zone two is large and certainly suggests that the pins at the 7 -mm level might also lie in the attenuation region. Figures 34 and 35 are the plots for the fit in table 7 with $x_{1}$ at $6.5 \mathrm{~mm}$. The velocity plot in figure 35 shows a more gradual decrease of the shock velocity in zone two than does the fit with $x_{1}$ at $7.5 \mathrm{~mm}$ but an even smoother velocity plot is produced when $x_{1}$ is moved to $5.5 \mathrm{~mm}$. This last plot shown in figure 36 is somewhat disturbing since it implies that the 6-mm pins should be in the attenuation region, which contradicts the assumption made about the maximum sour 1 speed in deriving the values for the minimum position of $x_{1}$ in table 5 . A discussion of this point will be reserved for chapter 4 .

Table 8 lists data for fits of experiment RS-2315, which was the last of the stainless-steel experiments conducted in this research. The situation for this experiment is somewhat similar to experiment RS-2309. The plots asscciated with the fit that has $x_{1}$ at $8.5 \mathrm{~mm}$ are shown in figures 37 and 38 . These plots indicate that both the 8 -and 9 -mm pin levels are in the attenuaiion region and not zone 
one. The $\delta t$ data and shock velocity graphs for the fit with $x_{1}$ at $7.5 \mathrm{~mm}$ are displayed in figures 39 and 40 . Even these plots look a little suspicious as though the 7 -mm pin level should be in the attenuation region. The plots for $x_{1}$ at 6.5 $\mathrm{mm}$ are in figures 41 and 42 . This fit gives a smoother shock velocity plot than the fit with $x_{1}$ at $7.5 \mathrm{~mm}$. As was the case in experiment RS-2309, these plots suggest that the overtaking point may occur before the minimum value jredicted in table 5 .

The first experiment using OFHC copper for the target plate was experiment RS-2306. The initial shock velocity and other quantities for some fits to the experimental data of RS-2306 are listed in table 9. The respective $\delta t-x$ plot and the associated shock velocity plot for each of these fits are shown in figures 43 through 50. The uninimum value for $x_{1}$ listed in table 5 is $6.4 \mathrm{~mm}$. The fit with $x_{1}$ at $6.5 \mathrm{~mm}$ gives as small a siandard deviation as seen in table 9 and the shock velocity curve in figure 46 is the smoothest of the velocity curves derived from these fits. The fit with $x_{1}$ at $5.5 \mathrm{inm}$ shows an acceleration of the shock wave in zone two, which implies that not enough pin data have been included in the first zone. The fit with $x_{1}$ at $8.5 \mathrm{~mm}$ shows the large deacceleration of the siock wave in zone two that seems to be typical of these plots when too man, pin levels are included in the unperturbed region. The fit with $x_{1}$ at $7.5 \mathrm{~mm}$ is sonewhat similar to the fit with $x_{1}$ at $8.5 \mathrm{~mm}$ but the shock velocity in sone two does not 
show as great or rapid a drop in the veiocity, as is seen in figure 50 , which makes it difficult to jidge whether the 7-mm pin level should be in zone one or not.

Due to a slight error in machining the copper target plate for experiment RS-2308, the first pin level was nominally set at $1.3 \mathrm{~mm}$ with subsequent levels machined at 1-rmm increments; thus the midway point between successive pin levels orcurs at .8 instead of at .5 as in the previous experiments. The first fit listed in table 10 for RS-2308 starts with a value for $x_{1}$ that is iess than the minimum value of $x_{1}$ lister in table 5 for RS-2308. The $\delta t$ and shock velocity plots in figures 51 and 52 for this fit certainly seem to agree with table 5 that a $4.8 \mathrm{~mm}$ position for $x_{1}$ is too small. When the first break point is moved to 5.8 $\mathrm{mm}$, the calculated value for the initial shock velocity increases from $7.19 \mathrm{~km} / \mathrm{s}$ to $7.24 \mathrm{~km} / \mathrm{s}$. The $\delta t$ plot for $x_{1}$ at $5.8 \mathrm{~mm}$ is shown figure 53 . The interesting thing about the shock velosity plot in figure 54 is that zone two shows only a very gradual decrease in the shock volocity. This is certainly suggestive that a $5.8-\mathrm{mm}$ position for $x_{1}$ may not be great enough. Figures 55 and 56 are for the ît with $x_{1}$ at $6.8 \mathrm{~mm}$. Figure 56 for this fit shows an extremely smooth shock velocity curve. The plots for the $8.8-\mathrm{mm}$ fit are shown in figures 57 and 58 . These piots indicate that the $8.3-\mathrm{mm}$ pin le el should be in zone two or three. The next to the last fit $\left(x_{1}\right.$ at $\left.7.8 \mathrm{~mm}\right)$ is plotted in figures 59 and 60 . 'This fit cioes not give as smooth as a shock velocity curve as the $6.8-\mathrm{mm}$ fit but there is enough ambiguity 
in the plots that one cannot say in a definitive manner whether the $7.3-\mathrm{mm}$ pin level should be in the attenuation region o: not.

Figure 61 through figure 68 are the plots associated with f's given in table 11 for experiment RS-2314. This experiment is quite similar to the other two copper experiments. The fit using $x_{1}$ at $8.5 \mathrm{~mm}$ indicate that the 8 - $\mathrm{mm}$ pin level is in the attenuation region and not cone one. The plots with the first break point at $5.5 \mathrm{~mm}$ seem to indicate that indeed this is too small a value for the overtaking position. The better overali fits are achieved with $x_{1}$ at 6.5 and $7.5 \mathrm{~mm}$.

The analyses of the shock wave experiments have been intended to accurately find the position at which the shock wave is overtaken by the release wave in each experiment. Unfortunately, the scatter in the data has preventr.: al1 accurate determination of the point $x_{1}$ in $3.1 y$ of the experiments. There is, however, sufficient resolution of the data that some conclusions can be drawn about a region or interval in which the overtaking of the shock wave by the release wave probably occurred. This zegion or interval for each experiment can be determined using only the analysis teshniques in this chapter. A brief summary of the results extracted from these experiments is given in table 12 . i his table ists the interval or region for each experiment in which the releasr wave probably overtook the shock wave as well as the speeds of the flytr plate and the shock wave as calculated from the pin measurements. The values for the shock speeds are actually 
weighted values associated with the unperturbed region of each experiment. For the copper experinents the point $x_{1}$ could only be determined within a $2-\mathrm{mm}$ interval with the region being essentially the same for each experiment. In the stainless-steel experiments there is a little more variation of the overtaking region and in experiment RS-2315 the overtaking interval appears to be determined to within 1 mm as opposed to a 2 -mm interval for the other experiments. The experiments seem to suggest that the speed of the lead characteristic in stainless steel may be greater thas the maximum sound speed given by the siope of the principal Hugoniot while in copper this is probably not the case.

There are some anomalies in the data that need more investigation. The shock speeds measured in these experiments are generally one to two per cent above the values predicted by the impedance-matching calculations using the measured velocities of the flyer plates. The one exception to this is experimen.t RS-2308, which has a measured shock velocity about one per cent lower than the predicted value in table 5 . This is not all that suprising when one considers the uncertainty in the flyer plate velocities but this could be intimately connected to one of the basic assumptions being made in the anlyses of these experiments. An examination of the $\delta t$ plots for the data in zone one shows that in most of these plots there appears to be some curvature of the data in this region. This could be caused by a small pressure gradient and hence a small density gradient existing in the 
flyer plate at the time it collides with the target plate. This would mean that the assumpition that the flyer plate has uniform properties is erroneous. These questions concerning the state of the flyer and the curvature of the data will be addressed in more detail in the next chapter. 


\section{COMPUTER MODELING OF THE EXPERIMENTS}

\section{A. Preliminaries}

In the analyses of the shock trajectory data in section 3D some questions arose that could not be answered based on that simplified approach to the data. A more thorough examination of the experimental data can be accomplished by resorting to computer modeling of the experiments in which the trajectory of the shock wave was measured. Problems concerning the curvature of the data or the shape of the velocity curve in the attenuation region can all be addressed in a calculational investigation.

Of the questions raised in section $3 \mathrm{D}$, one of the most fundamental concerns the state of the flyer at the time it collides with the target plate. The basic assumptions that the flyer plate has uniform properties and is at normal density at the time it collides with the target plate must be questioned in light of the apparent curvature exhibited in many of the $\delta t$ plots for the data in the supposedly undisturbed region. The importance of the initial state of the fiyer plate is difficult to emphasize too much since the modeling of the experiments is dependent upon the initial conditions.

When the flyer plate is assumed to have uniform properties, it explicitly follows that the shocks introduced into the flyer plate and target plate initially move at constant velocity. It also follows that the rarefaction wave created when 
the shock wave in the flyer plate reaches the free surface of the flyer will be a simple-centered wave. If the target and flyer are made of the same material and have the same initial states, then the rarefaction fan will remain a simplecentered wave until it overtakes the shor.k wave in the target plate. The modeling of shock attenuation is considerably simplified when one can consider the problem as a simple-centered wave overtaking a shock wave. Even when the target and flyer are dissimilar materials, the assumption that the Ayer plate initially has no gradients in it means that analytic methods may still be used to calculate the overtaking point as was done in section 3D. Numerical methods must still be used to calculate the decay of the shock wave, but the fact that an analytic expression exists for the overtaking position is a great benefit.

For the case when gradients do exist in the flyer plate at the time it collides with the target plate, shock waves cannot propiagate in either the flyer or the target plate at constant velocities but must show accelerations as a result of the gradients in the flyer. Once the shock wave traveling in the flyer plate has reached the back surface of the flyer, the release wave no longer travels into a constant state region and thus the rarefaction fan cannot be a simple wave. There is also no simple analytic expression for the overtaking point and the whole problem from the propagation of the shock waves to the subsequent decay of the shock wave in the target plate must be solved numerically. The modeling of these experiments 
will use both assumptions concerning the initial state of the flyer. When the assumption is made that the initial state in the flyer is not uniform, the initial condition of the flyer plate will be calculated in the manner shown in appendix $\mathrm{E}$.

Another question of fundamental importance is one concerning the maximum speed of the release wave. In section 3D a simple argument was made that the slope of the principal Hugoniot in the $P-\rho$ plane is the limiting factor in determining the maximum speed in the rarefaction fan. The stainless-steel experiments seemed to contradict this hypothesis. The argument of section $3 \mathrm{D}$ concering the maximum speed of the release wave is valid for a material behaving as an inviscid fluid, but it is not valid for a material described by the constitutive models of section $2 \mathrm{~F}$. These constitutive models have non-zero deviatoric stresses and a discontinuity in the wave speeds at an elastic-plastic boundary. This results in a release process that is fundamentally different from that of a material that cannot support a deviatoric stress. Making the assumption that on the principal Hugoniot the normal component of the deviatoric stress is at an upper yield limit implies that in the initial stages of the unloading process the material will be in an elastic state. In this elastic state the slope of the release path in the $P-\rho$ or $\sigma-\rho$ plane may be greater than the slope of the principal Hugoniot. Eventually the material goes into a plastic state and the sound speeds are then essentially determined by bulk properties alone. For such material models, a compressive wave for a state already 
on the principal Hugoniot would not take the material into an elastic regime but into a plastic state and the wave speeds would once again be determined by brik properties only.

There is some difficulty in sorting out the influence of the strength nodels and the influence of only the equations of state on the attenuation of a shock wave Even the consideration of the simpler case in which strength effects are not modeled presents a rather complicated flow in the attenuation of a shock wave. An heuristic approach to the problem may be taken by considering the situation of a simple-centered wave overtaking a shock wave. A diagram for such a flow is shown in figure 69. This figure is similar to figure 1 but certain regions have been labeled and some additional characteristic lines have been drawn. Region $I$ is the region which has not yet been hit by the shock wave. The line labeled " $U_{s}$ " is the path or trajectory of the shock wave. Region II represents a material region sitting at peak shock pressure, particle velocity, etc. The state of the material is uniform throughout region II. The simple-centered release wave occupies region III. Region IV is a final state which is characterized by zero pressure in this region. The line labeled " $\mathrm{u}-\mathrm{c}$ " is a left-going characteristic given by equation (2.19) which originates from the overtaking point $x_{1}, t_{1}$. This line is also the left boundary for region $\mathrm{V}$. The right boundary for region $\mathrm{V}$ is the line labeled $" u$, which is the material or particle path for the point at which the shock wave 
was intercepted by the lead characteristic of the release wave. In region III the right-going characteristics have a constant slope; but, once the release wave enters region $V$, the wave no longer remains a simple wave. The specific entropy can be considered to be constant in regions II, III, IV, and V. In region VI the entropy is different along each particle path since each particle has been shocked to a different state on the principal Hugoniot. The right-going characteristics are not shown in regions $\mathrm{V}$ and VI except for the solid line, which is the hypothetical continuation of the dashed line of region III.

In region III each right-going characteristic has a constant value of $u$ and c. The particle velocity on each right-going characteristic can be found from the equation

$$
u=u_{2}+\int_{P_{2}}^{P} \frac{1}{\rho c} d P
$$

In this equation $u_{2}$ and $P_{2}$ are the particle velocity and pressure in region II. Equation (4.1) is the compationility equation for the left-going characteristics of region III. Every left-going characteristic in region III originated in regicn II. Because all the material in region II and region III has seen the same peak shock conditions, equation (4.1) may be written as

$$
u=u_{2}+\int_{\rho_{2}}^{\rho^{\prime}} \frac{c}{\rho} d \rho .
$$

By the same token the specific internal energy on each right-going characteristic 
may be found from the equation

$$
E=E_{2}+\int_{\rho_{3}}^{\rho} \frac{P}{\rho^{2}} d \rho
$$

For a general equation of state in which the pressure is given as a function of specific internal energy and density, the sound speed will in general be a function of these same quantities as well. Equations (4.2) and (4.3) may be integrated numerically to find the conditions along earh right-going characteristic of region III.

In region $\mathrm{V}$, the simple-wave solution is no longer appropriate because the leftgoing characteristics no longer originate in region II but in region VI. Any point in region $\mathrm{V}$ may be considered as being intersected by three different characteristics, a right-going characteristic, a left-going characteristic, and a particle path. The right-going characteristic may be considered as originating from the left boundary of region $\mathrm{V}$ and thus the compatibility equation for the right-going characteristic may be written as

$$
u=u_{3}+\int_{P_{3}}^{P} \frac{1}{\rho c} d P,
$$

where $u_{3}$ and $P_{3}$ are the particle velocity and pressure for the appropriate point on the left boundary. Along the left-going characteristic the particle velocity is given by

$$
u=u_{s}+\int_{P_{.}}^{P} \frac{1}{\rho c} d P .
$$


In this equation $u_{9}$ and $P_{9}$ represent the particle velocity and pressure for the point of origin of the left-going characteristc on the decaying portion of the shock wave. The last characteristic direction is the particle path and the compatibility equation along this path in region $V$ is again given by equation (4.3). The fact that the material in region $\mathrm{V}$ has all been shocked to the same peak pressure implies that the right-going characteistic can be considered as being isentropic and equation (4.4) can be written as

$$
u=u_{3}+\int_{p_{3}}^{\rho} \frac{c}{\rho} d \rho
$$

The replacement of $d P$ by $c^{2} d \rho$ cannot be made in equation (4.5) because part of the integration occurs in region VI. The left-going characteristics in region VI traverse different particle paths which are also different isentropes. The integral in equation (4.5) can be split into two integrals, one for region $\mathrm{V}$ and one for region VI. For that integral in region $V$ the replacement of $d P$ by $c^{2} d \rho$ may be made.

The integrals presented thus far are only the compatibility equations and they hold only along the characteristic directions or paths. In order to integrate them numerically it is necessary to know the specific paths of the characteristics. The differential forms for these paths are given by equations $(2.17)-(2.19)$. The complexity of the problem is apparent and this is why it is necessary to resort to numerical techniques to solve the problem of shock attenuation. In some special 
cases the problem is amenable to analytic methods but for a general equation of state the problem is only tractable using numerical tichniques.

A further understanding of the problem may be accomplished by resorting to a model in which the equation of state is a tunction of densicy only. When the equation of state is a function of density alone, the energy equation is no longer necessary and a particle path is not a characteristic direction. There are just two characteristic directions and they are given by equations (2.18) and (2.19). For such an equation of state, regions $V$ and $V I$ of figure 69 can be considered as one region since the specific internal energy is not needed in determining the pressure.

Some interesting questions concerning the behavior of the characteristics in the wave interaction region can be addressed with this model. One question is whether the right-going characteristics of the simple wave can remain lines of constant slope in region $V$ with constant values of $u, c$, and $\rho$ ? The first step in answering this question is to assume that the characteristics are straight lines everywhere and find what requirements are placed on the equation of state by this assumption. The changing conditions at the shock front must be related to the changing conditions in the wave interaction region. For initial conditions of zero pressure and zero particle velocity ahead of the shock, equations (2.1) and (2.2) can be solved to give

$$
u^{2}=P\left(\frac{1}{\rho_{o}}-\frac{1}{\rho}\right)
$$


Equation (4.7) holds at the shock front. Along a left-going chraracteristic behind the shock, the change of the particle velocity with respect to the density is given by

$$
\frac{d u}{d \rho}=\frac{c}{\rho} .
$$

Equation (4.8) is valid for any left-going characteristic in any region because it is explicitly assumed that the pressure is a function of density only. If the right-going characteristics are to remain straight lines in region $\mathrm{V}$ with constant values of $u$, $c$, and $o$, then equations (4.7) and (4.8) may be used to arrive at a differential equation for $P$ :

$$
\frac{1}{P} \frac{d P}{d \rho}=\frac{\rho_{\circ}}{\rho\left(\rho-\rho_{0}\right)}
$$

The solution of equation (4.9) results in an equation of state which does not satisfy the Bethe-Weyl conditions in that the second derivative of pressure with respect to density is less than zero. A material tha: has such an equation of state cannot support a compressive sheck wave. The conclusion is that for an equation of state with pressure as a function of density only and satisfying the Bethe-Weyl conditions, the $u+c$ characteristics cannot maintain constant values of $u, c$, and $\rho$ in region $\mathrm{V}$.

Such conclusions cannot be so easily reached when the equation of state exhibits an energy dependence. When the equation of state exhibits an energy dependence, then equation (2.11) must be included in the system of partial dif- 
ferential equations that govern the flow. The additional complications that arise from the inclusion of the energy equation make a simple analysis impossible. Another analysis of the attenuation problem using a highly specialized equation of

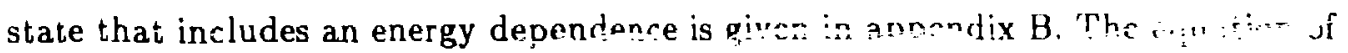
state discussed in appendix $B$ results in an analytical solution for the trajectory of the shock wave.

\section{B. Hydrodynamic and Simple Strength Modeling}

The experiments using SS-304L for the target plate will be modeled first because the modeling of the copper experiments is dependent on knowing the behavior of the stainless-steel flyer plate. The first step in modeling these experiments is to start with a simple hydrodynamic model and ass ume that the flyer plate is at normal density and zers pressure at the time it collides with the target plate. This implies that figure 69 gives the correct qualitative description of the shock attentuation process. The program SHKSIM described in appendiz A can then be used to calculate the decay of the shock wave. The primary goal of this first step is to test the hypothesis of whether or nnt the attenuation of the shock wave can be suitably modeled as a simple-centered release wave overtaking a shock wave.

Important considerations in setting up calculations on the code SHKSIM are 
the initial ronditions. One input parameter is the thickness of the flyer plate. Another input parameter is the velocity of the flyer plate. This input parameter can be taken as the measured value of the velocity of the flyer but it is not. In general the pin data for the flying plate are not as good as the pin data for the trajectory of the shock wave in the target plate. For this reason the velocity of the flyer plate taken from the pin data monitoring the flver is not used for the calculations done with the code SHKSIM. Rather, the flyer plate velocity is adjusted as an input parameter so that the rinck velocity given by the code in the region before the release wave catches the shock, agrees with a shock velocity determined from the experimental data for the target plate.

The results of a calculation by the code SHKSIM for experiment RS-2302 are shown in figure 70. For the comparison of the calculational results to the experimental data some choices have to be made as to the location of the first and second break points for the analysis of the pin data. Whenever possible the first break point for the analysis of the pin data was chosen to coincide with the initial overtaking point given by the cocie calculation. In some instances such a choice for the first break point was not warranted. For each $\delta t-x$ plot that shows a comparison between a calculation and experimental data there is a table listing information about the analysis of the data and the calculation shown in the plot. Information pertaining to the code calculation and the data analysis for figure 70 
are listed in table 13.

In the last section some questions concerning the behavior of right-going characteristics in the wave interaction region were raised. For the particular calculation shown in figure 70 these questions can now be answered. This calculation used equation (2.44) for the equation of state. For the $u+c$ characteristics that were specifically followed in the calculation there was very little change in the slope of any characteristic through the wave interaction region. Referring to figure 69 , the hypothetical continuation of a right-going characteristic from region III through regions $\mathrm{V}$ and $\mathrm{VI}$ is shown with a decreasing value of $u+c$. The behavior of a right-going characteristic is just the opposite in the present calculation. The value of $u+c$ on a righi-going characteristic increases as the characteristic traverses the wave interaction region. A specific example of this is given in table 14. The starting values are the values of variables on the characteristic when it is at the left boundary of region $\mathrm{V}$ in figure 69 . The ending values for the characteristic occur when it intersects the shock front. The characteristic listed in table 14 intersected the sliock front at $19.27 \mathrm{~mm}$. The calculation ended when the shock front reached $29.0 \mathrm{~mm}$. The increase in $u+c$ from table 14 is well under one percent. As might be expected from the work in appendix $\mathrm{F}$, both the pressure and specific internal energy decrease along the characteristic while density increases. These results should not be generalized to other calculations using different equations of 
state since the details of how much the variables change is highly dependent on the equation of state.

Figure 70 shows a large discrepancy between the experimental data and the calculational results. The calculation does not show as much attenuation of the shock wave as is seen in the experiment. This discrepancy could have its origin in the equation of state used in the calculation or could be due to the basic asssumptions of the modeling. To address the question concerning the equation of state, comparisons between the data of RS-2302 and calculations using the other equations of state are shown in figures 71-73. Each of these calculations also shows a noticeable discrepancy in comparison with the experimental data. None of the equations of state predict as much attelıation as is seen experimentally. The best agreement of a calculation with experiment RS-2302 is shown in figure 71. This calculation used the Rice equation of state in which the sound speed is independent of the specific internal energy. The examination of the respective tables for these plots reveals that this equation of state is the only one that predicts the overtaking of the shock wave by the release wave before the 9 -mm pin level in this experiment.

The discrepancy between the calculations and the experimental data of RS2302 is indicative of a fundamental inadequacy of the modeling; however, it is possible that something could be wrong with the data of RS-2302. To answer this 
question a set of purely hydrodynamic calculations using the code SHKSIM was performed for experiments RS-2315 and RS-2309. The comparisons between these calculations and the experiments are shown in figures 74-81. Information for the data analysis in each figure and other information pertaining to the calculation are given in the appropriate table (tables 18-25). The results from these calculations are qualitatively the same as the calculaticnal results shown in figures $70-73$. None of these calculations show as much attenuation of the shock wave as is seen in either experiment. Again the best agreement betweeen a calculation and an experiment, is achieved when the Rice equation of state is used in the modeling of the experiment.

The fact that none of these preliminary calculations accurately match any of the experiments does suggest that something is indeed wrong with the modeling. There are other possible explanations for the lack of agreement between the calculations and the experiments. Again one such hypothesis for the source of the discrepancy is that the data are in error. If this is true, then it must be highly systematic since all the stainless-steel experiments show the same qualitative behavior in comparisons with code calculations. Another possible source of errors is numerical problems in the codes, but the work of appendix B contradicts this hypothesis. Assuming that these are not the sources of the discrepancies, then one is left with the original questions concerning the basic assumptions of the modeling. 
Either the equations of state are not giving the correct bulk compressibiblites at these hish pressures or the assumptions of modeling the attenuation of the shock wave as a sirictly hydodynamic process is incorrect. Now the equations of state in a good job reproducing the principal Hugoniot data and there is no a priori reason to expect that the equations of state would be grossly inadequate for computing bulk sound speeds. This suggests that the basic assumption of modeling the attenuation process as a simple-centered release wave in which strength is not important must be questioned.

Further illumination of this problem can be gained by an examination of the shock velocities from the code calculations and comparing them against, the shock velocities derived from the pin data. A comparison of the experimental and calculational shock velocities of figure 70 is shown in figure 82 . Similar comparisons of the shock velocities for figure 74 and figure 78 are shown in figures 83 and 84 . The solid line in each figure is the shock velocity from the fit to the data and the dashed line is from ine code calculation. For experiment RS-2302 shown in figure 82 , the velocity curva from the code calculation seems to parallel the experimentally determined shock curve but is just translated. For the other two experiments the calculated shock velocity curves appear to be converging toward the experimental curves. Anything in the modeling that would cause the release wave to come in earlier or faster would thus enhance the agreement between the 
calculations and the experiments.

The next step in modeling these experiments is to include strength effects. A suitable starting point is the strength modeling in section $2 \mathrm{~F}$. The constitutive equations for strength given in that section are examples of simple elastir-plastic models. As stated previously an elastic-plastic model implies there is a region defined by a yield surface within which the material behaves in an elastic manner. Outside this region the material behaves plastically. The material in this region behaves more like a fluid but one in which deviatoric stresses still exist and satisfy the yield condition. The effects of such a simple strength model in the code can be anticipated. The most noticeable effect of an elastic-plastic model is that the speed of the lead characteristic in the release wave should increase as discussed in section IIF. This is easily seen in equations (2.100) and (2.101), which give the longitudinal sound spetds for a material obeying the constitutive equations of section 2F. Another noticeable effect of the strength models will occur in a simple-centered release wave. The rarefaction or release wave will separate into two distinct parts. This happens because of the discontinuity in wave speeds when the material goes from an elastic state into a plastic state; thus, the elastic part of the rarefaction fan will outrun the plastic part.

Of the equations of state used in the purely hydrodynamic calculations for shock attenuation in stainless steel, the Rice equation of state gave the best agree- 
ment with the experiments. Using this equation of state and equation (2.93) for the stress rate, the effects of the constitutive equations for strength in a code calculation of the attenuation of a shock wave are snown in figures 85 and 86 . The c. 'ulation and data shown in these figures a"e: for experiment RS-2302. Table 26 lists parameters for the calculation and the analysis of the pin data for the plots displayed in these figures. The code employed for the calculation was the code SHKSII since the code SHKSIM cannot handle a strength model. The immediate consequences of using a simple strength model in a calculation for experiment RS-2302 are easily seen in these figures. The overtake position occur: much earlier and the plateau or shoulder caused by the discontinuity in the wave speeds between the elastic and plastic regions is clearly defined in the shock velocity plot of ligure 86 . Figure 85 can be direcly compared with figure 71 , which shows a purely hydrodynamic calculation for experiment RS-2302 that uses the same equation of state. Although the overtake position does occur much earlier in figure 85 than in figure 71 , the overall agreement between the data and the calculational results is not significantiy improved. Part of the explanation for this can be found from an examination of the shock velocity plots for the two calculations. Figure 87 shows the shock velocities associated with figure 71 . In comparing figures 86 and 87 , the calculation with strength rapidly approaches the solution which does not include strength. 
In making comparisons between the data and calculations and displaying them in $\delta t-x$ plots, some mention must be made of the sensitivity of the comparisons to the choice of the first break point for the analysis of the data. For figure 85 the first break point was chosen to be at $7.1 \mathrm{~mm}$. This meant that the first seven levels of pin data were used in determining the initial shock velocity in the target plate. This was done in spite of the fact that the calculation suggested a better choice for the first break point was at $6.9 \mathrm{~mm}$. If only the first six pin levels are used for the first zone of the data analysis, there is a significant reduction in the value for the initial shock velocity of experiment RS-2302. A calculation performed to match this value for the initial shock velocity does show better agreement with the data. An example of this is shown in figure 88 . Undoubtably the better agreement results from the lower value for the initial shock velocity of the data. An examination of the shock velocity plot in figure 89 still shows divergence of the calculated shock velocity curve from the experimental curve. For this reason the value of the shock velocity calculated with the first seven pin levels is probably the better value. Another clue for this assumption is that the pins at the 1-mm pin level do appear to be early. If these pins are deleied from the first zone of the data for the deturmination of the shork velocity, then the 2-6-mm pin levels give an initial shock velocity of $7.36 \mathrm{~km} / \mathrm{s}$. This agrees with the shock velocity calculated from the data for figure 85 . 
Another example of a calculation using a strength model is shown in figure 90 . The calculation shown in this figure for experiment RS-2315 uses the same equation for the stress rate and the same equation of state as the calculation shown in figure 85 . Figure 90 can be compared with figure 75 , which shows a calculation for the same experiment but one which does not have strength. Still another calculation using the same equation of state and strength model as the calculations plotted in figures 85 and 90 is given in figure 91 for RS-2309. Figure 79 shows a comparable calculation for RS-2309 in which strength was not used. Again the most noticeable effect of the strength model is the movement of overtaking point. The calculations with strength show a little better agreement with the data for the interniediate pin levels than the calculations without strength but at the higher pin levels there is little difference between the two types of calculations. This is also confirmed by an examination of the shock velocities for figures 90 and 91. The shock velocities for figure 90 are shown in figure 92 and those for figure 91 are plotted in figure 93.

Some interesting trends can be seen in the strength calculations that have been done thus far for the three stainless-steel experiments. These trends are most apparent in the shock velocity plots. In experiments RS-2315 and RS-2309, the initial attenuation of the shock wave shows a definite divergence of the experimental and computational shock velocity curves. Just the opposite behavior 
is seen in the velocity curves in figure 86 for RS-2302. Some reservation must be taken before making too much of this trend because the experimental shock velocity curves are highly dependent on the scatter of the data as well as the data arii!'sis.

To make sure there was nothing peculiar to these strength calculations, another set of strength calculations was done using a different equation of state. In figures 94-99 are shown experimental and calculational results for each of the stainless-steel experiments. The code calculations for these figures used the Tillotson equation of state but the exact same strength model as that used previously. The same qualitative effects are seen in going from calculations without strength to calculations with strength for the Tillotson equation of state as were seen for the calculations using the Mie-Gruneisen equation of state.

Yet another question that inust be addressed is the one concerning the condition of the flyer plate at the time it impacts the target plate. So far all the calculations that have been shown have heen done using the initial conditions that the flyer plate is at normal density, zero pressure, zero internal energy, and a uniform particle velocity. The work in appendix E shows how a density and pressure gradient may be estimated for the flyer plate. The effect of such gradients in the fiyer plate will now be examined by repeating a strength calculation with gradients in the flyer plate for the initial conditions. 
The initial conditions for the flyer plate are calculated in accordance with the procedure descibed in appendix E. For experiment RS-2302 it is assumed that the back boundary condition of the flyer plate is a pressure of 1.3 cipa. The front boundary condition is zero pressure. A linear density gradient in the flyer plate is assumed and each material point is assumed to be on a release isentrope from a shock pressure of $7.5 \mathrm{GP}$ a. This assumption plus equation $(e 9)$ fixes, $p, E$, and $P$ at each point in the Hyer. To fix the material or particle velocity in the Hyer, some value that lies within the error bars of the measured velocity of the flyer plate is used as the velocity at the front boundary of the flyer. The particle velocity at ach succeeding point in the plate is then determined by finding the difference in he Riemann integrals for each point and the front surface and subtracting this difference from the chosen velocity for the front surface.

An example of a calculation with these initial conditions in the flyer plate is shown in figure 100. A shock velocity plot from this calculation is shown in figure 101. These nonuniform initial conditions in the flyer plate do not have a large effect on the calculation of shock attenuation but there are definite effects. One effect is that the overtake position changes by about $0.1 \mathrm{~mm}$. Another effect is there is slightly more dispersion in the initial part of the release wave. Also the strength of the shock wave grows slightly before it is overtaken by the release wave. This last effect is readily seen as an increase in the shock velocity in figure 
101. This qualitatively explains why in many of the experiments an increase in the shock velocity of the first zone of the data is seen as more pin levels are added to this zone. In estimating the initial conditions for the flyer plate, the greatest uncertainty is probably the choice of the particle velocity gradient across the Hyer plate at the moment of impact. There is some ambiguity in calculating ot for code calculations in which the flyer plate is given nonuniform conditions. This occurs because the shock velocity is not constant in the region before the release wave overtakes the shock. In general, $\delta t$ for the code calculations is found from the equation

$$
\delta t=t \quad \begin{gathered}
I \\
U_{s}
\end{gathered}
$$

The choice of $L_{a}$ in equation (4.10) when the initial conditions of the flyer plate are uniform and the same as the target plate, except for particle velocity, is unambiguous. In this case, it is just the intial shock velocity calrulated from an impedance rnatch solution for the impact of the flyer plate into the target plate. For a calculation with nonuniform initial conditions in the flyer, a nominal value of the shock velocity in the region free from attenuation is used in eq ation (4.10).

The modeling that has been done up to this point for the stainless-steel experiments shows that strength is important in the code calculations. The simple strength model does produce better agreement between the code calculations and the data. The inclusion of nonuniform initial conditions in the flyer plate does seem 
to qualitatively explain the behavior of the pin data in the first zone. Before going to more complicated or elaborate strength models for use in the computations for the stainiess-steel experiments, it is worth while to do some calculations for the copper experiments to see if they follow the same trend as the calculations for the stainless-steel : xperiments. 'This will 'e done even though none of the stainlesssteel experiments has been closely matched by calculations. In the calculations for the copper experiments, ihe Rice equation of state and equation (2.93) will be used as the constitutive equations for the flyer plate because these equations produced the best match with the stainless-steel data.

The logical calculation for the first of the copper experiments is to use the same type of equation of state and strength model that were the most successful in the modeling of the stainless-steel experiments. For copper the rigidity modulus and yield strength are much smaller than for stainless steel so it is expected that the simple strength model used thus far will have less of an effect in calculations for the attenuation of a shock wave in copper than in the modeïng of shockwave attenuation in stainless steel. A $\delta:-\tau$ plot for the pin data of experiment RS-2306 as well as a calculation from SHKSJB that models this experiment are shown in figure 102. The code calculation does a fair job in matching the data. A plot of the shock velocities from figure 102 is shown in figure 103. It is very apparent from this figure that the strength model in the calculation for RS-2306 
is responsible for only a very small amount of the attenuation of the shock wave. When the calculation is reperathout a strength model for the copper target plate, the calculation matches the data nearly as well as with a strength model. The repeated caiculation is shown in figure 104.

The calculations for experiment RS-2306 are much like the calculations for the stainless-steel experiments in that the calculations that come the closest in matching the data use the Rice equation of state. Calculations for RS-2306 using the other equations of state are shown in figures 105-107. These calculations also use a strength model for the copper target. The fact that the yield strength for copper is only $0.12 \mathrm{GPa}$ and is treated as a constant in these calculations means that the strength model has an almost negligible effect on the attenuation of a shock wave. The only appreciable effect of the strength model is to move the locatior. of the overtaking point. Another calculation for RS-2306 using the Tillotson equation of state but no strength in the copper is shown in figure 108 . Comparison of figures 108 and 106 again demonstrates the very small effect of the strength model in the copper calculations.

The next set of copper calculations were for experiment RS-2314. Since the strength model has been shown to have such a small effect in the calculations for the attenuation of a shock wave in the copper target, no strength model was used in the modeling of the target plate in these calculations. The surprising thing 
about this set of calculations for experiment RS-2314 is that the equation of state that worked best in matching the data of RS-2306 did not give the best agreement for RS-2314. The results of the calculations for experiment RS-2314 are shown in figures 109-112. Additional information abcit the calculations and the analysis of the data for the figures can be found in the appropriate tables. The striking feature of the calculation shown in figure 109 is that it shows greater attenuation of the shock wave than is seen in the experiment. This is quite a reversal of the calculational results for experiment RS-2306. Altough the equation of state used for the calculation in figure 109 gave the best agreement with the data of any of the calculations for experiment RS-2306, it definitely did not predict too much attenuation for that experiment. In experiment RS-2314 this equation of state definitely gives too much attenuation. The better agreement between the calculational results and the data of RS-2314 is seen in figures 110 and 112 . The calculations shown in these plots use the Mie-Gruneisen equation of state with $\gamma \rho$ a constant and the Gray equation of state, respectively. : :hese equations of state give very similar results. Lastly, the calculation using the Tillotson equation of state shown in figure 111 still produces too little attenuation of the shock.

Further insight into the behavior of the code calculations in comparison to the experimental data can be gained by examining the shock velocity curves. The shock velocity curves for the calculation and the data of figure 109 are shown 
in figure 113. The calculation shows a more rapid and precipitous drop in the shock velocity from the initial overtake point than the data. This is in contrast to figure 114, which shows good agreement between the shoik velocity curves of the calculation and the data for the initial part of the attenuation. The $\delta t-x$ plot for figure 114 is shown in figure 110 .

Plots of $\tilde{x} t$ versus $x$ and shock velocities versus $x$ for experiment RS-2308 are shown in figures 115-122. Figure 115 shows that the calculation using the Rice equation of state again predicts too much attenuation of the shock wave. The calculation using the Mie-Gruneisen equation of state in which $\gamma \rho$ is constant is shown in figure 117 and does a better job of matching the data. This calculation is very similar to the calculation using the Gray equation of state shown in figure 121 , but these calculations now seem to be showing a little too much attenuation. The best job of matching the data of RS-2308 with a calculation is seen in figure 119. The calculation in this figure uses the Tillotson equation of state.

\section{C. Additional Modeling}

A!l the calculations shown thus far have been done with the parameters of the strength models and the equations of state fixed by zero pressure data and principal Hugoniot data. No attempt has been made to adjust any of the parameters to make the calculations fit the shock-attenuation data. The calculations in 
section $4 \mathrm{~B}$ were done to show the general behavior of the material models in the codes and to give some insight in determining what may need improvement.

It must be remembered that these experiments follow the attenuation of a shock wave over a limited pressure range. Maximum pressure in any of the experiments is not above $150 \mathrm{GPa}$ and the minimum pressule at a shock front is around $50 \mathrm{GPa}$. In this pressure regime the comparisons between the calculations and the experimental data showed some interesting trends. Within the limited modeling done in section $4 \mathrm{~B}$, quite different behavior is seen in the stainless-steel experiments as compared to the copper experiments. Qualitatively, the results of the iast section indicate that the combination of a strength model with the equation of state that gives the greatest isentropic bulk modulus along the principal Hugoniot does the best job in matching the data of the stainless-steel experiments. In the copper experiments this is not the case. The general trend seen in the copper experiments is to go to material models that give lower wave velocities as the peak pressures in the experiments increase. This response suggests the copper may be in a melt phase in experiments RS-2314 and RS-2308. If the copper is not in a mixed phase region on the principal Hugoniot, then it may well be melting on the release isentrope. This would agree with work reported on the phase line of copper. $^{45}$

The possibility of a material melting on the principal Hugoniot or on a release 
isentrope points out a rather glaring deficiency in three of the four equations of state discussed in chapter 2 . Of the equations of state discussed in that chapter only the Gray equation of state takes into account the phase transfo:mation of melting. Also the Gray equation of state is the only one that explicitly includes electronic effects, although in a relatively simple fashion. None of the calculations using the Gray equation of state showed the copper entering a melt region. This could be because the Gray equation of state uses an approximation of the Lindemann law for melting and for some materials this melting law has been found to give rather high values for the pressure at which melting begins on the principal Hugoniot. ${ }^{4 \mathrm{C}}$

The other aspect of the modeling that is lacking is the treatment of the rigidit $y^{\prime}$ modulus and the yield strength. In the code calculations performed in the last section, the strength parameters were simply treated as constants whenever a strength model was employed. On the basis of elasticity theory, it is probably a better assumption to consider the rigidity modulus as being proportional to the bulk modulus. In many high pressure experiments, increases in the rigidity modulus as well as the yield strength have been reported. ${ }^{47-49}$ Also some account of the reduction of the yield strength along an isobar as the specific internal energy or temperature increases should be considered. A good example of a more comprehensive treatment of strength parameters is given in reference 49 . 
Before actually invoking any new material models it is interesting to explore in greater detail the interplay of the simple strength models and the equation of state. While many experiments have been done that report values of the yield strength and the rigidity or shear modulus at high pressure, it is important to remember that in most cases the values that are reported are dependent on the equation of state used to interpret the data or upon other assumptions made about the bulk response of the material. In analyzing such experiments, the difficulty lies in trying to sort the bulk proprerties from those that give rise to deviatoric terms. The present experiments suffer from the same problem but the main problem with the present experiments is the ambigıity in the determination of the initial overtaking point. There are times when it is clear that strength models are not needed. This is the situation for experiment RS-2308 in which it is fairly obvious that no strength model is needed to explain the attenuation of the shock wave in the copper target. In making this statement, the implicit assumption is made that one of the equations of state is correctly mc eling the bulk response of the material.

In the modeling performed in section $4 \mathrm{~B}$ in which the rigidity modulus and the yield strength were treated as constants with values appropriate for ambient conditions, it was seen that only one of the four equations of state given in chapter II gave what can be considered as adequate agreement with the experimental data 
for stainless steel. If the rigidity modulus and the yield strength are now treated as adjustable parameters then better agreement between the data and calculations using the other equations of state can be obtained. This then is a curve-fitting exercise that is intended to give insight into the interplay of the constitutive equations for strength and the equations of state.

An example of changing the values for the strength parameters to achieve better agreement between the calculations and the data of experiment RS-2302 is shown in figures 123-125. For the three calculations in these figures, the strength rnodel is the same as that used previously. The Tillotson equation of state was used to model bulk response in each calculation. Ir each succeeding figure the yield strength in the calculation has been increased until a reasonably good fit to the data has been achieved. Shown in figures 126-128 are comparisons of the shock velocity plots for these calculations and the experiment. The interesting item is the behavior of the shock velocity in these new calculations as revealed in figures 126-128. As the yield strength increases, the elastic-plastic plateau or shoulder occurs at a lower value of the shock velocity. There is also the appearance of additional structure in the shock velocity curve beyond the plateau region that becomes more pronounced as the yield strength increases.

The influence of the increased yield strength can be split into two principal effects. The first effect is that in the simple-centered release wave, the elastic 
part of the ielease wave encompasses a larger drop or change in the normal stress and the density. For the strength model used in these calculations, the change in drisity arosis the elastic part of the release wave is given by

$$
\Delta \rho \quad Y_{\mu}^{\prime} \rho_{1} .
$$

The second effect is related to the first but now brings into play the plastic part of the release wave. Because of the increased yield strength and the increased attenuation of the shock wave by the elastic part of the release wave, there is a greater portion of the wave in seraction region that will support elastic wave speeds. For a release wave overtaking a shock wave moving to the right, this will mean that a $u+c$ characteristic of the rarefaction wave that has a plastic wave speed will encounter earlier that portion of the wave interaction region that supports elastic wave speeds. This in turn attenuates the shock at a faster rate.

In the work of section $4 \mathrm{~B}$, the calculations using the Tillotson equation of state produced the poorest agreement of any of the equations of state with the data of the stainless-steel experiments. The plots in figures 123-125 show that by adjusting the values of the strength parameters, the calculational modeling using the Tillotson equation of state can be made to fit the data of RS-2302 reasonably well. Although the comparison of the data and the calculation in figure 125 is pretty good, one might be hestitant to say that the comparison of 
the shock velocities in figure 128 is as good. When the same type of adjustinent of the strength parameters is done for the calculations for experiments RS-2315 and RS-2309 that use the Tillotson equation of staie, there is better agreement shown between the calculations and the data in the shock velocity plots than in figure 128 for experiment RS-2302. Figure 129 shows $\delta t-x$ plots of the calculational results and the experimental data for RS-2315. The shock velocity from the calculation and the shock velocity from the pin data are shown in figure 130 . Both the $\delta t$ data and the shock velocity from the pin data have been matched fairly well by the calculation. Another example of forcing a better match to the data by adjusting the strength parameters is shown in figures 131 and 132 for RS-2309. The calculation shown in these figures was also done using the Tillotson equation of state.

In order to achieve a closer match between the data of the stainless-steel experiments and the calculations using the Tillotson equation of state some rather large increases in the yield strength have been needed. The rigidity modulus has not been increased by more than a factor of two but the yield strength has been increased by more than an order of magnitude. The same type of curve fitting that has been done using the Tillotson equation of state can be done with any of the other equations of state. When this is done it is found that the adjustment of the yield strength to force a fit to the data is dependent on the equation of state. A 
specific $2 x a m p l e$ of this is shown in figures 133-138. All the calculations shown in these figures use the equation of state in which the Gruneisen parameter is given by equation $(2.41)$. It is significant that in order to get the close match to the data in these new calculations the yield strength has been reduced by a factor of two as compared to the ralculations using the Tillotson equation of state. The fart that the rigidity or shear modulus has essentially the same values as those used in the calculations with the Tilltoson equation of state just reflects the uncertainty in the determination of the overtaking point $i$ these experiments.

These examples of adjusting the values of the parameters of the strength models to gain better agreement between the calculations and the experimental data, while to some extent are exercises in curve fitting, do show how the choice of an equation of state can influence the values of the parameters of the strength model. While the values of the rigidity modulus and the yield strength have been changed, at no time were the values of the parameters in the equations of state changed. In the calculations of section $4 \mathrm{~B}$ where the deviatoric strength was always less than one percent of the total stress, there was little influence of the strength model on the calculation of the principal Hugoniot when it is based on the equation of state and the strength model. When the yield strength is allowed to go as high as $4.76 \mathrm{GPa}$, the deviatoric stress can be nearly four or five percent of the total stress at the shock front in the pressure regime of interest in these 
experiments. This large yield strength can cause a significant change or shift in the calculation of the Hugoniot. An example of this is given by considering a 100 GPa shock in stainless steel using the Tillotson equation of state. For the purely hydrodynamic case, the values for the density, specific internal energy, shock velocity, and particle velocity on the Hugoniot are, respectively, $10.16 \mathrm{~g} / \mathrm{cc}$, $1.546 \mathrm{GPa}-\mathrm{cc} / \mathrm{g}, 7.196 \mathrm{~km} / \mathrm{s}$, and $1.243 \mathrm{~km} / \mathrm{s}$. If the yield strength is now increased to $4.5 \mathrm{GPa}$, then the values for the same quantities are respectively, $10.39 \mathrm{~g} / \mathrm{cc}$, $1.515 \mathrm{GPa}-\mathrm{cc} / \mathrm{g}, 7.268 \mathrm{~km} / \mathrm{s}$, and $1.231 \mathrm{~km} / \mathrm{s}$. Although the deviatoric stress is $3 \%$ of the total stress when the yield strength is $4.5 \mathrm{GPa}$, none of the other quantities change by $3 \%$. The density and particle velocity each decrease by about $1 \%$. The internal energy decreases by about $2 \%$ and the shock velocity increases by about $1 \%$. In general, principal Hugoniot data is thought to be good to about $1 \%$. Thus the influence of such a high yield strength is just great enough that one should consider making changes to the equations of state to allow for the increased yield strength; however, no changes will be made to the equations of state at this time.

In yet other experiments ${ }^{50}$ where a material has been both released and reshocked from the same high pressure state, there is some hope in trying to sort the bulk properties from the deviatoric porperties; but even these experiments have their problems. Most experiments at pressures greater than $10 \mathrm{GPa}$ that investigate strengths of materials have been done using wave propagation tech- 
niques. Whenever these techniques are employed, the interpretation of the data is again dependent on the equation of state and other aspects of the constitutive modeling. For the present experiments not all corstitutive models pertinent to the examination or modeling of the experiments will be investigated. Such possible material response as viscoelasticity and viscoplasticity will be ignored.

It would be desirable if a single equation of state and strength model could be found that fits all the stainless-steel and copper data. Up to now the constitutive equations for strength have been added to the hydrodynamic model in a rather ad hoc fashion. There has been no attempt to relate the strength parameters to the equation of state in any manner except to treat the strength parameters as constants. The first problem that arises is to find a model that relates the strength parameters to the equation of state in a consistent manner. One such model that does this is given by Steinberg, Cochran, and Guinan. ${ }^{49}$ An alternative to that model is to generalize linear elasticity theory. For an isotropic material, linear e'asticity theory gives a relation between the rigidity modulus and the isothermal bulk modulus:

$$
\mu=\frac{3}{2}\left(\frac{1-2 \nu}{1+\nu}\right) K
$$

In this equation $\nu$ is Poisson's ratio and $K$ is the isothermal bulk modulus. Equation (4.12) is appropriate for small isothermal deformations. The analogue of equation (4.12) for the propagation of waves in a linear elastic medium occurs 
when the isentropic bulk modulus is substituted for the isothermal bulk modulus in equation (4.12). Because the isentropic bulk modulus can be directly calculated from the equation of state, equation (4.12) becomes

$$
\mu=\frac{3}{2}\left(\frac{1-2 \nu}{1+\nu}\right) \rho c^{2}
$$

where $c^{2}$ is given by equation (2.23). In doing this, $\nu$ in equation $(1.13)$ can now be iden:ified as the isentropic value of Poisson's ratio. To continue with this model it is necessary to relate che yield strength to other parameters. The yield strength can be related to the rigidity modulus by assuming that the ratio of the yield strengtis to the rigidity modulus is constant:

$$
Y=Y_{0} \frac{\mu}{\mu_{0}}
$$

This equation is taken from reference 49 when work hardening and strain rate effects are ignornd. If the yield strength increases at a faster rate than the rigidity modulus, ti.en equation (4.14) can be changed to

$$
Y=Y_{0}\left(\frac{\mu}{\mu_{0}}\right)^{b}
$$

In equation (4.15), the parameter $b$ may be assumed to be a constant. With these equations for $\mu$ and $Y$, the strength parameters are intimately connected to the equation of state.

The problem of treating thermal effects and how they should be incorporated in the constitutive equations for strength still remains. Two different approaches 
may be taken with respect to the calculation of temperature. Temperature may be directly incorporated into the equation of state as is done in the Gray equation of state or a temperature may be calculated from the equation of state solely for use in calculating the parameters $\mu$ and $Y$ in the strength model. Obviously in the latter case, the phenomenom of melting cannot be properly treated with an "ipe thom of state hased only on a single phase of the material. In this latter situation, the temperature is being used as a means to turn off strength effects as the Lemperature approaches the melting temperature. When the temperature is not dirctly calculated in the equation of state, then a separate reference curve such is a zero-degree isotherm must be found to allow the calculation of temperature at higher densities and pressures.

One manner of incorporating temeperature effects in the strength model is to ussume that $\nu$ in equation (4.13) depends on the temperature. One requirement of the strength model is that $\mu$ and $Y$ should be zero when the material is at the melt temperature. This implies that $\nu$ should be one-half when the temperature $T$ is equal to $T_{m}$, the temperature at which melting begins. This in turn suggests that $\nu$ has a functional dependence on the ratio of $T$ to $T_{m}$ :

$$
\nu=\nu\left(\frac{T}{T_{m}}\right)
$$

The melting temperature, $T_{m}$, can be calculated from one of the melting formulas discussed in appendix C. Equation (4.16) may be expanded in a power series but 
a particularly simple form for $\nu$ may be written as

$$
\nu \nu_{1}+\nu_{2}\left(\begin{array}{c}
T \\
T, \ldots
\end{array}\right)^{x}
$$

Equation (4.17) is intended to approximate the general behavior of Poisson's ratio.

If there is sufficient isobaric data, one can assume a truncated powar series for equation (4.16) and calculate the coefficients for the power series. If there is not sufficient data, then equation (-1.17) should qualitatively discribe the behavinof $\nu$. While a material might show a highly skewed dependence of $\nu$ on the temperature at zero pressure there is the possiblity that $\nu$ might exhibit a more linear dependence at higher pressures. Thus one could make the exponent in equation (4.17) dependent on the pressure.

In order to evaluate the parameters $\nu_{1}$ and $\nu_{2}$ in equation (4.17), one needs to know the value of ihe exponent, $I$, and an initial vaule of $\nu$. If $\nu$ has an initial value of $\nu_{0}$ at the temperature $T_{0}$, then $\nu_{1}$ and $\nu_{2}$ must satisfy the following:

$$
\nu_{1}+\nu_{2}=\frac{1}{2}
$$

and

$$
\nu_{1}+\nu_{2}\left(\frac{T_{o}}{T_{m}}\right)^{x}=\nu_{0}
$$

The difficult problem lies in trying to determine the value of the exponent, $x$, in equation (4.17). A metal such as a steel might show less than a $1 \%$ change in $\nu$ for 
a temperature rise of $100 \mathrm{~K}$. This might represent a 20 to $30 \%$ change in the ratio of the temperature to the melting temperature. Thus for steel, a minimum value for $x$ is probably in the range of 3 to 4 . Longitudinal ar, $i$ tranverse sound speed data for $\operatorname{copper}^{51}$ indicate a minimum value for $x$ that is in the same range. These values for $x$ are taken from data measlired under ambient pressure conditions.

Examples of calculations using a strength model given by equations (4.13), (4.15), and (4.17), are plotted against the appropriate stainless-steel experiment in f.gures 139-144. The equation of state used for this set of calculations was the one by Rice given by equation (2.42). This strength model has a significant dependence on the calculation of Poisson's ratio, which in turn depends on the calculation of the temperature and the melting temperature. The melting temperature was calculated using the Kraut-Kennedy melting formula discussed in appendix C. Instead of using a value of 2.9 for $A$ in equation(c11) as suggested in the appendix, a higher value of 4.9 was used. The reason for doing this was that work on iron gives the intersection of the phase boundary of the soild with the principal Hugoniot at around $240 \mathrm{GPa}^{52}$ If the stainless steel hits the melting region in the same pressure regime, then a value of 2.9 for $A$ is too low. For this equation of state and a value of $A$ in the Kraut-Kennedy formula of 4.9, melting begins on the principal Hugoniot at abust $220 \mathrm{GPa}$. Equally important in this strength model is the calculation of the temperature. The temperature for equation (4.17) was 
calculated from equation (2.51). The cold curve or zero-degree isotherm needed for equation (2.51) and the room-temperature isotherm needed for the KrautKennedy melting formula were both directly calculater from the Rice equation of state. The value for $c_{v}$ in equation (2.51) was the Dulong and Petit value and the value of $g$ for stainless steel was taken to be $1.74 \times 10^{-8} \mathrm{GPa}-\mathrm{cc} /\left(\mathrm{K}^{2}-\mathrm{g}\right)$. After calculating both $T$ and $T_{m}$ one still needs the exponents for equation (4.17) and (4.15). Instead of using an exponent of 3 or greater, a value of 2 was used for $x$ in equation (4.17). A value of 1.5 was used for $b$ in equation (4.15).

The plots in figures 139-144 show that this model for the strength parameters does a fairly good job of matching the data for the stainless-steel experiments. In the previous calculations using constant values for $\mu$ and $Y$ it was found that these parameters had to be adjusted for each experiment in order to obtain a reasonably good fit between a calculation and the data. In this new model for the strength parameters, some fine-tuning of the modei can be achieved by adjusting the exponents in equations (4.15) and (4.17). When $x$ is changed in equation (4.17), $\nu_{1}$ and $\nu_{2}$ must also be adjusted according to equations (4.18) and (4.19). In this model for the strength parameters the magnitude of the yield strength can be controlled to some extent by the value of $b$ in equation (4.15). Thus if the equation of state is changed and it is found that it is necessary to increase the value of the yield strength, this can be accomplished by increasing the value of $b$. 
When the Kraut-Kennedy formula was used for the calculation of the melting temperature in the code calculations shown in figures 139-144, it was not possible to match both the zero pressure data for the phase line and still have the phase linte intersect the principal Hugoniot of stainless-steel at $220 \mathrm{GPa}$. The melting formula given by equation (c16) overcomes this problem. It is clear from the manner in which this strength model is formulated that the yield strength and the rigidity modulus are sensitive to the calculation of Poisson's ratio. When the melting formula is changed, this of course has an influence on the calculation Poisson's ratio an 1 hence the rigidity modulus and the yield strength. The effects "f changing from the $\ddot{r}$ raut-Kennedy melting formula to equation (c16) are shown in figures 145-150. In general the two melting laws produce about the same results hut when equation $(c 16)$ is used for the melting law, the value of Poisson's ratio is slightly higher. The examination of the appropriate tables shows that the use of equation(c!6) moves the overatake position about $0.1 \mathrm{~mm}$.

Although the melting formula does have an effect on the calculations, a relatively larger effect is seen when the exponent in equation (4.17) is changed. When this exponent for the calculations shown in figures 145-150 is changed from 2 to 1, there is a significant increase in the value of Poisson's ratio along the principal Hugoniot. This produces lower values of the rigidity modulus and the yield strength. Examples of using a value of 1 instead of 2 for the exponent in equation 
(4.17) are shown in figures 151-156. The comparisons show that the match to the data is actualiy a little better for the intermediate pin levels of experiment RS-2302 when the exponent is 1 instead of 2; however, for experiments RS-2315 and RS-2309 the new calculations shown in figures 153 and 155 do not agree with the data quite as well as the previous calculations.

There is one aspect of wave propagation at high pressure that has not yet been discussed. In the many experiments performed at high pressures that examine release waves, the elastic-plastic plateau or shoulder that is soclearly defined in the calculations using strength, has not been observed experimentally. ${ }^{49}$ The lack of a clear separation between the elastic and plastic regions of the release wave has been attributed to the Bauschinger effect. The Bauschinger effect ${ }^{53}$ is a phenomenom noted in stress-strain tests where there is reverse loading or cyclic loading of the test specimen. The modeling of this phenomenom in a hydodynaraics code can be quite complicated. ${ }^{54}$ Suffice it to say that there are two effects in the modeling of this phenomenom that have an influence on the present problem. The first effect is that the discontinuity in wave speeds in going from the elastic regime to the plastic regime is effectively eliminated. This wipes out the plateau region seen in the shock velocity plots. The second effect is there is more dispersion to the elastic regime of the release wave. This in turn obviates the need for the yield strengths to be as large as they are in the calculations that use the adjustment 
of the yield strength to match experimental data. For the calculations shown in figures 139-144, the modeling of the Bauschinger effect would decrease the value of $b$ from 1.5 for these calculations, but just how much this decrease would be is hard to say.

With this new strength modal for the stainless steel, attention can now be turned to the modeling of the copper experiments. Changing to the strength model of stainless steel used for the calculations in figures 145-150 does not change any of the conclusions reached previously about the copper experiments. The modeling that was done indicated that each of the separate equations of state did a fair job of matching one of the copper experiments without the need of a strength model. The Rice equation of state matched the data of experiment RS-2306 while the Gray and the Mie-Gruneisen equation of state matched the data of experiment RS-2314. For the copper experiment with the highest pressure, RS-2308, the Tillotson equation of state did the best job of matching the experimental data. The fact that a different equation of state is used in matching the data of each succeeding copper experiment and each successive equation of state gives a higher compressibility would seem to indicate that the copper is indeed undergoing a phase change.

The most difficult aspect of the modeling situation for copper is trying to include a licuid phase of the material in the equation of state. In lieu of a sat- 
isfactory procedure for doing this, parameters in the equation of state can be adjusted to achieve a match between the calculations and the experimental data. Up to this point no parameters in the equation of state have been adjusted to force an agreement between calculations and experiments. The strength parameters, $\mu$ and $Y$, have been used in this manner, but no parameters in the equations of state have been used to do this. If either the Rice or the Mie-Gruneisen equation of state is used to do this, there are only four parameters in the equation of state to work with. If the equation of state is to reproduce the principal Hugoniot, then all parameters in the Rice or the Mie-Gruneisen equation of state except one are fixed by the principal Hugoniot. The one exception is the Gruneisen parameter, $\gamma_{0}$. By adjusting this parameter for the calculations of each of the copper experiments, then the general trend for the sound speeds of the release waves in the copper experiments can be ascertained.

A particularly interesting calculational exercise can be carried out using the Mie-Gruneisen equation of state to match the data of the copper experiments. A Mie-Gruneisen form based on a linear $U_{a}-u_{p}$ relation is quite useful for doing this. What makes it so useful is that the Gruneisen parameter can be adjusted in such an equation of state without having any influence on the principal Hugoniot; that is, the principal Hugoniot derived from the equation of state is independent of the Gruneisen parameter. Using equation (2.44) for the equation of state of copper it 
is found that a value of 1.3 for $\gamma_{0}$ in a code calculation matches the data for experiment RS-2306. The experiment next higher in pressure, RS-2314, is matched yutt w.ll with a value of 20 for the cirmeisen parameter. For experiment RS2308 it is found that a value of 2.5 is needed for the Gruneisen parameter in order 10 match the data. The comparisons between these calculations and the experimental data are shown in ligures $157 \quad 162$. The material model for the stainless

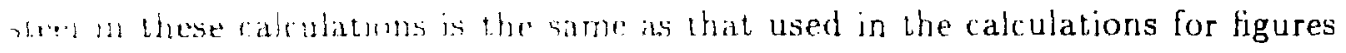
145150

\section{\D. Further Discussion}

The original goal of the experiments has been stated as being an accurate measurement of the sound speed in copper and stainless steel at high pressures. Although this primary goal was not obtained because of the scatter in the data, the experimental data proved to have sufficient resolution to be very useful for comparisons with the calculational modeling. The experiments have enough resolution and are simple enough conceptually that any model of material behavior can be checked against the present data.

Two rather general statements can be made about the behavior of copper and stainless steel at the pressures in these experiments. For the stainless steel, it appears that the modeling of strength parameters is important to shock pressures 
of at least $150 \mathrm{GPa}$ and probably higher. For the OFHC copper, strength modeling is not as important in the same pressure range; but it does appear that the copper is undergoing a phase transition to a liquid state.

The basis for making these statements about strength for stainless steel and the melting of copper is founded on comparisons of the calculational results with the experimental data. It was clear from the calculations of the last chapter that a match to the data of the stainless-steel experiments could not be done without invoking a strength model. This must be qualified in that a match could not be obtained with a purely hydrodynamic calculation unless one were willing to alter the parameters in the equation of state. An example of this is the calculation for experiment RS-2302 shown in figure 70. This calculation used a Mie-Gruneisen equation of state and a value of 2.07 for $\gamma_{0}$. There was no strength model in the calculation and the calculation did not match the experiment. If this calculation is now repeated but with a value of 0.8 for the Gruneisen parameter, a very good match to the data is achieved. The calculation for this new value of $\gamma_{0}$ is shown in figures 163 and 164 . The point that must be made is that calculations for RS-2315 and RS-2309 using the same equation of state and the same value of 0.8 for $\gamma_{o}$ do not match the experimental data. Thus a simple change of $\gamma_{0}$ from its nominal value at zero pressure in purely hydrodynamic calculations cannot account for the variability seen in the speeds of the release waves in the three experiments. By 
switching to a strength model in which the strength parameters are related to the equation of state and by using the Rice equation of state, a moderately good fit to all three stainless-steel experiments was obtained (see figures 145-150).

It is of course possible to make the argument that strength effects are not important for the stainless steel and that the lack of agreement between the experiments and the hydrodynamic calculations in which there is no strength model merely points out the deficiencies in the equations of state. There are reasons for not accepting this argument. One reason is that strength effects are seen unequivocally in static pressure experiments and low pressure dynamic experiments. Another reason for rejecting the argument that the whole problem lies with the equation of state is that the present data can be explained by using a quite reasonable strength model. As long as the material remains in the solid phase, there is no reason not to expect possible strength effects. These arguments must not be interpreted as saying there are no deficiencies $i .1$ the equations of state. There are still questions concerning the bulk response of mateirial at high pressure that must be addressed.

A principal question raised by this work is, "Which equation of state is the most appropriate for the description of a metallic solid under high pressures?". To some extent the answer to this question is subjective and much depends upon the assumptions made about such diverse quantities as the strength parameters, the 
zero-degree isotherm, phase lines, and temperatures along the principal Hugoniot.

The calculations in this chapter demonstrate the interplay of the constitutive equations for strength and the equations of state. When $Y_{o}$ was used as an adjustable parameter for the calculations, it was found that the different equations of state required different values of the yield strength in order to match the same data. If one believed that the equation of state that needed the lowest value of the yield strength was the correct or most appropriate equation of state, then one would use the Rice equation of state.

Another consideration in choosing an equation of state is the behavior of the lattice Gruneisen parameter. In both the Mie-Gruneisen and the Rice equations of state the lattice definition of the Gruneisen parameter and the thermodynamic definition are the same. In the Gray and the Tillotson equations of state the thermodynamic definition is not assumed to be a function of density only. Of the three forms (equations $(2.41),(2.43)$, and $(2.48))$ for the lattice definition of the Gruneisen parameter, the form (equation (2.48)) used for the Gray equation of state agrees the best with the work done in appendix D. On this basis alone one might choose the Gray equation of state over the others but there are other aspects of the Gruneisen parameter that are also important. Both the form given by equation (2.41) and the form in which $\gamma \rho$ is a constant give lower values of $\gamma$ at densities greater than normal density than equation (2.48). As shown in appendix 
1), the form for the Rice equation of state gives much lower values of the Gruneisen paramerer than the other forms and as a consequence the prossure and specific internal turgy along the zero-degret isother are greater for compressed states Whan along the zero-degree isotherms given by the other equations of state. As a result of the higher entergies for the zero-degree isotherm, when equation (2.51) is ised lwatrahate temperatures aloug the principal Hugoniot, the Rice equation of state gives appreciably lower values of the temperature than the other three "(p)tations of state discussed in chapter 2.

liact of the equations of state has its own idiosyncracies and arguments can he made for or against using a particular equation of state. The Rice equation of state gives lower temperatures and compressibilities along the principal Hugor iot than the other equations of state. In matching code calculations to the stainlesssteel data, the Rice equation of state needs lower values of the yield strength and the rigidity modulus than the other equations of state. Although these facts suggest that the Rice equation of state may be better for the description of a metal in a highly compressed solid state than the other equations of state discussed in section $2 \mathrm{D}$, there are other pressure regimes and thermodynamic states where a different equation of state is clearly preferable.

One area in which this is true is the region where Thomas-Fermi theory is believed to hold. A deficiency that the Mie-Gruneisen, Rice, and Gray equations 
of state all have is the density singularity in the zero-degree isotherm. This singularity is a direct result of a linear $U_{s}-u_{p}$ relation. The limit of the maximum density for these three equations of state is given by

$$
\rho=\rho_{12} \sum_{i}
$$

Because the value of $s$ for most materials is around 1.5 , equation $(4.20)$ limits the maximium density to about three times the normal density. This limit is well below the densities allowed in the Tillotson equation of state and so the Tilltoson equation of state is expected to be more appropriate for pressure regimes in the Thomas-Fermi region.

The example just presented for preferring one equation of state over another is not that pertinent to the present study but is shown for illustrative purposes. The reason that it is not relevant is that pressures in the Thomas-Fermi region are at least one or two orders of magnitude greater than the pressures generated in these experiments. It does show, however, that at times a theoretical basis for a choice about the equation of state exists. It cannot be emphasized too greatly that of the equations of state used in these calculations, there are no clearcut theoretical justifications for saying one equation of state is preferable over another in the pressure regimes of these experiments. At best, one can place an emphasis on a certain aspect of the equation of state such as the behavior of the lattice Gruneisen 
parameter and then use this as a basis for making a choice about the equation of state.

Many of the shortcomings of the equations of state have been presented in chapter 2. A farther elaboration of some of the probiems and uncertainties of the equations of state can only be discussed in the context of the manner in which the equations of state are being used. The equations of state in the computer calculations are used to give two quantities of critical importance, the pressure as a function of the density and the specific internal energy, and the bulk sound speed or isentropic compressibility as a functı'n of the same quantities. The uncertainties in the equations of state do not lie so much in the pressure as in the bulk sound speed.

The measurements in the experiments are the trajectories of shock fronts and the shock fronts are followed from maximum pressures of less than $150 \mathrm{GPa}$ to minimum shock pressures of around $50 \mathrm{GPa}$. Because of the range of pressures followed in the experiments, the states of interest in the $P$ - $\rho$ plane are never very far from the principal Hugoniot. The farthest poins of interest off the Hugoniot may be taken as a presisure of approximately $50 \mathrm{GPa}$ on a release isentrope that intersects the principal Hugoniot at $150 \mathrm{GPa}$. This holds for both the copper experiments and the stainless-steel experiments. All the equations of state faithfully reproduce the principal Hugoniot data for both copper and stainless steel 
and they all give release isentropes from $150 \mathrm{GPa}$ that are quite similar. The are some noticeable differences on the release isentropes in the $P$ - $\rho$ plane but they occur as the isentrupes approach zero pressure. It is for these reasor that one is led to the conclusion that the calcilation of the pressure from the equations of state is not a problem.

Even though all four equations of state give release isentropes in the $P$ - $\rho$ plane that agree quite well in the pressure range of these experiments, the situation for the bulk sound speed is not as good. Restricting the loci of states to be considered to the principal Hugoniot, the agreement of the bulk sound speed among the different equations of state is pretty good for pressures below $50 \mathrm{G}$ pa. The laigest difference is seen between the Rice and the Tillotson equations of state. At 50 $\mathrm{SPa}$ the difference is $1.5 \%$ for stainless steel and for copper the diffence is $1.8 \%$. As the shock pressure increaser, the difference in sound speeds between the Rice equation of state and the Tillotson equation of state increases. When the shock pressure reaches $130 \mathrm{GPa}$, the difference in the sound speed has grown to $5.3 \%$ for stainless steel and $6.5 \% \tilde{H}_{\text {- }}$ opper. It is the differencus in the bulk sound speeds when integrated over time that accounts for the very appreciable differences seen in the calculations of the attenuation of a shock wave when different equations of state are used.

All the equations of state used in this work can be considered as being semi- 
empirical equations of state since all are based on matching the principal Hugoniot of a material. This means all the equations of state will give reliable answers for shock states. The differences among the equations of state arise in the calculation of the compressibility or the bulk sound speed. This uncertainty or variation in the sound speeds actually exists as well in the calculation of the pressure from the equations of state but is masked to a certain exterit because of the semi-empirical nature of the equations of state. Consider for the moment equation (2.26). If the pressure on the principal Hugoniot is substituted for the left-hand side of equation (2.26), the known quantities are the total pressure, density, and specific internal energy along the prinicpal Hugoniot. What is not known is the correct partitioning of the right-hand side of equation (2.26) between the cold pressure, $P_{c}$, and the thermal pressure, $P_{t}$. Each equation of state has a different partition between the cold pressure and the thermal pressure and it is this aspect of the equations of state that leads to the variations seen in the sound speeds.

The general ideas of modeling strength for the calculations pertaining to these high pressure experiments are extrapolations and generalizations of the phenomenologic descriptions of elasticity and plasticity. In this respect the correct modeling of strength parameters at high pressures is much less certain than the equations of state.

Difierent models or treatments of the strength parameters have been used 
in the computer calculations. One treatment has been simply to tieat the yield strength, $Y$, and the rigidity modulus, $\mu$, as constants. The comparison of the calculational results with the experimental data has shown this to be unsatisfactory and some type of model that allows $Y^{\prime}$ and $\mu$ to vary as the pressure changes is needed. One method or treatment for the strength parameters that allows this is the model given in reference 49. Another model for the strength parameters is the generalization of the Steinberg, Cochran, and Guinan model that is given in section $4 \mathrm{C}$ as embodied in equation (4.13) through equation (4.17). In the Steinberg, Cochran, and Guinan model the rigidity modulus has a linear dependence on the pressure. In the model for the strength parameters given in section $4 \mathrm{C}$, the rigidity modulus is related to the isentropic compressibility as is done in linear elasticity. One might assume that the model in which the pressure is used instead of the compressibility in calculating the rigidity modulus would be less sensitive to the vagaries of the equation of state but this is not the case. The rigidity modulus in both models is used in calculating the longitudinal sound speed and in both models the bulk sound speed is needed for the calculation of the longitudinal sound speed. This shows that in order to match experimental data on longitudinal sound speeds, both models for the rigidity modulus are still dependent on the isentropic compressibility.

The combination of the strength model given in section $4 \mathrm{C}$ and the Rice 
equation of state has proven to give a satisfactory fit to all three stainless-steel experiments. A more stringent test of this material model is to compare it with actual sound speed data taken at high pressures. In making such a comparison no inferences can be drawn about the yield strength but by making a comparison with such data, a good quantitative check on the sound speed given by the material model can be made.

A good set of sound speed data for tantalum has been reported by Brown and Shaner. ${ }^{55}$ In order to see if the model embodied in equations (4.13)-(4.17), which has been successful in modeling the behavior of stainless steel, will be equally successful for tantalum, it is necessary to first determine the many parameters needed in the Rice equation of state and in the strength model. The parameters applicable to tantalum in the Rice equation of state will be taken as follows; $\rho_{o}=16.68 \mathrm{~g} / \mathrm{cc}, c_{o}=3.293 \mathrm{~km} / \mathrm{s}, s=1.307$, and $\gamma_{o}=1.65$. The values for $c_{0}$ and $s$ have been reported by Nellis and Mitchell. ${ }^{56}$ The value for the Gruneisen parameter is a nowinal value taken from the literature.

The next set of parameters for which values are needed are for equation $(c 16)$, which gives the melting temperature as a function of the density. The density of tantalum in the solid phase is $15.22 \mathrm{~g} / \mathrm{cc}$ at a melting temperature of $3270 \mathrm{~K}$. A value of -0.05 for $\delta_{0}$ gives excellent agreement with the experimental value of 58 $\mathrm{K} / \mathrm{GPa}$ for the slope of the phase line in the $T-P$ plane. ${ }^{57}$ The parameter, $\delta_{1}$, in 
equation (c16) determines where the phase line of the solid intersects the principal Hugoniot. The value for this parameter cannot be determined from experiments done under normal pressure conditions. If $\delta_{1}$ is assumed to be zero, then the model gives the intersection of the Hugoniot and the phase line at $219 \mathrm{GPa}$. Based on their sound speed measurements, Brown and Shaner determined the intersection of the phase line and the Hugoniot to occur at around $295 \mathrm{GPa}$. A value of 1.66 for $\delta_{1}$ give an intersection at $294 \mathrm{GPa}$.

The last piece of information that is needed has to do with the determination of the parameters in equation (4.17). It may be assumed that Poisson's ratio is linear in the ratio of the temperature to the melting temperature. This fixes the exponent in equation (4.17) and leaves only $\nu_{1}$ and $\nu_{2}$ to be determined. Any one of three pieces of data may be used to determine these parameters. The three pieces of data are the longitudinal sound speed, Poisson's ratio, and the rigidity modulus. There is a discrepancy between the bulk sound speed given by the Rice equation of state at zero pressure and the bulk sound speed of tantalum based on longitudinal and transverse sound speed measurements. The bulk sound speed derived from the longitudinal and transverse sound speed measurements is about $3.40 \mathrm{~km} / \mathrm{s}$ which is somewhat higher than the value of $3.293 \mathrm{~km} / \mathrm{s}$ for $c_{o}$ in the Kice equation of state. This implies that all three pieces of data (longitudinal sound speed, rigidity modulus, and Poisson's ratio) cannot be matched simultaneously 
at zero pressure in this model. If the measured value of the rigidity modulus is used to determine the values of $\nu_{1}$ and $\nu_{2}$, then one cannot match either the longitudinal sound speed or Poisson's ratio. If one uses a value of $69.5 \mathrm{GPa}$ for the rigidity modulus in this modei, then the calculated value for Poisson's ratio is 0.329 and the calculated value for the longitudinal sound speed is $4.04 \mathrm{~km} / \mathrm{s}$. This compares with reported values of 0.342 for Poisson's ratio and $4.14 \mathrm{~km} / \mathrm{s}$ for the longitudinal sound speed. ${ }^{58}$

The calculated sound speeds from this model are compared with the experimental data of Brown and Shaner in table 68 . Also shown in this table are sounı speeds using an exponent of $\frac{3}{2}$ in equation (4.17) instead of 1 . This produces somewhat higher sound speeds but both sets of calculated sound speeds agree farly well with the measured values. No attempt has been made to compare calculated sound speeds with the measured values in the melt region. This was not done because the model given in section $4 \mathrm{C}$ does not address any questions concerning the equation of state in the liquid region.

The greatest difficulty in treating the liquid region is knowing what the com. pressibility of the material in the liquid state should be. Few definitive statements about the equation of state in the liquid region can be ade and the statements that can be mare :se more qualitative in nature than quantitative. In many cases the assumption is made that the same equation of state for the solid phase may 
be used in the liquid phase as well at high pressures. By making this assumption, there is no change in the isentropic compressibility of the material in going from the solid phase into the mixed phase region. Some questions about the mixed phase region can be answered based on knowing the phase line of the solid. Using equation $(c 13)$ for the change in the specific entropy along the phase line of the solid, it can be determined if there should be a change in the isentropic comprussibility or equivalently the sound speed of the material on entering the coexistence or mixed phase region. If the change in the specific entropy along the boundary of the solid and the coexistence region is zero, then there is no change in the sound speed on going from the solid to the mixed phase region. If the specific entropy increases on the phase line of the solid with increasing pressure and

$$
\gamma T_{m}<\rho c^{2} \frac{d T_{m}}{d P_{m}}
$$

then the sound speed will show a discontinuity on entering the mixed phase region and will be lower than in the sulid phase. If the specific entropy change is negative and the inequality given by equation $(4.21)$ is true, then the sound speed in the mixed phase region will be greater than the sound speed in the solid phase.

It is interesting to apply these arguments to the material model that has been used for calculating the sound speeds of tantalum given in table 68 . At $297 \mathrm{GPa}$ on the principal Hugoniot the reported sound speed of tantalum is $6.37 \mathrm{~km} / \mathrm{s}$ and at $293 \mathrm{GPa}$ the reported sound speed is $6.65 \mathrm{~km} / \mathrm{s}$. If it is assumed that the state 
at $293 \mathrm{GPa}$ is a pure solid phase with almost no contribution from the rigidity modulus to the sound speed, then the model given for the solid phase of tantalum has the correct qualitative features. The tantalum data supports the idea that the specific entropy along the phase of the solid is increasing with increasing pressure. One consequence of this interpretation is that the Gruneisen parameter as defined by equation (2.28) must undergo a discontinuous change from the solid to the mixed phase region since the sound speed is undergoing a discontinuous change. Whether the Gruneisen parameter increases or decreases under this phase chinge can be determined from the work in section $2 \mathrm{E}$. When the sound speed decreases from the solid to the mixed phase region, the Gruneisen parameter must increase.

The general discussion that has been given for tantalum pertains equally well to copper. The unfortunate aspect of the present copper experiments is that the sound speeds at the peak shock pressures are not well determined and all the data for the shock wave in the target plate must be examined. In reviewing the set of calculations that are shown in figures $157-162$, the fact that $\gamma_{0}$ was increased to 2.5 in order to match the data of experiment RS-2308 certainly implies that the copper has at least partially melted in this experiment. It is intrigueing that the value of 2.5 for $\gamma_{o}$ is close to the value of the Gruneisen parameter used in the equation of state for copper in the liquid state in reference 45 . The value of the Gruneisen parameter was also increased in going from the calculation for 
experiment RS-2306 shown in figures 157 and 158 to the calculation shown in figures 159 and 160 for experiment RS-2314. However, the low value for $\gamma_{0}$ in the calculation for RS-2306 can be explained as compensating for the lack of a strength model in the calculation.

In modeling the copper experiments different conclusions may be reached depending on the form of the equation of state used to model the bulk response of copper. Now according to the Mie-Gruneisen equation of state in which $\gamma \rho$ is a constant, the modeling of the present copper experiments indicates the phase line of the solid for copper intersects the principal Hugoniot between 131 and $144 \mathrm{GPa}$. If the Rice equation of state is used to model the copper experiments, the modeling indicates that incipient melting occurs slightly before $131 \mathrm{GPa}$ on the principal Hugoniot. The conculsions that result from the modeling performed with the Mie-Gruneisen equation of state agree somewhat better with the reported value of $133 \mathrm{GPa}$ for the intersection of the phase line and the principal Hugoniot of copper than when the Rice equation of state is used; however, the modeling performed with the Rice equation of state is not inconsistent with a two-phase region beginning at 130 or $131 \mathrm{GPa}$ on the principal Hugoniot. The magnitude of the differences between the results of these two equations of state is not great but the impact on the interpretation of experiment RS-2314 is quite dramatic. For the Mie-Gruneisen equation of state, the conclusion is that the copper has not yet 
entered a mixed phase region where the liquid phase is present but is still in a pure solid phase. For the Rice equation of state the conclusion is that the peak shuck pressure has taken the copper into the melt region in this experiment.

When using the Rice equation of state a very pronounced difference is seen in the phase line of copper as opposed to the phase line of tantalum. In appendix $C$ a value of 0.56 for $b_{1}$ in equation $(c 16)$ gives the intersection of the phase line with the principal Hugoniot at approximately 140 CPa for copper. If this intersection point is now lowered to $131 \mathrm{GPa}$, then $\delta_{1}$ must be lowered to -0.11 . In the modeling of tantalum the specific entropy along the phase line is increasing with increasing pressure near the intersection point of the phase line with the Hugoniot. In copper with the value of -0.11 for $\delta_{1}$, the specific entropy along the phase line of the solid is always decreasing with increasing pressure. This would seem to imply that the sound speed might actually increase in going to the melt region but whether this happens or not depends on the inequality given in equation (4.21). For tantalum, equation (4.21) holds true, but for copper this inequality at $131 \mathrm{GPa}$ on the Hugoniot does not hold true and thus the sound speed is still expected to decrease on going from the pure solid phase to the mixed phase region.

One last sidelight to modeling with the Rice equation of state and the MieGruneisen equation of state for the copper experiments is that the expected change 
in the sound speed in going from the pure solid phase to the mixed phase region may be estimated. For the Rice equation of state the sound speed would be expected to change from about $7.5 \mathrm{~km} / \mathrm{s}$ in the solid phase to about $7.2 \mathrm{~km} / \mathrm{s}$. With the Mie-Gruneisen equation of state the change would be from about 7.2 $\mathrm{km} / \mathrm{s}$ in the solid phase to about $7.0 \mathrm{~km} / \mathrm{s}$ in the mixed phase region. When more accurate data become available, it may well be possible to distinguish which equation of state is more appropriate for copper in this pressure regime. 


\section{SUMMARY}

When the shock attenuation data are examined closely and compared with the results from the calculational modeling, different trends are seen in the decay of shock waves in stainless steel as compared against the attenuation of shork waves in copper. It is found that the experimental data for the stainless steel cannot be explained without invoking strength effects and that strength effects are important to shock pressures of at least $150 \mathrm{GPa}$. Althoug t there is no evidence of a melt phase in the experiments for the stainless steel, it can be inferred from the modeling done in section $4 \mathrm{C}$, that the intersection of the phase line of the solid. with the principal H"goniot in the neighborhood of $220 \mathrm{GPa}$ is probably a good estimate for SS-304L. In copper it is found that strength effects are not needed in order to explain the experimental results. The data for copper is consistent with copper entering a melt state on the principal Hugoniot slightly above $130 \mathrm{GPa}$. This is in substantial agreement with the value of $133 \mathrm{GPa}$ reported in reference 45. The intersection point given in reference 45 was determined from Hugoniot data taken on porous copper samples.

The experimental data has proved valuable when used in conjunction with the characteristic codes to analyze material behavior. The experimental data can be used to test any proposed material descriptions Material models have been discussed in some detail in chapters 2 and 4 . One of the most intrigueing aspects 
of this present work is the differences seen in modeling with the Rice equation of state and the modeling with the standard Mie-Grunessen equation of state. Significant differences are implied in the strength modeling depending on which equation of state is used to model bulk response. Further work still needs to be done on the equation of state of copper in the liquid state. 


\section{REFERENCES}

1 Muris hinhtir. "Some Aspects of High Pressure Research" in Proceedings of the Furth hoternutonal conference on High Presuure. (The Physico-Chemical sociely of dapin. Kyoto, 1976): pp. 15.

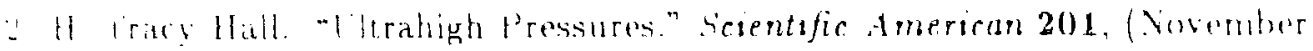
lusil He ill tio.

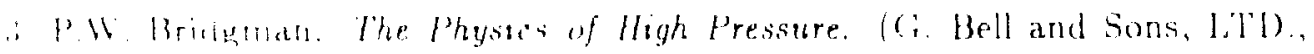

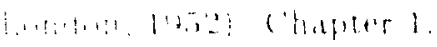

1. W. Waylor. "Thunder in the Momuains" in Shock Waves in Condensed Matter lass. J.R. Asay, R.A. Graham, and G.K. Straub, editors. (NorthHollami, Antoterdati, 1983): pap. 315.

; Ruy W. Curansun, Dennison Bancroft, Blendin L. Burton, Thodore Blechar, Filwin F. Houston, Elisabeth F. Gittings, and Stanley A. Landeen. "Dynamic hetermination of the Compressibilty of Metals." Journal of Applied Physics 26. (1)ecember 1955): pp. 1472 1479.

(i. Fdward F. Cireene and J. Peter Toennies. Chemical Reactions in Shock Wues. (Academic K'ress Inc., New York, 1964): pp. 11-12.

7. Lee Davison and R.A. Graham. "Shock Compression of Solids." Phusics Reports 55. (1979): pp. $255-379$.

Q I. V. Al'tshuler, S.B. Kormer, M.I. Brazhnik, L.A. Vladimirov, M.P. Speranskaya. and A.I. Funtikov. "The Isentropic Compressibility of Aluminum, Copper, Lead, and Iron at High Pressures." Soviet Physics JETP 11, (October 1960): pp. 766-775.

9. I..R. Veeser and J.C. Solem. "Studies of Laser-Driven Shock Waves in Aluminum." Physical Review Letters 40, (22 May 1978): pp. 1391-1394.

10. M.H. Rice, R.G. McQueen, and J.M. Walsh. "Compression of Solids by Strong Shock Waves" in Solid State Physics Vol. 6. Frederick Seitz and David Tarnbull, (Academic Press Inc, New York, 1958): pp. 1-63.

11. J.M. Walsh. Introduction to Fluid Dynamics (Class Notes). Los Alamos Vational Laboratory, Group M-4, (October 1981): pp. 100-107.

12. W.J. MacQuorn Rankine. "XV. On the Thermodynamic Theory of Waves of Finite Longitudinal Disturbance." Philosophical Transacions of the Royal Society of London 160, (1870): pp. 277-288. 
13. I.C. Skidmore. "An Introduction to Shock Waves in Solids." Appited Irate rials Research 4, (July 1965): pp. 131-147.

1. R.G. McQueen and S.P. Marsh. "Equation of State for Vinetern Metallic Elements from Shock-Wave Measurements to Two Megithars.' linural of Applied Physics 31, (Juiy 1960): pp. 1253-1269

15. John M. Walsh, Melvin H. Rice, Robert G. McQueen, and Froderick l.. Yarger. "Shock-Wave Compressions of Twenty-Seven Metals. Fuplutiuas w State of Metals." Physical Review 108, (October 15, 195T): pp. (96: :li.

16. R. Courant and K.O. Friedrichs. Supersonic Flow and shock Wauts.luterscience Publishers, Inc., New Yori, 1948): pp. 141-146.

17. R. Karpp and P.C. Chou. "The Method of Sharacteristics" i. Dynamic Response of Materials to Iniense Implusive Loading. Pei Chi Chou and Alan K. Hopkins, editors. (Air Force Materials Laboratory, Wright Patterson AFB, Ohio, 1972): pp. 283-362.

18. See chapter II in reference 16.

19. Robert D. Richtmeyer and K.W. Morton. Difference Methods for InitialValue Problems, second edition. (Inlerscience Publishers, New York, 1967): pp. 293-295.

20. Ya.B. Zel'dovich and Yu.P. Raizer. Physics of Shock Waves and High-Temperature Hydrodynamic Phenomena Vol. II. (Academic Press, New York, 1967): pp. 693-694.

21. E.B. Royce. "The Gruneisen Equation of State" in Hysics of High Energy Density, Proceedings of the International Sciool of Physics Enrico Fermi. P. Caldirola and H. Knoepfel, editors. (Academic. Press, New York, 1971), pp. $80-86$.

22. Albert Haug. Thzoretical Solid State Physics Vol. 1. (Perginon Press, Oxford, 1972): p. 448.

23. L.A. Girifalco. Statistical Physics if Muterials, (John Wiley and Sons, New York, 1973): pp. 59-61.

24. L.V. Al'tsiuler. "Use of Shock Waves inHigh Pressure Physics." Soviet Physics USPEKHI 8, (July-August 1965): pp. 52-91.

25. M.H. Rice "Pressure-Volume Relations for the Alkaii Metals from ShockWave Meialcanirements." Journal of the Physics and Chemistry of Solids 8, (1965): pp. 483-492. 
26. T. Neal. "Dynamic Determination of the Gruneisen Coefficient in Aluminum Alloys for Densities up to $6 \mathrm{Mg} / \mathrm{m}^{3}$." Physical Review $B$ 14, (15 December 1976): pp. 5172-5181.

27. J.H. Tillotson. Metallic Equations of State for Hypervelocity Impact. General A tomic report GA-3216, (July 18, 1962).

28. N.H. March. "The Thomas-Fermi Approximation in Quantum Mechanics." Advances in Physics, A Quarterly Supplement of the Philosophical Magazine 6, (January 1957): pp. 1-101.

29. R.P. Feynman, N. Metropolis, and E. Teller. "Equations of State of Elements Based on the Generalized Fermi-Thomas Thenry." Physical Review 75, (May 15, 1949), pp. 1561-1573.

30. E.B. Royce. Gray, a Three-Phase Equation of State for Metals. Lawrence Livermore National Laboratory report, UCRL-51121, (September 3, 1971).

31. William O. Wray. Analysis of Hot Metal Alloys with the Modified Gray Equation of State. Systems, Science, and Software report, SSS-R-78-3521, (December 1977).

32. R.G. McQueen, S.P.Marsh, J.W. Taylor, J.N. Fritz, and W.J. Carter. "The Equation of State of Solids from Shock. Wave Studies" in High-Velocity Impact Phenomena. Ray Kingslow, editor. (Academic Press, New York, 1970): pp. 293-417.

33. Wlliam irager. Introduction to Mechanics of Continuua. (Dover Publications, New York, 1973).

34. Duane C. Wallace. Themoelastic-Plastic Fow in Solids. Los Alamos National Laboratory report, LA-10119, (June 1985).

35. Y.C. Fung. Foundations of Solid Mechanics. (Prentice-Hall, Inc., Englewood Cliffs, New Jersey, 1965).

36. George E. Mase, Schaum's Outline of Theory and Problems of Continuum Mechanics. (McGraw-Hill Book Company, New York, 1970).

37. James B. Kelly. "Plasticity." Physics Today 4, (March 1951): pp. 17-23.

38. Hilda Geiringer. "Ideal Plasticity" in Mechanics of Solids III, volume VIa/3 of the Encyclopedia of Physics. C. Truesdell, editor. (Springer-Verlag, Berlin, 1973): p. 405 and p. 411.

39. L.D. Landau and E.M. Lifshitz. Theory of Elasticity. (Pergamon Press, London, 1959): pp. 13-15. 
40. P. Germain and E.H. Lee. "On Shock Waves in an Elastic-Plastic Solid." Journal of the Mechanics and Physics of Solids 21, (1973): pp. 359-382.

41. G. E. Duvall. "Shock Waves in Condensed Media" in Physics of High Energy Density, Proceedings of the International School of Physics Enrico Fermi. P. Caldirola and H. Knoepfel, editors. (Acadenic Press, New York, 1971): pp. 23-28.

42. Richard D. Dick and Thomas E. Gould. "Two Electrical Contactor Pin Designs." The Review of Scientific Instruments 36, (February 1965): pp. 143145.

43. Terry R. Gibls and Alphonse Popolato, editors. LASL Explosive Property Data. (University of Caiifornia Press, Berkeley, California, 1980).

44. Wildon Fickett and William C. Davis. Detonation, (University of California Press, Berkeley, California, 1979): pp. 16-20.

45. R.G. McQueen, W.J. Carter, J.N. Fritz, and S.P. Marsh. "The Solid-Liquid Phase Line in $\mathrm{Cu}^{\prime \prime}$ in Accurate Characterization of the High-Pressure Environment, (NBS, Gaithersburg, Maryland, 1968): pp. 219-227.

46. J.M. Brown and J.W. Shaner. "Rarefaction Velocities in Shocked Tantalum and the High Pressure Melting Point ${ }^{n}$ in Shock Waves in Condensed Matter1989. J.R. Asay, R.A. Graham, and G.K. Straub, editors. (North-Holland, Amsterdam, 1983): pp. 91-98.

47. Donald R. Curran. "Nonhydrodynamic Attenuation of Shock Waves in Aluminum." Journal of Applied Physics 34, (September 1963): pp. 2677-2685.

48. L.V. Al'tshuler, M.I. Brazhnik, and G.S. Telegin. "Strength and Elasticity of Iron and Copper at High Shock-Wave Compression Pressures." Zhurnal Prikladnoi Mekhaniki I Tekhnicheskoi Fiziki, english translation 12, (1971): pp. 921-926.

49. D.J. Steinberg, S.G. Cochran, and M.W. Guinan. "A Constitutive Model for Metals Applicable at High-Strain Rate." Journal of Applied Physics 51 , (March 1980): pp. 1498-1504.

50. J. Lipkin and J.R. Asay. "Reshock and Release of Shock-Compressed 6061-T6 Aluminum." Journal of Applied Physics 48, (Januarry 1977): pp. 182-189.

51. Yong-Shan Austen Chang. Dilation Contribution to Heat Capacity of Met_is and Alloys. dissertation, University of California, Berkeley, (1963).

52. J.M. Brown R.G. McQueen. "Meltir; of Iron under Core Conditions." Geophysical Research Letters 7, (July 1980): pp. 533-536. 
53. See refrerences 35 and 36 , also reference 49 .

54. W.C. Moss and L.A. Glenn. "A Bauschinger Effect Model Suitable for Use in Large Computer Codes" in Shock Waves in Condensed Matter-1989. J.R. Asay, R.A. Graham, andi G.K. Straub, editors. (North-Holland, Amsterdam, 1983): pp. 133-136.

55. J.M. Brown. (private communication), see also reference 46 .

56. A.C. Mitchell and W.J. Nellis. "Shock Compression of Aluminum, Copper, and Tantalum." Journal of Applied Physics 52, (May 1981): pp. 3363-3374.

57. John W. Shaner, G Roger Gathers, and Camille Minichino. "Thermophysical Properties of Liquid Tantalum and Molybdenum." High Temperatures-High Pressures 9, (1977): pp. 331-343.

58. Gene Simmons and Herbert Wang. Single Crystal Elastic Constants and Calculated Aggregate Properties: A Handbook, second edition. (The M.I.T. Press, Cambridge, Massachusetts, 1971).

59. J.M Walsh. On Nonlinear Plane Waves in Metal Plates (Pulse Attenuation and Pulse-Free Surface Interactions). General Atomic report, GAMD-2115, (March 10, 1961).

60. Mark W. Zemansky. Heat and Thermodynamics, fifth edition. (McGraw-Hill Book Company, New York, 1968): pp. 375--377.

61. B.I. Bennett. Melting Under Shock Compression. Los Alamos National Laboratory report, LA-8567-MS, (october 1980).

62. Edgar A. Kraut and George C. Kennedy. "New Melting Law at High Pressures." Physical Review 151, (11 November 1966): pp. 668-675.

63. Edgar A. Kraut and George C. Kennedy. "New Melting Law at High Pressures." Physical Review Letters 16, (4 April 1966): p. 608.

64. J.C. Slater. Introduction to Chemical Physics. (McGraw-Hill Book Company, New York, 1939): pp. 238-240.

65. J.S. Dugdale and D.K.C. MacDonald. "The Thermal Expansion of Solids." Physical Review 89, (February 15, 1953): pp. 832-834.

66. Stanley P. Marsh, editor. LASL Shock Hugoniot Data. (University of California Press, Berkeley, California, 1980): p. 214. 


\section{APPENDICES}

\section{A. Characteristic Codes for One-Dimensional Flow}

Two computer codes were written to specifically help in analyzing and modeling the present experiments. The codes are based on applying differen. s techniques to the equations for the characteristic directions and their associated compatibility equations. The main difficulty in using computer cocies based on the method of characteristics is that they often require much specialized logic. The advantage of characteristic codes is that they can treat discontinuities, such as shocks, exactly. The codes were written to solve the problem of shock attenuation in a semi-infinite target plate impacted by a thin flyer plate. The target is assumed tc occupy the space $x>0$. The codes use a constant time step technique. $\dagger$ In this technique, initial conditions are specified at, different spatial positions or grid points but at the same initial time. A time step is then calculated which is used in updating the flow variables and the state variables at each grid point along the characteristic direction given by equation (2.17). A new time step is then calculated to update the variables at each grid point, and thus the problem is advanced in time.

The same basic ideas and differencing techniques appertain to both the code

† The basic idea of using a constant time step technique in a charactristic code was taken from reference 17. 
SHKSIM and the code SHKSJB but there are some fundamental differences. The code SHKSIM is more specialized than the code SHKSJB and cannot handle anything but the hydrodynamic problem of a simple-centered release wave overtaking a shock wave. This means the code SHKSIM cannot handle the problem when the flyer plate is a different material from the target plate or when the flyer plate is the same material but has a different initial thermodynamic state than the target. The main aspect of the code SHKSIM, that the code SHKSJB does not have, is that the code SHKSIM can follow as many of the right-going characteristics given by equation (2.18) through the wave interaction region as needed. Tne code SHKSJB cannot follow more than two of the $u+c$ characteristics in the release wave.

In solving the problem of a simple-centered release wave overtaking a shock wave, the code SHKSIM makes extensive use of Riemann integrals. The rightgoing characteristics and all values for quantities at grid points throughout the release wave can simply be calculated from equations (4.1)-(4.3) and the characteristic equation (2.18). Thus the assumption that the problem can be treated as a simple-centered release wave overtaking a shock means that the initial configuration (that is, the configuration at the moment the lead chracteristic of the release wave just catches ihe shock front) can be calculated without resorting to finite differencing techniques applied to the characteristic equations and the 
compatibility equations. To farther calculate the time evolution of the problem, the code SHKSIM resorts to the same type of differencing techniques as the code SHKSJB.

The code SHKSJB is more versatile than the code SHKSIM in that it can do the problem of a dissimilar material impacting the target and it can also handle problems with material strength. The main disadvantage of the code SHKSJB is that finite differencing techniques are applied to the problem the moment the flyer plate makes contact with the target plate. In the code SHKSIM such techniques are not employed until the release wave actually overtakes the shock wave. Because of this difference the code SHKSJB takes more computer time to run a problem than the code SHKSIM.

The structure of the coding in which strength effects are modeled is slightly different and more complicated than the purely hydrodynamic case. Since the coding for the hydrodynamic problem is so similar in hoth SHKSIM and SHKSJB, it will be discussed first. After the shock wave has been overtaken by the release wave, there are just two principal algorithms that need to be considered. One of these algorithms updates the variables at each material or grid point while the other algorithm updates the trajectory of the shock front in the target.

For updating variables at material grid points, the characteristic direction given by equation (2.17) is followed. Consider at time $t^{0}$ a grid point at the 
spatial position $x_{j}^{0}$. The superscript will refer to a time index and the subscript to a spatial index. The material or particle velocity at the grid point is $u_{j}^{u}$. The density and specific internal energy are $\rho_{j}^{0}$ and $E_{j}^{0}$. The pressure and sound speed can be calculated from the equation of state. For a time step given by $\Delta t$, the new time is

$$
t^{1}=t^{0}+\Delta t
$$

A simple forward differencing scheme is used to update the position of the grid point:

$$
x_{j}^{1}=x_{j}^{0}+u_{j}^{0} \Delta t
$$

This is the difference equation associated with equation (2.17). To find the new material velocity, use is made of equations (2.18) and (2.19) and their associated compatibility equations (2.21) and (2.22). For a point to the left of $x_{j}^{0}$, a rightgoing characteristic can be found that eminates from the point $x_{l}$ and ends at the point $x_{j}^{1}$. The difference equat:on for the right-going characteristic is

$$
x_{l}+\left(u_{l}+c_{l}\right) \Delta t=x_{j}^{1} .
$$

If the nearest grid point to the left of $x_{j}^{0}$ is $x_{i}^{0}$ then a simple linear inerpolation formula can be used to calculate a particle velocity and sound speed at the point $x_{l}$. The interpolation formula for $u_{l}$ is given by

$$
u_{l}=\frac{\left(u_{j}^{0}-u_{i}^{0}\right)\left(x_{l}-x_{i}^{0}\right)}{\left(x_{j}^{0}-x_{i}^{0}\right)}+u_{i}^{0} .
$$


A similar equation is used to calculate $c_{l}$. Using these interpolation formulas, squation $(a 3)$ can be solved for $x_{l}$. For a point to the right of $x_{j}^{0}$, a left-going characteristic can be found that emanates from the point $x_{\tau}$ and ends at the point $x_{j}^{1}$. The difference equation that corresponds to equation $(2.19)$ is

$$
x_{r}+\left(u_{r}-c_{r}\right) \Delta t=x_{j}^{1} .
$$

Linear interpolation can be used to find $u_{r}$ and $c_{r}$ in terms of $x_{r}$ and then equation (ai) can be used to solve for $x_{r}$.

To find the new particle velocity at the grid point $x_{j}^{l}$, it is necessary to invoke the compatibility equations. Along the right-going characteristic, a forward differencing equation that corresponds to equation (2.21) can be written:

$$
P_{j}^{1}-P_{l}=-\rho_{l} c_{l}\left(u_{j}^{1}-u_{l}\right)
$$

Since $x_{l}$ is known, linear interpolation can be used to solve for the pressure, density, sound speed, and particle velocity at $x_{l}$. Along the left-going, the difference equation analogous to equation $(2.22)$ is

$$
P_{j}^{1}-P_{r}=\rho_{r} c_{r}\left(u_{j}^{1}-u_{r}\right)
$$

Subtracting equation (a7) from equation $(a 6)$ one arrives at the following equation for the new particle velocity:

$$
u_{j}^{1}=\frac{\rho_{l} c_{l} u_{l}+\rho_{r} c_{r} u_{r}+P_{l}+P_{r}}{\rho_{r} c_{r}+\rho_{l} c_{l}}
$$


Once the new particle velocity is found, it is an easy matter to use either equation (a6) or (a7) to solve for the pressure at the new grid position $x_{j}^{1}$.

It only remains to calulate the new values of density and specific internal energy. The fact that the new pressure at $x_{j}^{1}$ is known, can be used to find the new density and specific internal energy. From the equation of state, any infinitesimal change in pressure can be written as

$$
d P=\left(\frac{\partial P}{\partial \rho}\right)_{E} d \rho+\left(\frac{\partial P}{\partial E}\right)_{\rho} d E
$$

When the characteristic equation (2.17) is followed, the compatibility equation given by equation (2.20) must hold. Since the beginning and ending pressure for the material point $x_{j}$ are known, then equations $(a 9)$ and $(2.20)$ can be considered as a system of first-order partial differential equations which may be solved for $d \rho$ and $d E$. An algorithm using a fourth-order Runge-Kutta method is employed to solve for $d E$ and $d \rho$.

In tracking shock fronts, the code SHKSJB must follow two shock fronts while the code SHKSIM must track only one shock. Both codes use an impedance-match solution for finding the shock conditions in the target and the flyer but because of the initial conditions that are assumed in the code SHKSIM, the solution is considerably more simple. The code SHKSIM only follows the shock front in the target plate and the shock velocity is constant until the shock is overtaken by the 
release wave. In the code SHKSJB, the left-going shock in the flyer plate must be followed as well as the right-going shock wave in the target plate. Because SHKSJB allows for nonuniform initial conditions, numerical techniques for advancing the shocks are employed from the time the flyer impacts the target. The following descripition of the algorithm for advancing the right-going shock in the target is essentially the same for both codes. The one difference is that the state of the material ahead of the shock is uniform in the code SHKSIM while the code SHKSJB allows for a nonuniform state.

The algorithm for the advancement of the shock keeps track of the shock velocity and, of course, the position of the shock front. The algorithm also tracks the particle velocity, density, and specific internal energy immediately ahead of the shock and immediately behind it. The advancement of the shock front employs a forward differencing scheme similar to the main algorithm for the advancement of the position of a grid point. At time $t^{0}$, the position of the shock front is $x_{s}^{0}$. At time $t^{1}$ the new position of the shock front is given by

$$
x_{s}^{1}=x_{s}^{0}+U_{s}^{0} \Delta t
$$

where $U_{a}^{0}$ is the shock velocity at time $t^{0}$.

The new position of the shock front is the starting point for updating all other quantities at the shock front. The next step is to update the particle velocity, 
density, and specific internal energy immediately ahead of the shock front to the new position of the shock. The algorithm for doing this follows the algorithm for updating a material grid point and does not involve the shock-jump or RankineHugoniot equations.

After the quantities immediately ahead of the shock front have been updated, it is necessary to find the new values of the same quantities immediately behind the shock front. For a right-going shock wave, only $u+c$ characteristics from points to the left of the shock wave can overtake the shock. Neither $u$ characteristics nor $u-c$ characteristics from any point to the left of the shock front can intercept the shock front. A right-going characteristic can be found that emanates from a position, $x_{\text {, that }}$ is to the left of the old position of the shock front and ends at the new position of the shock front. This is given by the equation

$$
x_{a}^{1}=x_{l}+\left(u_{l}+c_{l}\right) \Delta t
$$

When linear interpolation is used to write $u_{l}$ and $c_{l}$ in terms of $x_{l}$, equation (a11) can be used to solve for $x_{l}$. The compatibilty equation that holds along this right-going characteristic can be written as

$$
P_{s}^{1}-P_{l}=-\rho_{l} c_{l}\left(u_{s}^{1}-u_{l}\right)
$$

where $P_{s}^{1}$ and $u_{s}^{1}$ are, respectively, the pressure and particle velocity at the new position of the shock front. The quantities $P_{l}, \rho_{l}, u_{l}$, and $c_{l}$ are all known from 
linear intrepolation. There are now five unknowns at the shock front, the shock velocity, the particle velocity, the pressure, the density, and the specific internal energy. Equation ( $a 12$ ) and the Rankine-Hugoniot equations form a system of four equations. To find the shock and particle velocities at the new shock pusition, a fifth equation must be used. This fifth equation is the equation of statc.

To solve for the new values at the shock front let $x$ be the new shock velocity at $x_{d}^{1}$. The quantities subscripted with a will refer to the known quantities immediately ahead of the shock front. The quantities subscripted with $b$ will refer to the new unknown variables immediately behind the shock front. The quantities with the subscript ! are the same ones as those defined in equations $(a 11)$ and (a12). On the basis of the shock-jump conditions, four relations can be written:

$$
\begin{gathered}
"_{b}=u_{a}+\frac{P_{l}-p_{a}+\rho_{l} c_{l}\left(u_{l}-u_{a}\right)}{\rho_{a}\left(\chi-u_{a}\right)+\rho_{l} c_{l}}, \\
\rho_{b}=\rho_{a} \frac{\chi-u_{a}}{\chi-u_{b}}, \\
E_{b}=E_{a}+\frac{P_{a}}{\rho_{a}} \frac{u_{b}-u_{a}}{\chi-u_{a}}+\frac{1}{2}\left(u_{b}-u_{a}\right)^{2},
\end{gathered}
$$

and

$$
P_{b}=P_{a}+\rho_{a}\left(\chi-u_{a}\right)\left(u_{b}-u_{a}\right) .
$$

As stated previously, the necessary fifth equation to solve for the five unknowns is the equation of state in which the pressure, $P_{b}$, is written as a function of the density, $\rho_{b}$, and the specific internal energy, $E_{b}$. 
The solution for the new value of the shock velocity, $\chi$, and hence the other quantities, proceeds by defining a new function $g(\chi)$ which is the pressure given by the equation of state substracted from equation (a16):

$$
y(x)=P_{b}-P\left(\rho_{b}(x), E_{b}(x)\right)
$$

Starting with the old value of the shock velocity, a Newton-Rhapson search method can be applied to equation $(a 17)$ to find the new value of the shock velocity. Then the new values for other quantities can be found from the shock-jump conditions.

The method of characteristics can still be used when strength is modeled but the differencing equations become a little more involved. When strength is being modeled there is a fourth equation governing the flow. The difficuliy lies in the fact that this fourth equation has abrupt changes in it depending on whether the flow is in an elastic regime or a plastic one. In an elastic regime the fourth equation is given by an equation such as equation (2.92) or (2.93) and in a plastic regime the fourth equation is one such as equation (2.76). With the modeling of strength effects, the characteristic directions are given by the equations

$$
\begin{gathered}
\frac{d x}{d t}=u, \\
\frac{d x}{d t}=u+c_{\ell},
\end{gathered}
$$

and

$$
\frac{d x}{d t}=u-c_{\ell}
$$


When there are four equations governing the flow, there should be four characteristic directions. When strength is modeled, equaticn (a18) is actually a double root of the solution for the characteristic directions. The terni $c_{2}$ in these equations is not the bulk sound speed but rather the lengitudinal sound speed. Again the term $c_{\ell}$ depends on whether the flow is elastic or plastic. For flow in an elastic regime, $c_{\ell}$ is given by equation $(2.100)$ or equation (2.101) while for flow in a plastic regime, uquation (2.102) is appropriate for $c_{\ell}$.

When strength is modeled there are still only three distinct characteristic directions to deal with which are the same as in the hydrodynamic problem, but the compatibility equations are increased to four in number. These equations are altered by the addition of the deviatoric stress term. Along the characteristic direction given by equation (a18) there are now two compatibility equations. One equation is for the specific internal energy and is given by

$$
d E=\frac{\left(P+\tau_{n}\right)}{\rho^{2}} d \rho
$$

The other equation is for the change in the deviatoric stress and is given by an equation such as equation (2.92) when the flow is ir an elastic region. If allowance is made for a variable yield strength, then the compatibility equation for equation (a18) in a plastic flow region is

$$
d \tau_{n}= \pm \frac{2}{3} d Y
$$


The plus sign is appropriate when the deiviatoric stress is at an upper yield limit and the minus sign applies when the deviatoric stress is at a lower yield limit. The compatibility equation for equation $(a 19)$ is

$$
d P+d \tau_{n}+\rho c_{\ell} d u=0
$$

and for the last characteristic equation, the compatibility equation is

$$
d P+d \tau_{n}-\rho c_{\ell} d u=0
$$

The obvious complication in the compatibility equations is the addition of the stress deviator and the question of when tise flow is in a plastic regime or in an elastic one. One interesting sidelight about writing the compatibility equations in this manner is that there is no additional conceptual difficulty in assuming that $\mu$ and $Y$ may be functions of $\rho$ and $E$ as opposed to assuming they are constants.

With the inclusion of strength, the code also keeps track of the stress deviator at each material point or grid point as well as the other hydrodynamic variables such as density, specific internal energy, and pressure. The procedure for updating the variables at a grid point begins in the same manner as in the hydrodynamic case. The new position of the grid Frint is found by using equation $(a 2)$. The code then uses forward differencirg equations $(c 19)$ and $(a 20)$ and linear interpolation tc find the interpolation points $x_{l}$ and $x_{r}$. Difference equations appropriate for equations ( $a 23$ ) and $(a 24)$ are then used to find the rew particle velocity. Next 
the new total stress is found from these equations. It must be einphasized that the new stress is now known, neither the new pressure nor the new value of the stress deviator is known. At this point the code checks to see if the flow is such that the change in the total stress for this material point will be an elastic flow or not. If the flow is not elastic, then equation $(a 22)$ is used to calculate the change in the stress deviator. If the yield strength is constant, then there is no change in the stress deviator and knowing the new total stress is equivalent to knowing the new value of the pressure. In this situation, the same type of Runge-Kutta algoithm as for the hydrodynamic case can then be used to solve for $d \rho$ and $d E$. If the yield stiength is no: constant or the flow is elastic, a Runge-Kutta method can still be used. The reason such a method can still be employed is that in either of these two cases, $d \tau_{n}$ may be written as

$$
d \tau_{n}=g(\rho, E) d \rho .
$$

Of course in writing $\boldsymbol{d} \tau_{n}$ in this manner, the assumption is being made that if $Y$ and $\mu$ are not constants they may be assumed to be functions of $\rho$ and $E$.

In advancing the shock front in the strength case, the assumption is made that the shock process always takes a material to a stress $s$ tate such that the stress deviator is at the upper yield limit. Witin this assumption, the stress deviator at the shock front is always given by

$$
\tau_{n}=\frac{2}{3} Y
$$


The attenuation process for shocks occurs in the sarne manner as in the hydrodynamic case except it is now complicated by tho effects of material strength. The new position of the shock front is given by equation (a10). The compatibility equation for the right going characteristic that overtakes the shock is

$$
P_{s}^{1}+\frac{2}{3} Y-P_{l}-\tau_{l}+\rho_{l} c_{l}\left(u_{s}^{1}-u_{l}\right)=0 \text {. }
$$

The interpolation point $x_{l}$ is found from equation (a11) where $c_{l}$ is calculated as an elastic sound speed. Equation (a27), the equation of state, and the RankineHugoni' 。 equations in which the pressure has been replaced by the normal component of the stress tensor form a system of equations which is used to solve for the new shock velocity at the new postion of the shock front. The system of equations is formulated in the exact same manner as in the hyurodynamic case so that a Newton-Rhapson search may be used : find the new value of the shock velocity.

The codes SHKSIM and SHKSJE are written in Fortran. Listings of the codes can be obtained by writing to

William B. Harvey

Box 1663

M.S. F664

LANL

Los Alamos, N.M., 87545. 


\section{B. An Analytical Solution for Shock Attenuation}

To check on both the logic and accuracy of the computer codes SHKSIM and Siiis SJB, results from the codes can be compared to an analytical solution ${ }^{59}$ of the problem of a shock being attenuated by a release wave. To arrive at this exact solution, the problem must be carefully formulated. Consider a homogeneous material initially at rest that occupies the half-space $x \geq 0$. At time $t=t_{1}$, a pressure is instantaneously applied to the material boundary at $x=0$. This sudden application of pressure produces a shock wave of constant velocity in the body. Assumirig the material obeys equation (2.4) relating shock velocity to particle velocity, then equations $(2.1),(2.2)$, and $(2.3)$ can be used to solve for the oth.er thermodynamic and kinematical variables if any one of the five variatles, $\rho, E$, $P, U_{s}$, or $u_{p}$, is given. These steady-state conditions are maintained until $t=0$, when the pressure at the material boundary is reduced to zero. This introduces a rarefaction that eventually overtakes and decays the strength of the shock wave. The position of the shock wave and the pressure and particle velocity of any point in the body can be found analytically at any time if the flow equations are written in terms of Lagrangian cocrdinates, and the proper equation of state is chosen. This problem is directly analogous to the problem of a thin flyer plate striking a target plate, provided the flyer and the target plate have the same equations of state and the same initiai dorsities and specific internal energies. 
The equation of state that must be used for this analytical solution is the Walsh equation of state. ${ }^{59}$ It is interesting to note that although Walsh found an analytical solution for the problem of shock attenuation, he never invoked the specific internal energy and in fact did not need an equation of state giving $P$ as a function of $\rho$ and $E$. Rather, he postulated that the isentropic compressibility is given by

$$
\rho^{2} c^{2}=\rho_{0}^{2} c_{0}^{2}+4 s \rho_{o} P
$$

To obtain results irom the computer codes, $P$ must be given as a function of $\rho$ and $E$. From equation (bl), an equation of state can be formulated. Equation (b1) can be integrated along an isentrope to give the pressure as a function of the density:

$$
P=\frac{\rho_{o} c_{o}}{4 s}\left[A \exp \left(-\left(\frac{4 s \rho_{o}}{\rho}\right) i-1\right]\right.
$$

In equation ( 22$), A$ is a constant of integration that varies from isentrope to isertrope. The constant $A$ can be evaluated from the fact tisat equation $(b 2)$ must agree with equation (2.5) when the isentrope intersects the principal Hugoniot; thus,

$$
A=\left(\frac{1+s \eta_{h}}{1-s \eta_{h}}\right) \exp \left(\frac{4 s \rho_{o}}{\rho_{h}}\right)
$$

where $\eta_{h}$ is given by

$$
\eta_{h}=1-\frac{\rho_{o}}{\rho_{h}}
$$


and $\rho_{h}$ is the density at which the isentrope intersects the Hugoniot. To find the intersection density $\rho_{h}$, the specific internal energy can be integrated along the isentrope from the point $\rho, E$ to the point $\rho_{h}, E_{h}$ where $E_{h}$ is the specific internal energy at which the isentrope intersects the principal Hugonict. This leads to the following equation fo- $n_{t}$.

$$
\begin{aligned}
2 s \eta_{h}+\left(\frac{2 s \eta_{h}}{1-s \eta_{h}}\right)^{2} & -\left(\frac{1+s \eta_{h}}{1-s \eta_{h}}\right)^{2}\left(1 \cdots \exp \left(4 s\left(\eta-\eta_{h}\right)\right)\right) \\
& =2 s \eta+8 E\left(\frac{s}{c_{v}}\right)^{2} .
\end{aligned}
$$

Once $\eta_{h}$ is found from equation (h5), then $A$ and hence $P$ are easily calculated. The equation of state algorithm used in the codes uses an iterative scheme to calculate $\eta_{h}{ }^{r}$ 'm equation (b5).

The test problem chosen for checking the code was a flyer plate $1.5-\mathrm{mm}$ thick impacting a $20-\mathrm{mm}$ thick target plate of the same material. Physical parameters appropriate for SS-30 re used in the equation of state. Problems were run using two different impact velocities for the flying plate and these velocities approximately bracketed the actual velocities of the flying plates seen in the experiments. One set of calculations was run using an impact velocity of $3.7 \mathrm{~km} / \mathrm{s}$ and another set of calculations was run using a velocity of $4.65 \mathrm{~km} / \mathrm{s}$. An examination of the effects of zoning or distance between material points was also made.

A comparison between a numerical solution fiom the code SHKSIM and the analytical solution for shock attenuation is shown in figure 163. The particular 
problem shown is for an impact velocity of $3.7 \mathrm{~km} / \mathrm{s}$ for the flyer plate. The initial zoning used for the code calculation was $0.05 \mathrm{~mm}$ between points. The plot shows that the numerical results from SHKSiM are in excellent agreement with the analytical solution. For this problem, the overtaking point or the point at which the lead characteristic of the release wave intersects the shock wave occurs at $9.486 \mathrm{~mm}$. There is of course exact agreement between the code calculation and the analytical solution on the value of the overtaking point. For the intersection of other characteristics with the shock wave the agreement between the numerical solution and the analytical solution is no longer exact but it is still very good. For a shock position of $20 \mathrm{~mm}$, the $\delta t$ value from the numerical solution is approximately $0.3 \mathrm{~ns}$ less than the $\delta t$ value from the analytical solution.

Although the plot is not shown, the agreement between the numerical solution from SHKSIM and the analytical solution is equally as good when the impact velocity of the flying plate is raised to $4.65 \mathrm{~km} / \mathrm{s}$. There is a slight increase in the difisence between the $\delta \dot{t}$ values at $20 \mathrm{~mm}$ but only by about $0.1 \mathrm{~ns}$. All the colculatiuns performed in chapter 4 using the code SHKSIM had finer zoning than the calculation shown in figure 163 . The initial zoning for the calculations in chapter 4 using the code SHKSIM! was $0.025 \mathrm{~mm}$. From the test problems it was found that when the distance between the points was cut in halî, the difference or the discrepancy between the $\delta t$ values of the numerical solution and the analytical 
solution was roughly cut in half. Because of the fine zoning used for the SHKSiM calculations in chapter 4 and the good agreement between code results and the analytica! solutions shown here, the numerical errors for the calculations in figures 71-87 are probably negligible.

The test calculations using the code SHKSJB do not show as good agreement with the analytical solutions as the calculations using SHKSIM. Undoubtably, this occurs because of the difference in the initial or starting conditions for the two codes. In the code SHKSIM, the code starts from the point at which the lead characteristic of the release wave has just intersected the shock. For the code SHKSJB, the entire evolution of the release wave must be numerically calculated by applying a finite differencing scheme to the system of characteristic equations. An example comparing the numerical results from SHKSJB to an analytical solution is plotted in figure 164 . The test problem for this figure is the same as that in figure 163. The initial zonia. for the code calculation is $0.05 \mathrm{~mm}$ between material points. The difference in the $\delta t$ values at a shock position of $20 \mathrm{~mm}$ is approximately $6 \mathrm{~ns}$, which is a considerably larger discrepancy than the result from SHKSMi. In going to a greater velocity of $4.65 \mathrm{~km} / \mathrm{s}$ for the flyer plate the difference between the code result and the analytical solution at $20 \mathrm{~mm}$ grows to approsimately $8 \mathrm{~ns}$. When the zoning is made finer for the code SHKSJB, there is better agreement beiween the code results and the analytical solutiun but the 
agreement is still not as good as that seen by using the code SHKSIM. Figure 165 shows the results for the test problem with an impact velocity of $4.65 \mathrm{~km} / \mathrm{s}$ but one in which the initial zoning for the code calculation has been decreased from $0.05 \mathrm{~mm}$ to $0.025 \mathrm{~mm}$. The difference in the $\delta t$ values at a shock position of 20 $\mathrm{mm}$ is now about $4 \mathrm{~ns}$. The difference between the numerically calculated shock velocity and the shock velocity from the analytical solution as a function of shock position can be seen in figure 166 . The numerical results shown in this figure are from the same code calculation shown in figure 165.

In the calculations performed in chapter 4 using the code SHKSJB, the width between point for the initial zoning was never greater than $0.025 \mathrm{~mm}$. Whenever a calculation involved a strength model, the initial width of tine zoning was decreased to $0.02 \mathrm{~mm}$. From these results, the numerical effects of the zoning on the calculations can be estimated. A conservatire estimate is the effects of the zoning are never greater than $5 \mathrm{~ns}$ in any of the $\delta t$ plots based on the computer calculations of chapter 4. Effects this great would only occur at or around the 20-mm level. For calculations er.ding at a $16-\mathrm{mm}$ shock position, the effect of the zoning would be less than 3 ns. The initial overtaking point in the SHKSJB calculations shows almost no dependence on the initial zoning as long as the flyer p'ate has uniform initial conditions. 


\section{Melting on the Principal Hugoniot}

In section $2 F$ the effects of strength were described in terms of an elasticplastic model of a solid Central to this model was the concept of a yield strength. Once a material has melted it will permanently deform under any shear stress no matter how small. This implies the material has zero yield strength. If the peak shuck pressure is above the pressure on the principal Hugoniot at which melting occurs, then one would imagine that the hydrodynamic description of the shock attenuation process would be valid.

Shock processes are not isentropic and irreversible work is associated with the shocking of any material. After a material has released from a shocked state to zero or ambient pressure, this irreversibie work is manifested as a heating of the material. The shock heating leaves the material with a residual temperature $T_{r}$ which is greater than the initial temperature $T_{o}$. The rise in temperature also leaves the material with a density $\rho_{r}$, which for normal materials is less than the initial density $\rho_{o}$.

Iwo different thermodynamic paths can be considered in reaching the final terr perature $T_{r}$. If the initial pressure can be taken as zero, then a zero-pressure isobar can be considered as the first path. Along this path the change in the specific internal energy in going from the temperature $T_{o}$ to the temperature $T_{r}$ 
is given by

$$
\Delta E=\int_{T_{r}}^{T_{r}} c_{p} d T
$$

where $c_{p}$ is the specific heat at constant pressure. The second path can be broken into two steps. The first step is the shock itself and the second step is the subsequent release to zero pressure. The energy change associated with the shock can be found from equation (2.3). Since the release process is isentropic, the change in the specific internal energy in going from a density $\rho$ on the principal Hugoniot to the final density $\rho_{r}$ is given by

$$
\Delta E_{2}=\int_{\rho}^{\rho_{\mathrm{r}}} \frac{P}{\rho^{2}} d \rho
$$

If $\Delta E_{1}$ is the the change in the specific internal energy due to the shock, then the following equation must hold:

$$
\Delta E=\Delta E_{1}+\Delta E_{2}
$$

By means of equation (c3), any final state on the zero-pressure isobar can be related to a state on the principal Hugoniot.

For most solid materials, the melting point increases with increasing pres:ure. For such materials, equation ( $c 3$; and a simple equation of state such as equation (2.44) can be used to calculate a minimum pressure on the principal Hugoniot below wiicis one would expect the substance to always remain in the solid phase. 
Let $T_{m o}$ be the melting temperature for a material at zero pressure. The maximum change in the specific internal energy that can be tolerated by the material without entering into a mixed phase region can be determined from equation $(c 1)$ with $T_{\mathrm{r}}$ replaced by $T_{m o}$. By knowing $\Delta E, \rho_{r}$, the principal Hugoniot, and the equation of state, it is possible to calculate a pressu:e on the principal Hugoniot that results in the final or residual temperature $T_{m o}$. This calculation is sensitive to the equation of state and the parameters used in the equation of state. In table 69 are listed some results of calculating pressures on the principal Hugoniot that produce the residual temperature $T_{m o}$. The pressures that are calculated for the copper used in these experiments are in the same range as the experimental pressures. For the stainless steel, the calculated pressures are higher than the pressures generated in these experiments.

The pressures calculated in table 69 are only an estimate of where melting might be imnortant in the release stage. To see if melting might occur in the high pressure state, it is necessary to extend the solid-liquid phase lines and determine where they intersect the principa! Hugoniot. A simple first step is to use the Clapeyron-Clausius formula. Along the solid-liquid phase line in the $P-T$ plane one has that

$$
\frac{d_{i}}{d T}=\frac{\Delta S}{\Delta v}
$$

where $\Delta S$ and $\Delta v$ are the change in the specific entrupy and specific volume on 
melting at constant presiure. The easiest assumption to make is that the slope of the phase line is constant. This implies that

$$
d P=B d^{\prime} T
$$

where $B$ is nuv a constant. Liquation $(c 5)$ can be integrated to yield the melting temperature as function of pressure:

$$
T_{m} T_{m n}+\frac{p}{B}
$$

It is now necessary to know the temperature along the principal Hugoniot in order to determine the intersection point between the phase line (equation(c6)) and the principal Hugoniot.

To salculate temperatures along the principal Hugoniot consider ine specific entropy sxpressed in terms of the specific volume and temperature. The thermodynamic equation is

$$
T d S=c_{v} d T+T\left(\frac{\partial S}{\partial v}\right)_{T} d v
$$

Using equation (2.28) for the definition of the Gruneisen parameter, equation ( $c 7$ ) may be written as

$$
T d S=c_{v} d T+c_{s} T \frac{\gamma}{v} d v
$$

Equation $(c 8)$ is to be integrited along the principal Hugoniot. The left-hand side of equation $(c 8)$ can be found from equation (2.55) and for a material that 
obeys a linear $U_{3}-u_{p}$ relation, $d P$ along the principal Hugoniot can be found from equation (2.5). Thus, along the principal Hugoni t equation (c8) becomes

$$
\frac{\rho_{0} c_{0}^{2}}{\rho^{2}} \frac{s \eta^{2}}{(1-s \eta)^{3}}=c_{u} d T-c_{v}{ }^{T} \frac{\gamma \rho}{\rho^{2}} d \rho
$$

Equation $(k 9)$ can be integrated numerically; but, before it can be integrated, the functional form for the Gruneisen parameter and the specific heat must be specified. Using equation (2.43) for the Gruneise: parameter and the classical Dulong and Petit value for the specific heat at ronstant volume, the phase line for copper intersects the ?rincipal Hugoniot above $400 \mathrm{GPa}$. When the assumption is made that the percentage volume change and the entropy change are constant on melting, the phase line for copper intersects the principal Hugoriot at a considerably lower pressure of approximately $230 \mathrm{GPa}$. Effecis of the electren gas can be included by writing the specific heat in equation ( $c 9)$ as in equation (2.51). When this is done the calculated temperatures along the principal Hugoniot are lowered and the intersection of the phase line and the principal Hugoniot occurs at a higher pressure.

Yet another way of exterding the phase line in the $P-T$ plane is to use the Lindemann equation for meliing. ${ }^{60,61}$ The Lindemann formula can be expressed as

$$
T_{m}=A \rho^{2 / 3} \Theta^{2}
$$


where $A$ is a material constant and $\Theta$ is the Debye temperature. If $\Theta$ and $\rho$ are known, then the melting temperature can be determined from equation (clu). The results given in appendix D can be used to find the Debye temperature as a function of $\eta$. Because the internal energy along the principal Hugoniot is known as a function of $\eta$, it is possible to calculate the temperature on the principal Hugoniot. Figures 167 and 168 are temperature calculations for copper and stainless steel based on the results given in appendix D. In general the temperature calculations based on the results of appendix $D$ give a higher temperature for a given Hugoniot pressure than does a temperature calculation based on equation (c8). The melting curve given by equation $(c 10)$ and temperature calculations based on appendix $\mathrm{D}$ predict that melting begins on the principal Hugoniot at about $180 \mathrm{GPa}$ for copper and at about $250 \mathrm{GPa}$ for stainless steel.

Another melting formula that has had some success describing melting is the Kraut-Kennedy law. ${ }^{62,63}$ The formulation of this melting law relates the melting temperature to the compression on the room-temperature isotherm. It is given by the equation

$$
T_{m}=T_{m o}(1+A \eta)
$$

$T_{m o}$ is the melting temperature at zero pressure. The parameter $A$ is a constant characteristic of the material in question. The term $\eta$ in equation $(c 11)$ is given by equation (2.6) but $\rho$ is now the density on the isotherm at room temperature 
for a given pressure and thus $\eta$ in equation $(c 11)$ is actually a function of the pressure.

Since $\eta$ is to be tren along the isotherm at room temperature, knowledge of $\Delta S$ and $\Delta v$ at melting allows the determination of the constant $A$ in equation (c11). From these considerations the value of $A$ can be approximated as

$$
A \approx \frac{\rho_{o} c_{o}^{\wedge}}{T_{m o}} \frac{\Delta v}{\Delta S}
$$

If values of $33 \mathrm{~K} / \mathrm{GPa}$ and $31 \mathrm{~K} / \mathrm{GPa}$ are used for $\Delta v / \Delta S$ for copper and stainless steel, respectively, then $A$ for copper is 3.4 and $A$ for stainless steel is 2.9 . The value of $T_{\mathrm{m} \text { o }}$ for copper is $1356 \mathrm{~K}$ and for SS-304L a value of $1700 \mathrm{~K}$ was used. When electronic effects are included, the copper phase line intersects the principal Hugoniot at about $140 \mathrm{GPa}$. For the stainless steel the intersection occurs at about $175 \mathrm{GPa}$.

Yet another melting formula can be derived by using equation $(c 8)$. For the solid along the melting boundary, equation (c8) can be written as

$$
T_{m} \frac{d S}{d \eta}=c_{v} \frac{d T_{m}}{d \eta}-c_{v} T_{m} \frac{\gamma \rho}{\rho_{o}}
$$

In equation (c13) all derivatives are taken along the phase line of we solid. Both $c_{v}$ and $\gamma$ are known for the solid. It is informative to rewrite equation $(c 13)$ as

$$
\frac{1}{T_{m}} \frac{d T_{m}}{d \eta}=\frac{\gamma_{\rho}}{\rho_{0}}+\frac{1}{z_{v}} \frac{d S}{d \eta}
$$


Now make the assumption that the following expansion may be made for the mtinpy of the solid along the phase line:

$$
\frac{1}{c_{v}} \frac{d S}{d \eta}-\delta_{0}+2 \delta_{1}\left(\eta \cdots \eta_{0^{i}}\right)
$$

The expansion has been taken around $\eta_{0}$, where $\eta_{0}$ is the value for the solid at melting under normal conditions. With $\gamma$ given by equation $(2.41)$, equation $(c 14)$ may now be integrated to yield

$$
T_{m}=T_{m 0} \frac{1+\gamma_{0} \eta}{1+\gamma_{0} \eta_{0}} \exp \left(\delta_{0}\left(\eta-\eta_{0}\right)+\delta_{1}\left(\eta-\eta_{0}\right)^{2}\right)
$$

The inclusion of two constants in equation (c15) allows for a good deal of flexibility in fitting the data. The constant $\delta_{0}$ aan be chosen from knowing the values of $\Delta S$ and $\Delta v$ at the normal melting temperature and then $\delta_{1}$ can be chosen on the basis of the intersection of the phase line with the principal Hugoniot. For copper with a value of -0.07 for $\delta_{0}$ and a value of 0.56 for $\delta_{1}$, equation $(c 16)$ predicts an intersection of the copper phase line with the principal Hugoniot at 140 GPa. For the stainless steel with values of -1.09 for $\delta_{0}$ and 3.31 for $\delta_{1}$, equation (c16) prericts an intersection of the phase line and the prinripal Hugoniot at 200 GPa. If the phase line for SS-304L intersects the principal Hugoniot at $220 \mathrm{GPa}$, then $\delta_{1}$ must be changed from a value of 3.21 to 3.89 . The temperature on the Hugoniot was calculated using the cold curves from the Rice equation of state and equation (2.51) to calculate the temperature. 
Perhaps the most satisfying way of calculating the pressure on the principal Hugoniot at which melting begins would be to know the equation of state for the liquid phase as well as the solid phase. By knowing the equation of state for the liquid state, one could formulate the Gibbs free energy for each phase and thus calculate the phase lines. McQueen and others used experimental data on porous copper to empirically formulate an cquation of state for the liquid phase of copper. ${ }^{45}$ Based on this equation of state, they estimated that melting would begin on the principal Hugoniot for OFHC copper at $133 \mathrm{GPa}$. This pressure is within the range of the present experiments. Both the Kraut-Kennedy and the Rice melting formula (equation $(c 16)$ ) agree fairly well with this value. 


\section{The Gruneisen Parameter and the Cold Curve}

When a solid is modeled as a collection of harmonic oscillators, then the Ciruneisen parameter can be defined by equation (2.38). In Debye theory all irequencies are assumed to be independent of temperature or specific internal energy. This means that the maximum or cutoff frequency postulated in Debye theory may be substituted for $\nu_{i}$ in equation (2.38). Next the question arises about the relation between the maximum frequency and bulk properties. Three prevalent relations that express the Debye cutoff frequency, $\nu_{x}$, in terms of quantities on the cold compression curve can all be expressed by the relation ${ }^{24}$

$$
\nu_{x} \propto \rho^{(q-2) / 3}\left(\rho^{2} \frac{d}{d \rho}\left(P_{c} \rho^{-2 q / 3}\right)\right)^{\frac{1}{2}} .
$$

In equation $(d 1) P_{c}$ is the pressure on the cold compression curve or zero-degree isotherm.

With $\nu_{x}$ replacing $\nu_{i}$ in equation (2.38), the equation for the Gruneisen parameter can be written as

$$
\gamma=\frac{\eta}{2} \frac{\left(P_{c}(1-\eta)^{2 q / 3}\right)^{\prime \prime}}{\left(P_{c}(1-\eta)^{2 q / 3}\right)^{\prime}}
$$

In this equation the single prime represents the first derivative of the quantity within the brackets with respect to $\eta$ and the double prime is the second derivative. When $q=0$ in equation $(d 2)$, the equation gives the Slater formula ${ }^{64}$ for the 
Grumeisen parameter. When $q=1$ and $q=2$, the equation gives lite DugdaleMcDonald formula ${ }^{65}$ and the "free-volume" formula respectively for the Gruneisen parameter.

Equations $(2.33),(2.64)$, and $(d 2)$ can be used to form a system of equations that can be numerically integrated to determine the cold curve and the Ciruneisen parameter at high pressure. The system of equations is

$$
\begin{gathered}
E_{c}^{\prime}=\frac{P_{H}}{\rho_{o}}-B\left(E_{H} \cdots E_{c}\right), \\
G^{\prime}=2 B-\frac{2}{3} \frac{q-2}{1-\eta}
\end{gathered}
$$

and

$$
B^{\prime}=\left(\frac{c_{H}^{2}}{(1-\eta)^{2}}-E_{c}^{\prime \prime}\right)\left(E_{H}-E_{c}\right)^{-1}-B^{2}
$$

In equation $(d 3)-(d 5)$ the prime again denotes differentiation with respect to $\eta$. All variables subscripted by $H$ refer to quantities on the principal Hugoniot. The quantity $B$ is given by the equation

$$
B=\frac{\gamma}{1-\eta}
$$

and the variable $G$ is given by

$$
G=\ln \left(A E_{c}^{\prime}(1-\eta)^{2 q / 3}\right)^{\prime}
$$

where the parameter $A$ is a constant. 
Before this system of equations can be integrated, it is necessary to specify intial values for the quantities appearing in these equations. The initial value for $\therefore H$ is $\Sigma_{1}$ as given in equation $(2,4)$. The thermodynamic definition of the Gruneisen parameter can be used to find the initial value for it and hence the initial value for $B$. The initial value for $E_{c}$ is a little more difficult to calculate. The reference energy can chosen such that $E_{M}$ has an initial value of zero. This choice for $E_{I I}$ implies that the initial value for $E_{c}$ will be given by the equation

$$
E_{c}=-\frac{3 N k \Theta}{a_{w}} D_{E}\left(x_{D}\right)
$$

In equation $(d 8) D_{E}$ is the Debye energy function, $a_{w}$ is the atomic weight, $N$ is Avogadro's number, and $\Theta$ is the Debye temperature.

The variable for which it is difficult to calculate an initial value is $E_{c}^{\prime \prime}$. Instead of trying to calculate $E_{c}^{\prime \prime}$ on the basis of zero-pressure isobar data, $E_{c}^{\prime \prime}$ can be calculated from equation $(d 5)$ if the initial value of $B^{\prime}$ is known. The initial value $B^{\prime}$ can be estimated from zero-pressure data or values of $B^{\prime}$ can be calculated based on assumptions about the initial behavior of the Gruneisen parameter. One assumption that can be made is that the derivative of $\gamma$ with respect to $\rho$ is zero at $\rho=\rho_{o}$. In this case one would have that $B^{\prime}$ has an initial value equal to $B$. A second assumption that can be made is that the derivative of $\gamma \rho$ with respect to $\rho$ or $\eta$ is zero at $\rho=\rho_{\mathrm{o}}$ in which case the initial value for $B^{\prime}$ would be zero. 
The last quantity that must still be specified is $q$. This quantity is treated as a constant and if one of the three models is invoked in equation $(d 2)$ then it is treated as an integer constant; however, the value for $q$ must not be chosen arbitrarily. The reason that $q$ must not be arbitrarily chosen is that there is a second-order contact between the Hugoniot and the isentrope that goes through the foot of the Hugoniot. This continuity between the isentrope and the Hugoniot forces constraints on many of the initial values of the variables in equations $(d 3)-$ $(d 5)$ and the value of $q$. The second-order contact between the Hugoniot and the isentrope can be expressed by the equation

$$
\left(\frac{\partial^{2} P}{\partial \rho^{2}}\right)_{S}=\left(\frac{\partial^{2} P}{\partial \rho^{2}}\right)_{H}
$$

where equation $(d 9)$ holds for $\rho=\rho_{0}$. For a material that has a linear $U_{s}-u_{p}$ relation, the initial value for the right-hand side of equation $(d 9)$ is given by

$$
\left(\frac{\partial^{2} P}{\partial \rho^{2}}\right)_{H}=\frac{(4 s-2) c_{o}^{2}}{\rho_{\circ}}
$$

By making use of these equations and the additional assumption that $E_{c}$ may be ignored in comparison to $c_{o}^{2}$, an equation for $q$ can be derived ${ }^{66}$ :

$$
q=\frac{3}{2}\left(4 s-2 \gamma_{0}-\frac{4}{3}\right) .
$$

There are many different thermodynamic quantities that can be examined by the integration of equations $(d 3)-(d 5)$. Examples of calculating the temperature 
along the principal Hugoniot for copper and stainless steel are shown in figures 167 and 168. The initial values necessary for the integration of equations $(d 3)-(d 5)$ are given in table 70. In figure 169 the Gruneisen parameter for copper is plotted as a function of $\eta$. Also shown in this figure are curves calculated from equations (2.41), $(2.43)$ and $(2.48)$. As can be seen in these plots equation (2.41) gives much lower values for the Gruneisen parameter at high densities than does equation (2.43). The numerical ir.tegration of equations $(d 3)-(d 5)$ predicts yet higher values for the Gruneisen parameter than does equation (2.43). The analytic form for $\gamma$ given by equation (2.48) agrees somewhat better with the integration of equations (d3)(d5) than does equation (2.43). Lastly the pressure on the zero-degree isotherm is plotted against $\eta$ in figure 170. Three different zero-degree isotherms are drawn in this figure. One curve is derived from the integration of equations $(d 3)-(d 5)$, another from the Rice equation of state, and the last from the Mie-Gruneisen equation of state. For reference purposes the principal Hugoniot is also shown. Similar plots for stainless-steel SS-304L are shown in figures 171 and 172. 


\section{E. Initial Conditions in the Flyer Plate}

The initial conditions in the fyer plate can be estirnated by taking advantage of what is measured in each experiment. Specitically, these measurements are the thickness of the flyer plate, the speed of the plate at the time it impacts the target, and the distance the flyer plate has traveied. The distance the lyer plate has traveled is nominally the same in each experiment at $25.4 \mathrm{~mm}$. From these measurements an average driving pressure for the flyer plate can be calculated. This average pressure is given by the equation

$$
\tilde{P}=\rho_{o} h \frac{u_{f}^{2}}{2 d}
$$

In this equation $h$ is the initial thickness of the flyer plate, $d$ is the distance the Hyer plate has traveled, $\rho_{o}$ is initial density of the flyer, and $u_{f}$ is velocity of the flyer just before it impacts the target.

The derivation of equation (e1) assumes a constant acceleration of the flyer plate. It would then be expected that equation (e1) would overestimate the pressure on the back side of the flyer plate at the time it collides with the target plate. To refine the estimate, the detonation products of the HE may be treated as an ideal gas. The pressure in the gas may be related to the average driving pressure by the equation

$$
\frac{1}{d} \int_{l}^{l+1} P d x=\tilde{P}
$$


The term $d$ in equation (e2) is the same as that in equation (e1). Assuming that the expansion of the gas is adiabatic, equation (e2) may be integrated to yield the equation

$$
\frac{P_{1}}{1-\gamma}\left((y+1)\left(\frac{y}{y+1}\right)^{\gamma}-y\right)=\tilde{P},
$$

where

$$
y=\frac{l}{d} .
$$

The term $P_{\mathrm{i}}$ represents an initial pressure and the term $l$ represents some thickness of the $\mathrm{HE}$ gas that actually takes part in driving the flyer. Throughout this appendix, the parameter $\gamma$ in equation $(e 3)$ is the ratio of specific heats for an ideal gas and is not the Gruneisen parameter as it is elsewhere.

In order to calculate the final pressure at the interface of the flyer plate and the detonation products of the explosive, it is necessary to know either $P_{l}$ or $l$. The term $l$ can be estimated as

$$
l=\tilde{c} t
$$

The time $t$ in this equation can be taken $\Rightarrow$ s the time it takes the flyer plate to travel the distance $d$. The term $\dot{c}$ is an average sound speed in the HE gas and may be found from the average pressure. All this leads to the equation

$$
\left(\frac{l}{d}\right)^{2}=\frac{2 \gamma h}{d} \frac{\rho_{o}}{\mu_{g}}
$$


where $\rho_{y}$ is a density of the HE detonation products. To finc a minimum value for $l$ and hence $y$, a maximum value for $\rho_{y}$ can be found from the equation

$$
\rho_{y}=\rho_{1} \frac{\gamma+1}{\gamma} .
$$

The quantity $\rho_{\mathrm{i}}$ in equation $(e 7)$ is the densicy of tue in beïre it is deconated. After $l$ is found from equation (e6) then $P_{1}$ can be calculated from equation (e3). The final pressire is then given by the equation

$$
P_{f}=P_{i}\left(\frac{y}{y+1}\right)^{\gamma}
$$

With the calculation of $P_{f}$, the bourdary conditions for the pressure on the flyer plate are known. The initial pressure $P_{i}$ may be assumed to be a shock pressure from which the lyer plate has released. Instead of assuming or choosing a specific type of pressure gradient in the flyer plate, a linear density gradient in the flyer plate is assumed. The density gradient in the flyer plate is chosen such that

$$
\int \mu d x=\rho_{o} h .
$$

The density and specific internal energy at the front surface of the flyer plate are such that the calculation of the pressure from the equation of state for the Hyer will give zero pressure. The density and specific internal energy at the back surface will give the pressure $P_{f}$ for the same calculation. 
To evaluate $P_{\mathbf{i}}$ and $P_{f}$ it is necessary to know the value of $\gamma$ for the HE detonation products. The value of $\gamma$ does depend on the type of high explosive but a typical value for a generic $\mathrm{HE}$ is 2.7 . Table 71 lists values for $P_{\mathrm{i}}$ and $P_{f}$ for each of the different type explosiv s used in these experiments. A nominal thickness of $1.5 \mathrm{~mm}$ was used for the thickness of the flyer plate in calculating the pressures $P_{i}$ and $P_{f}$. 


\section{F. Equation of State Parameters and Sound Speeds}

As stated previously, the parameters for the equations of state are mainly determined from principal Hugoniot data and zero pressure data. Principal Hugoniot data for both SS-304L stainless steel and OFHC copper are described by equation (2.4) very well. In the Mie-Gruneisen, the Rice, and the Gray equations of state, a linear $U_{s}-u_{p}$ relation is assumed in deriving the equation of :iate. The parameters $c_{0}$ and $s$ are explicitly used in these equations of state. Only in the Tillotson equation of state is the parameter $s$ not explicitly used. The parameter $c_{o}$ is used in the Tillotson equation of state in determining the adiabatic bulk modulus at normal density and zero pressure. Reported values, ${ }^{32}$ of $3.940 \mathrm{~km} / \mathrm{s}$ and 1.489 were used for $c_{0}$ and $s$, respectively, for copper. For the stainless steel $c_{0}$ and $s$ were derived from a fit of $U_{s}$ to $u_{p}$ data. ${ }^{66}$ The values used for $c_{0}$ and $s$ for stainless steel were $4.566 \mathrm{~km} / \mathrm{s}$ and 1.485 , respectively.

Other parameters needed for the equations of state are the normal density of the materis's and the value of the Gruneisen $\gamma$ at zero pressure. For the stainless steel $\rho_{0}$ was taken to be $7.903 \mathrm{gm} / \mathrm{cc}$ and $\rho_{0}$ for copper was taken to be 8.930 $\mathrm{gm} / \mathrm{cc}$. The initial values of the Gruneisen $\gamma$ or $\gamma_{0}$ were determined from the thermodynamic definition which leads to the equation

$$
\gamma_{\circ}=\frac{c_{o}^{2} \beta}{c_{p}}
$$


In equation $(f 1) \beta$ is the coefficient of thermal expansion and $c_{p}$ is the specific heat capacity at constant pressure. Using values of $45.5 \times 10^{-6} / \mathrm{K}$ for $\beta$ and $3.839 \times 10^{6}$ $\mathrm{ergs} / \mathrm{gm}$ for $c_{p}$, equation $(f 1)$ gives a value of 2.002 for the Gruneisen parameter tor copper. Using values or $44.1 \times 10^{-6}$ for $\beta$ and $4.442 \times 10^{6} \mathrm{ergs} / \mathrm{gm}$ for $c_{p}, \gamma_{0}$ for SS-304 L is 2.070 . With the specification of $\gamma_{0}$, ali the equation of state parameters are Letermined for the Rice and the Mie-Gruneisen equations of state. Additional infurmation about the other parameters needed for the Gray and the Tillotson equations of state can be found in the appropriate reference.

There is one aspect of the formulation of the Gray equation of state in the present work that slightly deviates from that given by Royce ${ }^{30}$ Instead of assuming that $\alpha$ in equation (2.48) is determined from tr.. equation

$$
\gamma_{o}-\alpha=\frac{1}{2}
$$

it is assumed shat $\alpha$ is determined from the singularity produced by the linear $U_{s}-u_{p}$ relation. More particularly, this singularity leads to a limiting value for $\gamma$ in equations $(d 3)-(d 5)$. When equation $(2.48)$ is forced to agree with this limiting value, $\alpha$ can be found from the equation

$$
\gamma_{0}-\frac{\alpha}{s}=2(s-1)
$$

The principal Hugoniot calculated from these equations of state agree very weil with experimental data. Comparisons of the principal Hugoniot calculated 
from each equation of state and shock data for stainless stee ${ }^{66}$ and copper ${ }^{32}$ are shown in figures 173-180. These comparisons are plotted in the $P-\rho$ plane and each plot is lin ited in magnitude to $200 \mathrm{GPa}$. This more than covers the pressure range of interest in the present experiments. Also shown in each plot are releasc isentropes from $150 \mathrm{GPa}$ and $80 \mathrm{GPa}$. In figures $181-188$ the specific internal energy is plotted as a function of the pressure for the principal Hugoniot and for the release isentropes from $150 \mathrm{GPa}$ and from $80 \mathrm{GPa}$. Each of the equations of state does a good job of matching the experimental Hugoniot data, but this is to be expected. What is more interesting than the calculatiuons of the Hugoniots is the behavior of the isentropes. There are slight but definite differences in the release isentropes. These differences are the most noticeable in the $E-P$ plots.

In calculating the decay of a shock wave, two quantities of principal importance are the bulk sound speed and the particie velocity. As figures 173-188 show, all the equations of state give nearly identical shock loci and release isentropes that are all very similar in the $P-\rho$ and the $E-P$ planes. The one quantity in which significant differences arise occurs in the calculation of $c$, the bulk sound speed. Although $\left(\frac{\partial P}{\partial \rho}\right)_{H}$ along the principal Hugoniot is essentially the same for each equation of state, this is not true for $\left(\frac{\partial P}{\partial_{F}}\right)_{S}$ along the Huconiot. This term is, of course, identical to $c^{2}$. Calculations of the bulk sound speed as a function of the shock pressure from each equation of state are shown in figure 189 for staili- 
less steel. Figure 190 shows the corresponding plots for copper. At low shock pressures, there is little difference among the values of $c$ calculated from the different equations of state. As the pressure increases on the principal Hugoniot, the differences in the calculated sound speeds grow appreciably.

It is clear from figures 189 and 190 that above zero pressure on the Hugoniot, the Rice equation of state gives the largest sound speeds on the Hugoniot for a given pressure. A markedly different behavior is seen in the release isentropes. It nust be remembered that the P.ice equation of state is the only one of the equatinns of state in which the sound speed has no dependence on the specific internal energy. As the pressure decreases on the release isentropes, the effects of shock heating on the calculation of the bulk sound speed in the other equations of state become very apparent. Figure 191 shows the calculated sound speeds for SS-304L on the release isentropes from $150 \mathrm{GPa}$ for each of the equations of state. ln the pressure range of 60 to $80 \mathrm{GPa}$ the Rice isentrope is intersected by all the other isentropes so that belr.w about $60 \mathrm{GPa}$ the Rice equation of state gives the lowest sound speed of any of the equations of state for a given pressure. Figure 198 reveals that the calculated sound speeds for copper exhibit the same behavior. In figures 193 and 194 the sound speeds along the release isentropes from $80 \mathrm{GPa}$ are shown. 


\section{G. Pin Data}

The pin data for the experiments in which the attenuation of a shock was measured are given in tables 72-77. For every pin there is a pin identification number, a measured time, and a measured position given in terms of $x, y$, and $z$. Those pin numbers appended by a small ' $c$ ' identifies the pin as a capped pin. The capped pins were used to measure the trajectory of the flyer plate just before impact with the target plate. In some instances a pin failed to give a signal. In such cases the measured tiree is replace by 'np' (no pulse). In still other cases pin times were recorded but were so far out line with other times that they were dropped from the data analysis. Such times are marked by an asterisk.

In experiment RS-2302 some pins were used solely to check on the planarity of the shock and were not used in the actual analysis of the decay of the shock wave. These pins are marked by a dagger. The reference system for the pin positions is given in chapter 3 . 


\section{H. Tables}

Table 1.

Brief Description of the Experiments

$\begin{array}{lll}\text { Experiment } & \text { Driving } & \text { Shot } \\ \text { Number } & \text { Explosive } & \text { Description } \\ \text { RS-2301 } & \text { TNT } & \text { Flying Plate } \\ \text { RS-2302 } & \text { TNT } & \text { STM* in SS-304L } \\ \text { RS-2306 } & \text { TNT } & \text { STM in OFHC-Cu } \\ \text { RS-2307 } & \text { PBX-9404 } & \text { Flying Plate } \\ \text { RS-2308 } & \text { PBX-9404 } & \text { STM in OFHC-Cu } \\ \text { RS-2309 } & \text { PBX-9404 } & \text { STM in SS-304L } \\ \text { RS-2310 } & \text { Comp. B } & \text { Flying Plate } \\ \text { RS-2314 } & \text { Comp. B } & \text { STM in OFHC-Cu } \\ \text { RS-2315 } & \text { Comp. B } & \text { STM in SS-304L }\end{array}$

* Shock Trajectory Measuremeni 
Table 2a.

RS-2301: Parameters for the Fit

$$
t=a_{0}+a_{x} x+a_{y} y+a_{z} z+a_{r} r^{2}
$$

Nominal Planes

No. of Data Points

$a_{\circ}(\mu \mathrm{s})$

$a_{x}\left(10^{-1} \mu \mathrm{s} / \mathrm{mm}\right)$

$a_{y}\left(10^{-3} \mu \mathrm{g} / \mathrm{mm}\right)$

$a_{x}\left(10^{-3} \mu \mathrm{s} / \mathrm{mm}\right)$

$a_{r}\left(10^{-5} \mu \mathrm{s} / \mathrm{mm}\right)$

$\sigma(\mu \mathrm{s})$

$u(k m / s)$
$-2,-1,0$

94

$1.643 \pm .004$

$2.765 \pm .022$

$-0.213 \pm .036$

$-0.125 \pm .035$

$-0.694 \pm .072$

0.011

$3.617 \pm .029$
$0,15.22$

96

$1.643 \pm .005$

$1.155 \pm .063$

$-0.237 \pm .045$

$-0.112 \pm .043$

$-0.695 \pm .072$

0.013

$8.656 \pm .021$

Table $2 b$.

RS-2301: Parameters for the Fit

$$
t=a_{0}+a_{y} y+a_{z} z+a_{r} r^{2}
$$

Nominal Plane

No. of Data Points

$a_{0}(\mu \mathrm{s})$

$1.645 \pm .005$

$a_{y}\left(10^{-3} \mu \mathrm{s} / \mathrm{mm}\right)$

$-0.264 \pm .036$

$a_{x}\left(10^{-3} \mu \mathrm{s} / \mathrm{mm}\right)$

$-0.130 \pm .052$

$a_{r}\left(10^{-5} \mu \mathrm{s} / \mathrm{mm}\right)$

$-0.734 \pm .096$

$\sigma(\mu \mathbf{s})$
0

49

0.011
15.22

47

$3.400 \pm .006$

$-0.237 \pm .045$

$-0.093 \pm .073$

$-0.666 \pm .109$

0.014 
Table 3a.

RS-2307: Parameters for the Fit

$$
t=a_{0}+a_{x} x+a_{y} y^{\prime}+a_{z} z
$$

$\begin{array}{lll}\text { Nominal Planes } & -1.5,0 & 0,5 \\ \text { No. of Data Points } & 75 & 74 \\ a_{o}(\mu \mathrm{s}) & 1.937 \pm .004 & 1.937 \pm .004 \\ a_{x}\left(10^{-1} \mu \mathrm{s} / \mathrm{mm}\right) & 2.404 \pm .037 & 1.081 \pm .011 \\ a_{y}\left(10^{-3} \mu \mathrm{s} / \mathrm{mm}\right) & 0.106 \pm .054 & -0.156 \pm .052 \\ a_{x}\left(10^{-3} \mu \mathrm{s} / \mathrm{mm}\right) & -0.141 \pm .055 & -0.138 \pm .051 \\ \sigma(\mu \mathrm{s}) & 0.015 & 0.014 \\ u(\mathrm{~km} / \mathrm{s}) & 4.159 \pm .065 & 9.248 \pm .091\end{array}$

Table 3b.

RS-2307: Parameters for the Fit

$$
t=a_{0}+a_{y} y+a_{z} z
$$

$\begin{array}{lll}\text { Nominal Plane } & 0 & 5 \\ \text { No. of Data Points } & 37 & 37 \\ a_{o}(\mu \mathrm{s}) & 1.937 \pm .004 & 2.477 \pm .004 \\ a_{y}\left(10^{-3} \mu \mathrm{g} / \mathrm{mm}\right) & 0.161 \pm .077 & 0.156 \pm .078 \\ a_{s}\left(10^{-3} \mu \mathrm{s} / \mathrm{mm}\right) & -0.136 \pm .077 & -0.156 \pm .076 \\ \sigma(\mu \mathrm{s}) & 0.014 & 0.014\end{array}$


Table 4a.

RS-2310: Parameters for the Fit

$$
t=a_{0}+a_{1}-a_{y} y+a_{z} z+a_{1} r+a_{2} r^{2}
$$

Nominal Planes

$-2,-1,0$

$0,15.22$

No. of Data Points

94

96

$\begin{array}{lll}a_{\mathrm{o}}(\mu \mathrm{s}) & 3.312 \pm .013 & 3.305 \pm .015 \\ a_{x}\left(10^{-1} \mu \mathrm{s} / \mathrm{mm}\right) & 2.179 \pm .034 & 1.050 \pm .010 \\ a_{y}\left(10^{-3} \mu \mathrm{s} / \mathrm{mm}\right) & 0.164 \pm .041 & 0.114 \pm .047 \\ a_{x}\left(10^{-4} \mu \mathrm{s} / \mathrm{mm}\right) & 0.166 \pm .409 & 0.298 \pm .462 \\ a_{1}\left(10^{-3} \mu \mathrm{s} / \mathrm{mm}\right) & 1.537 \pm .439 & 1.719 \pm .474 \\ a_{2}\left(10^{-5} \mu \mathrm{s} / \mathrm{mm}\right) & -1.132 \pm .319 & -1.235 \pm .350 \\ \sigma(\mu \mathrm{s}) & 0.011 & 0.012 \\ u(\mathrm{~km} / \mathrm{s}) & 4.590 \pm .072 & 9.520 \pm .087\end{array}$

Table 4b.

RS-2310: Parameters for the Fit

$$
t=a_{0}+a_{y} y+a_{z} z+a_{1} r+a_{2} r^{2}
$$

$\begin{array}{lll}\text { Nominal Plane } & 0 & 5 \\ \text { No. of Data Points } & 37 & \ddots 4 \\ a_{o}(\mu \mathrm{s}) & 3.296 \pm .018 & 3.831 \pm .024 \\ a_{y}\left(10^{-3} \mu \mathrm{s} / \mathrm{mm}\right) & 0.107 \pm .060 & 0.119 \pm .076 \\ a_{z}\left(10^{-4} \mu \mathrm{s} / \mathrm{mm}\right) & 0.074 \pm .589 & 0.482 \pm .757 \\ a_{1}\left(10^{-3} \mu \mathrm{s} / \mathrm{mm}\right) & 1.997 \pm .596 & 1.590 \pm .786 \\ a_{2}\left(10^{-5} \mu \mathrm{s} / \mathrm{mm}\right) & -1.414 \pm .439 & -1.136 \pm .582 \\ \sigma(\mu \mathrm{s}) & 0.011 & 0.013\end{array}$


Table 5.

\section{Measured Flyer Plate Velocities and \\ Predicted Shock Speeds}

$\begin{array}{lllll}\text { Experiment } & \text { Flyer } & \text { Shock } & x_{1} & x_{1} \\ & \text { Velocity } & \text { Speed } & \text { minimum } & \text { maximum } \\ & (\mathrm{km} / \mathrm{s}) & (\mathrm{km} / \mathrm{s}) & (\mathrm{mm}) & (\mathrm{mm}) \\ \text { RS-2302 } & 3.65 & 7.28 & 7.8 & 10.6 \\ \text { RS-2309 } & 4.53 & 7.93 & 6.6 & 9.5 \\ \text { RS-2315 } & 4.15 & 7.65 & 7.0 & 9.8 \\ \text { RS-2306 } & 3.69 & 6.65 & 6.4 & 8.8 \\ \text { RS-2308 } & 4.63 & 7.34 & 5.6 & 8.3 \\ \text { RS-2314 } & 4.10 & 6.95 & 7.0 & 9.8\end{array}$


'able 6.

$\delta t$ Fits for RS-2302

$\begin{array}{cllclll}U_{0} & x_{1} & x_{2} & \text { Spline } & \sigma_{1} & \sigma_{2} & \sigma \\ (\mathrm{km} / \mathrm{s}) & (\mathrm{mm}) & (\mathrm{mm}) & \text { Option } & (\mathrm{ns}) & (\mathrm{ns}) & \text { (ns) } \\ 7.25 \pm 0.06 & 4.5 & 8.5 & 2-2 & 4.9 & 8.6 & 8.2 \\ 7.24 \pm 0.05 & 5.5 & 8.5 & 2-2 & 5.9 & 9.1 & 8.5 \\ 7.31 \pm 0.05 & 6.5 & 8.5 & 2-2 & 7.9 & 7.6 & 7.4 \\ 7.35 \pm 0.04 & 7.1 & 8.5 & 2-2 & 7.8 & 6.7 & 7.1 \\ 7.35 \pm 0.04 & 7.8 & 8.5 & 3-3 & 7.8 & 6.7 & 7.1 \\ 7.35 \pm 0.04 & 7.8 & 11.5 & 3-3 & 7.8 & 6.8 & 7.1 \\ 7.34 \pm 0.03 & 8.2 & 12.5 & 2-3 & 7.8 & 6.6 & 7.1 \\ 7.36 \pm 0.03 & 9.2 & 10.6 & 2-2 & 7.9 & 6.5 & 7.2 \\ 7.34 \pm 0.03 & 10.2 & 10.6 & 2-2 & 8.0 & 6.5 & 7.3\end{array}$




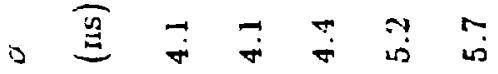

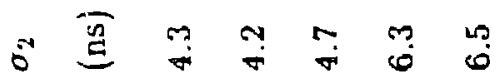

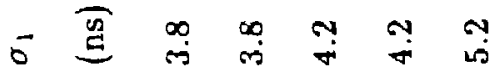

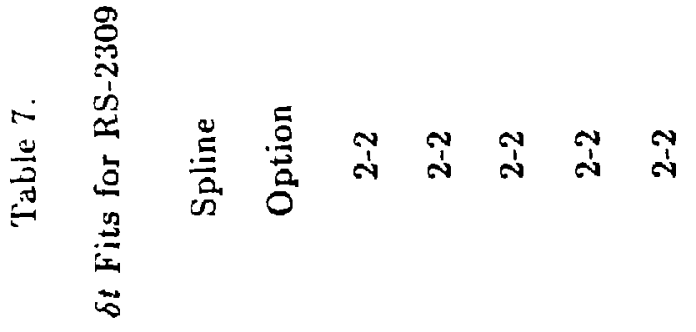

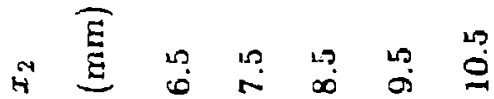

$$
\begin{aligned}
& \vec{H} \text { 昜 }
\end{aligned}
$$

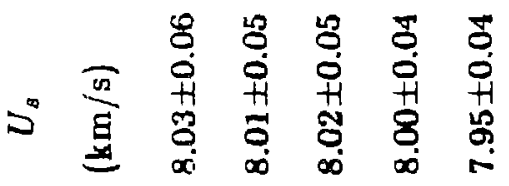




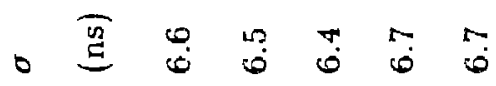

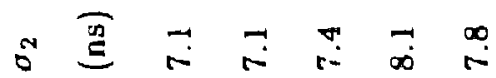

$$
\begin{aligned}
& 0 \text { ह } \\
& \text { 1120202 }
\end{aligned}
$$

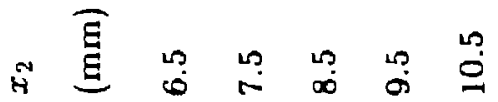

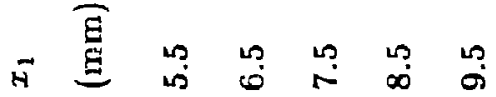

$$
\begin{aligned}
& \text { ऽ. }
\end{aligned}
$$




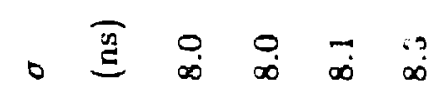

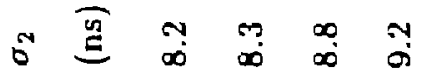

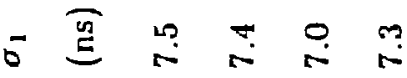

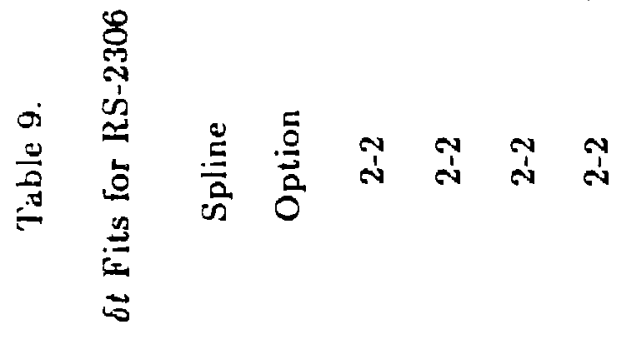

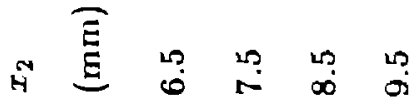

$$
\begin{aligned}
& \vec{H} \text { 急 }
\end{aligned}
$$

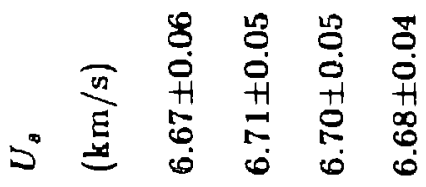


Table 10.

$\delta t$ Fits for RS-2308

$\begin{array}{lllclll}U_{s} & x_{1} & x_{2} & \text { Spline } & \sigma_{1} & \sigma_{2} & \sigma \\ (\mathrm{km} / \mathrm{s}) & (\mathrm{mm}) & (\mathrm{mm}) & \text { Option } & (\mathrm{ns}) & \text { (ns) } & \text { (ns) } \\ 7.19 \pm 0.06 & 4.8 & 5.8 & 2-2 & 3.9 & 6.0 & 5.3 \\ 7.24 \pm 0.05 & 5.8 & 6.8 & 2-2 & 4.0 & 6.1 & 5.4 \\ 7.24 \pm 0.05 & 6.8 & 7.8 & 2-2 & 3.9 & 6.4 & 5.4 \\ 7.22 \pm 0.04 & 7.8 & 8.8 & 2-2 & 4.2 & 6.5 & 5.4 \\ 7.19 \pm 0.04 & 8.8 & 9.8 & 2-2 & 4.7 & 6.2 & 5.4\end{array}$




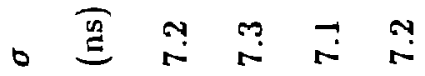

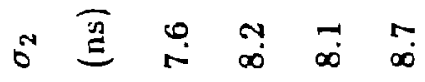

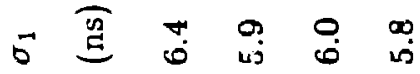

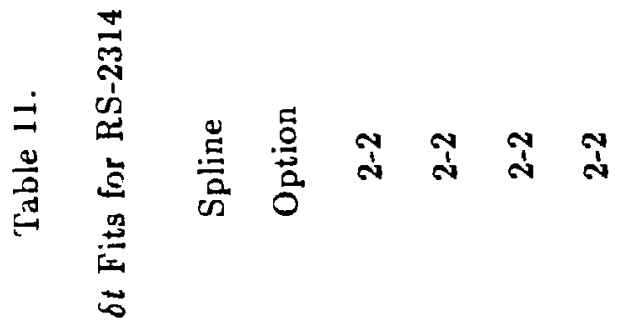

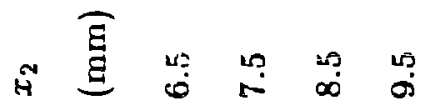

$$
\begin{aligned}
& \text { स 息 嵑 }
\end{aligned}
$$

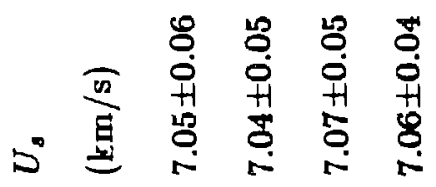


Table 12.

Probable Overtake Region

$\begin{array}{lllll}\text { Experiment } & \text { Flyer } & \text { Shock } & \text { Shock } & \text { Overtake } \\ & \text { Velocity } & \text { Speed } & \text { Pressure } & \text { Region } \\ & (\mathrm{km} / \mathrm{s}) & (\mathrm{km} / \mathrm{s}) & (\mathrm{GPa}) & (\mathrm{mm}) \\ \text { RS-2302 } & 3.65 \pm 0.09 & 7.35 \pm 0.07 & \sim 109 & 7-9 \\ \text { RS-2309 } & 4.54 \pm 0.15 & 8.02 \pm 0.05 & \sim 147 & 5-7 \\ \text { RS-2315 } & 4.15 \pm 0.10 & 7.72 \pm 0.05 & \sim 130 & 6-7 \\ \text { RS-2306 } & 3.69 \pm 0.09 & 6.71 \pm 0.06 & \sim 111 & 6-8 \\ \text { RS-2308 } & 4.63 \pm 0.14 & 7.23 \pm 0.04 & \sim 143 & 6.3-8.3 \\ \text { RS-2314 } & 4.10 \pm 0.10 & 7.06 \pm 0.04 & \sim 132 & 6-8\end{array}$


Table 13.

RS-2302: Data Analysis and Code Parameters for Figure 70

Data Analysis Parameters

First Break Point

Second Break Point

$U_{s}$ for Zone 1

Polynomial Fit for Zone 2

Polynomial Fit for Zone 3

Code Parameters-SHKSIM

Flyer Plate Velocity

Mie-Gruneisen EOS for Flyer

No Strength Model for Flyer

Shock Velocity in Target

Overtake Position

Mie-Gruneisen EOS for Target

No Strength Model for Target
$8.5 \mathrm{~mm}^{*}$

$9.5 \mathrm{~mm}$

$7.34 \mathrm{~km} / \mathrm{s}$

Quadratic

Cubic

$3.73 \mathrm{~km} / \mathrm{s}$

$7.34 \mathrm{~km} / \mathrm{s}$

$9.5 \mathrm{~mm}$

- The first break point for the data analysis does not coincide with the overtake position from the code calculation. 
Table 14 .

Variables on a Right-Going Characteristic

$\begin{array}{lll} & \text { Starting } & \text { Ending } \\ & \text { Values } & \text { Values } \\ u(\mathrm{~km} / \mathrm{s}) & 1.287 & 1.289 \\ c(\mathrm{~km} / \mathrm{s}) & 6.656 & 6.700 \\ \rho(\mathrm{g} / \mathrm{cc}) & 9.763 & 9.866 \\ E(\mathrm{GPa}-\mathrm{cc} / \mathrm{g}) & 1.058 & 0.831 \\ P(\mathrm{GPa}) & 66.33 & 66.02\end{array}$


Table 15.

RS-2302: Ỹata Analysis and Code Parameters for Figure 71

Data Analysis Parameters

First Break Point $\quad 8.8 \mathrm{~mm}$

Second Break Point $\quad 9.5 \mathrm{~mm}$

$U_{s}$ for Zone $1 \quad 7.34 \mathrm{~km} / \mathrm{s}$

Polynomial Fit for Zone 2 Quadratic

Polynomial Fit for Zone $3 \quad$ Cubic

Code Parameters-SHKSIM

Fiyer Plate Velocity

$3.73 \mathrm{~km} / \mathrm{s}$

Rice EOS for Flyer

No Strength Model for Flyer

Shock Velocity in Target

$7.34 \mathrm{~km} / \mathrm{s}$

Overtake Position

$8.78 \mathrm{~mm}$

Rice EOS for Target

No Strength Model for Target 
Table 16.

RS-2302: Data Analysis and Code Parameters for Figure 72

Data Analysis Parameters

First Break Point

$8.5 \mathrm{~mm}^{*}$

Second Break Point

$9.9 \mathrm{~mm}$

$U_{\text {, for Zone } 1}$

$7.34 \mathrm{~km} / \mathrm{s}$

Polynomial Fit for Zone 2

Quadratic

Polynomial Fit for Zone 3

Cubic

Code Parameters-SHKSIM

Flyer Plate Velocity

$3.71 \mathrm{~km} / \mathrm{s}$

Tillotson EOS for Flyer

No Strength Model for Flyer

Shock Velocity in Target

$7.34 \mathrm{~km} / \mathrm{s}$

Overtake Fosition

$10.01 \mathrm{~mm}$

Tillotson EOS for Target

No Strength Model for Target

* The first break point for the data analysis does not coincide with the overtake position from the code calculation. 
Table 17.

RS-2302: Data Analysis and Code Parameters foi Figure 73

Data Analysis Parameters

First Break Point

$8.5 \mathrm{~mm}^{*}$

Second Break Point

$9.8 \mathrm{~mm}$

$U$, for Zone 1

$7.34 \mathrm{~km} / \mathrm{s}$

Polynomial Fit for Zone 2

Quadratic

Polynomial Fit for Zone 3

Cubic

Code Parameters-SHKSIM

Flyer Plate Velocity

$3.74 \mathrm{~km} / \mathrm{s}$

Gray FOS for Flyer

No Strength Model for Flyer

Shock Velocity in Target

$7.34 \mathrm{~km} / \mathrm{s}$

Overtake Position

$9.80 \mathrm{~mm}$

Gray EOS for Target

No Strength Model for Target

- The first break point for the data analysis does not coincide with the overtake position from the code calculation. 


\section{Table 18.}

RS-2315: Data Analysis and Code Parameters for Figure 74

Data Analysis Parameters

First Break Point

Second Break Point

$U_{a}$ for Zone 1

Polynomial Fit for Zone 2

Polynomial Fit for Zone 3

Code Parameters-SHKSIM

Flyer Plate Velocity

Mie-Gruneisen EOS for Flyer

No Strength Model for Flyer

Shock Velocity in Target

Overtake Position

Mie-Gruneisen EOS for Target

No Strengtii Model for Target
$7.2 \mathrm{~mm}^{*}$

$8.6 \mathrm{~mm}$

$7.73 \mathrm{~km} / \mathrm{s}$

Quadratic

Quadratic

$4.26 \mathrm{~km} / \mathrm{s}$

$7.73 \mathrm{~km} / \mathrm{s}$

$8.64 \mathrm{~mm}$

- The first break point for the data analysis does not coincide with the overtake position from the code calculation. 
Table 19.

RS-2315: Data Analysis and Code Parameters for Figure 75

Data Analysis Parameters

First Break Point

Second Break Point

$U_{s}$ for Zone 1

Polynomial Fit for Zone 2

Polynomial Fit for Zone 3

Code Parameters-SHKSIM

Flyer Plate Velocity

Rice EOS for Flyer

No Strength Model for Flyer

Shock Velocity in Target

Overtake Position

Rice EOS for Target

No Strength Model for Target
$7.1 \mathrm{~mm}^{\bullet}$

$7.9 \mathrm{~mm}$

$7.73 \mathrm{~km} / \mathrm{s}$

Quadratic

Quadratic

$4.26 \mathrm{~km} / \mathrm{s}$

$7.73 \mathrm{~km} / \mathrm{s}$

$7.86 \mathrm{~mm}$

- The first break point for the data analysis does not coincide with the overtake position from the code calculation. 
Table 20.

RS-2315: Data Analysis and Code Parameters for Figure 76

Data Analysis Parameters

First Break Point

Second Break Point

$U_{s}$ for Zone 1

Polynomial Fit for Zone 2

Polynomial Fit for Zone 3

Code Parameters-SHKSIM

Flyer Plate Velocity

Tillotson EOS for Flyer

No Strength Model for Flyer

Shock Velocity in Target

Overtaks Position

Tillutson EOS for Target

No Strength Model for Target
$7.1 \mathrm{~mm}^{*}$

$9.1 \mathrm{~mm}$

$7.73 \mathrm{~km} / \mathrm{s}$

Quadratic

Quadratic

$4.25 \mathrm{~km} / \mathrm{s}$

$7.73 \mathrm{~km} / \mathrm{s}$

$9.13 \mathrm{~mm}$

- The first break point for the data analysis does not coincide with the overtake position from the code calculation. 
Table 21.

RS-2315: Data Analysis and Code Parameters for Figure 77

Data Analysis Parameters

First Break Point

$7.2 \mathrm{~mm}^{*}$

Second Break Point

$8.9 \mathrm{~mm}$

$U_{s}$ for Zone 1

$7.73 \mathrm{~km} / \mathrm{s}$

Polynomial Fit for Zone 2

Quadratic

Polynomial Fit for Zone 3

Quadratic

Code Parameters-SHKSLM

Flyer Plate V-locity

$4.28 \mathrm{~km} / \mathrm{s}$

Gray EOS for Flyer

No Strength Model for Flyer

Shock Velocity in Target

$7.73 \mathrm{~km} / \mathrm{s}$

Overtake Position

$8.91 \mathrm{~mm}$

Gray EOS for Target

No Strength Model for Target

- The first break point for the data analysis does not coincide with the overtake position from the cod $\varepsilon$ calculation. 
Table 22.

RS-2309: Data Analysis and Code Parameters for Figure 78

Data Analysis Parameters

First Break Point

$6.9 \mathrm{~mm}^{*}$

Second Break Point

$8.3 \mathrm{~mm}$

$U_{\text {o }}$ for Zone 1

$8.02 \mathrm{~km} / \mathrm{s}$

Polynomial Fit for Zone 2

Quadratic

Polynomial Fit for Zone 3

Quadratic

Code Parameters-SHKSIM

Flyer Plate Velocity

$4.65 \mathrm{~km} / \mathrm{s}$

Mie-Gruneisen EOS for Flyer

No Strength Model for Flyer

Shock Velocity in Target

$8.02 \mathrm{~km} / \mathrm{s}$

Overtake Position

$8.26 \mathrm{~mm}$

Mie-Gruneisen EOS for Target

No Strength Model for Target

- The first break point for the data analysis does not coincide with the overtake position from the code calculation. 
Table 23.

RS-2309: Data Analysis and Code Parameters for Figure 79

Data Analysis Parameters

First Break Point

$6.9 \mathrm{~mm}^{*}$

Second Break Point

$7.5 \mathrm{~mm}$

$U_{s}$ for Zone 1

$8.02 \mathrm{~km} / \mathrm{s}$

Polynomial Fit for Zone 2

Quadratic

Polynomial Fit for Zone 3

Quadratic

Code Parameters-SHKSLM

Flyer Plate Velocity

$4.65 \mathrm{~km} / \mathrm{s}$

Rice EOS for $\mathrm{Flyer}$

No Strength Model for Flyer

Shock Velocity in Target

$8.02 \mathrm{~km} / \mathrm{s}$

Overtake Position

$7.46 \mathrm{~mm}$

Rice EOS for Target

No Strength Model for Target

- The first break point fo: the data analysis does not coincide with the overtake position from the code calculation. 
Table 24.

RS-2309: Data Analysis and Code Parameters for Figure 80

Data Analysis Parameters

First Break Point

$6.9 \mathrm{~mm}^{*}$

Second Break Point

$8.8 \mathrm{~mm}$

$U_{\text {. for Zone } 1}$

$8.02 \mathrm{~km} / \mathrm{s}$

Polynomial Fit for Zone 2

Quadratic

Polynomial Fit for Zone 3

Quadratic

Code Parameters-SHKSIM

Flyer Plate Velocity

$4.65 \mathrm{~km} / \mathrm{s}$

Tillotson EOS for Flyer

No Strength Model for Flyer

Shock Velocity in Target

$8.02 \mathrm{~km} / \mathrm{s}$

Overtake Position

$8.77 \mathrm{~mm}$

Tillotson EOS for Target

No Strength Model for Target

* The first break point for the data analysis does not coincide with the overtake position from the code calculation. 
Table 25.

RS-2309: Data Analysis and Code Parameters for Figure 81

Data Analysis Parameters

First Break Point

$6.9 \mathrm{~mm}^{*}$

Second Break Point

$8.5 \mathrm{~mm}$

$U_{\text {. for Zone } 1}$

$8.02 \mathrm{~km} / \mathrm{s}$

Polynomial Fit for Zone 2

Quadratic

Polynomia! Fit for Zone 3

Quadratic

Code Parameiers-SHñsLM

Flyer Plate Velocity

$4.67 \mathrm{~km} / \mathrm{s}$

Gray EOS for Flyer

No Strength Model for Flyer

Shock Velocity in Target

$8.02 \mathrm{~km} / \mathrm{s}$

Overtake Position

$8.55 \mathrm{~mm}$

Gray EOS for Target

No Strength Model for Target

- The first break point for the data analysis does not coincide with the overtake position from the code calculation. 
Table 26.

RS-2302: Data Analysis and Code Parameters for Figure 85

Data Analysis Parameters

First Break Point

$7.1^{*} \mathrm{~mm}$

Second Break Point

$8.5 \mathrm{~mm}$

$U_{a}$ for Zone 1

$7.35 \mathrm{~km} / \mathrm{s}$

Polynomial Fit for Zone 2

Quadratic

Polynomial Fit for Zone 3

Quadratic

Code Parameters-SHKSJB

Flyer Plate Velocity

$3.74 \mathrm{~km} / \mathrm{s}$

Rice EOS for Flyer

Strength Equation (2.93) for Flyer

Constant $\mu$

$75.13 \mathrm{GPa}$

Constant $Y_{0}$

$0.34 \mathrm{GPa}$

Shock Velocity in Target

$7.35 \mathrm{~km} / \mathrm{s}$

Overtake Position

$6.94 \mathrm{~mm}$

Rice EOS for Target

Strength Equation (2.93) for Target

Constant $\mu$

$75.13 \mathrm{GPa}$

Constant $Y_{0}$

$0.34 \mathrm{GPa}$

- The first break point for the data analysis does not coincide with the overtake position from the code calculation. 
Table 27.

RS-2302: Data Analysis and Code Parameters for Figure 88

Data Analysis Parameters

First Break Point

$6.9 \mathrm{~mm}$

Second Break Point

$8.5 \mathrm{~mm}$

$U_{a}$ for Zone 1

$7.31 \mathrm{~km} / \mathrm{s}$

Polynomial Fit for Zone 2

Quadratic

Polynomial Fit for Zone 3

Quadratic

Code Parameters-SHKSJB

Flyer Plate Velocity

$3.69 \mathrm{~km} / \mathrm{s}$

Rice EOS for Flyer

Strength Equation (2.93) for Flyer

Constant $\mu$

$75.13 \mathrm{GPa}$

Constant $Y_{0}$

$0.34 \mathrm{GPa}$

Shock Velocity in Target

$7.31 \mathrm{~km} / \mathrm{s}$

Overtake Position

$6.97 \mathrm{~mm}$

Rice EOS for Target

Strength Equation (2.93) for Target

Constant $\mu$

$75.13 \mathrm{GPa}$

Constant $Y_{0}$

$0.34 \mathrm{GPa}$ 
Table 28.

RS-2315: Data Analysis and Code Parameters for Figure 90

Data Analysis Parameters

First Break Point

$6.5 \mathrm{~mm}$

Second Break Point

$7.5 \mathrm{~mm}$

$U_{a}$ for Zone 1

$7.72 \mathrm{~km} / \mathrm{s}$

Polynomial Fit for Zone 2

Quadratic

Polynomial Fit for Zone 3

Quadratic

Code Parameters-SHKSJB

Flyer Plate Velocity

$4.23 \mathrm{~km} / \mathrm{s}$

Rice EOS for Flyer

Strength Equation (2.93) for Flyer

Constant $\mu$

$75.13 \mathrm{GPa}$

Constant $Y_{0}$

$0.34 \mathrm{GPa}$

Shock Velocity in Target

$7.71 \mathrm{~km} / \mathrm{s}$

Overtake Position

$6.47 \mathrm{~mm}$

Rice EOS for Target

Strength Equation (2.93) for Target

Constant $\mu$

$75.13 \mathrm{GPa}$

Constant $Y_{0}$

$0.34 \mathrm{GPa}$ 
Table 29.

RS-2309: Data Analysis and Code Parameters for Figure 91

Data Analysis Parameters

First Break Point

$6.3 \mathrm{~mm}^{*}$

Second Break Point

$7.5 \mathrm{~mm}$

$U_{s}$ for Zone 1

$8.01 \mathrm{~km} / \mathrm{s}$

Polynomial Fit for Zone 2

Quadratic

Polynomial Fit for Zone 3

Quadratic

Code Parameters-SHKSJB

Flyer Plate Velocity

$4.64 \mathrm{~km} / \mathrm{s}$

Rice EOS for Flyer

Strength Equation (2.93) for Flyer

Constant $\mu$

$75.13 \mathrm{GPa}$

Constant $Y_{0}$

$0.34 \mathrm{GPa}$

Shock Velocity in Target

$8.02 \mathrm{~km} / \mathrm{s}$

Overtake Position

$6.26 \mathrm{~mm}$

Rice EOS for Target

Strength Equation (2.93) for Target

Constant $\mu$

$75.13 \mathrm{GPa}$

Constant $Y_{0}$

$0.34 \mathrm{GPa}$ 
Table 30 .

RS-2302: Data Analysis and Code Parameters for Figure 94

Data Analysis Parameters

First Break Point

$7.5 \mathrm{~mm}$

Second Break Point

$8.5 \mathrm{~mm}$

$U_{s}$ for Zone 1

$7.35 \mathrm{~km} / \mathrm{s}$

Polynomial Fit for Zone 2

Quadratic

Polynomial Fit for Zone 3

Cubic

Code Parameters-SHKSJB

Flyer Plate Velocity

$3.71 \mathrm{~km} / \mathrm{s}$

Rice EOS for Flyer

Strength Equation (2.93) for Flyer

Constant $\mu$

$75.13 \mathrm{GPa}$

Constant $Y_{0}$

$0.34 \mathrm{GPa}$

Shock Velocity in Target

$7.34 \mathrm{~km} / \mathrm{s}$

Overtake Position

$7.54 \mathrm{~mm}$

Rice EOS for Target

Strength Equation (2.93) for Target

Constant $\mu$

$75.13 \mathrm{GPa}$

Constant $Y_{0}$

$0.34 \mathrm{GPa}$ 
Table 31.

RS-2315: Data Analysis and Code Parameters for Figure 96

Data Analysis Parameters

First Break Point

$7.1 \mathrm{~mm}$

Second Break Point

$8.5 \mathrm{~mm}$

$U_{\text {a }}$ for Zone 1

$7.73 \mathrm{~km} / \mathrm{s}$

Polynomial Fit for Zone 2

Quadratic

Polynomial Fit for Zone 3

Quadratic

Code Parameters-SHKSJB

Flyer Plate Velocity

$4.25 \mathrm{~km} / \mathrm{s}$

Tillotson EOS for Flyer

Strength Equation (2.93) for Flyer

Constant $\mu$

$75.13 \mathrm{GPa}$

Constant $Y_{0}$

$0.34 \mathrm{GPa}$

Shock Velocity in Target

$7.74 \mathrm{~km} / \mathrm{s}$

Overtake Position

$7.12 \mathrm{~mm}$

Tillotson EOS for Target

Strength Equation (2.93) for Target

Constant $\mu$

$75.13 \mathrm{GPa}$

Constant $Y_{0}$

$0.34 \mathrm{GPa}$ 
Table 32.

RS-2309: Data Analysis and Code Parameters for Figure 98

Data Analysis Parameters

First Break Point

$6.9 \mathrm{~mm}$

Second Break Point

$7.5 \mathrm{~mm}$

$U$, for Zone 1

$8.01 \mathrm{~km} / \mathrm{s}$

Polynomial Fit for Zone 2

Quadratic

Polynomial Fit for Zone 3

Quadratic

Code Parameters-SHKSJB

Flyer Plate Velocity

$4.63 \mathrm{~km} / \mathrm{s}$

Tillotson EOS for Flyer

Strength Equation (2.93) for Flyer

Constant $\mu$

$75.13 \mathrm{GPa}$

Constant $Y_{0}$

$0.34 \mathrm{GPa}$

Shock Velocity in Target

$8.01 \mathrm{~km} / \mathrm{s}$

Overtake Position

$6.99 \mathrm{~mm}$

Tillotson EOS for Target

Strength Equation (2.93) for Target

Constant $\mu$

$75.13 \mathrm{GPa}$

Constant $Y_{0}$

$0.34 \mathrm{GPa}$ 
Table 33.

RS-2302: Data Analysis and Code Parameters for Figure 91

Data Analysis Parameters

First Break Point

$6.8 \mathrm{~mm}$

Second Break Point

$7.5 \mathrm{~mm}$

$U_{\text {s }}$ for Zone 1

$7.36 \mathrm{~km} / \mathrm{s}^{*}$

Polynomial Fit for Zone 2

Quadratic

Polynomial Fit for Zone 3

Quadratic

Code Parameters-SHKSJB

Flyer Plate Velocity

variable

Rice EOS for Flyer

Strength Equation (2.93) for Flyer

Constant $\mu$

$75.13 \mathrm{GPa}$

Constant $Y_{0}$

$0.34 \mathrm{GPa}$

Shock Velocity in Target

variable

Overtake Position

$6.84 \mathrm{~mm}$

Rice EOS for Target

Strength Equation (2.93) for Target

Constant $\mu$

$75.13 \mathrm{GPa}$

Constant $Y_{0}$

$0.34 \mathrm{GPa}$

- The 1-mm pin level has been deleted for the calculation of $U_{a}$. 
Table 34.

RS-2306: Data Analysis and Code Parameters for Figure 102

Data Analysis Parameters

First Break Point

$6.1 \mathrm{~mm}$

Second Break Point

$7.5 \mathrm{~mm}$

$U_{a}$ for Zone 1

$6.71 \mathrm{~km} / \mathrm{s}$

Polynomial Fit for Zone 2

Quadratic

Polynomial Fit for Zone 3

Cubic

Code Parameters-SHKSJB

Flyer Plate Velocity

$3.77 \mathrm{~km} / \mathrm{s}$

Rice EOS for Flyer

Strength Equation (2.93) for Flyer

Constant $\mu$

$75.13 \mathrm{GPa}$

Constant $Y_{0}$

$0.34 \mathrm{GPa}$

Shock Velocity in Target

$6.71 \mathrm{~km} / \mathrm{s}$

Overtake Position

$6.07 \mathrm{~mm}$

Rice EOS for Target

Strength Equation (2.93) for Target

Constant $\mu$

$47.7 \mathrm{GPa}$

Constant $Y_{0}$

$0.12 \mathrm{GPa}$ 
Table 35.

RS-2306: Data Analysis and Code Parameters for Figure 104

Data Analysis Parameters

First Break Point

$6.9 \mathrm{~mm}$

Second Break Point

$7.5 \mathrm{~mm}$

$U$, for Zone 1

$0.71 \mathrm{~km} / \mathrm{s}$

Polynomial Fit for Zone 2

Quadratic

Polynomial Fit for Zone 3

Quadratic

Code Parameters-SHKSJB

Flyer Plate Velocity

$3.76 \mathrm{~km} / \mathrm{s}$

Rice EOS for Flyer

Strength Equation (2.93) for Flyer

Constant $\mu$

$75.13 \mathrm{GPa}$

Constant $Y_{0}$

$0.34 \mathrm{GPa}$

Shock Velocity in Target

$6.71 \mathrm{~km} / \mathrm{s}$

Overtake Position

$6.90 \mathrm{~mm}$

Rice EOS for Target

No Strength Model for Target 
Table 36.

RS-2306: Data Analysis and Code Parameters for Figure 105

Data Analysis Parameters

First Break Point

$6.4 \mathrm{~mm}$

Second Break Point

$7.5 \mathrm{~mm}$

$U$. for Zone 1

$6.71 \mathrm{~km} / \mathrm{s}$

Polynomial Fit for Zone 2

Quadıatic

Polynomial Fit for Zone 3

Quadratic

Code Parameters-SHKSJB

Flyer Plate Velocity

$3.77 \mathrm{~km} / \mathrm{s}$

Rice EOS for Flyer

Strength Equation (2.93) for Flyer

Constant $\mu$

$75.13 \mathrm{GPa}$

Constant $Y_{0}$

$0.34 \mathrm{GPa}$

Shock Velocity in Target

$6.71 \mathrm{~km} / \mathrm{s}$

Overtake Position

$6.41 \mathrm{~mm}$

Mie-Gruneisen EOS for Target

Strength Equation: :.93) for Target

Constant $\mu$

47.7 GPa

Constant $Y_{0}$

$0.12 \mathrm{GPa}$ 
Table 37.

RS-2306: Data Analysis and Code Parameters for Figure 106

Data Analysis Parameters

First Break Point

$6.6 \mathrm{~mm}$

Second Break Point

$7.5 \mathrm{~mm}$

$U_{a}$ for Zone 1

$6.71 \mathrm{~km} / \mathrm{s}$

Polynomial Fit for Zone 2

Quadratic

Polynomial Fit for Zone 3

Quadratic

Code Parameters-SHKSJB

Flyer Plate Velocity

$3.76 \mathrm{~km} / \mathrm{s}$

Rice EOS for Flyer

Strength Equation (2.93) for Flyer

Constant $\mu$

$75.13 \mathrm{GPa}$

Constant $Y_{0}$

$0.34 \mathrm{GPa}$

Shock Velocity in Target

$6.72 \mathrm{~km} / \mathrm{s}$

Overtake Position

$6.65 \mathrm{~mm}$

Tillotson EOS for Target

Strength Equation (2.93) for Target

Constant $\mu$

47.7 GPa

Constant $Y_{0}$

$0.12 \mathrm{GPa}$ 
Table 38.

RS-2306: Data Analysis and Code Parameters for Figure 107

Data Analysis Parameters

First Break Point

$6.5 \mathrm{~mm}$

Second Break Point

$7.5 \mathrm{~mm}$

$U_{a}$ for Zone 1

$6.71 \mathrm{~km} / \mathrm{s}$

Polynomial Fit for Zone 2

Quadratic

Polynomial Fit for Zone 3

Quadratic

Code Parameters-SHKSJB

Flyer Plate Velocity

$3.77 \mathrm{~km} / \mathrm{s}$

Rice EOS for Flyer

Strength Equation (2.93) for Flyer

Constant $\mu$

$75.13 \mathrm{GPa}$

Constant $Y_{0}$

$0.34 \mathrm{GPa}$

Shock Velocity in Target

$6.71 \mathrm{~km} / \mathrm{s}$

Overtake Position

$6.49 \mathrm{~mm}$

Gray EOS for Targat

Strength Equation (2.93) for Target

Constant $\mu$

$75.13 \mathrm{GPa}$

Constant $Y_{0}$

$0.34 \mathrm{GPa}$ 
Table 39.

RS-2306: Data Analysis and Code Parameters for Figure 108

Data Analysis Parameters

First Break Poin

$6.8 \mathrm{~mm}$

Second Break Point

$7.5 \mathrm{~mm}$

$U_{a}$ for Zone 1

$6.70 \mathrm{~km} / \mathrm{s}$

Polynomial Fit for Zone 2

Quadratic

Polynomial Fit for Zone 3

Quadratic

Code Parameters-SHKSJB

Flyer Plate Velocity

$3.75 \mathrm{~km} / \mathrm{s}$

Rice EOS for Flyer

Strength Equation (2.93) for Flyer

Constant $\mu$

$75.13 \mathrm{GPa}$

Constant $Y_{0}$

$0.34 \mathrm{GPa}$

Shock Velocity in Target

$6.71 \mathrm{~km} / \mathrm{s}$

Overtaire Position

$7.84 \mathrm{~mm}$

Tilltoson EOS for Target

No Strength Model for Target 
Table 40.

RS-2314: Data Analysis and Code Parameters for Figure 109

Data Analysis Parameters

First Break Point

$6.4 \mathrm{~mm}$

Second Breał Point

$7.5 \mathrm{~mm}$

$U_{a}$ for Zone 1

$7.04 \mathrm{~km} / \mathrm{s}$

Polynomial Fit for Zone 2

Quadratic

Polynomial Fit for Zone 3

Quadratic

Code Parameters-SHKSJB

Flyer Plate Velocity

$4.23 \mathrm{~km} / \mathrm{s}$

Rice EOS for Flyer

Strength Equation (2.93) for Flyer

Constant $\mu$

$75.13 \mathrm{GPa}$

Constant $Y_{0}$

$0.34 \mathrm{GPa}$

Shock Velocity in Target

$7.05 \mathrm{~km} / \mathrm{s}$

Overtake Position

$6.42 \mathrm{~mm}$

Rice EOS for Target

No Strength Model for Target 
Table 41.

RS-2314: Data Analysis and Code Parameters for Figure 110

Data Analysis Parameters

First Break Point

$6.9 \mathrm{~mm}$

Second Break Point

$7.5 \mathrm{~mm}$

$U$, for Zone 1

$7.04 \mathrm{~km} / \mathrm{s}$

Polynomial Fit for Zone 2

Quadratic

Polynomial Fit for Zone 3

Quadratic

Code Parameters-SHKSJB

Flyer Plate Velocity

$4.23 \mathrm{~km} / \mathrm{s}$

Rice EOS for Flyer

Strength Equation (2.93) for Flyer

Constant $\mu$

$75.13 \mathrm{GPa}$

Constant $Y_{0}$

$0.34 \mathrm{GPa}$

Shock Velocity in Target

$7.05 \mathrm{~km} / \mathrm{s}$

Overtake Position

$6.97 \mathrm{~mm}$

Mie-Gruneisen EOS for Target

No Strength Model for Target 
Table 42.

RS-2314: Data Analysis and Ccde Parameters for Figure 111

Data Analysis Parameters

First Break Point

$7.4 \mathrm{~mm}$

Second Break Point

$8.5 \mathrm{~mm}$

$U$. for Zone 1

$7.07 \mathrm{~km} / \mathrm{s}$

Polynomial Fit for Zone 2

Quadratic

Polynomial Fit for Zone 3

Quadratic

Code Parameters-SHKSJB

Flyer Plate Velocity

$4.25 \mathrm{~km} / \mathrm{s}$

Rice EOS for Flyer

Strength Equation (2.93) for Flyer

Constant $\mu$

$75.13 \mathrm{GPa}$

Constant $Y_{0}$

$0.34 \mathrm{GPa}$

Shock Velocity in Target

$7.06 \mathrm{~km} / \mathrm{s}$

Overtake Position

$7.37 \mathrm{~mm}$

Tillotson EOS for Target

No Strength Model for Target 
Table 43.

RS-2314: Data Analysis and Code Parameters for Figure 112

Data Analysis Parameters

First Break Point

Second Break Point

$U_{a}$ for Zone 1

Polynomial Fit for Zone 2

Polynomial Fit for Zone 3

Code Parameters-SHKSJB

Flyer Plate Velocity

Rice EOS for Flyer

Strength Equation (2.93) for Flyer

Constant $\mu$

Constant $Y_{0}$

Shock Velocity in Target

Overtake Position

Gray EOS for Target

No Strength Model for Target
$7.1 \mathrm{~mm}$

$8.5 \mathrm{~mm}$

$7.07 \mathrm{~km} / \mathrm{s}$

Quadratic

Quadratic

$4.25 \mathrm{~km} / \mathrm{s}$

$75.13 \mathrm{GPa}$

$0.34 \mathrm{GPa}$

$7.06 \mathrm{~km} / \mathrm{s}$

$7.10 \mathrm{~mm}$ 
Table 44.

RS-2308: Data Analysis and Code Parameters for Figure 115

Data Analysis Parameters

First Break Point

$6.4 \mathrm{~mm}$

Second Break Point

$7.8 \mathrm{~mm}$

$U_{\mathrm{a}}$ for Zone 1

$7.24 \mathrm{~km} / \mathrm{s}$

Polynomial Fit for Zone 2

Quadratic

Polynomial Fit for Zone 3

Quadratic

Code Parameters-SHKSJB

Flyer Plate Velocity

$4.49 \mathrm{~km} / \mathrm{s}$

Rice EOS for Flyer

Strength Equation (2.93) for Flyer

Constant $\mu$

$75.13 \mathrm{GPa}$

Constant $Y_{0}$

$0.34 \mathrm{GPa}$

Shock Velocity in Target

$7.24 \mathrm{~km} / \mathrm{s}$

Overtake Position

$6.26 \mathrm{~mm}$

Rice EOS for Target

No Strength Model for Target 
Table 45.

RS-2308: Data Analysis and Code Parameters for Figure 117

Data Analysis Parameters

First Break Point

$6.8 \mathrm{~mm}$

Second Break Point

$7.8 \mathrm{~mm}$

$U_{\text {, for Zone } 1}$

$7.24 \mathrm{~km} / \mathrm{s}$

Polynomial Fit for Zone 2

Quadratic

Polynomial Fit for Zone 3

Quadratic

Code Parameters-SHKSJB

Flyer Plate Velocity

$4.49 \mathrm{~km} / \mathrm{s}$

Rice EOS for Flyer

Strength Equation (2.93) for Flyer

Constant $\mu$

$75.13 \mathrm{GPa}$

Constant $Y_{0}$

$0.34 \mathrm{GPa}$

Shock Velocity in Target

$7.24 \mathrm{~km} / \mathrm{s}$

Overtake Position

$6.82 \mathrm{~mm}$

Mie-Gruneisen EOS for Target

No Strength Model for Target 
Table 46.

RS-2303: Data Analysis and Code Parameters for Figure 119

Data Analysis Parameters

First Break Point

$7.2 \mathrm{~mm}$

Second Break Point

$7.8 \mathrm{~mm}$

$U_{s}$ for Zone 1

$7.24 \mathrm{~km} / \mathrm{s}$

Polynomial Fit for Zone 2

Quadratic

Polynomial Fit for Zone 3

Quadratic

Code Parameters-SHKSJB

Flyer Plate Velocity

$4.50 \mathrm{~km} / \mathrm{s}$

Rice EOS for Flyer

Strength Equation (2.93) for Flyer

Constant $\mu$

$75.13 \mathrm{GPa}$

Constant $Y_{0}$

$0.34 \mathrm{GPa}$

Shock Velocity in Target

$7.24 \mathrm{~km} / \mathrm{s}$

Overtake Position

$7.25 \mathrm{~mm}$

Tillotson EOS for Target

No Strength Model for Target 
Table 47.

RS-2308: Data Analysis and Code Parameters for Figure 121

Data Analysis Parameters

First Break Point

$6.9 \mathrm{~mm}$

Second Break Point

$7.8 \mathrm{~mm}$

$U_{\text {o for Zone } 1}$

$7.24 \mathrm{~km} / \mathrm{s}$

Polynomial Fit for Zone 2

Quadratic

Polynomial Fit for Zone 3

Quadratic

Code Parameters-SHKSJB

Flyer Plate Velocity

$4.50 \mathrm{~km} / \mathrm{s}$

Rice EOS for Flyer

Strer.gth Equation (2.93) for Flyer

Constant $\mu$

$75.13 \mathrm{GPa}$

Constant $Y_{0}$

$0.34 \mathrm{GPa}$

Shock Velocity in Target

$7.24 \mathrm{~km} / \mathrm{s}$

Overtake Position

$6.97 \mathrm{~mm}$

Gray EOS for Target

No Strength Model for Target 
Table 48.

RS-2302: Data Analysis and Code Parameters for Figure 123

Data Analysis Parameters

First Break Point

$7.1 \mathrm{~mm}$

Second Break Point

$8.5 \mathrm{~mm}$

$U_{a}$ for Zone 1

$7.35 \mathrm{~km} / \mathrm{s}$

Polynornial Fit for Zone 2

Quadratic

Polynomial Fit for Zone 3

Cubic

Code Parameters-SHKSJB

Flyer Plate Velocity

$3.71 \mathrm{~km} / \mathrm{s}$

Tillotson EOS for Flyer

Strength Equation (2.93) for Flyer

Constant $\mu$

$100.17 \mathrm{GPa}$

Constant $Y_{0}$

$0.85 \mathrm{GPa}$

Shock Velocity in Target

$7.36 \mathrm{~km} / \mathrm{s}$

Overtake Position

$7.06 \mathrm{~mm}$

Tillotson EOS for Target

Strength Equation (2.93) for Target

Constant $\mu$

$100.17 \mathrm{GPa}$

Constant $Y_{0}$

$0.85 \mathrm{GPa}$ 
Table 49.

RS-2302: Data Analysis and Code Parameters for Figure 124

Data Analysis Parameters

First Break Point

$7.1 \mathrm{~mm}$

Second Break Point

$8.5 \mathrm{~mm}$

$U_{\text {s }}$ for Zone 1

$7.35 \mathrm{~km} / \mathrm{s}$

Polynomial Fit for Zone 2

Quadratic

Polynomial Fit for Zone 3

Cubic

Code Parameters--SHKSJB

Flyer Plate Velocity

$3.68 \mathrm{~km} / \mathrm{s}$

Tillotson EOS for Flyer

Strength Equation (2.93) for Flyer

Constant $\mu$

$100.17 \mathrm{GPa}$

Constant $Y_{0}$

$1.70 \mathrm{GPa}$

Shock Velocity in Target

$7.35 \mathrm{~km} / \mathrm{s}$

Overtake Position

$7.12 \mathrm{~mm}$

Tillotson EOS for Target

Strength Equation (2.93) for Target

Constant $\mu$ $100.17 \mathrm{GPa}$

Constant $Y_{0}$

$1.70 \mathrm{GPa}$ 
Table 50.

RS-2302: Data Analysis and Code Parameters for Figure 125

Data Analysis Parameters

First Break Point

$7.2 \mathrm{~mm}$

Second Break Point

$8.5 \mathrm{~mm}$

$U$, for Zone 1

$7.35 \mathrm{~km} / \mathrm{s}$

Polynomial Fit for Zone 2

Quadratic

Polynomial Fit for Zone 3

Cubic

Code Parameters-SHKSJB

Flyer Plate Velocity

$3.64 \mathrm{~km} / \mathrm{s}$

Tillotson EOS for Flyer

Strength Equation (2.93) for Flyer

Constant $\mu$

100.17 GPa

Constant $Y_{0}$

$3.40 \mathrm{GPa}$

Shock Velocity in Target

$7.36 \mathrm{~km} / \mathrm{s}$

Overtake Position

$7.24 \mathrm{~mm}$

Tillotson EOS for Target

Strength Equation (2.93) for Target

Constant $\mu$

$100.17 \mathrm{GPa}$

Constant $Y_{0}$

$3.40 \mathrm{GPa}$ 
Table 51 .

RS-2315: Data Analysis and Code Parameters for Figure 129

Data Analysis Parameters

First Break Point

$6.5 \mathrm{~mm}$

Second Break Point

$7.5 \mathrm{~mm}$

$U$, for Zone 1

$7.72 \mathrm{~km} / \mathrm{s}$

Polynomial Fit for Zone 2

Quadratic

Polynomial Fit for Zone 3

Quadratic

Code Parameters-SHKSJB

Flyer Plate Velocity

$4.13 \mathrm{~km} / \mathrm{s}$

Tillotson EOS for Flyer

Strength Equation (2.93) for Flyer

Constant $\mu$

$125.22 \mathrm{GPa}$

Constant $Y_{0}$

$4.08 \mathrm{GPa}$

Shock Velocity in Target

$7.71 \mathrm{~km} / \mathrm{s}$

Overtake Position

$6.51 \mathrm{~mm}$

Tillotson EOS for Target

Strength Equation (2.93) for Target

Constant $\mu$

125.22 GPa

Constant $Y_{0}$

$4.08 \mathrm{GPa}$ 
Table 52.

RS-2309: Data Analysis and Code Parameters for Figure 131

Data Analysis Parameters

First Break Point

$6.1 \mathrm{~mm}$

Second Break Point

$7.5 \mathrm{~mm}$

$U_{\text {, for }}$ Zone 1

$8.01 \mathrm{~km} / \mathrm{s}$

Polynomial Fit for Zone 2

Quadratic

Polynomial Fit for Zone 3

Quadratic

Code Parameters-SHKSJB

Flyer Plate Velocity

$4.53 \mathrm{~km} / \mathrm{s}$

Tillotson EOS for Flyer

Strength Equation (2.93) for Flyer

Constant $\mu$

$150.26 \mathrm{GPa}$

Constant $Y_{0}$

$4.76 \mathrm{GPa}$

Shock Velocity in Target

$8.01 \mathrm{~km} / \mathrm{s}$

Overtake Position

$6.12 \mathrm{~mm}$

Tillotson EOS for Target

Strength Equation (2.93) for Target

Constant $\mu$

$150.26 \mathrm{GPa}$

Constant $Y_{0}$

$4.76 \mathrm{GPa}$ 
Table 53.

RS-2302: Data Analysis and Code Parameters for Figure 133

Data Analysis Parameters

First Break Point

Second Break Point

$U_{s}$ for Zone !

Polynomial Fit for Zone 2

Polynomial Fit for Zone 3

Code Parameters-SHKSJB

Flyer Plate Velocity

Rice EOS for Flyer

Strength Equation (2.93) for Flyer

Constant $\mu$

Constant $Y_{0}$

Shock Velocity in Target

Overtake Position

Rice EOS for Target

Strength Equation (2.93) for Target

Constant $\mu$

Constant $Y_{\mathrm{O}}$
$6.6 \mathrm{~mm}$

$7.5 \mathrm{~mm}$

$7.37 \mathrm{~km} / \mathrm{s}^{*}$

Quadratic

Cubic

$3.74 \mathrm{~km} / \mathrm{s}$

$100.17 \mathrm{GPa}$

$1.36 \mathrm{GPa}$

$7.37 \mathrm{~km} / \mathrm{s}$

$6.59 \mathrm{~mm}$

$100.17 \mathrm{GPa}$

$1.36 \mathrm{GPa}$

- The 1-mm pin level has been deleted for the calculationof $U_{A}$. 
Table 54.

RS-2315: Data Analysis and Code Parameters for Figure 135

Data Analysis Parameters

First Break Point

$6.2 \mathrm{~mm}$

Second Break Poinc

$7.5 \mathrm{~mm}$

$U_{\text {s }}$ for Zone 1

$7.71 \mathrm{~km} / \mathrm{s}$

Polynomial Fit for Zone 2

Quadratic

Polynomial Fit for Zone 3

Quadratic

Code Parameters-SHKSJB

Flyer Plate Velocity

$4.21 \mathrm{~km} / \mathrm{s}$

Rice EOS for Flyer

Strength Equatio: (2.93) for Flyer

Constant $\mu$

$100.17 \mathrm{GPa}$

Constant $Y_{0}$

$1.70 \mathrm{GPa}$

Shock Velocity in Targe:

$7.72 \mathrm{~km} / \mathrm{s}$

Overtake Position

$6.20 \mathrm{~mm}$

Rice EOS for Target

Strength Equation (2.93) for Target

Constant $\mu$

$100.17 \mathrm{GPa}$

Constant $Y_{0}$

$1.70 \mathrm{GPa}$ 
Table 55.

RS-2309: Data Analysis and Code Parameters for Figure 137

Data Analysis Parameters

First Break Point

$5.5 \mathrm{~mm}$

Second Break Point

$6.5 \mathrm{~mm}$

$U_{s}$ for Zone 1

$8.03 \mathrm{~km} / \mathrm{s}$

Polynomial Fit for Zone 2

Quadratic

Polynomial Fit for Zone 3

Quadratic

Code Parameters-SHKSJB

Flyer Plate Velocity

$4.63 \mathrm{~km} / \mathrm{s}$

Rice EOS for Flyer

Strength Equation (2.93) for Flyer

Constant $\mu$

$150.26 \mathrm{GPa}$

Constant $Y_{0}$

$2.04 \mathrm{GPa}$

Shock Velocity in Target

$8.03 \mathrm{~km} / \mathrm{s}$

Overtake Position

$5.55 \mathrm{~mm}$

Rice EOS for Target

Strength Equation (2.93) for Target

Constant $\mu$

$150.26 \mathrm{GPa}$

Constant $Y_{0}$

$2.04 \mathrm{GPa}$ 
Table 56.

RS-2302: Data Analysis and Code Parameters for Figure 139

Data Analysis Parameters

First Break Point

$5.7 \mathrm{~mm}$

Second Break Point

$8.5 \mathrm{~mm}$

$U$, for Zone 1

$7.37 \mathrm{~km} / \mathrm{s}^{*}$

Polynomial Fit for Zone 2

Quadratic

Polynomial Fit for Zone 3

Cubic

Code Parameters-SHKSJB

Flyer Plate Velocity

$3.73 \mathrm{~km} / \mathrm{s}$

Rice EOS for Flyer

Strength Equation (2.92) for Flyer

Equations (4.13), (4.15), and (4.17) for $\mu$ and $Y$

$$
\mu_{0}, Y_{0}
$$

0.34 , and $75.13 \mathrm{GPa}$

Melting Formula (c11) for $T_{m}$

Shock Velocity in Target

$7.37 \mathrm{~km} / \mathrm{s}$

Overtake Position

$5.68 \mathrm{~mm}$

Rice EOS for Target

Strength Equation (2.92) for Target

Equations (4.13), (4.15), and (4.17) for $\mu$ and $Y$

$$
\mu_{0}, Y_{0}
$$

0.34 , and 75.1 ? GPa

Melting Formula (c11) for $T_{m}$

- $U_{a}$ is calculated from the $2-\mathrm{mm}$ through $6-\mathrm{mm}$ pin levels. 
Table 57.

RS-2315: Data Analysis and Code Parameters for Figure 141

Data Analysis Parameters

First Break Point

$5.4 \mathrm{~mm}$

Second Break Point

$7.5 \mathrm{~mm}$

$U_{\text {a }}$ for Zone 1

$7.72 \mathrm{~km} / \mathrm{s}^{*}$

Polynomial Fit for Zone 2

Quadratic

Polynomial Fit for Zone 3

Quadratic

Code Parameters-SHKSJB

Flyer Plate Velocity

$4.23 \mathrm{~km} / \mathrm{s}$

Rice EOS for Flyer

Strength Equation (2.92) for Flyer

Equations (4.13), (4.15), and (4.17) for $\mu$ and $Y$

$$
\mu_{0}, Y_{0}
$$

0.34 , and $75.13 \mathrm{GPa}$

Melting Formula (c11) for $T_{m}$

Shock Velocity in Target

$7.72 \mathrm{~km} / \mathrm{s}$

Overtake Position

$5.45 \mathrm{~mm}$

Rice EOS for Target

Strength Equation (2.92) for Target

Equations (4.13), (4.15), and (4.17) for $\mu$ and $Y$

$$
\mu_{0}, Y_{0}
$$

0.34 , and $75.13 \mathrm{GPa}$

Melting Formula (c11) for $T_{m}$

* $U_{\text {}}$ is calculated from the $1-\mathrm{mm}$ through 6-mm pin levels. 
Table 58.

RS-2309: Data Analysis and Code Parameters for Figure 143

Data Analysis Parameters

First Break Point

$5.4 \mathrm{~mm}$

Second Break Point

$6.5 \mathrm{~mm}$

$U$. for Zone 1

$8.03 \mathrm{~km} / \mathrm{s}$

Polynomial Fit for Zone 2

Quadratic

Polynomial Fit for Zone 3

Quadratic

Code Parameters-SHKSJB

Flyer Plate Velocity

$4.63 \mathrm{~km} / \mathrm{s}$

Rice EOS for Flyer

Strength Equation (2.92) for Flyer

Equations (4.13), (4.15), and (4.17) for $\mu$ and $Y$

$$
\mu_{0}, Y_{0}
$$

0.34 , and $75.13 \mathrm{GPa}$

Melting Formula (c11) for $T_{m}$

Shock Velocity in Target

$8.03 \mathrm{~km} / \mathrm{s}$

Overtake Position

$5.43 \mathrm{~mm}$

Rice EOS for Target

Strength Equation (2.92) for Target

Equations (4.13), (4.15), and (4.17) for $\mu$ and $Y$

$$
\mu_{0}, Y_{0}
$$

0.34 , and $75.13 \mathrm{GPa}$

Melting Formula (c11) for $T_{m}$ 
Table 59.

RS-2302: Data Analysis and Code Parameters for Figure 145

Data Analysis Parameters

First Break Point

$5.8 \mathrm{~mm}$

Second Break Point

$8.5 \mathrm{~mm}$

$U_{a}$ for Zone 1

$7.37 \mathrm{~km} / \mathrm{s}^{*}$

Polynomial Fit for Zone 2

Quadratic

Polynomial Fit for Zone 3

Cubic

Code Parameters-SHKSJB

Flyer Plate Velocity

$3.73 \mathrm{~km} / \mathrm{s}$

Rice EOS for Flyer

Strength Equation (2.92) for Flyer

Equations (4.13), (4.15), and (4.17) for $\mu$ and $Y$

$$
\mu_{0}, Y_{0}
$$

0.34 , and $75.13 \mathrm{GPa}$

Melting Formula (c16) for $T_{m}$

Shock Velocity in Target

$7.37 \mathrm{~km} / \mathrm{s}$

Overtake Position

$5.79 \mathrm{~mm}$

Rice EOS for Target

Strength Equation (2.92) for Target

Equations (4.13), (4.15), and (4.17) for $\mu$ and $Y$

$$
\mu_{0}, Y_{0}
$$

Melting Formula (c16) for $T_{m}$

* $U_{a}$ is calculated from the 2-mm through 6-mm pin levels. 
Table 60.

RS-2315: Data Analysis and Code Parameters for Figure 147

Data Analysis Parameters

First Break Point

$5.6 \mathrm{~mm}$

Second Break Point

$7.5 \mathrm{~mm}$

$U_{s}$ for Zone 1

$7.72 \mathrm{~km} / \mathrm{s}^{*}$

Polynomial Fit for Zone 2

Quadratic

Polynomial Fit for Zone 3

Quadratic

Code Parameters-SHKSJB

Flyer Plate Velocity

$3.73 \mathrm{~km} / \mathrm{s}$

Rice EOS for Flyer

Strength Equation (2.92) for Flyer

Equations (4.13), (4.15), and (4.17) for $\mu$ and $Y$

$$
\mu_{0}, Y_{0}
$$

0.34 , and $75.13 \mathrm{GPa}$

Melting Formula (c16) for $T_{m}$

Shock Velocity in Target

$7.72 \mathrm{~km} / \mathrm{s}$

Overtake Position

$5.56 \mathrm{~mm}$

Rice EOS for Target

Strength Equation (2.92) for Target

Equations (4.13), (4.15), and (4.17) for $\mu$ and $Y$

$$
\mu_{0}, Y_{0}
$$

0.34 , and $75.13 \mathrm{GPa}$

Melting Formula (c16) for $T_{m}$

- $U_{s}$ is calculated from the $1-\mathrm{mm}$ through $6-\mathrm{mm}$ pin levels. 
Table 61.

RS-2309: Data Analysis and Code Parameters for Figure 149

Data Analysis Parameters

First Break Point

$5.6 \mathrm{~mm}$

Second Break Point

$7.5 \mathrm{~mm}$

$U$ for Zone 1

$8.03 \mathrm{~km} / \mathrm{s}$

Polynomial Fit for Zone 2

Quadratic

Polynomial Fit for Zone 3

Quadratic

Code Parameters-SHKSJB

Flyer Plate Velocity

$4.64 \mathrm{~km} / \mathrm{s}$

Rice EOS for Flyer

Strength Equation (2.92) for Flyer

Equations (4.13), (4.15), and (4.17) for $\mu$ and $Y$

$$
\mu_{0}, Y_{0}
$$

0.34 , and $75.13 \mathrm{GPa}$

Melting Formula (c16) for $T_{m}$

Shock Velocity in Target

$8.03 \mathrm{~km} / \mathrm{s}$

Overtake Position

$5.57 \mathrm{~mm}$

Rice EOS for Target

Strength Equation (2.92) for Target

Equations (4.13), (4.15), and (4.17) for $\mu$ and $Y$

$$
\mu_{0}, Y_{0}
$$

0.34 , and $75.13 \mathrm{GPa}$

Melting Formula (c16) for $T_{m}$ 
Table 62.

RS-2302: Data Analysis and Code Parameters for Figure 151

Data Analysis Parameters

First Break Point

$6.2 \mathrm{~mm}$

Second Break Point

$8.5 \mathrm{~mm}$

$U_{\text {s }}$ for Zone 1

$7.37 \mathrm{~km} / \mathrm{s}^{*}$

Polynomial Fit for Zone 2

Quadratic

Polynomial Fit for Zone 3

Cubic

Code Parameters--SHKSJB

Flyer Plate Velocity

$3.74 \mathrm{~km} / \mathrm{s}$

Rice $\mathrm{EOS}$ for Flyer

Strength Equation (2.92) for Flyer

Equations (4.13), (4.15), and (4.17) for $\mu$ and $Y$

$$
\mu_{0}, Y_{0}
$$

0.34 , and $75.13 \mathrm{GPa}$

Melting Formula (c16) for $T_{m}$

Shock Velocity in Target

$7.36 \mathrm{~km} / \mathrm{s}$

Overtake Position

$6.15 \mathrm{~mm}$

Rice EOS for Target

Strength Equation (2.92) for Target

Equations (4.13), (4.15), and (4.17) for $\mu$ and $Y$

$$
\mu_{0}, Y_{0}
$$

0.34 , and $75.13 \mathrm{GPa}$

Melting Formula (c16) for $T_{m}$

*The 1-mm pin level has been deleted in the calculation of $U_{0}$. 
Table 63.

RS-2315: Data Analysis and Code Parameters for Figure 153

Data Analysis Parameters

First Break Point

$5.9 \mathrm{~mm}$

Second Break Point

$7.5 \mathrm{~mm}$

$U_{a}$ for Zone 1

$7.72 \mathrm{~km} / \mathrm{s}^{*}$

Polynomial Fit for Zone 2

Quadratic

Polynomial Fit for Zone 3

Quadratic

Code Parameters-SHKSJB

Flyer Plate Velocity

$3.74 \mathrm{~km} / \mathrm{s}$

Rice EOS for Flyer

Strength Equation (2.92) for Flyer

Equations (4.13), (4.15), and (4.17) for $\mu$ and $Y$

$$
\mu_{0}, Y_{0}
$$

0.34 , and $75.13 \mathrm{GPa}$

Melting Formula (c16) for $T_{m}$

Shock Velocity in Target

$7.72 \mathrm{~km} / \mathrm{s}$

Qvertake Position

$5.95 \mathrm{~mm}$

Rice EOS for Target

Strength Equation (2.92) for Target

Equations (4.13), (4.15), and (4.17) for $\mu$ and $Y$

$$
\mu_{0}, Y_{0}
$$

0.34 , and $75.13 \mathrm{GPa}$

Melting Formula (c16) for $T_{m}$

* $U_{o}$ is calculated from the 1-mm through 6-mm pin levels. 


\section{Table 64.}

RS-2309: Data Analysis and Code Parameters for Figure 155

Data Analysis Parameters

First Break Point

$6.0 \mathrm{~mm}$

Second Break Point

$7.5 \mathrm{~mm}$

$U$, for Zone 1

$8.03 \mathrm{~km} / \mathrm{s}$

Polynomial Fit for Zone :-

Quadratic

Polynnmial Fit for Zone 3

Quadratic

Code Parameters-SHKSJB

Flyer Plate Velocity

$4.65 \mathrm{~km} / \mathrm{s}$

Rice EOS for Flyer

Strength Equation (2.92) for Flyer

Equations (4.13), (4.15), and (4.17) for $\mu$ and $Y$

$$
\mu_{0}, Y_{0}
$$

0.34 , and $75.13 \mathrm{GPa}$

Melting Formula (c16) for $T_{m}$

Shock Velocity in Target

$8.03 \mathrm{~km} / \mathrm{s}$

Overtake Position.

$5.97 \mathrm{~mm}$

Rice EOS for Target

Strength Equation (2.92) for Taiget

Equations (4.13), (4.15), and (4.17) for $\mu$ and $Y$

$$
\mu_{0}, Y_{0}
$$

0.34 , and $75.13 \mathrm{GPa}$

Melting Formula (c16) for $T_{m}$ 
Table 65.

RS-2306: Data Analysis and Code Parameters for Figure 157

Data Analysis Parameters

First Break Point

$6.7 \mathrm{~mm}$

Second Break Point

$8.5 \mathrm{~mm}$

$U_{s}$ for Zone 1

$6.71 \mathrm{~km} / \mathrm{s}$

Polynomial $r$ it for Zone 2

Quadratic

Polynomial Fit for Zone 3

Quadratic

Code Parameters-SHKSJB

Flyer Plate Velocity

$3.76 \mathrm{~km} / \mathrm{s}$

Rice EOS for Flyer

Strength Equation (2.92) for Flyer

Equations (4.13), (4.15), and (4.17) for $\mu$ and $Y$

$$
\mu_{0}, Y_{0}
$$

0.34 , and $7=13 \mathrm{GPa}$

Melting Formula (c16) for $T_{m}$

Shock Velocity in Target

$6.71 \mathrm{~km} / \mathrm{s}$

Overtake Position

$6.65 \mathrm{~mm}$

Mie-Gruneisen EOS for Target

$\gamma_{0}$ changed to

No Str: agth Model for Target 
Table 66.

RS-2314: Data Analysis and Code Parameters fcr Figure 159

Data Analysis Parameters

First Break Point

$6.7 \mathrm{~mm}$

Second Break Point

$8.5 \mathrm{~mm}$

$U_{s}$ for Zone 1

$7.04 \mathrm{~km} / \mathrm{s}$

Poly nomial Fit for Zone 2

Quadratic

Polynomial Fit for Zone 3

Quadratic

Code Parameters-SHKSJB

Flyer Plate Velocity

$4.22 \mathrm{~km} / \mathrm{s}$

Rice EOS for Flyer

Strength Equation (2.92) for Flyer

Equations (4.13), (4.15), and (4.17) for $\mu$ and $Y$

$$
\mu_{0}, Y_{0}
$$

0.34 , and $75.13 \mathrm{GPa}$

Melting Formula (c16) for $T_{m}$

Shock Velocity in Target

$7.04 \mathrm{~km} / \mathrm{s}$

Overtake Position

$6.76 \mathrm{~mm}$

iMie-Gruneisen EOS for Target

$\gamma_{0}$ changed to

No Strength Model for Target 
Table 67.

RS-2308: Data Analysis and Code Parameters for Figure 161

Data Analysis Parameters

First Break Point

$7.1 \mathrm{~mm}$

Second Break Point

$8.8 \mathrm{~mm}$

$U$, for Zone 1

$7.24 \mathrm{~km} / \mathrm{s}$

Polynomial Fit for Zone 2

Quadratic

Polynomial Fit for Zone 3

Quadratic

vode Parameters-SHKSJB

Flyer Plate Velocity

$4.50 \mathrm{~km} / \mathrm{s}$

Rice EOS for Flyer

Strength Equation (2.92) for Flyer

Equations (4.13), (4.15), and (4.17) for $\mu$ and $Y$

$$
\mu_{0}, Y_{0}
$$

0.34 , and $75.13 \mathrm{GPa}$

Melting Formula (c16) for $T_{m}$

Shork Velocity in Target

$7.25 \mathrm{~km} / \mathrm{s}$

Overtake Position

$7.11 \mathrm{~mm}$

Mie-Gruneisen EOS for Target

$\gamma_{0}$ changed to

No Strength Model for Target 
Table 68.

Experimental and Calculated Sound Speeds for Tantalum

$\begin{array}{llll}\text { Pressure } & \text { Experimental } c_{l} & \text { Calculated } c_{l} & \text { Calculated } c_{l} \\ & & x=1 & x=1.5 \\ \mathrm{GPa} & \mathrm{km} / \mathrm{s} & \mathrm{km} / \mathrm{s} & \mathrm{km} / \mathrm{s} \\ 0 & & & \\ 152 & 4.14 & 4.04 & 4.05 \\ 211 & 6.23 & 6.23 & 6.38 \\ 229 & 6.55 & 6.50 & 6.64 \\ 255 & 6.61 & 6.56 & 6.68 \\ 293 & 6.75 & 6.63 & 6.71 \\ & 6.65 & 6.70 & 6.70\end{array}$


Table 69 .

Hugoniot Pressure and Residual Temperature

$\begin{array}{llll}\text { Material } & \text { EOS } & \text { Hugoniot } & \text { Residual } \\ & & \text { Pressure } & \text { Temperature } \\ & & & \\ \text { OF HC-Cu } & \text { Rice } & \sim 130 \mathrm{GPa} & \sim 1356 \mathrm{~K} \\ \text { OFHC-Cu } & \text { Mie-Gruneisen } & \sim 140 \mathrm{GPa} & \sim 1356 \mathrm{~K} \\ \text { SS-304L } & \text { Rice } & \sim 160 \mathrm{GPa} & \sim 1700 \mathrm{~K} \\ \text { SS-304L } & \text { Mie-Gruneiseil } & \sim 185 \mathrm{GPa} & \sim 1700 \mathrm{~K}\end{array}$


Table 70.

Initial Values for Equations $(d 3)-(d 5)$

Parameter

$E_{H}(\mathrm{GPa}-\mathrm{cc} / \mathrm{gm})$

$E_{c}(\mathrm{GPa} \mathrm{acc} / \mathrm{gm})$

R

$B^{\prime}$

$c_{H}(\mathrm{~km} / \mathrm{s})$
OF HC-Cu

0.0

$-0.0763$

2.002

0.0

3.940
SS-304L

0.0

$-0.0923$

2.070

0.0

4.566 
Table 71.

Pressures at the HE-Flyer Interface

HE

$\rho_{o}$

$u_{f}$

$\dot{P}$

$\frac{l}{d}$

$P_{\mathrm{i}}$

$P_{f}$

$\mathrm{gm} / \mathrm{cc}$

$\mathrm{km} / \mathrm{s}$

$\mathrm{GPa}$

$\mathrm{GPa} \quad \mathrm{GP} z$

TNT

1.6

3.7

3.2

1.1

7.5

1.3

Comp B

1.7

4.1

3.9

1.0

9.4

1.5

PBX-9404

1.8

4.6

4.9

1.0

12.0

1.9 
Table 72.

Pin Data for RS-2302

\begin{tabular}{|c|c|c|c|c|c|c|c|c|c|}
\hline $\begin{array}{l}\text { Pin } \\
\text { No. }\end{array}$ & $\begin{array}{l}t \\
(\mu s)\end{array}$ & $\begin{array}{l}x \\
(\mathrm{~mm})\end{array}$ & $\begin{array}{l}y \\
(\mathrm{~mm})\end{array}$ & $\begin{array}{l}z \\
(\mathrm{~mm})\end{array}$ & $\begin{array}{l}\text { Pin } \\
\text { No. }\end{array}$ & $\begin{array}{l}t \\
(\mu s)\end{array}$ & $\begin{array}{l}x \\
(\mathrm{~mm})\end{array}$ & $\begin{array}{l}y \\
(\mathrm{~mm})\end{array}$ & $\begin{array}{l}z \\
(\mathrm{~mm})\end{array}$ \\
\hline 1c & 1.111 & -1.498 & 62.5 & 0.0 & 38 & 3.315 & 12.97 & 48.2 & 57.5 \\
\hline $2 c$ & 1.318 & -0.746 & 62.2 & 6.5 & 39 & 3.469 & 13.98 & 44.1 & 60.7 \\
\hline $3 c$ & 1.538 & 0.0 & 81.1 & 13.0 & 40 & 3.620 & 14.95 & 39.7 & 63.6 \\
\hline $4^{\dagger}$ & 2.064 & 3.98 & 57.9 & 23.4 & 41 & 3.774 & 15.99 & 35.2 & 66.2 \\
\hline $5^{\dagger}$ & 2.212 & 4.97 & 54.1 & 31.2 & 42 & 3.919 & 16.96 & 30.5 & 68.5 \\
\hline $6^{\dagger}$ & 3.624 & 14.96 & 40.2 & 47.9 & 43 & $4.127^{\circ}$ & 17.96 & 25.7 & 70.5 \\
\hline $7^{\dagger}$ & 3.789 & 15.96 & 29.3 & 55.2 & 44 & 4.247 & 18.96 & 20.7 & 72.1 \\
\hline $8 c$ & 1.129 & -1.502 & -17.2 & 60.1 & 45 & 4.426 & 19.99 & 15.6 & 73.4 \\
\hline $9 c$ & 1.535 & 0.0 & -23.4 & 57.9 & 46 & 1.648 & 0.96 & -15.6 & 73.4 \\
\hline $10^{\dagger}$ & 2.071 & 3.97 & .33 .1 & 53.0 & 47 & 1.795 & 1.96 & -20.7 & 72.1 \\
\hline $11^{\dagger}$ & 2.213 & 5.02 & -40.2 & 47.9 & 48 & 1.926 & 2.98 & -25.7 & 70.5 \\
\hline $12^{\dagger}$ & 3.631 & 14.95 & .54 .1 & 31.3 & 49 & 2.073 & 3.97 & -30.5 & 68.5 \\
\hline $13^{\dagger}$ & 3.788 & 15.95 & -57.9 & 23.4 & 50 & 2.219 & 5.00 & -35.2 & 66.2 \\
\hline $14 t^{\dagger}$ & 3.783 & 15.96 & -57.9 & -23.4 & 51 & 2.350 & 5.99 & -39.7 & 63.6 \\
\hline $15^{\dagger}$ & 3.616 & 14.98 & -54.1 & -31.3 & 52 & 2.465 & 6.96 & .44 .1 & 60.7 \\
\hline $16^{\dagger}$ & 2.202 & 5.00 & -40.2 & -47.9 & 53 & 2.598 & 7.95 & -48.2 & 57.5 \\
\hline $17^{\dagger}$ & 2.062 & 3.96 & -33.1 & -.53 .0 & 54 & 2.732 & 8.99 & -52.1 & 54.0 \\
\hline $18 \mathrm{c}$ & 1.530 & 0.0 & -23.4 & -57.9 & 55 & 2.877 & 9.97 & -55.7 & 50.2 \\
\hline $19 c$ & $1.041^{\circ}$ & -1.509 & -17.2 & -60.1 & 56 & 3.024 & 10.56 & -59.1 & 46.2 \\
\hline $20^{\dagger}$ & 3.777 & 15.97 & 33.1 & -53.0 & 57 & 3.171 & 11.97 & -62.2 & 41.9 \\
\hline $21^{\dagger}$ & 3.621 & 14.95 & 40.2 & -47.9 & 58 & 3.320 & 12.98 & -65.0 & 37.5 \\
\hline $22^{\dagger}$ & 2.205 & 5.00 & 54.1 & -31.3 & 59 & 3.464 & 13.96 & -67.4 & 32.9 \\
\hline $23^{\dagger}$ & 2.065 & 3.96 & 57.9 & -23.4 & 60 & 3.616 & 14.94 & -69.5 & 28.1 \\
\hline $24 \mathrm{c}$ & 1.519 & 0.0 & 61.1 & -13.0 & 61 & 3.776 & 15.98 & -71.3 & 23.2 \\
\hline $25 c$ & 1.321 & -0.748 & 62.2 & -6.5 & 62 & 3.930 & 16.96 & -72.8 & 18.1 \\
\hline 26 & np & 0.96 & 75.0 & 2.6 & 33 & 4.081 & 17.96 & -73.9 & 13.0 \\
\hline 27 & 1.782 & 1.97 & 74.6 & 7.8 & 64 & 4.248 & 18.96 & -74.6 & 7.8 \\
\hline 29 & 1.923 & 2.96 & 73.9 & 13.0 & 65 & 4.404 & 19.99 & -75.0 & 2.6 \\
\hline 29 & 2.052 & 3.99 & 72.8 & 18.1 & 66 & 4.398 & 19.99 & -75.0 & -2.6 \\
\hline 30 & 2.192 & 4.96 & 71.3 & 23.2 & 67 & 4.233 & 18.96 & -74.6 & 7.8 \\
\hline 31 & 2.322 & 5.98 & 89.5 & 28.1 & 68 & 4.088 & 17.95 & -73.9 & -13.0 \\
\hline 32 & 2.466 & 6.96 & 67.4 & 32.9 & 69 & 3.918 & 16.95 & -72.8 & 18.1 \\
\hline 33 & 2.615 & 7.97 & 65.0 & 37.5 & 70 & 3.771 & 15.98 & -71.3 & -23.2 \\
\hline 34 & 2.715 & 8.99 & 52.2 & 41.9 & 71 & 3.603 & 14.95 & -69.5 & -28.1 \\
\hline 35 & 2.892 & 9.98 & 55.1 & 46.2 & 72 & 3.458 & 13.99 & -67.4 & -32.9 \\
\hline 35 & 3.033 & 10.99 & 55.7 & 50.2 & 73 & 3.304 & 12.96 & -65.0 & -37.5 \\
\hline 37 & 3.173 & $11.9^{\prime} \%$ & 52.1 & 54.0 & 74 & 3.161 & 11.87 & -62.2 & -41.9 \\
\hline
\end{tabular}


Table 72 corisnued

$\begin{array}{lllll}\text { Pin } & t & x & y & z \\ \text { No. } & (\mu \mathrm{s}) & (\mathrm{mm}) & (\mathrm{mm}) & (\mathrm{mm}) \\ 75 & 3.005 & 10.97 & -59.1 & -46.2 \\ 76 & 2.870 & 9.97 & -55.7 & -50.2 \\ 77 & 2.722 & 8.97 & -52.1 & -54.0 \\ 78 & 2.587 & 7.96 & -48.2 & -57.5 \\ 79 & 2.435 & 6.97 & -44.1 & -60.7 \\ 80 & 2.308 & 5.97 & -39.7 & -63.6 \\ 81 & 2.179 & 5.00 & -35.2 & -66.2 \\ 82 & 2.095 & 3.96 & -30.5 & -68.5 \\ 83 & 1.909 & 2.98 & -25.7 & -70.5 \\ 84 & 1.775 & 1.97 & -20.7 & -72.1 \\ 85 & 1.624 & 0.95 & -15.6 & -73.4 \\ 86 & 4.380 & 19.99 & 15.6 & -73.4 \\ 87 & 4.218 & 18.96 & 20.7 & -72.1 \\ 88 & 4.075 & 17.96 & 25.7 & -70.5 \\ 39 & 3.902 & 16.96 & 30.5 & -68.5 \\ 90 & 3.755 & 15.97 & 35.2 & -66.2 \\ 91 & 3.598 & 14.96 & 39.7 & -63.6 \\ 92 & 3.449 & 13.98 & 44.1 & -60.7 \\ 93 & 3.306 & 12.96 & 48.2 & -57.5 \\ 94 & 3.151 & 11.98 & 52.1 & -54.0 \\ 95 & 3.019 & 10.97 & 55.7 & -50.2 \\ 96 & 2.895 & 9.96 & 59.1 & -46.2 \\ 97 & 2.754 & 8.96 & 62.2 & -41.9 \\ 98 & 2.598 & 7.97 & 65.0 & -37.5 \\ 99 & 2.463 & 6.97 & 67.4 & -32.9 \\ 100 & 2.321 & 5.97 & 69.5 & -28 \\ 101 & 2.201 & 5.00 & 71.3 & -232 \\ 102 & 2.049 & 3.96 & 72.8 & -18.2 \\ 103 & 1.921 & 2.98 & 73.9 & -13.0 \\ 104 & 1.778 & 1.97 & 74.6 & -7.8 \\ 105 & 1.642 & 0.96 & 75.0 & -2.6\end{array}$

$\begin{array}{lllll}\text { Pin } & t & x & y & z \\ \text { No. } & (\mu \mathrm{g}) & (\mathrm{mm}) & (\mathrm{mm}) & (\mathrm{mm}) \\ 106 \mathrm{c} & 1.095 & -1.516 & 87.5 & 0.0 \\ 107 \mathrm{c} & 1.318 & -0.751 & 87.0 & 9.1 \\ 108 \mathrm{c} & 1.492 & 0.0 & 85.6 & 18.2 \\ 109^{\dagger} & 2.042 & 3.98 & 81.2 & 32.8 \\ 110^{\dagger} & 2.185 & 4.98 & 75.8 & 43.8 \\ 111^{\dagger} & 3.613 & 14.96 & 56.2 & 67.0 \\ 112^{\dagger} & 3.764 & 15.96 & 46.4 & 74.2 \\ 113 \mathrm{c} & 1.098 & -1.492 & -24.1 & 84.1 \\ 114 \mathrm{c} & 1.707 & 0.0 & -32.8 & 81.1 \\ 115^{\dagger} & 2.060 & 3.96 & -46.4 & 74.2 \\ 116^{\dagger} & 2.175 & 4.97 & -56.2 & 67.0 \\ 117^{\dagger} & 3.619 & 14.95 & -75.8 & 43.8 \\ 118^{\dagger} & 3.783 & 14.95 & -81.1 & 32.8 \\ 119^{\dagger} & 3.745 & 15.95 & -81.1 & -32.8 \\ 120^{\dagger} & 3.595 & 14.94 & -75.8 & -\mathbf{4 3 . 8} \\ 121^{\dagger} & 2.313 & 4.97 & -56.2 & -67.0 \\ 122^{\dagger} & 2.032 & 3.96 & -46.4 & -74.2 \\ 123 \mathrm{c} & 1.508 & 0.0 & -32.8 & -81.1 \\ 124 \mathrm{c} & 1.089 & -1.511 & -24.1 & -84.1 \\ 125^{\dagger} & 3.739 & 15.95 & 46.4 & -74.2 \\ 126^{\dagger} & \mathbf{7 P} & 14.97 & \mathbf{5 6 . 2} & -67.0 \\ 127^{\dagger} & 2.173 & 4.97 & 75.8 & -43.8 \\ 128^{\dagger} & 2.039 & 3.99 & 81.1 & -32.8 \\ 129 \mathrm{c} & 1.508 & 0.0 & 85.6 & -18.2 \\ 130 \mathrm{c} & 1.300 & -0.735 & 87.0 & -9.1\end{array}$


Table 73.

Pin Data for RS-2309

\begin{tabular}{|c|c|c|c|c|c|c|c|c|c|}
\hline $\begin{array}{l}\text { Pin } \\
\text { No. }\end{array}$ & $\begin{array}{l}t \\
(\mu s)\end{array}$ & $\begin{array}{l}x \\
(\mathrm{~mm})\end{array}$ & $\begin{array}{l}y \\
(\mathrm{~mm})\end{array}$ & $\begin{array}{l}z \\
(\mathrm{~mm})\end{array}$ & $\begin{array}{l}\text { Pin } \\
\text { No. }\end{array}$ & $\begin{array}{l}t \\
(\mu s)\end{array}$ & $\begin{array}{l}x \\
(\mathrm{~mm})\end{array}$ & $\begin{array}{l}y \\
(\mathrm{~mm})\end{array}$ & $\begin{array}{l}z \\
(\mathrm{~mm})\end{array}$ \\
\hline $1 c$ & 1.647 & -1.007 & 40.0 & 0.0 & 38 & 3.812 & 15.030 & -43.3 & -25.0 \\
\hline $2 c$ & np & 0.0 & 39.1 & 8.3 & $39 c$ & 1.851 & 0.0 & -25.0 & -43.3 \\
\hline $3 c$ & 1.643 & -1.011 & -20.0 & 34.6 & 40 & 1.977 & 1.016 & -20.3 & -45.7 \\
\hline $4 c$ & 1.857 & 0.0 & -26.8 & 29.7 & 41 & 2.103 & 2.013 & -15.5 & -47.6 \\
\hline $5 c$ & 1.619 & -0.991 & -20.0 & -34.6 & 42 & 2.222 & 3.010 & -10.4 & -48.9 \\
\hline $6 c$ & 1.844 & 0.0 & -12.4 & -38.0 & 43 & 2.347 & 4.010 & -5.2 & -49.7 \\
\hline $7 c$ & np & 0.0 & $50 . n$ & 0.0 & 44 & 2.478 & 5.028 & 0.0 & -50.0 \\
\hline 8 & 1.994 & 0.997 & 49.7 & 5.2 & 45 & 2.606 & 6.024 & 5.2 & -49.7 \\
\hline 9 & 2.119 & 2.012 & 48.9 & 10.4 & 46 & 2.727 & 7.041 & 10.4 & -48.9 \\
\hline 10 & 2.242 & 3.000 & 47.6 & 15.5 & 47 & 2.858 & 8.030 & 15.5 & -47.6 \\
\hline 11 & 2.365 & 3.988 & 45.7 & 20.3 & 48 & 2.992 & 9.017 & 20.3 & -45.7 \\
\hline 12 & 2.494 & 5.027 & 43.3 & 25.0 & 49 & 3.128 & 10.032 & 25.0 & -43.3 \\
\hline 13 & 2.620 & 6.009 & 40.5 & 29.4 & 50 & 3.258 & 11.029 & 29.4 & -40.5 \\
\hline 14 & 2.752 & 7.018 & 37.2 & 33.5 & 51 & 3.395 & 12.009 & 33.5 & -37.2 \\
\hline 15 & 2.871 & 8.022 & 33.5 & 37.2 & 52 & 3.540 & 13.023 & 37.2 & -33.5 \\
\hline $1 \theta$ & 3.003 & 9.014 & 29.4 & 40.59 & 53 & 3.681 & 14.010 & 40.5 & -29.4 \\
\hline 17 & 3.135 & 10.043 & 25.0 & 43.3 & 54 & 3.828 & 15.029 & 43.3 & -25.0 \\
\hline 18 & 3.270 & 11.028 & 20.3 & 45.7 & $55 c$ & 1.643 & -0.992 & 60.0 & 0.0 \\
\hline 19 & 3.406 & 12.005 & 15.5 & 47.6 & $56 c$ & 1.873 & 0.0 & 58.7 & 12.5 \\
\hline 20 & 3.539 & 13.012 & 10.4 & 48.9 & $57 \mathrm{c}$ & 1.643 & -1.001 & -30.0 & 52.0 \\
\hline 21 & 3.680 & 14.031 & 5.2 & 49.7 & $58 c$ & 1.858 & 0.0 & -40.1 & 44.6 \\
\hline 22 & 3.826 & 15.048 & 0.0 & 50.0 & $59 c$ & 1.640 & -0.994 & -30.0 & -52.0 \\
\hline $23 c$ & 1.860 & 0.0 & -25.0 & 43.3 & $B 0 c$ & 1.854 & 0.0 & -18.5 & -57.1 \\
\hline 24 & 1.983 & 1.023 & -29.4 & 40.5 & $61 \mathrm{c}$ & 1.863 & 0.0 & 70.0 & 0.0 \\
\hline 25 & 2.100 & 2.037 & -33.5 & 37.2 & 62 & 1.983 & 0.999 & 69.6 & 7.3 \\
\hline 26 & 2.187 & $2.7: 2$ & -37.2 & 33.5 & 63 & 2.107 & 2.019 & 68.5 & 14.6 \\
\hline 27 & 2.358 & 4.009 & -40.5 & 29.4 & 64 & 2.237 & 3.012 & 66.6 & 21.6 \\
\hline 28 & 2.482 & 5.029 & -43.3 & 25.0 & 65 & 2.360 & 4.016 & 63.9 & 28.5 \\
\hline 29 & 2.600 & 6.015 & -45.7 & 20.3 & 66 & 2.489 & 5.021 & 60.6 & 35.0 \\
\hline 30 & 2.725 & 7.018 & -47.6 & 15.5 & 67 & 2.608 & 6.015 & 56.6 & 41.1 \\
\hline 31 & 2.859 & 8.020 & -48.9 & 10.4 & 68 & 2.731 & 7.036 & 52.0 & 46.8 \\
\hline 32 & 2.989 & 9.026 & -49.7 & 5.2 & 69 & 2.860 & 8.015 & 46.8 & 52.0 \\
\hline 33 & 3.116 & 10.032 & -50.0 & 0.0 & 70 & 3.000 & 8.987 & 41.1 & 56.6 \\
\hline 34 & 3.246 & 11.036 & -49.7 & -5.2 & 71 & 3.132 & 10.030 & 35.0 & 60.6 \\
\hline 35 & np & 12.018 & -48.9 & -10.4 & 72 & 3.260 & 11.015 & 28.5 & 63.9 \\
\hline 36 & 3.525 & 13.038 & -47.8 & -15.5 & 73 & 3.389 & 11.982 & 21.6 & 66.6 \\
\hline 37 & 3.665 & 14.030 & -45.7 & -20.3 & 74 & 3.500 & 12.752 & 14.6 & 68.5 \\
\hline
\end{tabular}


Tuble 79 continued

$\begin{array}{lllll}\text { Pin } & t & x & y & z \\ \text { No. } & (\mu \mathrm{s}) & (\mathrm{mm}) & (\mathrm{mm}) & (\mathrm{mm}) \\ 75 & 3.671 & 14.006 & 7.3 & 69.6 \\ 76 & 3.816 & 15.022 & 0.0 & 70.0 \\ 77 \mathrm{c} & 1.845 & 0.0 & -35.0 & 60.6 \\ 78 & 1.974 & 1.013 & -41.4 & 56.6 \\ 79 & 2.105 & 2.005 & -46.8 & 52.0 \\ 80 & 2.221 & 2.998 & -52.0 & 46.8 \\ 81 & 2.339 & 4.006 & -56.6 & 41.1 \\ 82 & 2.173 & 5.037 & -60.8 & 35.0 \\ 83 & 2.590 & 6.016 & -63.9 & 28.5 \\ 84 & 2.715 & 7.038 & -66.6 & 21.6 \\ 85 & 2.842 & 8.035 & -68.5 & 14.6 \\ 86 & 2.980 & 9.024 & -69.6 & 7.3 \\ 87 & 3.116 & 10.049 & -70.0 & 0.0 \\ 88 & 3.250 & 11.047 & -69.6 & -7.3 \\ 89 & 3.380 & 12.011 & -68.4 & -14.6 \\ 90 & 3.518 & 13.027 & -66.6 & -21.6 \\ 91 & 3.666 & 14.029 & -63.9 & -28.5 \\ 92 & 3.800 & 15.042 & -60.6 & -35.0 \\ 93 \mathrm{c} & 1.829 & 0.0 & -35.0 & -60.6 \\ 94 & 1.960 & 1.023 & -28.5 & -63.9 \\ 95 & 2.090 & 2.044 & -21.6 & -66.6 \\ 96 & 2.220 & 3.019 & -14.6 & -68.5 \\ 97 & 2.339 & 4.014 & -7.3 & -69.6 \\ 98 & 2.457 & 5.012 & 0.0 & -70.0 \\ 99 & 2.599 & 6.022 & 7.3 & -69.6\end{array}$

\begin{tabular}{|c|c|c|c|c|}
\hline $\begin{array}{l}\text { Pin } \\
\text { No. }\end{array}$ & $\begin{array}{l}t \\
(\mu s)\end{array}$ & $\begin{array}{l}x \\
(\mathrm{~mm})\end{array}$ & $\begin{array}{l}y \\
(\mathrm{~mm})\end{array}$ & $\begin{array}{l}z \\
(\mathrm{~mm})\end{array}$ \\
\hline 100 & 2.716 & 7.027 & 14.6 & -68.4 \\
\hline 101 & 2.850 & 8.027 & 21.6 & -66.6 \\
\hline 102 & 2.981 & 9.022 & 28.5 & -63.9 \\
\hline 103 & 3.120 & 10.041 & 35.0 & -60.6 \\
\hline 104 & 3.256 & 11.045 & 41.1 & -56.6 \\
\hline 105 & 3.390 & 12.014 & 46.8 & -52.0 \\
\hline 108 & 3.525 & 13.034 & 52.0 & -46.8 \\
\hline 107 & 3.672 & 14.029 & 56.8 & -41.1 \\
\hline 108 & $n p$ & 15.042 & 60.6 & -35.0 \\
\hline $109 e$ & 1.632 & -1.015 & 80.0 & 0.0 \\
\hline $110 \mathrm{c}$ & $n p$ & 0.0 & 78.3 & 16.6 \\
\hline $111 \mathrm{c}$ & 1.620 & -0.990 & -40.0 & 69.3 \\
\hline $112 c$ & 1.836 & 0.0 & -53.5 & 59.5 \\
\hline $113 c$ & 1.608 & -0.995 & -40.0 & -69.3 \\
\hline $114 \mathrm{c}$ & 1.836 & 0.0 & -24.7 & -76.1 \\
\hline
\end{tabular}


Table 74.

Pin Data for RS-2315

\begin{tabular}{|c|c|c|c|c|c|c|c|c|c|}
\hline $\begin{array}{l}\text { Pin } \\
\text { No. }\end{array}$ & $\begin{array}{l}t \\
(\mu s)\end{array}$ & $\begin{array}{l}x \\
(\mathrm{~mm})\end{array}$ & $\begin{array}{l}y \\
\text { (.nm) }\end{array}$ & $\begin{array}{l}z \\
(\mathrm{~mm})\end{array}$ & $\begin{array}{l}\text { Pin } \\
\text { No. }\end{array}$ & $\begin{array}{l}t \\
(\mu s)\end{array}$ & $\begin{array}{l}x \\
(\mathrm{~mm})\end{array}$ & $\begin{array}{l}y \\
(\mathrm{~mm})\end{array}$ & $\begin{array}{l}z \\
(\mathrm{~mm})\end{array}$ \\
\hline $1 c$ & 1.394 & -1.012 & 40.0 & 0.0 & 38 & 3.680 & 15.000 & -43.3 & -25.0 \\
\hline $2 c$ & 1.637 & 0.0 & 39.1 & 8.3 & $39 c$ & 1.672 & 0.0 & -25.0 & -43.3 \\
\hline $3 c$ & 1.415 & -1.009 & -20.0 & 34.6 & 40 & 1.805 & 0.991 & -20.3 & -45.7 \\
\hline $4 c$ & 1.656 & 0.0 & -26.8 & 29.7 & 41 & 1.928 & 1.989 & -15.5 & -47.6 \\
\hline Jc & 1.424 & -1.002 & -20.0 & -34.6 & 42 & 2.056 & 2.982 & -10.4 & -48.9 \\
\hline $6 c$ & 1.670 & 0.0 & -12.4 & -38.0 & 43 & 2.183 & 3.972 & -5.2 & -49.7 \\
\hline $7 c$ & 1.641 & 0.0 & 50.0 & 0.0 & 44 & 2.317 & 4.985 & 0.0 & -50.0 \\
\hline 8 & 1.772 & 0.993 & 49.7 & 5.2 & 45 & 2.447 & 5.992 & 5.2 & -49.7 \\
\hline 9 & 1.903 & 2.012 & 48.9 & 10.4 & 4ิô & 2.571 & 7.015 & 10.4 & -48.9 \\
\hline 10 & 2.031 & 2.975 & 47.6 & 15.5 & 47 & 2.704 & 7.983 & 15.5 & -47.6 \\
\hline 11 & 2.148 & 3.936 & 45.7 & 20.3 & 48 & 2.841 & 8.979 & 20.3 & -45.7 \\
\hline 12 & 2.284 & 4.987 & 43.3 & 25.0 & 49 & 2.977 & 9.981 & 25.0 & -43.3 \\
\hline 13 & 2.411 & 5.984 & 40.5 & 29.4 & 50 & 3.111 & 10.979 & 29.4 & -40.5 \\
\hline 14 & 2.547 & 7.002 & 37.2 & 33.5 & 51 & 3.238 & 11.966 & 33.5 & -37.2 \\
\hline 15 & 2.672 & 7.998 & 33.5 & 37.2 & 52 & 3.391 & 12.994 & 37.2 & -33.5 \\
\hline 16 & 2.818 & 8.987 & 29.4 & 40.5 & 53 & 3.519 & 13.989 & 40.5 & -29.4 \\
\hline 17 & 2.940 & 9.993 & 25.0 & 43.3 & 54 & 3.666 & 14.993 & 43.3 & -25.0 \\
\hline 18 & 3.088 & 10.989 & 20.3 & 45.7 & $55 c$ & 1.396 & -1.008 & 60.0 & 0.0 \\
\hline 19 & 3.223 & 11.987 & 15.5 & 47.6 & $56 c$ & 1.629 & 0.0 & 58.7 & 12.5 \\
\hline 20 & 3.381 & 13.003 & 10.4 & 48.9 & $57 \mathrm{c}$ & 1.399 & -1.005 & -30.0 & 52.0 \\
\hline 21 & 3.516 & 14.000 & 5.2 & 49.7 & $58 c$ & 1.634 & 0.0 & -40.1 & 44.6 \\
\hline 22 & 3.663 & 15.009 & 0.0 & 50.0 & $59 c$ & 1.421 & -0.998 & -30.0 & -52.0 \\
\hline $23 c$ & 1.655 & 0.0 & -25.0 & 43.3 & $60 c$ & 1.661 & 0.0 & -18.5 & -57.1 \\
\hline 24 & 1.785 & 0.996 & -29.4 & 40.5 & $\theta 1 c$ & 1.638 & 0.0 & 70.0 & 0.0 \\
\hline 25 & np & 2.002 & -33.5 & 37.2 & 62 & 1.754 & 0.978 & 69.6 & 7.3 \\
\hline 26 & 2.038 & 3.008 & -37.2 & 33.5 & 63 & 1.881 & 2.004 & 68.5 & 14.6 \\
\hline 27 & 2.172 & 3.972 & -40.5 & 29.4 & 64 & 2.012 & 2.986 & 66.6 & 21.6 \\
\hline 28 & 2.308 & 4.984 & -43.3 & 25.0 & 65 & 2.143 & 3.990 & 63.9 & 28.5 \\
\hline 29 & 2.427 & 5.976 & -45.7 & 20.3 & 66 & 2.264 & 4.993 & 60.6 & 35.0 \\
\hline 30 & 2.553 & 6.997 & -47.6 & 15.5 & 67 & 2.390 & 5.972 & $5 e .6$ & 41.1 \\
\hline 31 & 2.697 & 7.997 & -48.9 & 10.4 & 68 & 2.521 & 6.983 & 52.0 & 48.8 \\
\hline 32 & 2.836 & 9.010 & -49.7 & 5.2 & 69 & 2.656 & 7.973 & 46.8 & 52.0 \\
\hline 33 & 2.965 & 9.99 & -50.0 & 0.0 & 70 & 2.791 & 8.991 & 41.1 & 56.6 \\
\hline 34 & 3.109 & 16.998 & -49.7 & -5.2 & 71 & 2.916 & 10.011 & 35.0 & 60.6 \\
\hline 35 & 3.258 & 11.913 & -48.9 & -10.4 & 72 & 3.060 & 10.972 & 28.5 & 63.9 \\
\hline 38 & 3.402 & 13.009 & -47.6 & -15.5 & 73 & 3.210 & 11.982 & 21.6 & 66.6 \\
\hline 37 & 3.531 & 14.000 & -45.7 & -20.3 & 74 & 3.352 & 12.991 & 14.6 & 68.5 \\
\hline
\end{tabular}


Table $7 f$ continued

\begin{tabular}{|c|c|c|c|c|}
\hline $\begin{array}{l}\text { Pirı } \\
\text { No. }\end{array}$ & $\begin{array}{l}t \\
(\mu s)\end{array}$ & $\begin{array}{l}I \\
(\mathrm{~mm})\end{array}$ & $\begin{array}{l}y \\
(\mathrm{~mm})\end{array}$ & $\begin{array}{l}z \\
(\mathrm{~mm})\end{array}$ \\
\hline 75 & 3.499 & 13.984 & 7.3 & 69.6 \\
\hline 76 & 3.656 & 15.000 & 0.0 & 70.0 \\
\hline $77 \mathrm{c}$ & 1.623 & 0.0 & -35.0 & 60.8 \\
\hline 78 & 1.752 & 0.994 & -41.4 & 56.8 \\
\hline 79 & $1 . \sin 8$ & 2.003 & -46.8 & 52.0 \\
\hline 80 & 2.022 & 2.992 & -52.0 & 46.8 \\
\hline 81 & 2.181 & 3.969 & -58.6 & $4 ! .1$ \\
\hline 82 & 2.288 & 5.009 & -60.6 & 35.0 \\
\hline 83 & 2.409 & 5.993 & -83.9 & 28.5 \\
\hline 84 & 2.549 & 7.001 & -66.6 & 21.6 \\
\hline 85 & 2.668 & 7.905 & -68.5 & 14.6 \\
\hline 86 & 2.816 & 8.992 & -69.6 & 7.3 \\
\hline 87 & 2.941 & 9.997 & -70.0 & 0.0 \\
\hline 88 & 3.084 & 10.972 & $-69 . \AA$ & -7.3 \\
\hline 89 & 3.233 & 11.981 & -68.4 & -146 \\
\hline 90 & 3.388 & 12.993 & -60.6 & -21.6 \\
\hline 91 & 3.530 & 13.998 & -63.9 & -28.5 \\
\hline 92 & 3.684 & 15.005 & -606 & -35.0 \\
\hline $93 c$ & 1.653 & 0.0 & -35.0 & -60.6 \\
\hline 94 & 1.774 & 0.978 & .28 .5 & -63.9 \\
\hline 95 & 1.902 & 1.996 & -21.6 & -68.5 \\
\hline 96 & 2.030 & 2.996 & -14.6 & -68.5 \\
\hline 97 & 2.168 & 3.977 & -7.3 & -69.6 \\
\hline 98 & 2.306 & $5.00 \mathrm{e}$ & 0.0 & -70.0 \\
\hline 99 & 2.426 & 5.992 & 7.3 & -69.8 \\
\hline
\end{tabular}

$\begin{array}{lllll}\text { Pin } & t & x & y & z \\ \text { No. } & (\mu \mathrm{s}) & (\mathrm{mm}) & (\mathrm{mm}) & (\mathrm{mm}) \\ : 00 & 2.549 & 8.982 & 14.6 & -68.4 \\ 101 & 2.086 & 7.982 & 21.6 & -68.6 \\ 102 & 2.823 & 8.991 & 28.5 & -63.9 \\ 103 & 2.956 & 9.995 & 35.0 & -60.6 \\ 104 & 3.093 & 10.957 & 41.1 & -56.6 \\ 105 & 3.227 & 11.962 & 46.8 & -52.0 \\ 106 & 3.374 & 12.986 & 52.0 & -46.8 \\ 107 & 3.507 & 13.974 & 56.6 & -41.1 \\ 108 & 3.648 & 14.974 & 60.6 & -35.0 \\ 109 \mathrm{c} & 1.378 & -1.006 & 80.0 & 0.0 \\ 110 \mathrm{c} & 1.615 & 0.0 & 78.3 & 16.6 \\ 111 \mathrm{c} & 1.396 & -1.007 & -40.0 & 69.3 \\ 112 \mathrm{c} & 1.624 & 0.0 & -53.5 & 59.5 \\ 113 \mathrm{c} & 1.404 & -0.996 & -40.0 & -69.3 \\ 114 \mathrm{c} & 1.648 & 0.0 & -24.7 & -76.1\end{array}$


Table 75.

Pin Data for RS-2306

\begin{tabular}{|c|c|c|c|c|c|c|c|c|c|}
\hline $\begin{array}{l}\mathrm{Pin} \\
\text { No. }\end{array}$ & $\begin{array}{l}t \\
(\mu-x)\end{array}$ & $\begin{array}{l}x \\
(\mathrm{~mm})\end{array}$ & $\begin{array}{l}y \\
(\mathrm{~mm})\end{array}$ & $\begin{array}{l}z \\
(\mathrm{~mm})\end{array}$ & $\begin{array}{l}\text { Pin } \\
\text { No. }\end{array}$ & $\begin{array}{l}t \\
(\mu \theta)\end{array}$ & $\begin{array}{l}x \\
(\mathrm{~mm})\end{array}$ & $\begin{array}{l}y \\
(\mathrm{~m} m)\end{array}$ & $\begin{array}{l}z \\
(\mathrm{~mm})\end{array}$ \\
\hline lc & 0.994 & -1.174 & 63.5 & 2.2 & 38 & 1.757 & 2.965 & -305 & 88.5 \\
\hline $2 c$ & 1.310 & 0.0 & 62.9 & 8.8 & 39 & 1.815 & 1.989 & -35.2 & 8. 2 \\
\hline $3 c$ & 1.323 & 0.0 & -27.8 & 57.1 & 40 & 1.190 & 0.955 & -39.1 & $\cdot 3.6$ \\
\hline $4 c$ & 1.009 & -1.207 & -33.6 & 53.9 & 41 & 1.310 & 1.990 & -44.1 & 60.7 \\
\hline $5 c$ & 1.333 & 0.0 & -39.1 & 50.0 & 42 & 1.771 & 2.969 & -48.2 & 57.5 \\
\hline $6 c$ & 1.322 & 0.0 & -35.5 & .52 .6 & 43 & 1.909 & 3.958 & -52.1 & 54.0 \\
\hline $7 c$ & 0.990 & -1.203 & -29.8 & -56.1 & 44 & 2.080 & 4.958 & -55.7 & 50.2 \\
\hline $8 c$ & 1.313 & 0.0 & -23.8 & -58.9 & 45 & 2.203 & 5.936 & -59.1 & 46.2 \\
\hline $9 c$ & 1.308 & 0.0 & 63.3 & -4.4 & 46 & $? 349$ & 6.927 & -62.2 & 41.9 \\
\hline 10 & 1.437 & 0.967 & 75.0 & 2.6 & 47 & 2.512 & 7.954 & $-u 5.0$ & 37.5 \\
\hline 11 & 1.599 & 1.973 & 74.6 & 7.8 & 48 & 2.686 & 8.952 & -67.4 & 32.9 \\
\hline 12 & 1.745 & 2.908 & 73.9 & 13.0 & 49 & 2.820 & 9.969 & -69.5 & 28.1 \\
\hline 13 & 1.903 & 3.964 & 72.8 & 18.1 & 50 & 2.982 & 10.950 & -71.3 & 23.2 \\
\hline 14 & 2.043 & 4.967 & $7: 3$ & 23.2 & 51 & 3.140 & 11.925 & -72.3 & 18.1 \\
\hline 15 & 2.189 & 5.939 & $6 y .5$ & 28.1 & 52 & 3.307 & 12.960 & -73.9 & 13.0 \\
\hline 16 & 2.349 & 6.934 & e7.1 & 32.9 & 53 & 3.474 & 13.912 & -74.0 & 7.8 \\
\hline 17 & 2.508 & 7.959 & 65.0 & 37.5 & 54 & 3.645 & 14.967 & .75 .0 & 2.6 \\
\hline 9 & 2.681 & 8.935 & 82.2 & 41.9 & 55 & 3.825 & 15.951 & -75.0 & -2.6 \\
\hline 19 & 2.817 & 9.954 & 59.1 & 46.2 & 56 & 3.041 & 14.923 & .74 .6 & -7.8 \\
\hline 20 & 2.973 & 10.953 & 55.7 & 50.2 & 57 & 3.468 & I3.905 & -73.9 & - . 2.0 \\
\hline 21 & 3.143 & 11.928 & 52.1 & 54.0 & 58 & 3.303 & i2.965 & -72.8 & $-18 . i$ \\
\hline 22 & 3.325 & 12.970 & 48.2 & 57.5 & 59 & 3.124 & 11.899 & -71.3 & -23.2 \\
\hline 23 & 3.494 & 13.927 & 44.1 & 60.7 & 80 & 2.973 & 10.937 & -69.5 & -28.1 \\
\hline 24 & 3.660 & 14.985 & 39.7 & 63.8 & 61 & 2.823 & 9.949 & .67 .4 & -32.9 \\
\hline 25 & 3.821 & 15.946 & 35.2 & 86.2 & 62 & 2.657 & 8.924 & -65.0 & 37.5 \\
\hline 26 & 3.652 & 14.968 & 30.5 & 68.5 & 83 & 2.499 & $? .951$ & -62.2 & -41.9 \\
\hline 27 & 3.469 & 13.925 & 25.7 & 70.5 & 64 & 2.349 & 6.924 & .59 .1 & -46.2 \\
\hline 28 & 3.295 & 12.979 & 20.7 & 72.1 & 65 & 2.197 & 5.921 & -55.7 & -50.2 \\
\hline 29 & 3.123 & 11.945 & 15.6 & 134 & 66 & 2.045 & 4.958 & -52.1 & -54.0 \\
\hline 30 & 2.972 & 10.971 & $10 . \dot{\varepsilon}$ & 74.3 & 67 & ipp & 3.940 & -48.2 & -57.5 \\
\hline 31 & 2.808 & 9.979 & 5.2 & 74.8 & 68 & 1.741 & 2.942 & -44.1 & .60 .7 \\
\hline 32 & 2.657 & 8.962 & 0.0 & 75.0 & 69 & 1.608 & 1.958 & -39.7 & -65.8 \\
\hline 33 & 2.492 & 7.965 & -5.2 & 74.8 & 70 & 1.452 & 0.941 & -35.2 & -66.2 \\
\hline 34 & 2.346 & 6.959 & -10.4 & 74.3 & 71 & 1.615 & 1.981 & -30.5 & -88.5 \\
\hline 35 & 2.194 & 5.951 & -15.6 & 7s.: & 72 & 1.734 & 2.960 & -25.7 & -70.5 \\
\hline 36 & np & 4.984 & -20.7 & 72.1 & 73 & 1.904 & 3.943 & -20.7 & -72.1 \\
\hline 37 & 1.912 & 3.965 & -25.7 & 70.5 & 74 & 2.046 & 4.965 & .15 .8 & -73.4 \\
\hline
\end{tabular}


Table 75 continued

$\begin{array}{lllll}\text { Pin } & t & x & y & z \\ \text { No. } & (\mu \mathrm{s}) & (\mathrm{mm}) & (\mathrm{mm}) & (\mathrm{mm}) \\ 75 & 2.192 & 5.933 & -10.4 & -74.3 \\ 76 & 2.345 & 6.947 & -5.2 & -74.8 \\ 77 & 2.497 & 7.957 & 0.0 & -75.0 \\ 78 & 2.642 & 8.934 & 5.2 & -74.8 \\ 79 & 2.803 & 9.958 & 10.4 & -74.3 \\ 80 & 2.965 & 10.948 & 15.6 & -73.4 \\ 81 & 3.125 & 11.926 & 20.7 & -72.1 \\ 82 & 3.299 & 12.962 & 25.7 & -70.5 \\ 83 & 3.460 & 19.930 & 30.5 & -68.5 \\ 84 & 3.643 & 14.961 & 35.2 & -68.2 \\ 85 & 3.818 & 15.956 & 39.7 & -63.6 \\ 86 & 3.827 & 14.953 & 44.1 & -60.7 \\ 87 & 3.486 & 13.925 & 48.2 & -57.5 \\ 88 & 3.309 & 12.982 & 52.1 & -54.0 \\ 89 & 3.128 & 11.939 & 55.7 & -50.2 \\ 90 & 2.973 & 10.967 & 59.1 & -46.2 \\ 91 & 2.817 & 9.979 & 62.2 & -41.9 \\ 92 & 2.658 & 8.968 & 65.0 & -37.5 \\ 93 & 2.508 & 7.979 & 67.4 & -32.9 \\ 94 & 2.338 & 6.957 & 69.5 & -28.1 \\ 95 & 2.181 & 5.950 & 71.3 & -23.2 \\ 96 & 2.060 & 4.977 & 72.8 & -18.1 \\ 97 & 1.894 & 3.957 & 73.9 & -13.0 \\ 98 & 1.743 & 2.971 & 74.6 & -7.8 \\ 99 & 1.596 & 2.003 & 75.0 & -2.6\end{array}$

$\begin{array}{lllll}\text { Pin } & t & x & y & z \\ \text { No. } & (\mu \mathrm{s}) & (\mathrm{rnm}) & (\mathrm{mm}) & (\mathrm{mm}) \\ 100 \mathrm{c} & 0.977 & -1.185 & 87.4 & 3.1 \\ 101 \mathrm{c} & 1.302 & 0.0 & 87.0 & 9.1 \\ 102 \mathrm{c} & 1.316 & 0.0 & -41.1 & 77.3 \\ 103 \mathrm{c} & 0.984 & -1.171 & -46.4 & 74.2 \\ 104 \mathrm{c} & 1.294 & 0.0 & -51.4 & 70.8 \\ 105 \mathrm{c} & 1.291 & 0.0 & =-46.4 & -74.2 \\ 106 \mathrm{c} & 0.972 & -1.168 & -41.1 & -77.3 \\ 107 \mathrm{c} & 1.287 & 0.0 & -35.6 & -79.9 \\ 108 \mathrm{c} & 1.310 & 0.0 & 87.4 & -3.1\end{array}$


Table 76.

Pin Data for RS-2308

\begin{tabular}{|c|c|c|c|c|c|c|c|c|c|}
\hline $\begin{array}{l}\text { Pin } \\
\text { No. }\end{array}$ & $\begin{array}{l}t \\
(\mu \mathrm{g})\end{array}$ & $\begin{array}{l}x \\
(\mathrm{~mm})\end{array}$ & $\begin{array}{l}y \\
(\mathrm{~mm})\end{array}$ & $\begin{array}{l}z \\
(\mathrm{~mm})\end{array}$ & $\begin{array}{l}\text { Pin } \\
\text { No. }\end{array}$ & $\begin{array}{l}t \\
(\mu 8)\end{array}$ & $\begin{array}{l}x \\
(\mathrm{~mm})\end{array}$ & $\begin{array}{l}y \\
(\mathrm{~mm})\end{array}$ & $\begin{array}{l}z \\
(\mathrm{~mm})\end{array}$ \\
\hline $1 c$ & 1.450 & -1.002 & 40.0 & 0.0 & 38 & 3.838 & 15.340 & -43.3 & -25.0 \\
\hline $2 c$ & 1.667 & 0.0 & 39.1 & 8.3 & $39 c$ & 1.671 & 0.0 & -25.0 & -43.3 \\
\hline $3 c$ & 1.463 & -0.995 & -20.0 & 34.6 & 40 & 1.802 & 1.320 & -20.3 & -45.7 \\
\hline $4 c$ & 1.689 & 0.0 & -26.8 & 29.7 & 41 & 1.937 & 2.342 & -15.5 & -47.6 \\
\hline $5 c$ & 1.468 & -0.996 & -20.0 & -34.6 & 42 & 2.076 & 3.342 & -10.4 & -48.9 \\
\hline $6 c$ & 1.680 & 0.0 & -12.4 & -38.0 & 43 & 2.210 & 4.326 & -5.2 & -49.7 \\
\hline $7 c$ & np & 0.0 & 50.0 & 0.0 & 44 & 2.348 & 5.323 & 0.0 & -50.0 \\
\hline 8 & 1.768 & 1.309 & 9.7 & 5.2 & 45 & 2.488 & 6.327 & 5.2 & .49 .7 \\
\hline 9 & 1.920 & 2.331 & 48.9 & 10.4 & 46 & 2.631 & 7.359 & 10.4 & -48.9 \\
\hline 10 & 2.046 & 3.321 & 47.6 & 15.5 & 47 & 2.771 & 8.324 & 15.5 & -47.6 \\
\hline 11 & 2.185 & 4.309 & 45.7 & 20.3 & 48 & 2.909 & 9.332 & 20.3 & -45.7 \\
\hline 12 & 2.314 & 5.302 & 43.3 & 25.0 & 49 & 3.057 & 10.361 & 25.0 & -43.3 \\
\hline 13 & 2.459 & 6.319 & 40.5 & 29.4 & 50 & 3.203 & 11.354 & 29.4 & -40.5 \\
\hline 14 & $2.589^{\circ}$ & 7.346 & 37.2 & 33.5 & 51 & 3.355 & 12.348 & 33.5 & -37.2 \\
\hline 15 & $2.724^{\circ}$ & 8.334 & 33.5 & 37.2 & 52 & 3.511 & 13.344 & 37.2 & -33.5 \\
\hline 16 & $2.858^{\circ}$ & 9.326 & 29.4 & 40.59 & 53 & 3.961 & 14.336 & 40.5 & -29.4 \\
\hline 17 & $n p$ & 10.319 & 25.0 & 43.3 & 54 & 3.829 & 15.350 & 43.3 & -25.0 \\
\hline 18 & 3.177 & 11.358 & 20.3 & 45.7 & $55 \mathrm{c}$ & 1.411 & -0.995 & 60.0 & 0.0 \\
\hline 19 & 3.332 & 12.340 & 15.5 & 47.6 & $56 c$ & 1.635 & 0.0 & 58.7 & 12.5 \\
\hline 20 & 3.493 & 13.351 & 10.4 & 48.9 & $57 \mathrm{c}$ & 1.429 & -0.992 & -30.0 & 52.0 \\
\hline 21 & 3.651 & 14.336 & 5.2 & 49.7 & $58 c$ & 1.661 & 0.0 & -40.1 & 44.6 \\
\hline 22 & 3.820 & 15.371 & 0.0 & 50.0 & $59 c$ & 1.450 & -0.988 & -30.0 & -52.0 \\
\hline $23 c$ & 1.664 & 0.0 & -25.0 & 43.3 & $60 c$ & 1.659 & 0.0 & -18.5 & -57.1 \\
\hline 24 & 1.784 & 1.300 & -29.4 & 40.5 & $61 \mathrm{c}$ & 1.614 & 0.0 & 70.0 & 0.0 \\
\hline 25 & 1.934 & 2.346 & -33.5 & 37.2 & 62 & 1.748 & 1.314 & 69.6 & 7.3 \\
\hline 26 & 2.077 & 3.329 & -37.2 & 33.5 & 63 & 1.883 & 2.344 & 68.5 & 14.6 \\
\hline 27 & 2.207 & 4.315 & -40.5 & 29.4 & 84 & 2.024 & 3.331 & 66.6 & 21.6 \\
\hline 28 & 2.348 & 5.339 & -43.3 & 25.0 & 65 & 2.164 & 4.325 & 63.9 & 28.5 \\
\hline 29 & 2.490 & 6.327 & -45.7 & 20.3 & 66 & 2.300 & $5 \therefore$. & 60.6 & 35.0 \\
\hline 30 & 2.637 & 7.339 & -47.6 & 15.5 & 67 & 2.435 & 6.314 & 56.6 & 41.1 \\
\hline 31 & 2.773 & 8.364 & -48.9 & 10.4 & 68 & 2.590 & 7.360 & 52.0 & 46.8 \\
\hline 32 & 2.906 & 9.332 & -49.7 & 5.2 & 69 & 2.732 & 8.348 & 46.8 & 52.0 \\
\hline 33 & 3.061 & 10.337 & -50.0 & 0.0 & 70 & 2.871 & 9.350 & 41.1 & 56.6 \\
\hline 34 & 3.214 & 11.346 & -49.7 & -5.2 & 71 & 3.015 & 10.354 & 35.0 & 60.6 \\
\hline 35 & 3.363 & 12.322 & -48.9 & -10.4 & 72 & 3.169 & 11.325 & 28.5 & 63.9 \\
\hline 36 & 3.520 & 13.347 & -47.6 & -15.5 & 73 & np & 12.331 & 21.6 & 66.6 \\
\hline 37 & 3.685 & 14.322 & -45.7 & -20.3 & 74 & 3.479 & 13.358 & 14.6 & 68.5 \\
\hline
\end{tabular}


Table 76 continuea

\begin{tabular}{|c|c|c|c|c|c|c|c|c|c|}
\hline $\begin{array}{l}\text { Pin } \\
\text { No. }\end{array}$ & $\begin{array}{l}t \\
(\mu s)\end{array}$ & $\begin{array}{l}x \\
(\mathrm{~mm})\end{array}$ & $\begin{array}{l}y \\
(\mathrm{~mm})\end{array}$ & $\begin{array}{l}z \\
(\mathrm{~mm})\end{array}$ & $\begin{array}{l}\text { Pin } \\
\text { No. }\end{array}$ & $\begin{array}{l}t \\
(\mu s)\end{array}$ & $\begin{array}{l}x \\
(\mathrm{~mm})\end{array}$ & $\begin{array}{l}y \\
(\mathrm{~mm})\end{array}$ & $\begin{array}{l}z \\
(\mathrm{~mm})\end{array}$ \\
\hline 75 & 3.632 & 14.365 & 7.3 & 69.6 & 100 & 2.601 & 7.343 & 14.6 & -68.4 \\
\hline 76 & 3.798 & 15.370 & 0.0 & 70.0 & 101 & 2.743 & 8.343 & 21.6 & -66.6 \\
\hline $77 \mathrm{c}$ & 1.643 & 0.0 & -35.0 & 60.6 & 102 & 2.880 & 9.338 & 28.5 & -63.9 \\
\hline 78 & 1.763 & 1.331 & -41.4 & 56.6 & 103 & 3.029 & 10.337 & 35.0 & -60.6 \\
\hline 79 & 1.923 & 2.367 & -46.8 & 52.0 & 104 & 3.188 & 11.346 & 41.1 & -56.6 \\
\hline 80 & 2.052 & 3.343 & -52.0 & 46.8 & 105 & 3.321 & 12.355 & 46.8 & -52.0 \\
\hline 81 & 2.201 & 4.348 & -56.6 & 41.1 & 106 & 3.489 & 13.359 & 52.0 & -46.8 \\
\hline 82 & 2.332 & 5.348 & -60.6 & 35.0 & 107 & 3.634 & 14.330 & 56.6 & -41.1 \\
\hline 83 & 2.478 & 6.342 & .63 .9 & 28.5 & 108 & 3.792 & 15.370 & 60.6 & -35.0 \\
\hline 84 & 2.613 & 7.352 & -60.6 & 21.6 & $109 \mathrm{c}$ & 1.393 & -0.987 & 80.0 & 0.0 \\
\hline 85 & 2.763 & 8.340 & -68.5 & 14.6 & $110 c$ & 1.602 & 0.0 & 78.3 & 16.8 \\
\hline 88 & 2.894 & 9.334 & -69.8 & 7.3 & $111 c$ & 1.426 & -0.988 & -40.0 & 69.3 \\
\hline 87 & 3.053 & 10.355 & -70.0 & 0.0 & $112 c$ & 1.643 & 0.0 & -53.5 & 59.5 \\
\hline 88 & $3.20 ?$ & 11.331 & -69.6 & -7.3 & $113 c$ & np & -0.998 & -40.0 & -69.3 \\
\hline 89 & 3.360 & 12.347 & -68.4 & -14.6 & $114 \mathrm{c}$ & 1.660 & 0.0 & .24 .7 & -76.1 \\
\hline 90 & 3.517 & 13.360 & -66.6 & -21.6 & & & & & \\
\hline 91 & 3.665 & 14.343 & -63.9 & -28.5 & & & & & \\
\hline 92 & ap & 15.355 & -60.6 & -35.0 & & & & & \\
\hline $93 c$ & 1.646 & 0.0 & -35.0 & -60.6 & & & & & \\
\hline 94 & 1.778 & 1.307 & -28.5 & -83.9 & & & & & \\
\hline 95 & 1.920 & 2.338 & -21.6 & -66.6 & & & & & \\
\hline 96 & 2.058 & 3.330 & -14.6 & -68.5 & & & & & \\
\hline 97 & 2.199 & 4.303 & -7.3 & -69.6 & & & & & \\
\hline 98 & 2.328 & 5.331 & 0.0 & -70.0 & & & & & \\
\hline 99 & 2.464 & 6.333 & 7.3 & -69.6 & & & & & \\
\hline
\end{tabular}


Table 17.

Pin Data for RS-2314

\begin{tabular}{|c|c|c|c|c|c|c|c|c|c|}
\hline $\begin{array}{l}\text { Pin } \\
\text { No. }\end{array}$ & $\begin{array}{l}t \\
(\mu s)\end{array}$ & $\begin{array}{l}x \\
(\mathrm{~mm})\end{array}$ & $\begin{array}{l}y \\
(\mathrm{~mm})\end{array}$ & $\begin{array}{l}z \\
(\mathrm{~mm})\end{array}$ & $\begin{array}{l}\text { Pin } \\
\text { No. }\end{array}$ & $\begin{array}{l}t \\
(\mu \mathrm{s})\end{array}$ & $\begin{array}{l}x \\
(\mathrm{~mm})\end{array}$ & $\begin{array}{l}y \\
(\mathrm{~mm})\end{array}$ & $\begin{array}{l}z \\
(\mathrm{~mm})\end{array}$ \\
\hline $1 c$ & 1.422 & -0.999 & 40.0 & 0.0 & 38 & 3.857 & 15.000 & -43.3 & -25.0 \\
\hline $2 c$ & 1.666 & 0.0 & 39.1 & 8.3 & $39 c$ & 1.627 & 0.0 & -25.0 & -43.3 \\
\hline $3 c$ & 1.419 & -1.001 & -20.0 & 34.6 & 40 & 1.773 & 1.013 & -20.3 & -45.7 \\
\hline $4 c$ & 1.656 & 0.0 & -26.8 & 29.7 & 41 & 1.920 & 2.029 & -15.5 & -47.6 \\
\hline $5 c$ & 1.385 & -1.002 & -20.0 & -34.6 & 42 & 2.060 & 3.003 & -10.4 & -48.9 \\
\hline$B c$ & 1.633 & 0.0 & -12.4 & -38.0 & 43 & 2.202 & 4.002 & -5.2 & -49.7 \\
\hline $7 c$ & 1.675 & 0.0 & 50.0 & 0.0 & 44 & 2.347 & 5.003 & 0.0 & -50.0 \\
\hline 8 & 1.808 & 0.947 & 49.7 & 5.2 & 45 & 2.487 & 6.017 & 5.2 & -49.7 \\
\hline 9 & 1.959 & 1.998 & 48.9 & 10.4 & 48 & 2.621 & 7.016 & 10.4 & -48.9 \\
\hline 10 & 2.097 & 2.974 & 47.6 & 15.5 & 47 & 2.779 & 8.015 & 15.5 & -47.6 \\
\hline 11 & 2.236 & 3.967 & 45.7 & 20.3 & 48 & 2.925 & 8.984 & 20.3 & -45.7 \\
\hline 12 & 2.386 & 4.984 & 43.3 & 25.0 & 49 & 3.089 & 10.003 & 25.0 & -4 ง..3 \\
\hline 13 & 2.533 & 5.979 & 40.5 & 29.4 & 50 & 3.234 & 10.989 & 29.4 & -40.5 \\
\hline 14 & 2.672 & 6.988 & 37.2 & 33.5 & 51 & 3.397 & 11.995 & 33.5 & -37.2 \\
\hline 15 & 2.816 & 7.953 & 33.5 & 37.2 & 52 & $3.5 B 7$ & 13.006 & 37.2 & -33.5 \\
\hline 16 & 2.961 & 8.960 & 29.4 & 40.5 & 53 & 3.744 & 14.004 & 40.5 & -29.4 \\
\hline 17 & 3.118 & 9.977 & 25.0 & 43.3 & 54 & 3.913 & 15.007 & 43.3 & -25.0 \\
\hline 18 & 3.257 & 11.001 & 20.3 & 45.7 & $55 c$ & np & -0.977 & 60.0 & 0.0 \\
\hline 19 & 3.430 & 11.990 & 15.5 & 47.6 & $56 c$ & 1.693 & 0.0 & 58.7 & 12.5 \\
\hline 20 & 3.600 & 13.000 & 10.4 & 48.9 & $57 c$ & 1.418 & -0.988 & -30.0 & 52.0 \\
\hline 21 & 3.756 & 14.003 & 5.2 & 49.7 & $58 \mathrm{c}$ & 1.658 & 0.0 & -40.1 & 44.6 \\
\hline 22 & 3.893 & 15.016 & 0.0 & 50.0 & $59 c$ & 1.381 & -1.009 & -30.0 & -52.0 \\
\hline $23 c$ & 1.662 & 0.0 & -25.0 & 43.3 & $60 c$ & 1.628 & 0.0 & -18.5 & -57.1 \\
\hline 24 & 1.807 & 1.005 & -29.4 & 40.5 & $61 \mathrm{c}$ & 1.658 & 0.0 & 70.0 & 0.0 \\
\hline 25 & 1.937 & 2.005 & -33.5 & 37.2 & 62 & 1.802 & 1.018 & 69.8 & 7.3 \\
\hline 26 & 2.072 & 2.994 & -37.2 & 33.5 & 63 & 1.954 & 2.011 & 68.5 & 14.6 \\
\hline 27 & 2.209 & 3.971 & -40.5 & 29.4 & 64 & 2.098 & 2.999 & 66.6 & 21.6 \\
\hline 28 & 2.361 & 5.014 & -43.3 & 25.0 & 65 & 2.232 & 4.016 & 63.9 & 28.5 \\
\hline 29 & 2.491 & 5.998 & -45.7 & $20 . ?$ & 66 & 2.382 & 5.023 & 60.6 & 35.0 \\
\hline 30 & 2.626 & 7.008 & -47.6 & 15.5 & 87 & 2.528 & 8.008 & 56.6 & 41.1 \\
\hline 31 & $\mathrm{np}$ & 8.002 & -48.9 & 10.4 & 68 & 2.671 & 7.038 & 52.0 & 46.8 \\
\hline 32 & 2.925 & 9.000 & -49.7 & 5.2 & 69 & 2.810 & 8.020 & 46.8 & 52.0 \\
\hline 33 & 3.070 & 10.015 & -50.0 & 0.0 & 70 & 2.947 & 9.002 & 41.1 & 56.6 \\
\hline 34 & 3.223 & 11.008 & -49.7 & -5.2 & 71 & 3.114 & 10.020 & 35.0 & 60.6 \\
\hline 35 & 3.370 & 11.992 & -48.9 & -10.4 & 72 & 3.273 & 11.025 & 28.5 & 63.9 \\
\hline 36 & 3.539 & 13.008 & -47.6 & -15.5 & 73 & 3.423 & 12.043 & 21.6 & 66.6 \\
\hline 37 & 3.694 & 13.992 & -45.7 & -20.3 & 74 & 3.581 & 13.039 & 14.6 & 68.5 \\
\hline
\end{tabular}


Table 77 continued

$\begin{array}{lllll}\text { Pin } & t & x & l & z \\ \text { Vo. } & (\mu \mathrm{s}) & (\mathrm{mm}) & (\mathrm{mm}) & (\mathrm{mm}) \\ 75 & 3.754 & 14.036 & 7.3 & 69.6 \\ 76 & \text { np } & 15.083 & 0.0 & 70.0 \\ 77 \mathrm{c} & 1.658 & 0.0 & -35.0 & 60.6 \\ 78 & 1.782 & 1.022 & -41.4 & 56.6 \\ 79 & 1.920 & 2.021 & -46.8 & 52.0 \\ 80 & 2.045 & 3.007 & -52.0 & 46.8 \\ 31 & 2.192 & 4.035 & -56.6 & 41.1 \\ 82 & 2.334 & 5.031 & -60.6 & 35.0 \\ 83 & 2.478 & 6.032 & -63.9 & 28.5 \\ 84 & 2.609 & 7.040 & -66.6 & 21.6 \\ 85 & 2.747 & 8.011 & -68.5 & 14.6 \\ 86 & 2.887 & 9.002 & -69.6 & 7.3 \\ 87 & 2.014 & 10.031 & -70.0 & 0.0 \\ 88 & 3.192 & 11.042 & -69.6 & -7.3 \\ 89 & 3.348 & 11.995 & -68.4 & -14.6 \\ 90 & \mathrm{nP} & 13.026 & -66.6 & -21.6 \\ 91 & 3.513 & 13.012 & -63.9 & -28.5 \\ 92 & 3.684 & 14.057 & -60.6 & -35.0 \\ 93 \mathrm{c} & 1.609 & 0.0 & -35.0 & -60.6 \\ 94 & 1.737 & 0.998 & -28.5 & -63.9 \\ 95 & 1.902 & 2.027 & -21.6 & -66.6 \\ 96 & 2.041 & 2.994 & -14.6 & -68.5 \\ 97 & 2.181 & 3.978 & -7.3 & -69.6 \\ 98 & 2.322 & 5.009 & 0.0 & -70.0 \\ 99 & 2.467 & 5.998 & 7.3 & -69.6\end{array}$

\begin{tabular}{|c|c|c|c|c|}
\hline $\begin{array}{l}\text { Pin } \\
\text { No. }\end{array}$ & $\begin{array}{l}t \\
(\mu s)\end{array}$ & $\begin{array}{l}x \\
(\mathrm{~mm})\end{array}$ & $\begin{array}{l}y \\
(\mathrm{~mm})\end{array}$ & $\begin{array}{l}z \\
(\mathrm{~mm})\end{array}$ \\
\hline 100 & 2.615 & 7.023 & 14.6 & -68.4 \\
\hline 101 & 2.748 & 8.001 & 21.8 & -66.8 \\
\hline 102 & $n p$ & 9.009 & 28.5 & -63.9 \\
\hline 103 & 3.057 & 10.021 & 35.0 & -80.8 \\
\hline 104 & 3.215 & 11.011 & 41.1 & -56.6 \\
\hline 105 & 3.378 & 11.980 & 46.8 & -52.0 \\
\hline 100 & 3.532 & 13.012 & 52.0 & -46.8 \\
\hline 107 & np & 14.020 & 56.6 & -41.1 \\
\hline 108 & np & 15.037 & 60.6 & -35.0 \\
\hline $109 \mathrm{c}$ & 1.426 & -0.994 & 80.0 & 0.0 \\
\hline $110 c$ & 1.660 & 0.0 & 78.3 & 16.6 \\
\hline $111 \mathrm{c}$ & 1.418 & -0.986 & -40.0 & 69.3 \\
\hline $112 \mathrm{c}$ & 1.652 & 0.0 & -53.5 & 59.5 \\
\hline $113 i$ & 1.376 & -1.001 & -40.0 & -69.3 \\
\hline $114 \mathrm{c}$ & 1.6203 & 0.0 & -24.7 & -76.1 \\
\hline
\end{tabular}




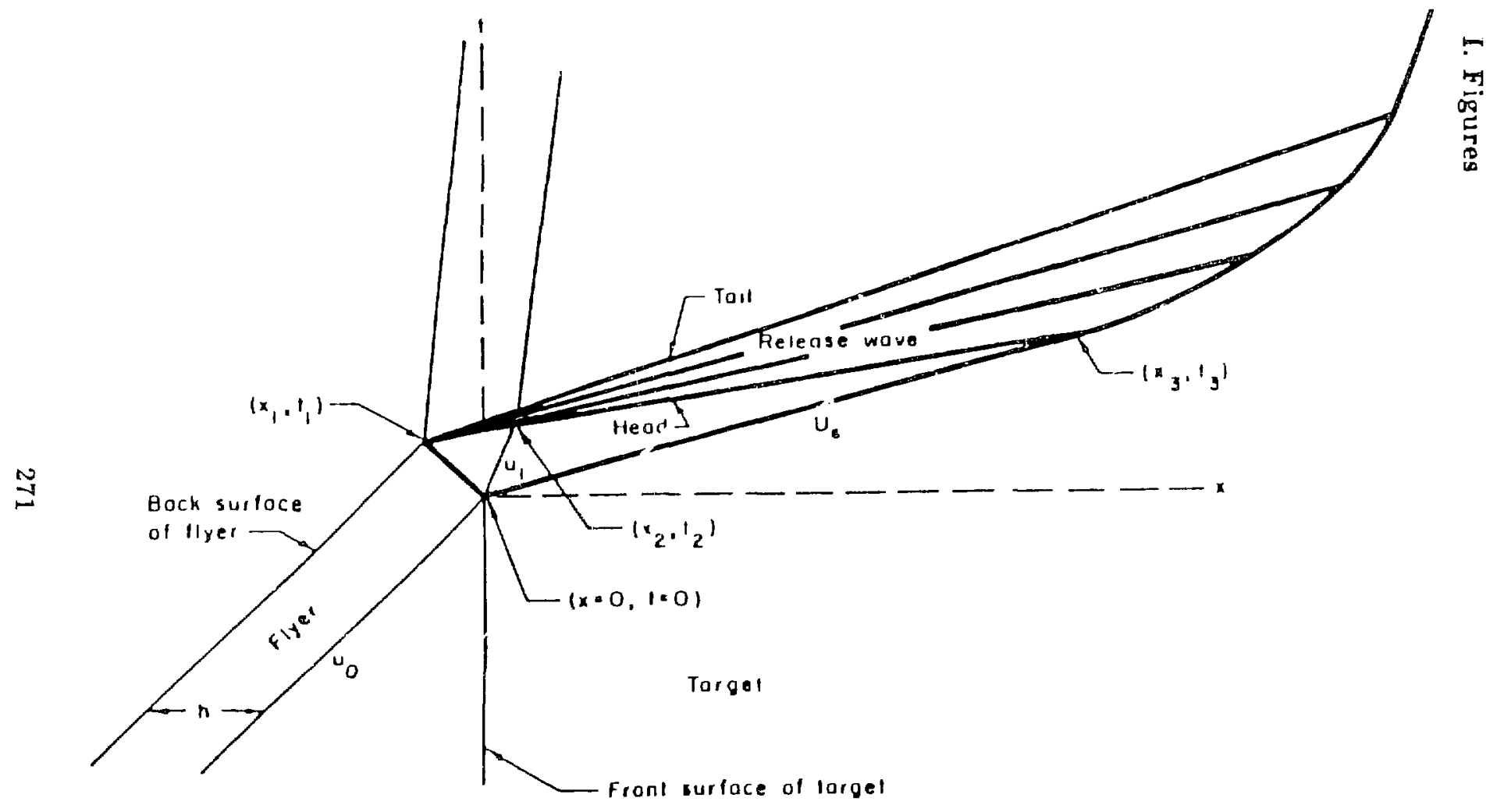

Figure 1: A diagram showing the wave interaction for a tyer plate hitting a semi-infiate tirget The figure is for the idealized case of a simple-centered release wave overtaking and attentuating a sizarp shock. 


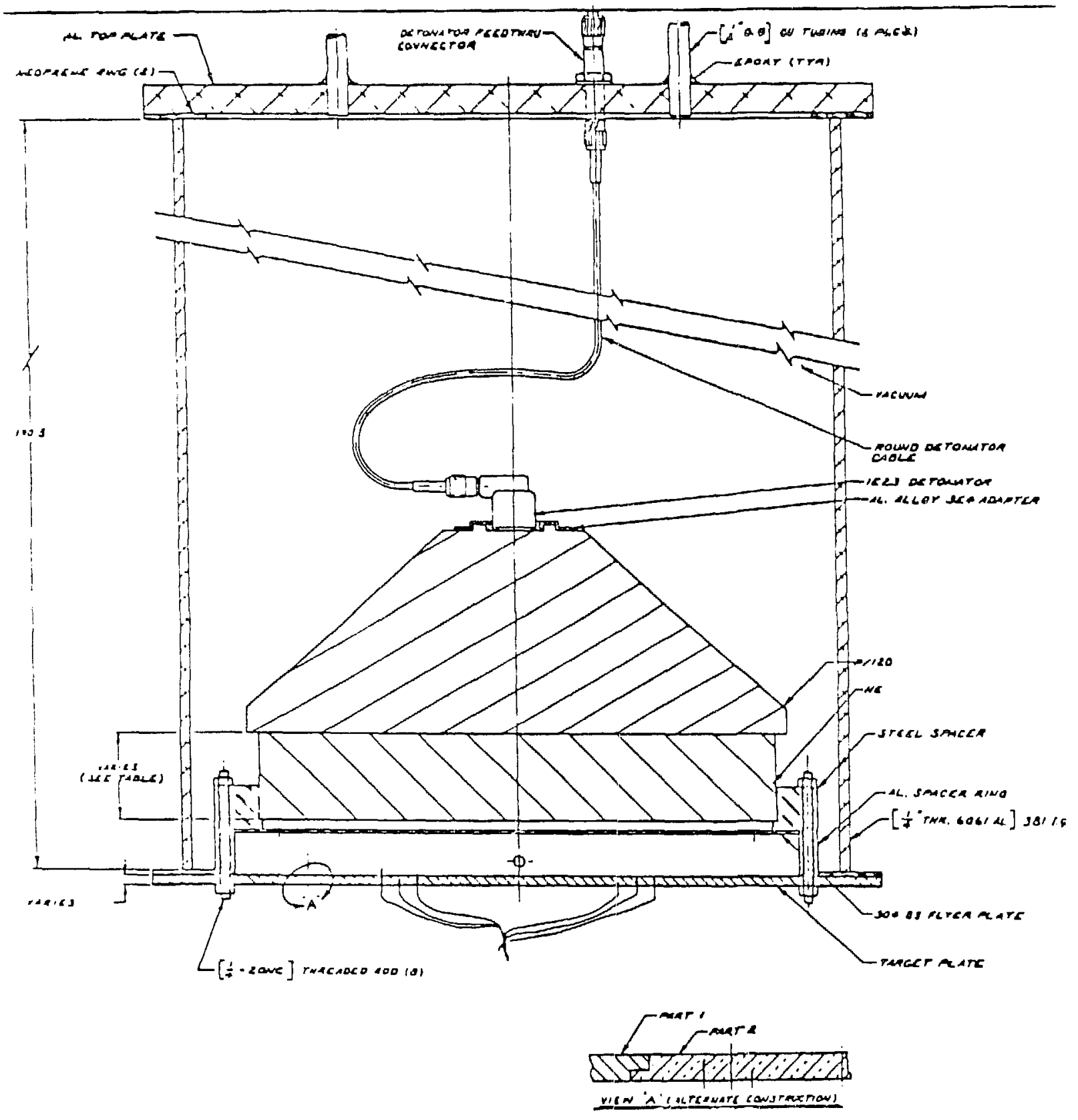

Figure 2: Schematic diagram for a typical experiment. 

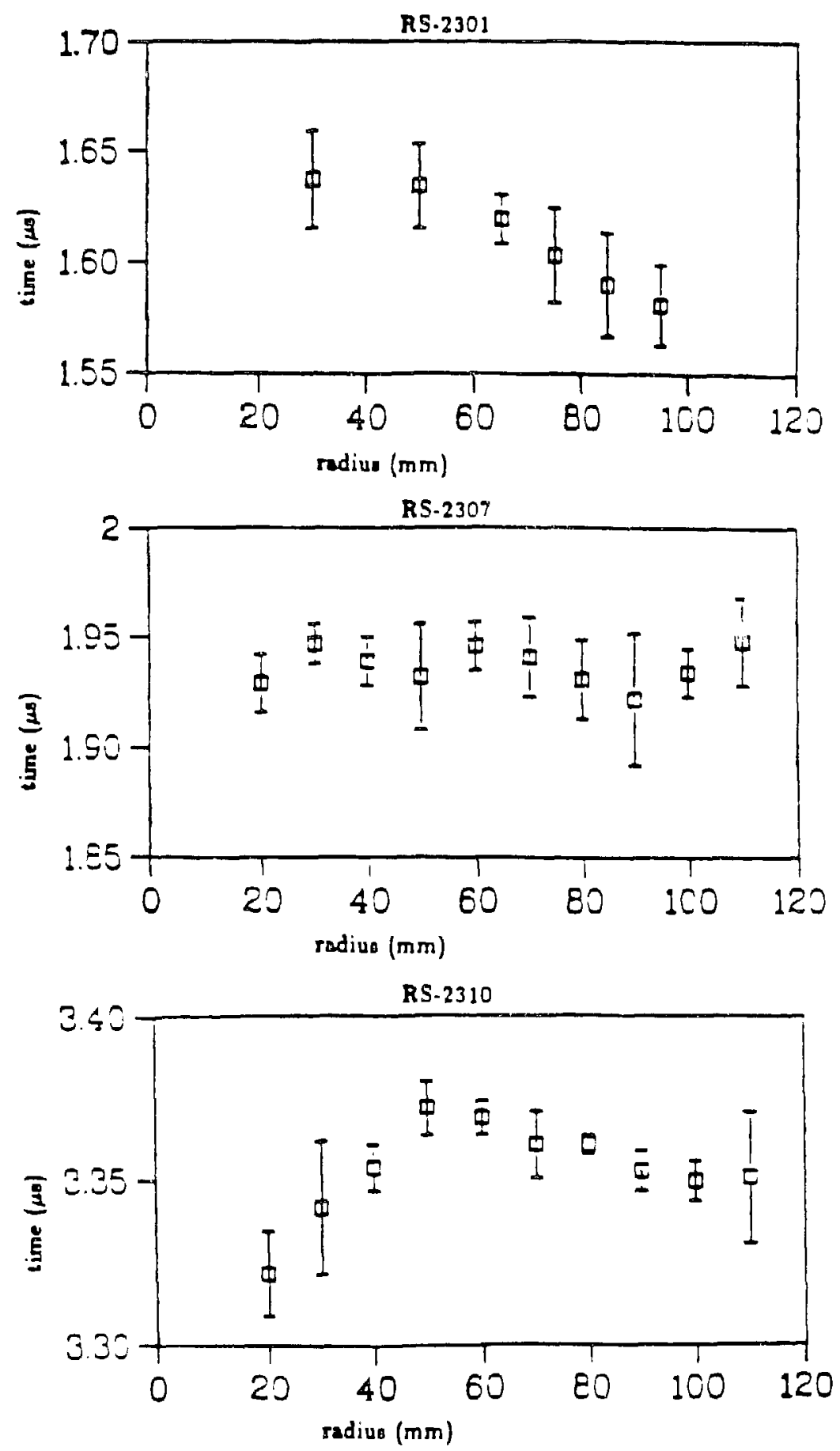

Figure 3: Average times for a given radiug in the plane $x=0$ for the three Aying plate experiments. 


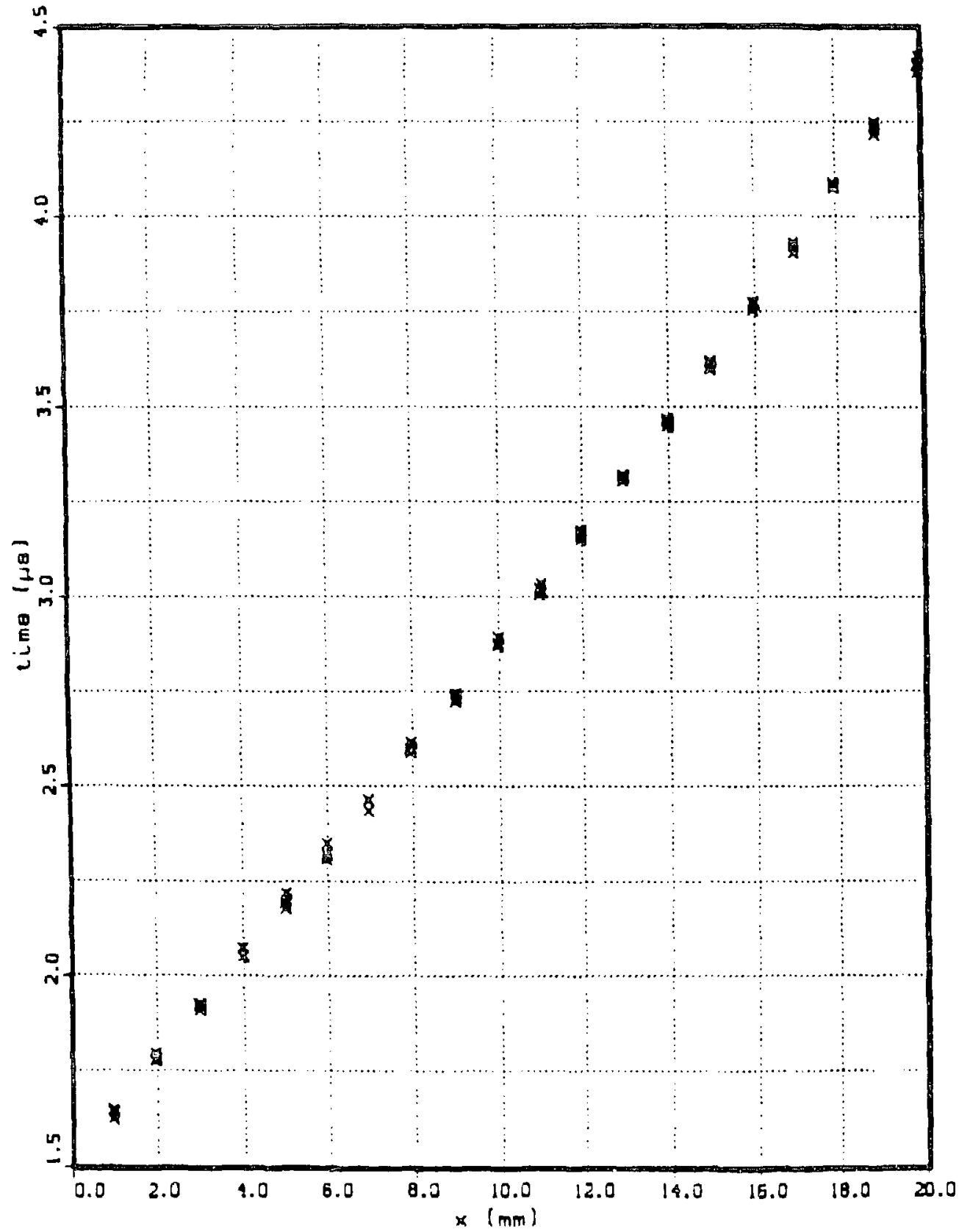

Figure 4: Observed times, $t_{i}$, plotted against pin positon, $x_{i}$, in the target plate for experiment RS-2302. 


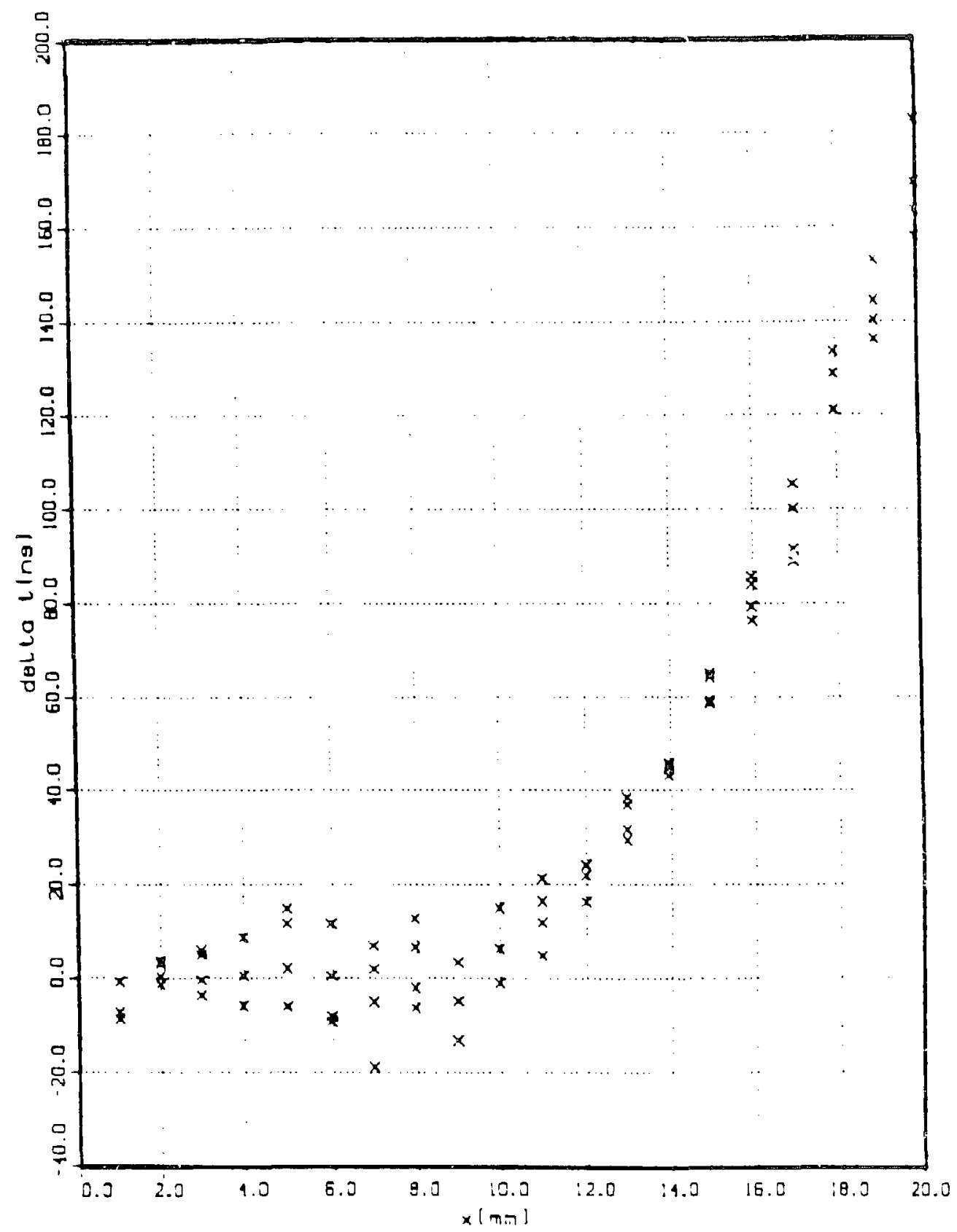

Figure 5: An example of the adjusted times, $\delta t_{i}$, plotted against pin position, $x_{i}$, for experiment RS-2302. 


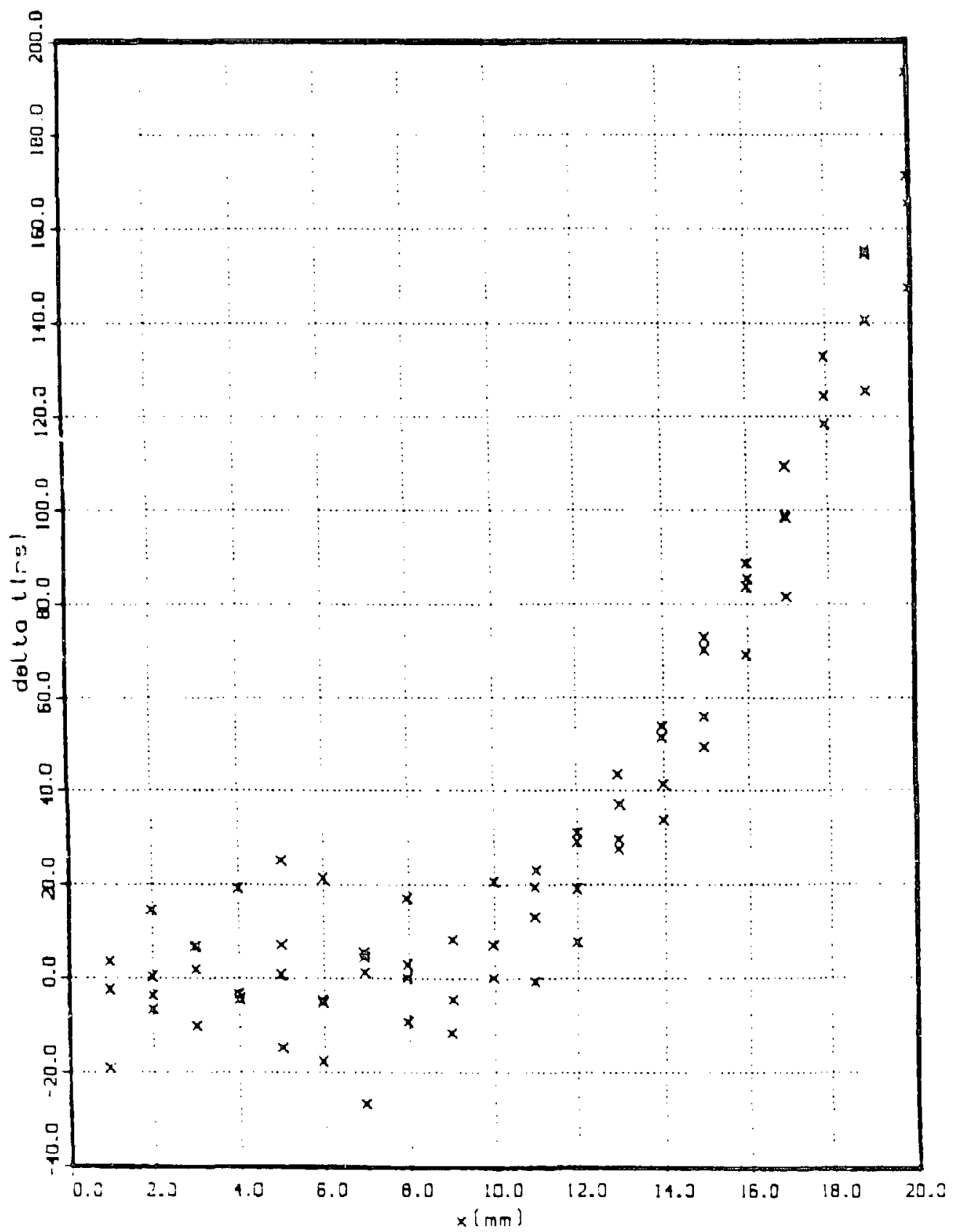

Figure 6: A plot of $\delta t$ rersus $x$ in which no attempt has been made to account for the nonflanarity of the shock wave for experiment RS-2.302. 


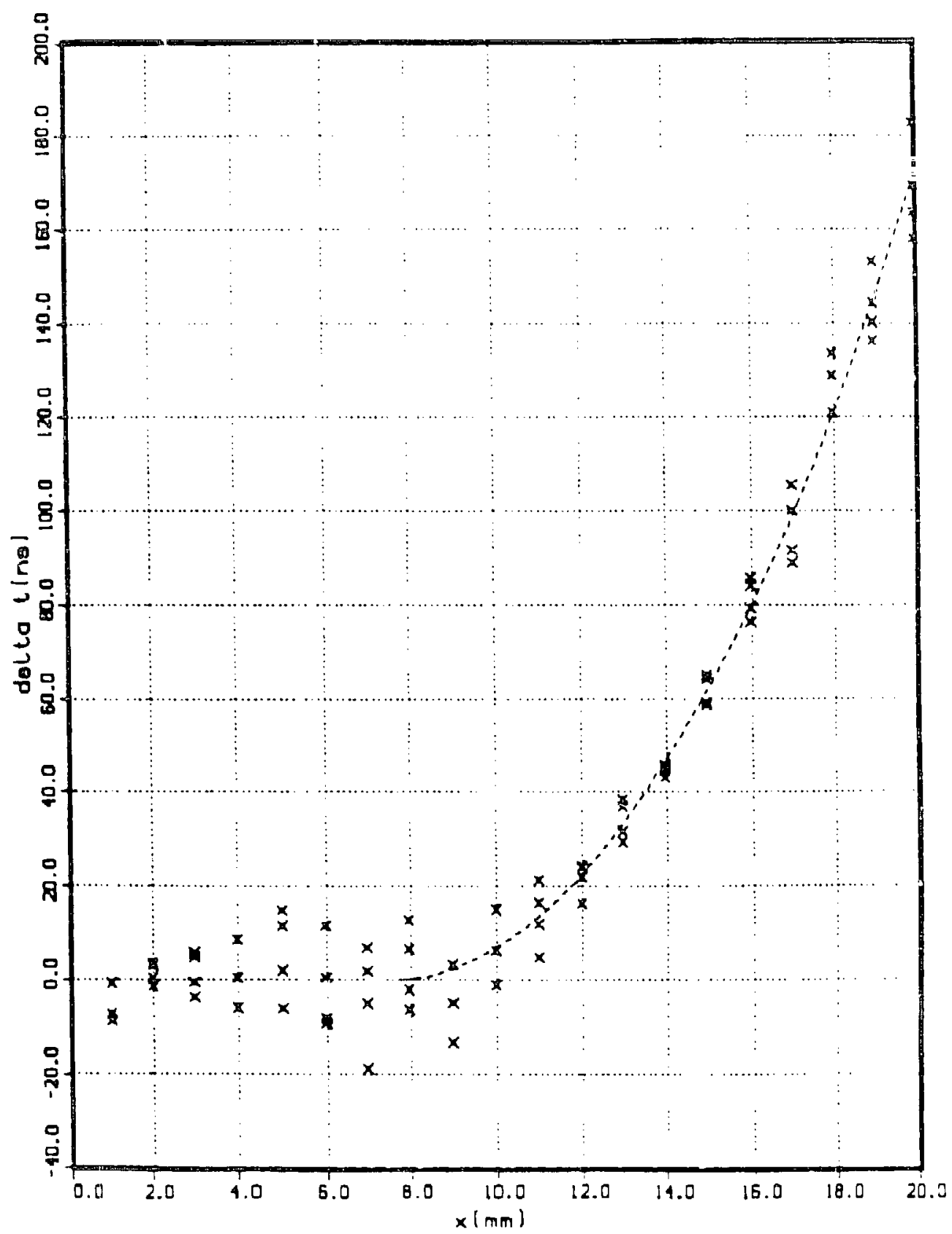

Figure 7: A $\delta t-x$ plot for experiment RS-2302. The dashed line is a fit to the adjusted $\delta t$ data. The firgt break point is at $x=7.8 \mathrm{~mm}$ and the second break poiut is at $8.5 \mathrm{~mm}$. Quadratic polynomials have been used for the firs in the second and third zones. 


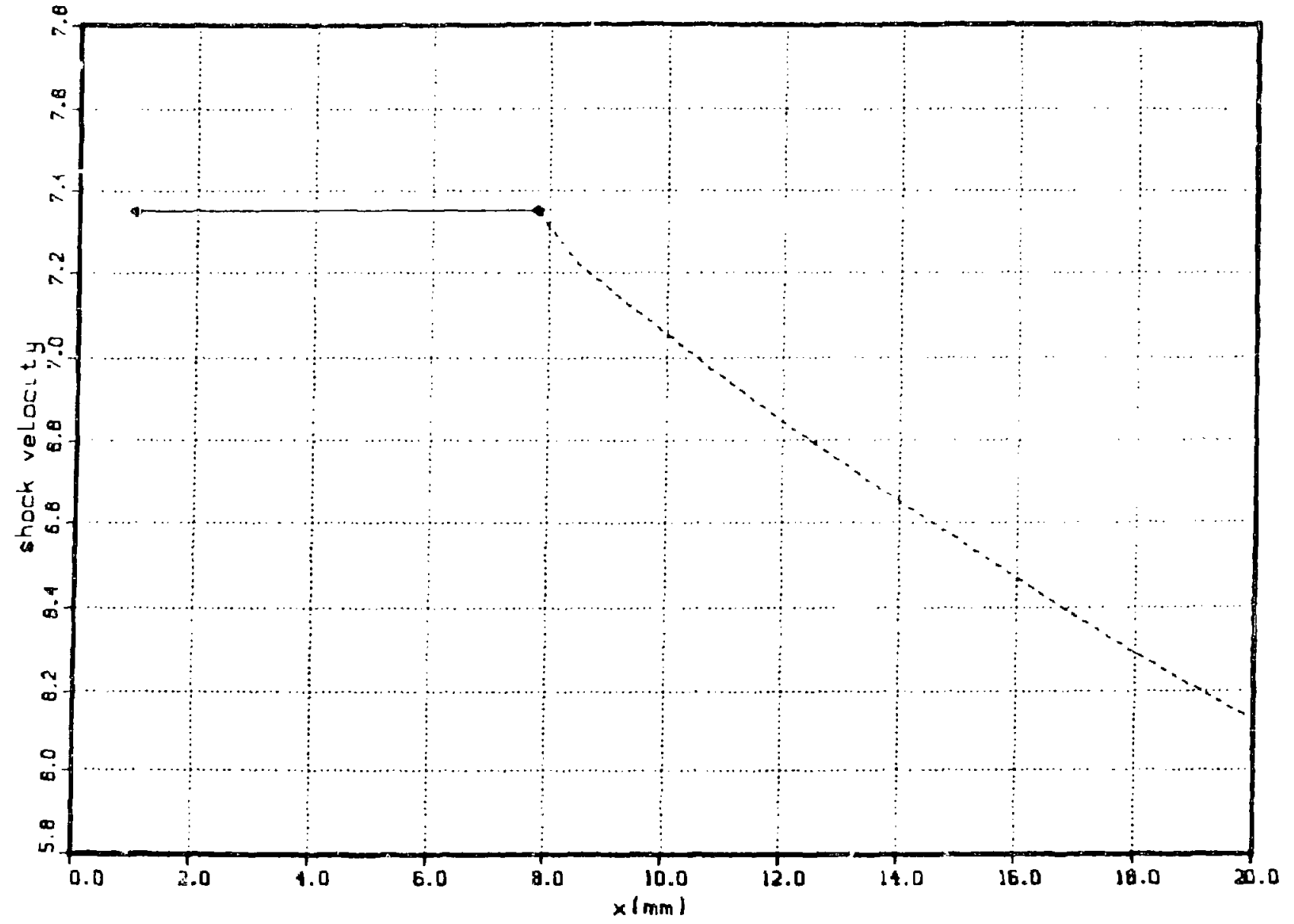

Figure 8: A plat of shock velocity, $U_{2}$, versus position, $x$, in the target plate taken from the fit to the data shown in Ggure 7 for experiment RS-2302. 


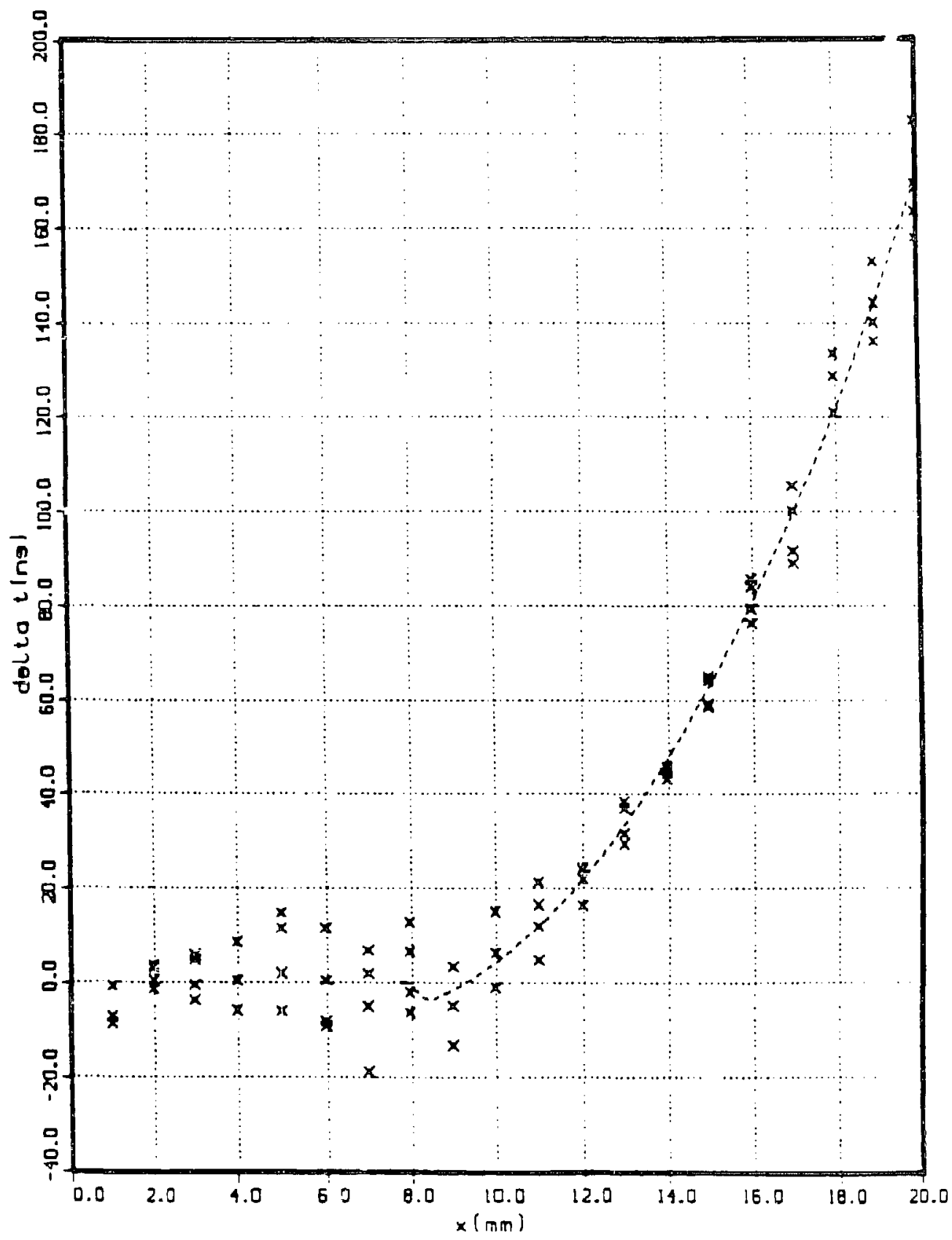

Figure 9: A $\delta t-x$ plot for experiment RS-2302. The dashed line is a fit to the adjusted $\delta t$ data. The first break point is at $x=7.8 \mathrm{~mm}$ and the second break point is at $8.5 \mathrm{~mm}$. Cubic polynomiais have been used for the fits in the second and third zones. 


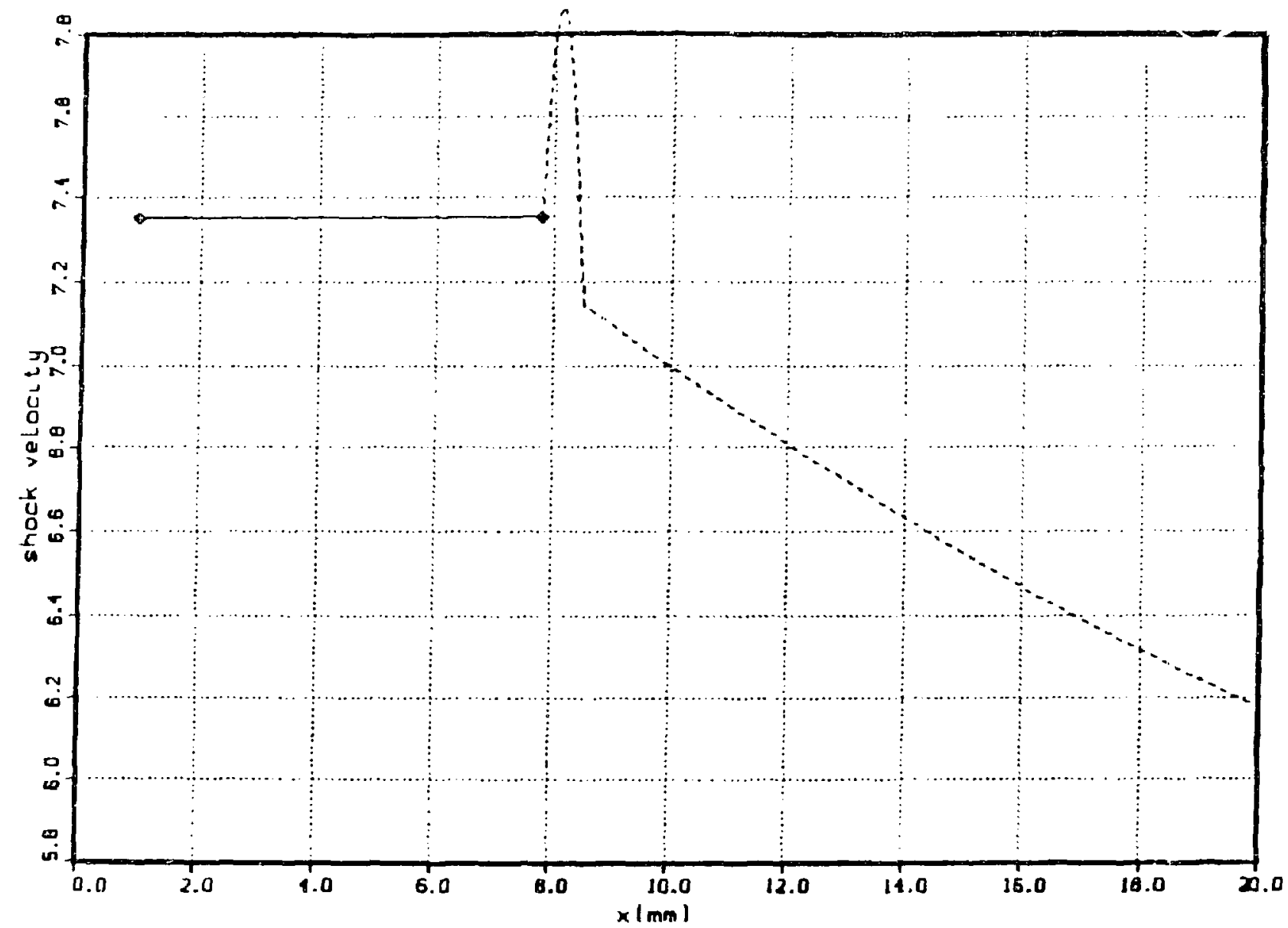

Figure 10: A $U$, versus $x$ plot for RS-2302 taken from the fit to the data shown in figure 9. 


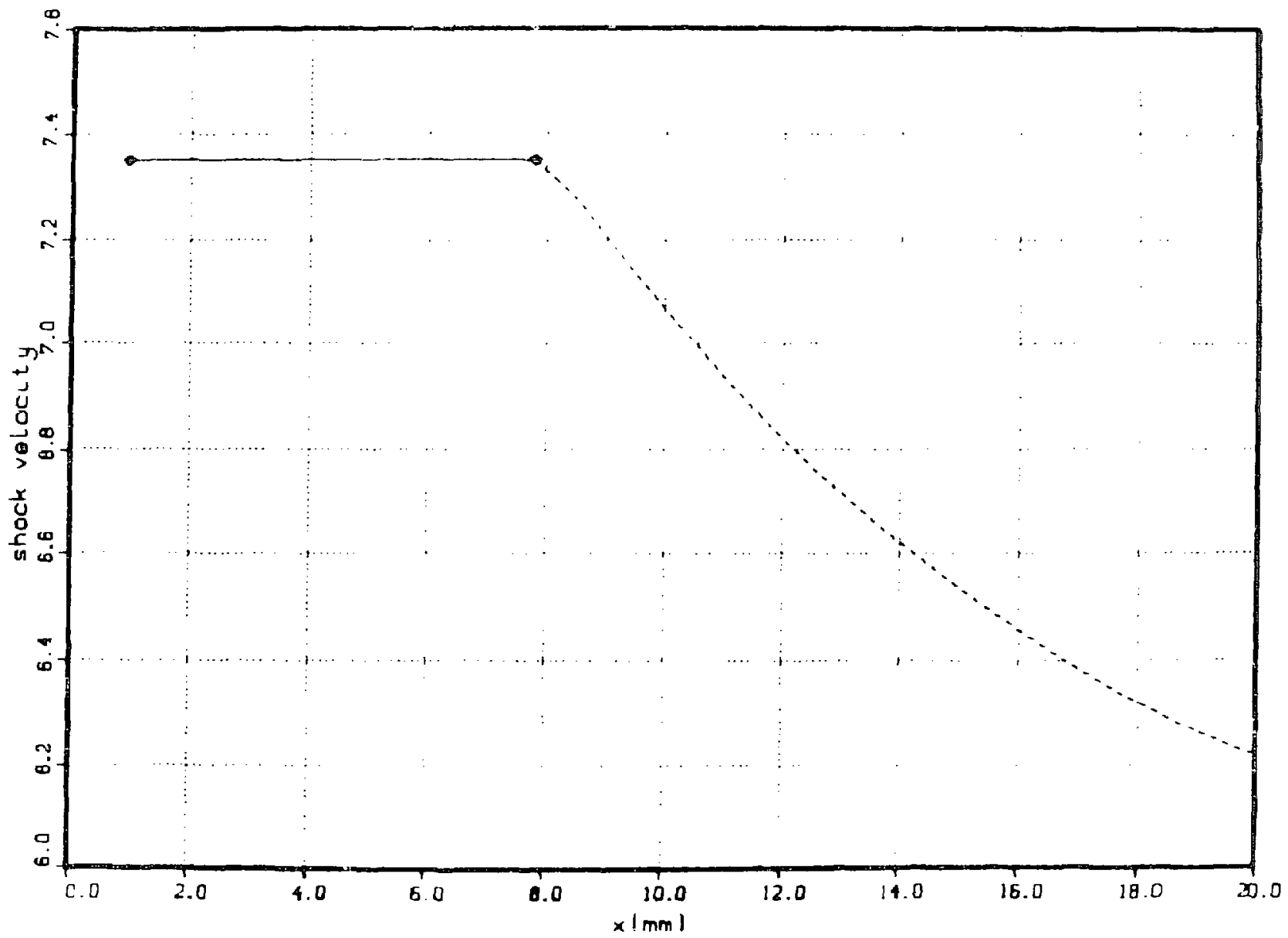

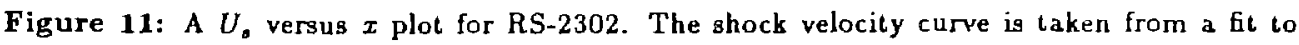
the data shown in figure 9 that has the same break points but with a quadratic polynomial in the second zone instead of a cubic polynomial. 


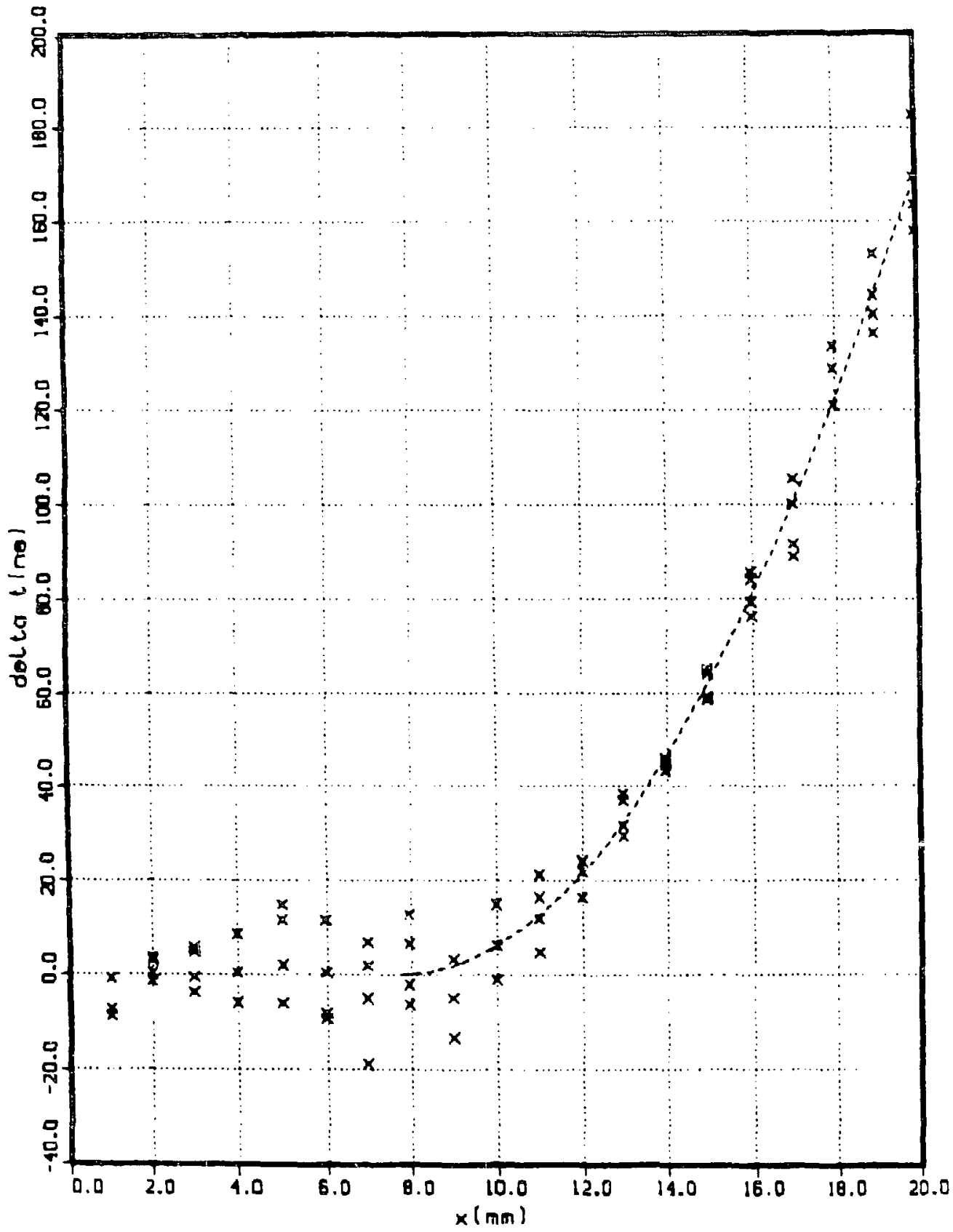

Figure 12: A $5 t-x$ plot for experiment RS-2302. The dashed line is a fit to the adjusted $\delta t$ data. The first break point is at $x=7.8 \mathrm{~mm}$ and the second break point is at $11.5 \mathrm{~mm}$. Cubic polynomials have been used for the fits in the second and third zones. 


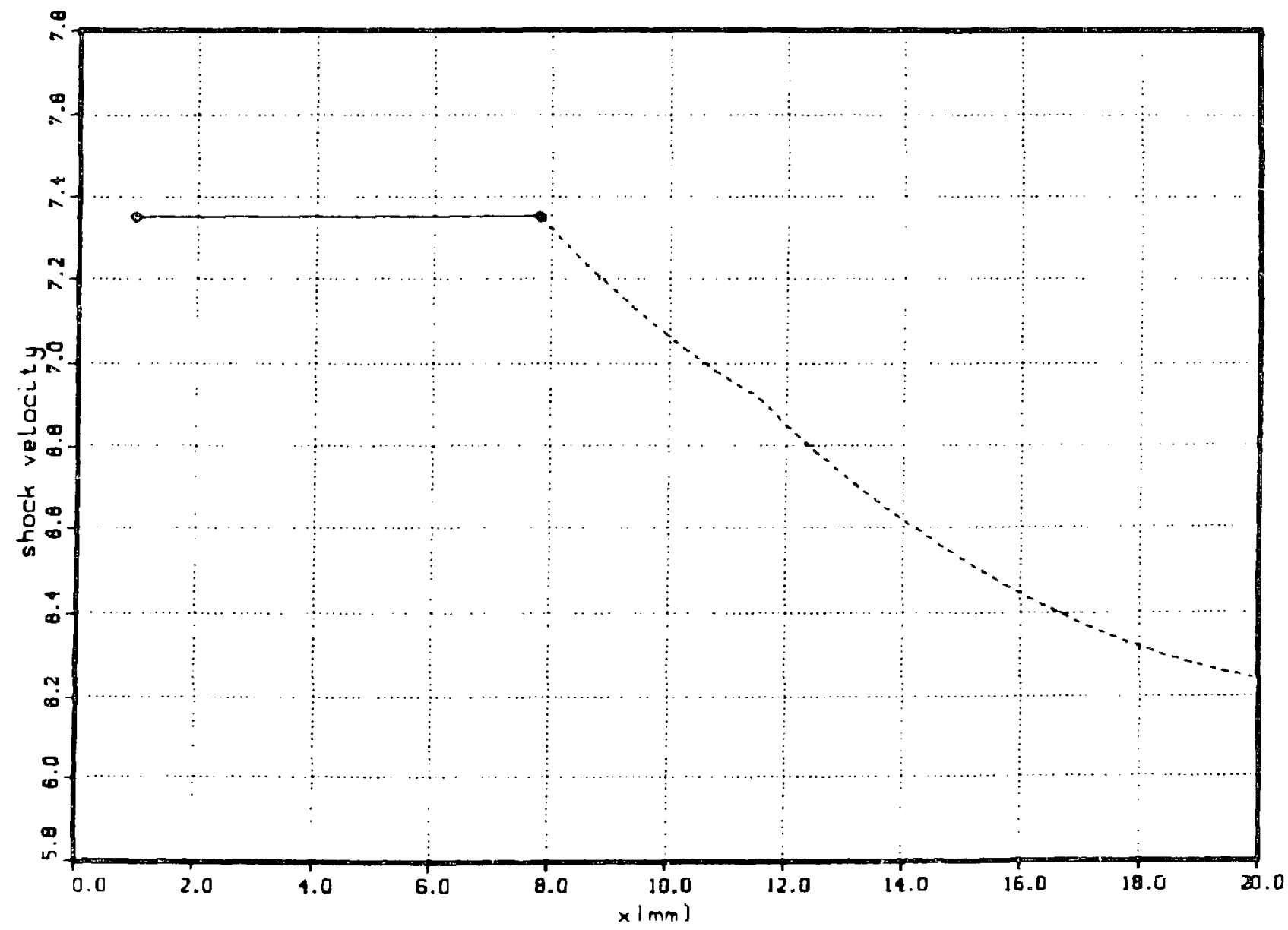

Figure 13: A $U$, versus $x$ plot for RS-2302 taken from the fit to the data shown in figure 12 . 


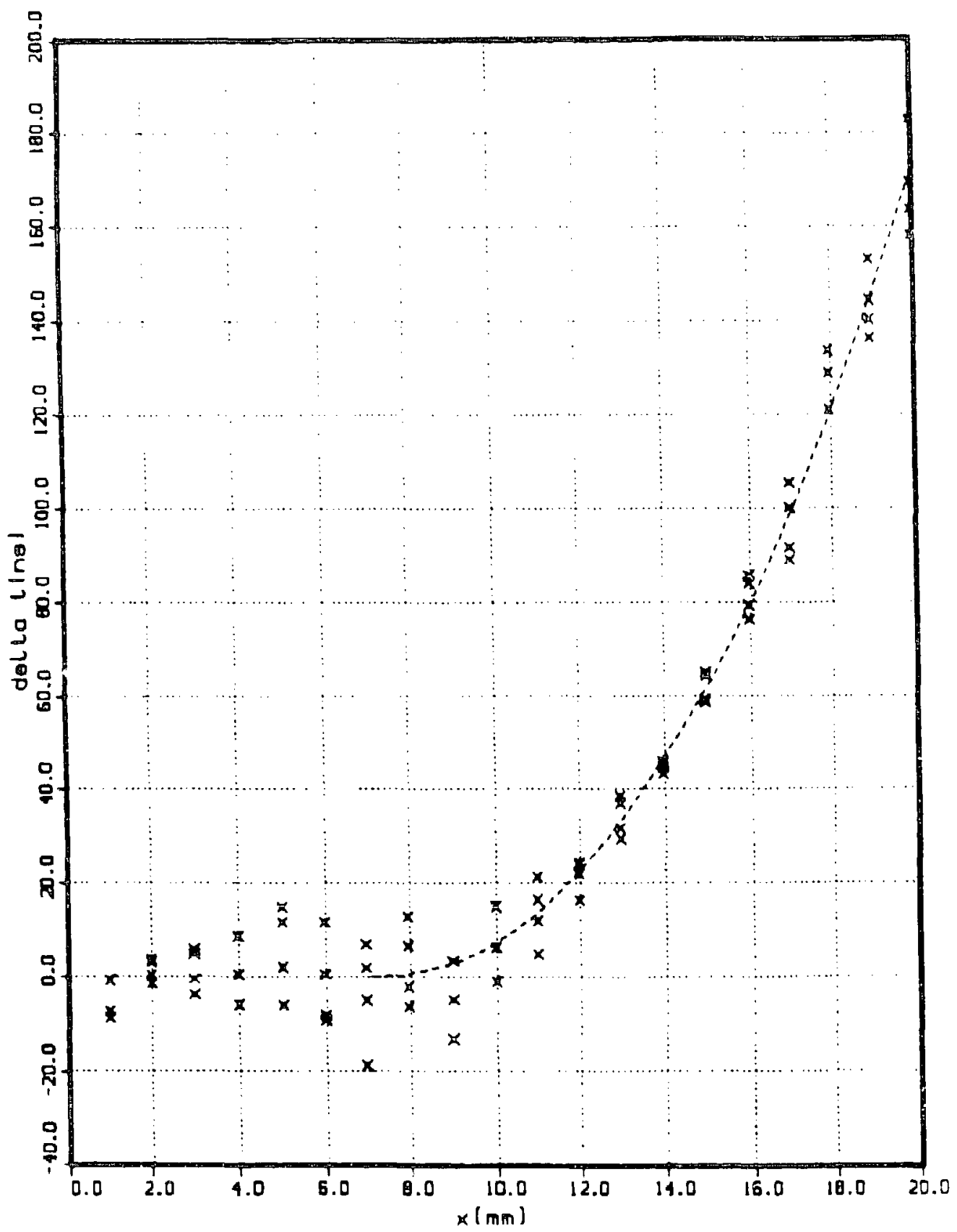

Figure 14: A $\delta t-x$ plot for experiment RS-2302. The dashed line is a fit to the adjusted $\delta t$ data. The first break point is at $x=7.1 \mathrm{~mm}$ and the second break point is at $8.5 \mathrm{~mm}$. Quadratic polynomials have been used for the fits in the second and third zones. 


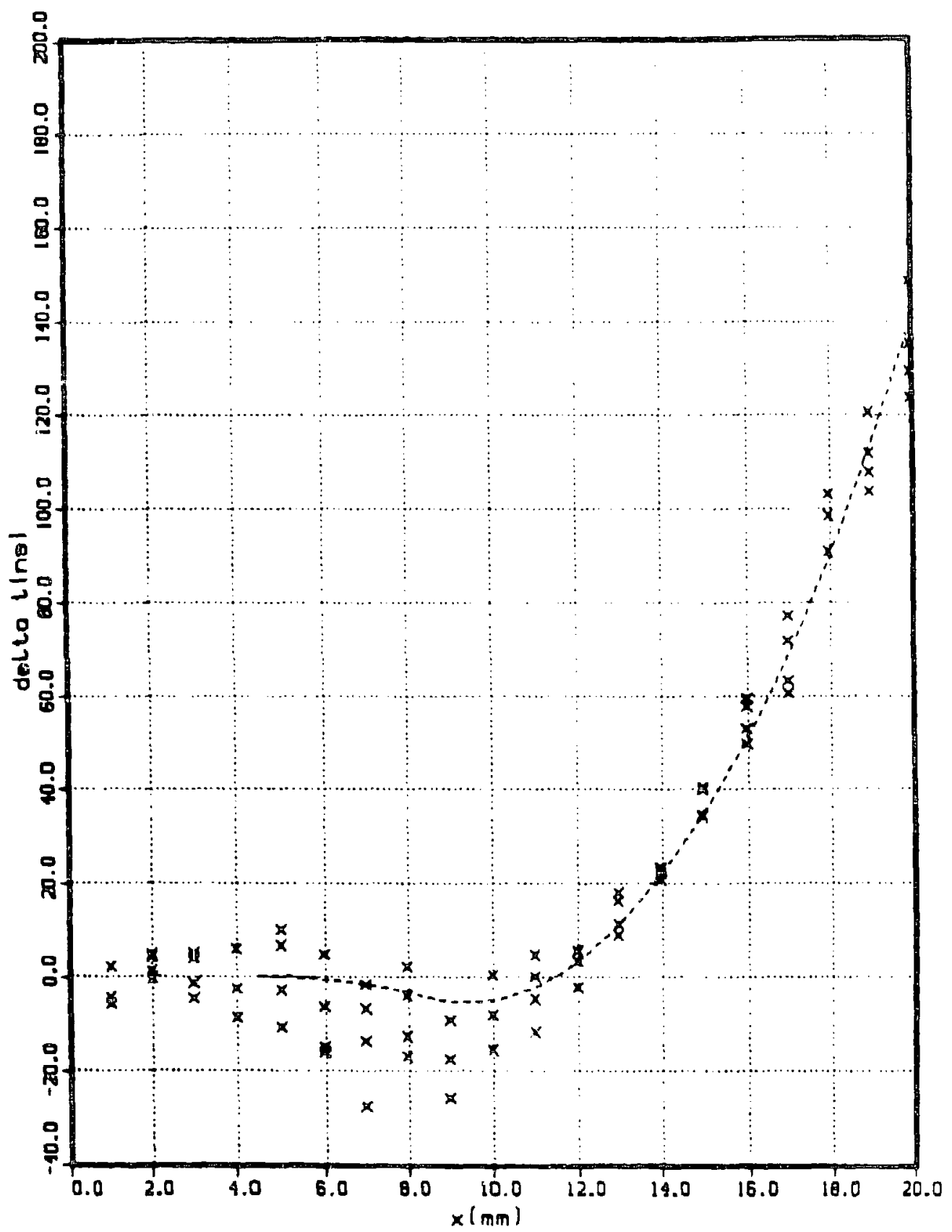

Figure 15: A $\delta t-x$ plot for experiment RS-2302. The dashed line is a fit to the adjusted $\delta t$ data. The frist break point is at $x=4.5 \mathrm{~mm}$ and the second break point is at $8.5 \mathrm{~mm}$. Quadratic polynomials have been used for the fits in the secord and third zones. 


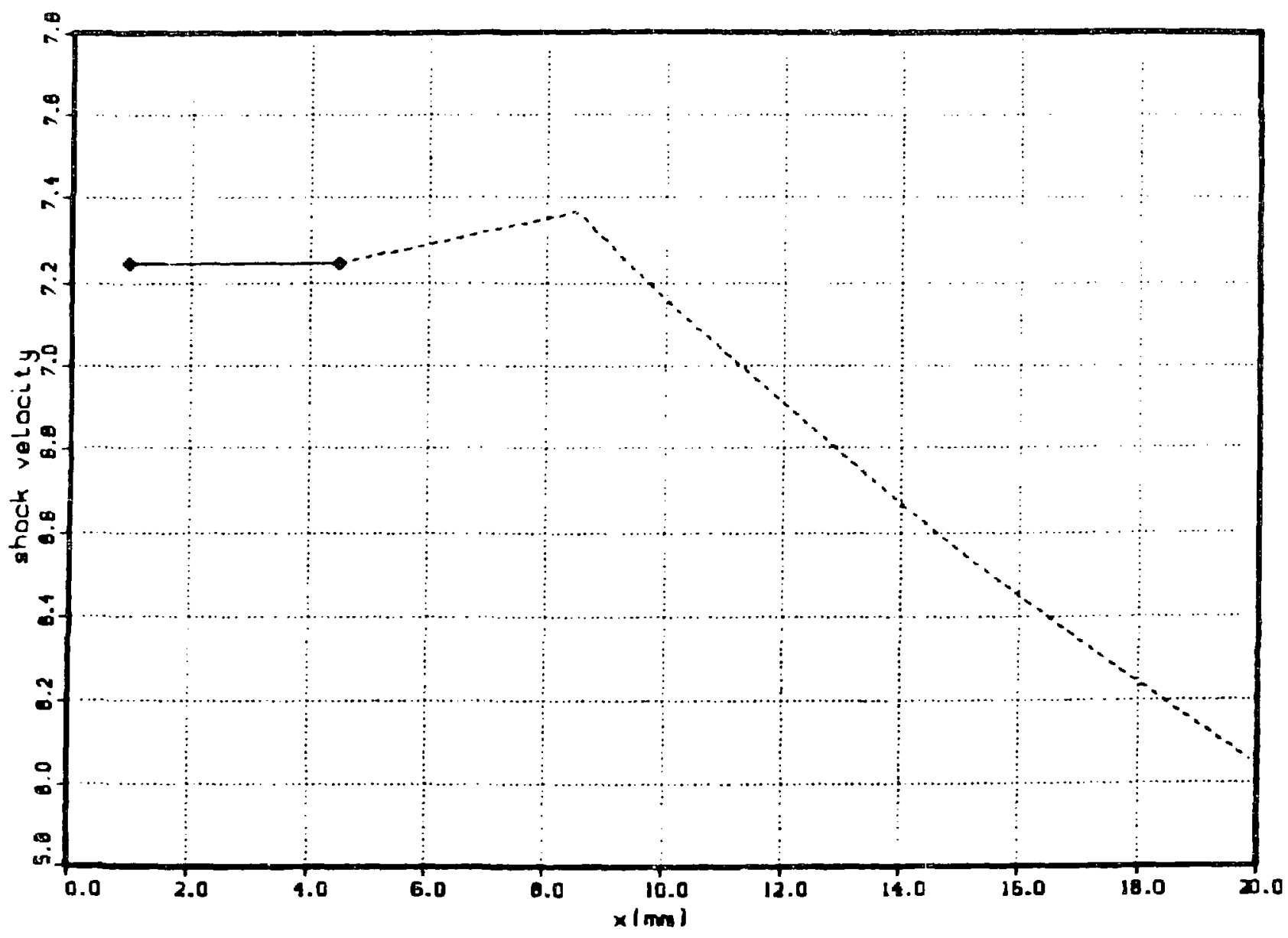

Figure 16: A $U$, versus $x$ plot for RS-2302 taken from the fit to the data shown in figure 15. 


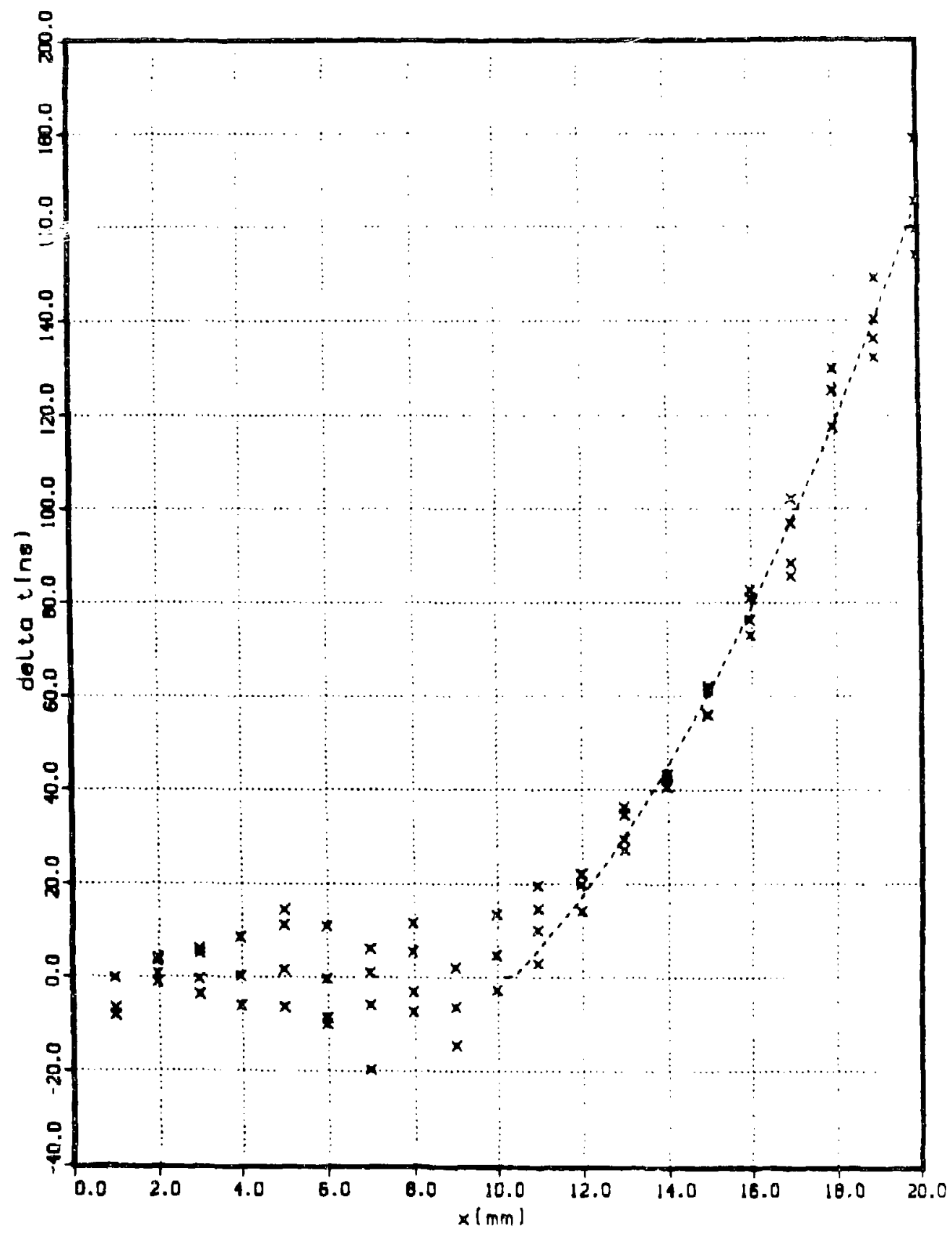

Figure 17: A $\delta t-x$ plot for experiment RS-2302. The dashed line is a fit to the adjusted $\delta t$ data. The first break point is at $x=10.2 \mathrm{~mm}$ and the second break point is at $10.6 \mathrm{~mm}$. Quadratic polynomials have been used for the fits in the seco $x \mathrm{c}$ and third zones. 


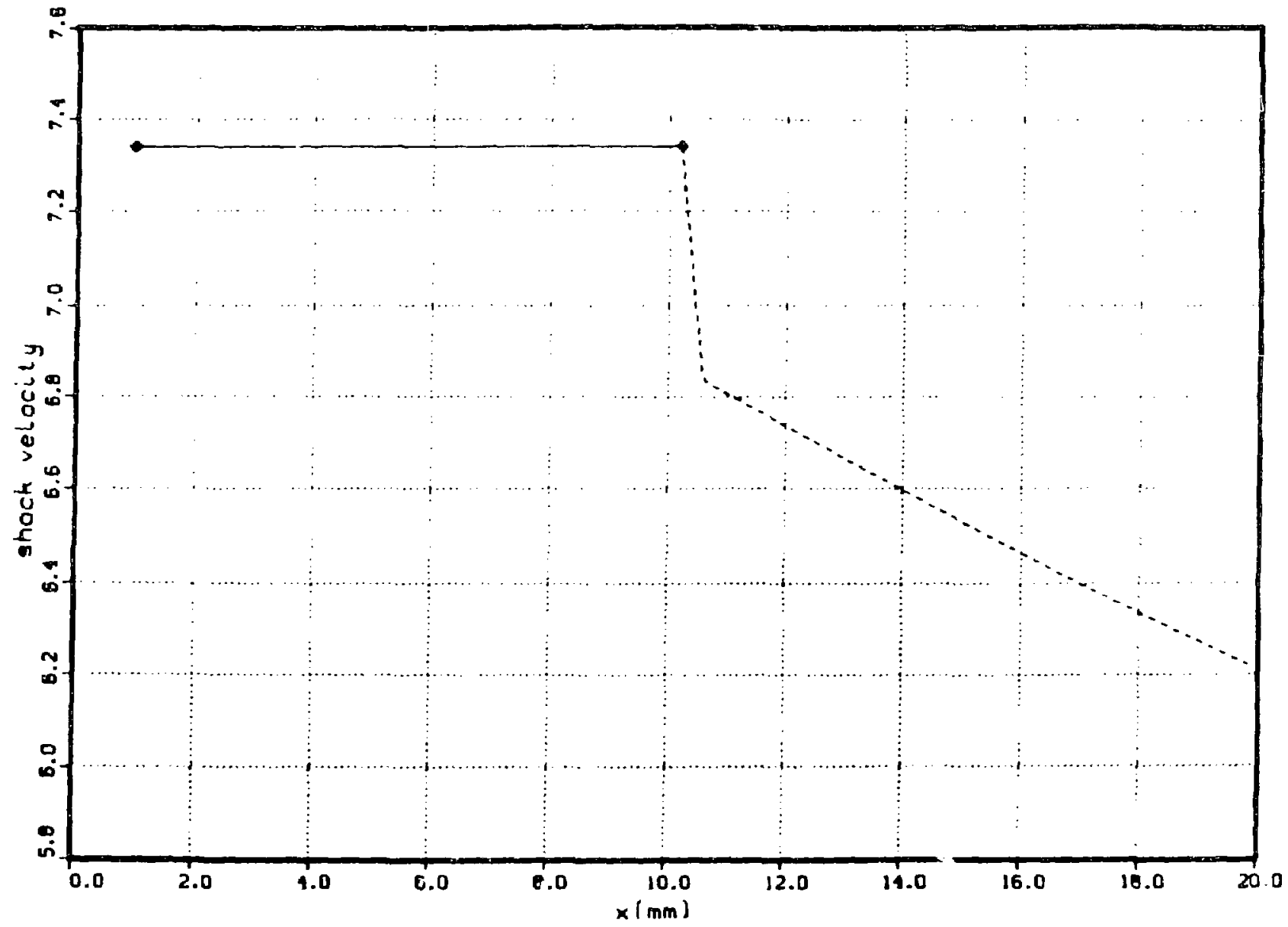

Figure 18: $: U$, versus $x$ plot for RS-2302 taken from the fit to the Jata shown in figure 17 . 


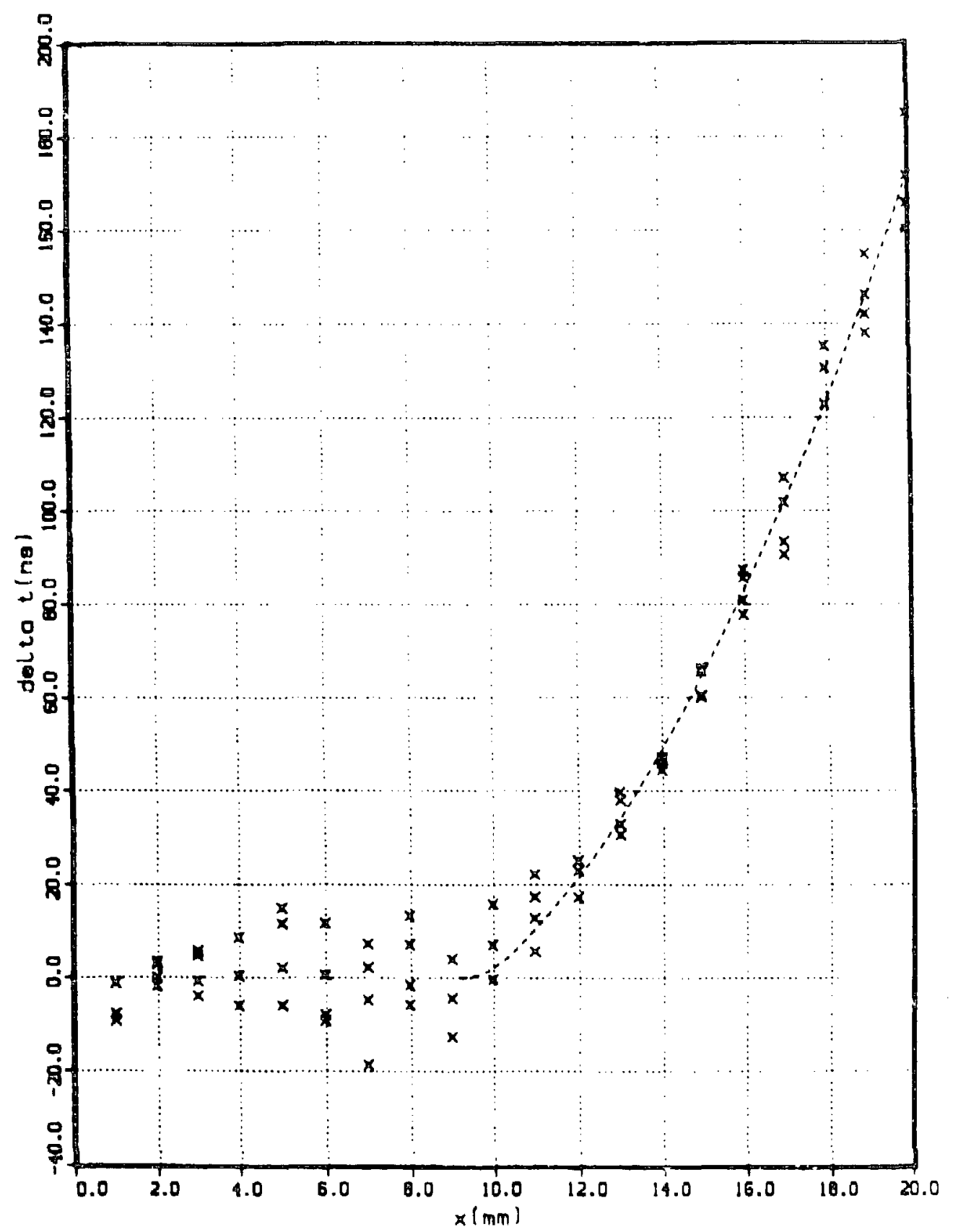

Figure 19: A $\delta t-x$ plot for experiment RS-2302. The dashed line is a fit to the adjusted $\delta t$ data. The fist break point is at $x=9.2 \mathrm{~mm}$ and the second break point is at $10.6 \mathrm{~mm}$. Quadratic polynomials have been used for the fits in the second and third zones. 


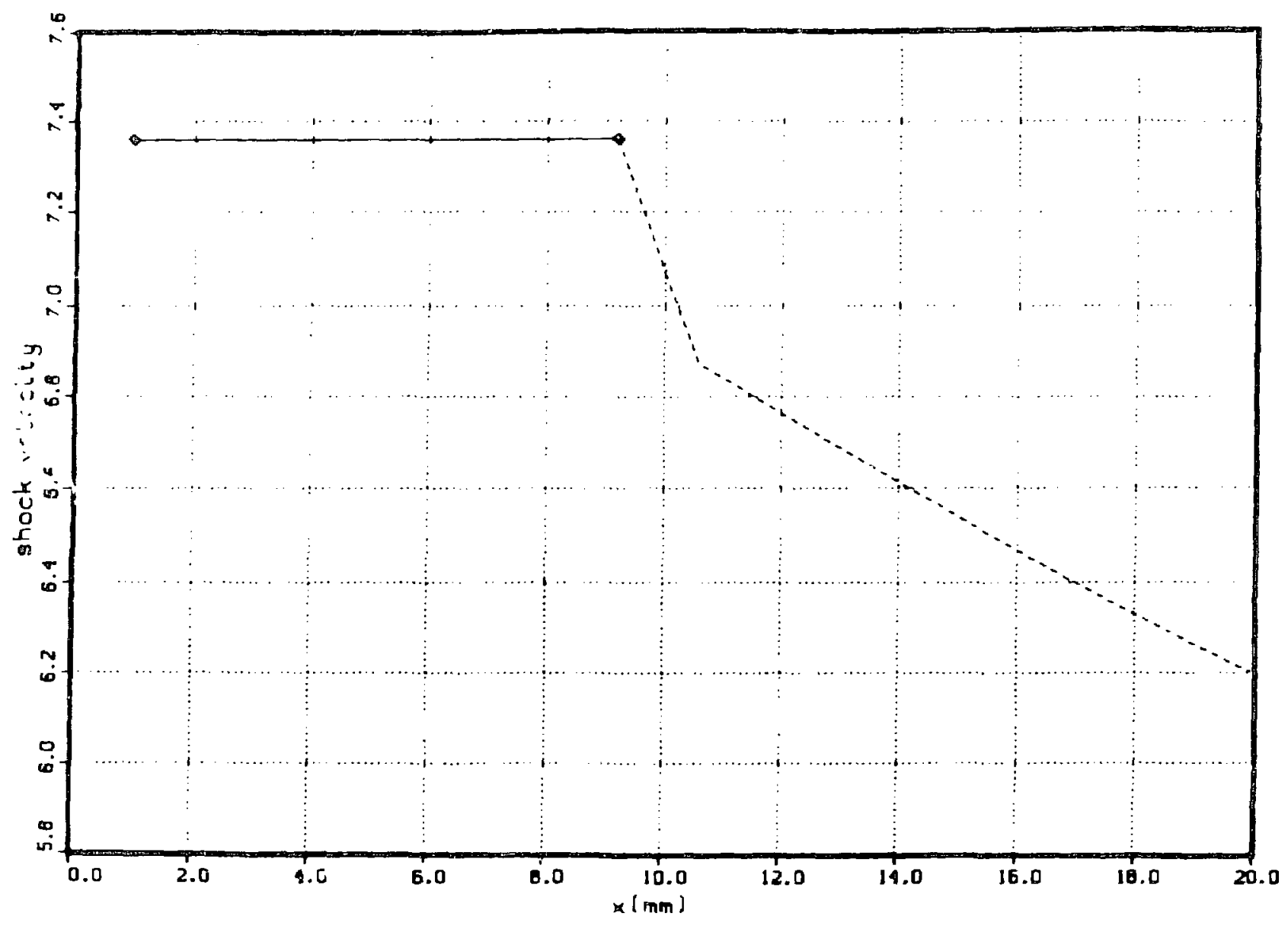

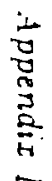

Figure 20: $\mathrm{A} U$, versus $x$ plot for RS-2202 taken from the fit to the data shown in figure 19. 


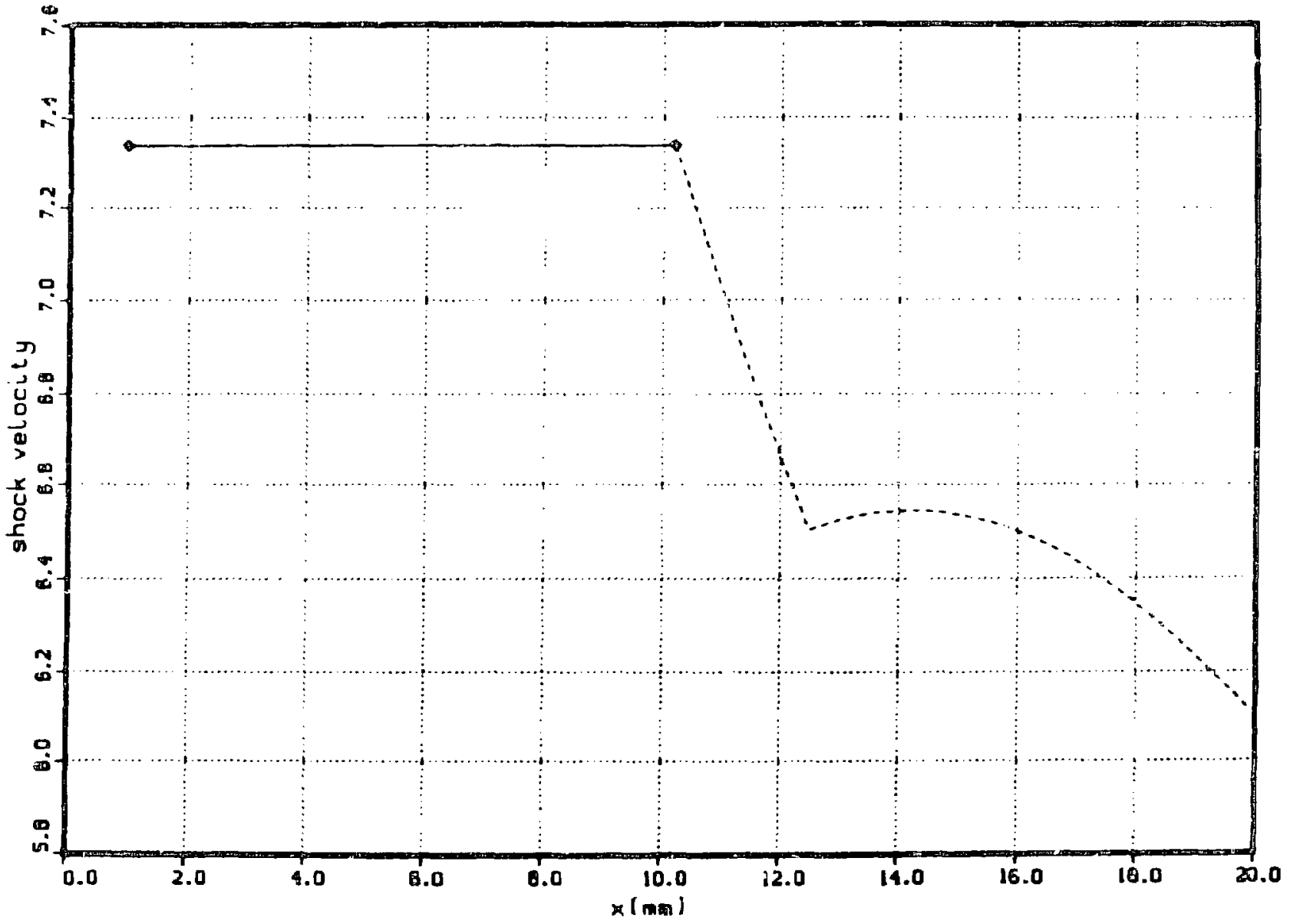

Figure 21: A $U$, versus $x$ plot taken from a fit to the data of RS-2302. The first break point is at $10.2 \mathrm{~mm}$ and the second at $12.5 \mathrm{~mm}$. A quadratic polynomial has been used for sone two anci a cubic polynomial in zone three. 


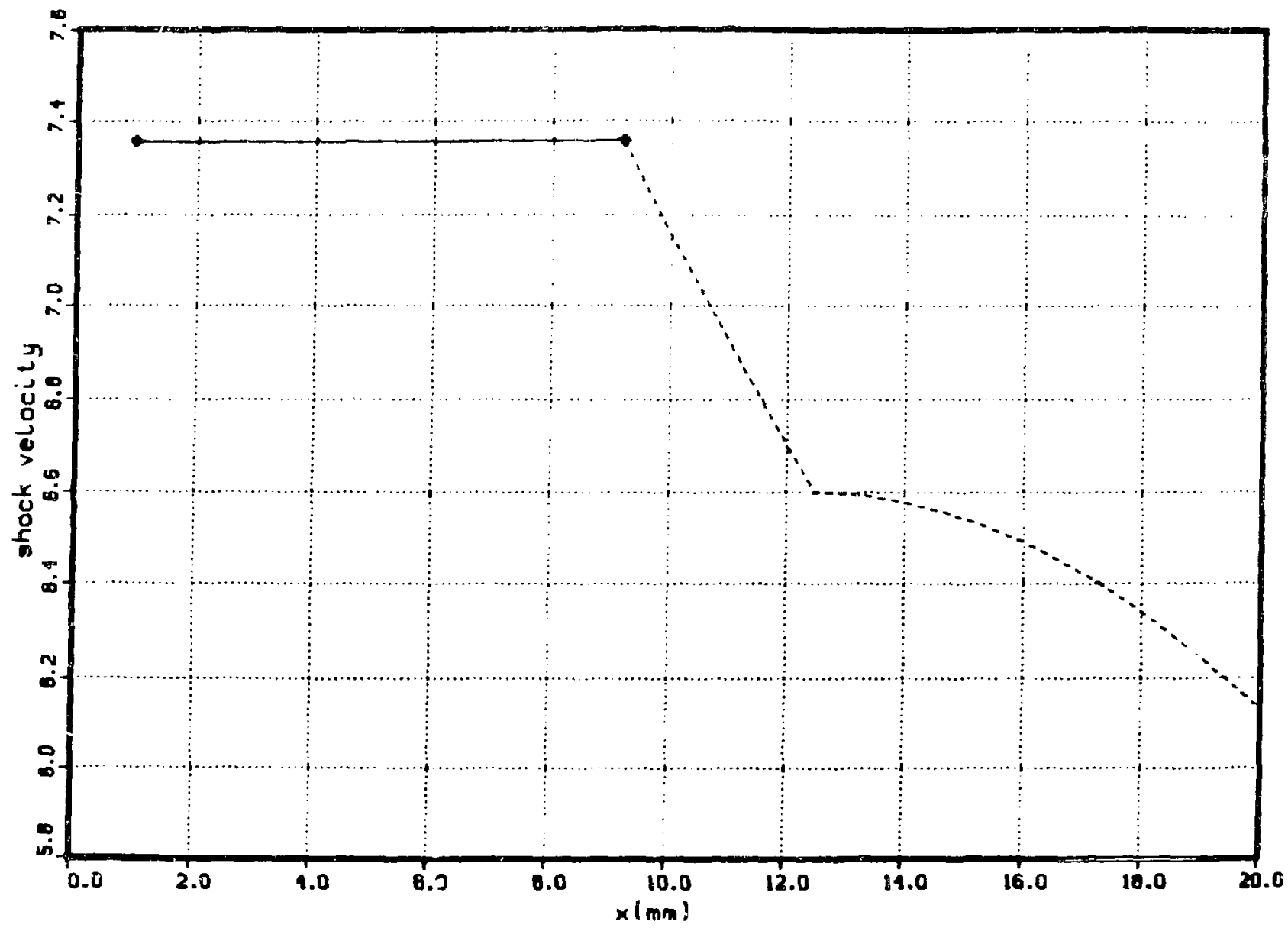

Figure 22: A $U$, versus $I$ plot $t$ dken from a tht to the data of RS-2302 using the same functional fiting forms as those used for figure 21 . The second break point is still at $12.5 \mathrm{~mm}$ but the first breals point has been moved to $9.2 \mathrm{~mm}$. 


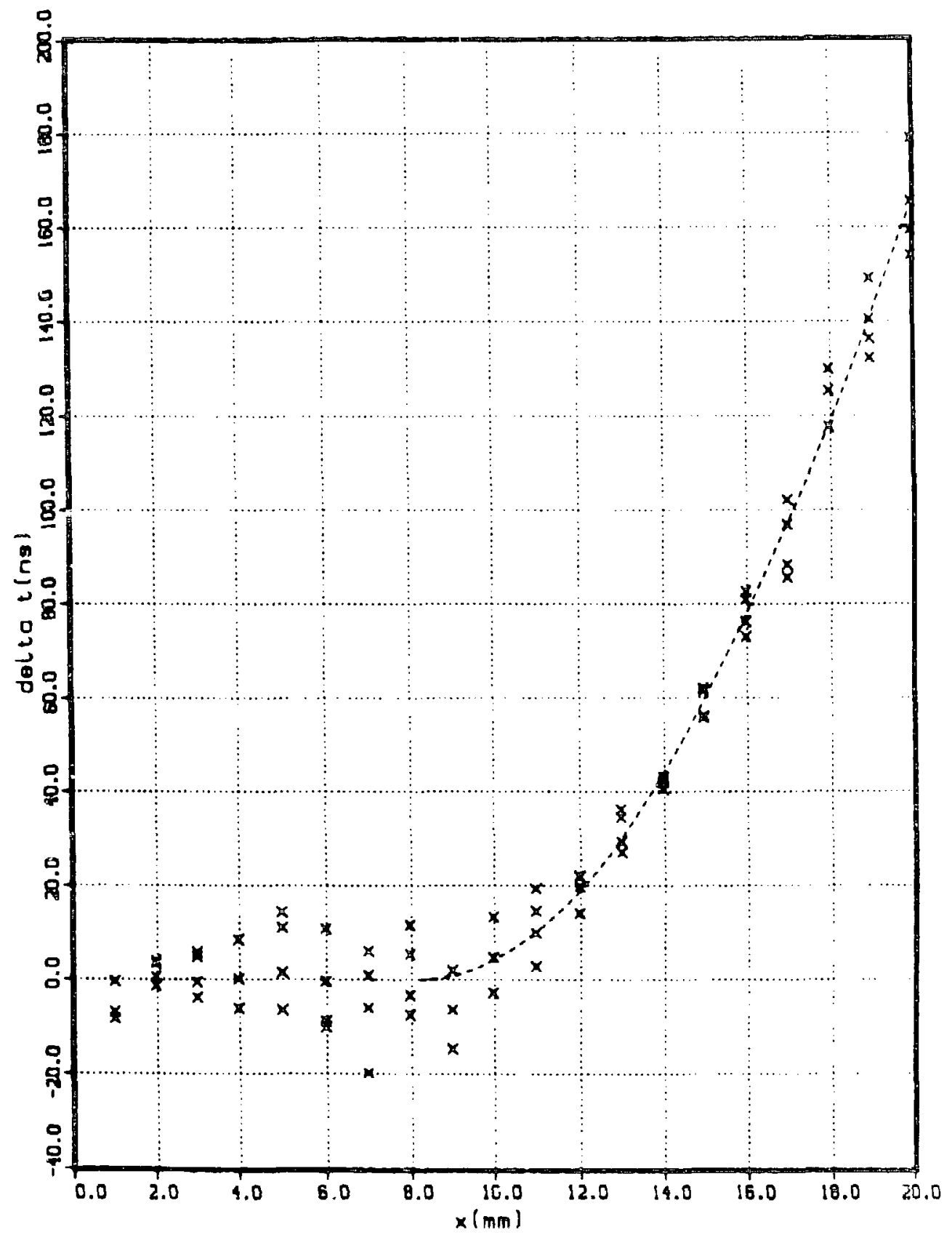

Figure 23: A $\delta t-x$ plot for experiment RS-2302. The dashed line is a fit to the adjusted $\delta t$ data. The first break point is at $x=8.2 \mathrm{~mm}$ and the second break point is at $12.5 \mathrm{~mm}$. A quadratic polynomial have been used for the fit in the second zone and a cubic polynomial in the third zone. 


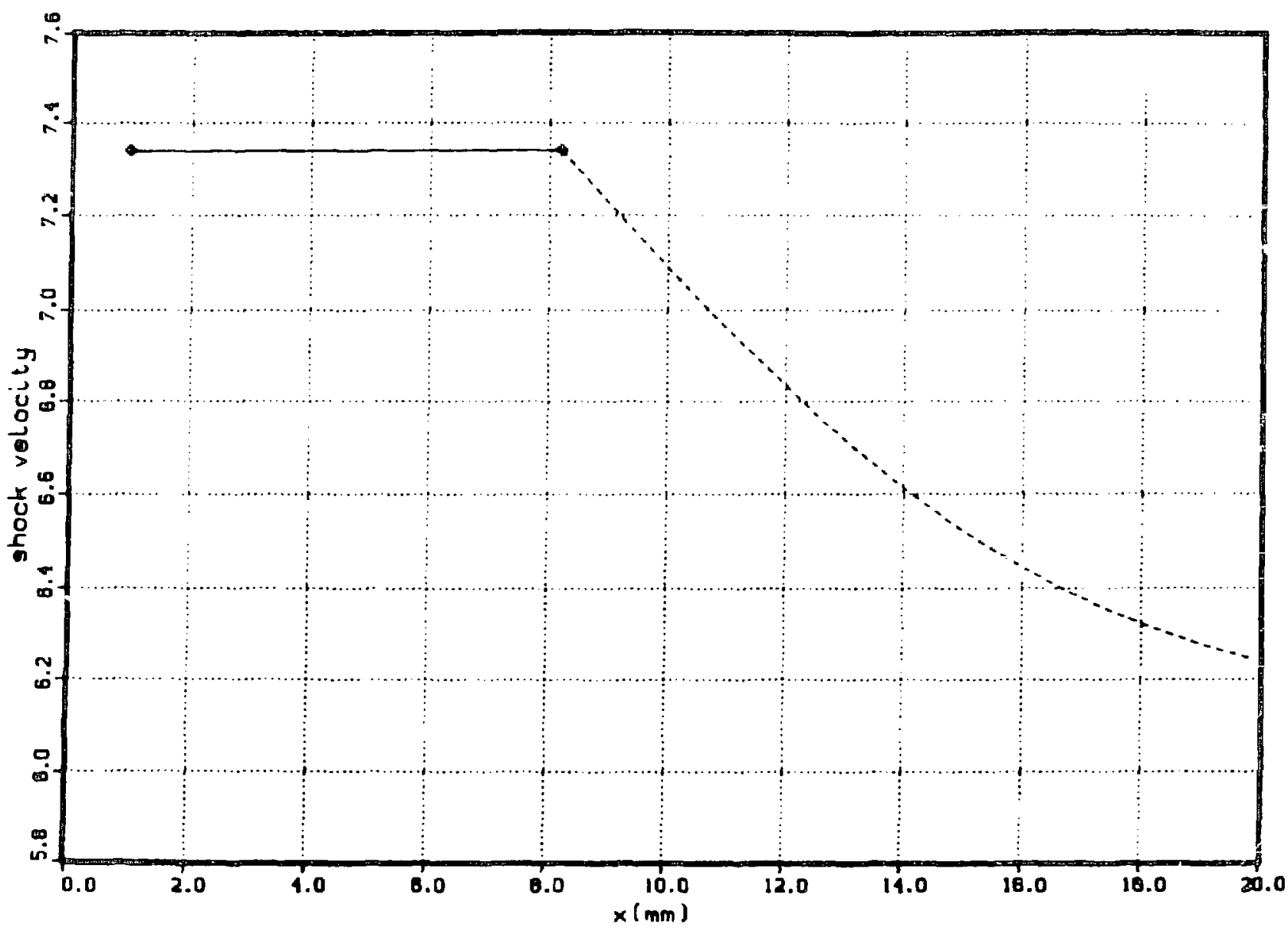

Figure 24: A $U$, versus $x$ plot for RS-2302 taken from the fit to the data shown in figure 23. 


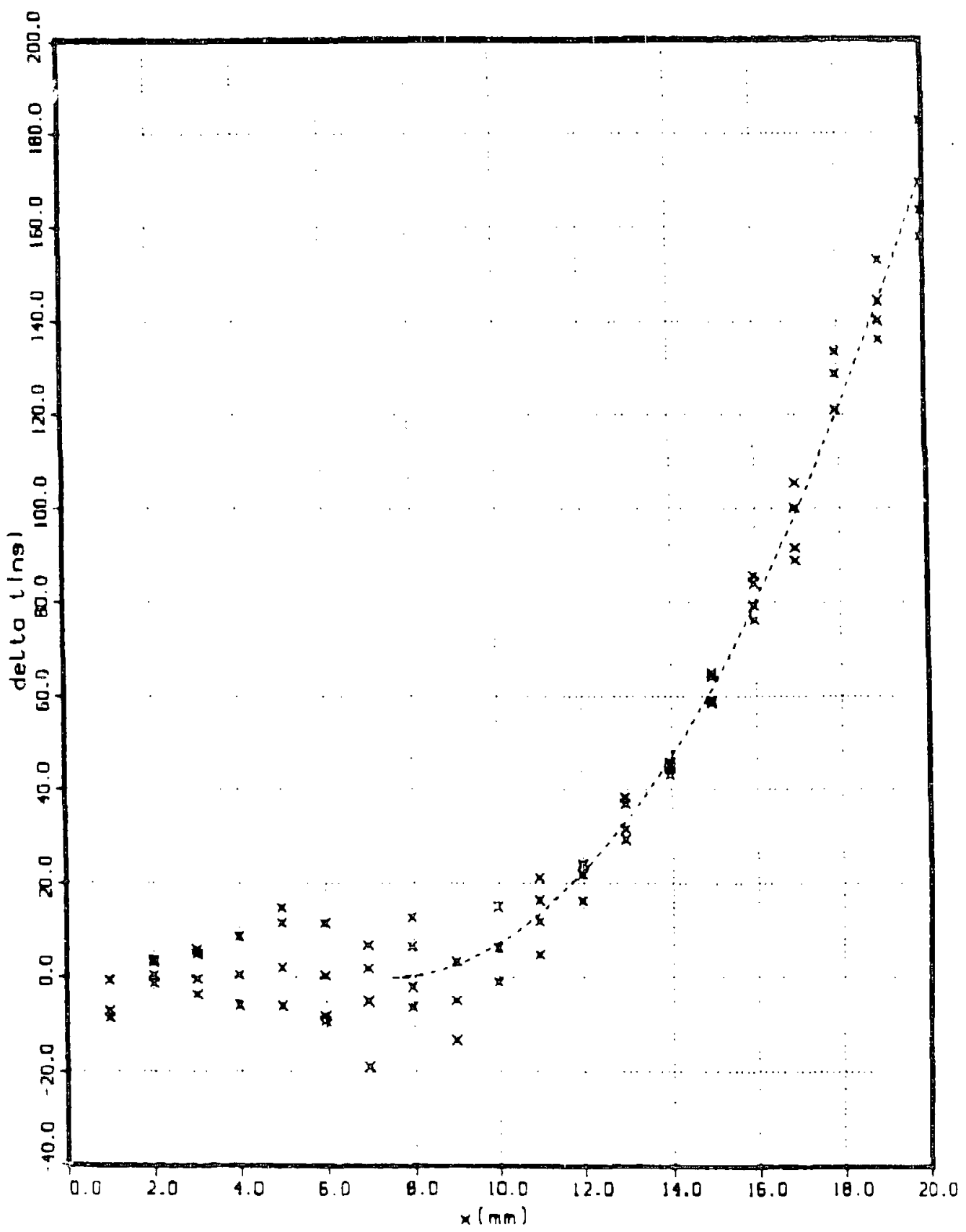

Figure 25: A $\delta t-x$ plot for experiment RS-2302. The dashed line is a fit to the adjusted $\delta t$ data. The first break point is at $x=7.5 \mathrm{inm}$ and the second break point is at $8.5 \mathrm{~mm}$. Quadratic polynomials have been used for the fits in the second and third zones. 


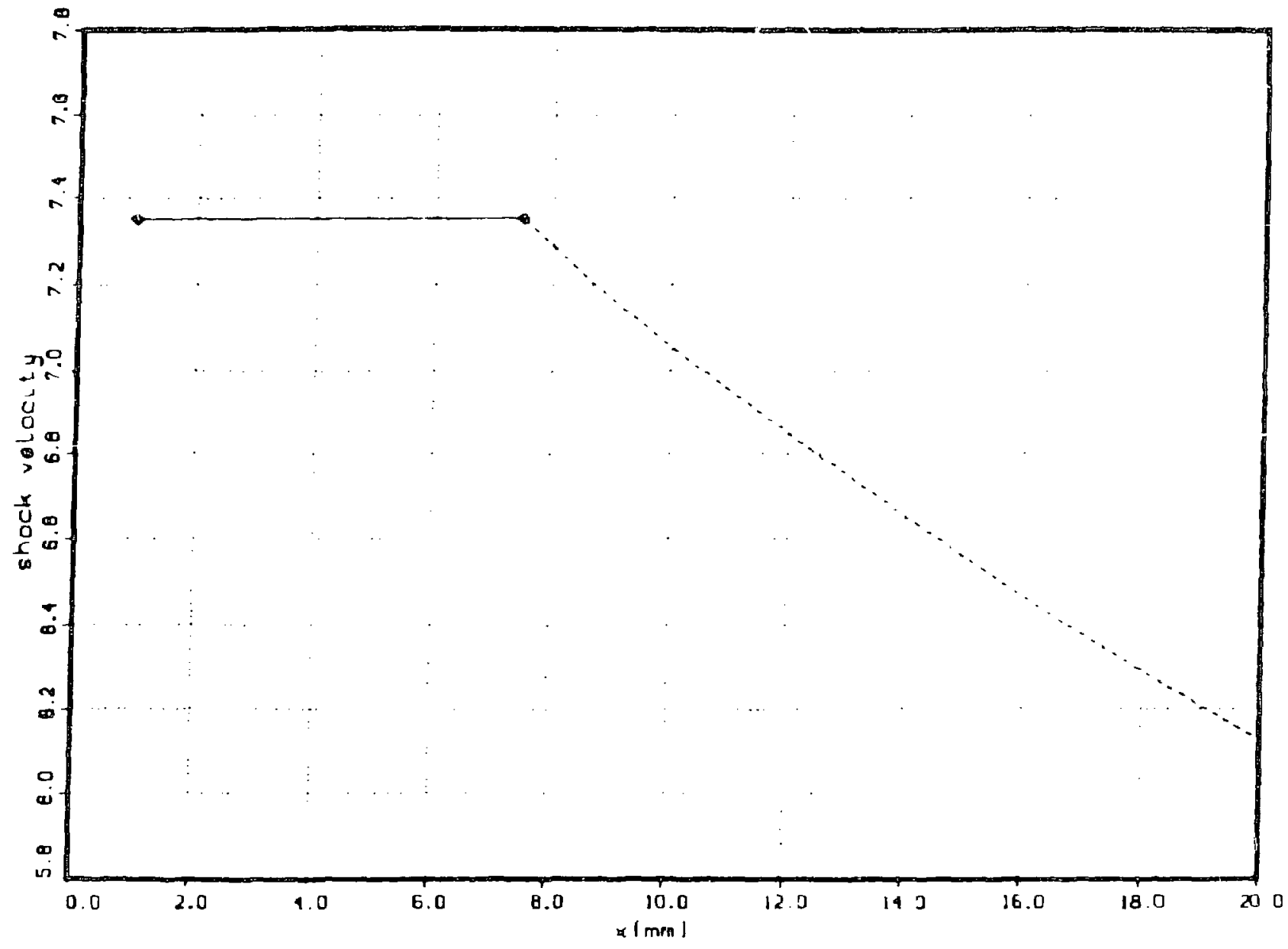

Figure 26: A $U$. versus $x$ plot for RS-2302 taken from the fit to the data shown in figure 25. 


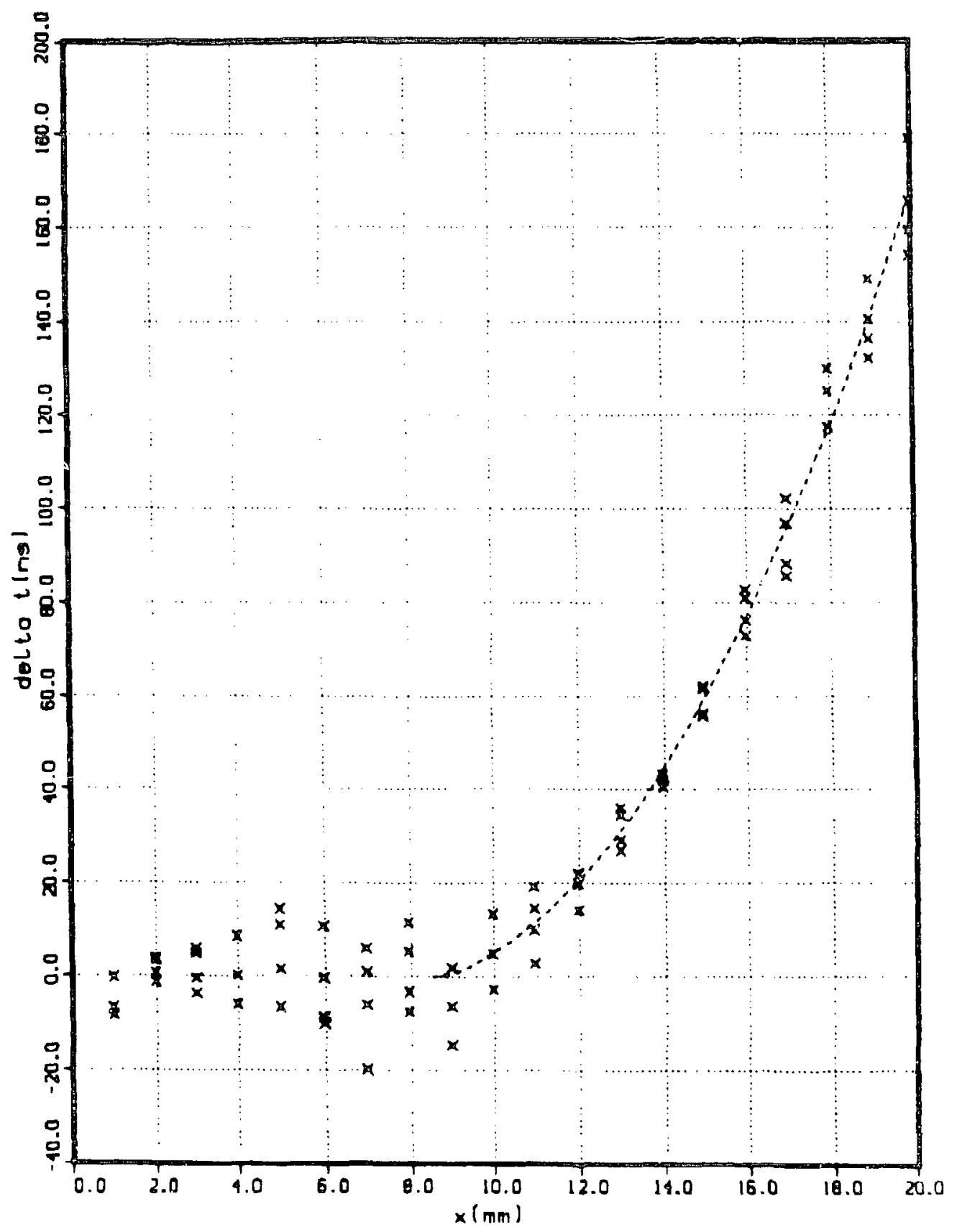

Figure 27: A $\delta t-x$ plot for experiment RS-2302. The dashed line is a fit to the adjusted $\delta t$ data. The first break point is at $x=8.5 \mathrm{~mm}$ and the second break point is at $9.5 \mathrm{~mm}$. Quadratic polynomials have been used for the fits in the second and third zones. 


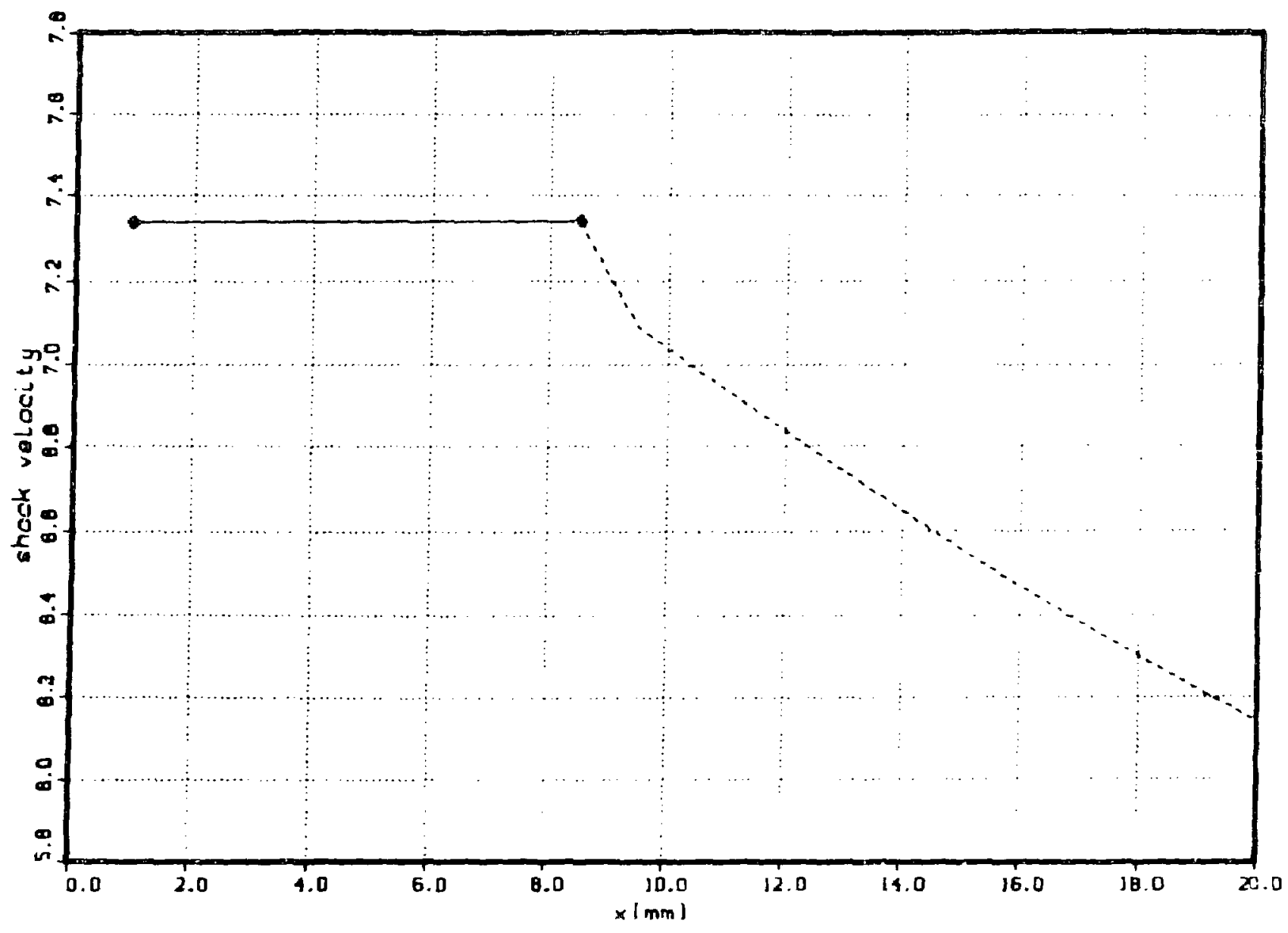

Figure 28: A $U$, versus $x$ plot fcr RS-2302 taken from the fit to the data shown in Ggure 27. 


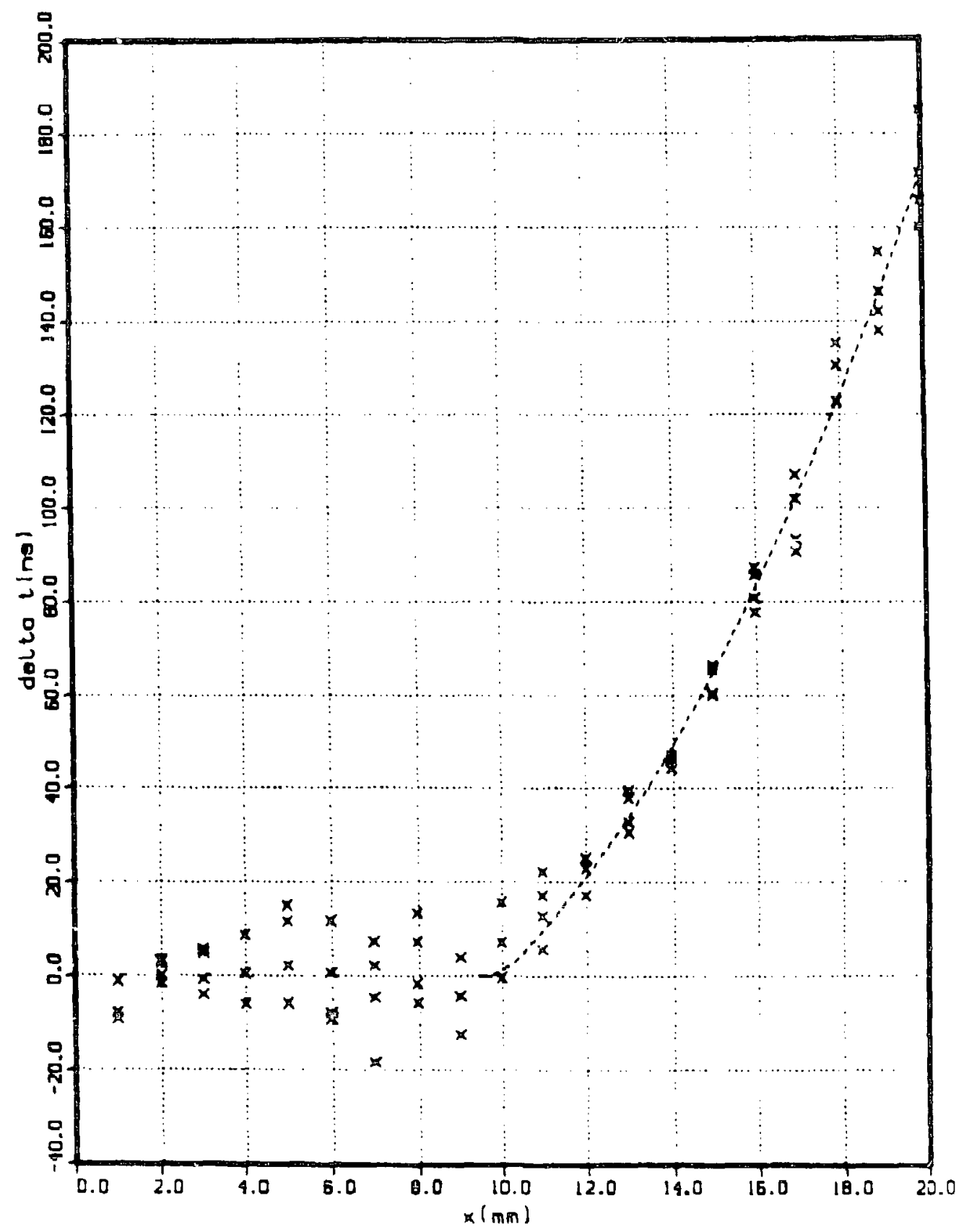

Figure 29: A $\delta t-x$ plot for experiment RS-2302 The dashed line is a fit to the adjusted $\delta t$ d.ata. The first break point is at $x=9.5 \mathrm{~mm}$ and the second break point is at $10.5 \mathrm{~mm}$. Quadratic polynomials have been used for the fits in the second and third zones. 


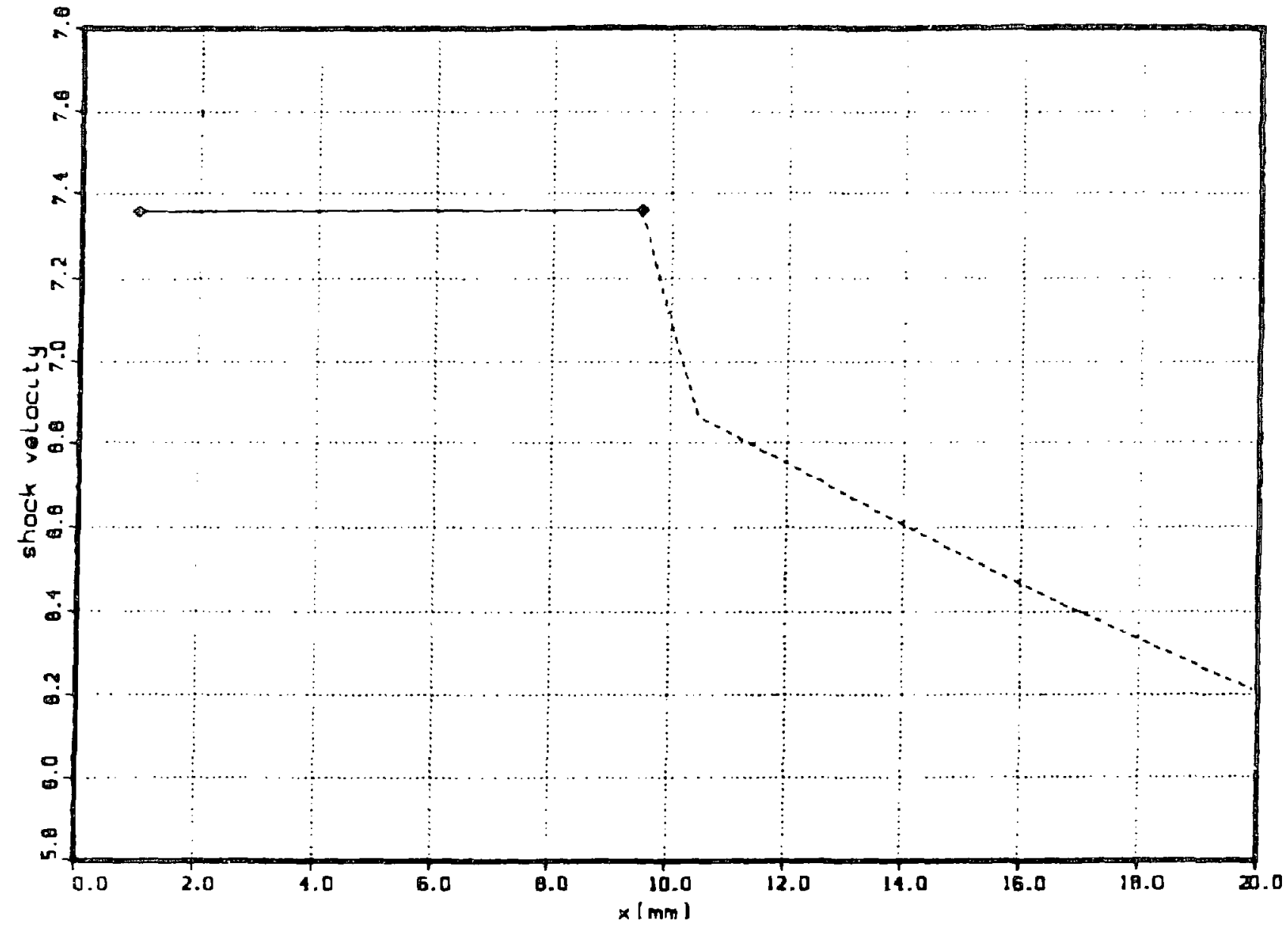

立

Figure 90: A $U$, versus $x$ plot for RS-2302 taken from the fit to the data shown in figure 29 . 


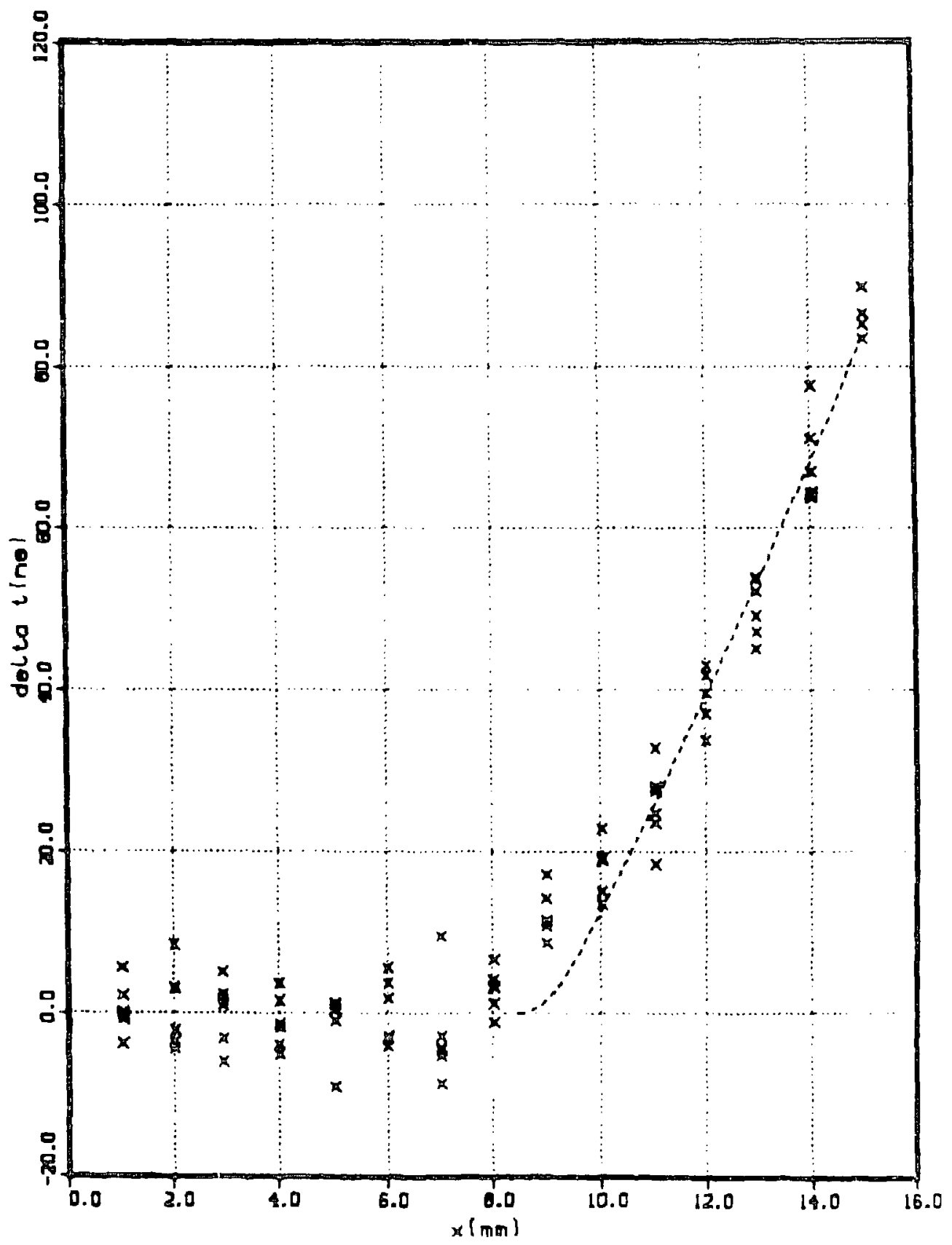

Figure 31: A $\delta t-x$ plot for experiment RS-2309. The dashed line is a fit to the adjusted $\delta t$ data. The first break point is at $x=8.5 \mathrm{~mm}$ and the second break point is at $9.5 \mathrm{~mm}$. Quadratic polynomials have been used for the fits in the seiond and thind rones. 


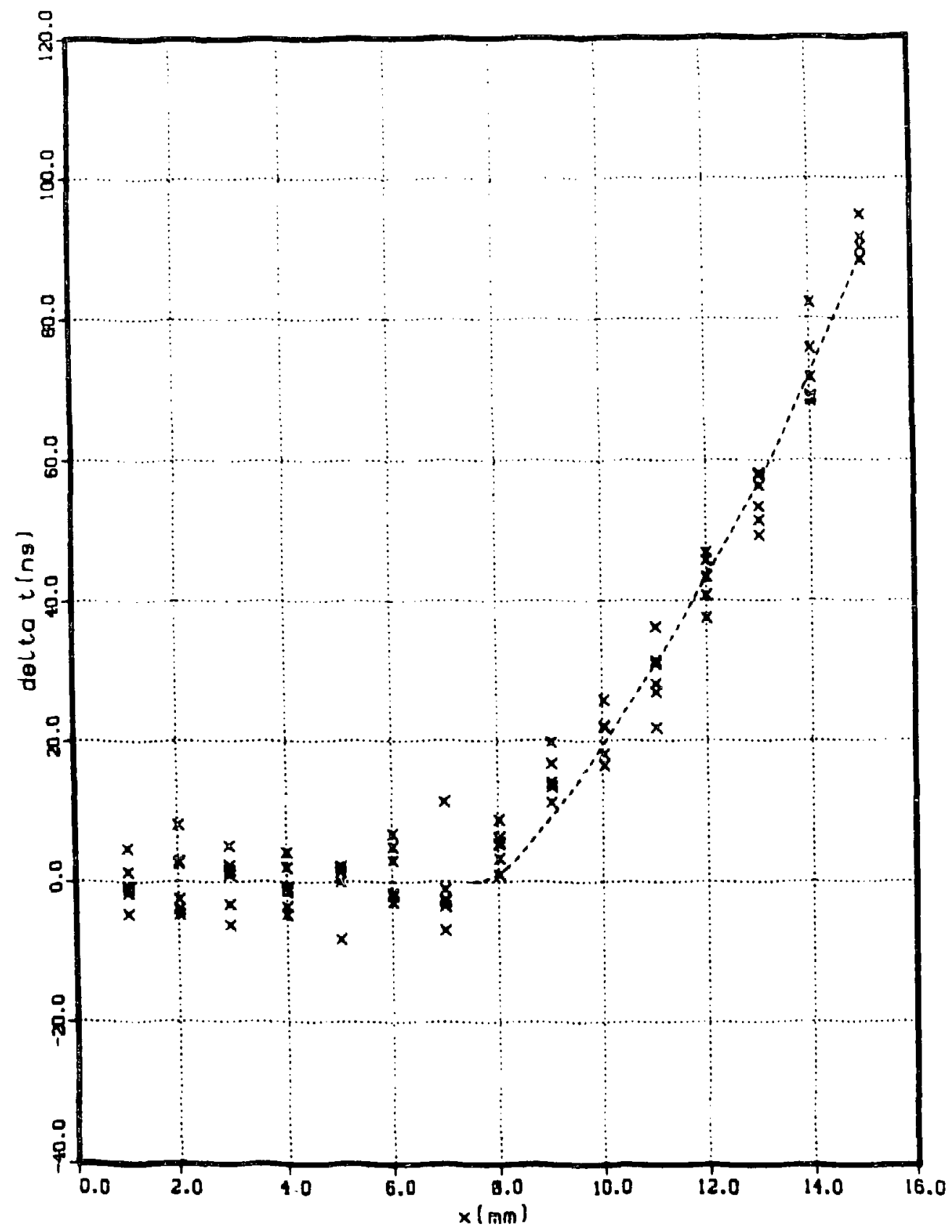

Figure 32: A $\delta t-x$ plot for experiment RS-2309. The dashed line is a fit to the adjusted $\delta t$ data. The first break point is at $x=7.5 \mathrm{~mm}$ and the second break point is at $8.5 \mathrm{~mm}$. Quadratic polynomials have been used for the fits in the second and third zones. 


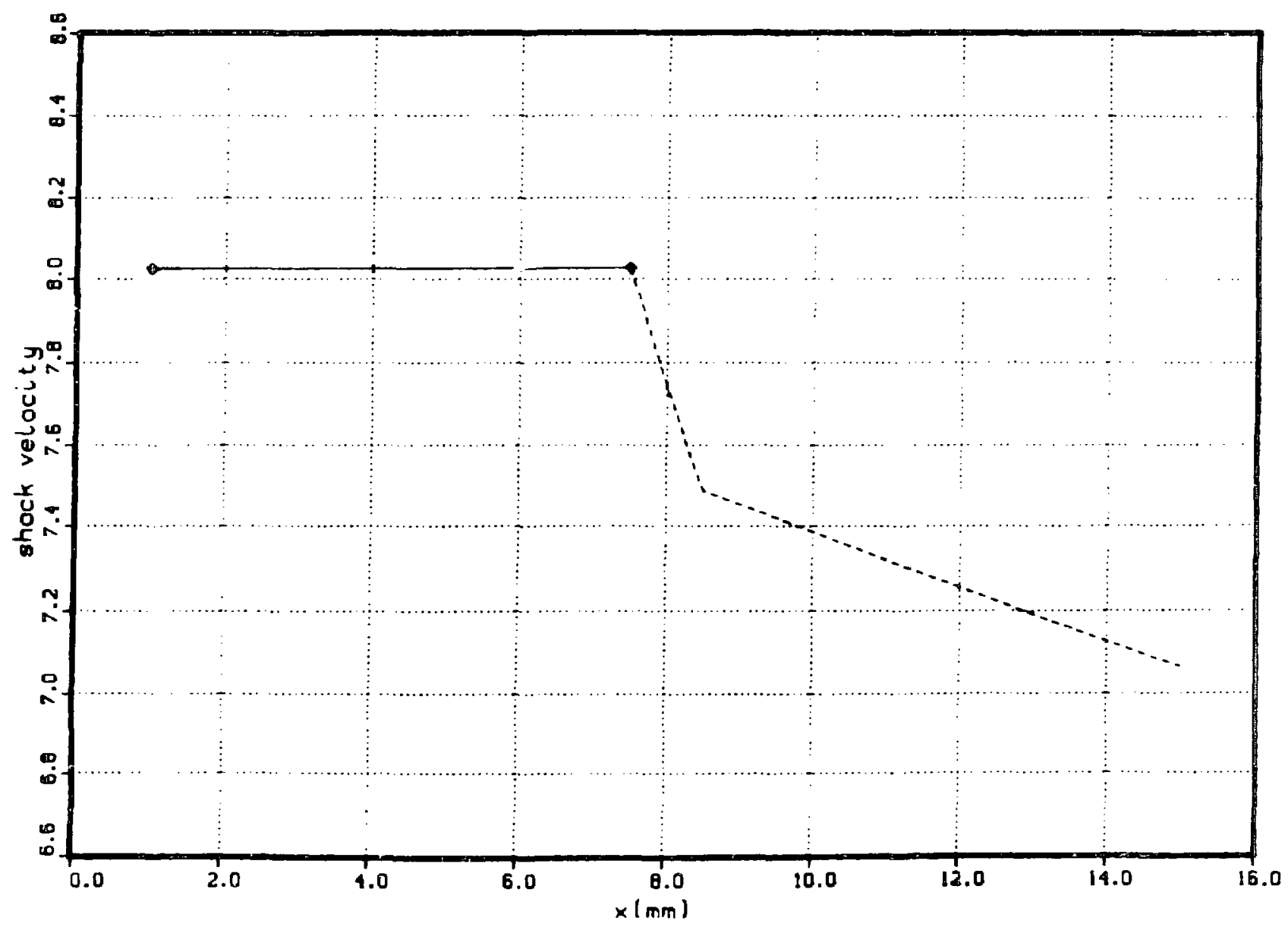

돈

Figure 33: A $U$, versus $x$ plot for RS-2309 taken from the fit to the data shown in figure 32 


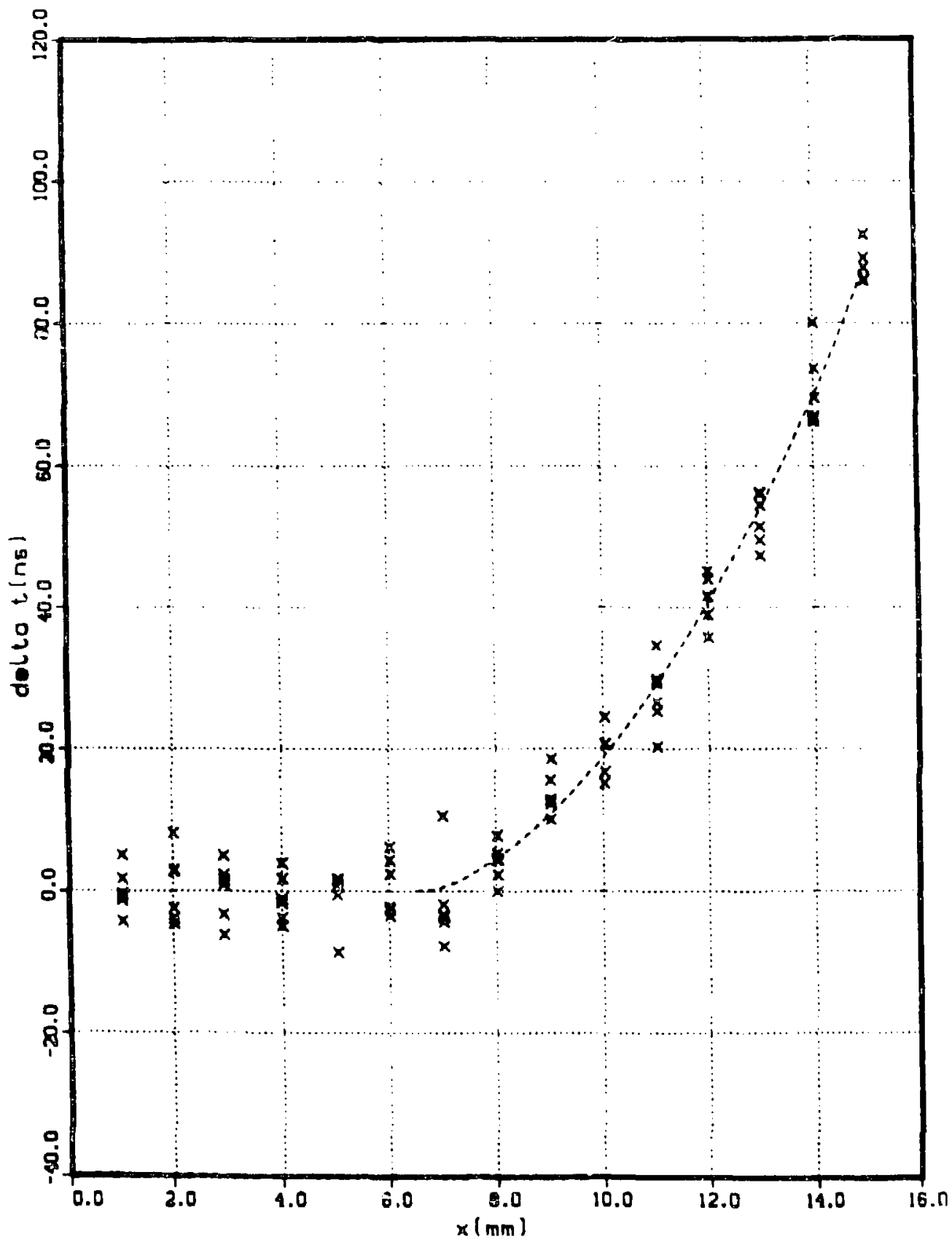

Figure 34: A $\delta t-x$ plot for experiment RS-2309. The dashed line is a fit to the adjusted $\delta t$ data. The first break point is at $x=6.5 \mathrm{~mm}$ and the second break point is at $7.5 \mathrm{~mm}$. Quadratic polynomials have been used for the fits in the second and third zones. 


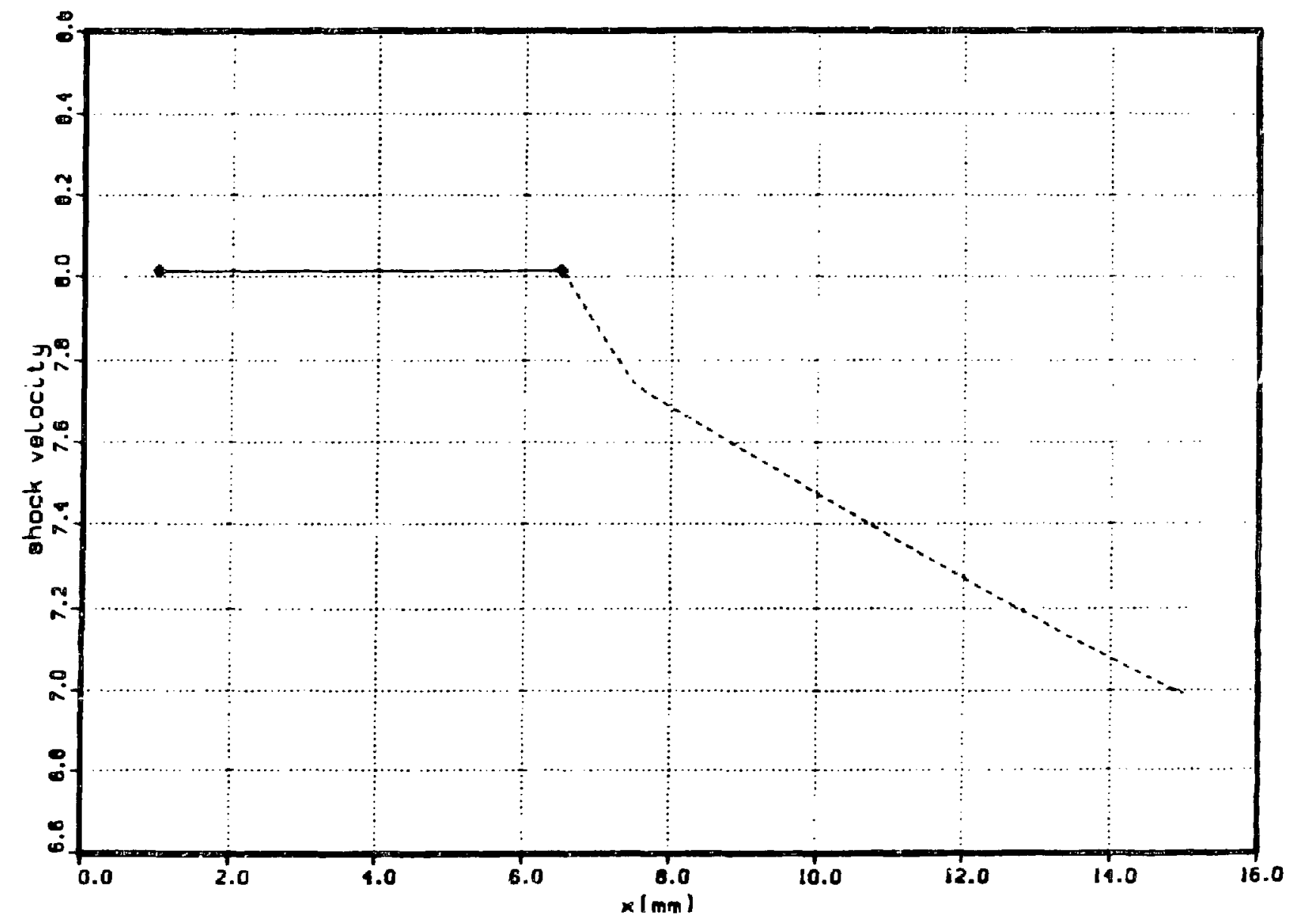

igure 85: A $U$, verJus I plot for RS-2309 taken from the fit to the data shown in fgure 34. 


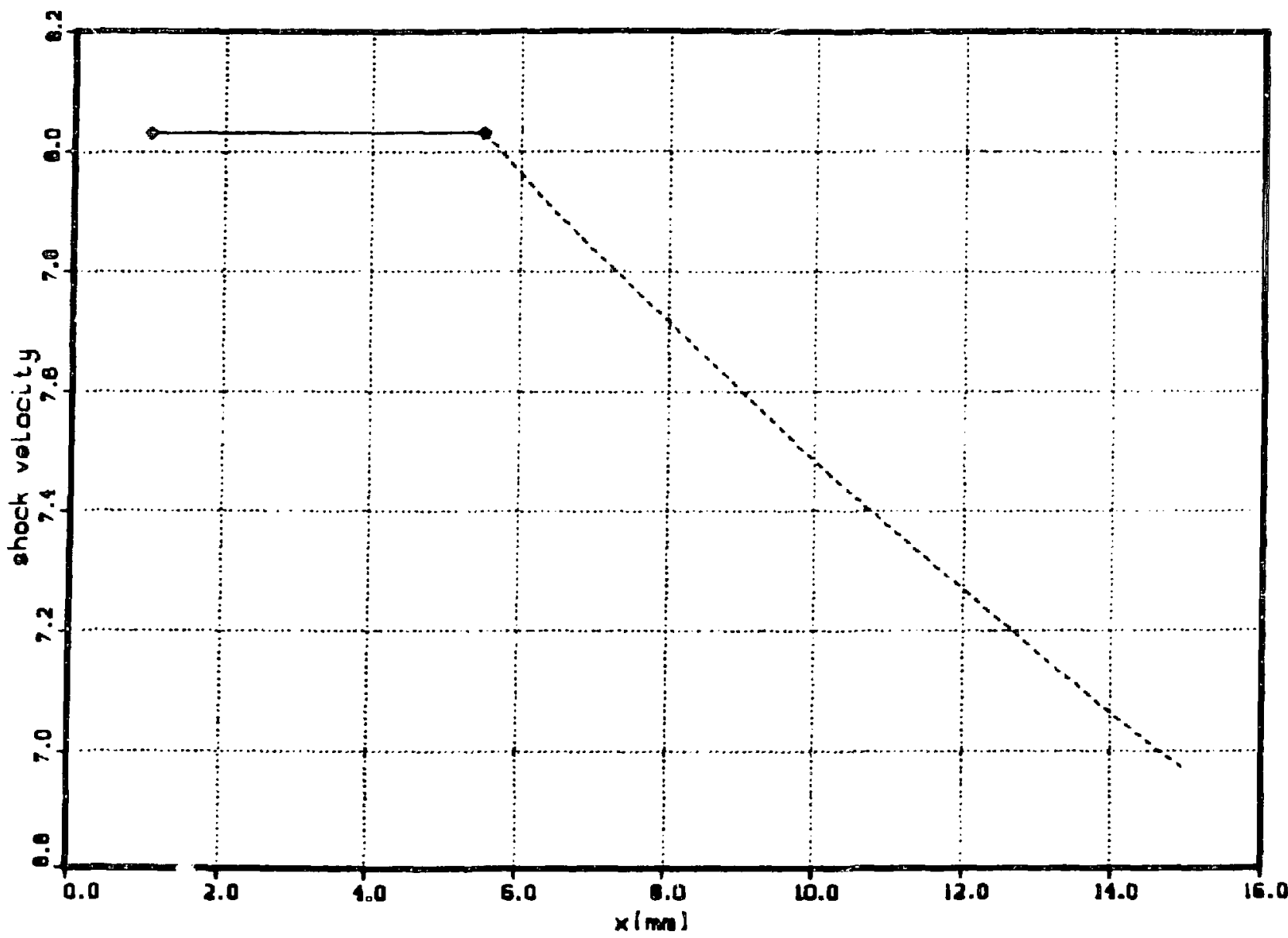

Figure 36: A $U$, versus $x$ plot taken from a fit to the data of RS-2309 using the same functional fitting forms as those used for figure 35 . The second break point is now at $6.5 \mathrm{~mm}$ but the first break point has been moved to $5.5 \mathrm{~mm}$. 


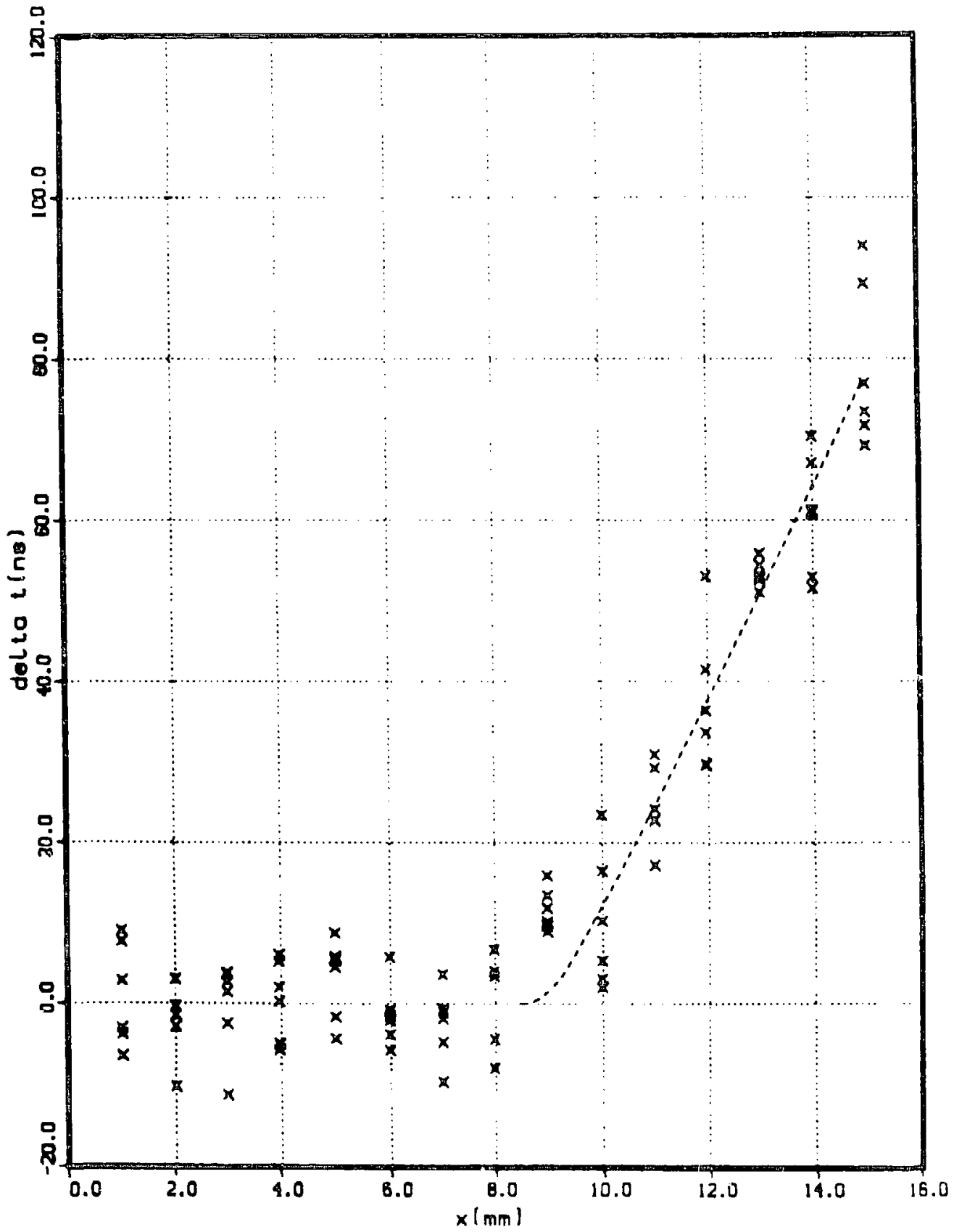

Higure 37: A $\delta t-x$ plot for experiment RS-2315. The dashed line is a fit to the adjusted $\delta$ t data. The first break point is at $x=8.5 \mathrm{~mm}$ and the second break point is at $9.5 \mathrm{~mm}$. Quadratic polynomials have been used for the fits in the second and third zones. 


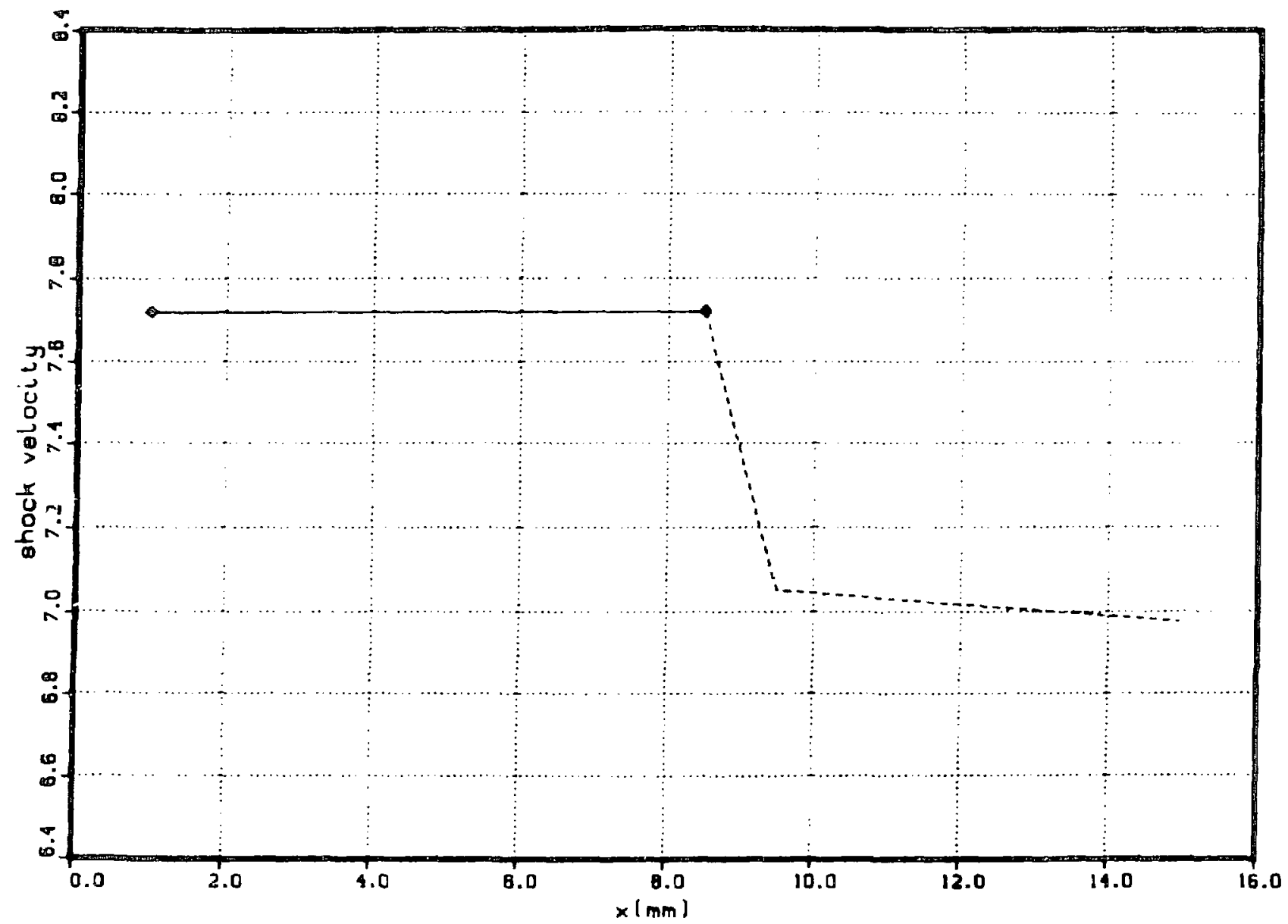

Figure 98: A $U_{\text {s }}$ versus $x$ plot for RS-2315 taken from the fit to the data shown in figure 37 . 


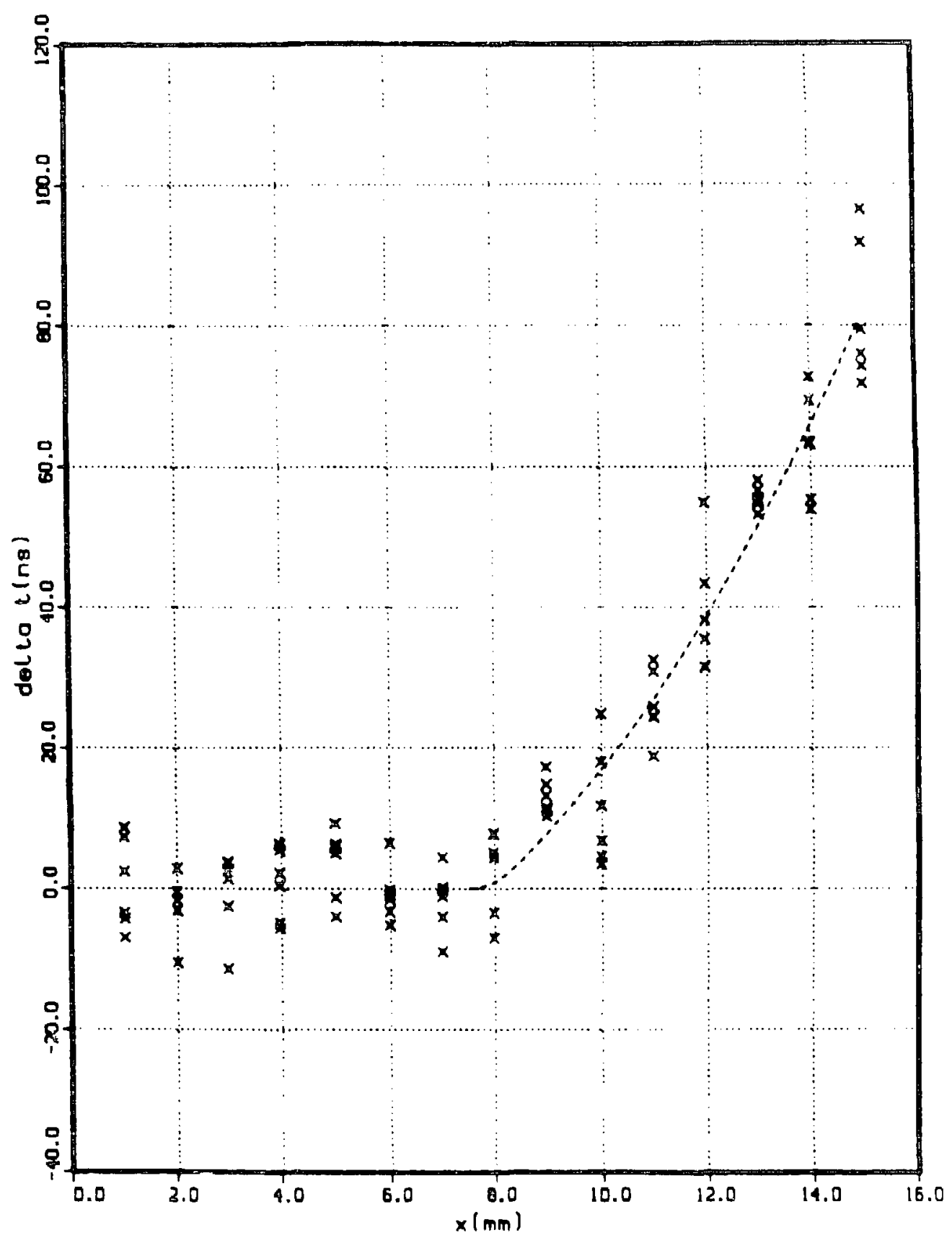

Figure 39: A $\delta t-x$ plot for experiment RS-2315. The dashed line is a fit to the adjusted $\delta t$ data. The first break point is at $I=7.5 \mathrm{~mm}$ and the second break point is at $8.5 \mathrm{~mm}$. Quadratic polynomials have been used for the fits in the second and third zones. 


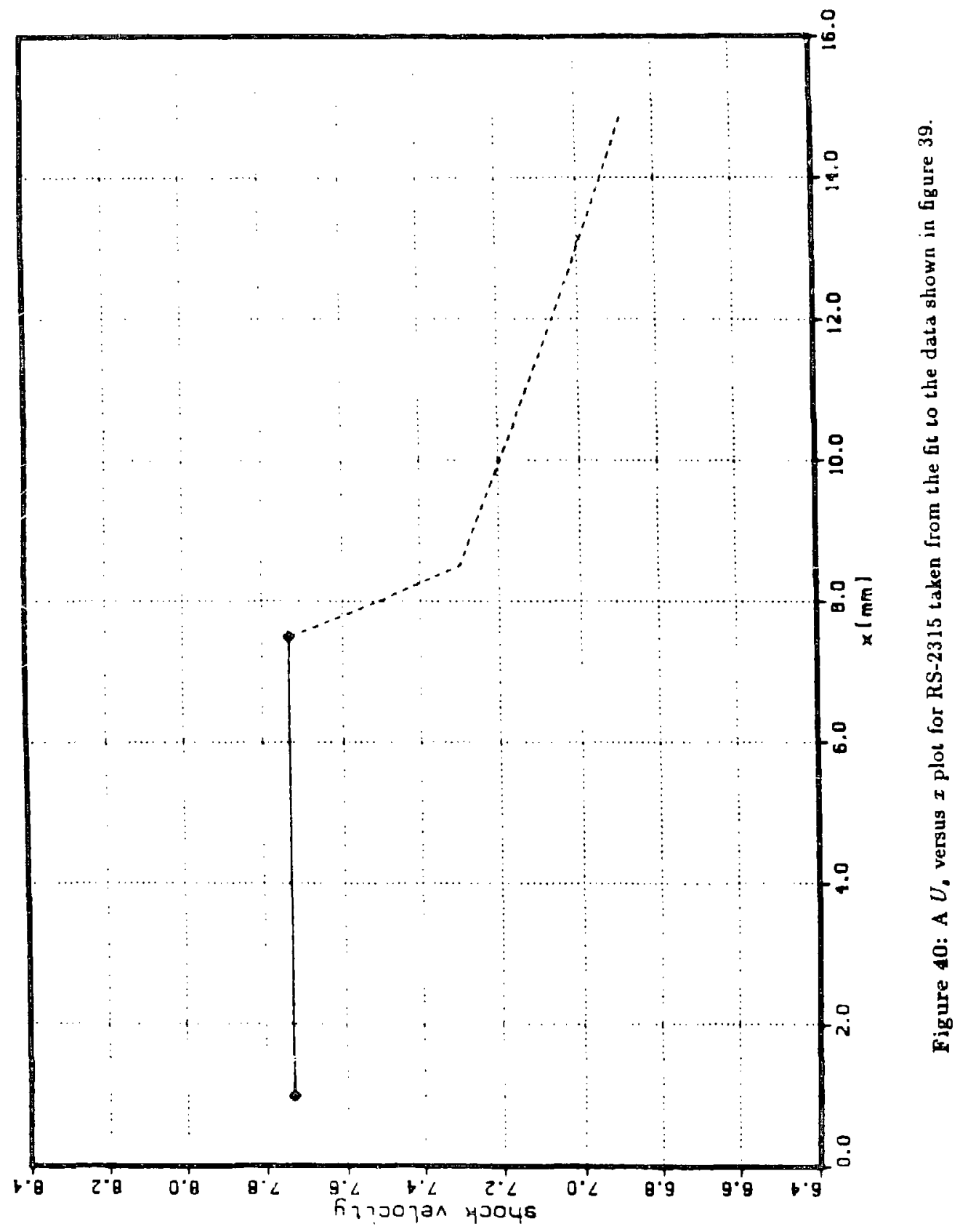




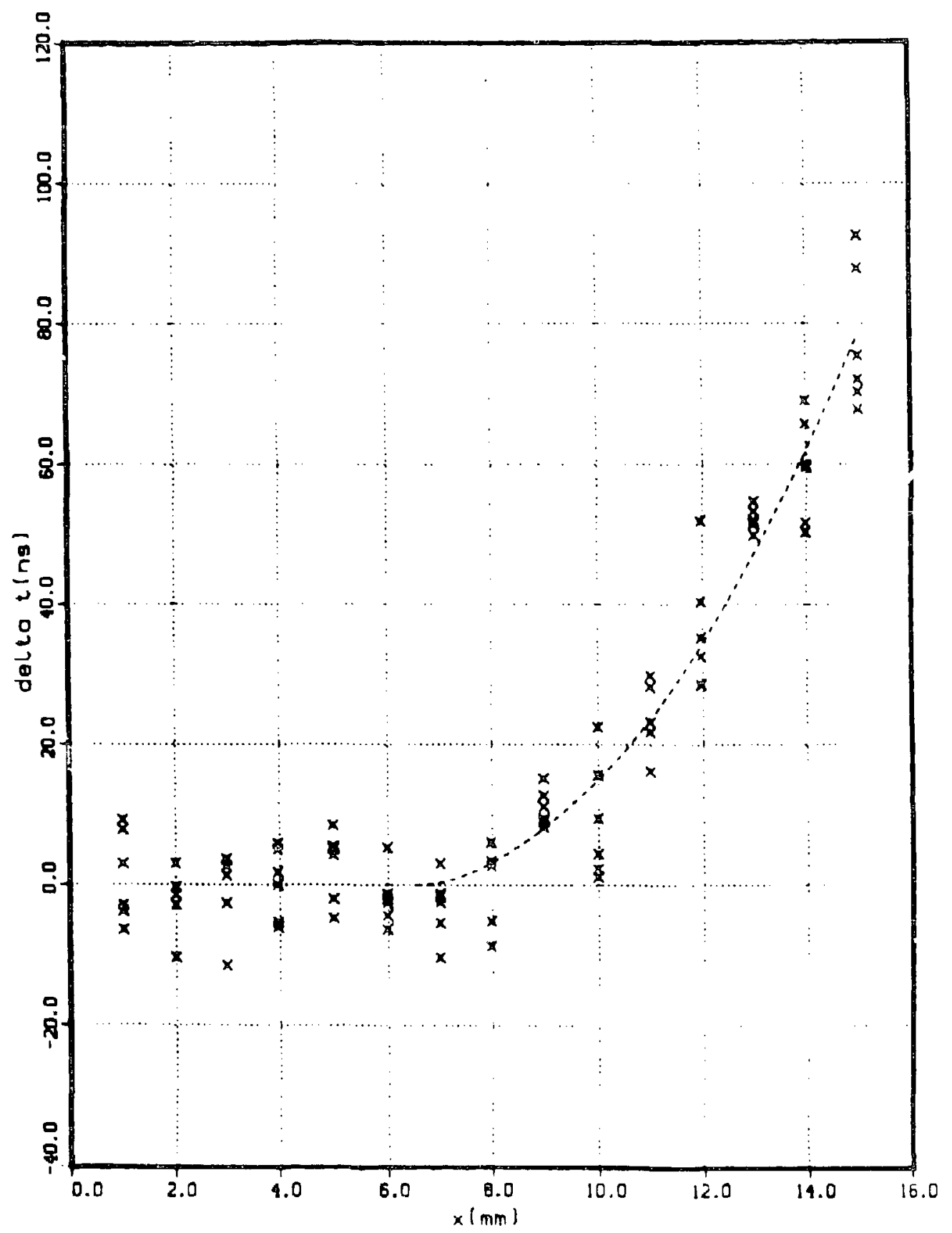

Figure 1: A $\delta t-x$ plot for experiment RS-2315. The dashed line is a fit to the adjusted $\delta t$ data. The first break point is at $x=6.5 \mathrm{~mm}$ and the second break point is at $7.5 \mathrm{~mm}$. Quadratic polynomials have been used for the fits in the second and third zones. 


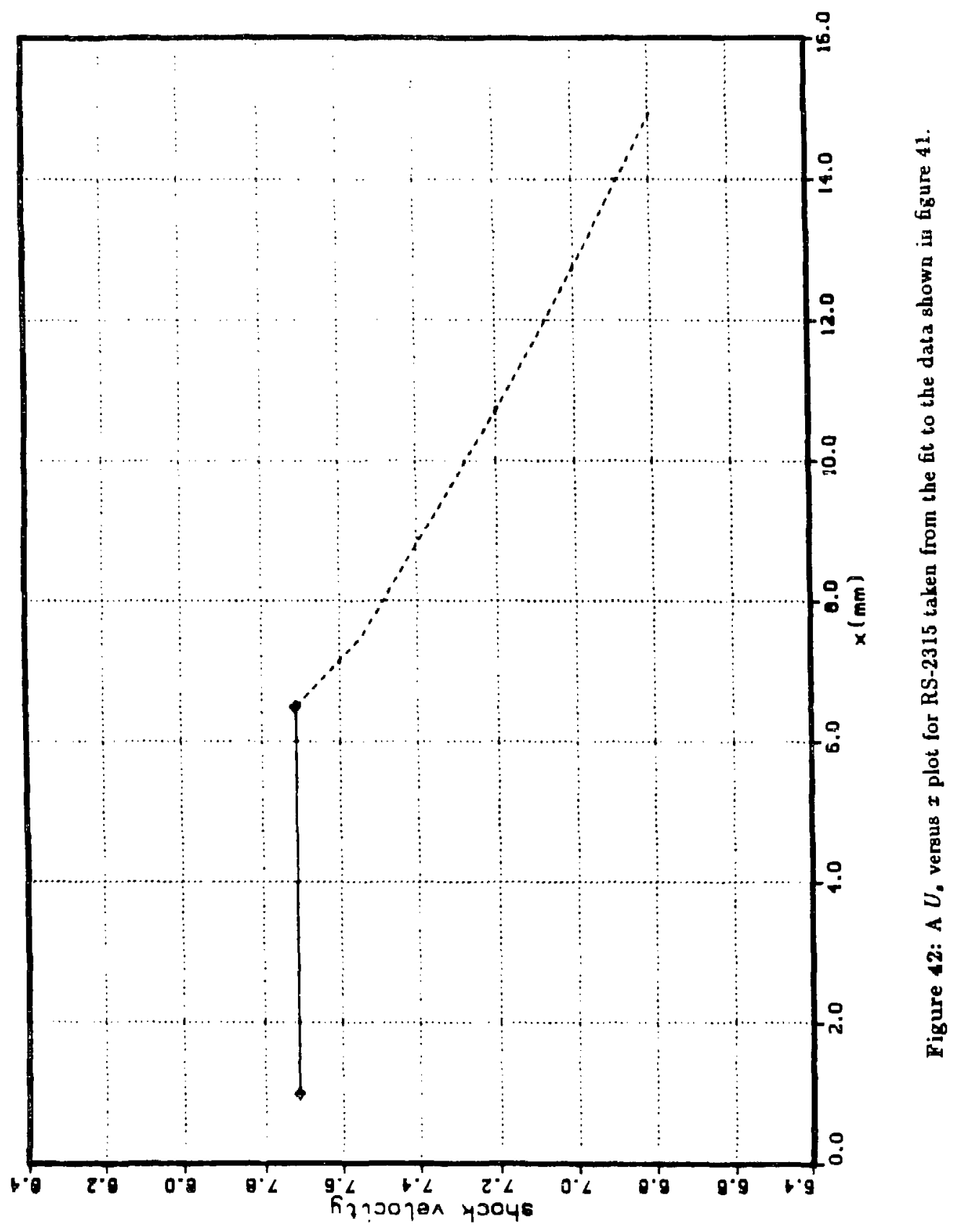




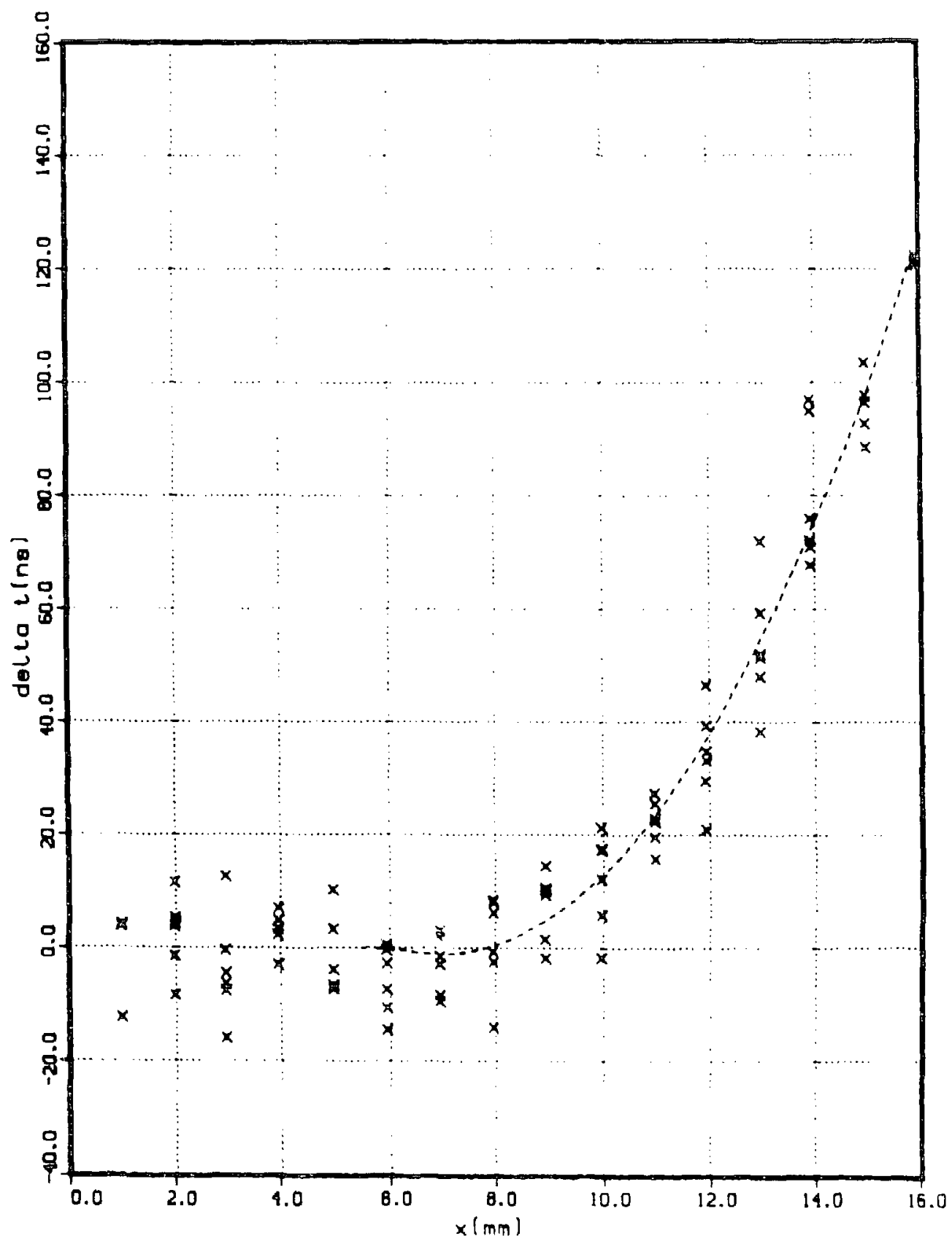

Figure 43: A $\delta t-x$ plot for experiment RS-2306. The dashed line is a fit to the adjusted $\delta t$ data. The first break point is at $x=5.5 \mathrm{~mm}$ and the second break point is at $6.5 \mathrm{~mm}$. Quadratic polynomials have been used for the fits in the second and third zones. 


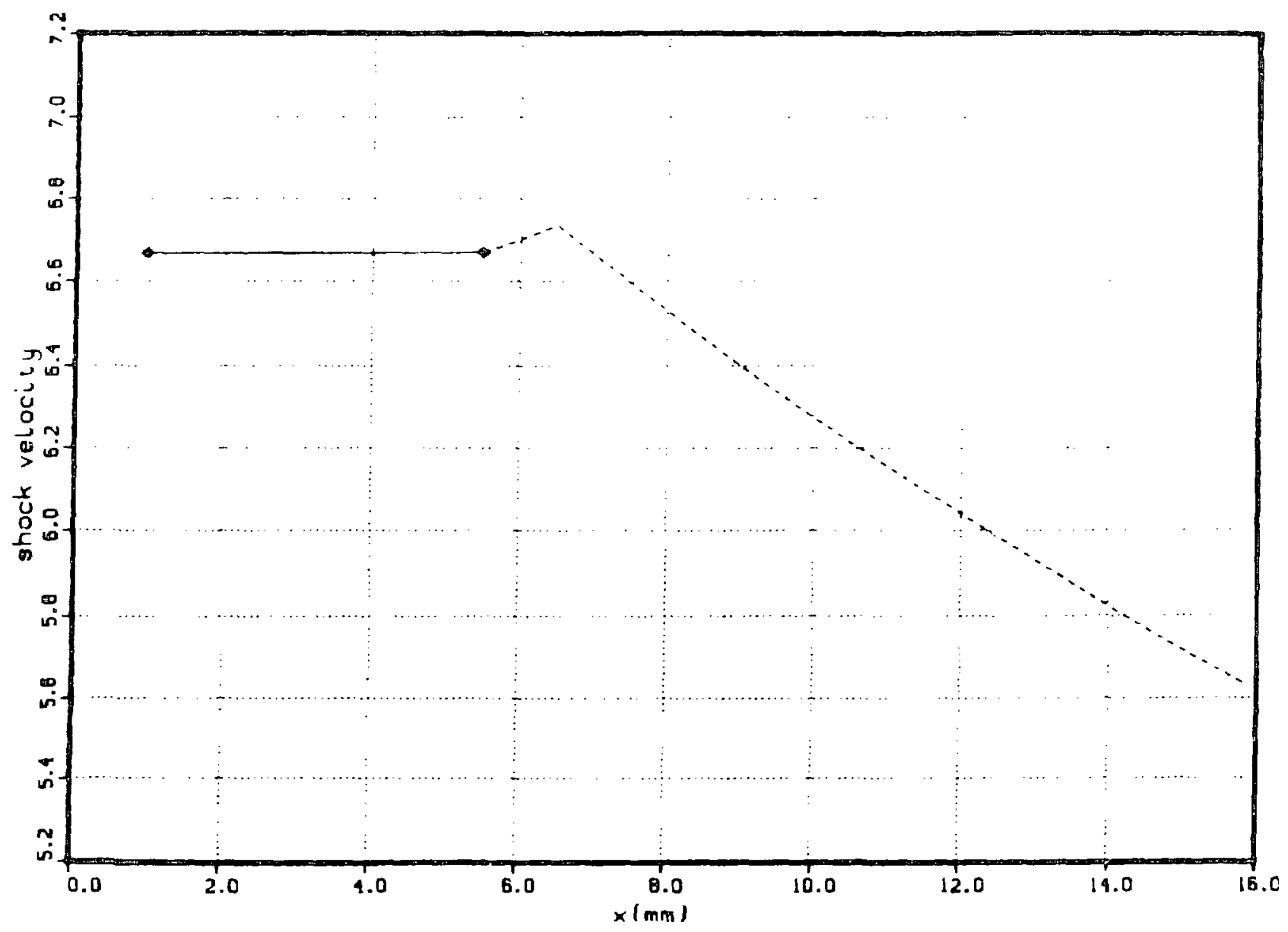

Figure 44: A $U$, versus $x$ plot for RS-2306 taken from the fit to the data shown in figure 43 . 


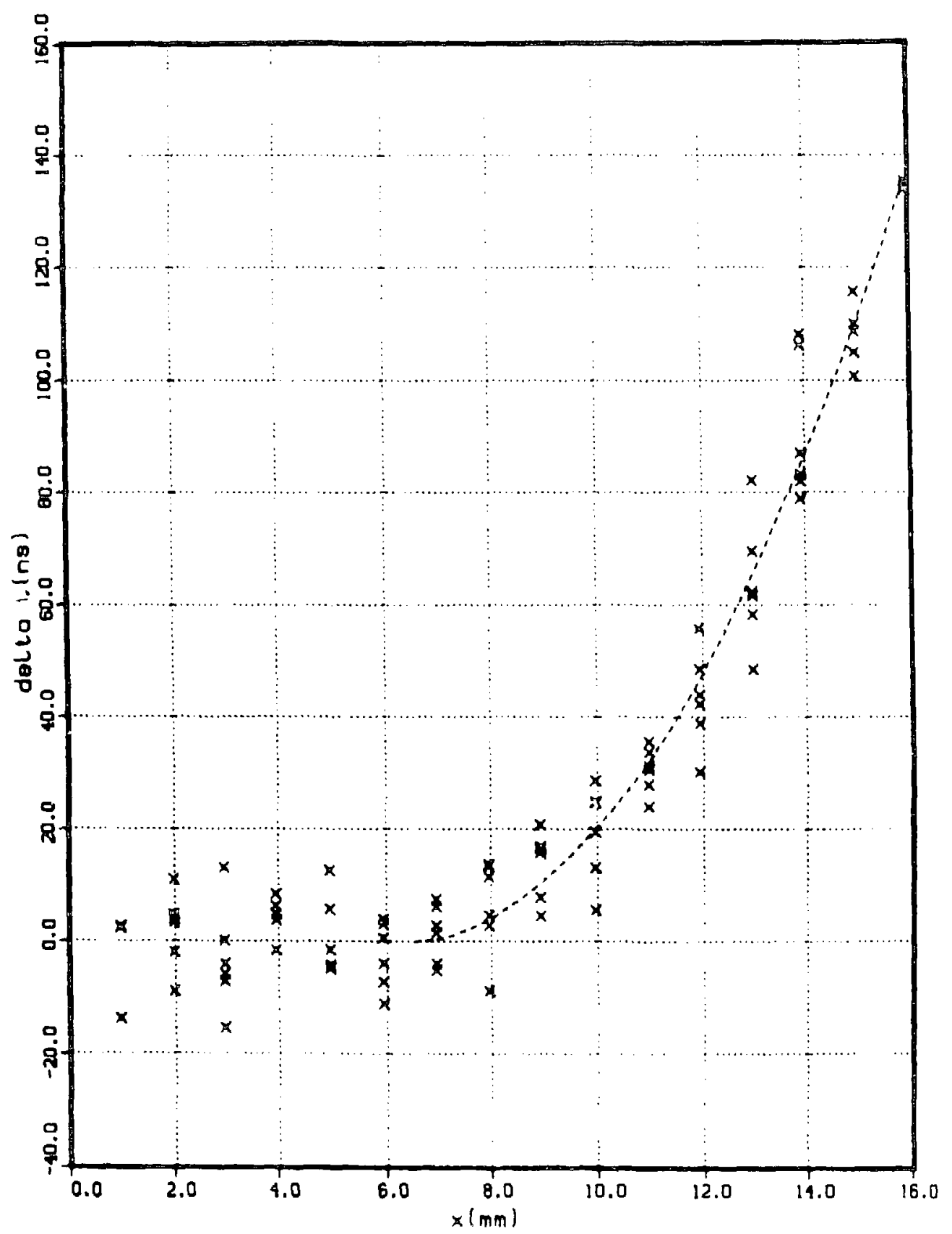

Figure 45: A $\delta t-x$ piot for experiment RS-2306. The dashed line is a fit to the adjusted $\delta t$ data. The first break point is at $x=6.5 \mathrm{~mm}$ and the second break point is at $7.5 \mathrm{~mm}$. Quadratic polynomials have been used for the fits in the second and third zones. 


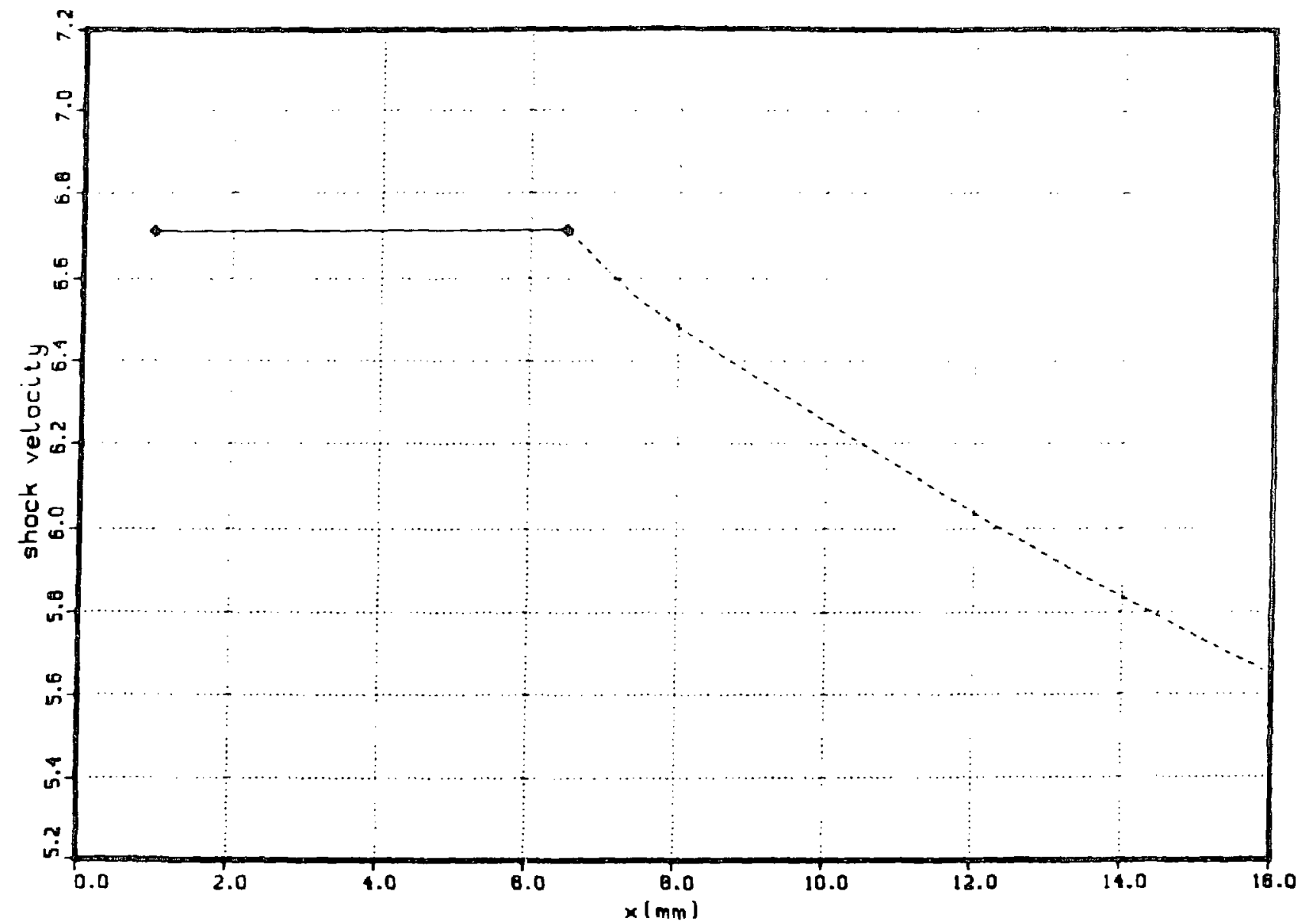

Figure 46: A $U$, versus $x$ plot for RS- ¿306 taken from the fit to the data shown in figure 45 


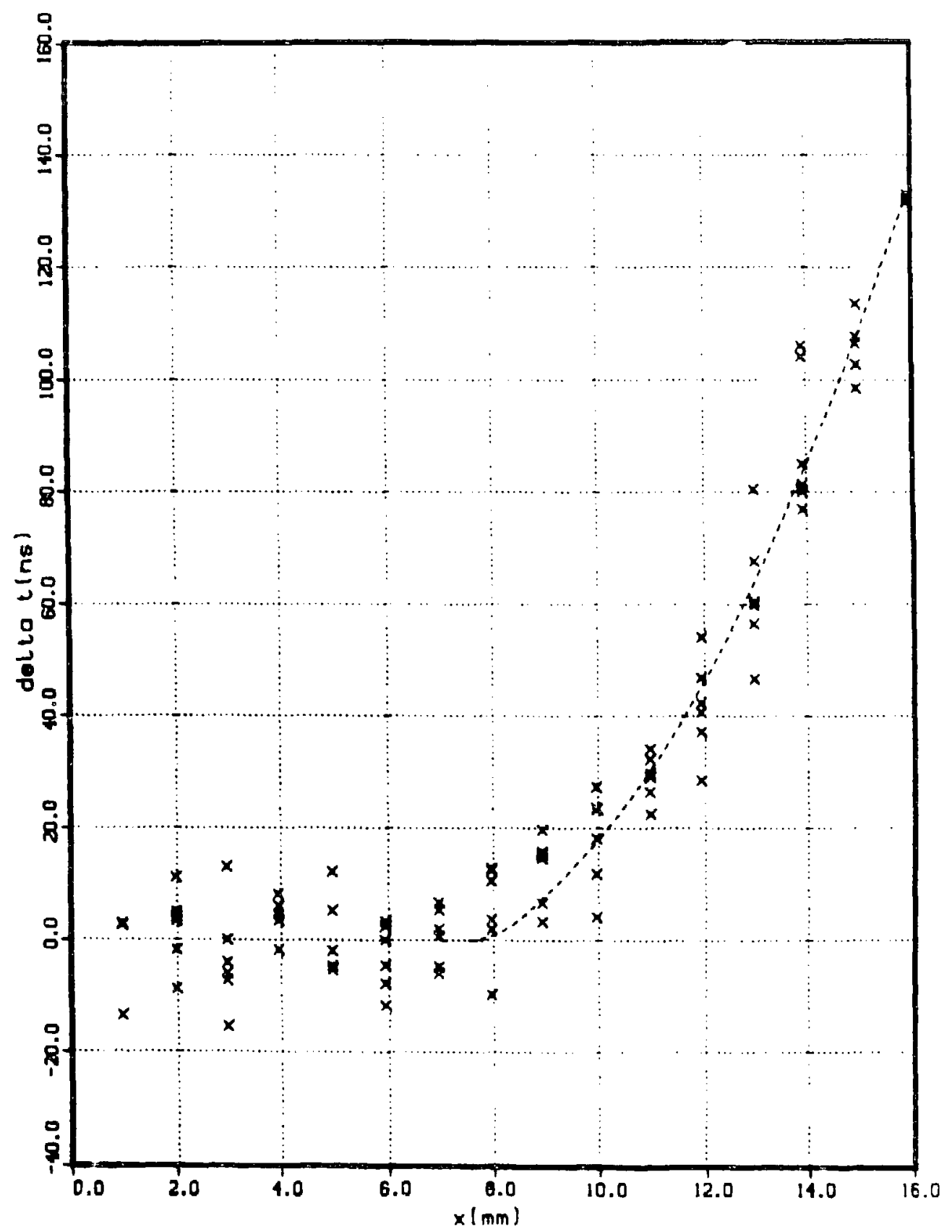

Figure 47: A $\delta t-x$ plot for experiment RS-2306. The dashed line is a fit to the adjusted $\delta t$ data. The first break point is at $x=7.5 \mathrm{~mm}$ and the second break point is at $8.5 \mathrm{~mm}$. Quadratic polynomials have been used for the fits in the second and third zones. 


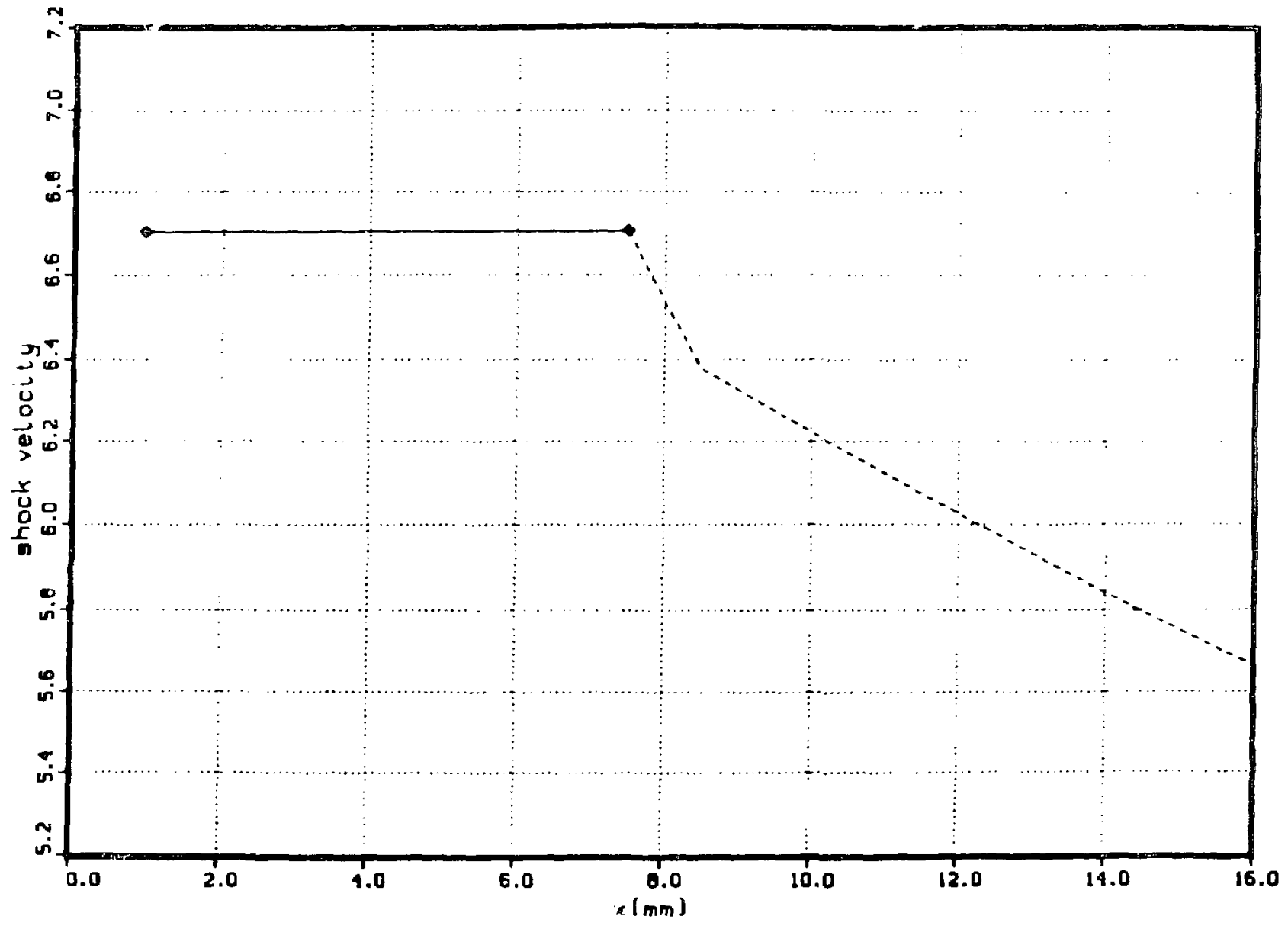

Figure 48: $A U$, versus $x$ plot for RS-2306 taken from the fit to the data shown in 6 gure 47 . 


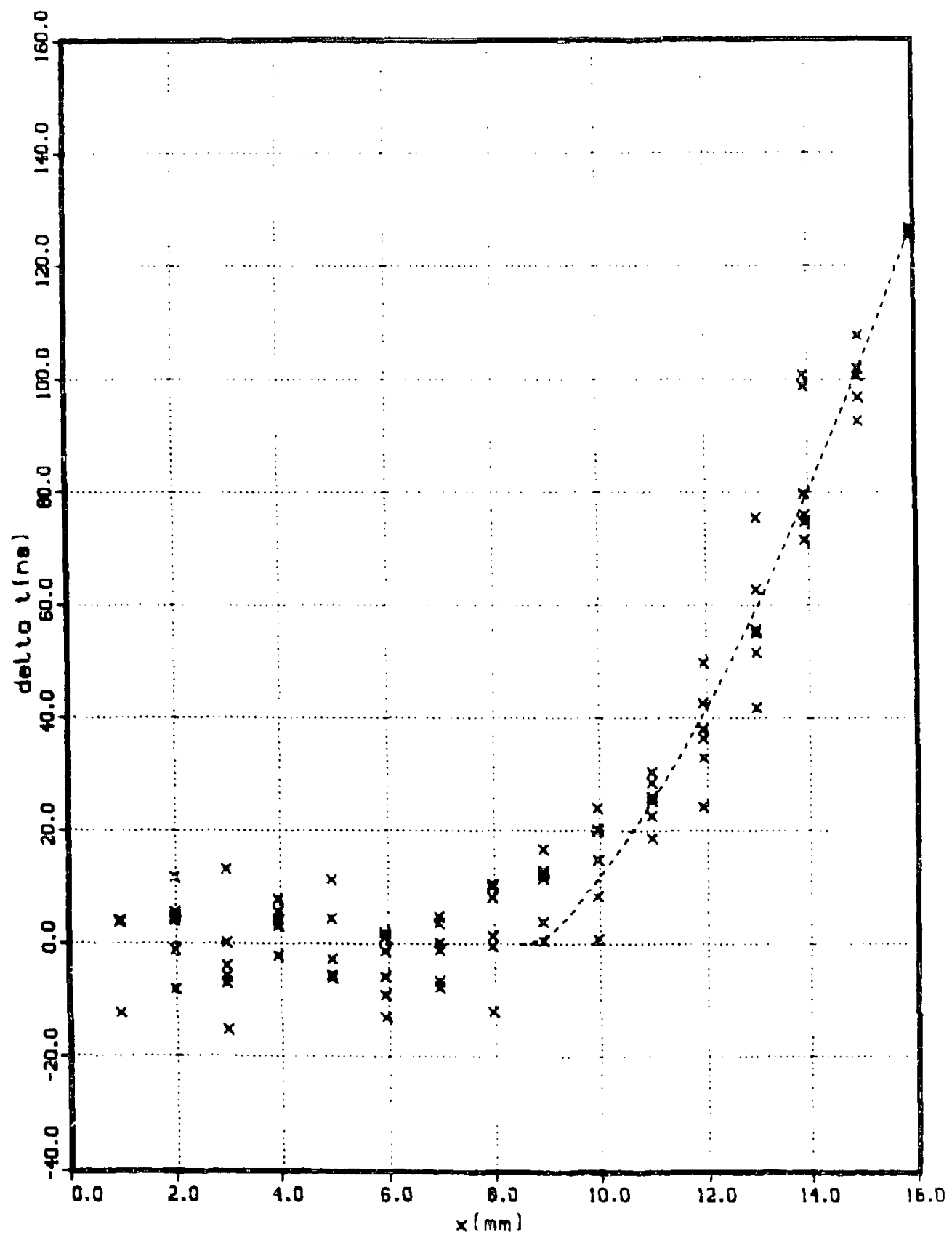

Figure 49: A $\delta t-x$ plot for experiment RS-2306. The dashed line is a fit to the adjusted $5 t$ data. The first break point is at $x=8.5 \mathrm{~mm}$ and the second break point is at $9.5 \mathrm{~mm}$. Quadratic polynomials have been used for the fits in the second and third zones. 


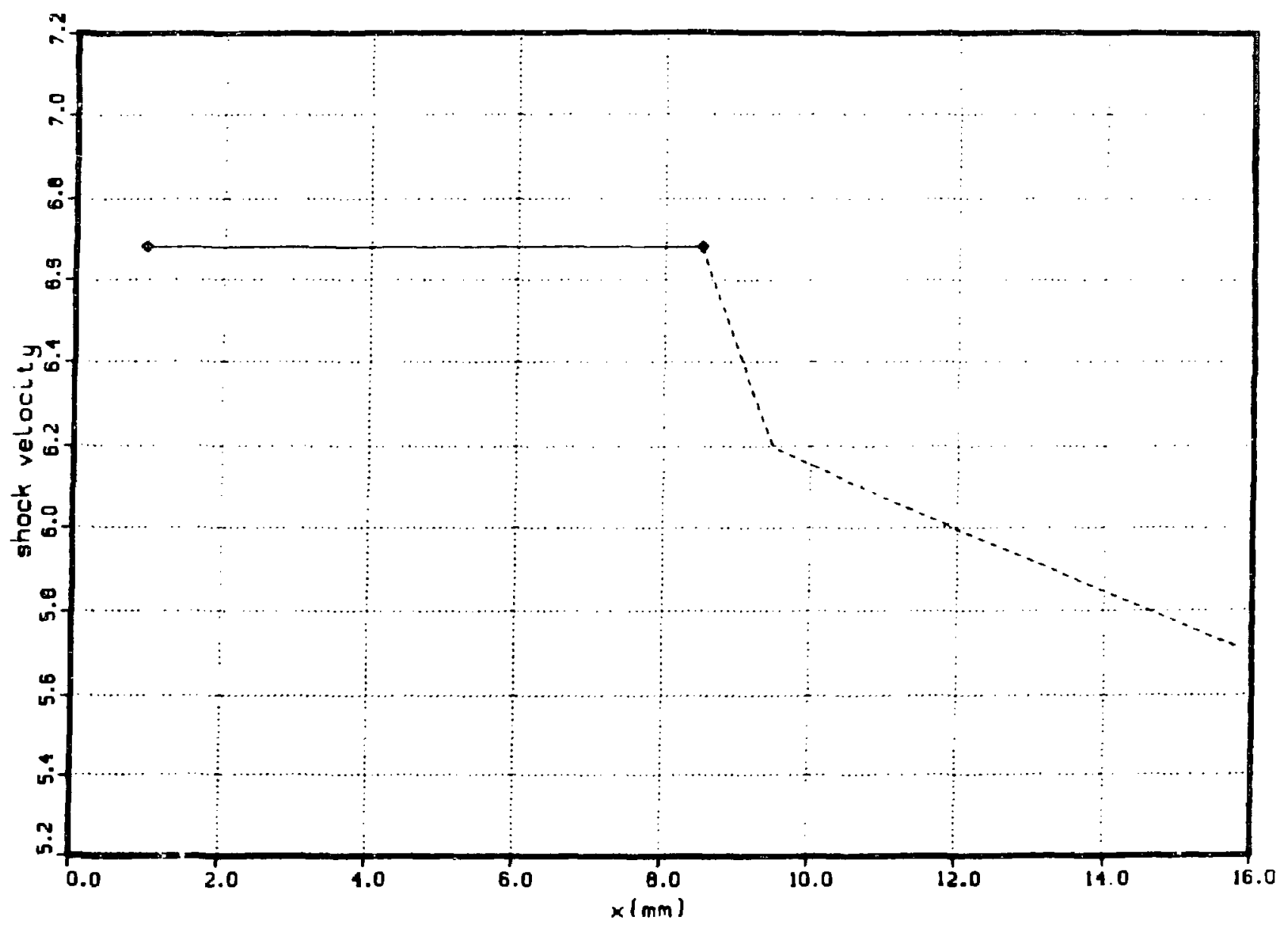

亩

Figure 50: A $U$, versus $x$ plot for RS-2306 taken from the fit to the data shown in figure 49 . 


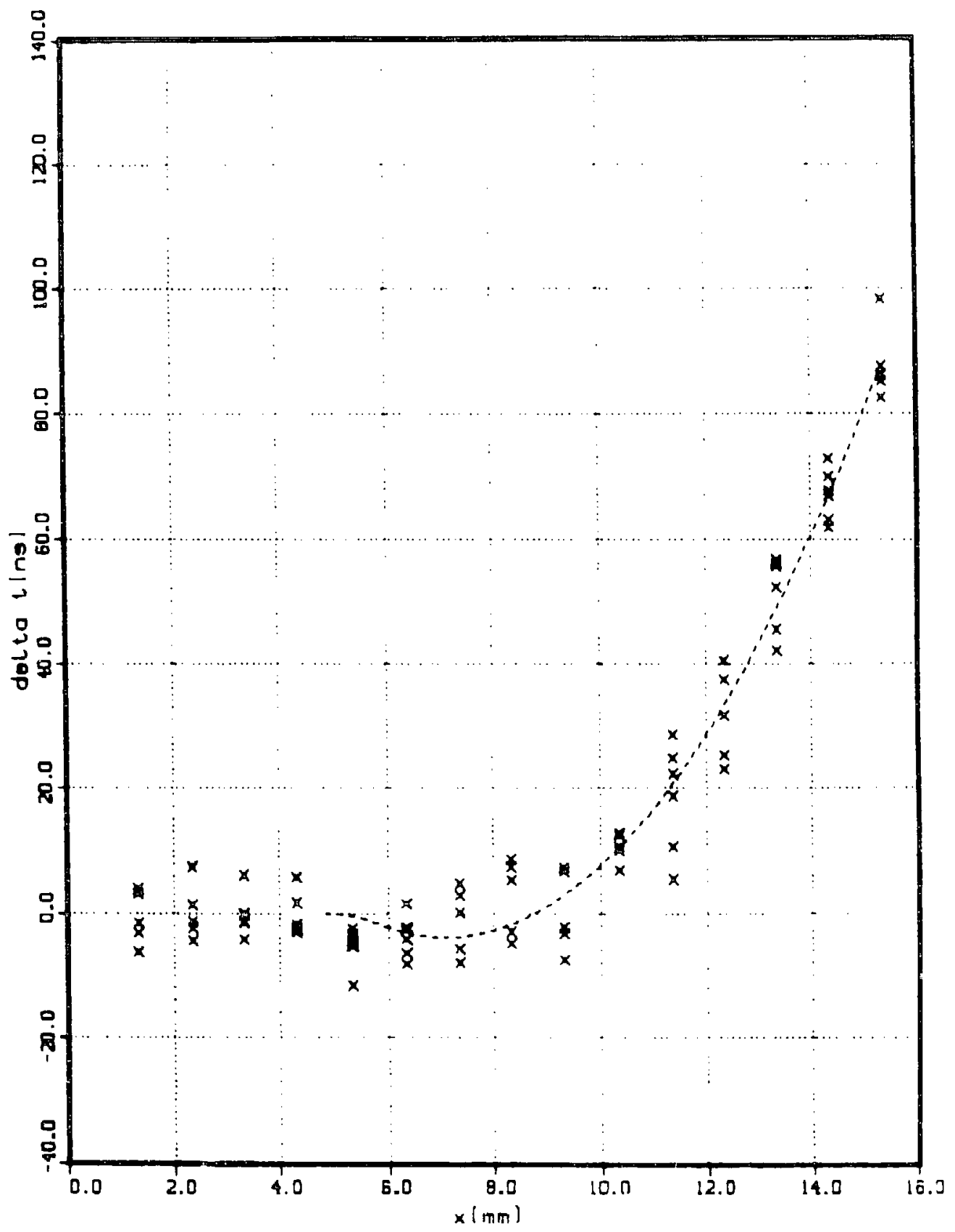

Figure 51: A $\delta t-x$ plot for experiment RS-2308. The dashed line is a fit to the adjusted $\delta t$ data. The first break point is at $x=4.8 \mathrm{~mm}$ and the second break point is at $5.8 \mathrm{~mm}$. Quadratic polynomials have been used for the fits in the second and third zones. 


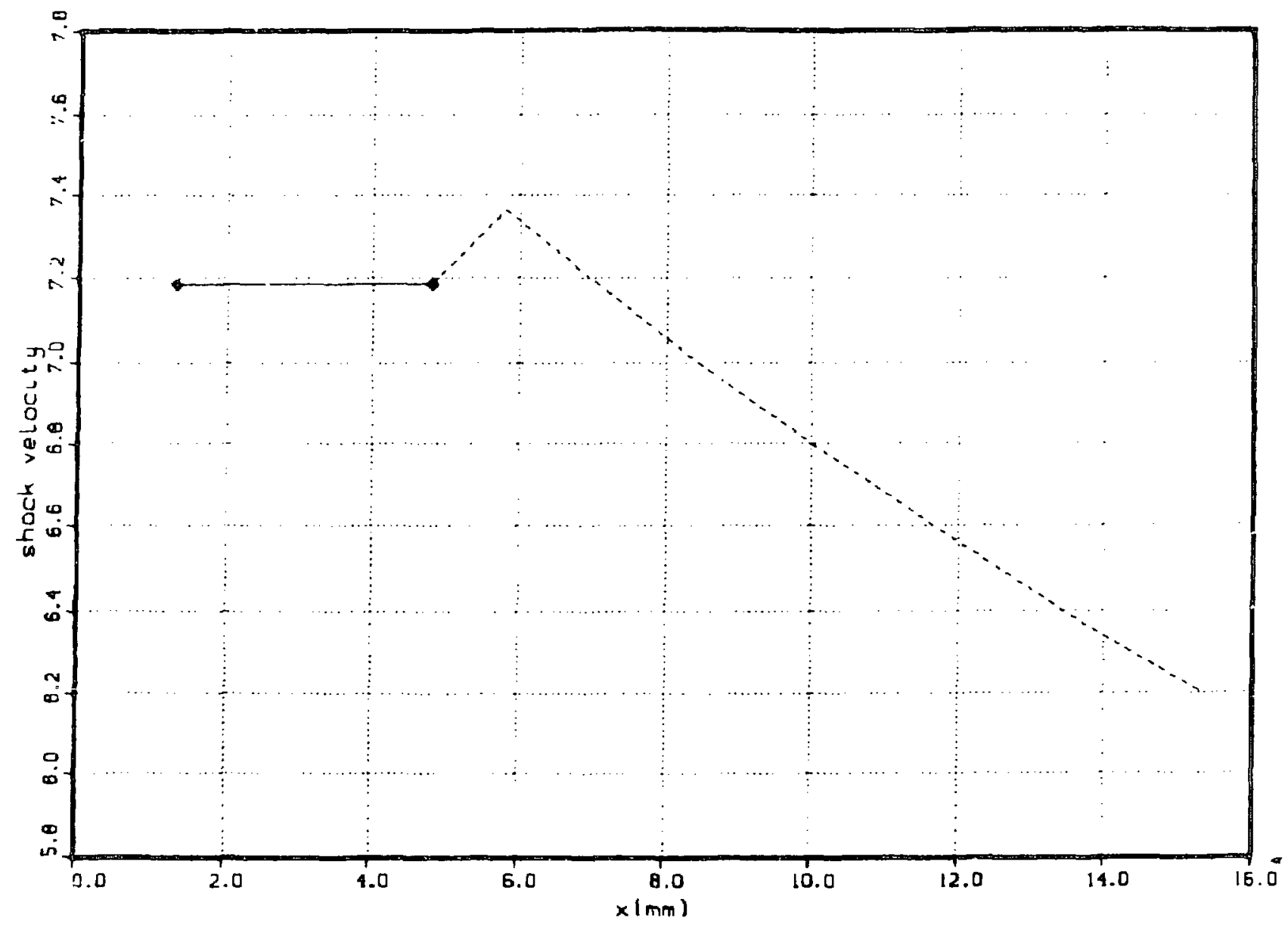

Figure 52: A $U$, versus $x$ plot for RS-2308 taken from the fit to the data shown in figure 51 . 


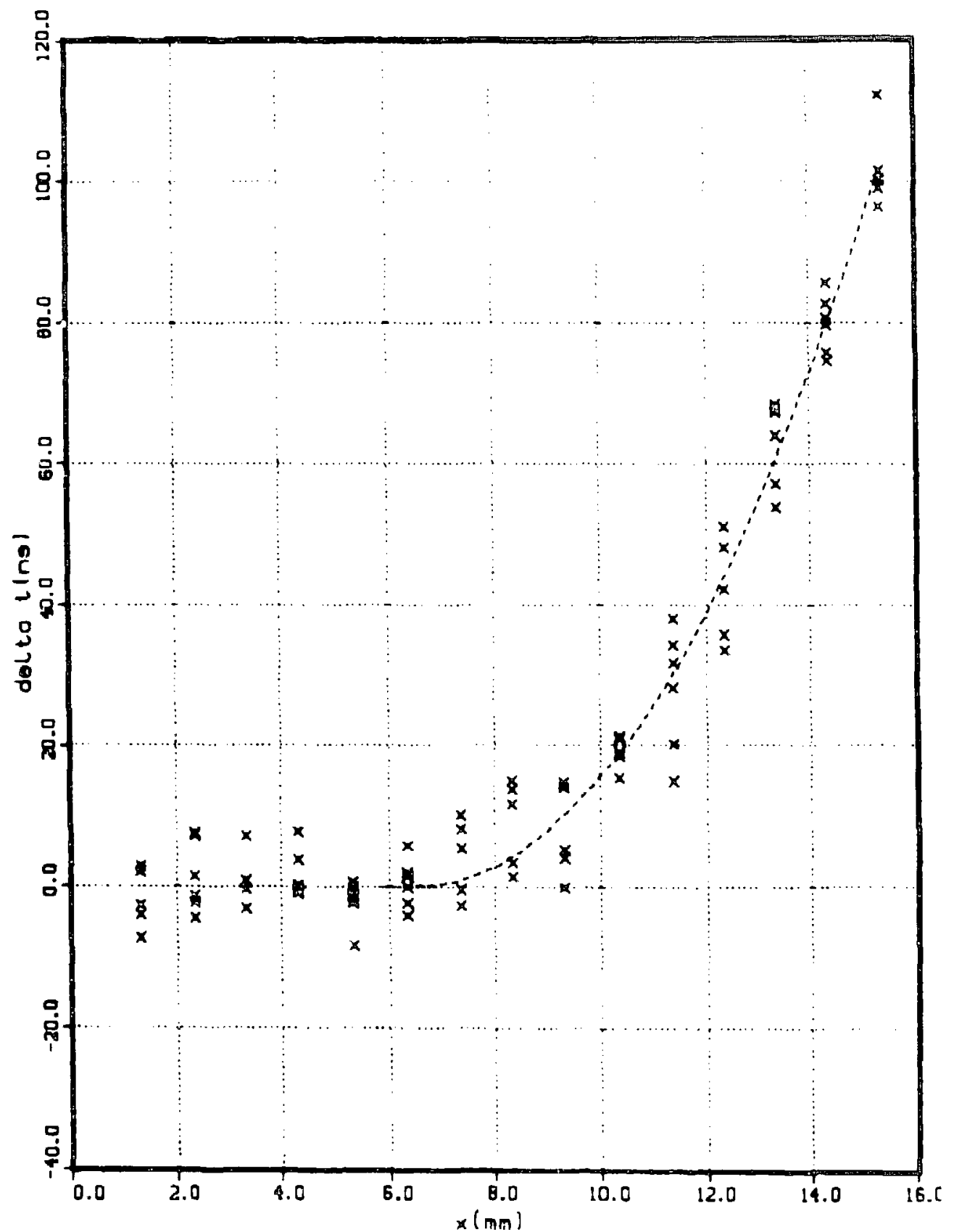

Figure 53: A $\delta t-x$ plot for experiment RS-2308. The dashed line is a fit to the adjusted $\delta t$ data. The first break point is at $\pi=5.8 \mathrm{~mm}$ and the second break point is at $6.8 \mathrm{~mm}$. Quadratic polynomials have been used for the fits in the second and third zones. 


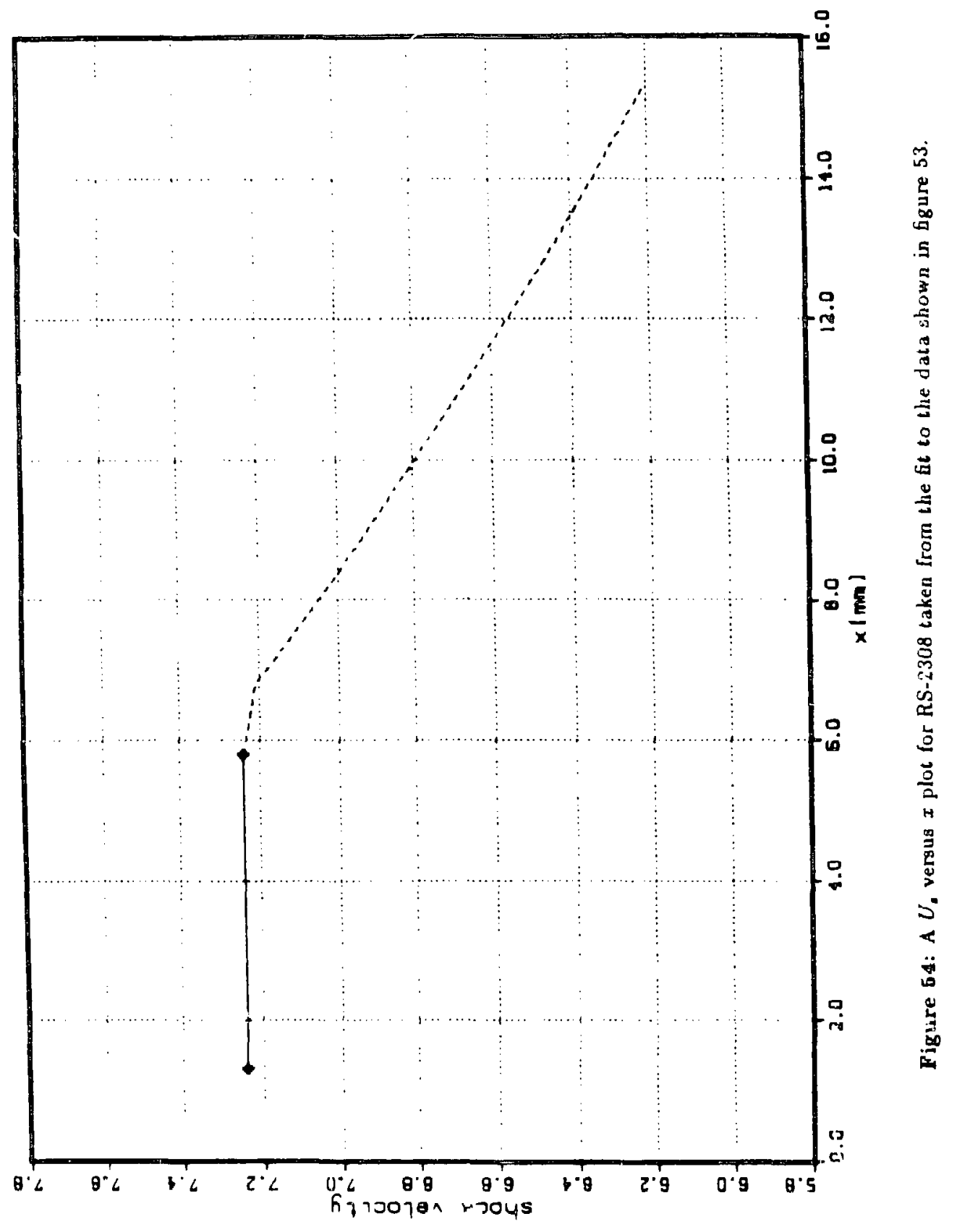




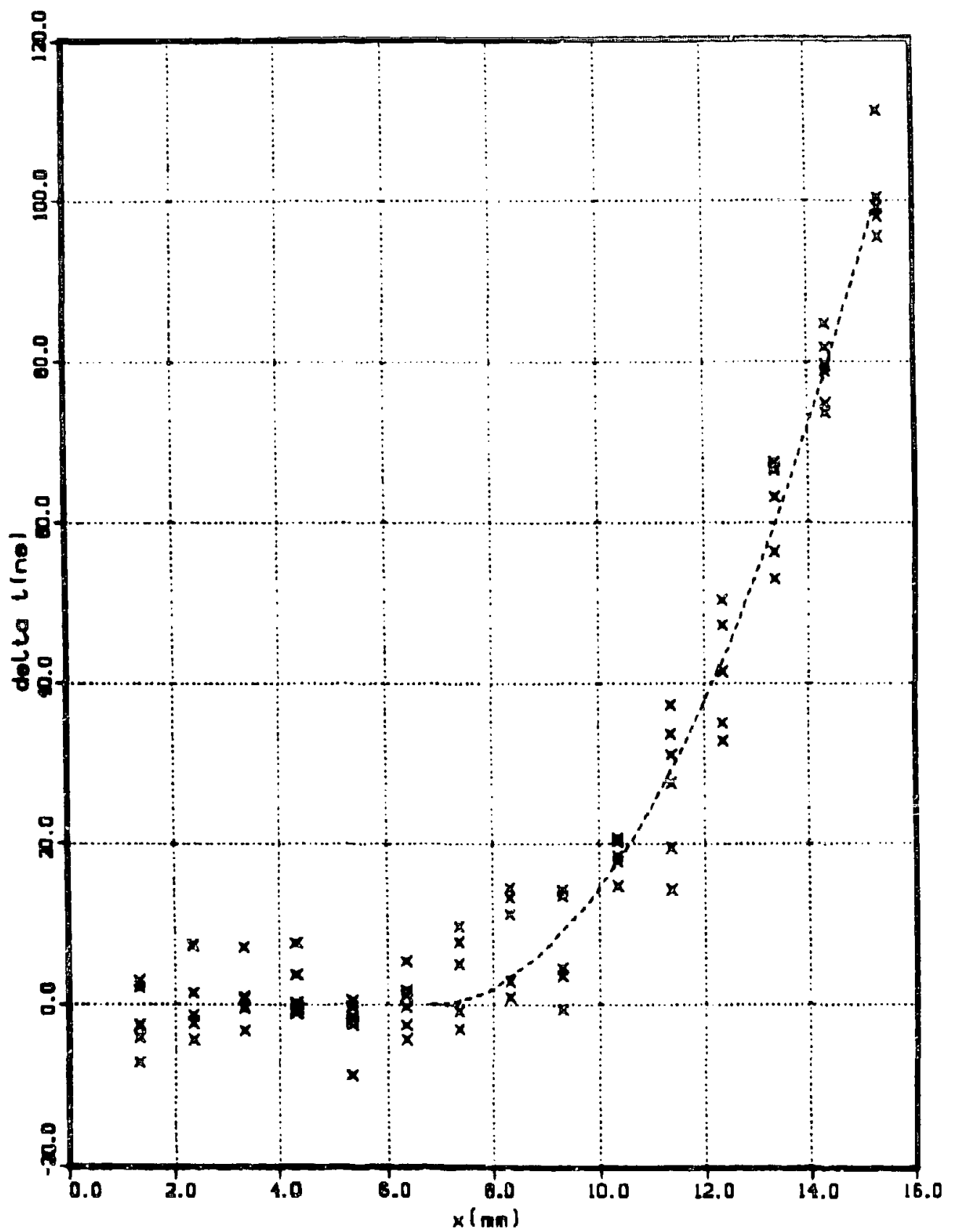

Figure 55: A $\delta t-x$ plot for experiment RS-2308. The dashed line is a fit to the adjugted $\delta t$ data. The 6 rst break poin: is at $x=6.8 \mathrm{~mm}$ and the second break point is at $7.8 \mathrm{~mm}$. Quadratic polynomials have been used for the fits in the second and third zones. 


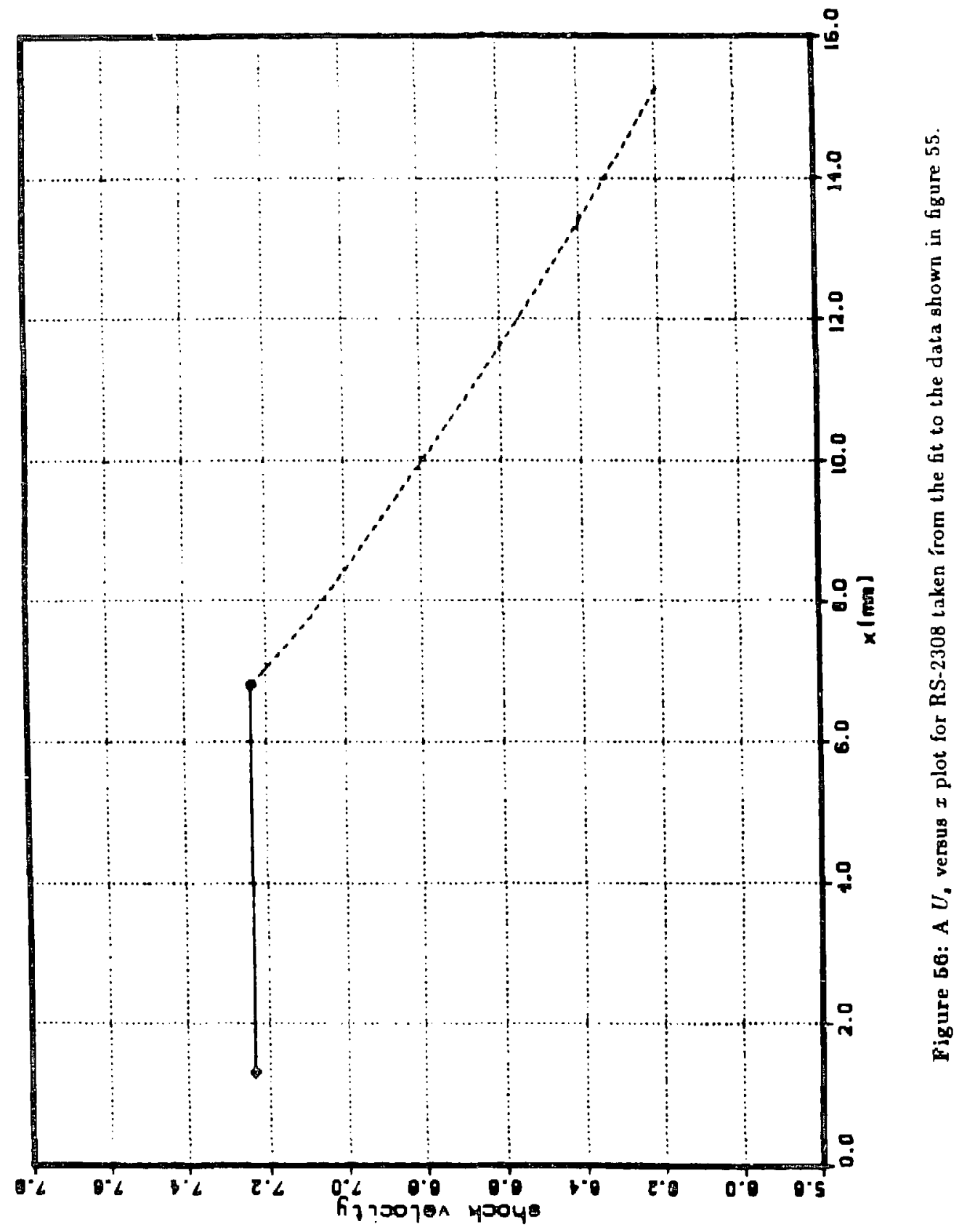




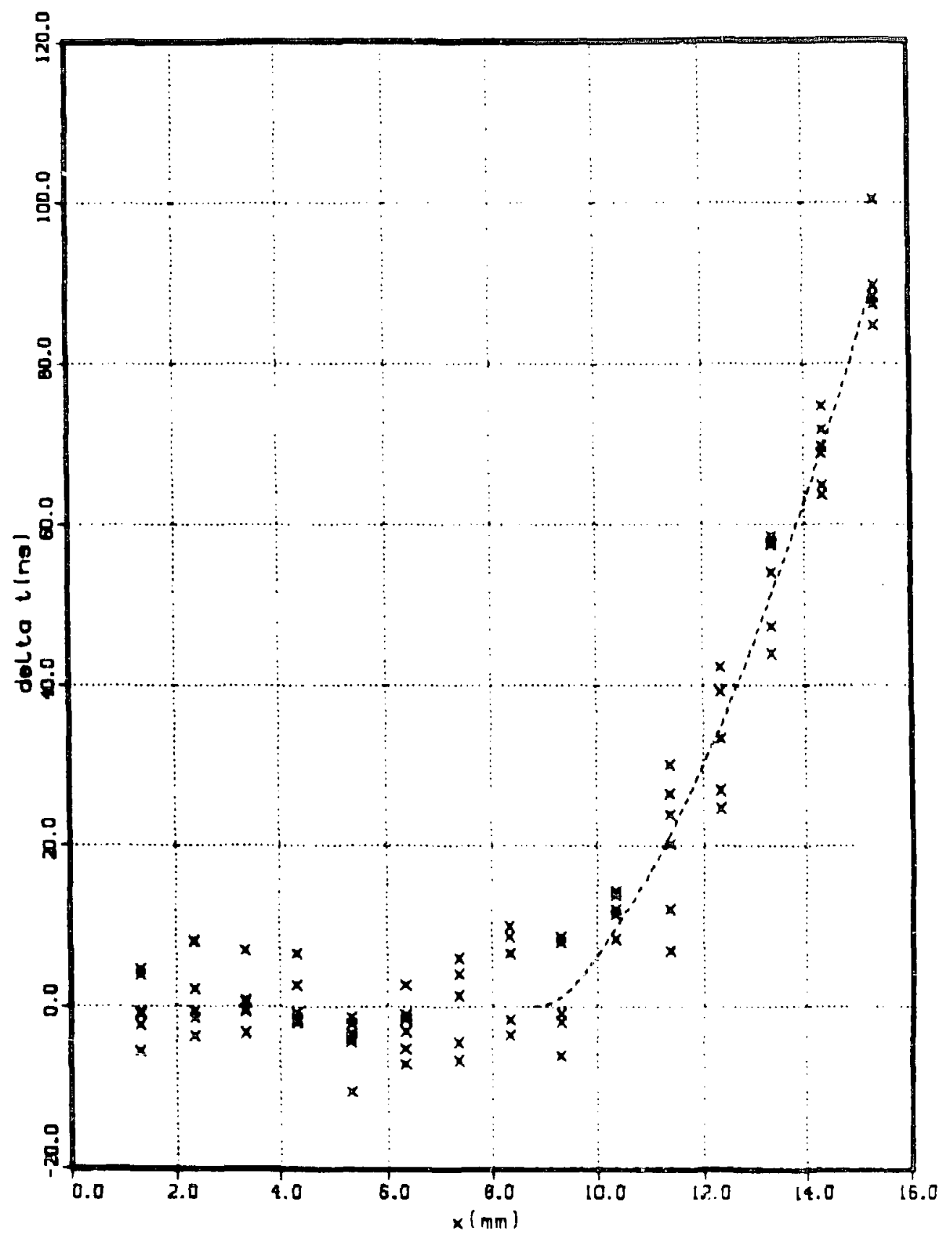

Figure 57: A $\delta t-x$ plot for experiment RS-2308. The dashed line is a fit to the adjusted $\delta t$ data. The first break point is at $x=8.8 \mathrm{~mm}$ and the second break point is at $9.8 \mathrm{~mm}$. Quadratic polynomials have been used for the fits in the second and third zones. 


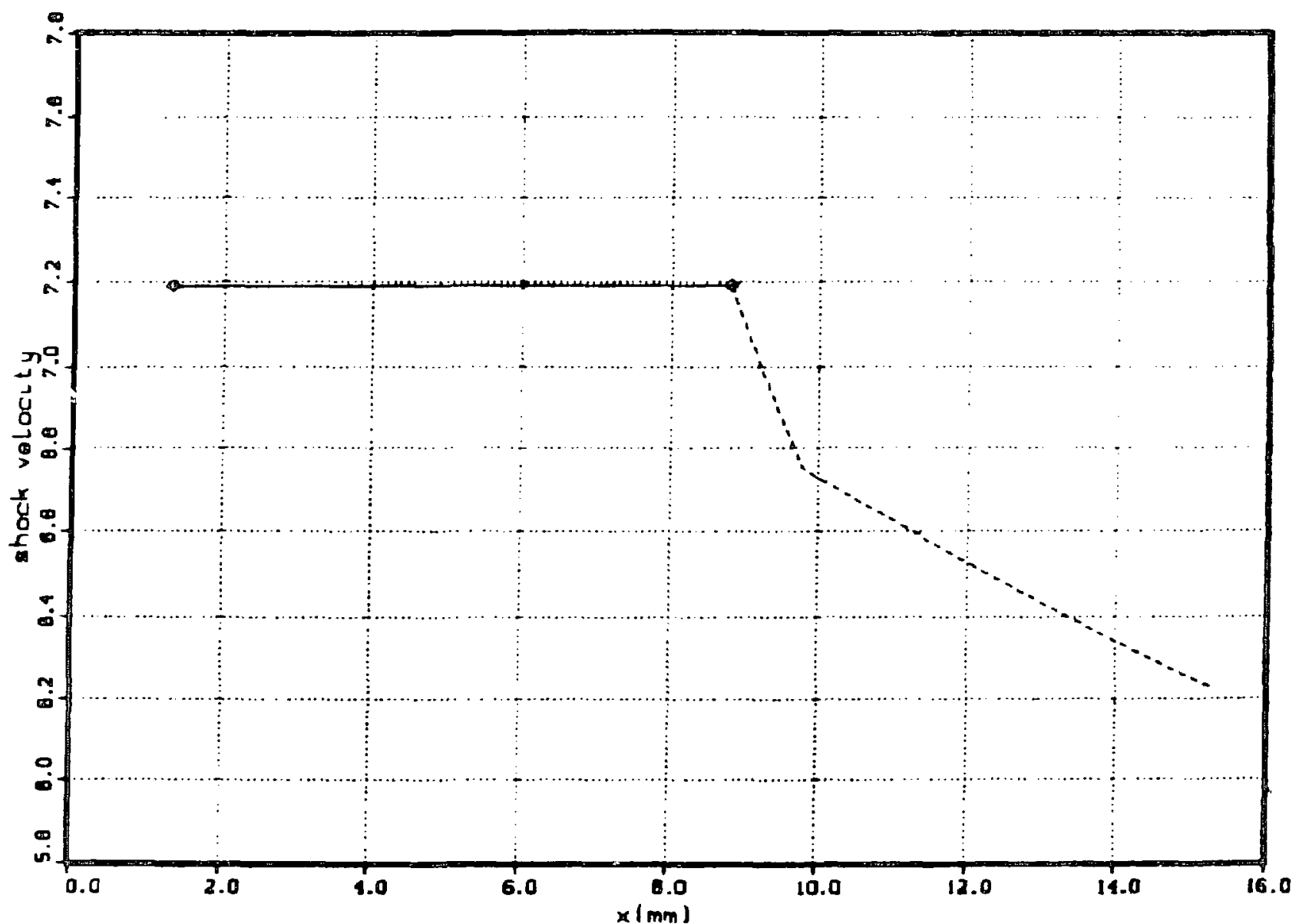

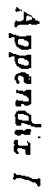

Figure 68: A $U$, versus $x$ plot for RS-2308 taken from the fit to the data ghown in figure 57 


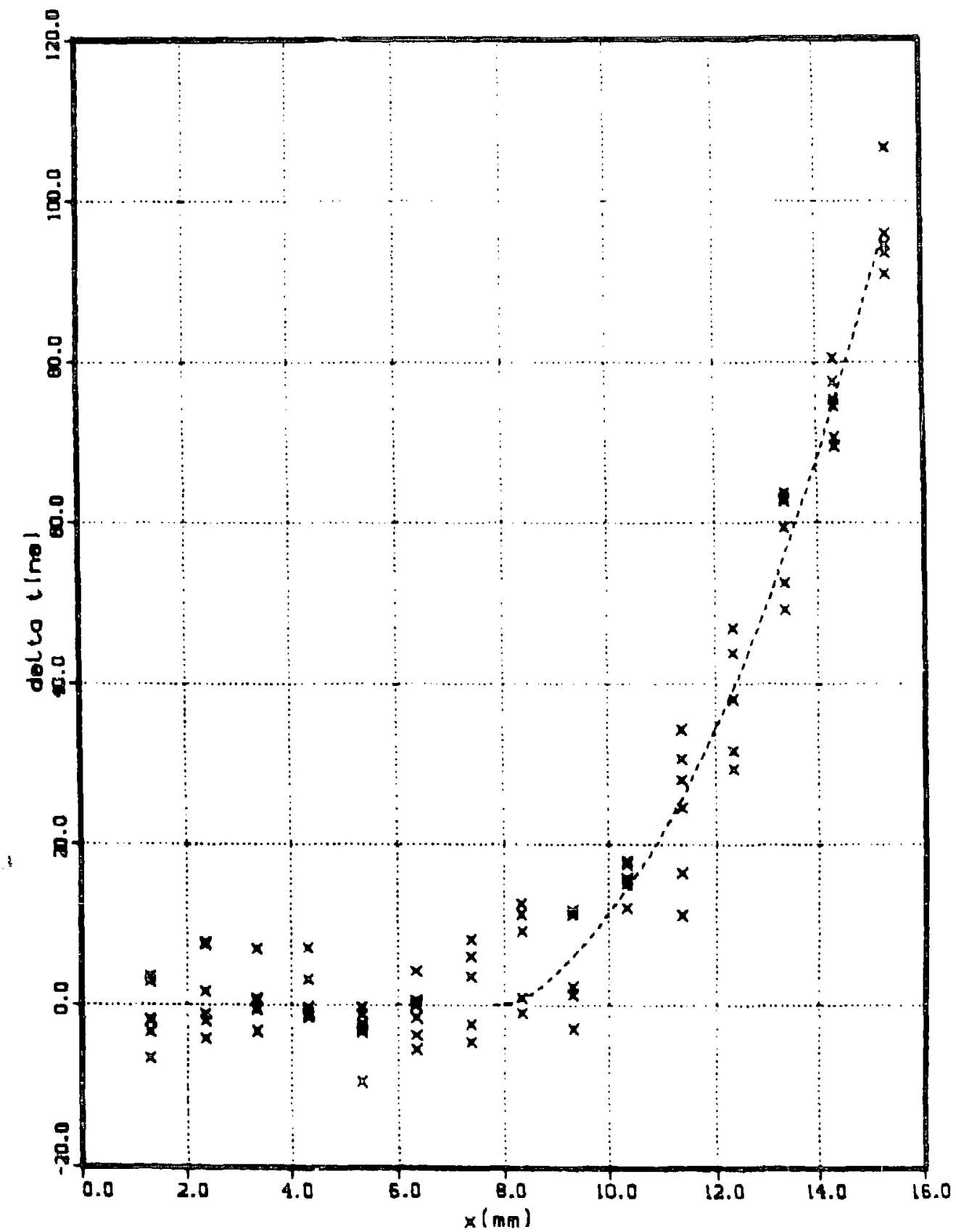

Figure 58: A $\delta t-x$ plot for experiment RS-2308. The dushed line is a fit to the adjusted $\delta t$ data. The first break point is at $x=7.8 \mathrm{~mm}$ and the second break point is at $8.8 \mathrm{~mm}$. Quadratic polynomials have been used for the fits in the second and third zones. 


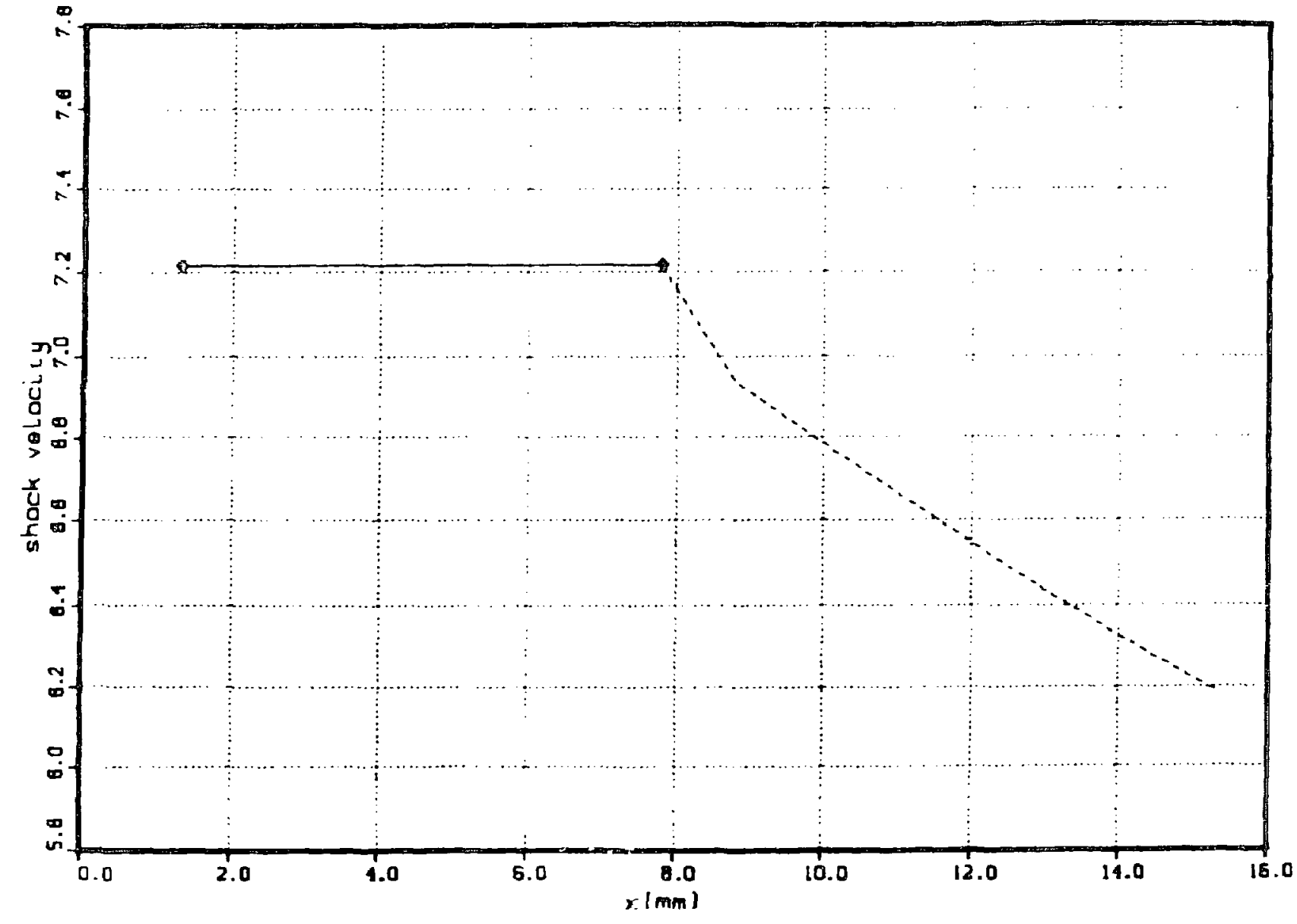

Figure 60: A $U$, versus $x$ plet for $[\mathrm{SS}-2308$ taken from the fit to the data shown in fgure 59 . 


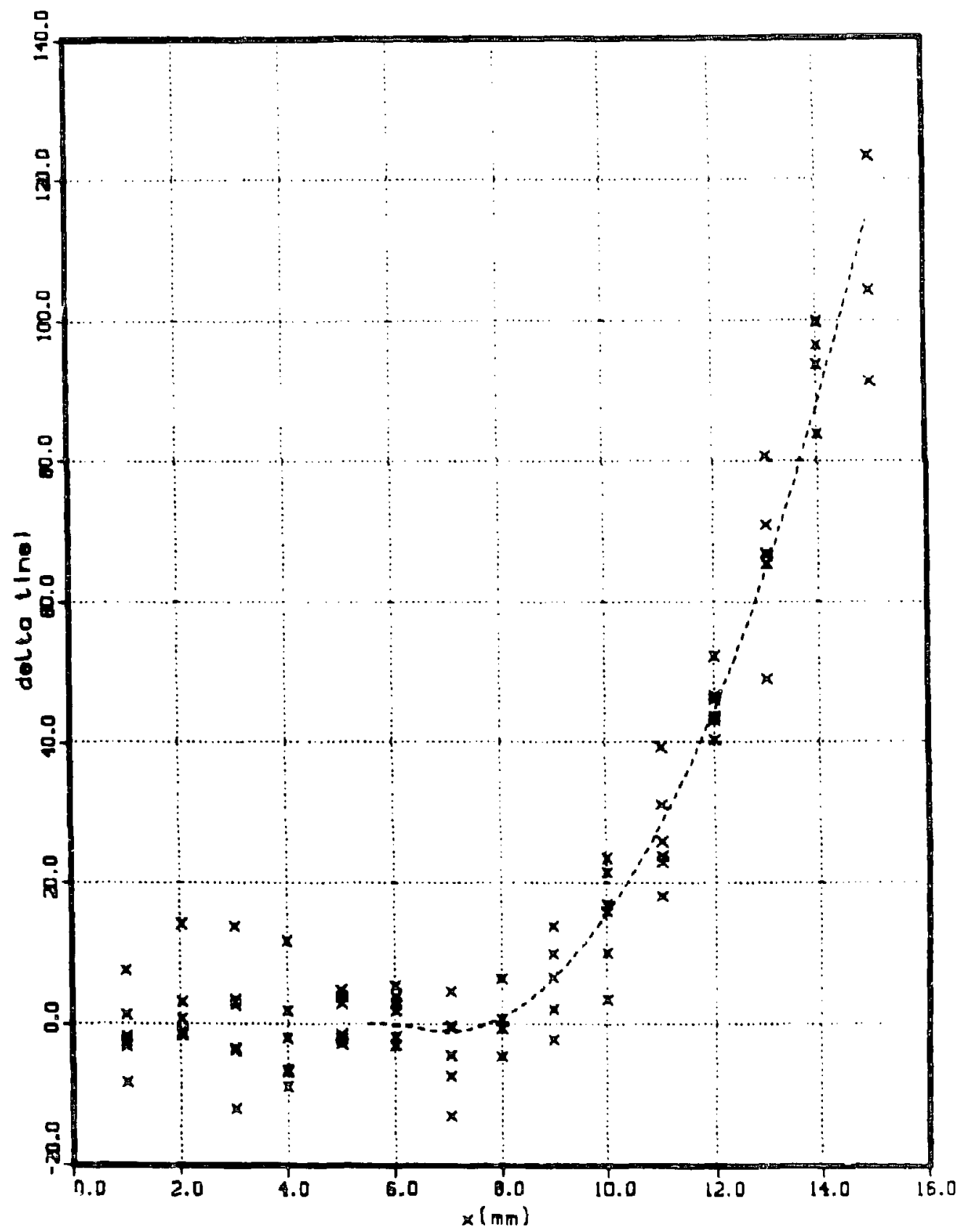

Figure 61: A $\delta t-x$ plot for experiment RS-2314. The dashed line is a fit to the adjusted $\delta t$ data. The first break point is at $x=5.5 \mathrm{~mm}$ and the second break point is at $6.5 \mathrm{~mm}$. Quadratic polynomiaic iave been used for the fits in the second and third zones. 


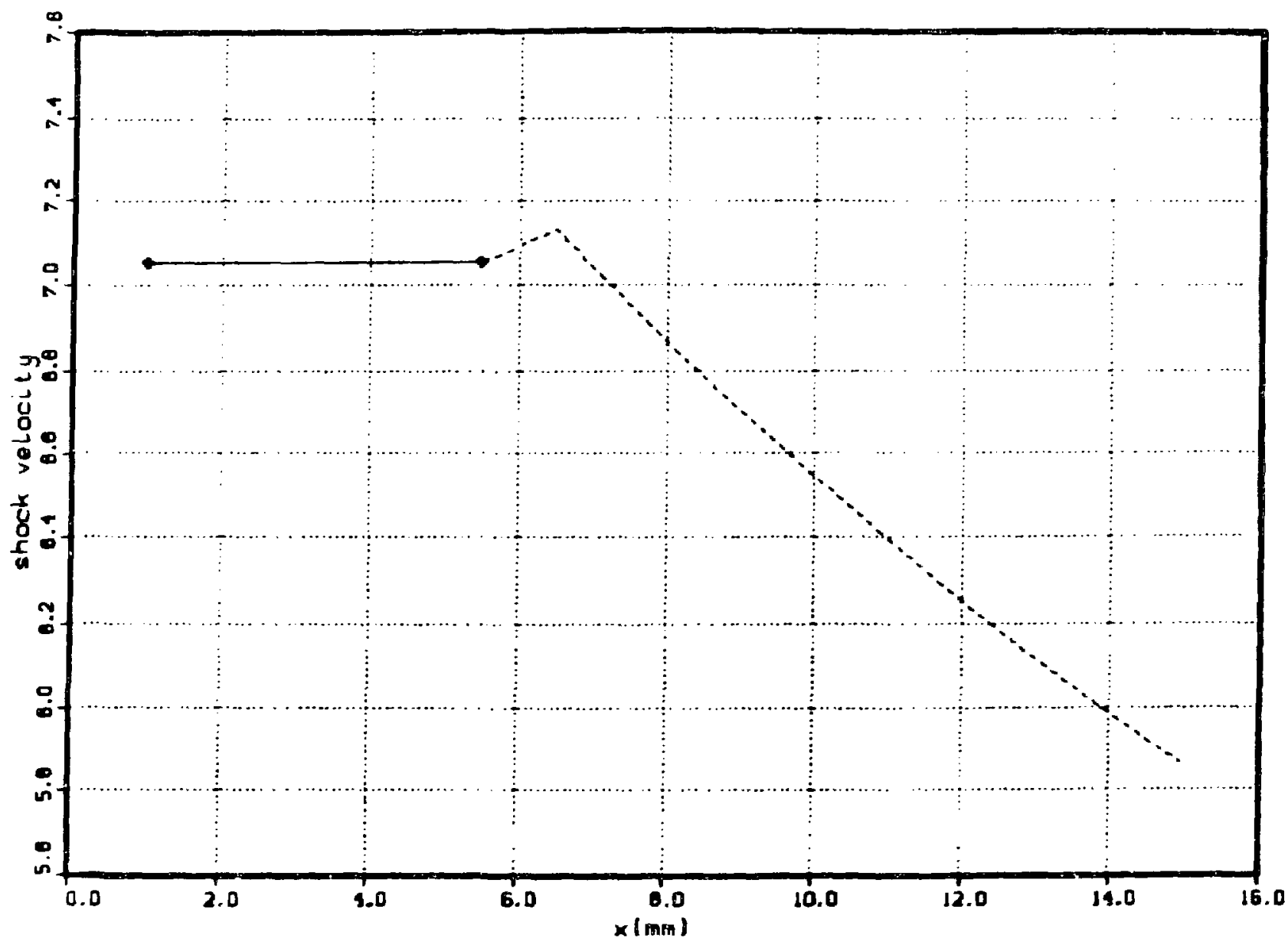

Figure 52: A $U$, versus $x$ plot for RS-2314 taken from the fit to the data shown in figure 61 


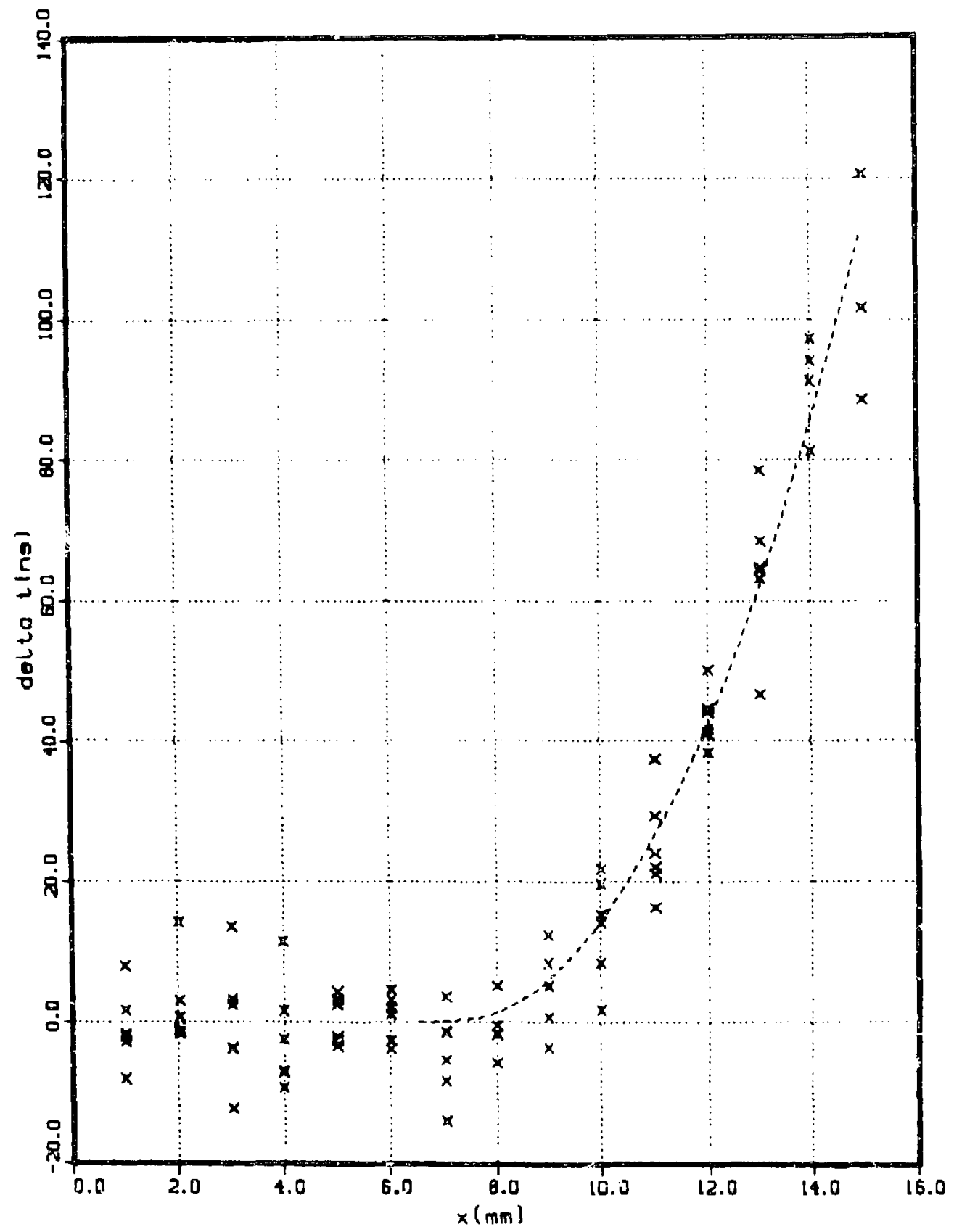

Figure BS. A $\delta t-x$ plot for experiment RS-2314. The dashed lire is a fit to the adjusted $\delta t$ data. The first breax point is at $x=6 . E \mathrm{~mm}$ and the second break point is at $7.5 \mathrm{~mm}$. Quadiatic polynomials have been used for the fits in the second and third zones. 


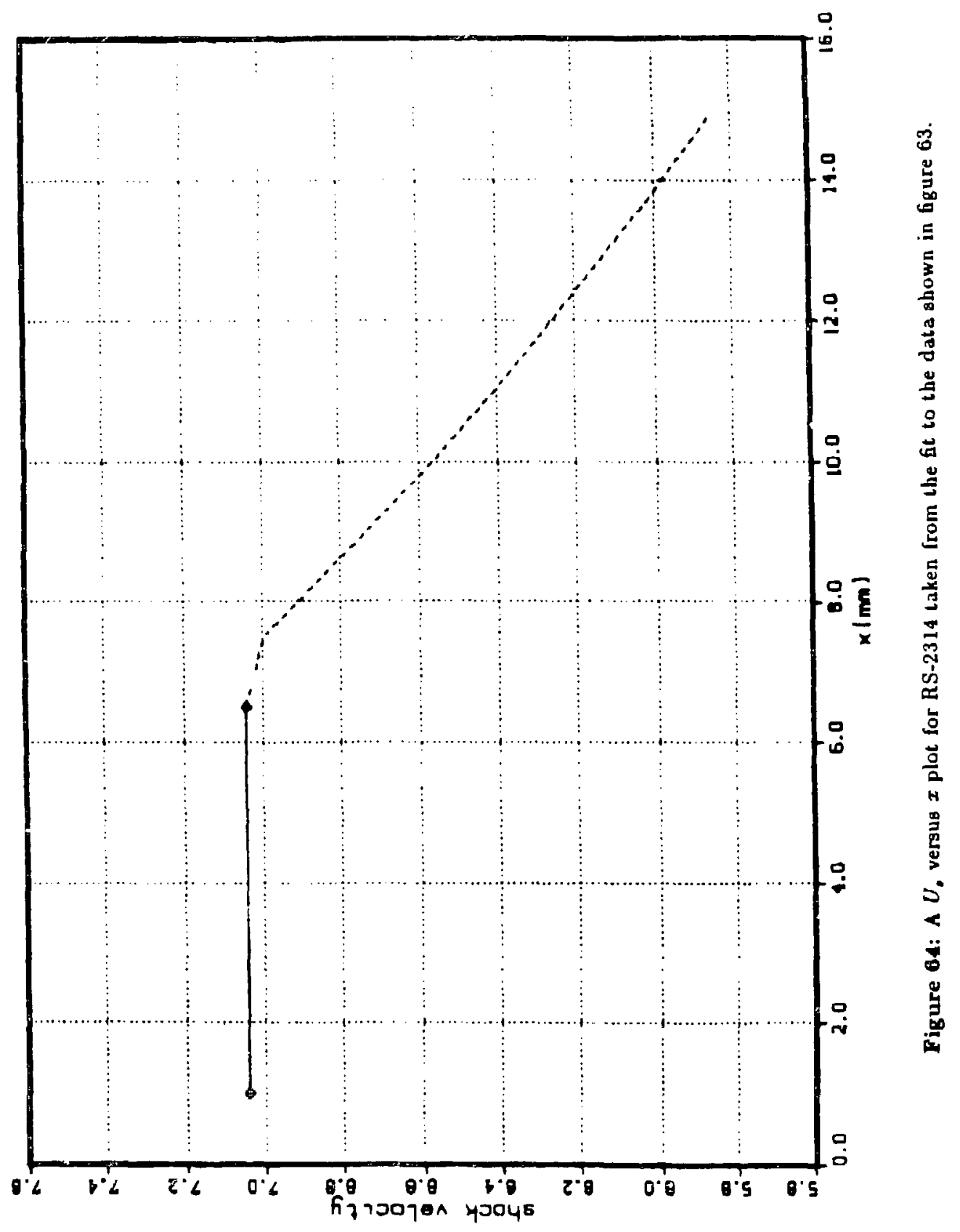




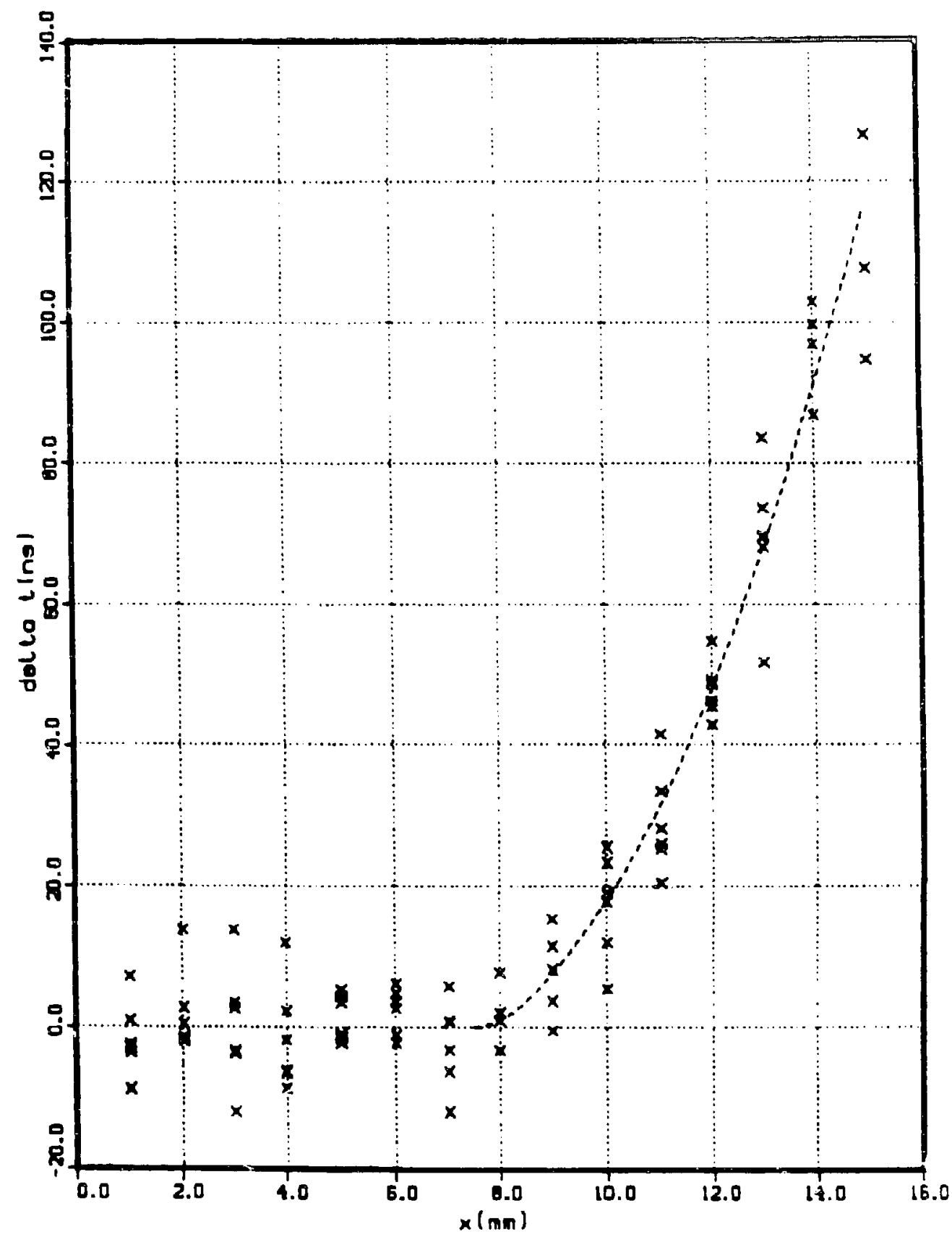

Figure 65: A $\delta t-x$ plot for experiment RS-2314. The dashed line is a fit to the adjusted $\delta t$ data. The first break poiat is at $x=7.5 \mathrm{~mm}$ and the second break point is at $8.5 \mathrm{~mm}$. Quadratic polynomials have been used for the fits in the second and third zones. 


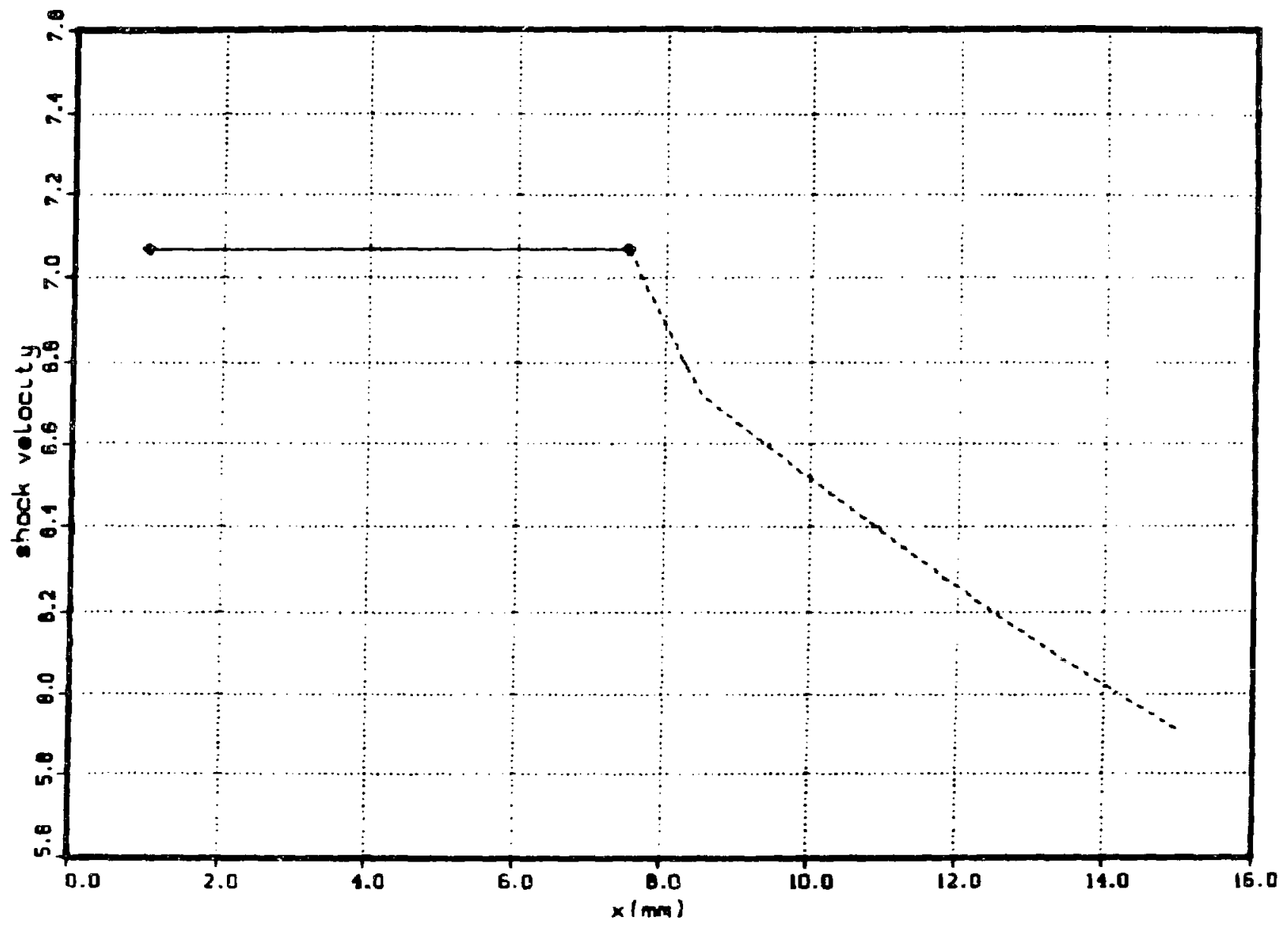

Figure 66: A $U$, versus $x$ plot for RS-2314 taken from the fit to the data shown in figure 65. 


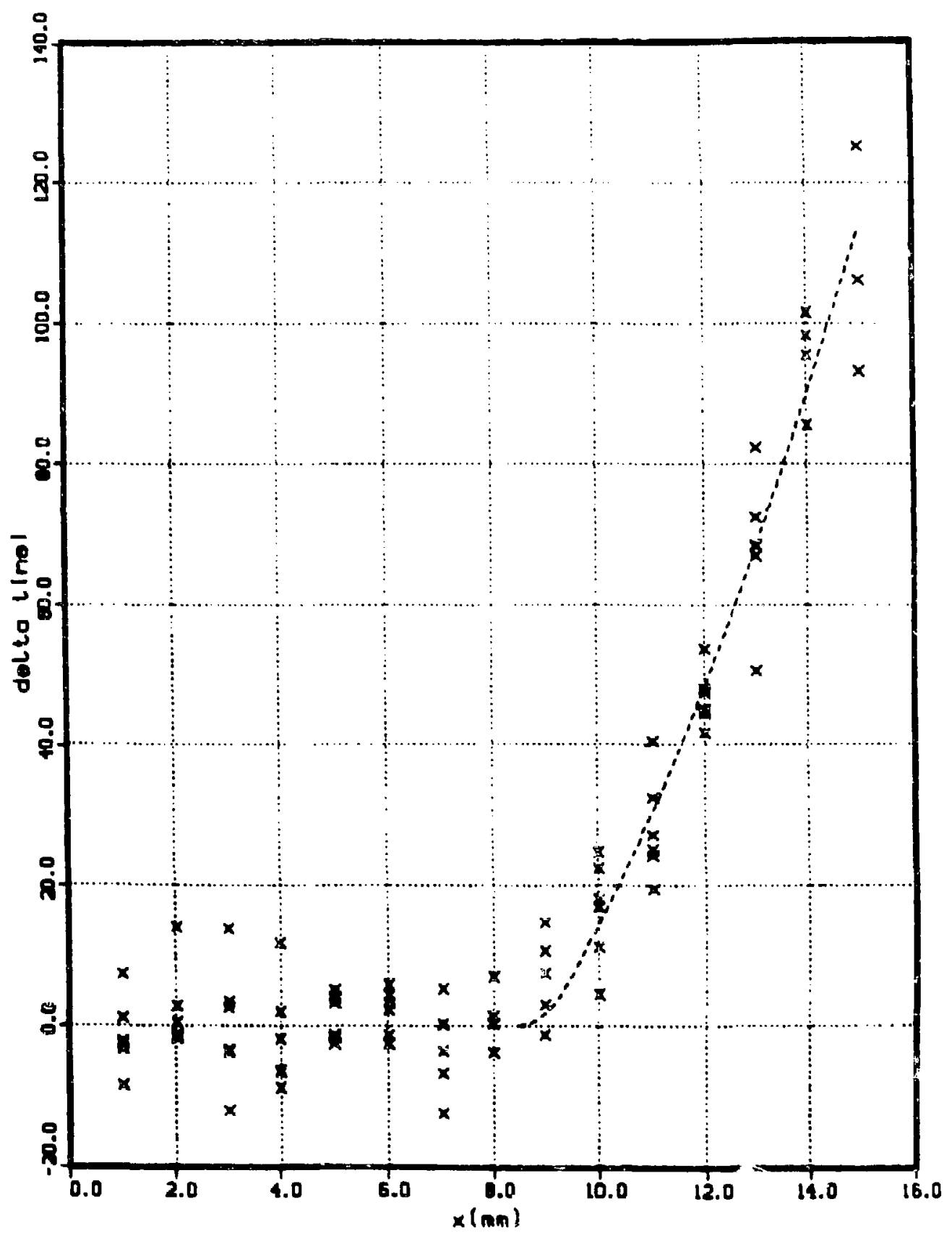

Figure 67: A $\delta t-x$ plot for experiment RS-2314. The dashed line is a fit to the adjusied $6 t$ data. The first break point is at $x=8.5 \mathrm{~mm}$ and the second break point is at $9.5 \mathrm{~mm}$. Quadratic polynomials have been used for the fits in the second and third zones. 


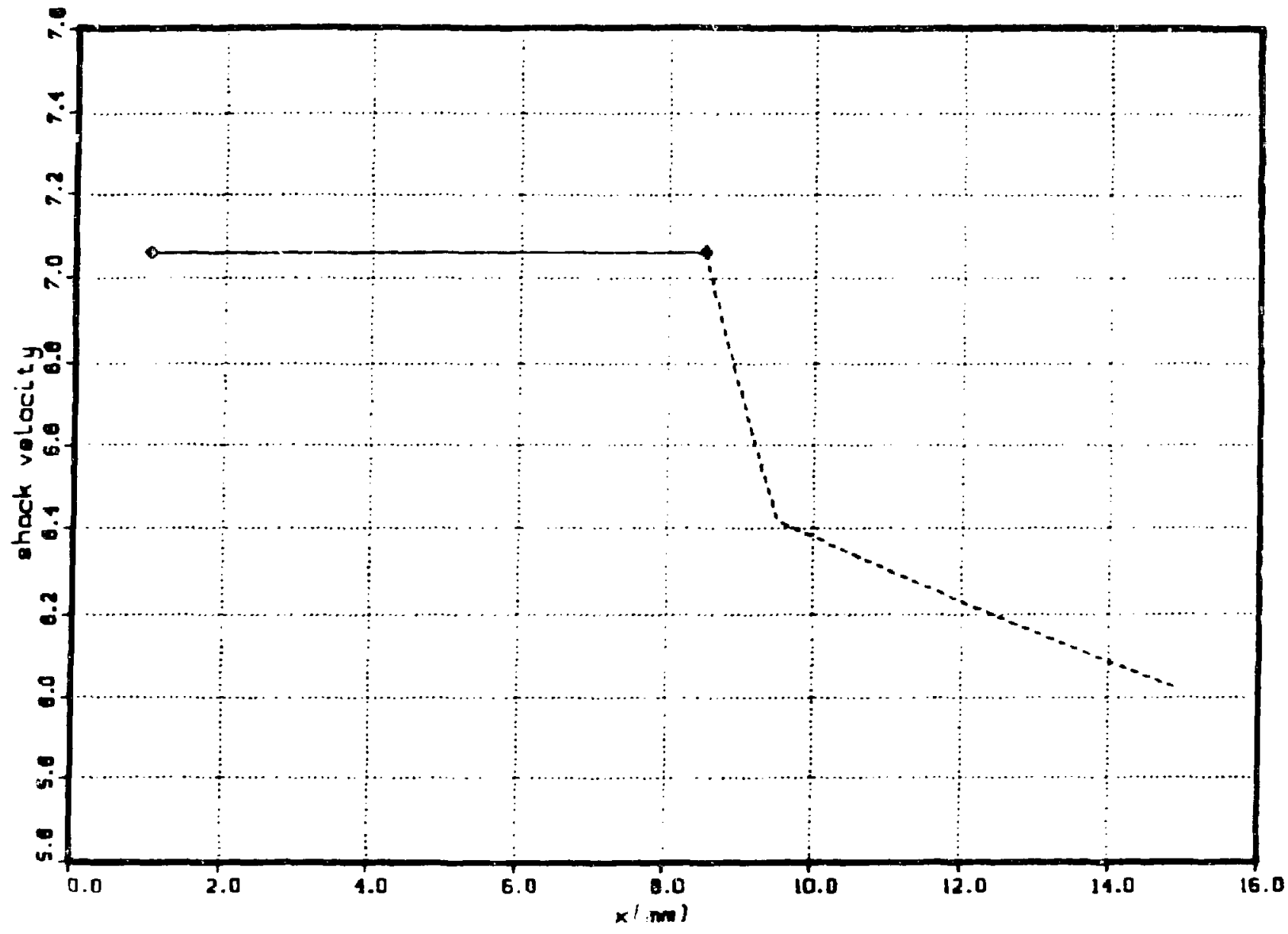

Figure 68: A $U$, versus $x$ plot for RS-2314 taken from the fit to the data shown in figure 67 . 


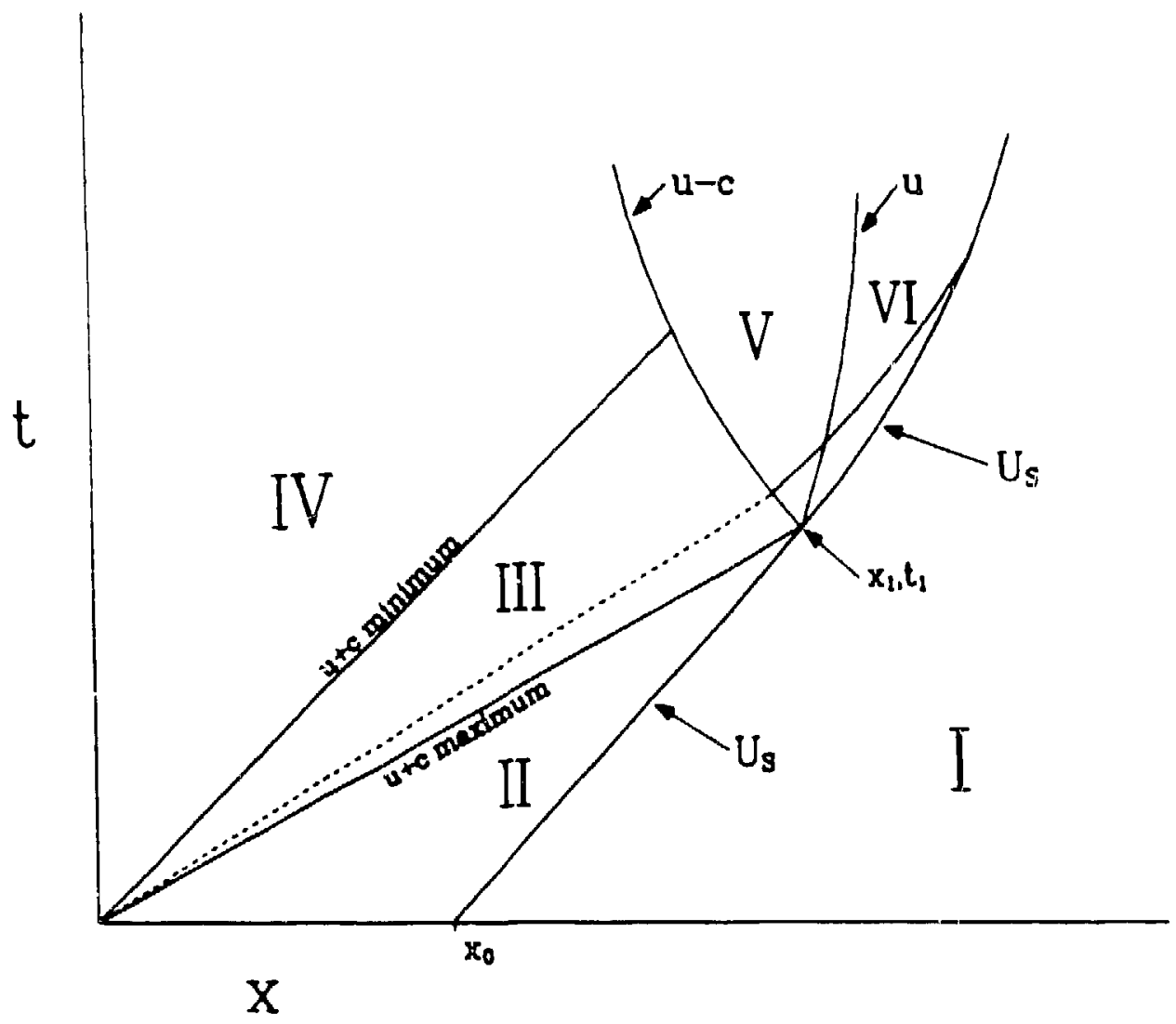

Figure 69: Detailed diagram of the different regions that occur when a simple-centered release wave attenuates a shock. 


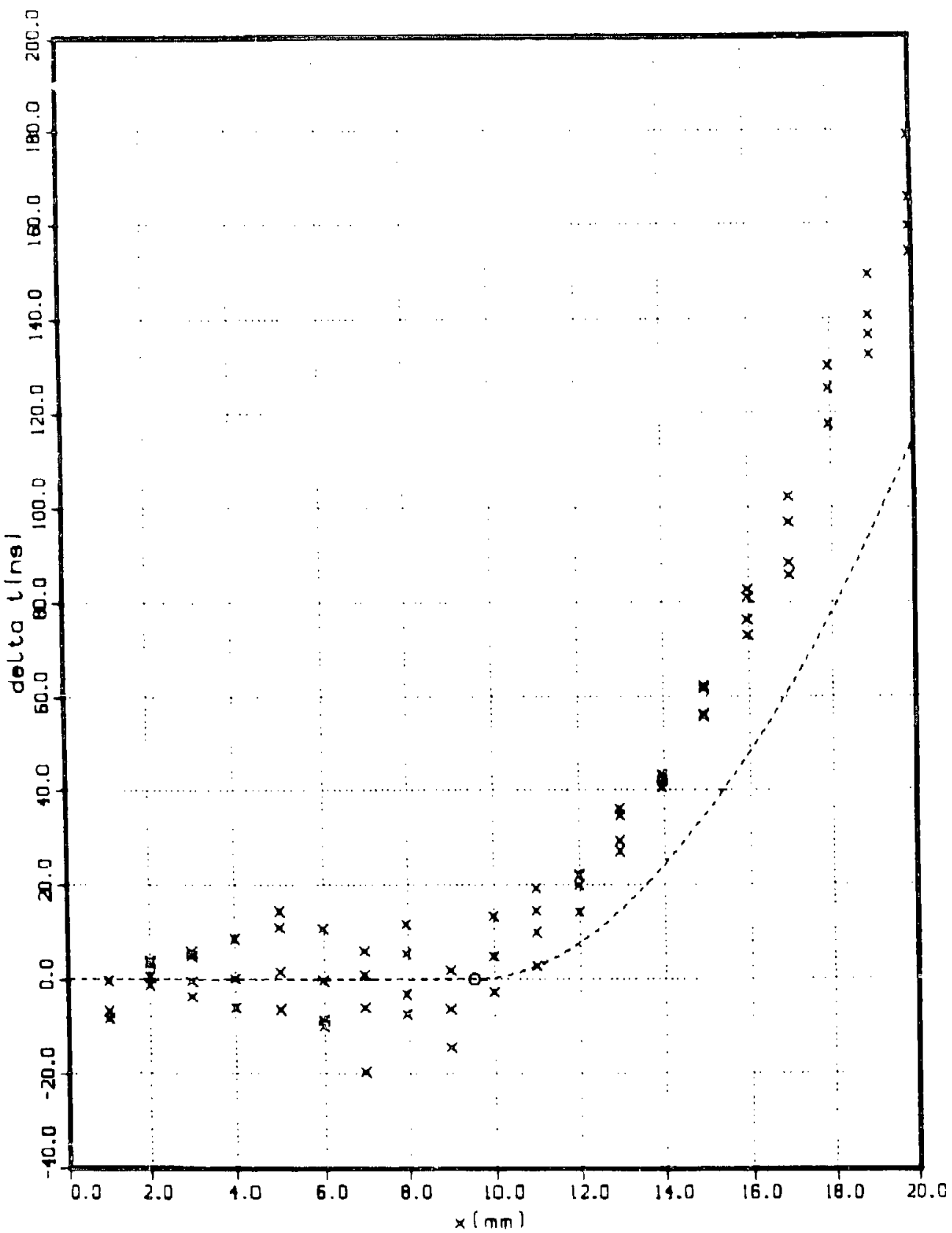

Figure 70: Comparison of the $\delta t$ data for RS-2302 and calculational results from the code SHKSIM. The dashed line is the $\delta t$ curve from the calculation. Parameterg for the calculational model and parameters for the data analysis are listed in table 13. 


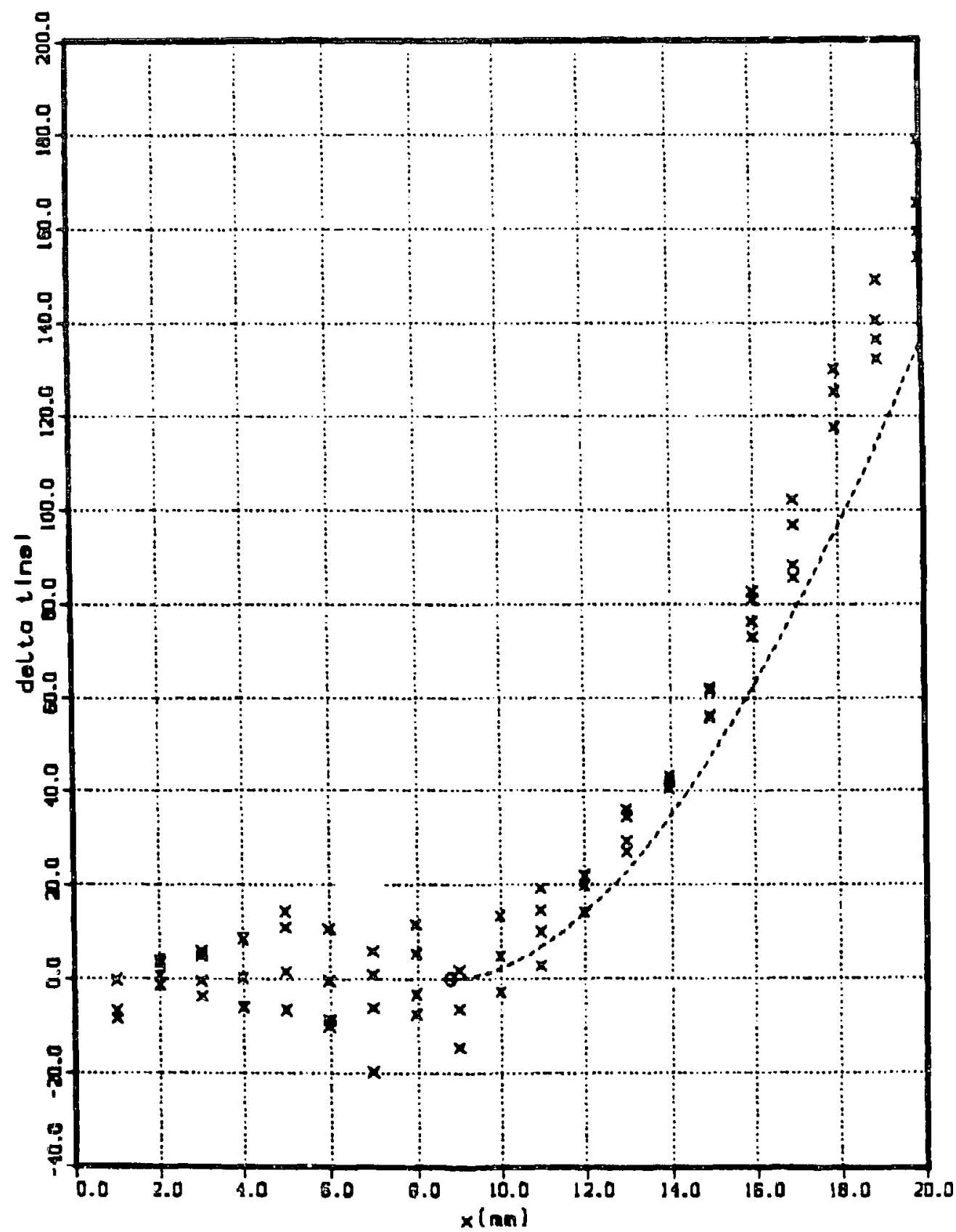

Figure 71: Comparison of the $\delta t$ data for RS-2302 and calculational resulis from the code SHKSIM. The dashed line is the $\delta t$ curve from the calculation. Parameters for the calculational model and parameters for the data analysis are listed in table 15. 


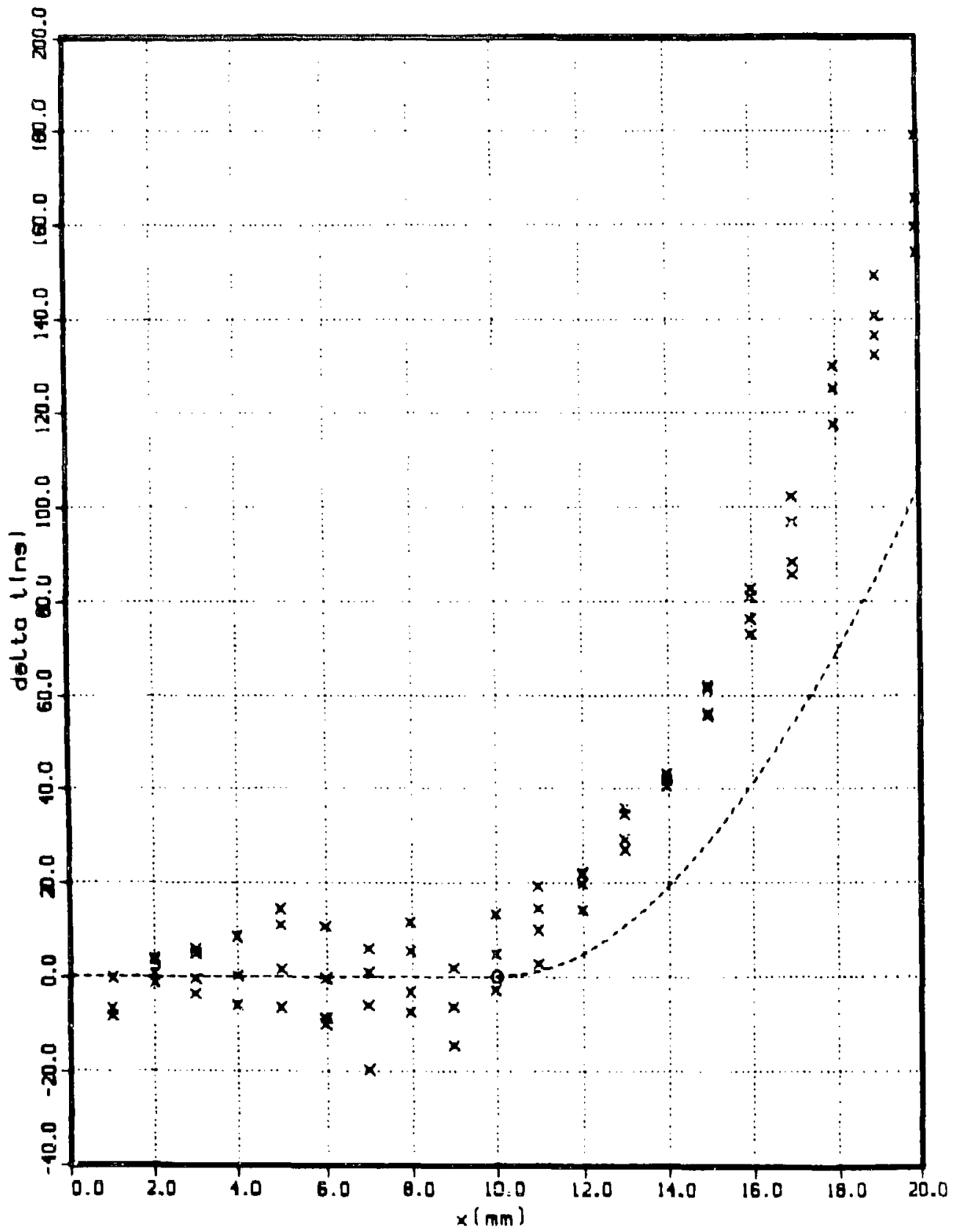

Figure 72: Comparison of the $\delta t$ data for RS-2302 and calculational results from the code SHKSIM. The dashed line is the $\delta t$ curve from the calculation. Parameters for the calculational model and parameters for the data analygis are listed in table 16. 


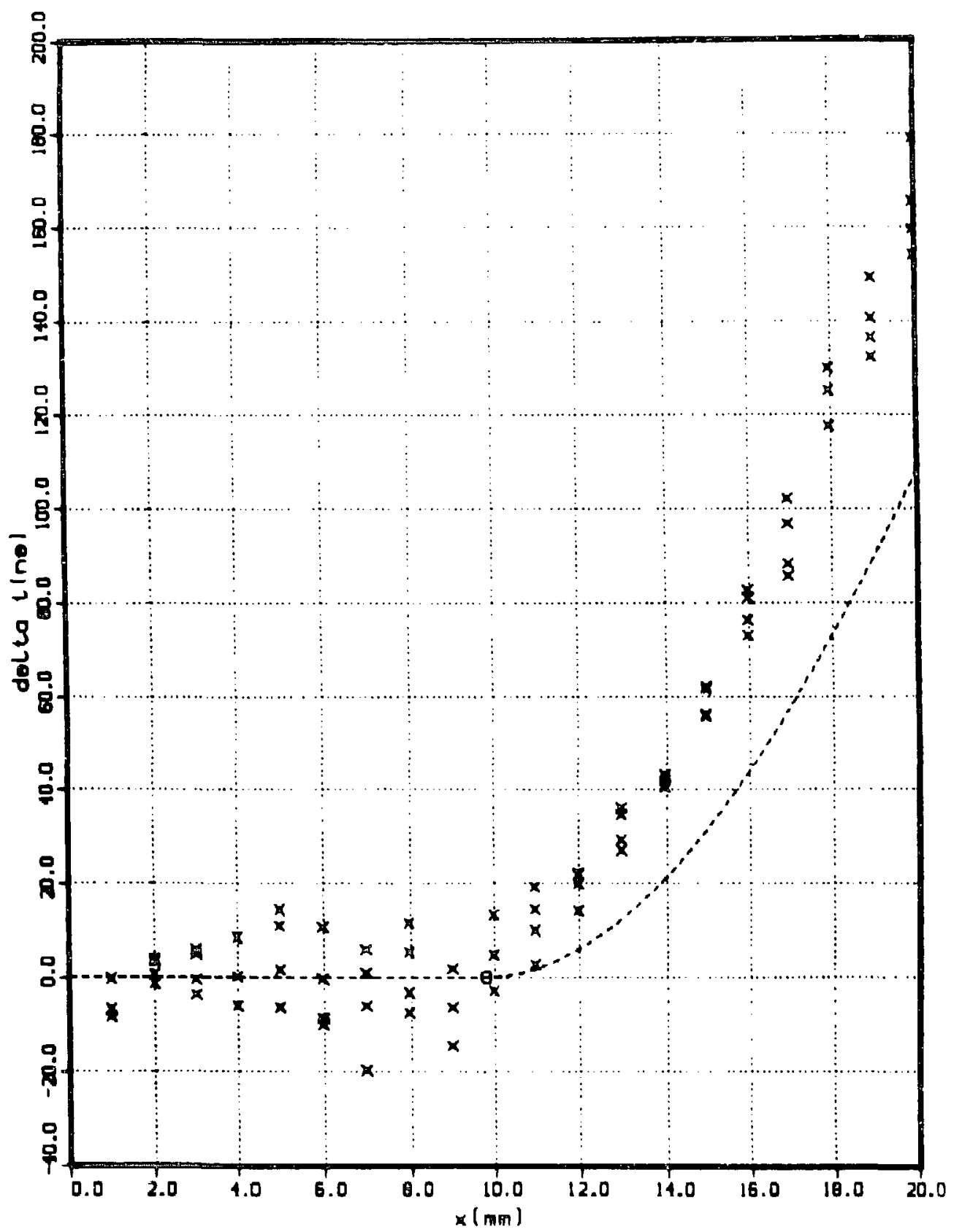

Figure 73: Comparion of the $\delta t$ data for RS-2302 and calculational results from the code SHKSIM. The dashed line is the $\delta t$ curve from the calculation. Parameters for the calculational model and parameters for the data analysis are listed in table 17. 


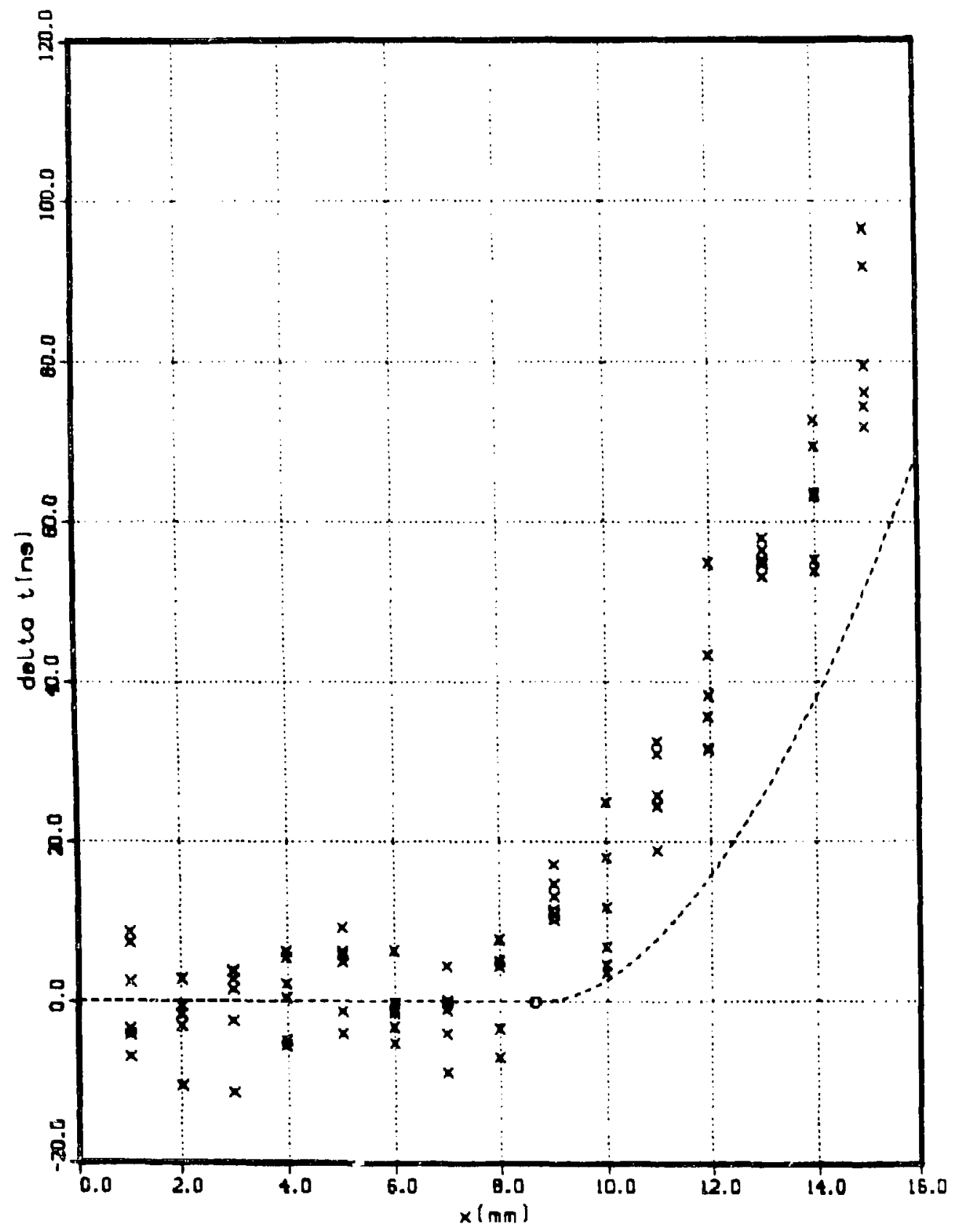

Figure T4: Comparison of the $\delta t$ data for RS-2315 and calculational resulta from the code SHKSIM. The dashed line is the $\delta t$ curve from the calculation. Parameters for the calculational model and parameters for the data analysis are listed in table 18. 


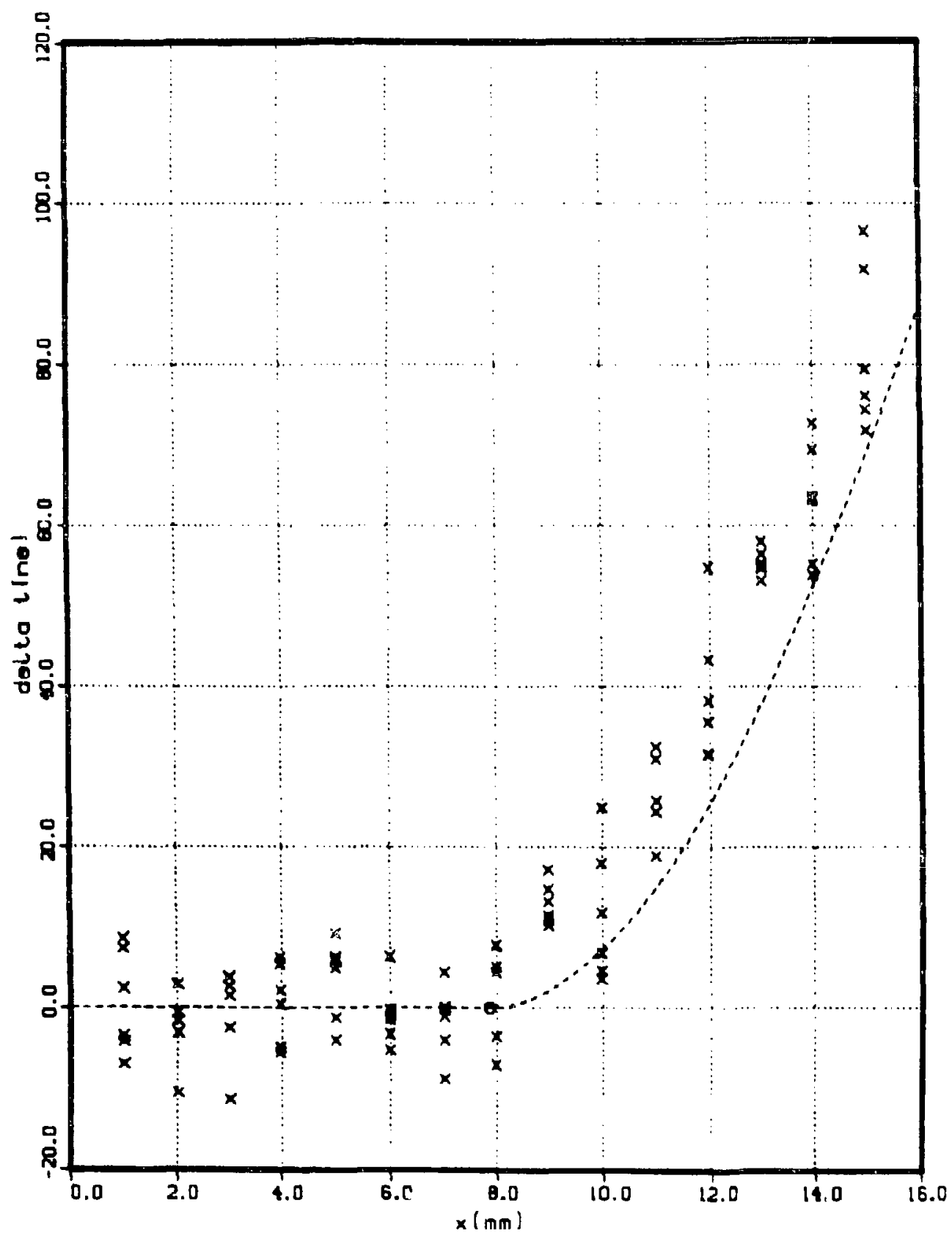

Figure 75: Comparion of the $\delta t$ data for RS-2315 and calculational results from the code SHKSIM. The dashed line is the $\delta t$ curve from the calculation. Parameters for the calculational model and parameters for the data analysis are listed in table 19. 


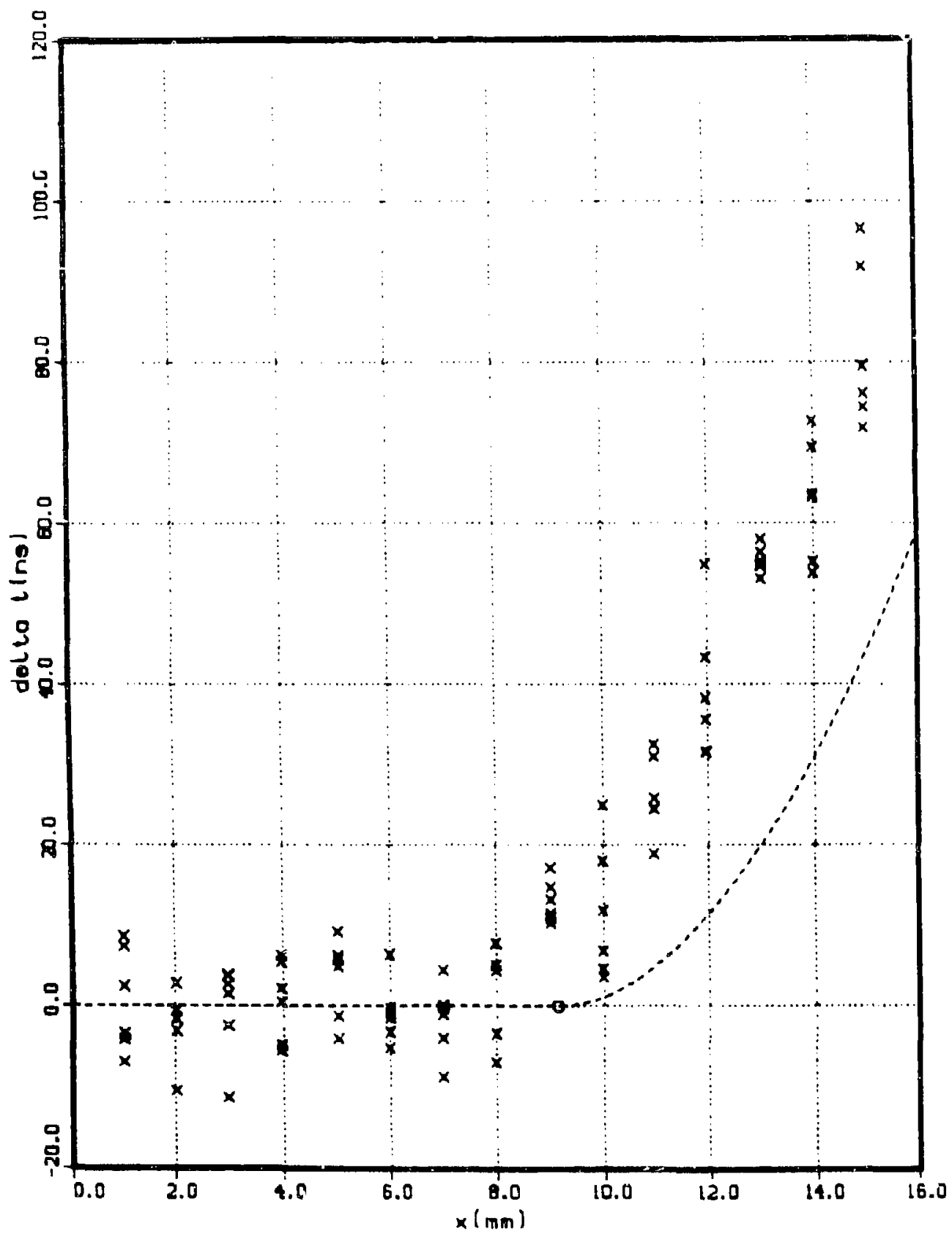

Figure 76: Comparisen of the $\delta t$ data for RS-2315 and calculational reeulta from the code SHKSIM. The dashed line is the $\delta t$ curve from the calculation. Parameters for the calculational model and parameters for the data analysis are listed in table 20. 


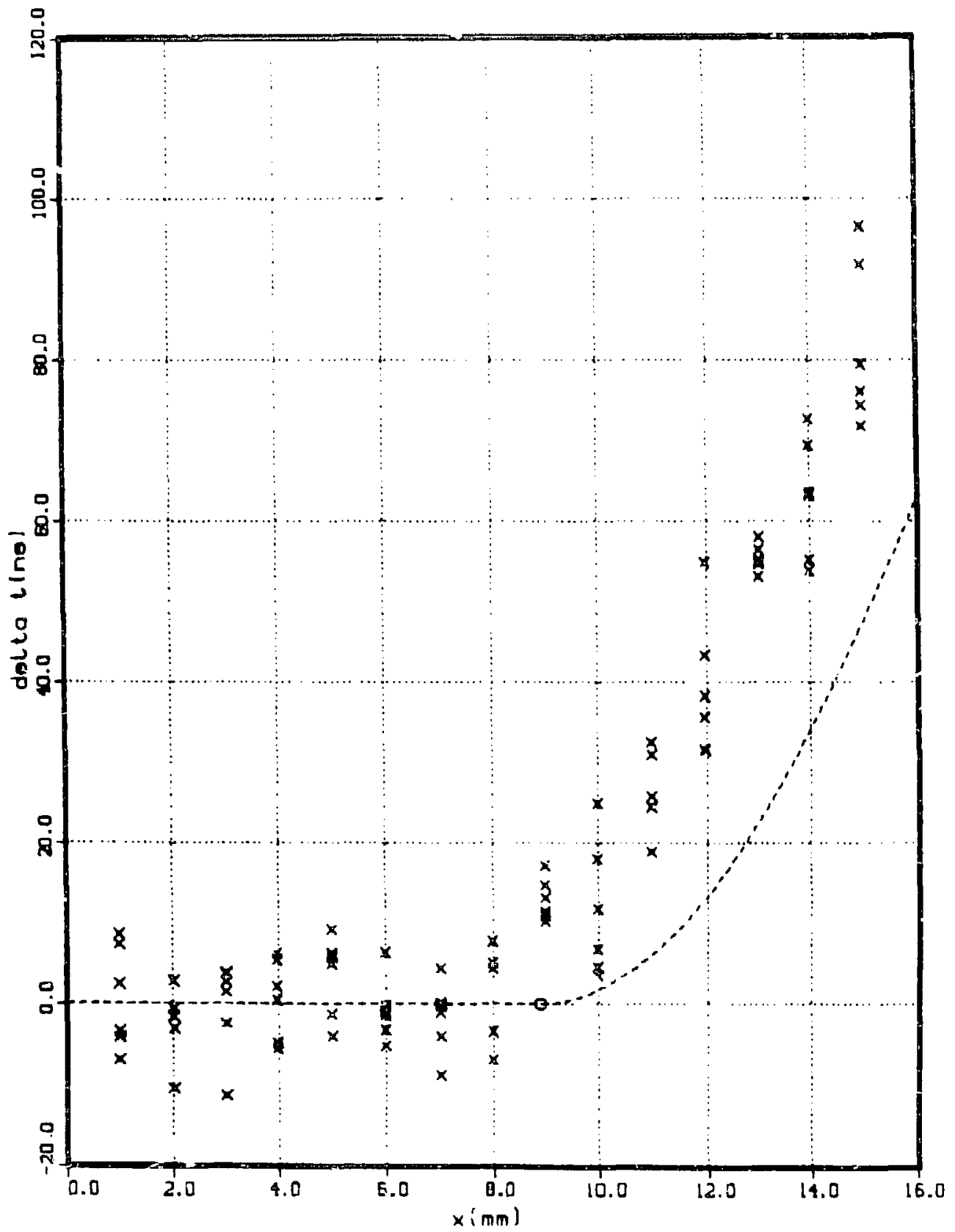

Figure 77: Comparison of the $\delta t$ ciata for RS-2315 and caiculistional reaults fron the code SHKSIM. The dashed line is the $\delta t$ curve from the salculation. Parameters for the rai:ulational model and parameters for the data analysis are listed in table 21. 


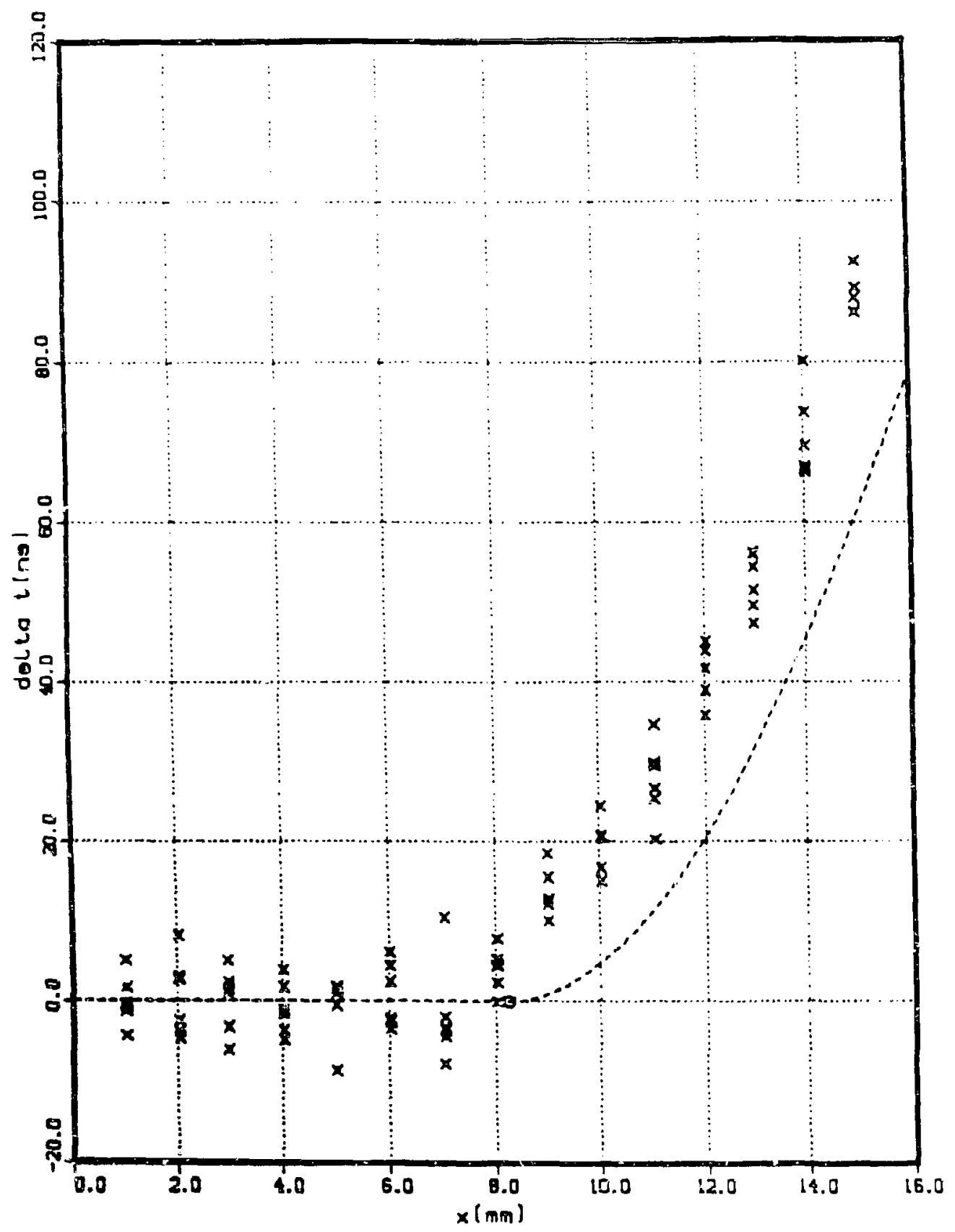

Rigure T8: Comparison of the $6 t$ data for KS-2309 and calculational results from the ccde SHKSIM. The dashed line is the $\delta t$ curve from the calculation. Parameters for the calculaitional model and parameters for the data analysis are listed in table 22. 


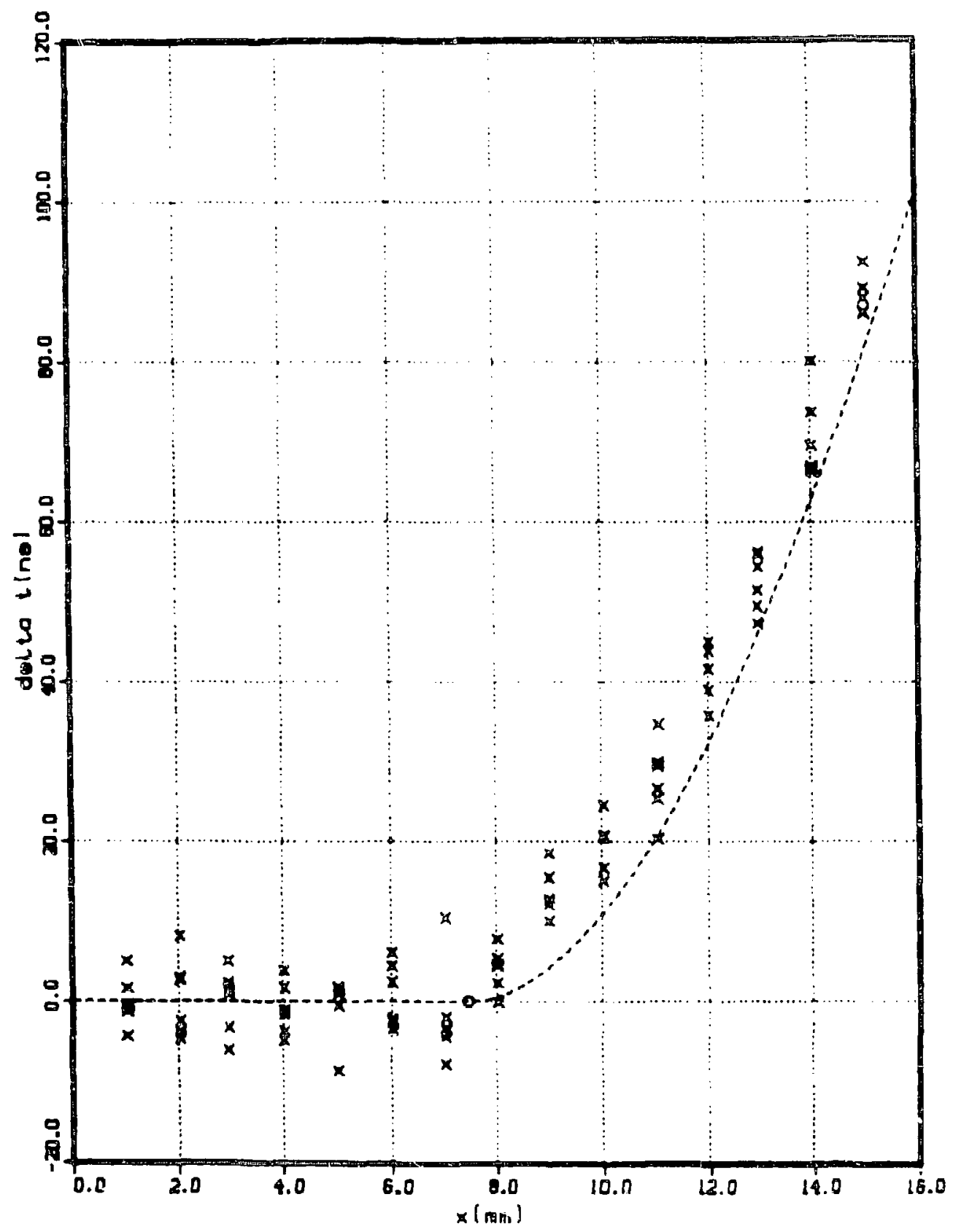

Figure 78: Comparisen of the $\delta i$ data for RS-2309 and calculational results from the code SHKSIM. The dashed line is the $\delta r$ curve from the calculation. Parameters tor the calculational model and farameters for the data analygis are listed in table 23. 


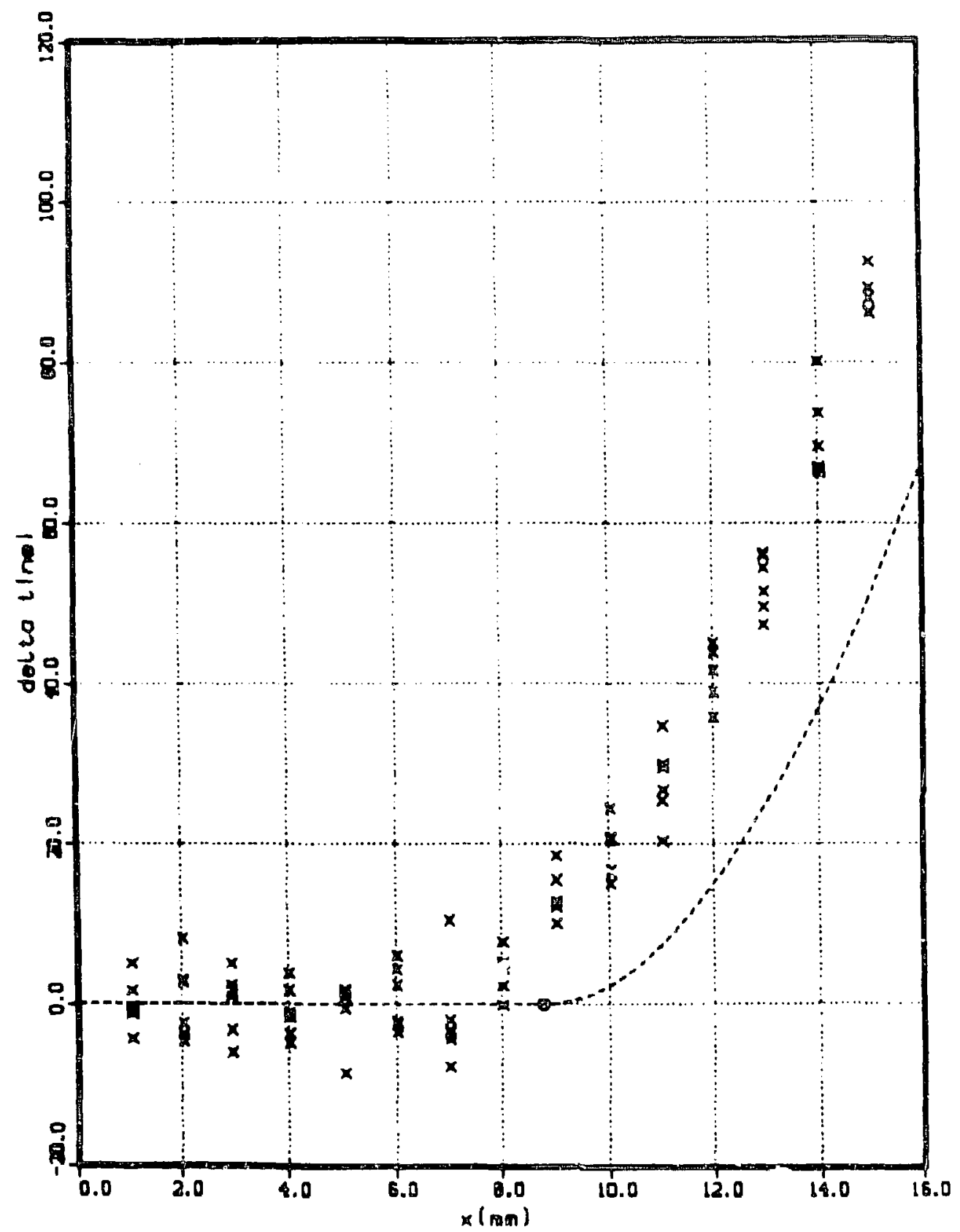

Figure 80: Comparison of the $6 t$ daia for RS-2309 and calculational results from the code SHKSIM. The dashed line is the $\delta t$ curve from the calculation. Parameterg for the calculational morlel and parameters for the data analysis are listed in table 24. 


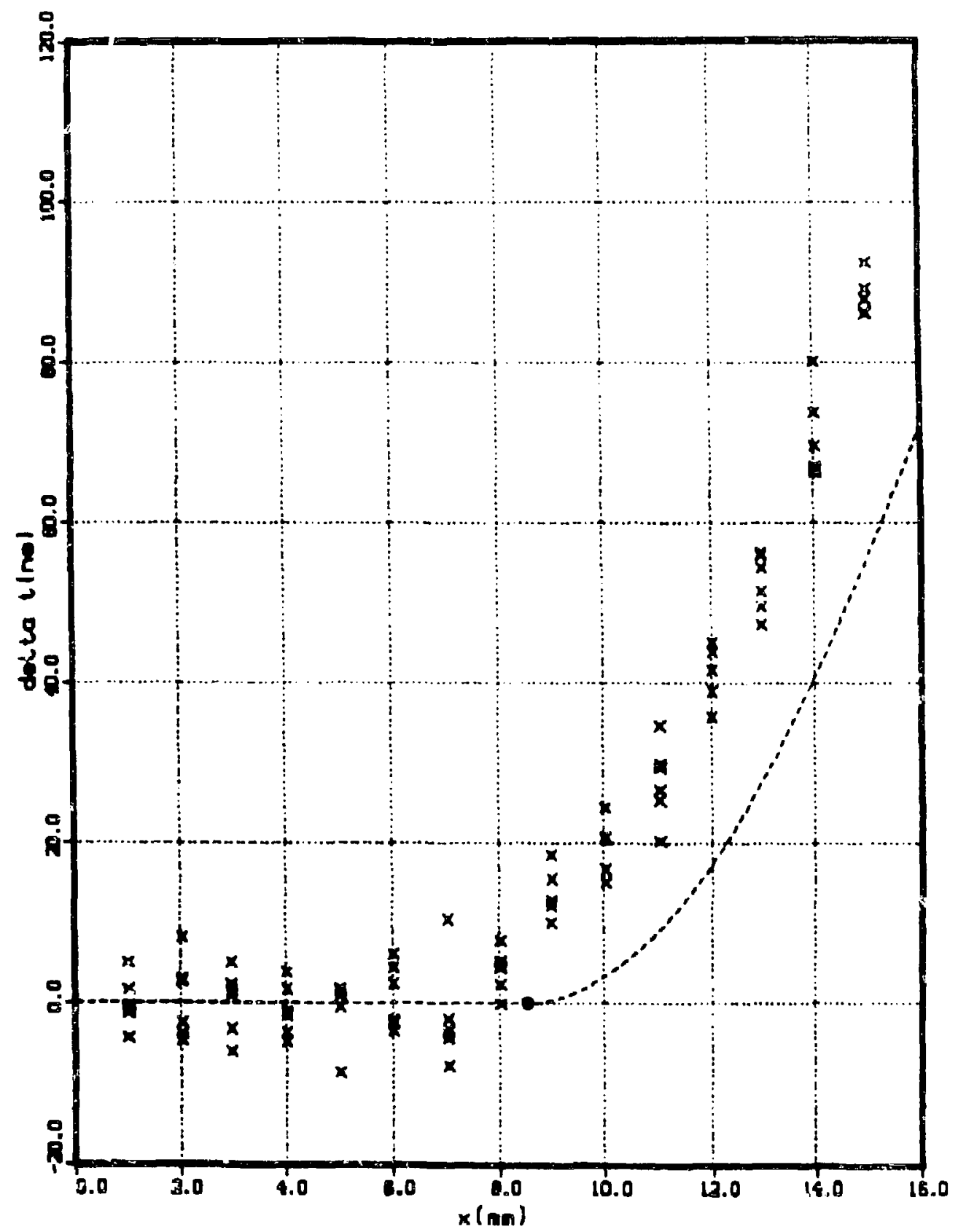

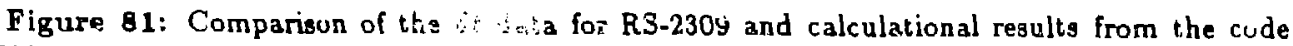
SHKSIM. The dashed line is the $\delta \hat{i}$ cus model and parameters for the data analysis are listed in table 25 . 


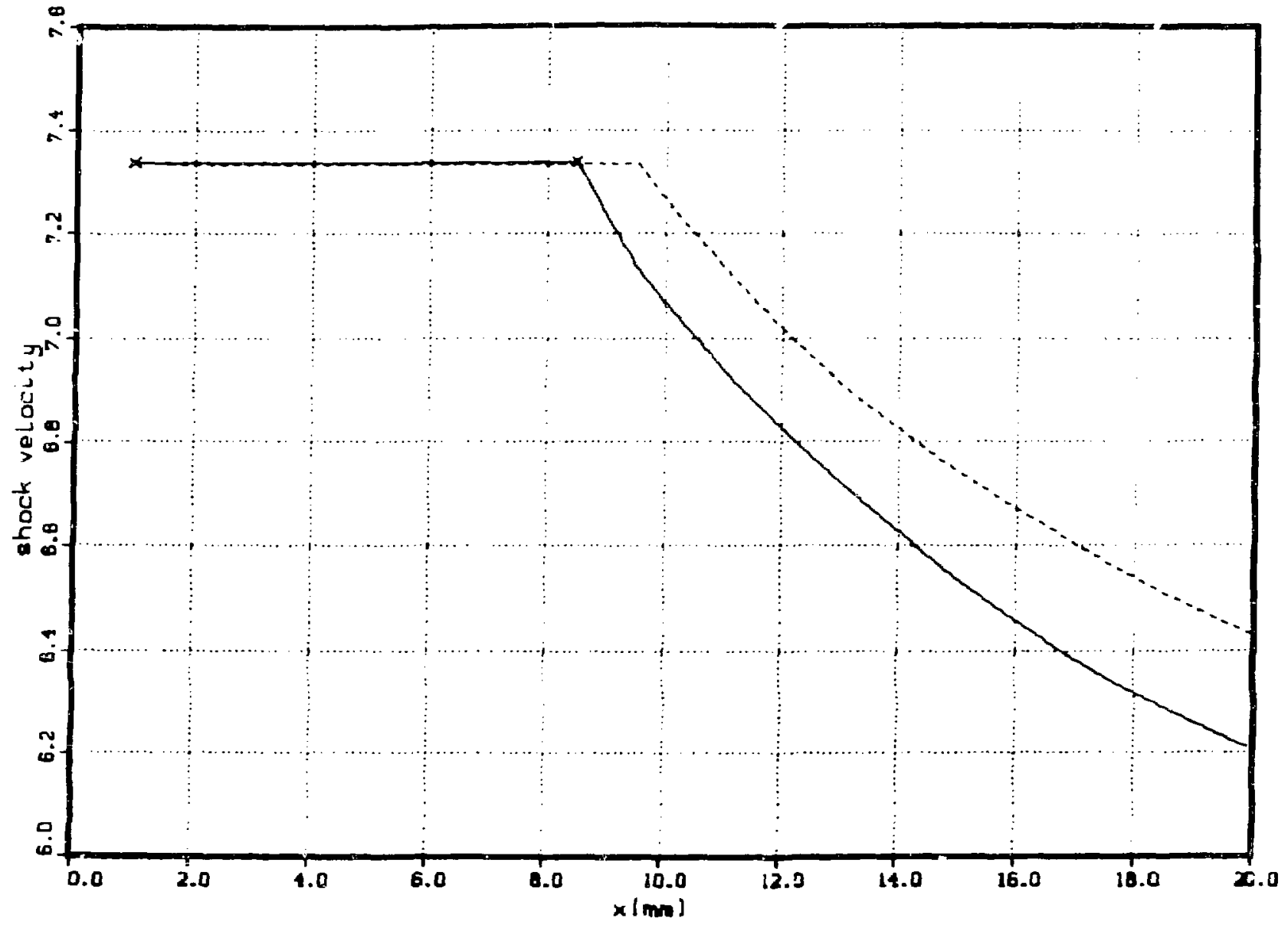

Figure 82: A comparison of the shock velocity from the code calculation and the shock velocity curve from a fit to the data for PS- 2302 . The solid line is the velocity curve taken from the fit to the data. The corresponding $\delta t$ plots are in figure 70 (see also table 13). 


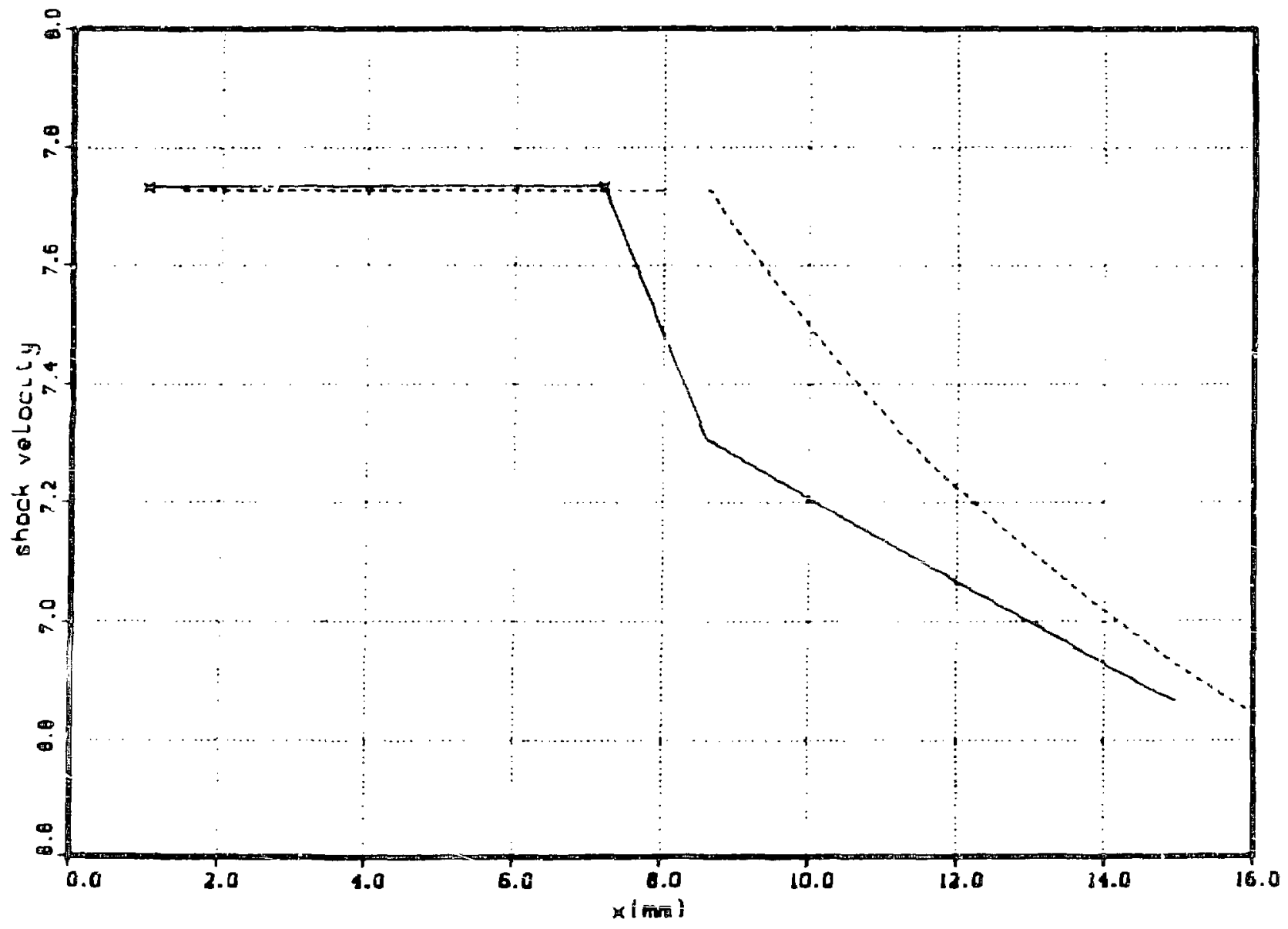

Pigure 83: A comparison of the shock velocity from the code calculation and the shock velocity curve from a fit to the data for RS-2315. The solid line is the velocity curve taken from the fit to the data. The corresponding ot plots are in tigure 74 (see also table 18). 


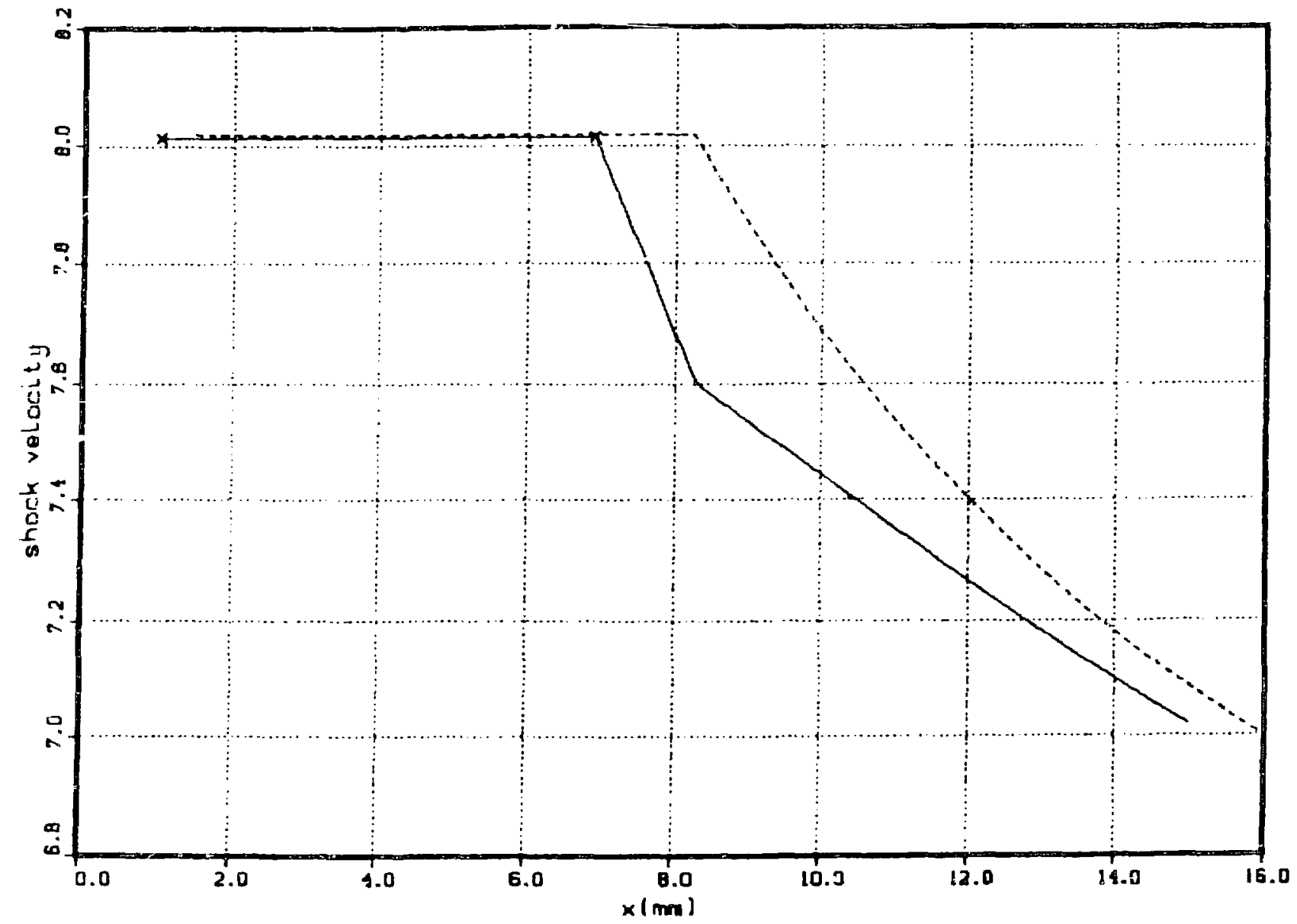

Figure 84: A comparison of the shock velocity from the code calculation and the shock velocity curve from a fit to the data for RS-2309. The solid line is the velocity curve taken from the fit to the data. The corresponding $\delta t$ plots are in figure 78 (see also table 22 ). 


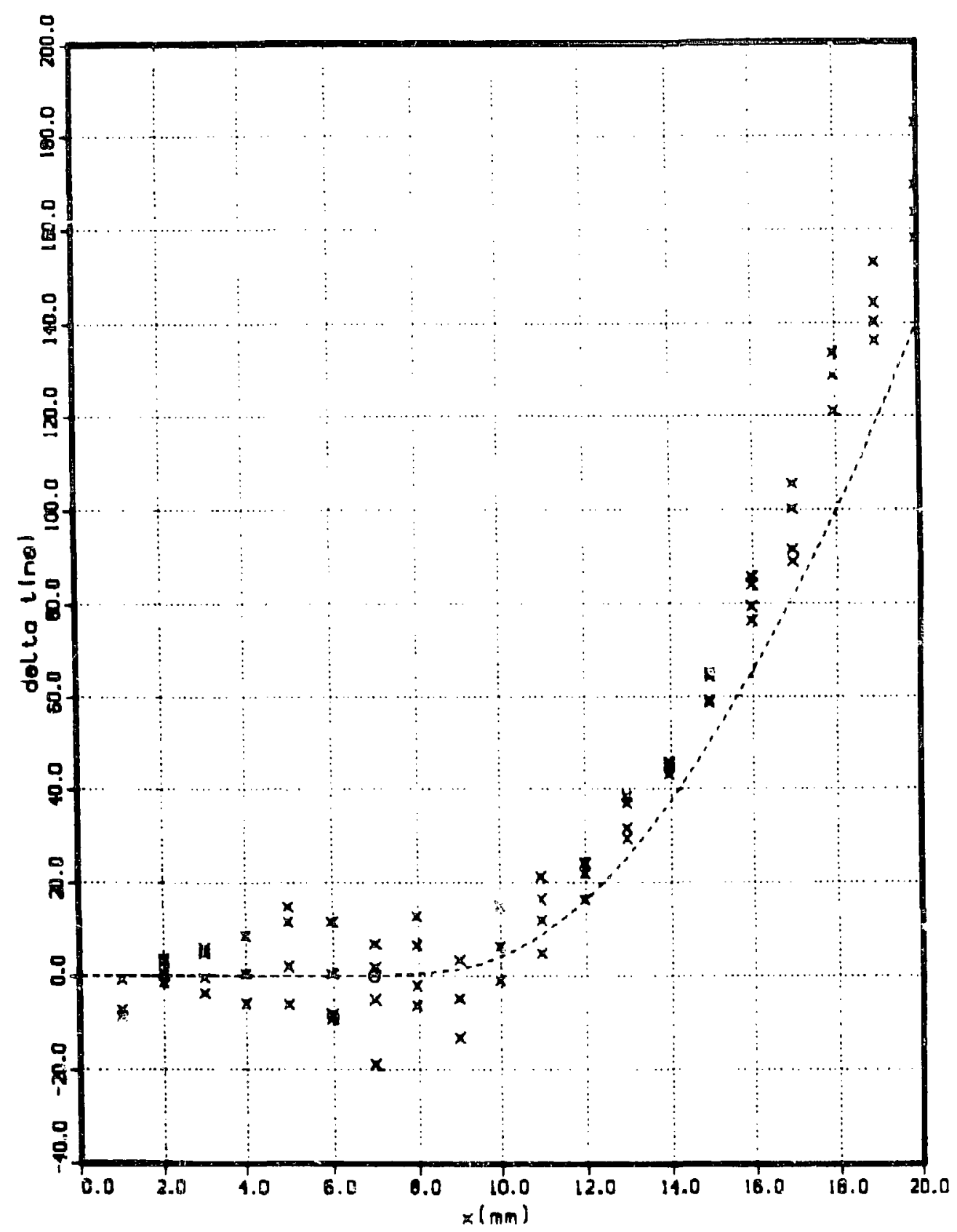

Figure 85: Comparison of the ht data for RS-2302 and calculational results from the code SHKSJB. The dashed line is the $\delta$ c curve from the calculation. Parameters for the calculational model and parameters for the data anslygis are lisied in table 26. 


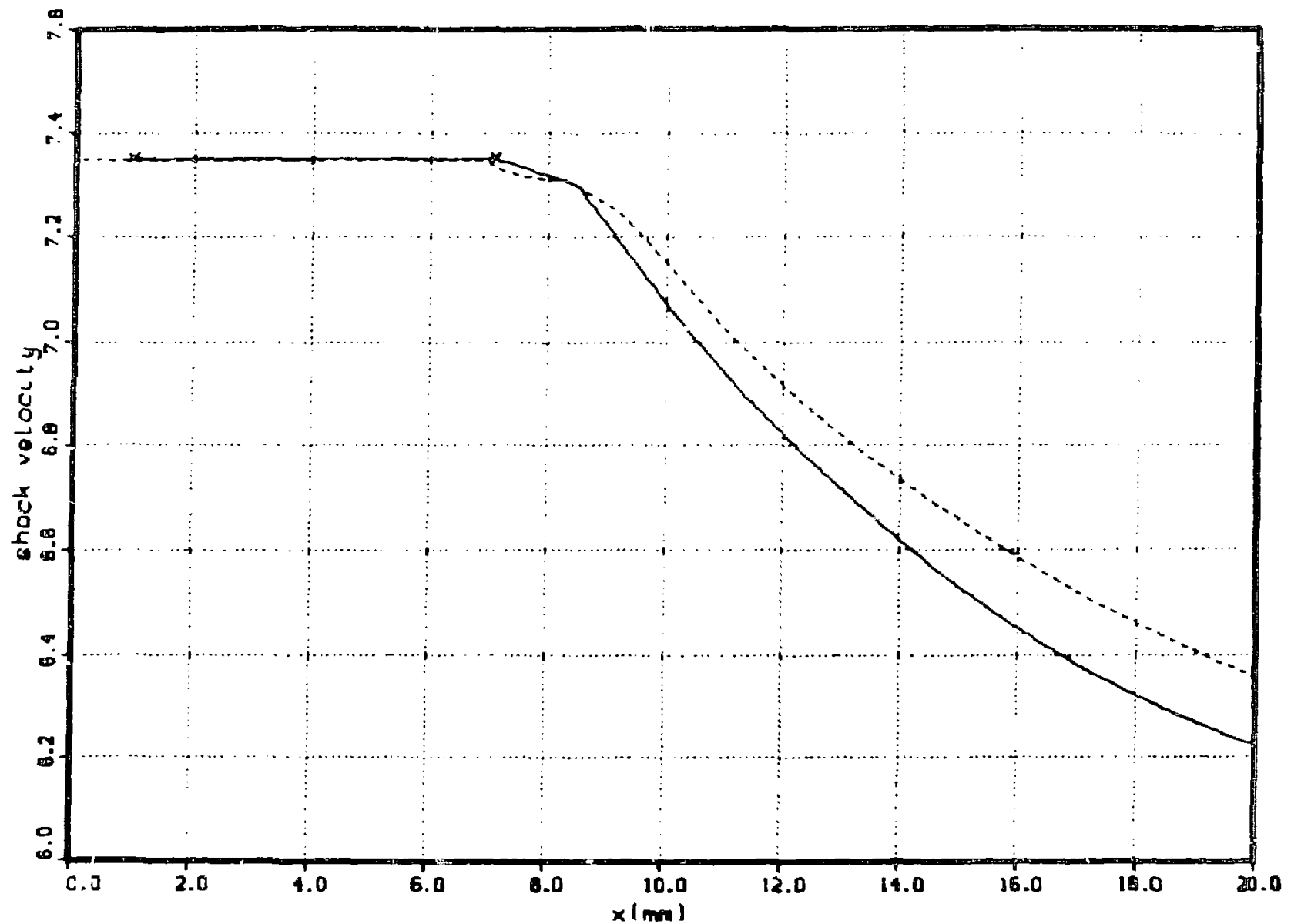

Figure 86: A comparioon of the shoch velocity from the code calculation and the shock velocity curve from a fit to the data for RS-2302. The solid line is the velocity curve takell from the fit to the data. The corresponding ot plots are in figure 85 (see also table 26j. 


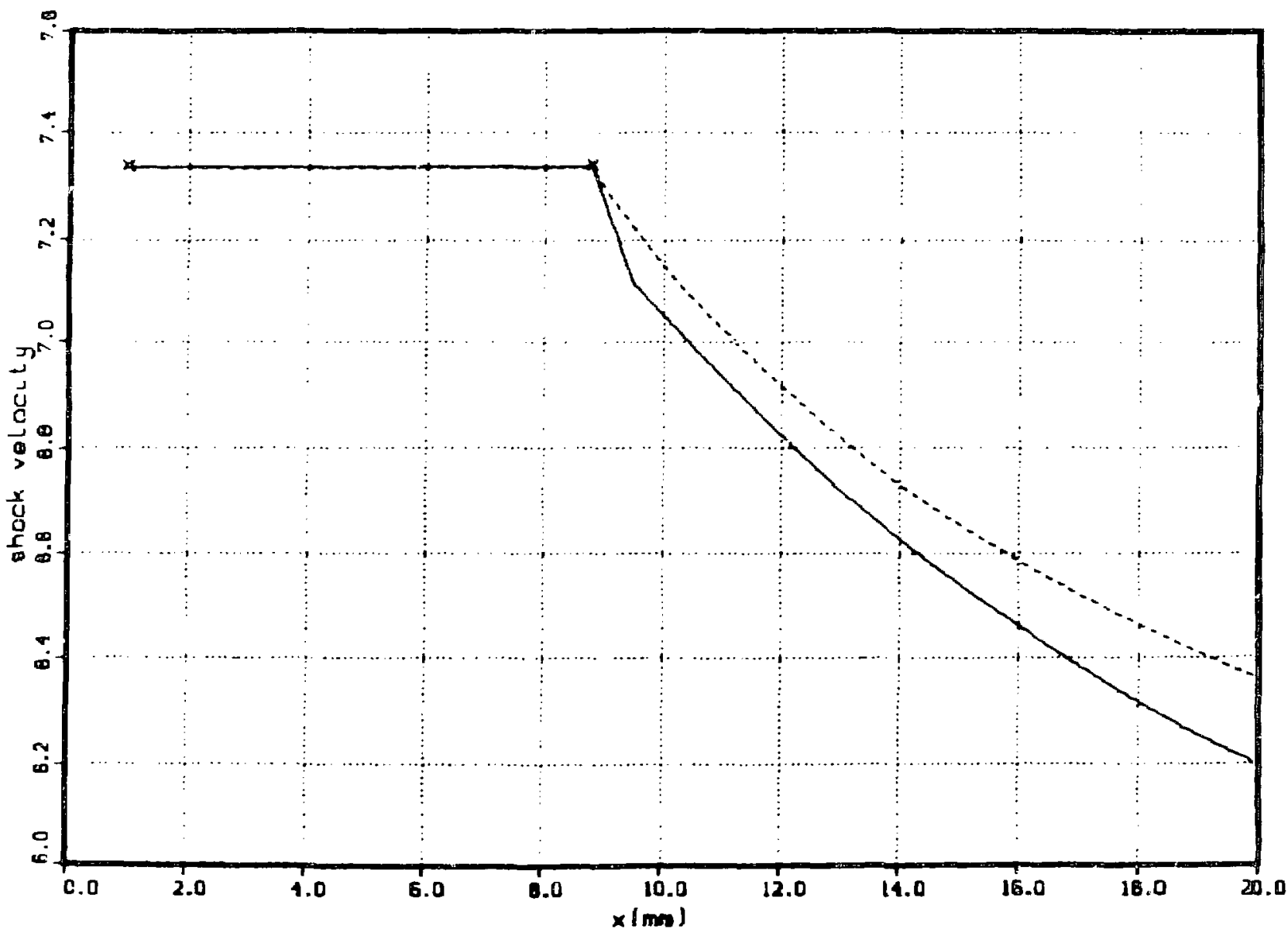

Figure 87: A comparison of the shock velocity from the code calculation and the shock velocity curve from a fit to the data for RS-2302. The solid line is the velocity curve taken from the fit to the data. The corresponding $\delta t$ plots are in figure 71 (see also table 15). 


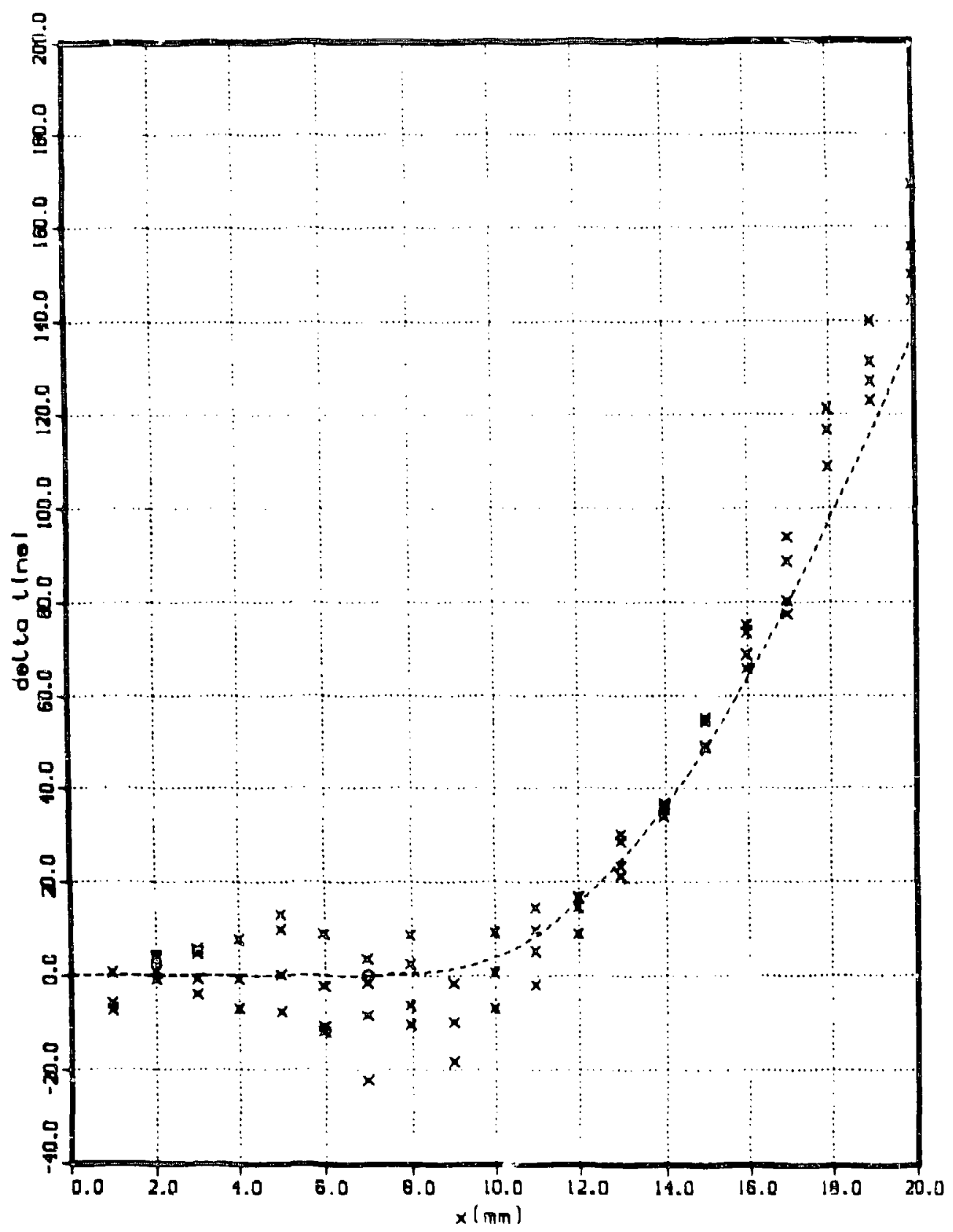

Figure 8g. Comparison of the $\delta t$ data for RS-2302 and calculational reaults from the code SHKSJB. The dasher line is the $\delta$ t curve from the calculation. Parameters for the calculational model and parameters for the data analysis are liated in table 27. 


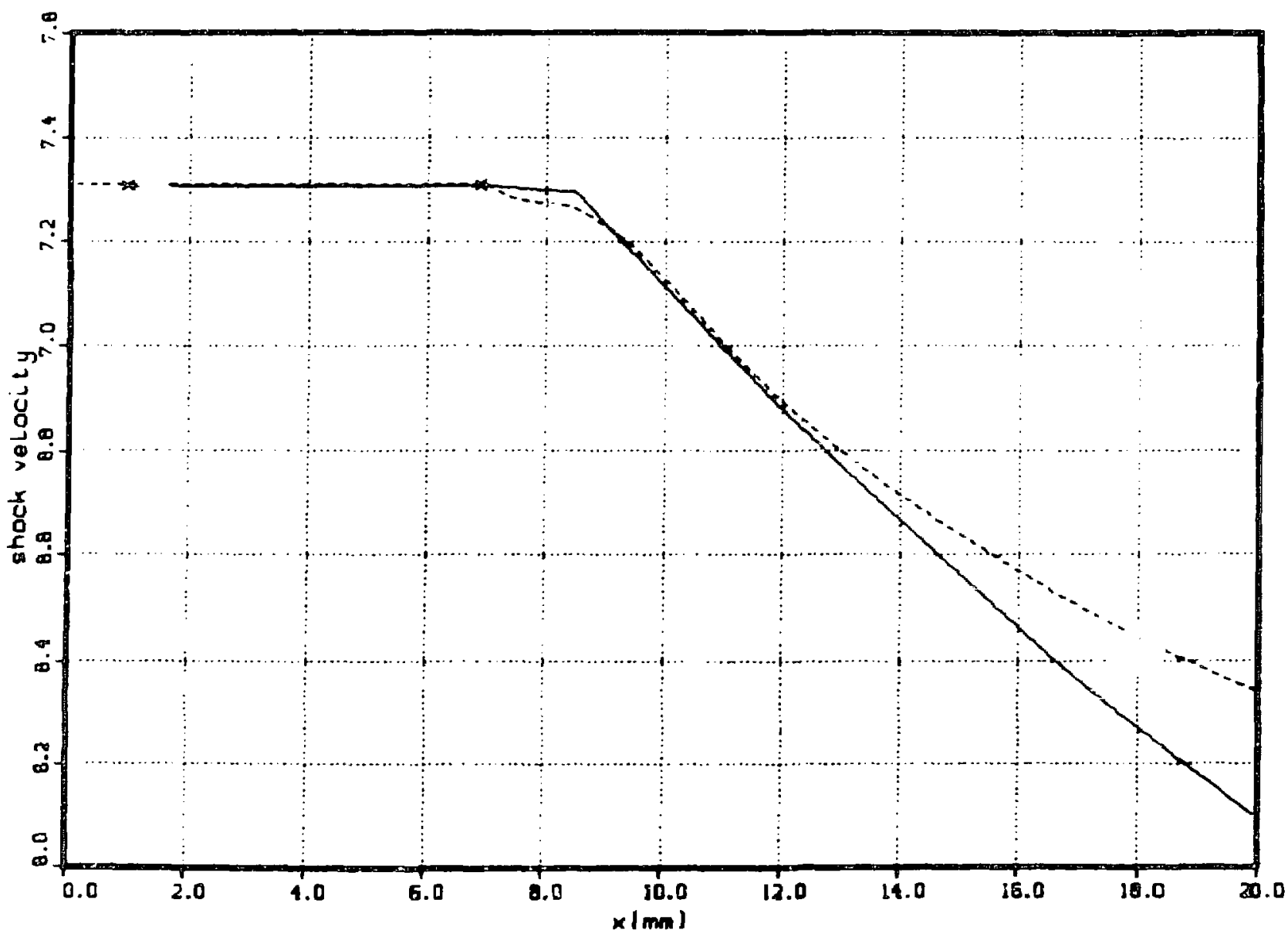

Figure 89: A comparison of the shock velority from the code calculation and the shock velocity curve from a fit to the data for RS-2302. The solid line is the velocity curve taken frnm the fit to the data. The corresponding $6 t$ plots are in figure 88 (see also table 27). 


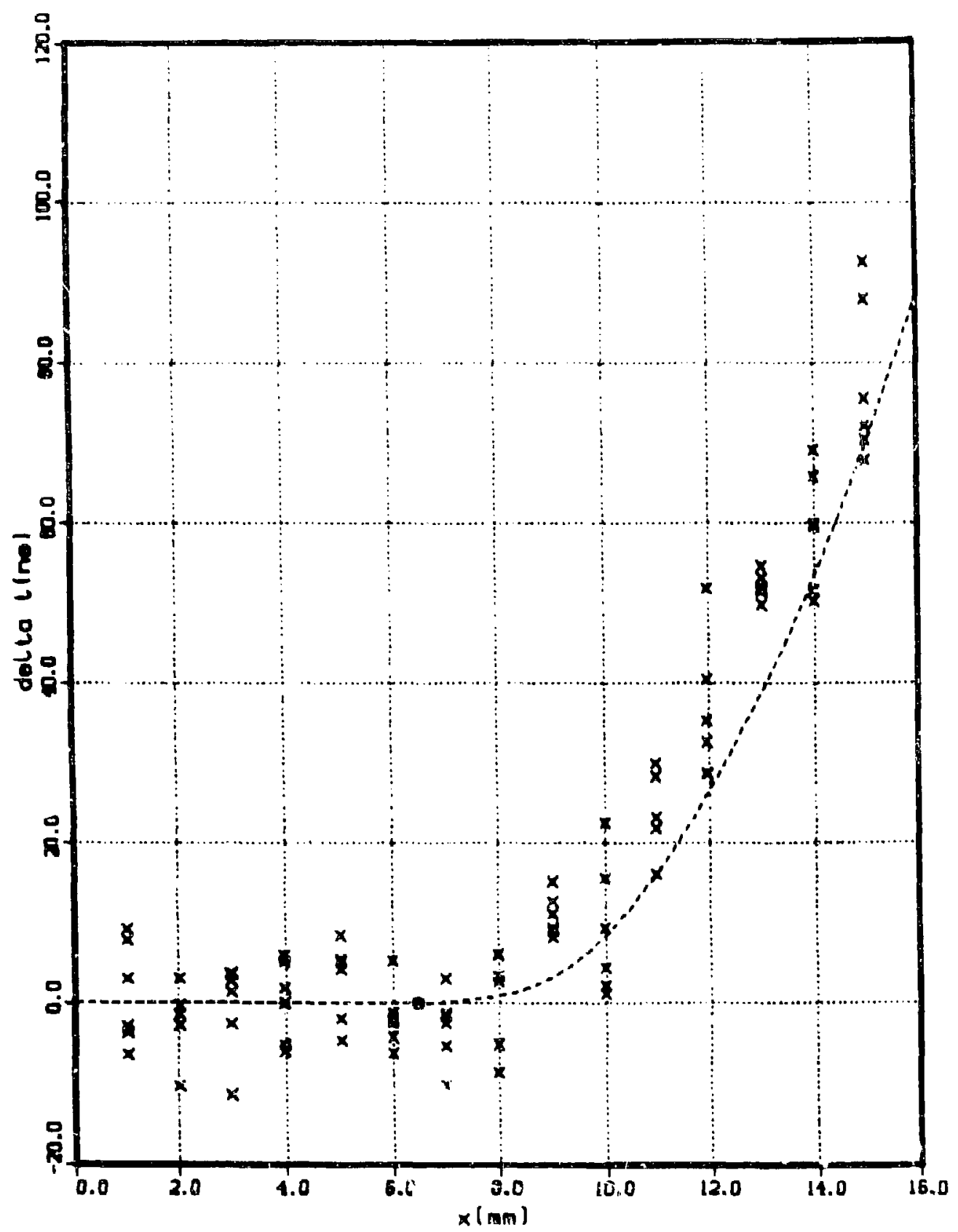

Figure 80: Comparison of the $\delta t$ data for RS-2315 and calcula. onzl resultn from the cod; SHKSJB. The dashed line is the $i t$ curve from the calculation. Parameters ror the calculational model and parameters for the data analysis are listed in table 28. 


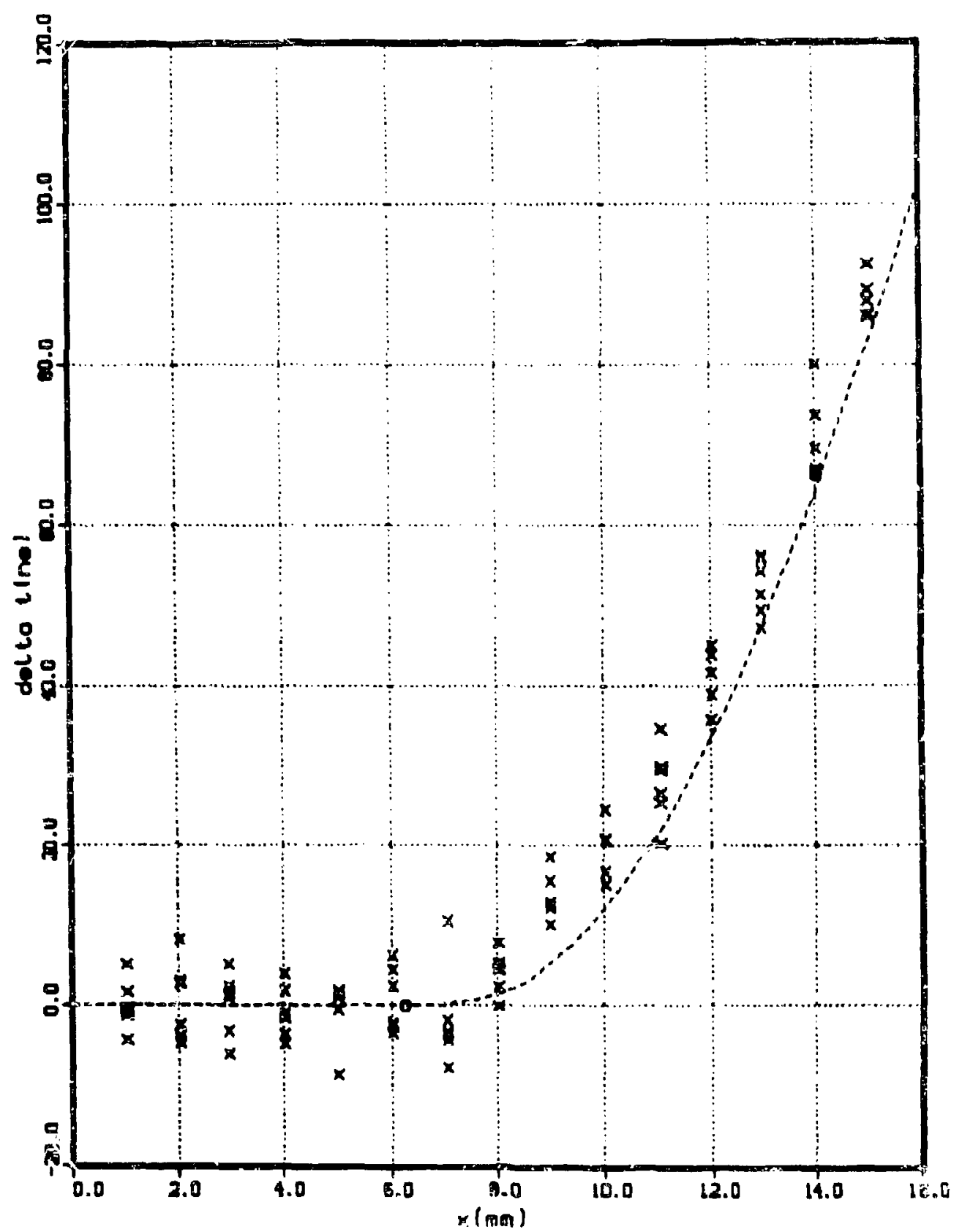

Figure 91: Comparison of the $\delta t$ data for RS-is09 anci calculational results from the colc SHKSJB. The $d=$ shed line is the $\delta t$ curve from the calculation. Parameter for tbe calculational medel and parameters for the data an alysia are liated in table 29. 


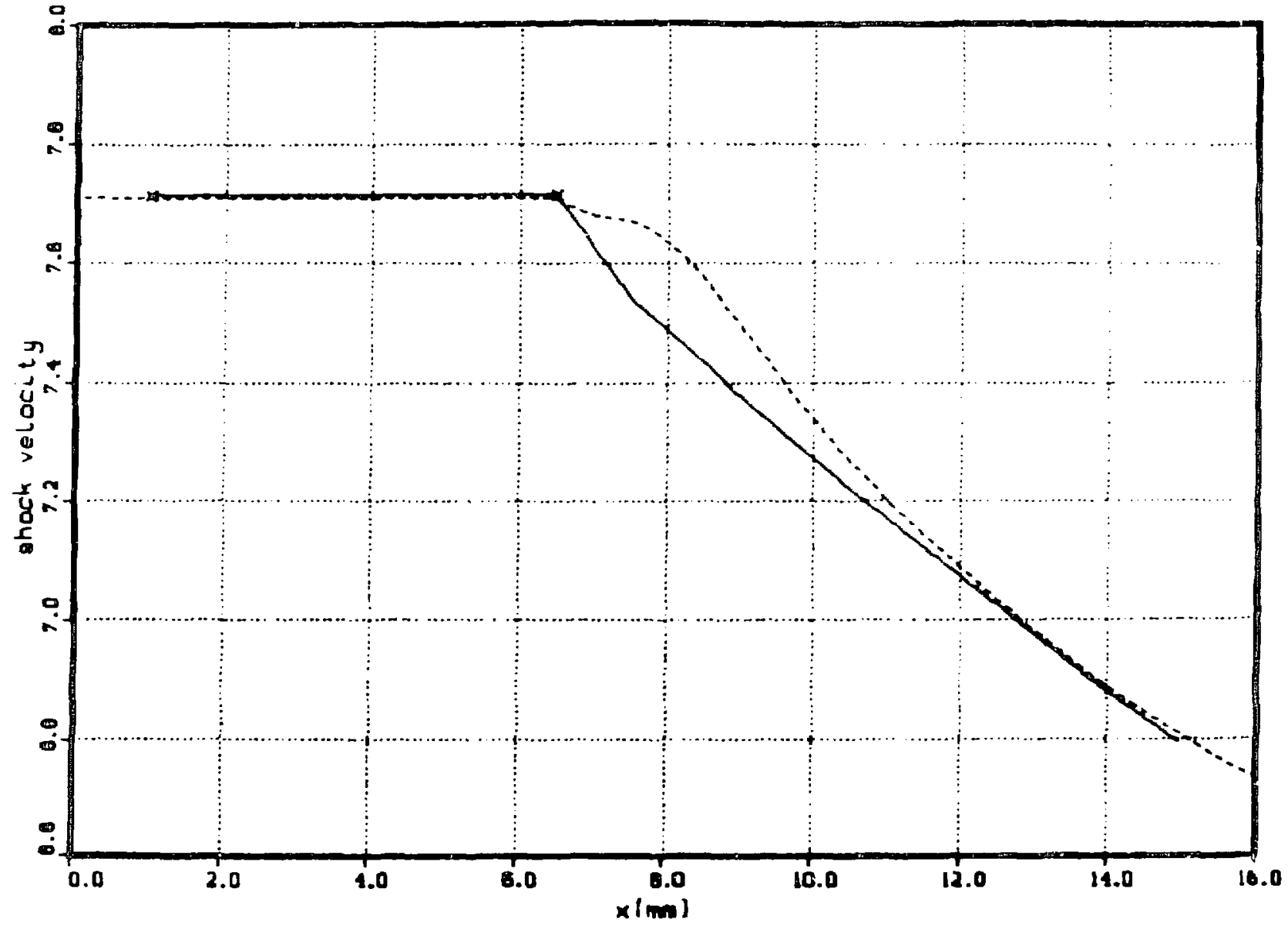

过

Figure 92: A comparison of the shock velocity from the code calculation and the shock velocity curve from a fit to the data for RS-2315. The solid line is the velocity curve taken from the at to the dat 3 . The corresponding $\delta t$ plots are in Ggure 90 (see also table 28). 


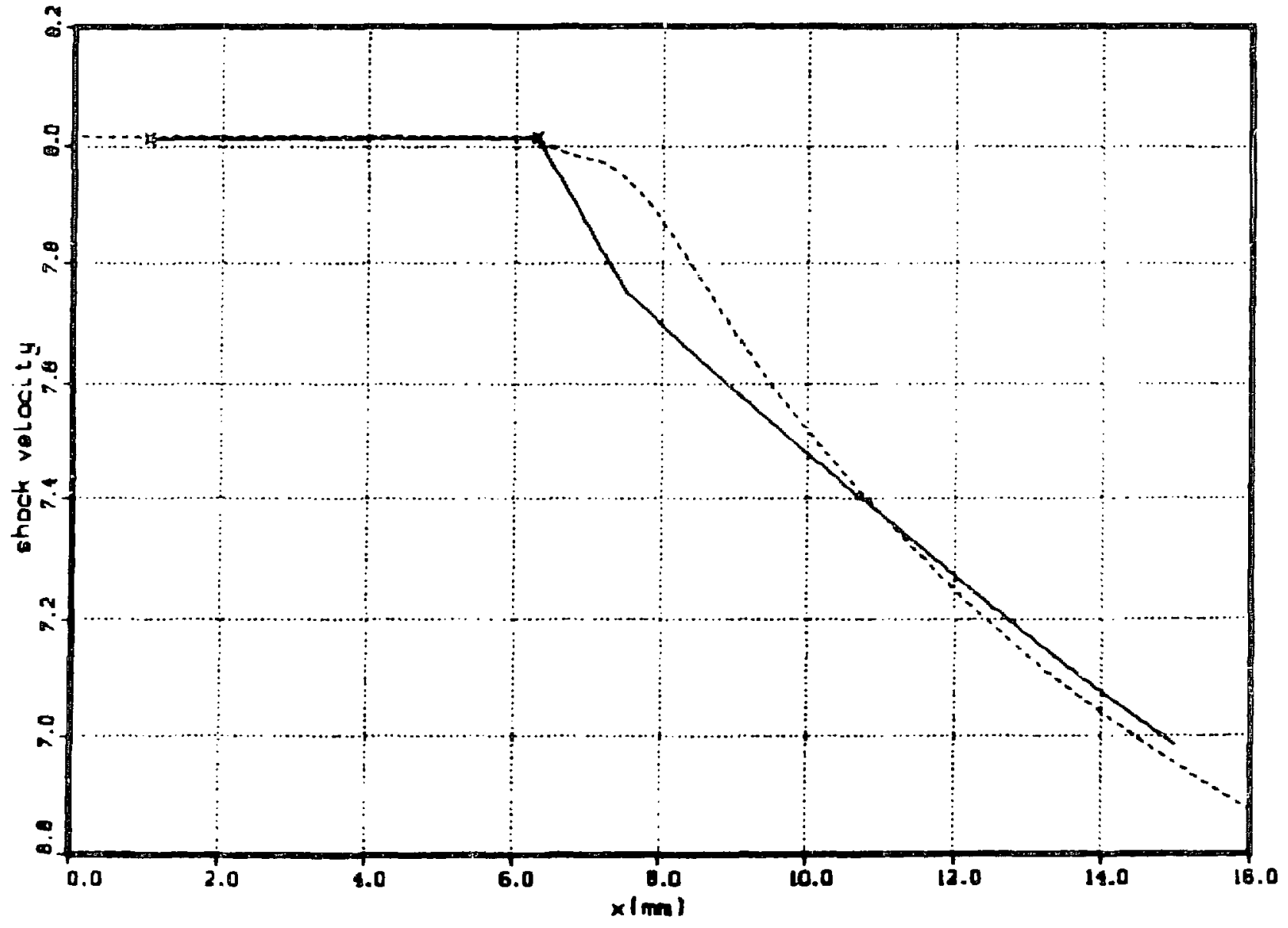

Figure 95: A comparison of the shock velocity from the code calculation and the shock velocity curve from a fit to the data for RS-2309. The solid line is the velocity curve taken from the fit to the data. The corresponding $\delta t$ plots are in figure 91 (see also table 29). 


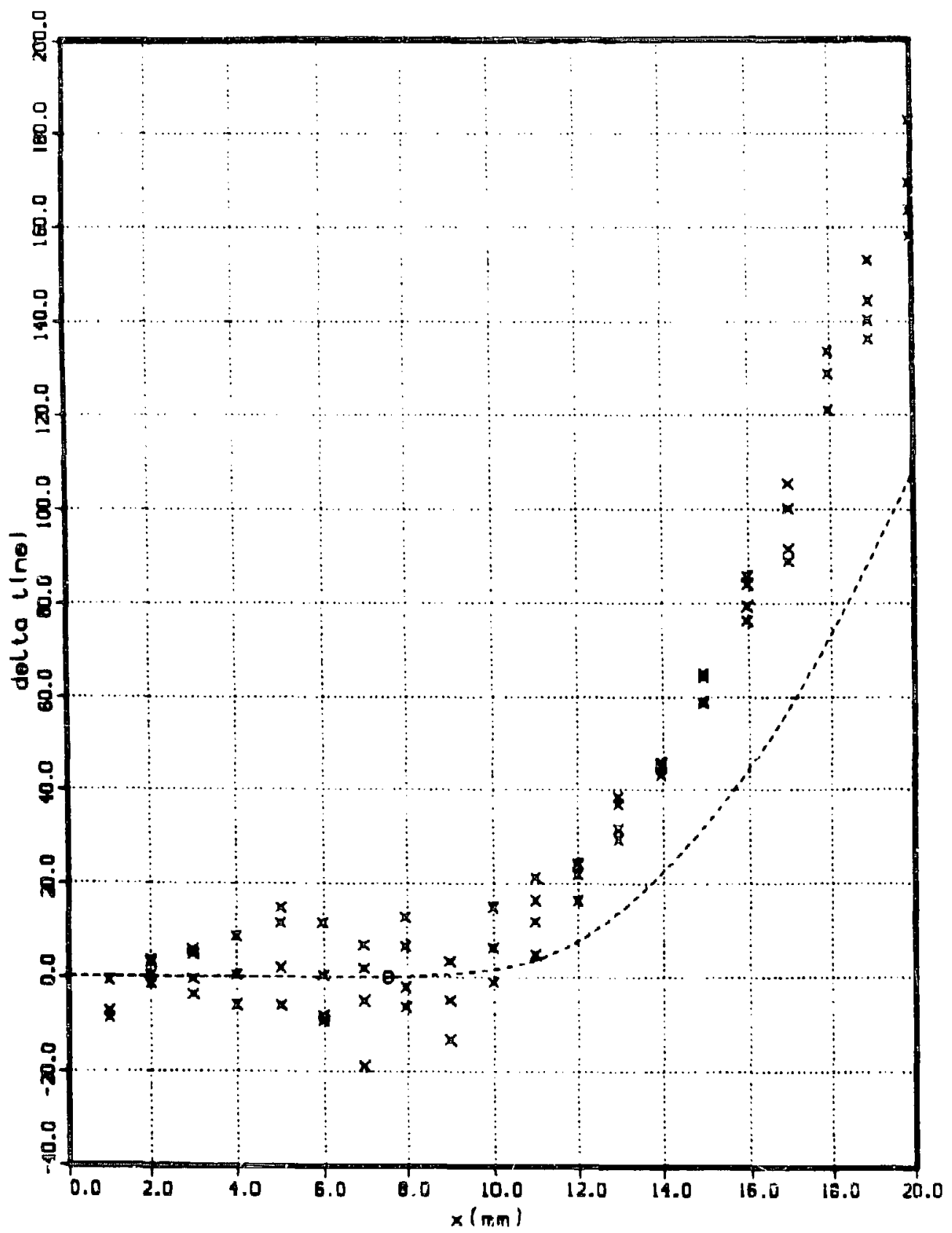

Figure 94: Comparison of the $\delta t$ data for RS-2302 and calculational results from the code SHKSJB. The dashed line is the $\delta t$ curve from the calculation. Parameters for the calculational model and parameters for the data analysis are listed in table 30. 


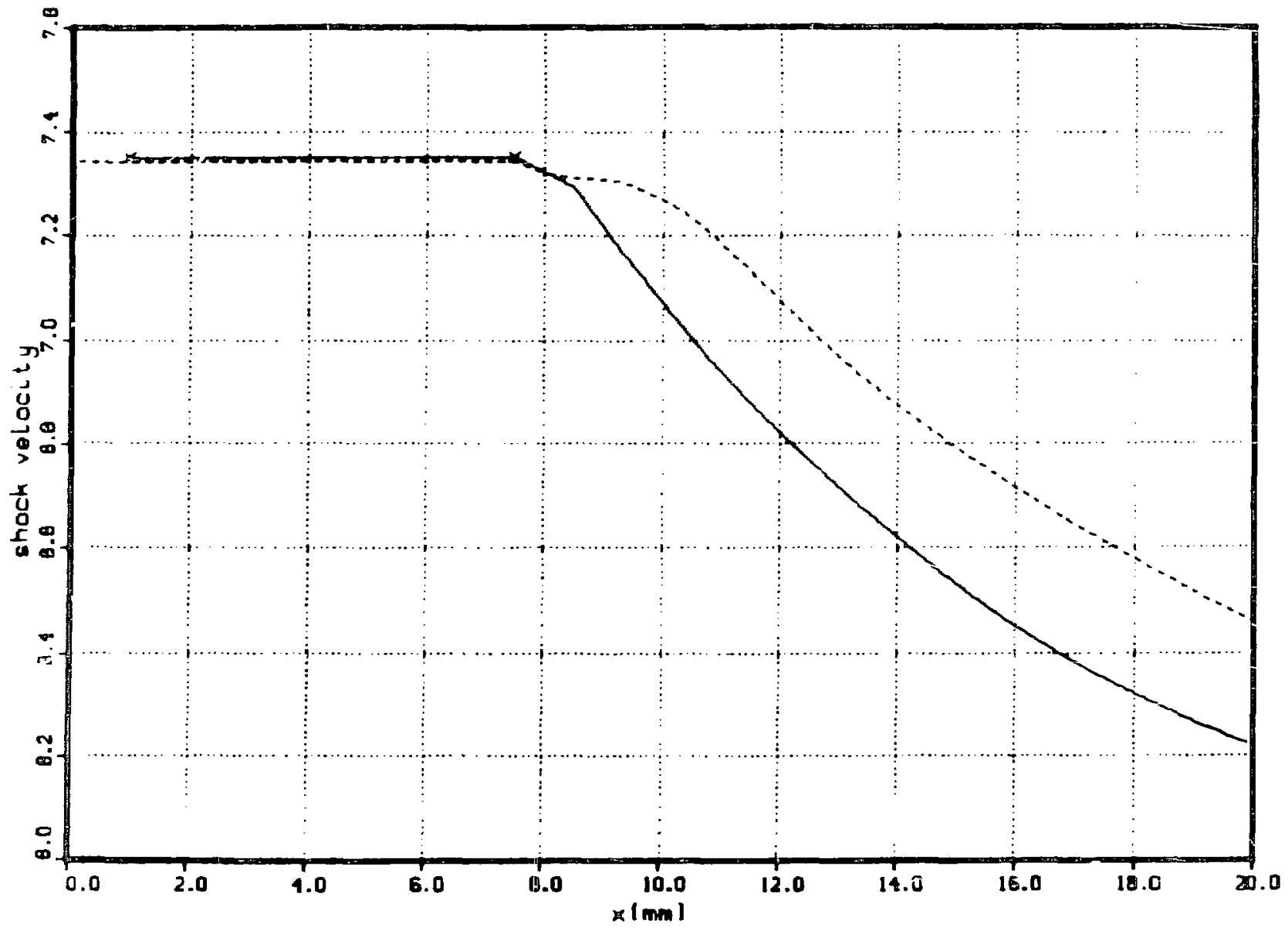

Figure 95: A comparison of the shock velocity from the code calculation and the shock velocity curve Irom a fit to the data for RS-2302. The sulid line is the velocity curve taken from the fit to the data. The corresponding $\delta t$ plots are in tigure 94 (see also table 30). 


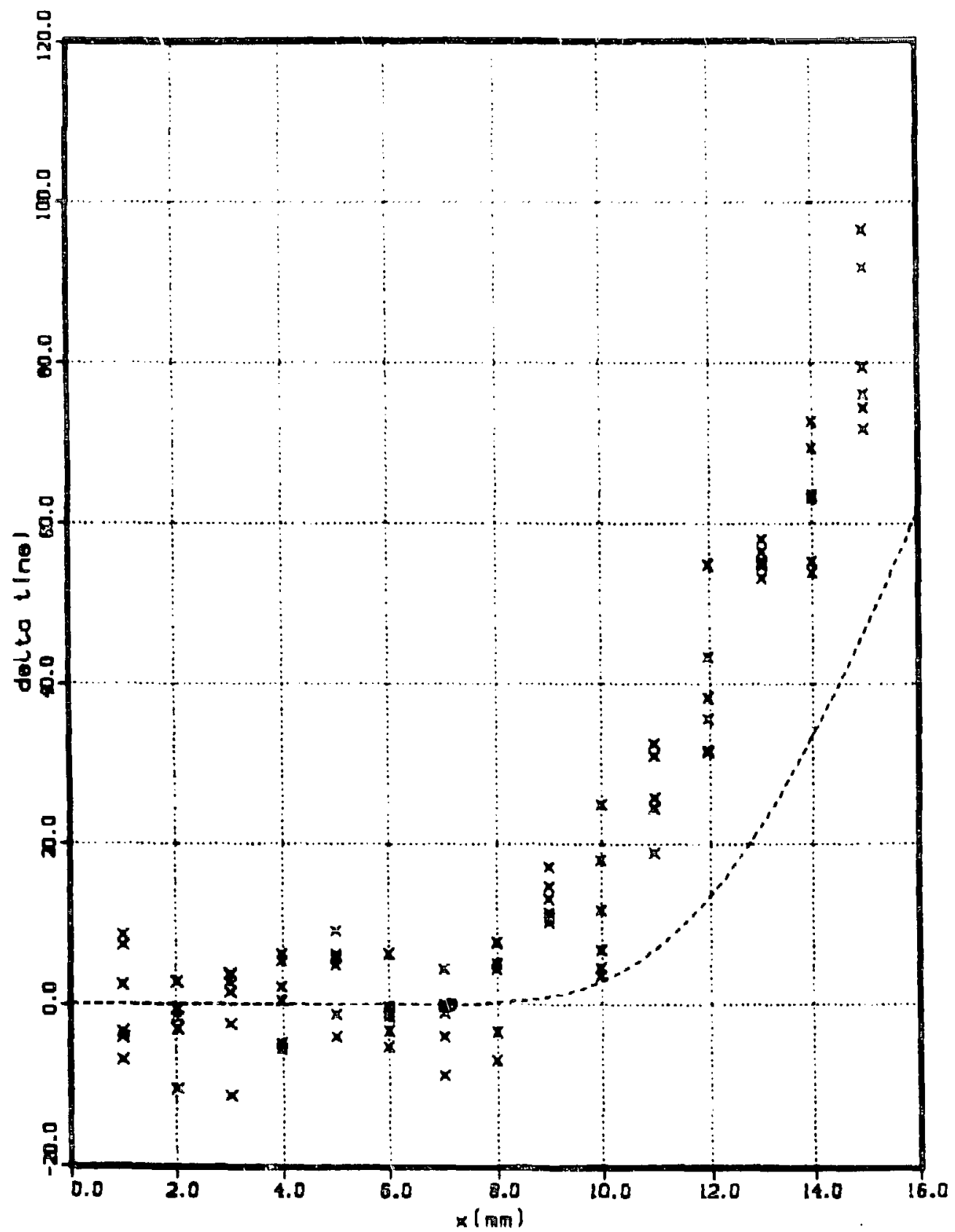

Figure 96: Comparison of the $\delta t$ data for RS-2315 and calculational results from the code SHKSJB. The dashed line is the $\delta t$ :urve from the calculation. Parameters for the calculational model and parameters for the data analysis are listed in table 31. 


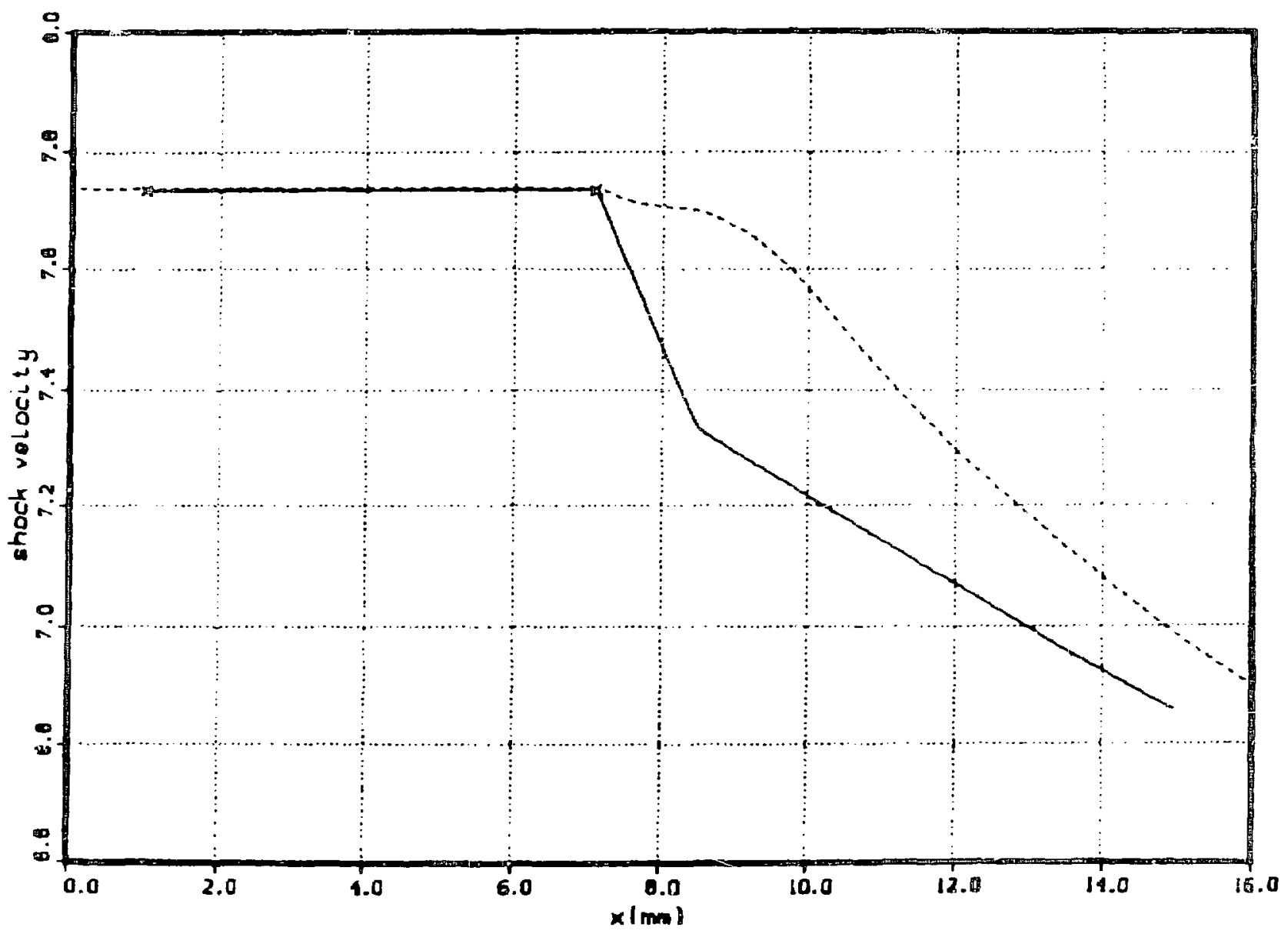

Figure 97: A comparison of the shock velocity from the code calculation and the shock velocity curve from a fit to the data for RS-2315. The solid line is the velocity curve taken from the fit to the data. The corresponding $\delta t$ plots are in Ggure 96 (see also table 31 ). 


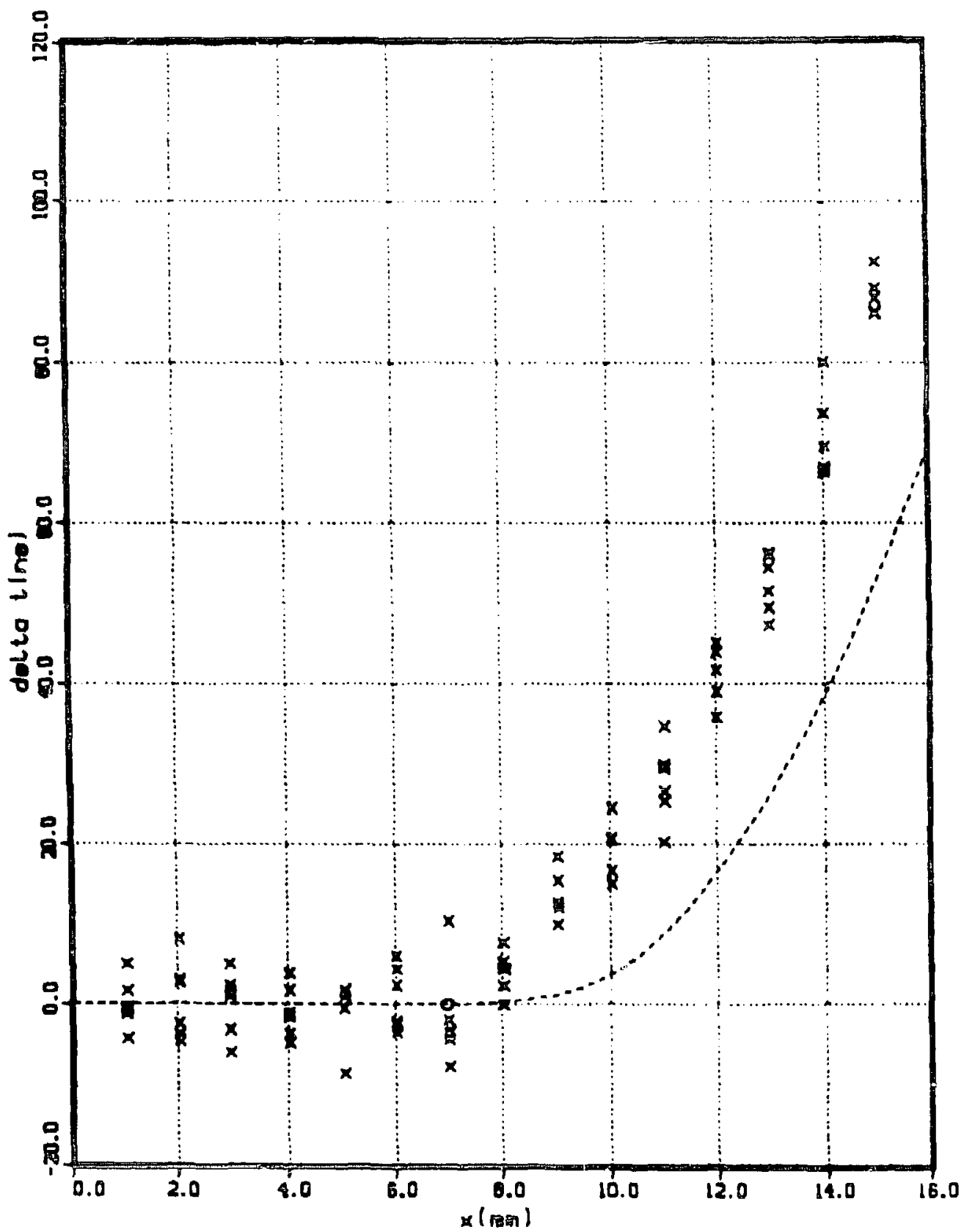

Figure 98: Comparison of the $\delta t$ data for RS-2309 and calculational results from the code SHKSJB. The dashed line is the $\delta t$ curve from the calculation. Parameters for the calculational model and parameters for the data analysis are listed in table 32. 


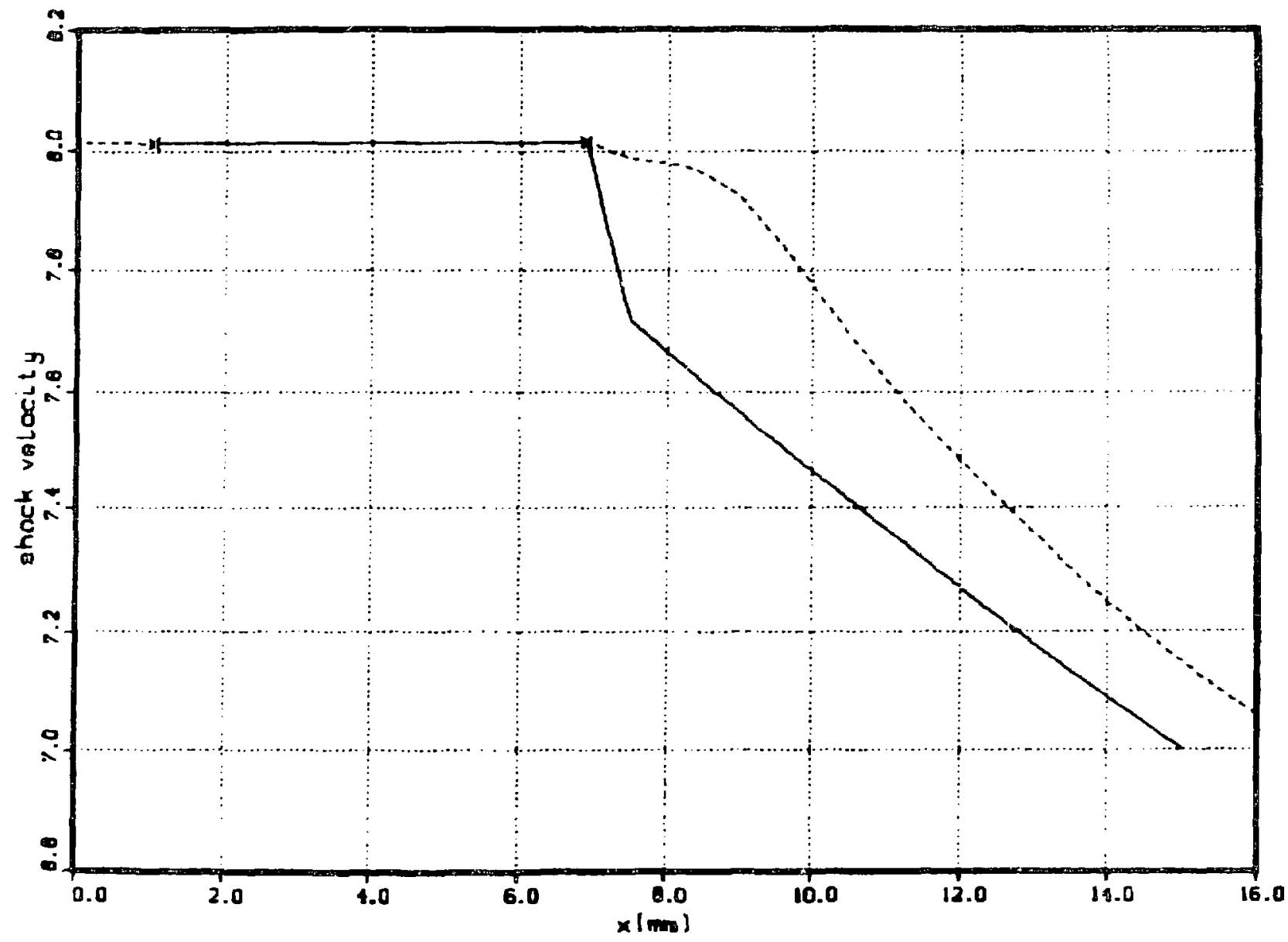

Figure 99: A comparison of the shock velocity from the code calculation and the shock velocity curve from a fit to the data for RS-2309. The solid line is the velocity curve taken from the fit to the data. The corresponding $\delta \ell$ plots are in figure 98 (see also table 32 ). 


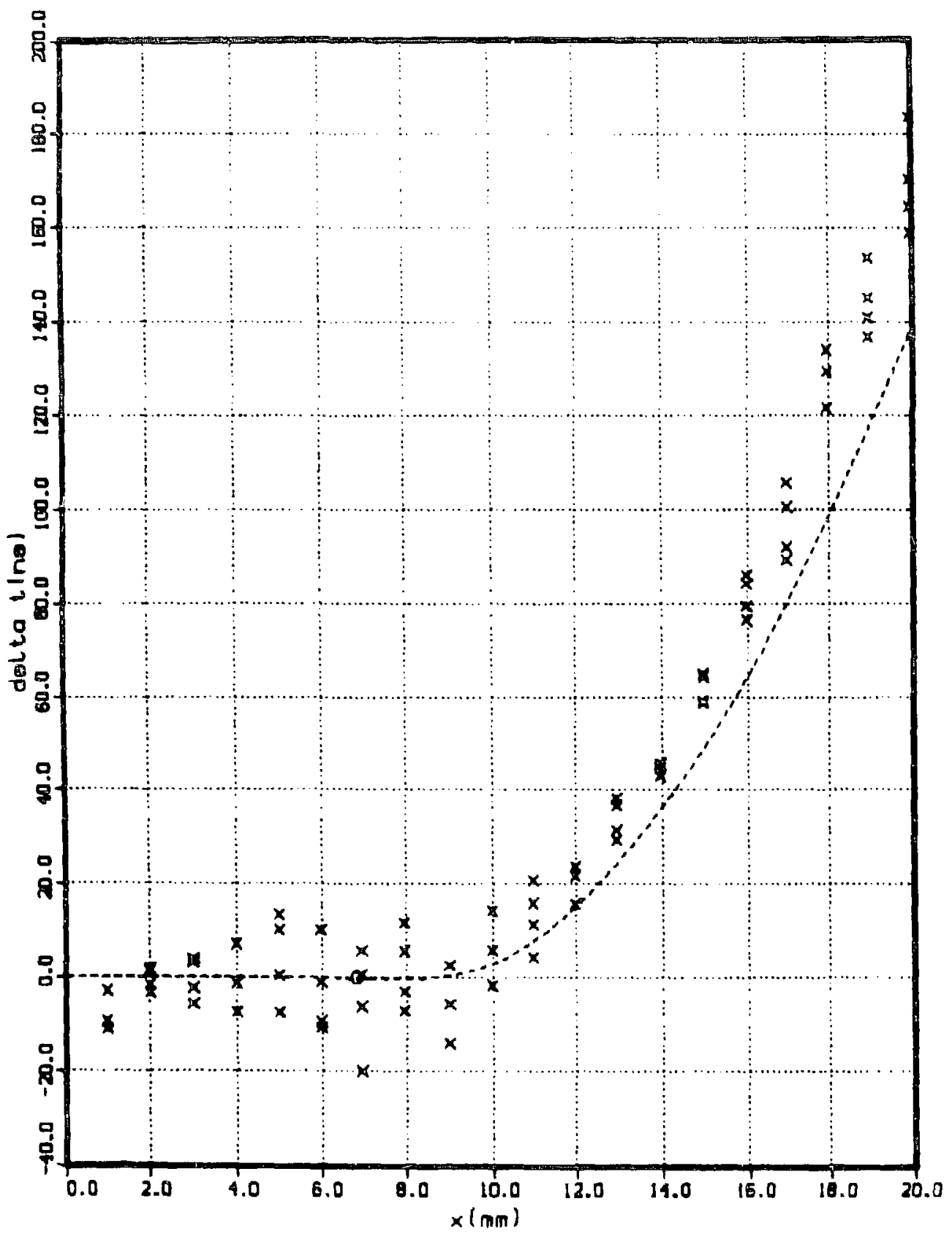

Figure 100: Comparison of the $\delta t$ data for RS-2302 and calculational results from the code SHKSJB. The dashed line is the $\delta t$ curve from the calculation. Parameters for the calculational model and parameters for the data analysis are listed in table 33. 


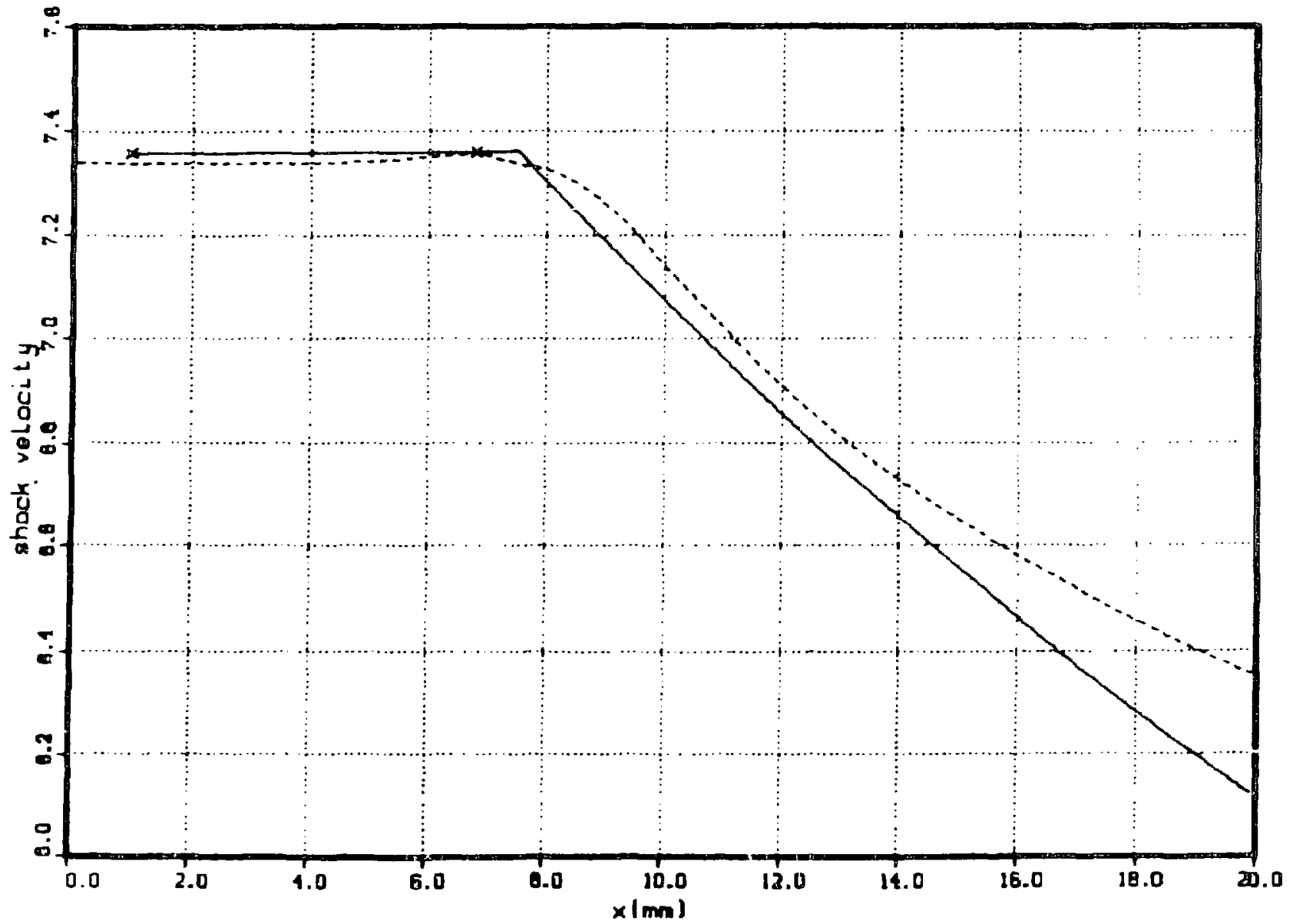

Figure 101: A comparison of the shock velocity from the code calculation and the shock velocity curve from a fit to the data for RS-2302. The solid line is the velocity curve taken from the fit to the data. The corresponding $\delta t$ plots are in figure 100 (see also table 33). 


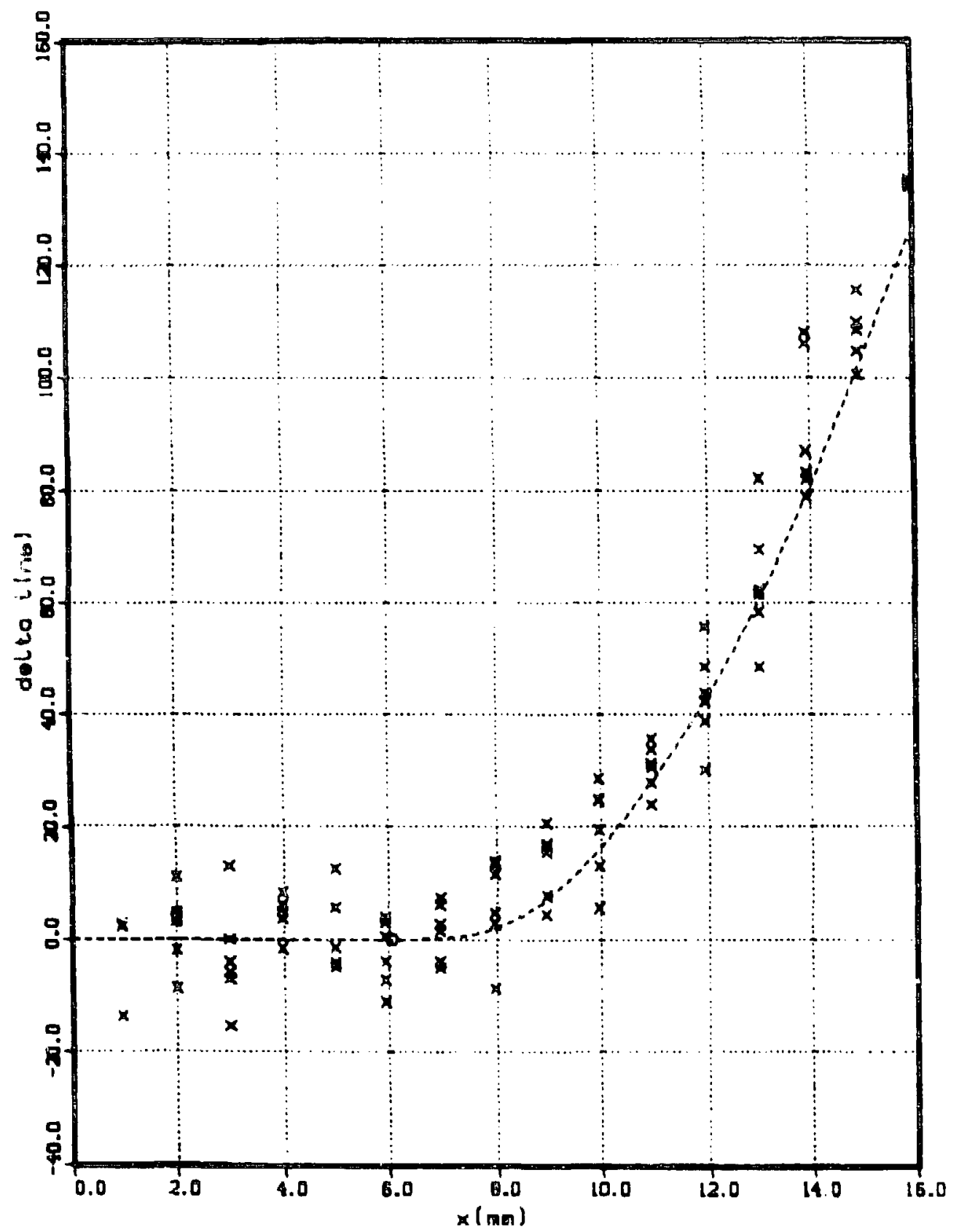

Figure 102: Comparison of the $\delta t$ data for RS-2306 and calculational results from the code SHKSJB. The dashed line is the $\delta t$ curve from the calculation. Parameters for the calculational model and parameters for the data analysis are listed in table 34. 


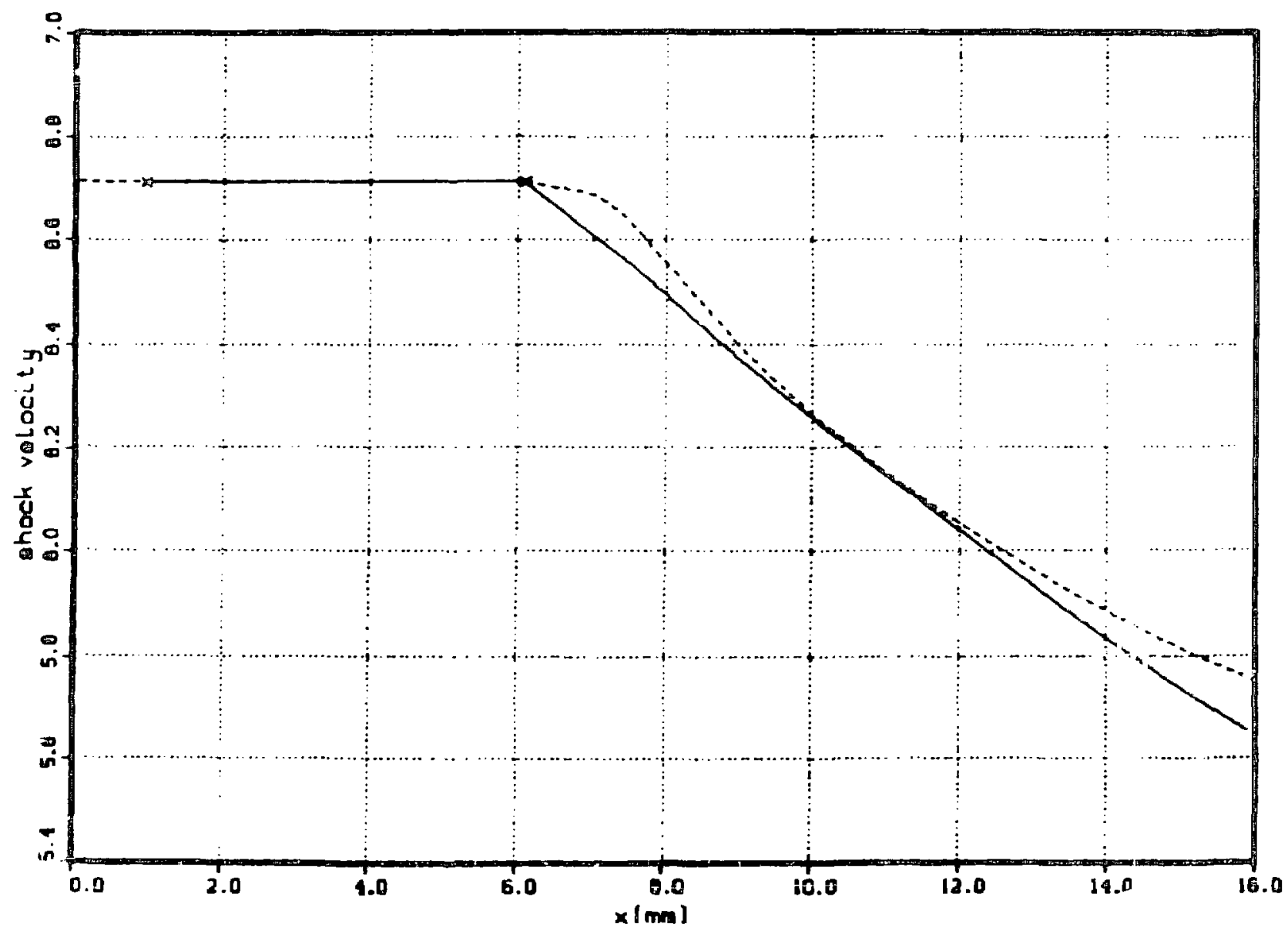

Figure 10S: A comparion of the shock velocity from the code calculation and the 1 xck velocity curve from a fit to the data for RS-2306. The solid line is the velocity curve taken frusl: the fit to the dala. The corresponding $6 t$ plots are in figure 102 (see also table 34). 


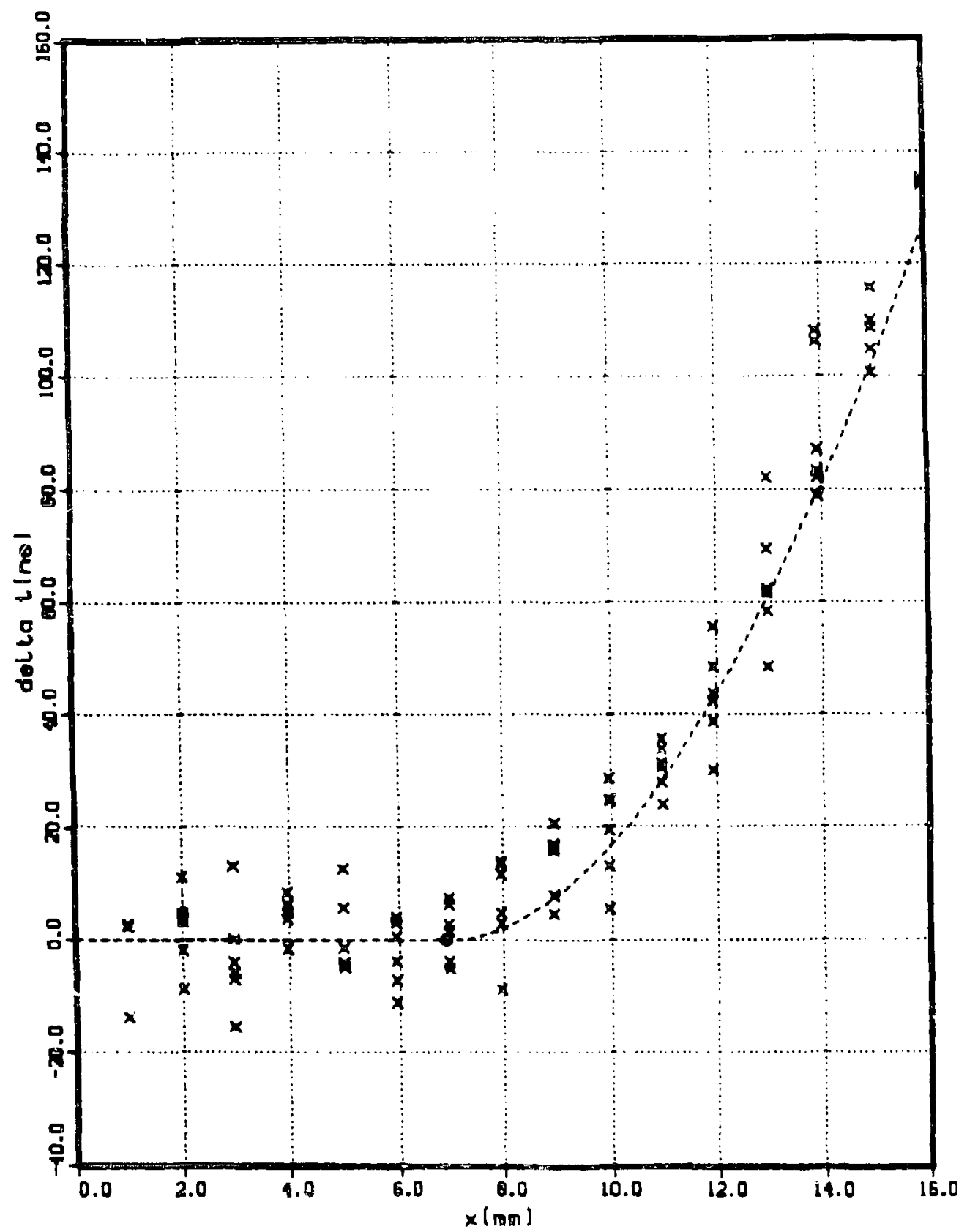

Figure 104: Comparion of the $\delta t$ data for RS-2306 and calculational results from the code SHKSJ日. The dashed line is the $6 t$ curve from the calculation. Parameters for the calculational model and parameters for the data analysis are listed in table 35. 


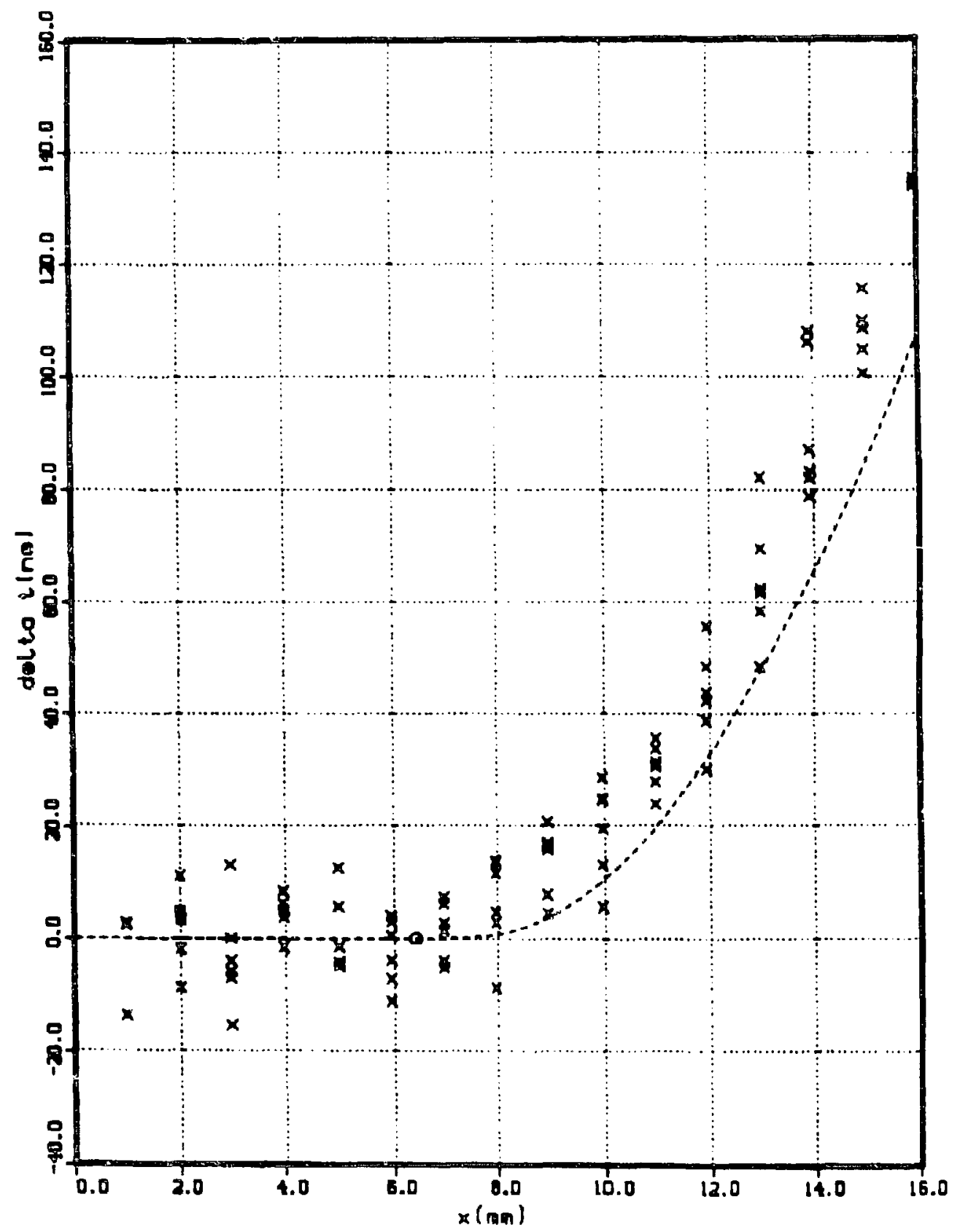

Figure 105: Comparison of the $\delta t$ data for RS-230 and calculational results from the code EFKSJB. The dashed line is the $\delta t$ curve from the calculation. Parameters for the calculational inodel and parameters for the data analysis are listed in table 36. 


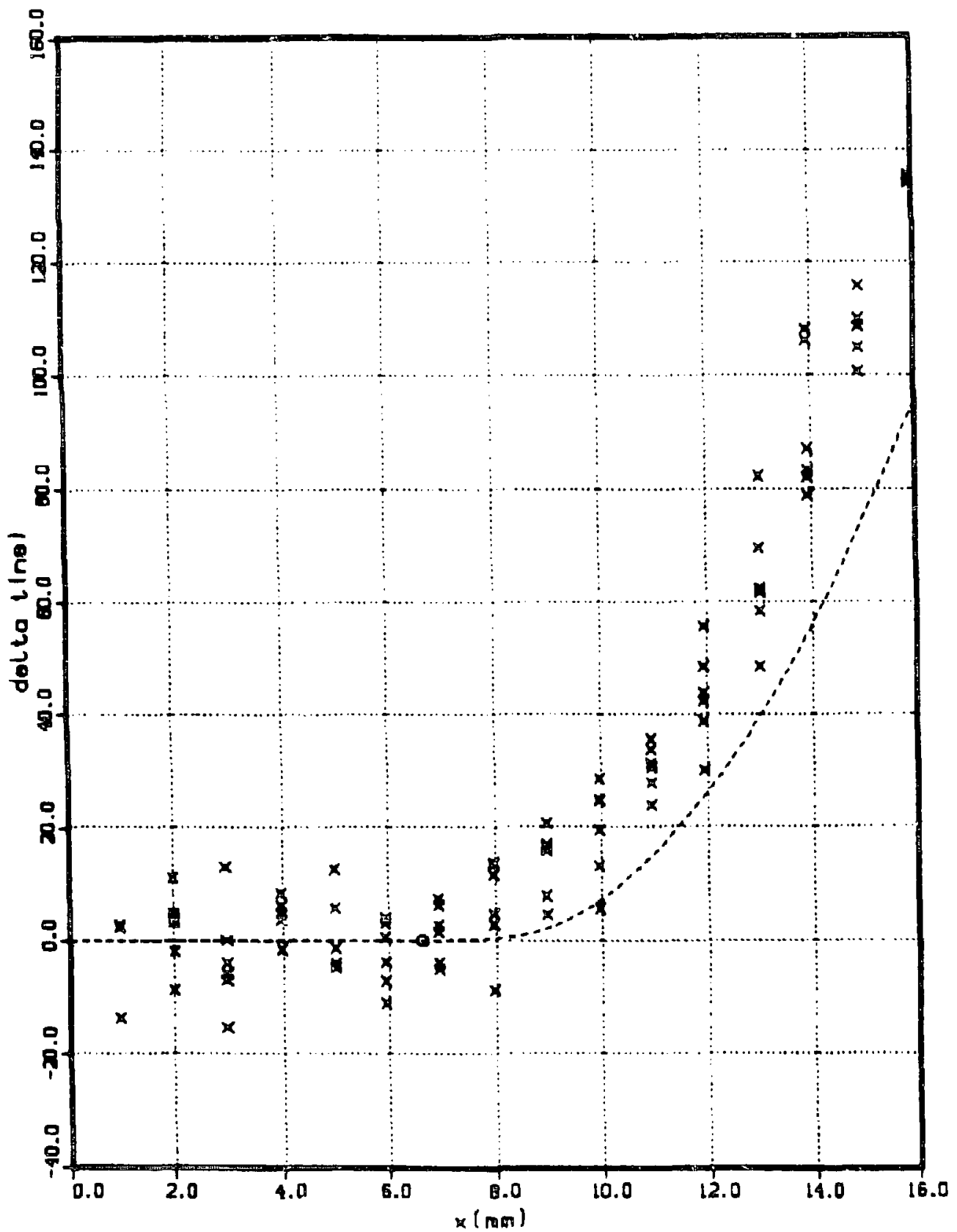

Figure 108: Comparison of the $\delta t$ data for RS-2306 and calculational regults from the code SHKSJB. The dashed line is the $\delta t$ curve from the calculation. Parameters for the calculational model and parameters for the data analysis are listed in table 37. 


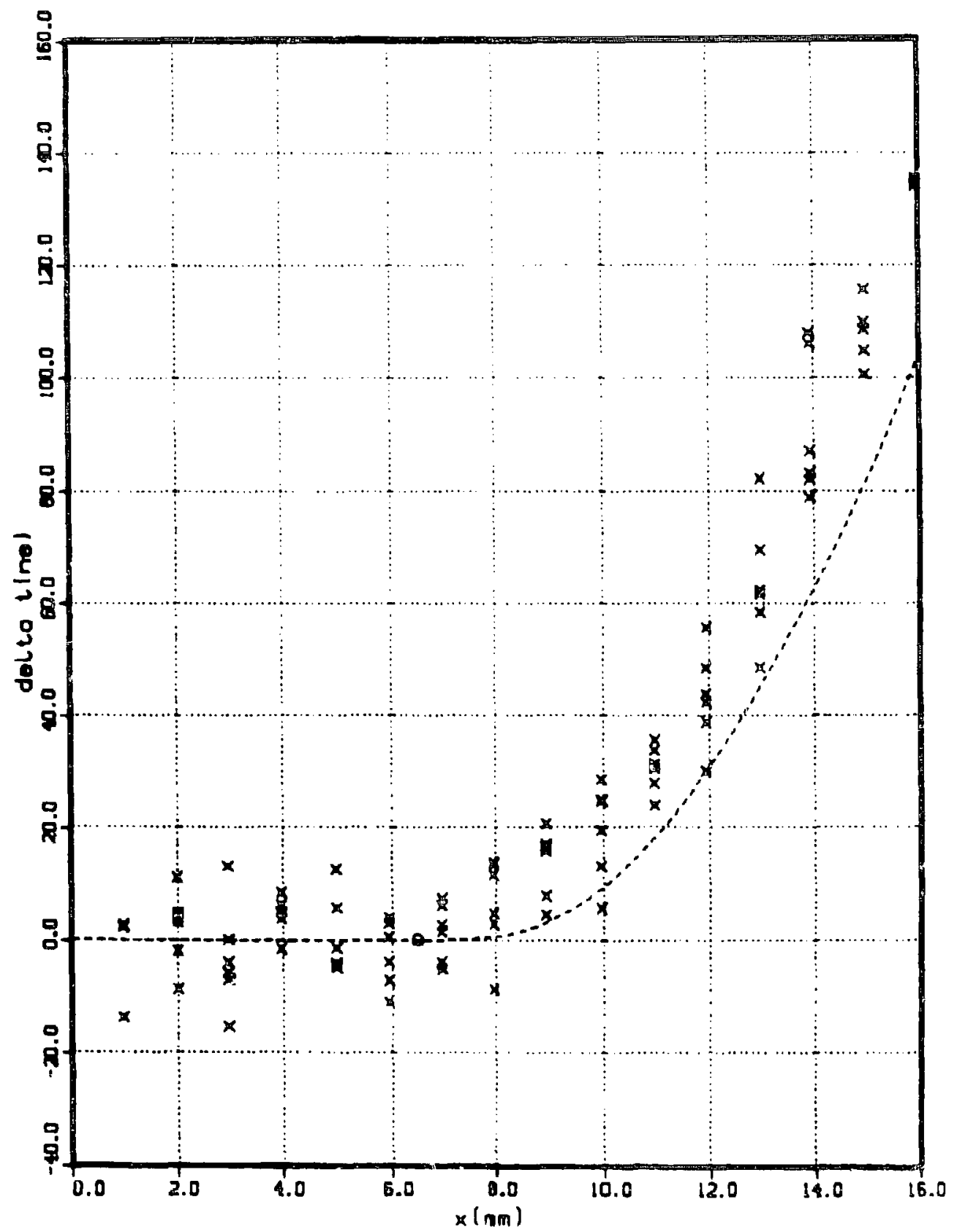

Figure 107: Comparison of the $\delta t$ date for RS-2308 and calculational results ftum the code SHKSJB. The dashed line is the $\delta t$ curve from the calculation. Parameter for the calculational model and parameters for the data analysis are listed in table 38 . 


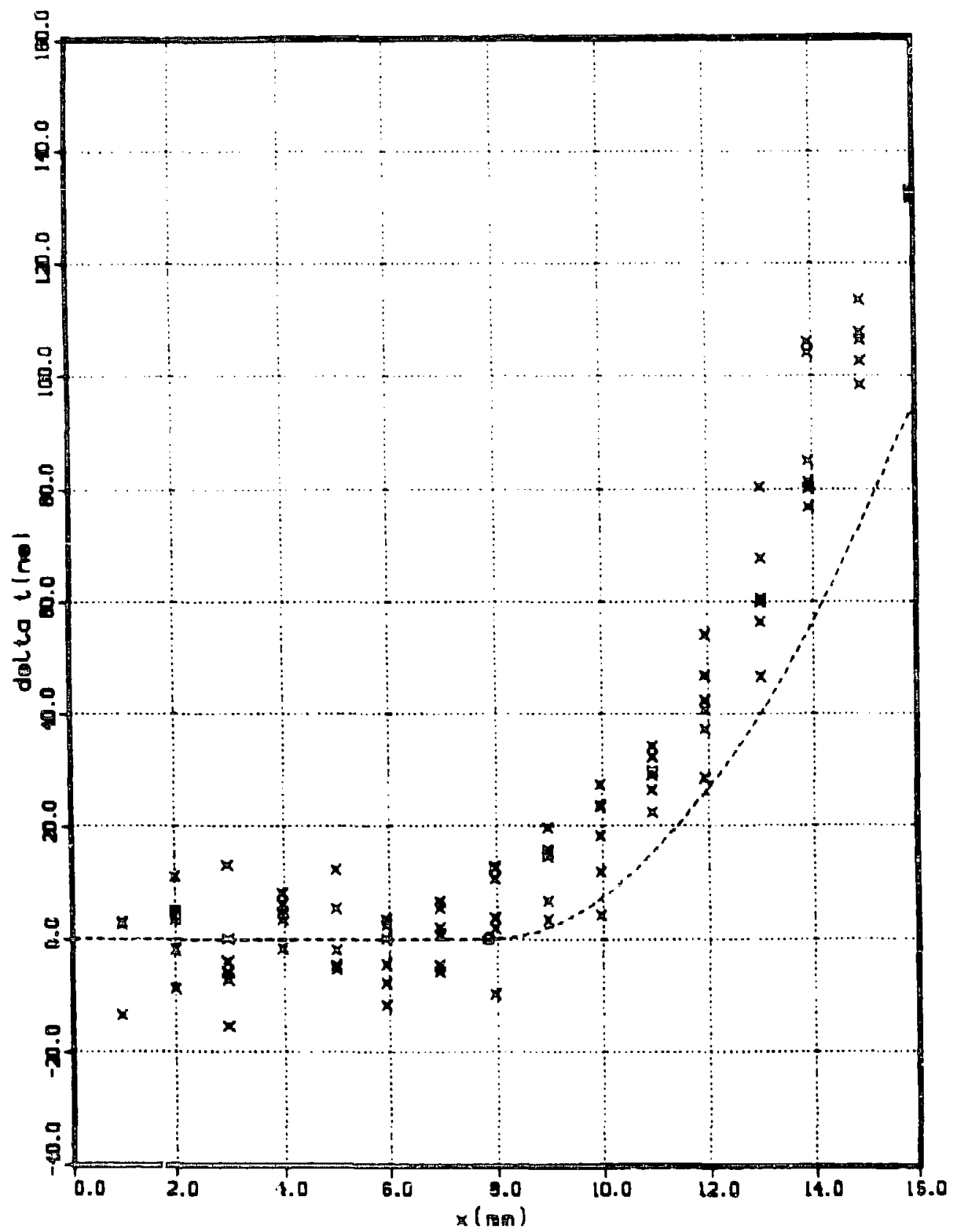

Figure 108: Comparison of the $\delta t$ data for RS-2306 and calculational results from the code SHKSJB. The dashed line is the $\delta t$ curve from the calculation. Parameters for the calculational model and parameters for the data andysis are listed in table 39. 


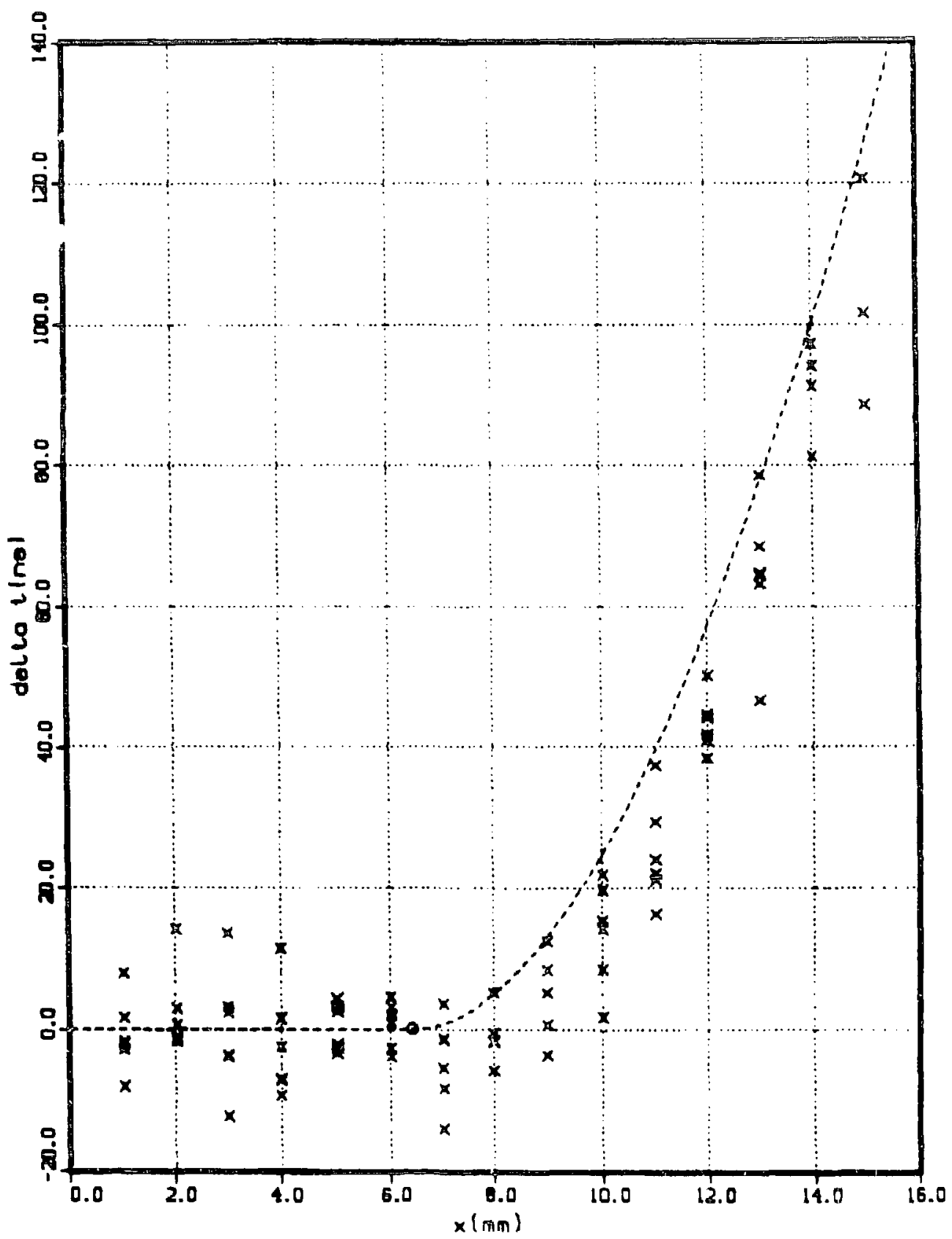

Figure 109: Comparison of the $\delta t$ data for RS-2314 and calculational results from the code SHKSJB. The dashed line is the $\delta t$ curve from the calculation. Parameters for the calculaticnal model and parameters for the data analysis are listed in table 40. 


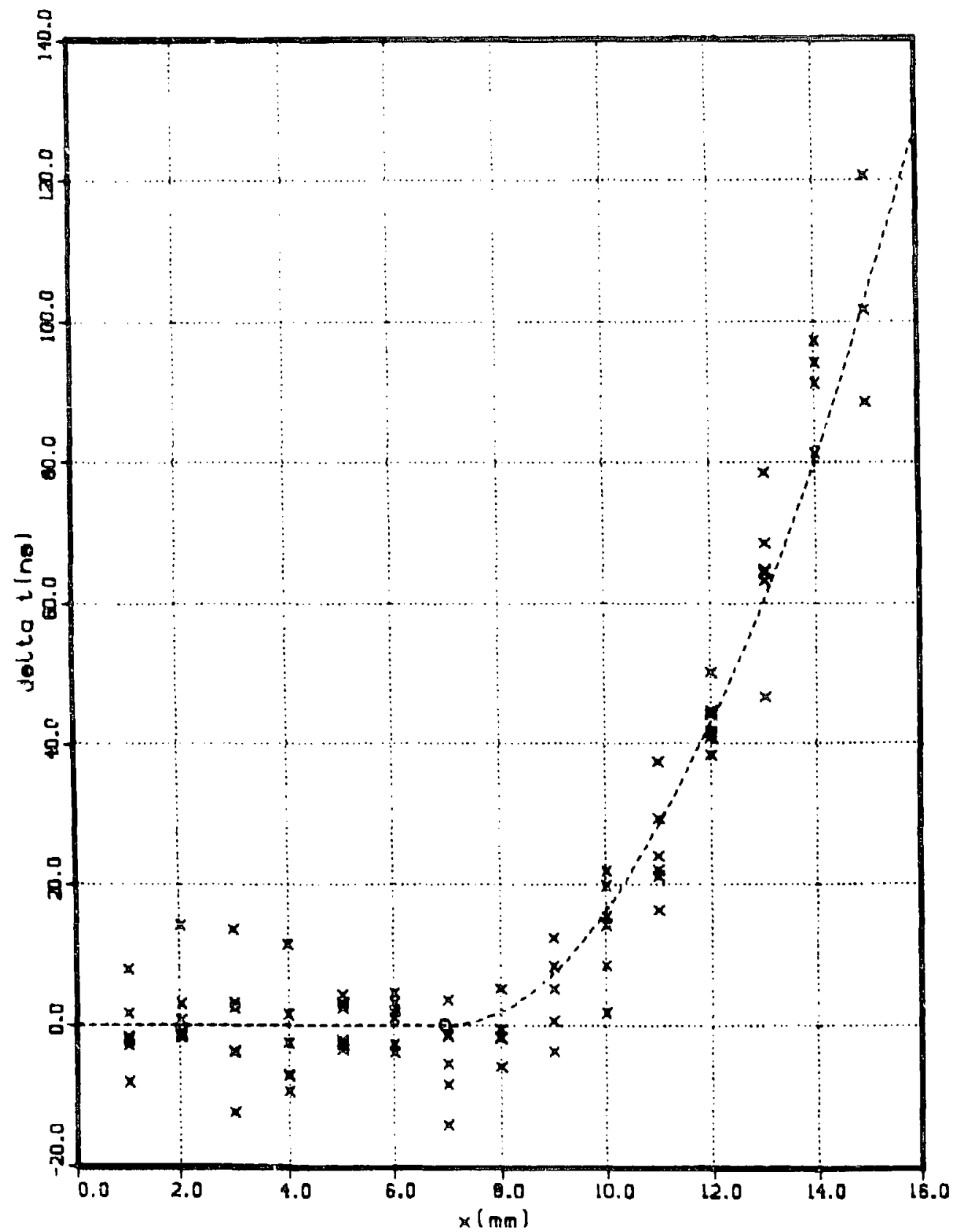

Figure 110: Comparison of the $\delta t$ data for RS-2314 and calculational results from the code SHKSJB. The dashed line is the $\delta t$ curve from the calculation. Parameters for the calculational model and parameters for the data analysis are listed in table $\mathbf{4 1 .}$ 


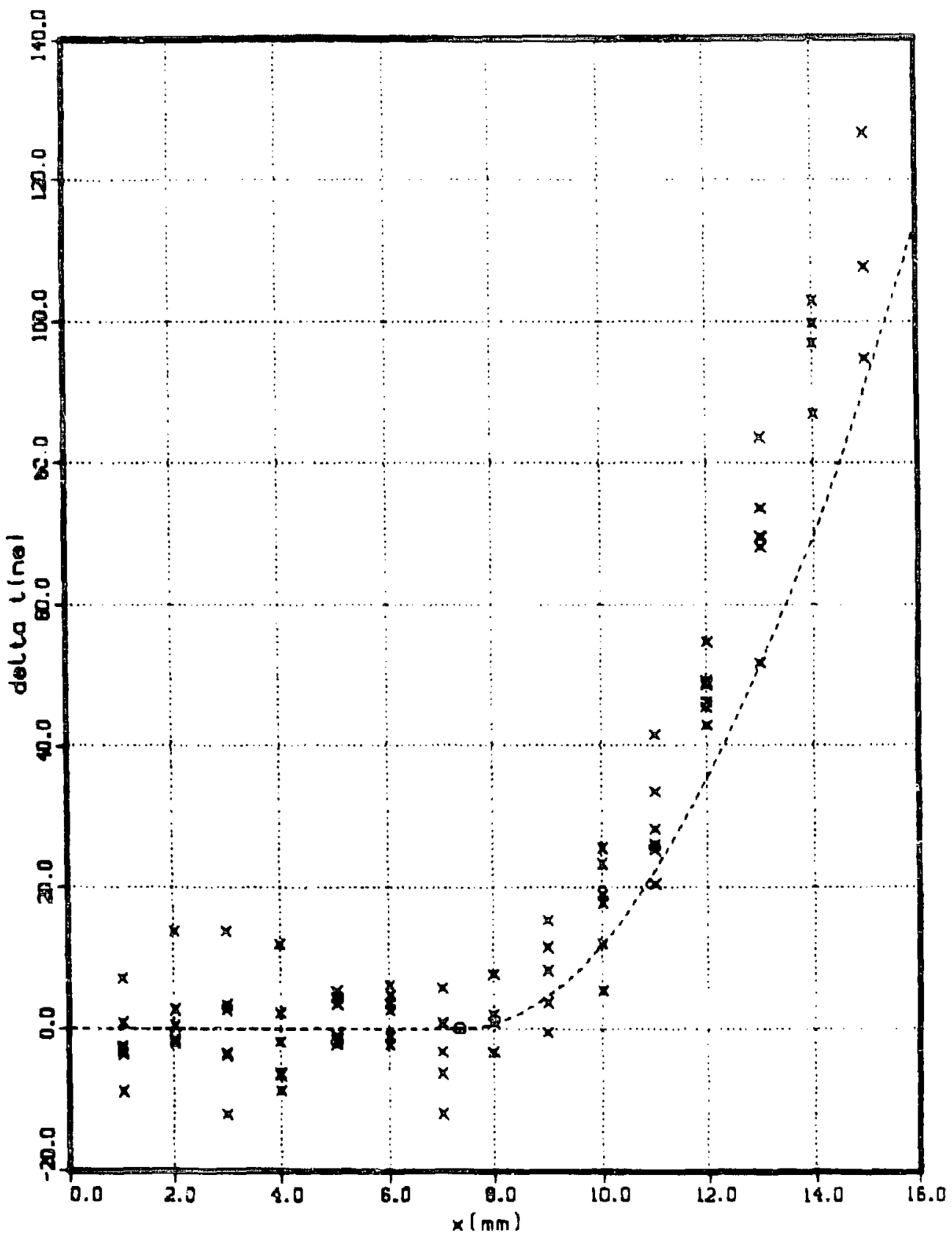

Figure 111: Comparison of the $\delta t$ data for RS-2314 and calculational resulta from the code SHKSJB. The dashed line is the $\delta t$ curve from the calculation. Parameters for the calculational model and parameters for the data analysis are listed in table $i 2$. 


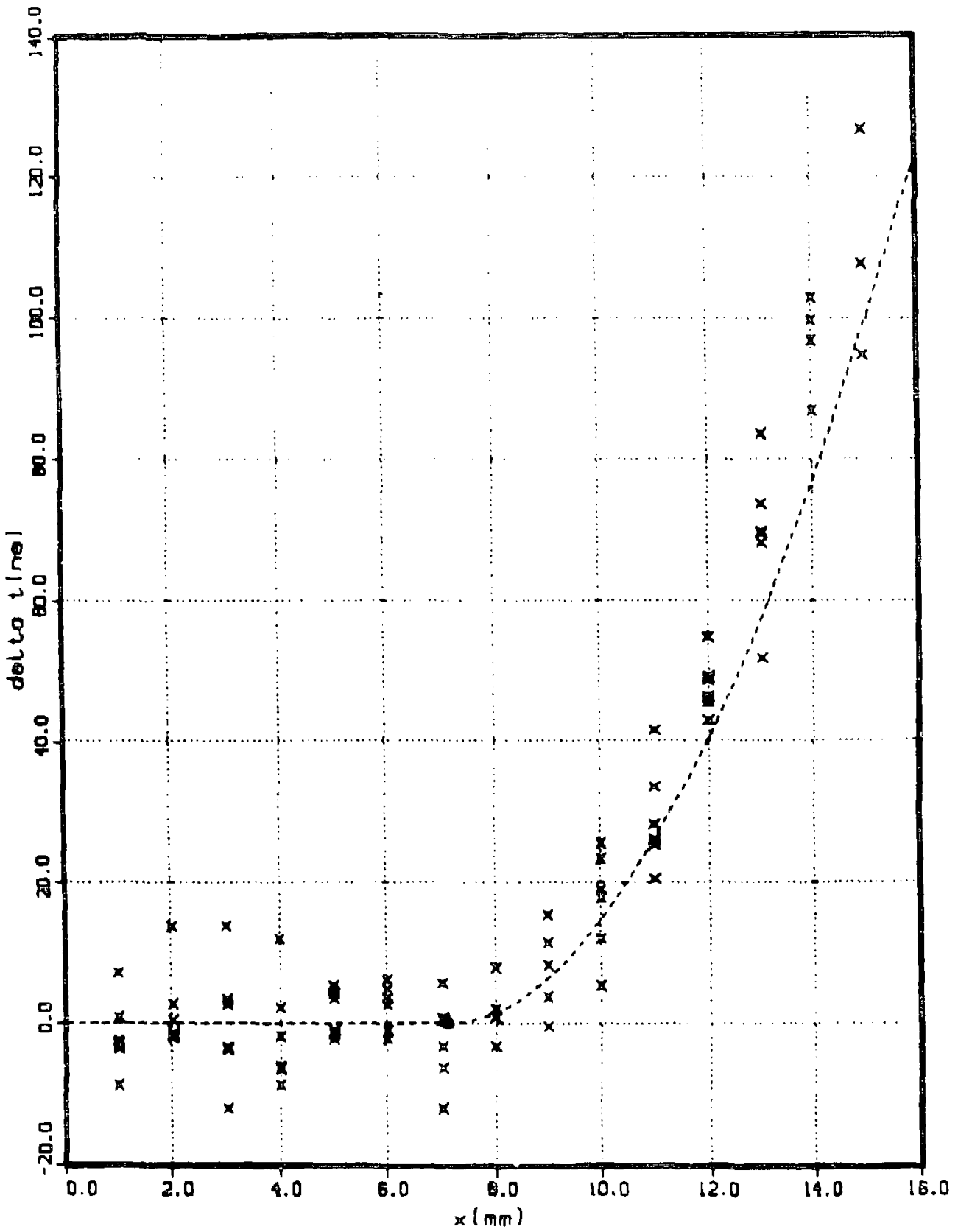

Figure 112: Comparion of the $\delta t$ dat a for RS-2314 and calculational results from the code SHKSJB. The dashed line is the $\delta t$ curve from the calculation. Parameters for the calculational model and parameters for the data analysis are listed in table 43. 


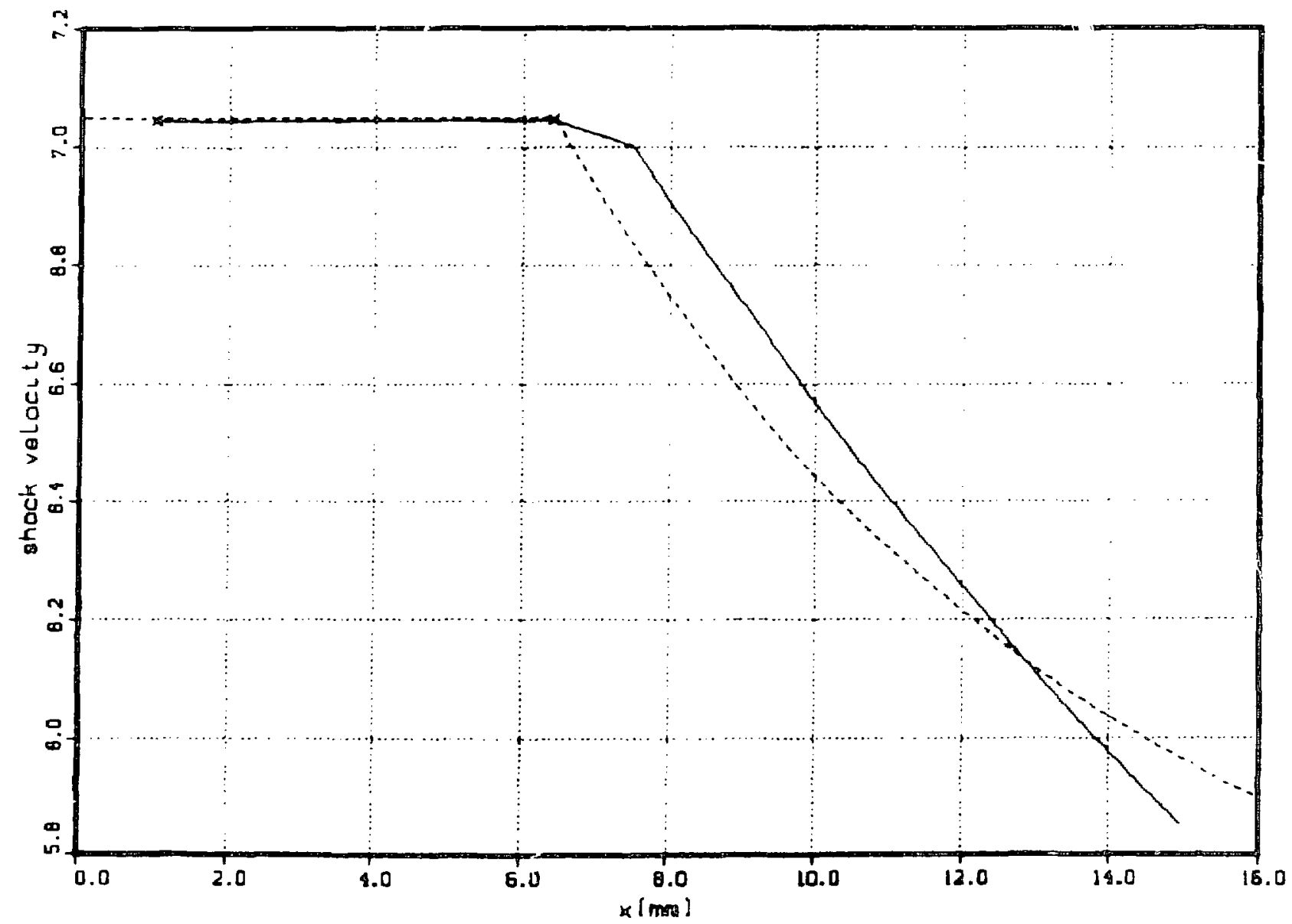

Figure 113: A comparison of the shock velocity from the code calculation and the shuck velocily curve from a fit to the data for RS-2314. The solid line is the velocity curve taken from the fit to the data. The corresponding $\delta t$ plots are in fgure 109 (see also table 40 ). 


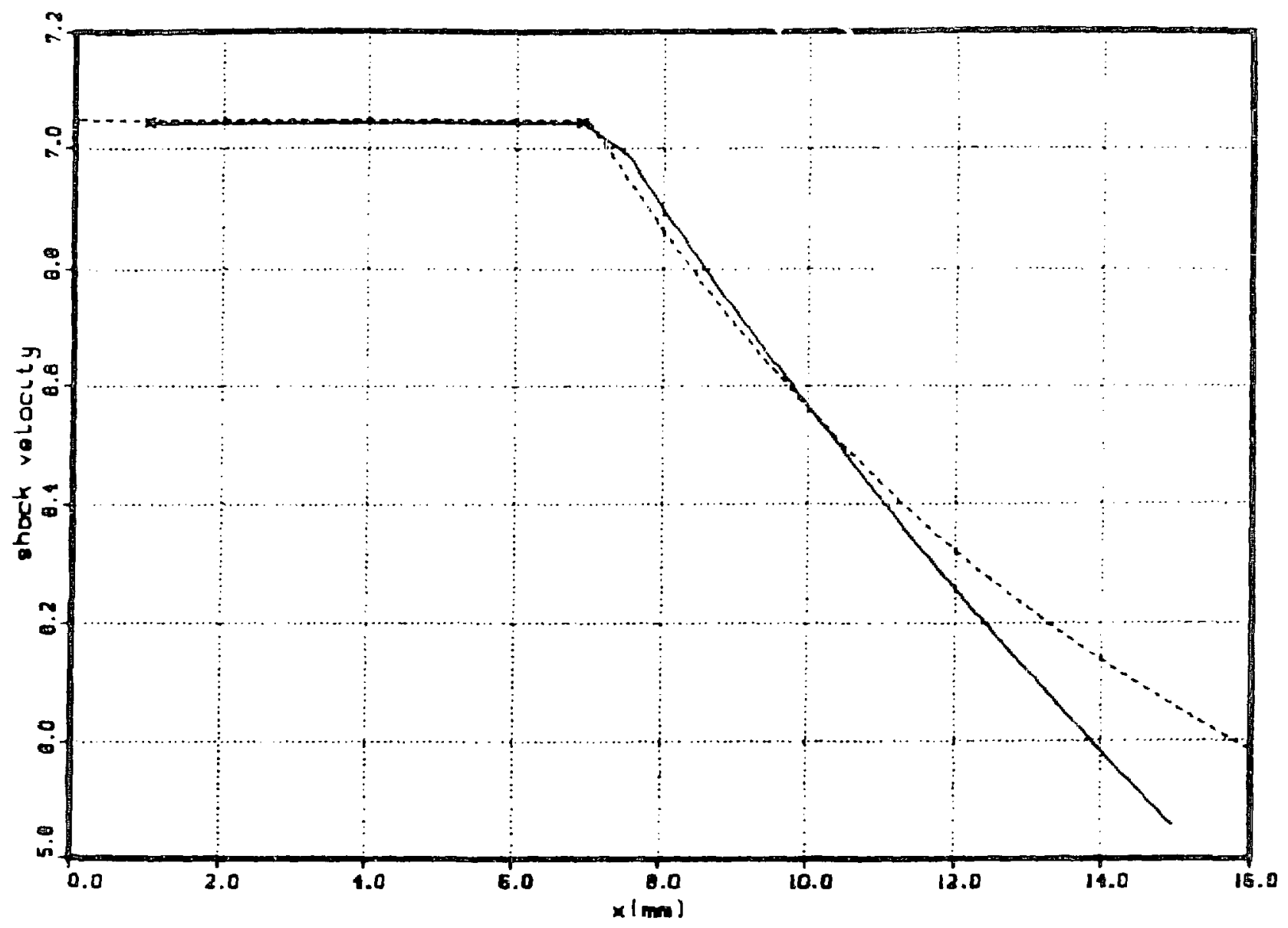

-1
0
0
8
3
0
0
-1
-1

Figure 114: A comparison of the shock velocity from the code calculation and the shock velocity curve from a fit to the dala for RS-2314. The solid line is the velocily curve caken from the fit to the data. The corresponding $\delta t$ plots are in Ggure 110 (see also table 41). 


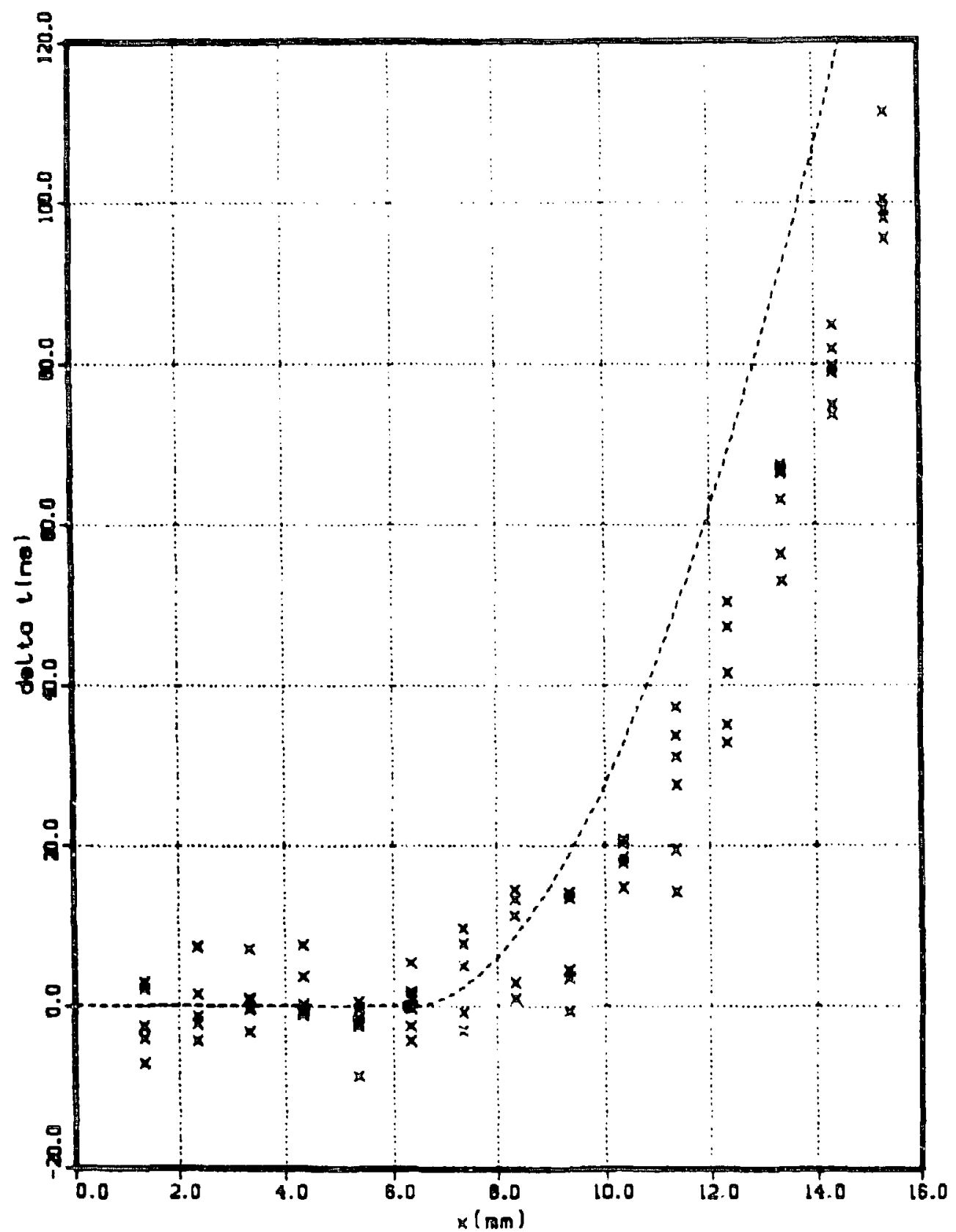

Figure 115: Comparion of the $\delta t$ data for RS-2308 and calculational results from the code SHKSJB. The dashed line is the $\delta$ t curve from the calculation. Parameters for the calculational model and parameters for the data analysis are listed in table 44. 




Figure 116: A comparison of the shock velocity from the code calculation and the shock velocity curve from a fit to the data for RS-2308. The solid line is the velocity curve taken from the fit to the data. The corresponding $\delta$ plots are in figure 115 (see also table 44). 


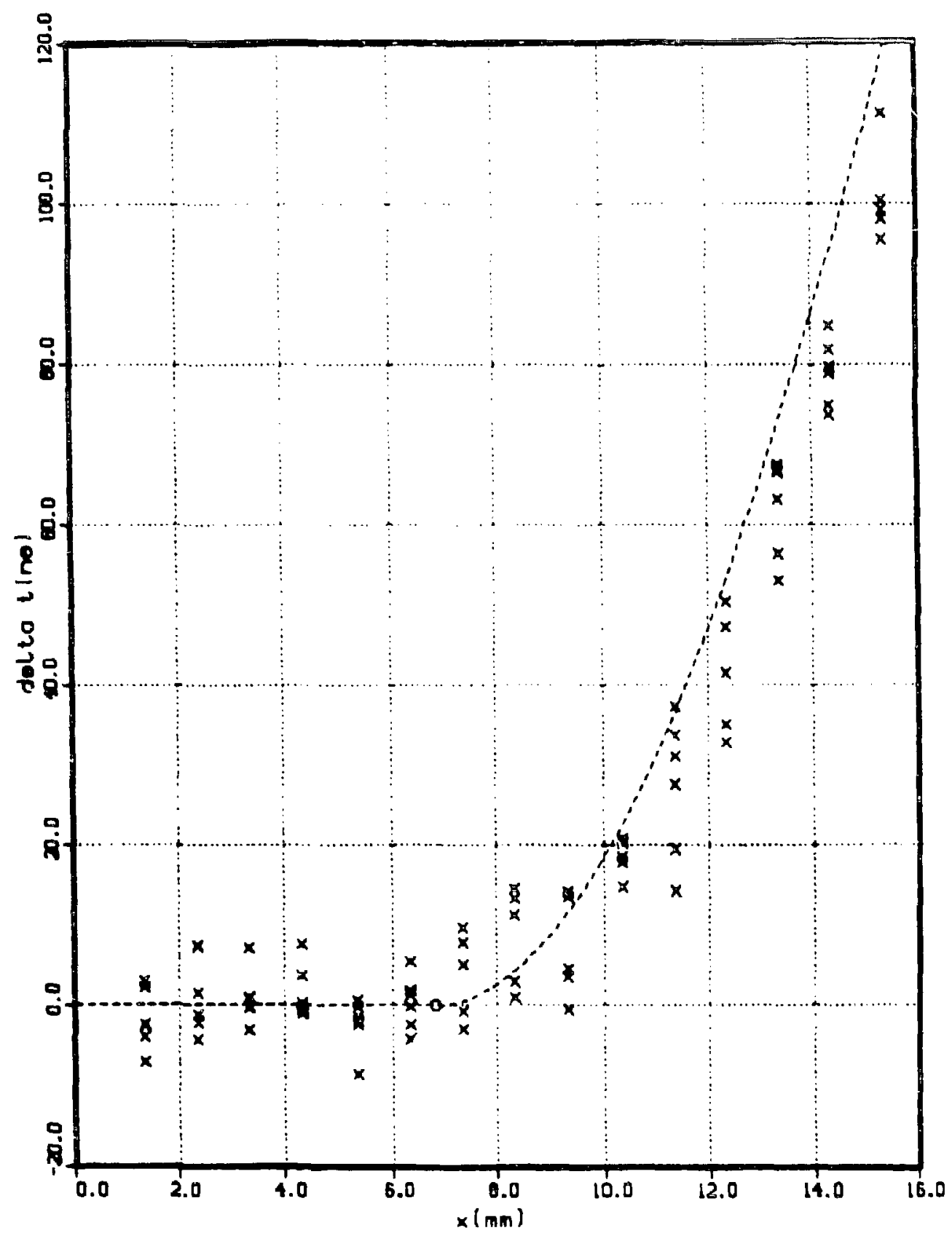

Fizure 117: Comparison of the $\delta t$ data for RS-2308 and calculationa! results from the code SHKSJB. The daghed line is the $\delta$ t curve from the calculation. Parameters for the calculational model and parameters for the data analysis are listed in table $\mathbf{4 5}$. 


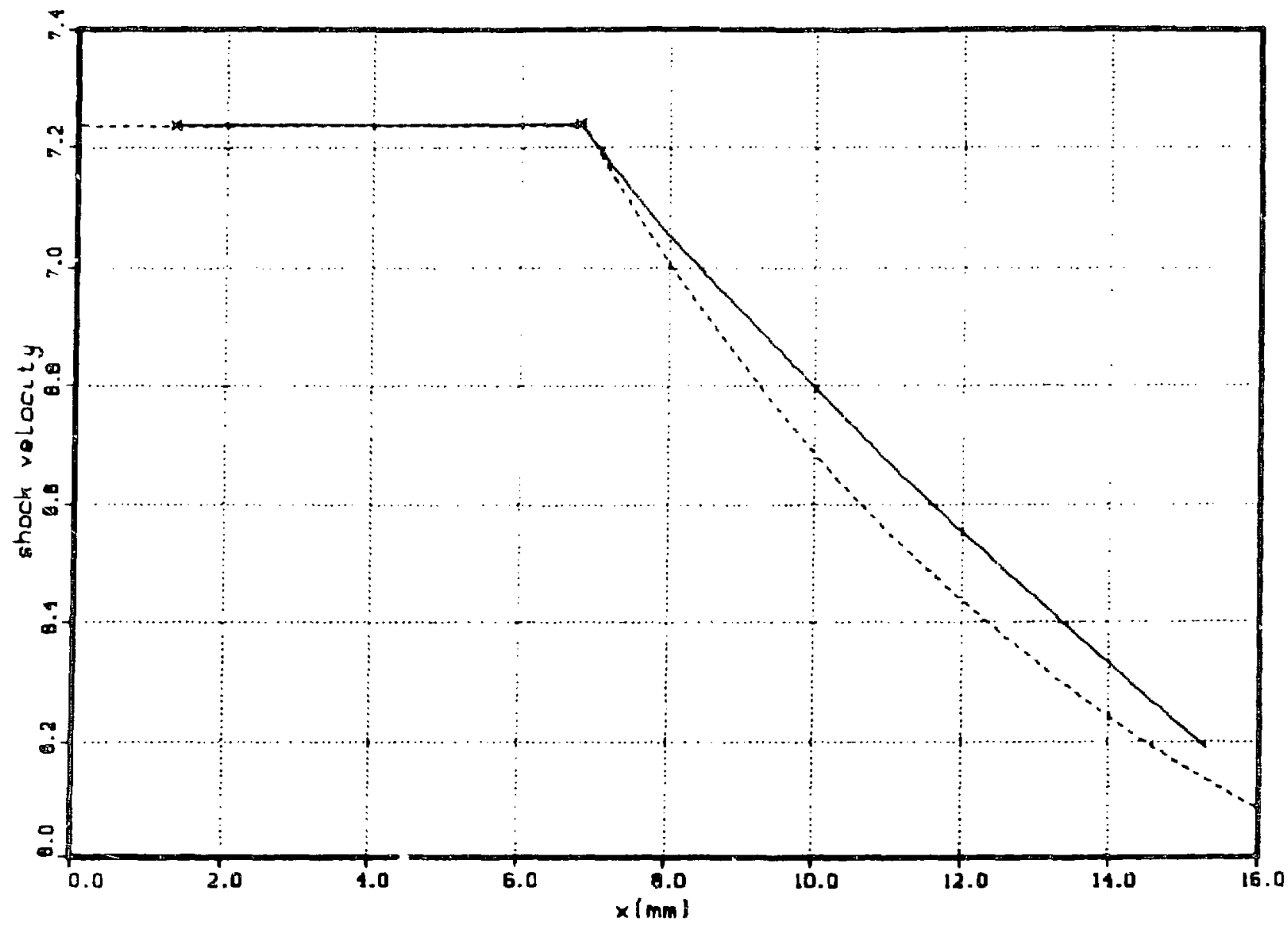

Figure 118: A comparison of the shock velocity from the code calculation and the shock velocity curve from a fit to the data for RS-2308. The solid line is the velocity curve taken from the fit to the data. The corresponding $\delta t$ plots are in figure 117 (see also table 45). 


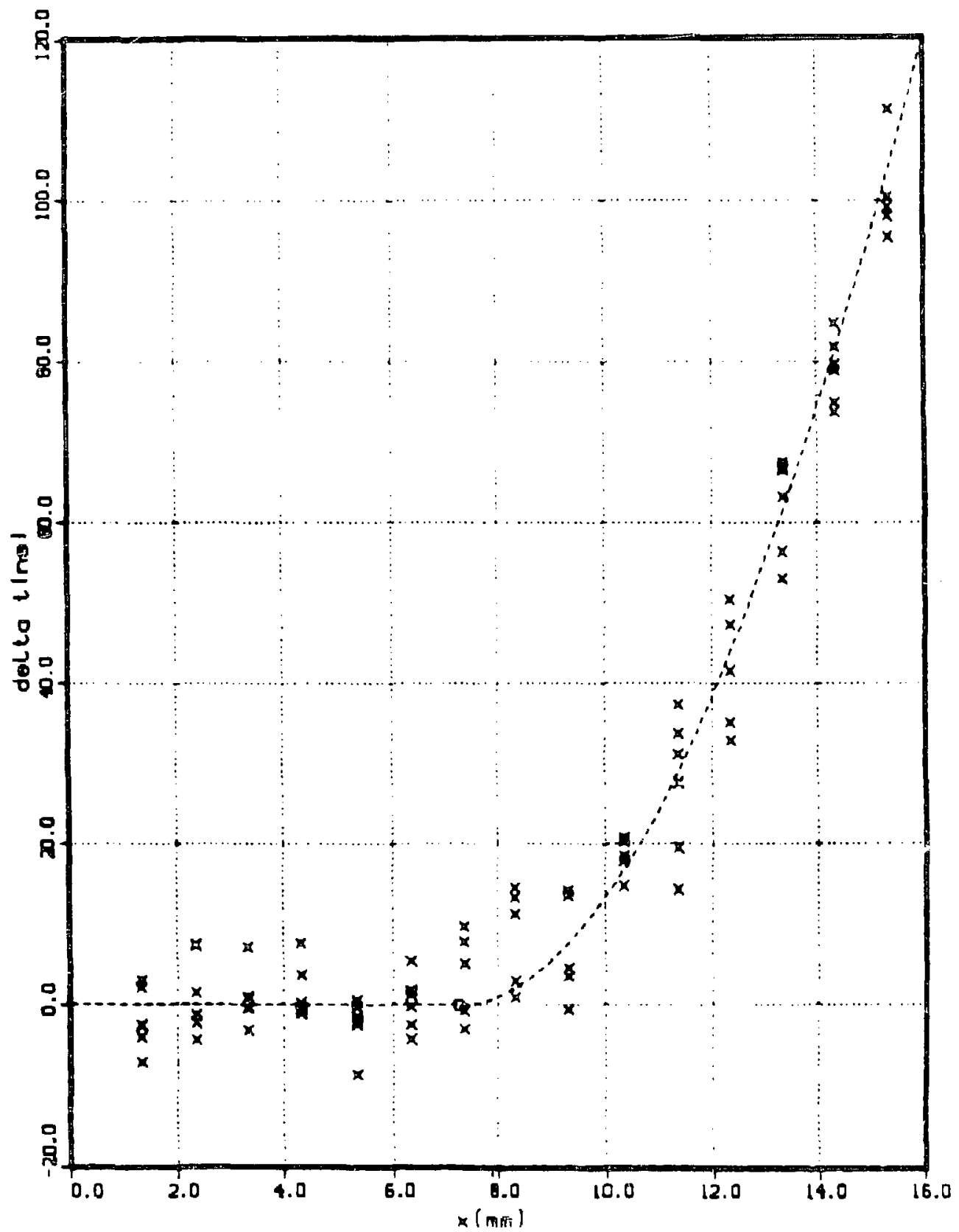

Figure 119: Comparion of the $\delta t$ data for RS-2308 and calculational results from the code SHKSJB. The dished line is the $\delta t$ curve from the calculation. Parameters for the calculational model and parameters for the data analysis are listed in table 46. 


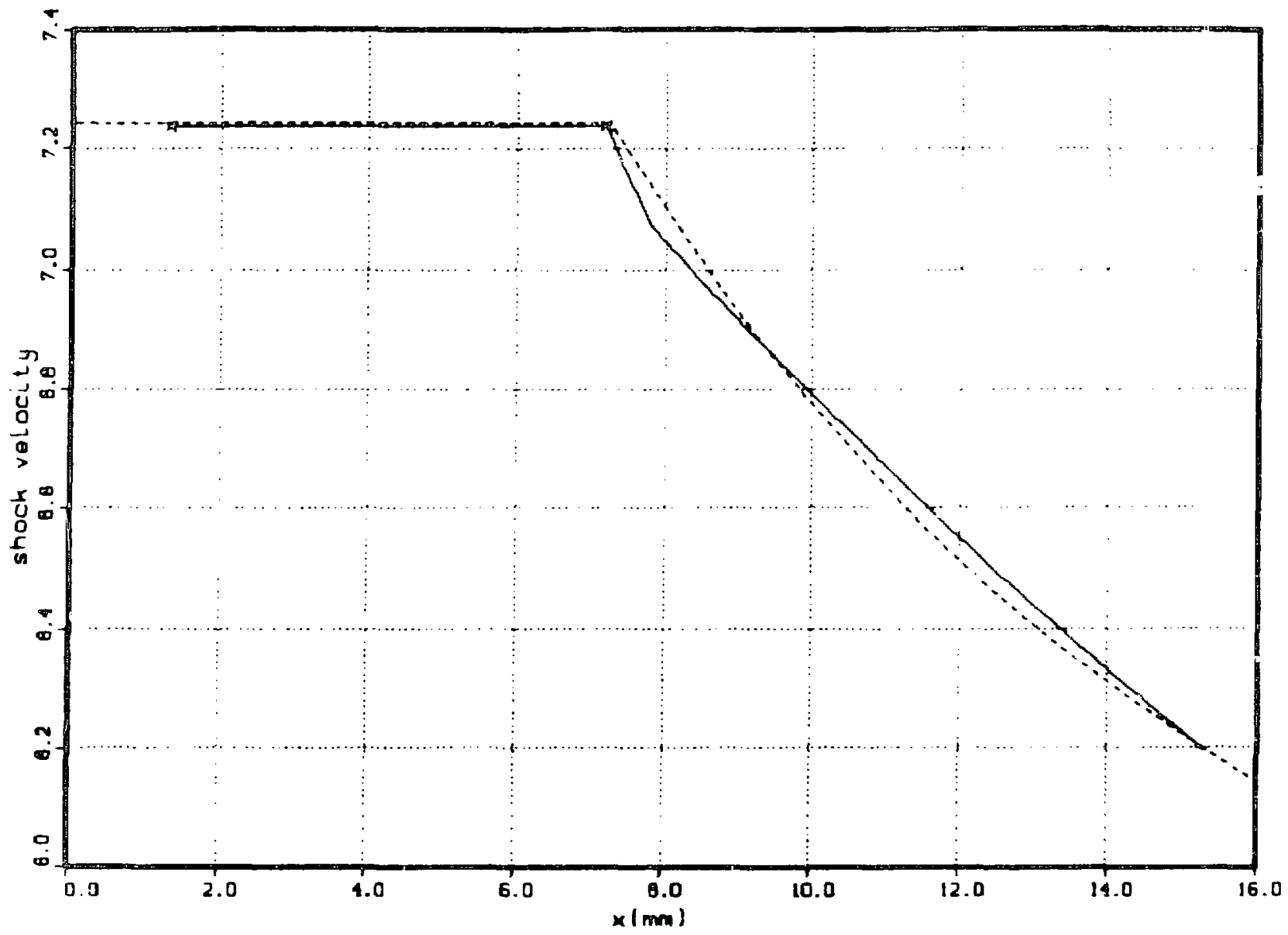

Figure 120: A comparison of the shock velocity from the code calculation and the shock velocity curve from a fit to the data for RS-2308. The solid line is the velocity curve taken from the fit to the data. The corresponding $\delta t$ plots are in figure 119 (see also table 46) 


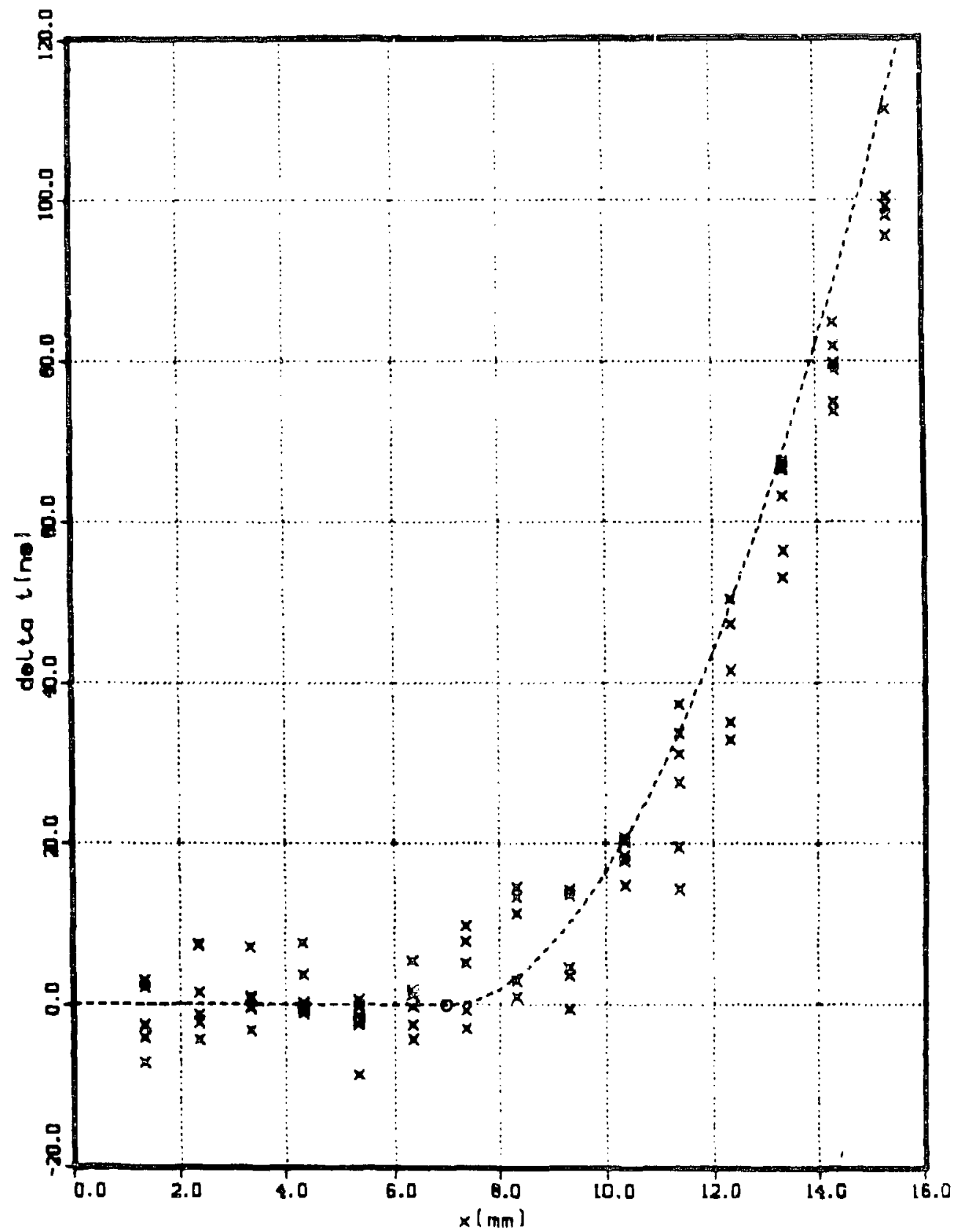

Figure 121: Comparison of the $\delta t$ data for RS-2308 and calculational regulta from the code SHKSJB. The dashed line is the $\delta t$ curve from the calculation. Parameters for the calculational model and parameters for the data analysis are listed in table 47. 


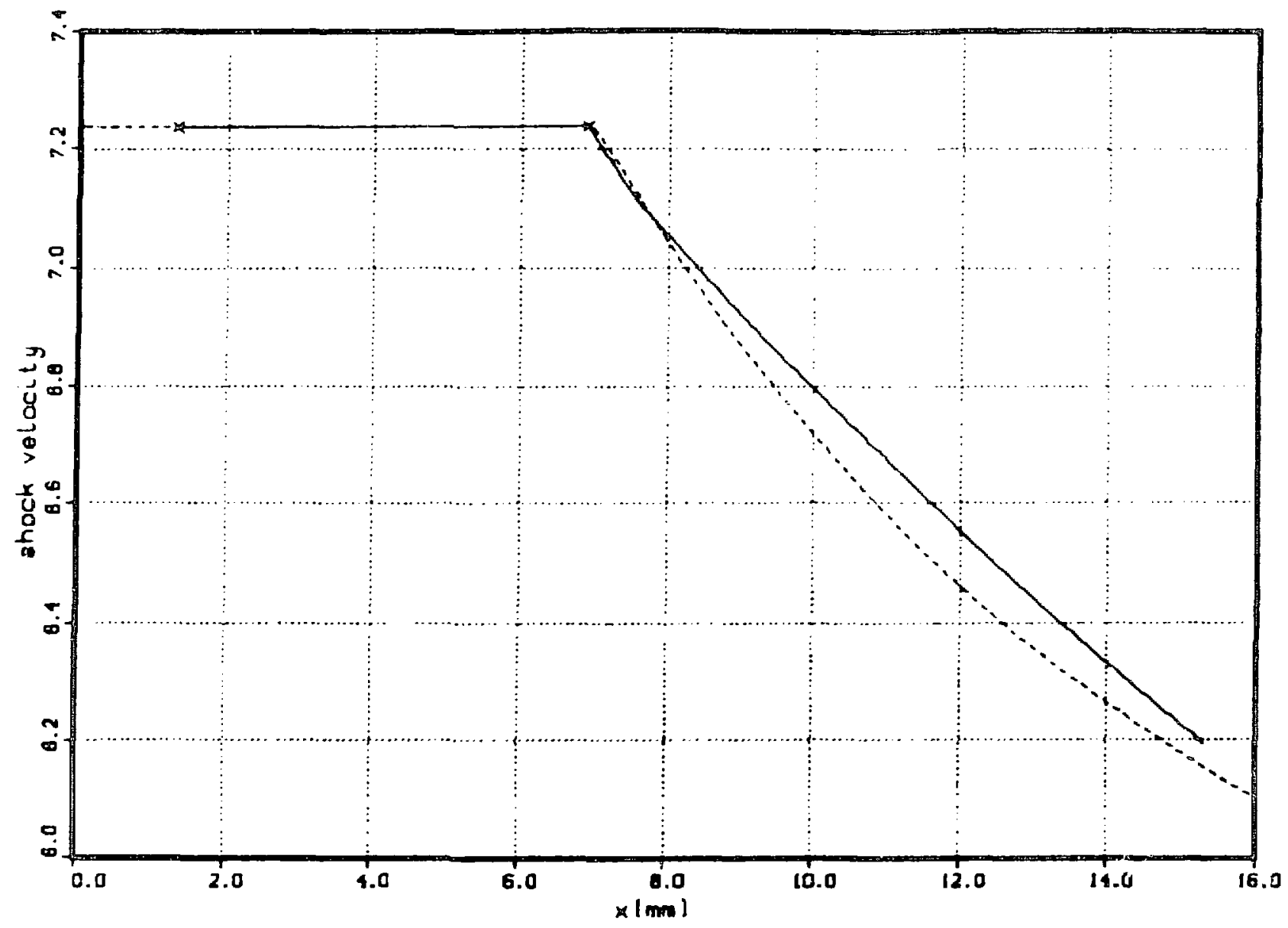

ํ.

Figure 122: A comparison of the shock velocity from the code caiculation and the shock velocity curve from a ft to the data for RS-2308. The solid line is the velocity curve taken from the fit to the data. The corresponding $\delta t$ plots are in figure 121 (see also table 47). 


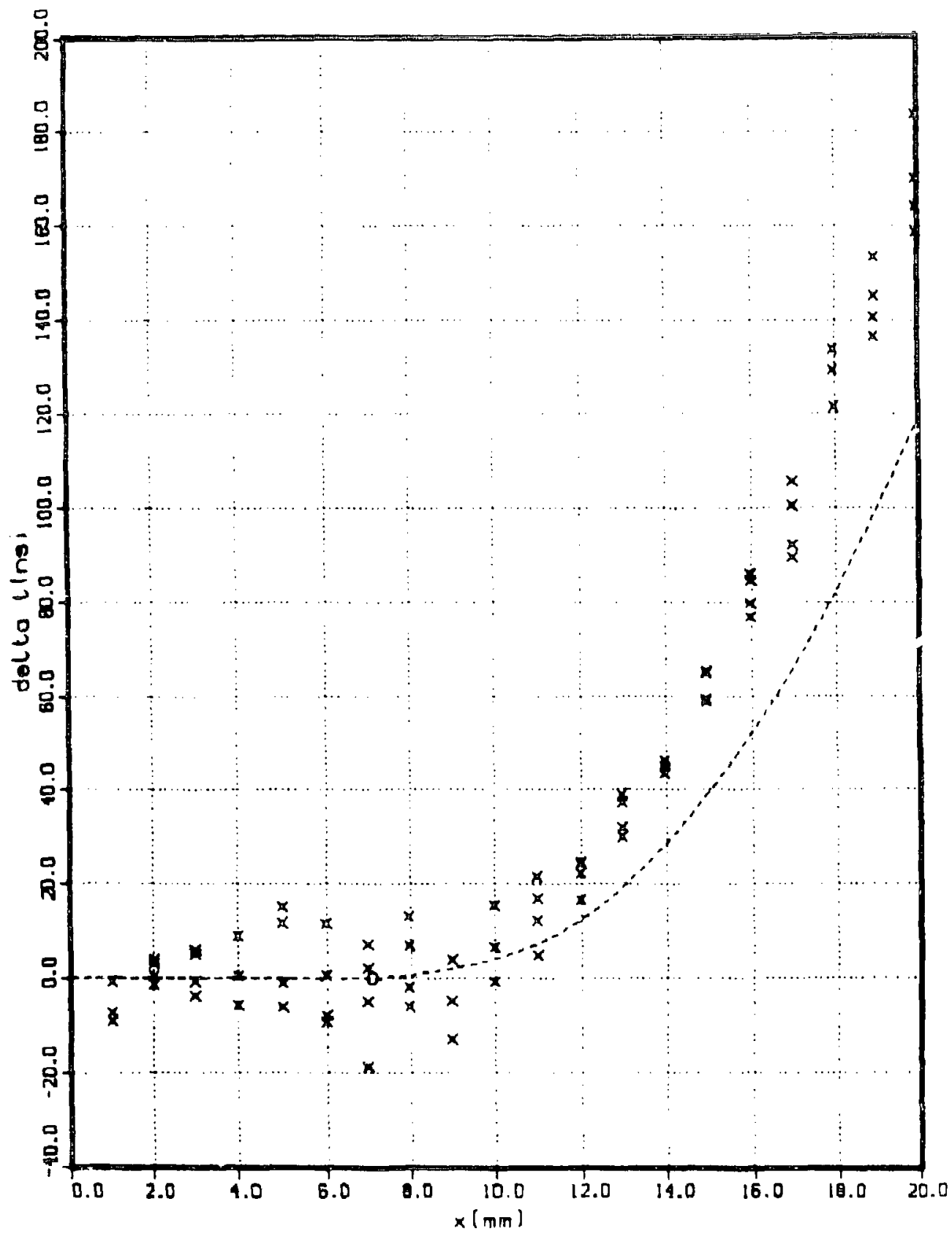

Figure 123: Comparison of the $\delta t$ data for RS-2302 and calculational results from the code SHKSJB. The dashed line is the $\delta t$ curve from the calculation. Parameters for the calculational model and parameters for the data analysis are listed in table 48. 


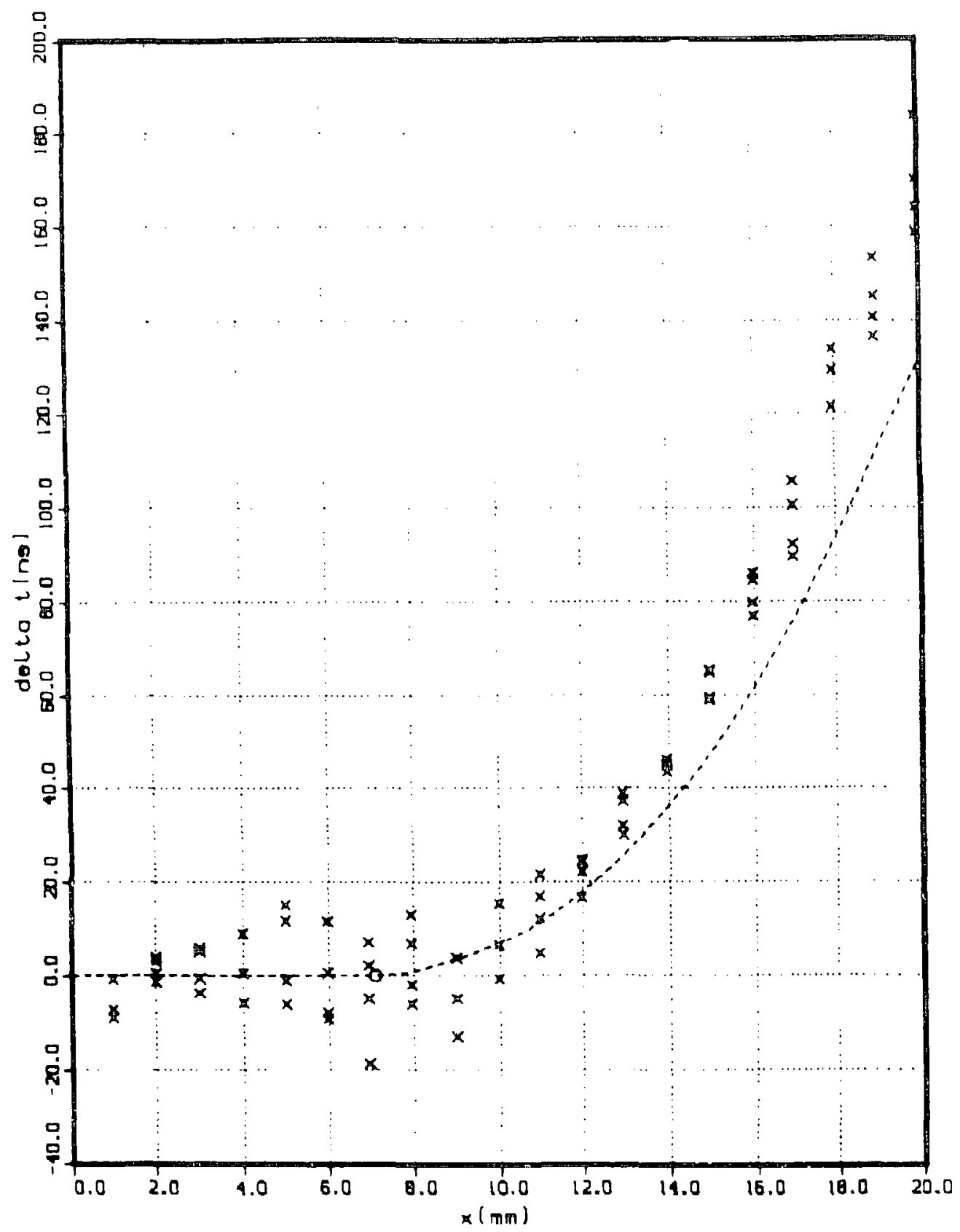

Figure 124: Comparison of the $\delta t$ data for RS-2302 and calculational results from the code SHKSJB. The dashed line is the $\delta t$ curve from the calculation. Parameters for the calculational model and parametere for the data analysis are listed in table 49 . 


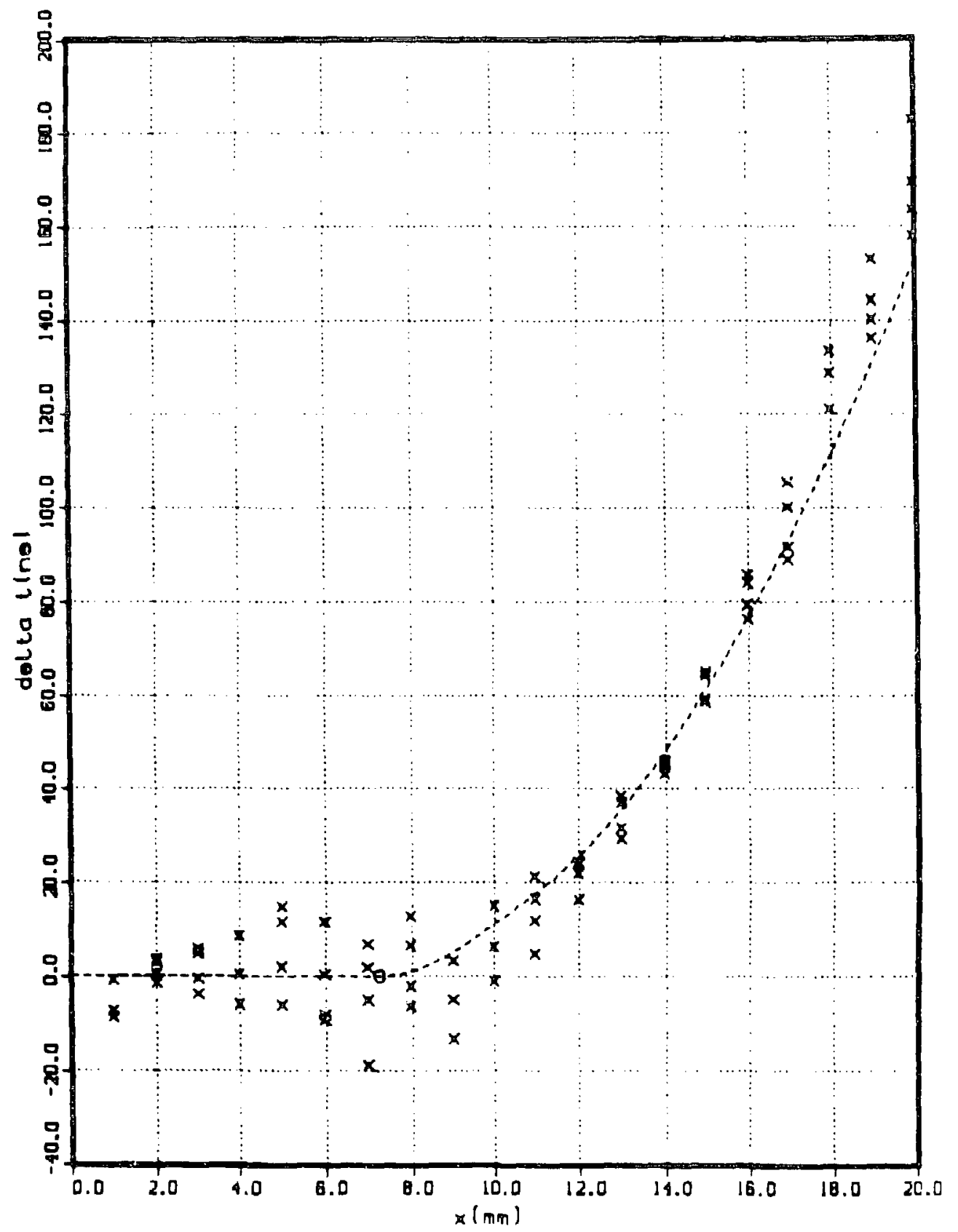

Figure 125: Comparion of the $\delta t$ data for RS-2302 and calculational results from the code SHKSJB. The dashed line is the $\delta t$ curve from the calculation. Parameters for the calculational model and parameters for the data analysis are listed in table 50. 


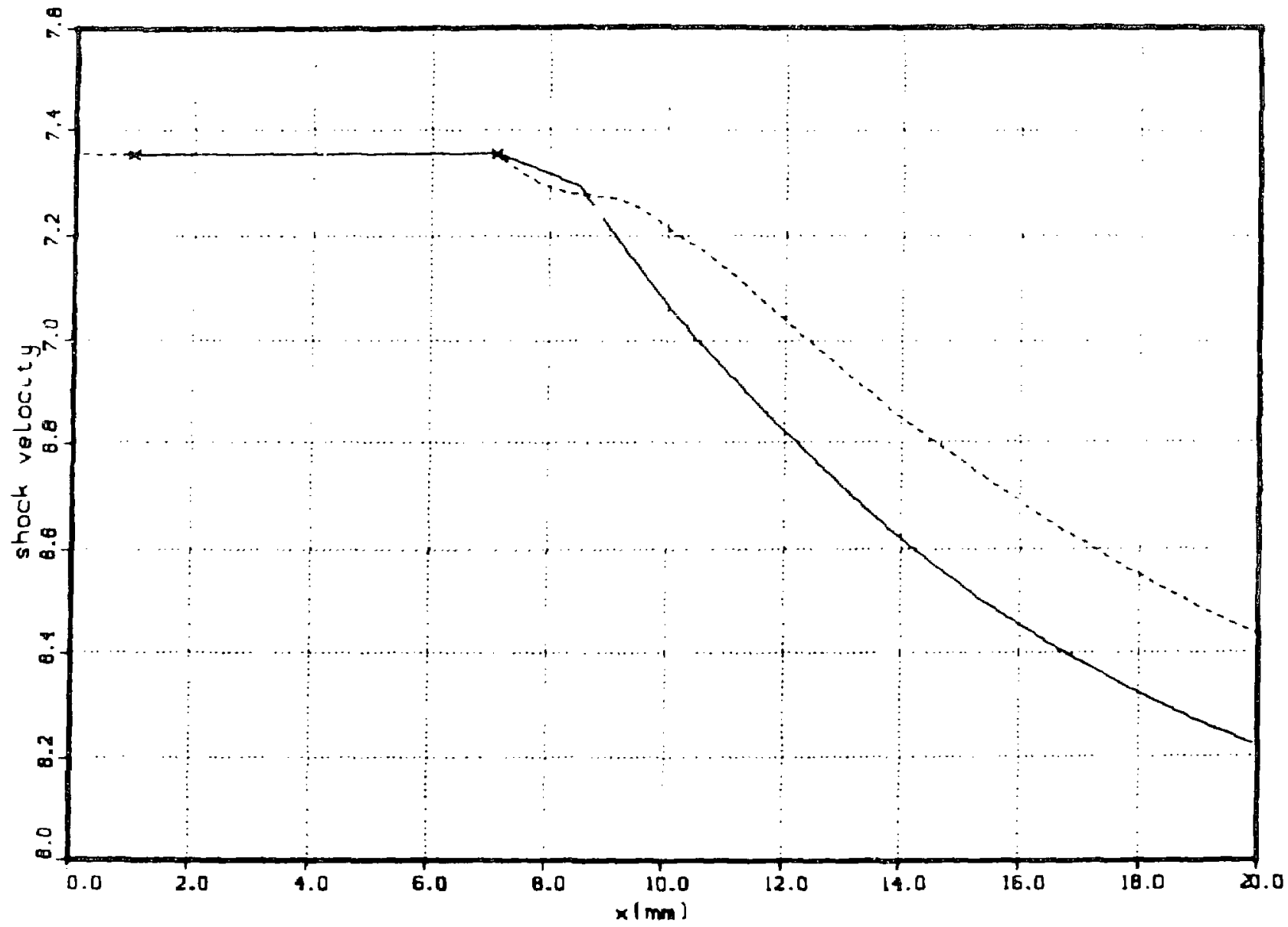

Figure 126: A comparison of the shock velocity from the code calculation and the shock velocity curve from a fit to the data for RS-2302. The solid line is the velocily curve taken from the fit to the data. The corresponding $\delta t$ plots are in figure 123 (see also table 48). 


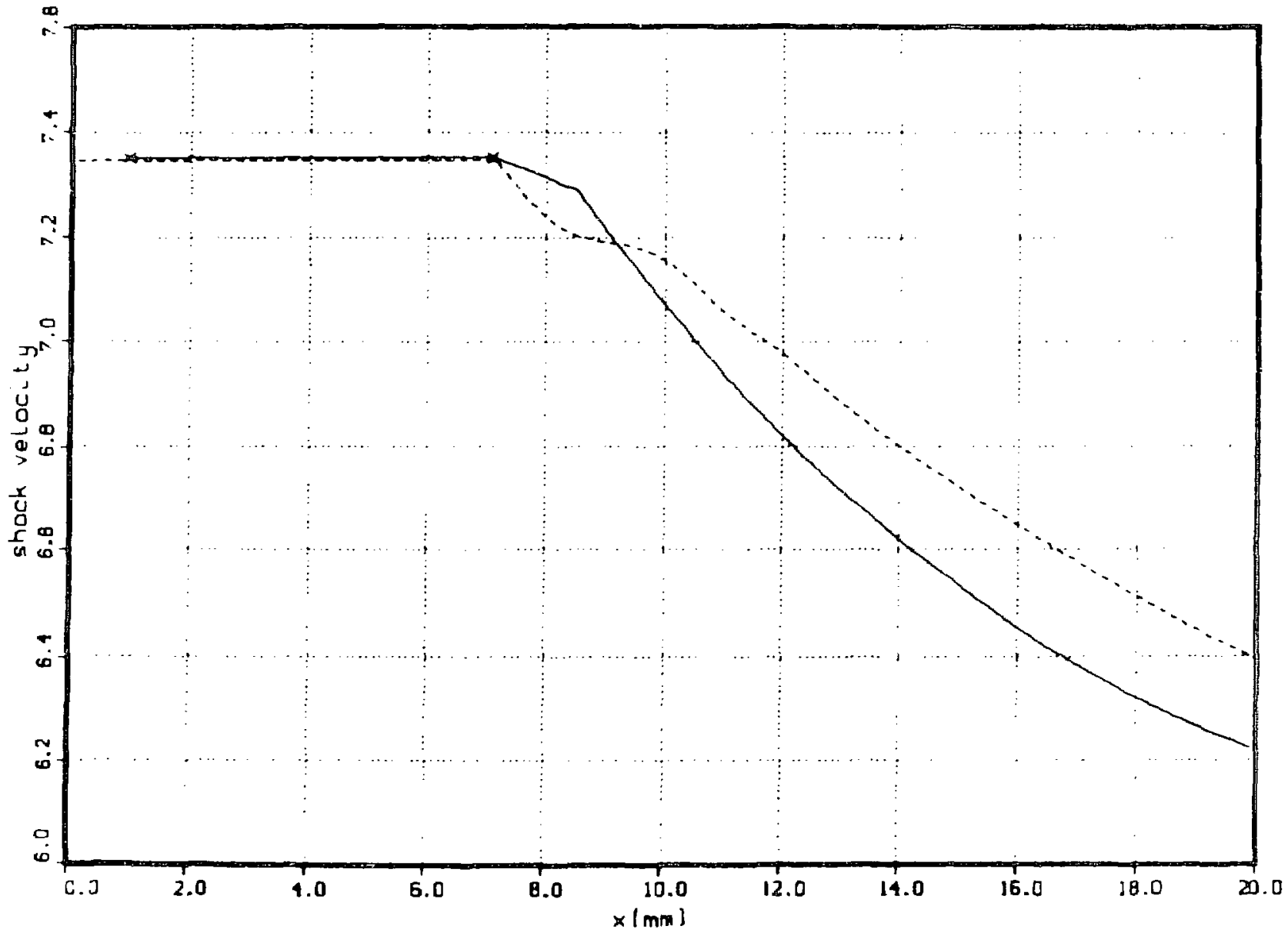

Figure 127: A comparison of the shock velocity from the code calculation and the shack velocity curve from a fit to the data for RS-2302. The solid line is the velocity curve taken from the fit to the data. The corresponding $\delta t$ plots are in Ggure 124 (see also table 49) 


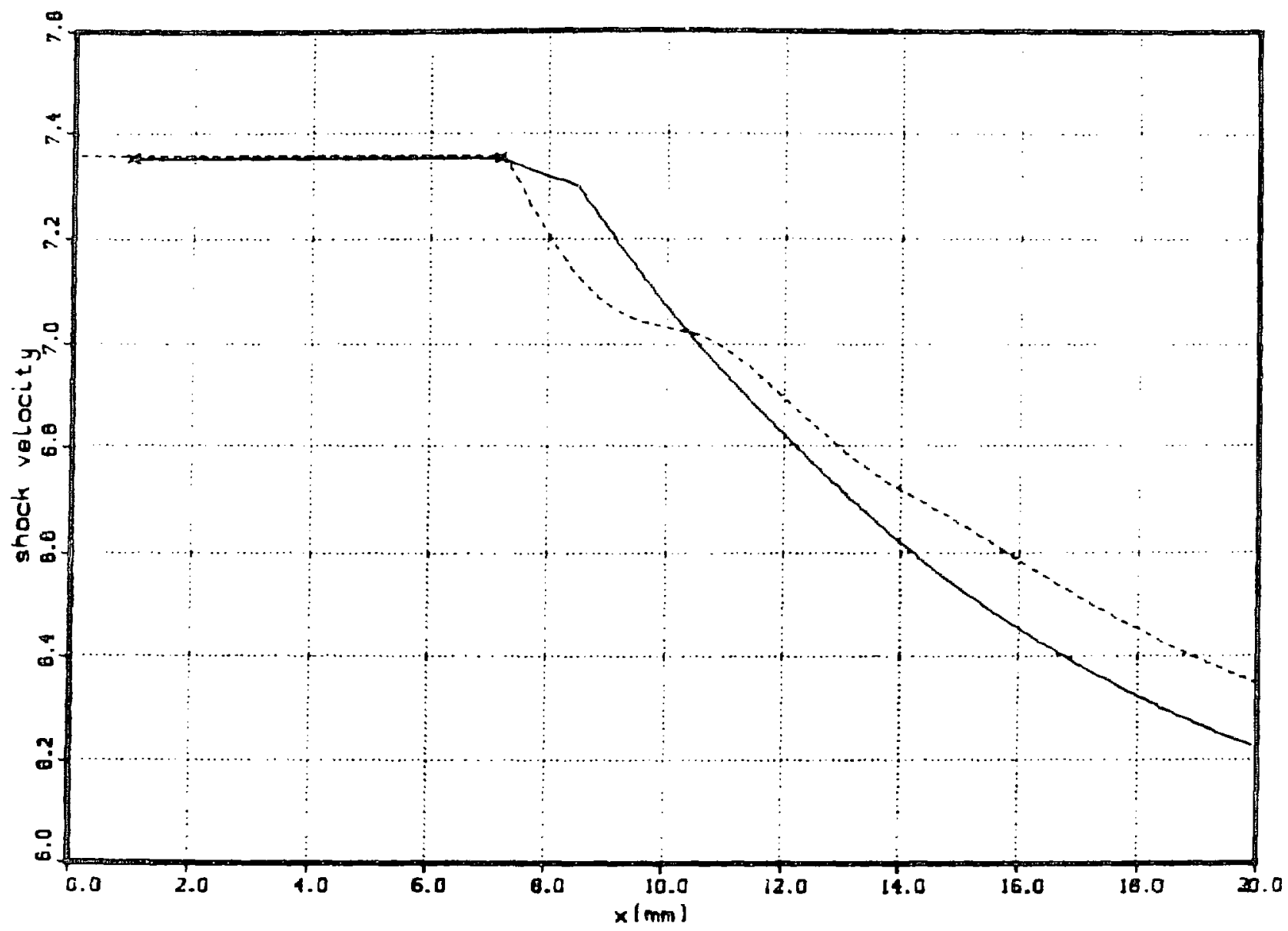

Figure 128: A comparison of the shock velocity from the code calculation and the shock velocity curve from a fit to the data for FS-2302. The solid line is the velocity curve taken from the fit to the data. The corresponding $\delta t$ plots are in figure 125 (see also table 50). 


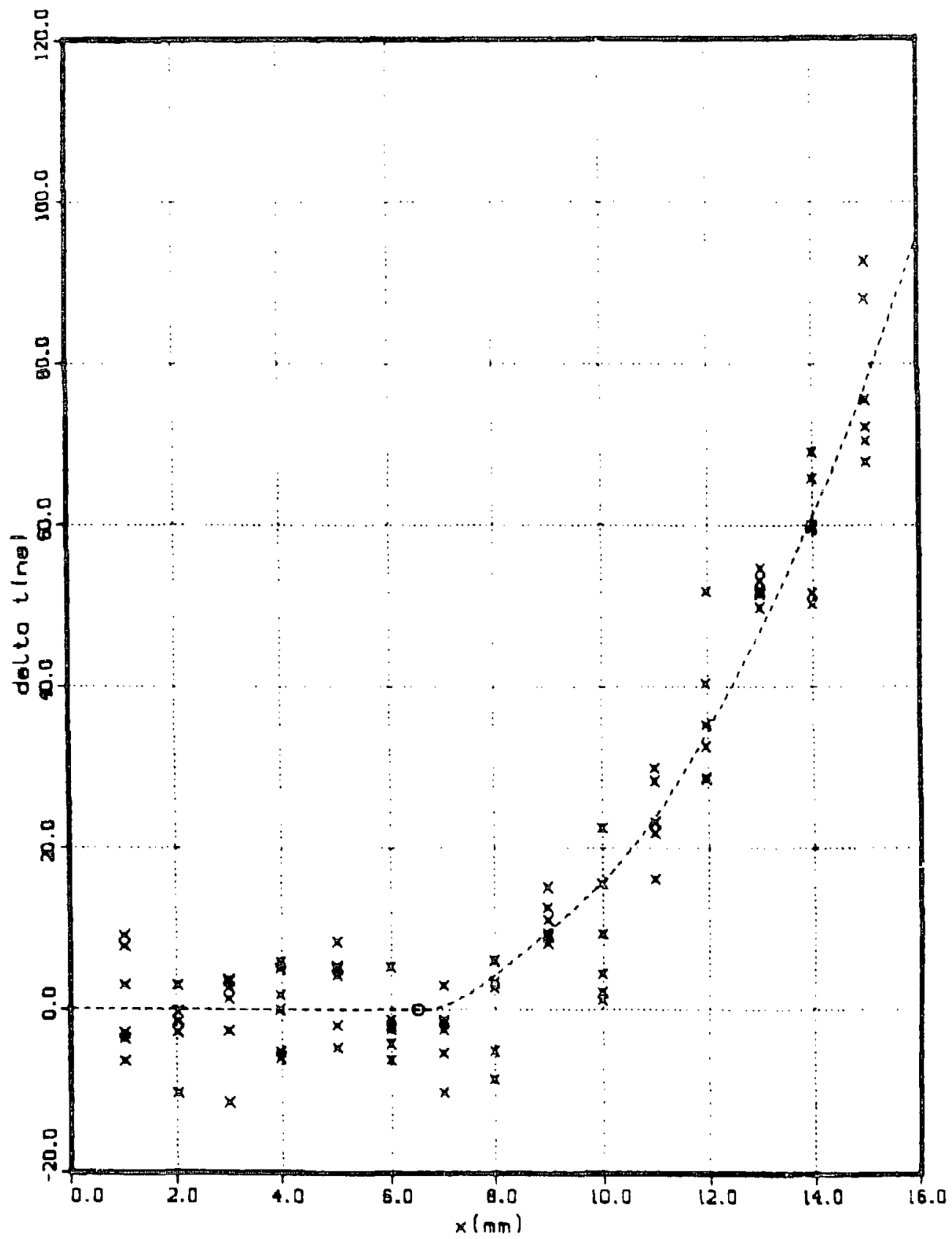

Figure 129: Comparison of the $\delta t$ data for RS-2315 and calculational regults from the code $\ulcorner$ HKSJB. The dashed line is the $\delta t$ curve from the calculation. Parameters for the calculational model and parameters lor the data analysis are listed in table $\tau 1$. 


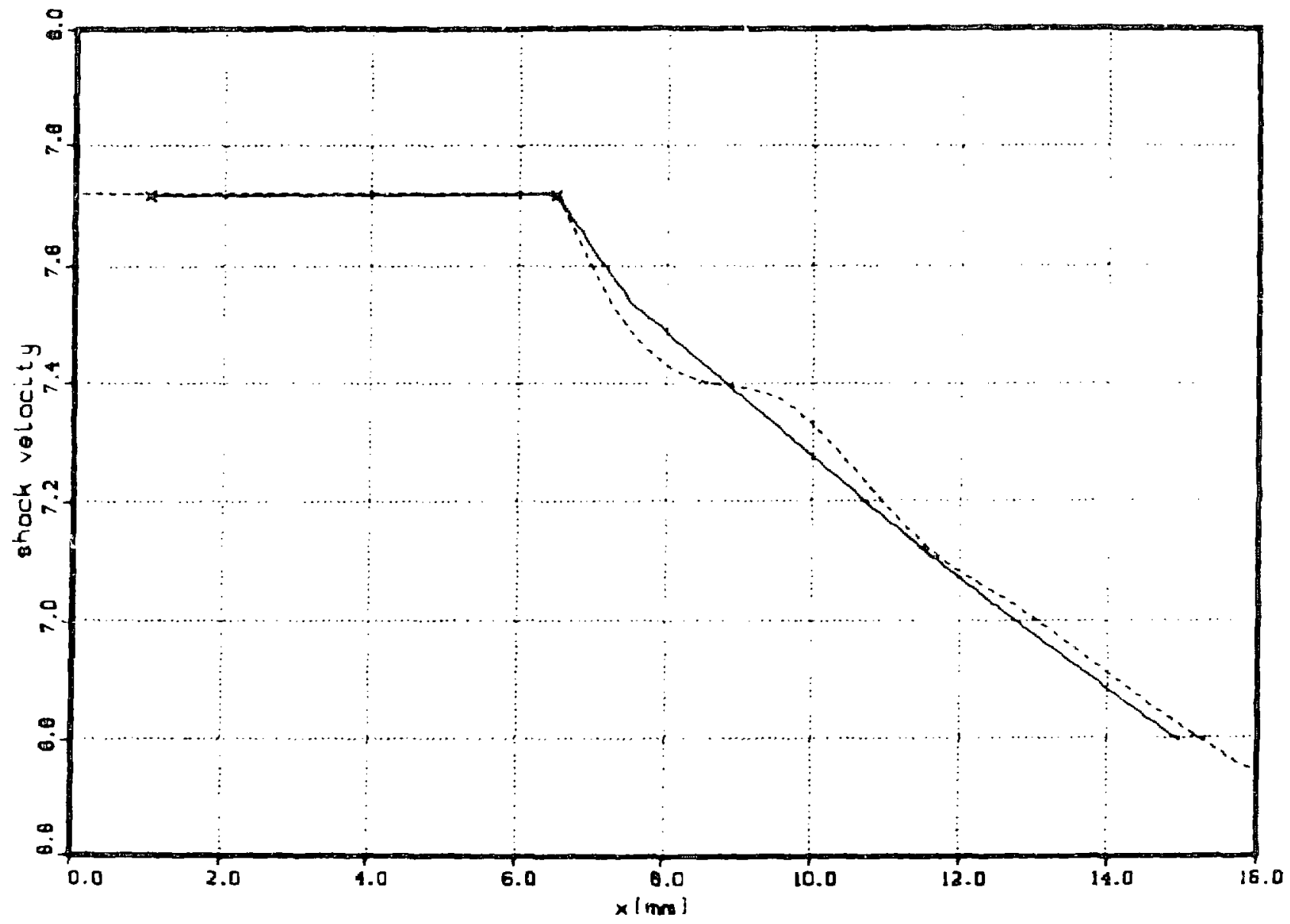

Figure 190: A comparison of the shoch velocity from the code calculation and the shock velocity curve from a fit to the data for RS-2315. The Bolid line is the velocity curve taken from the fit to the data. The corresponding $\delta \ell$ plots are in figure 129 (see also table 51). 


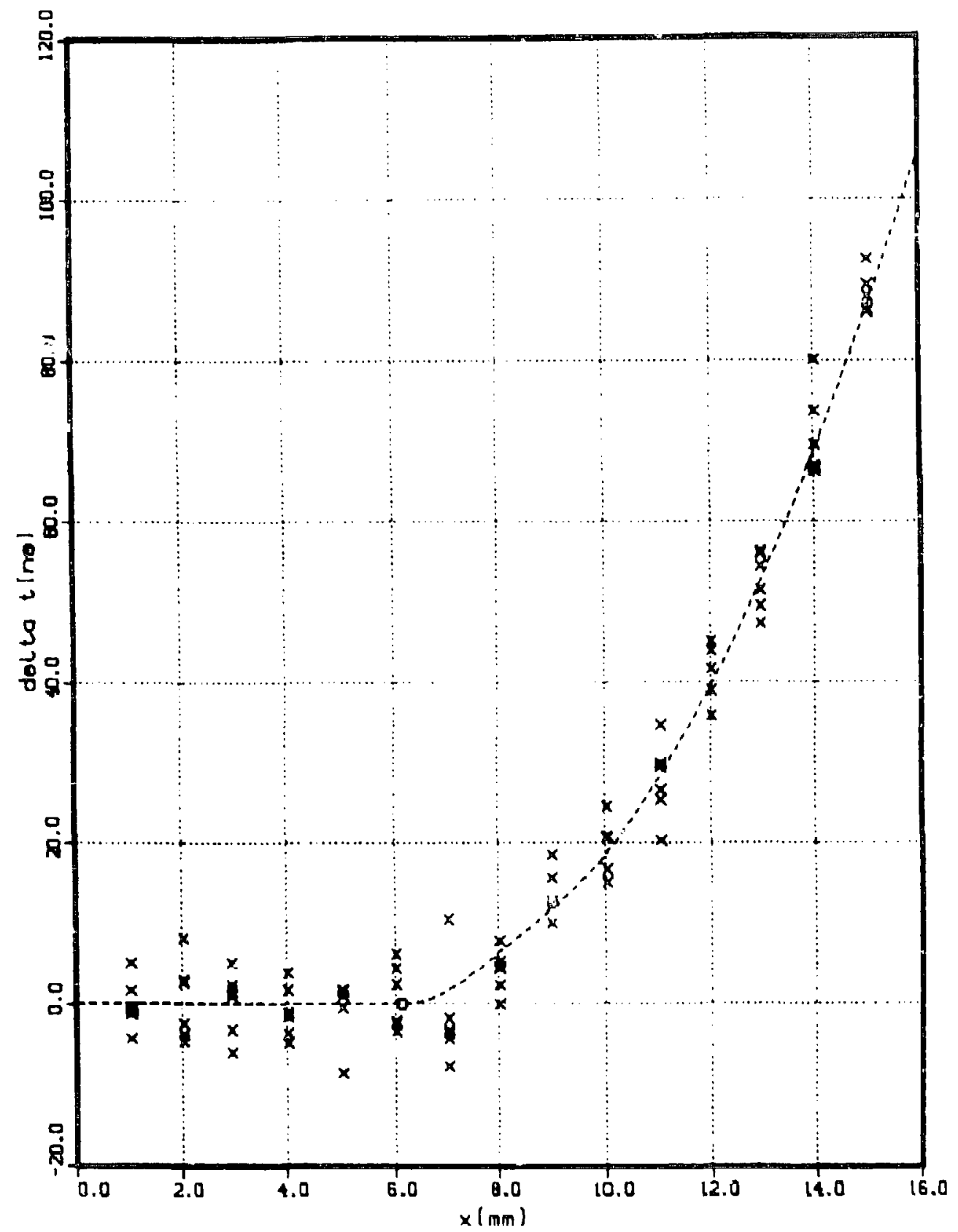

Figure 131: Comparison of the $\delta$ t data for RS-2309 and calculational results from the code SHKSJB. The dashed line is the $\delta t$ curve from the calculation. Parameters for the calculational model and parameters for the data analysis are listed in table 52. 


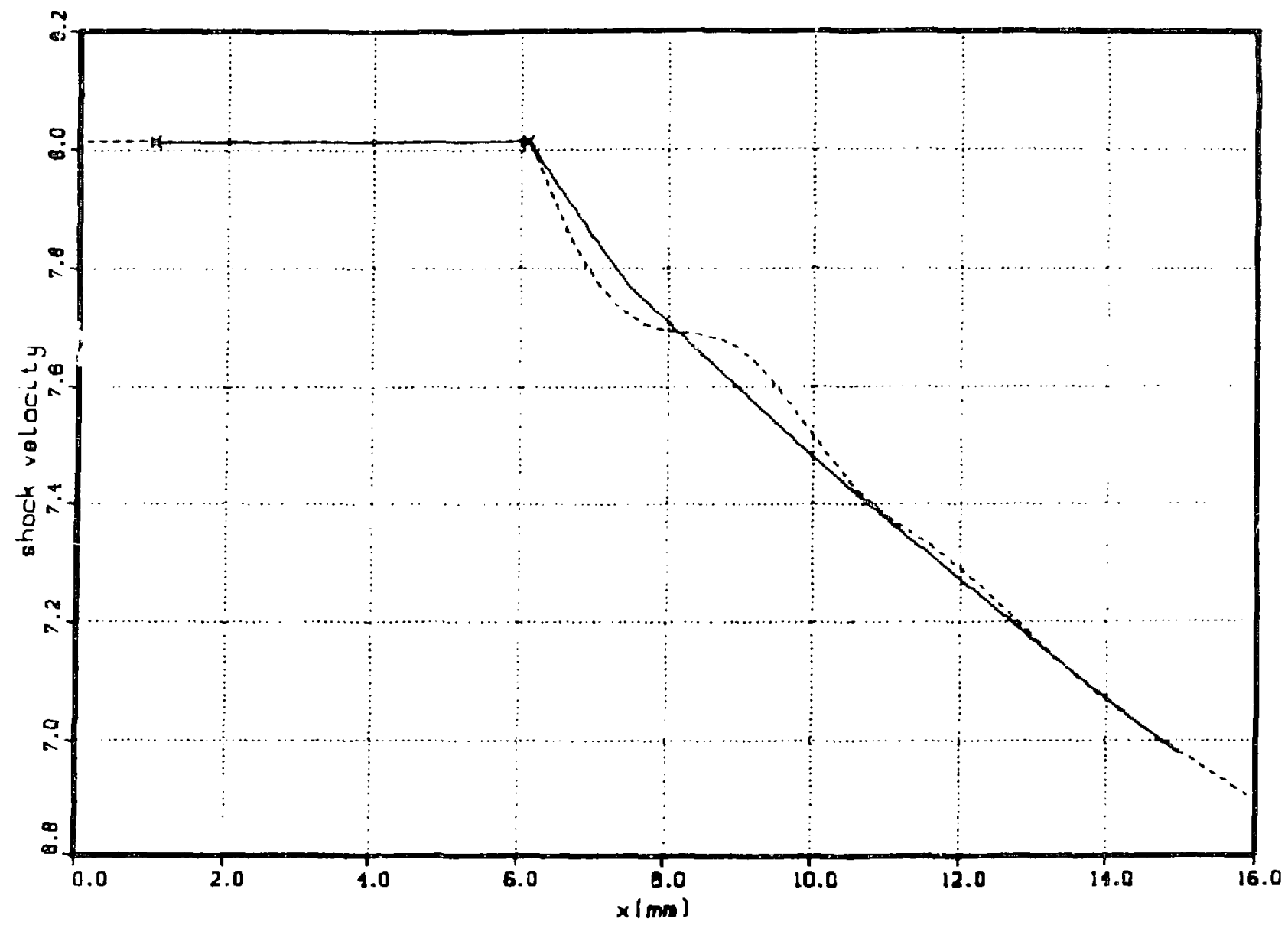

Figure 152: A comparison of the shock velocity from the code calculation and the shock velocity curve from a fit to the data for KS-2309. The solid line is the velocity curve taken from the fit to the data. The corresponding ot plots are in figure 131 (see also table 52). 


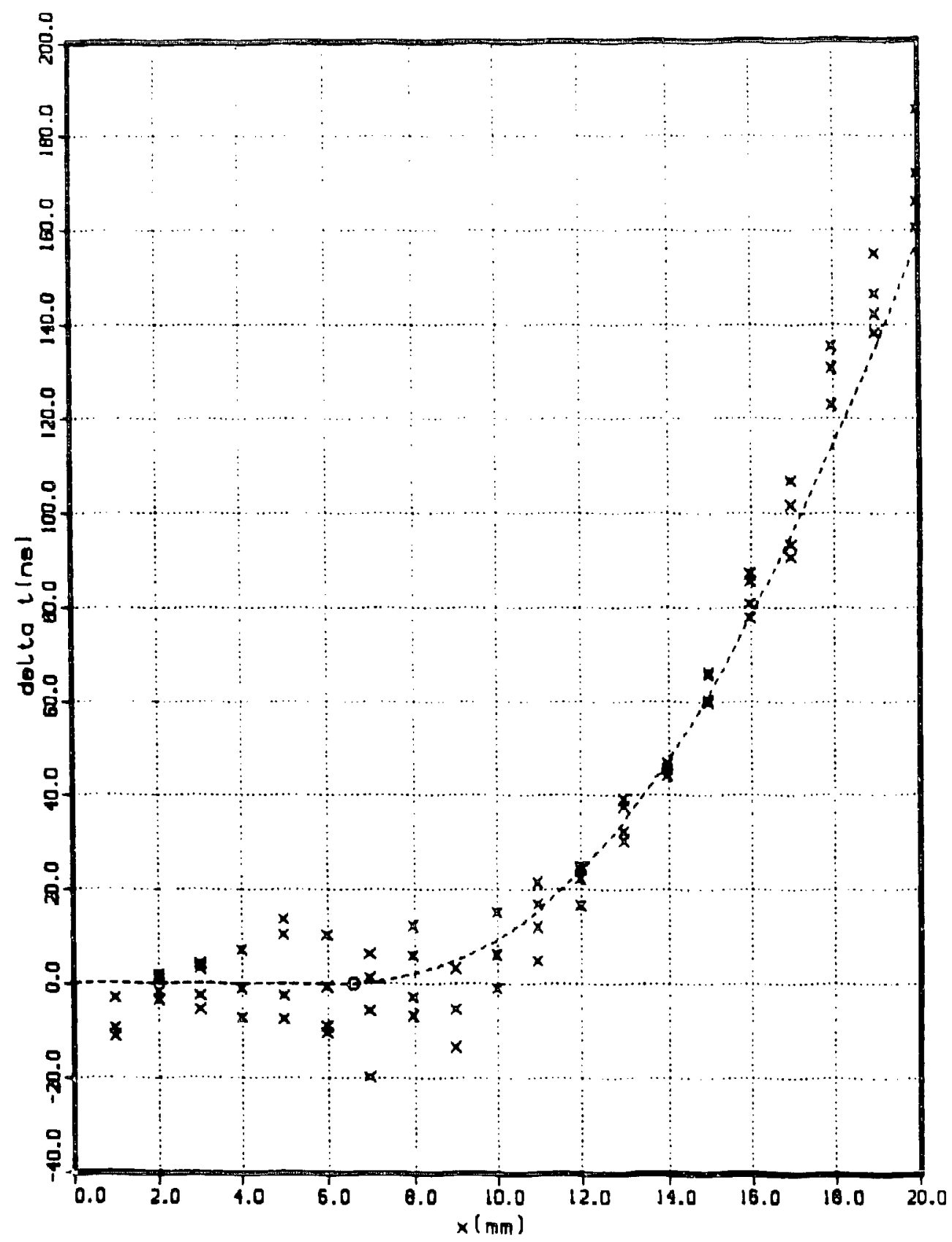

Figure 133: Comparion of the $\delta t$ data for RS-2302 and calculational results from the rode SHKSJB. The dashed line is the $\delta t$ curve from the calculation. Parameters for the calculational model and parameters for the data analysis are listed in table 53. 


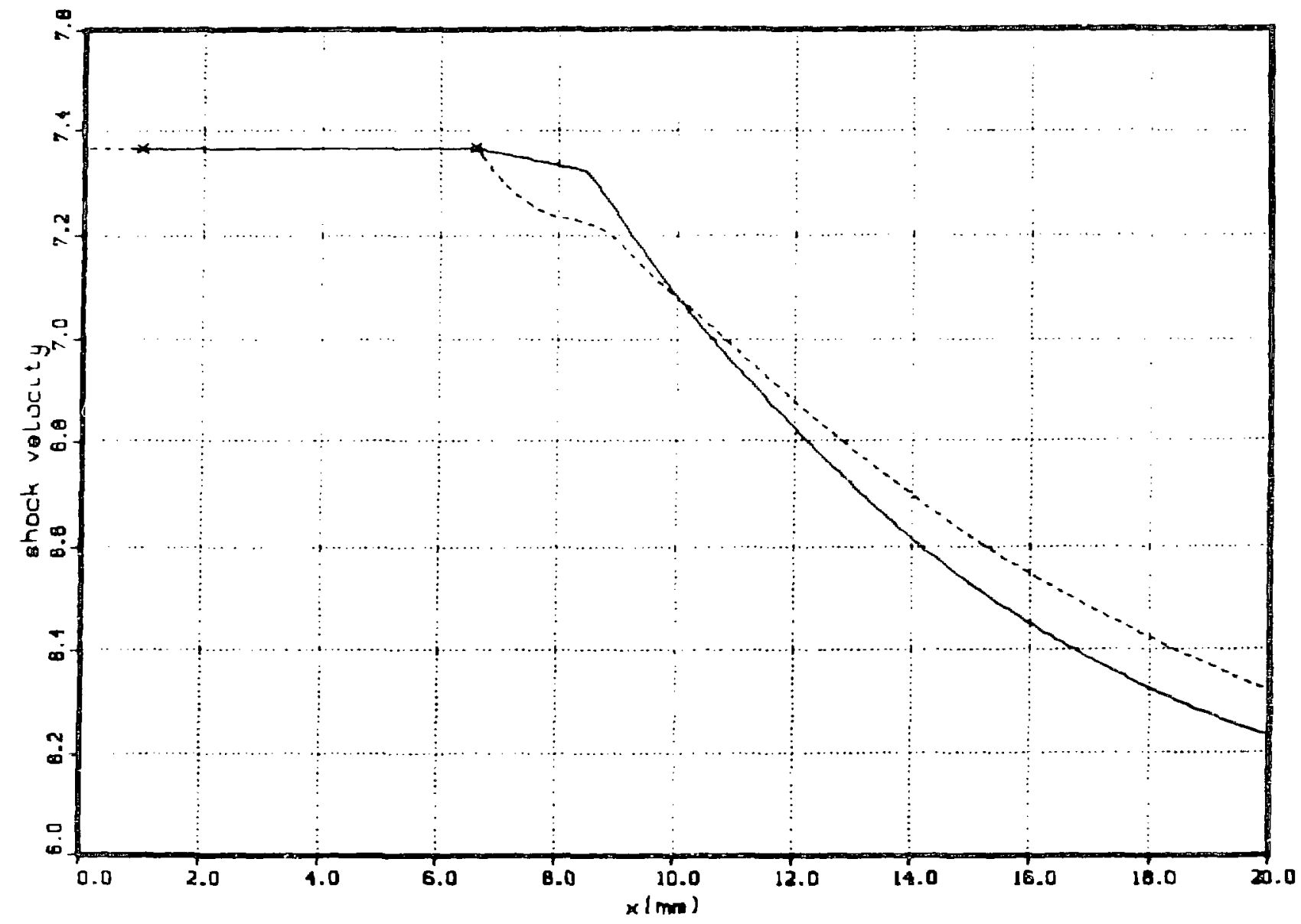

Figure 194: A comparison of the shock velocity from the code :alculation and the shock velocity curve from a fit to the data for RS-2302. The solid line is the veiocity curve taken from the fit to the data. The corresponding $\delta t$ plote are in tigure 133 (:iee also table 53). 


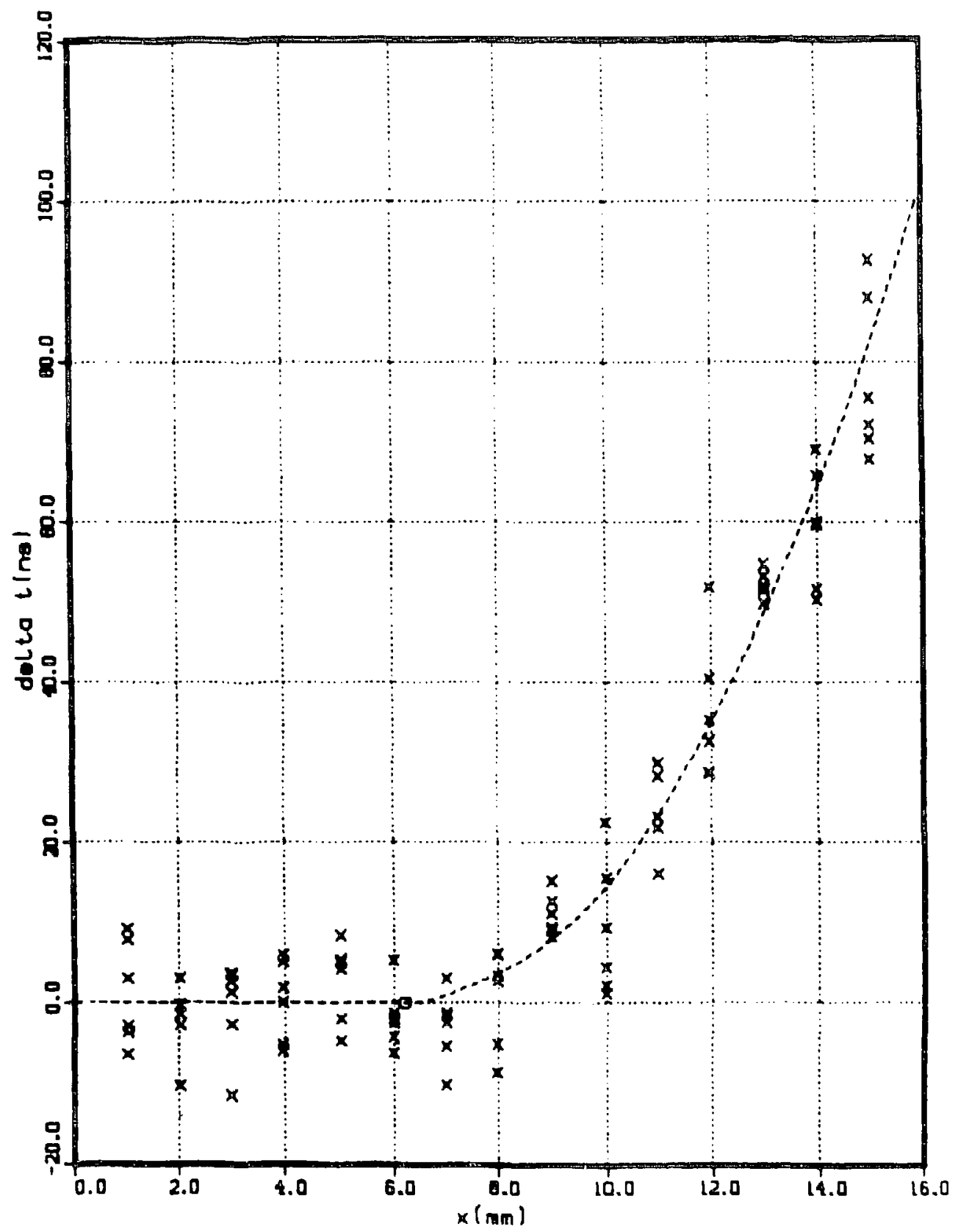

Figure 135: Comparison of the $\delta t$ data for RS-2315 and calculational results from the code SHKSJB. The dashed line is the $\delta t$ curve from the calculation. Parameters for the calculational model and parameters for the data analysis are listad in table 54. 


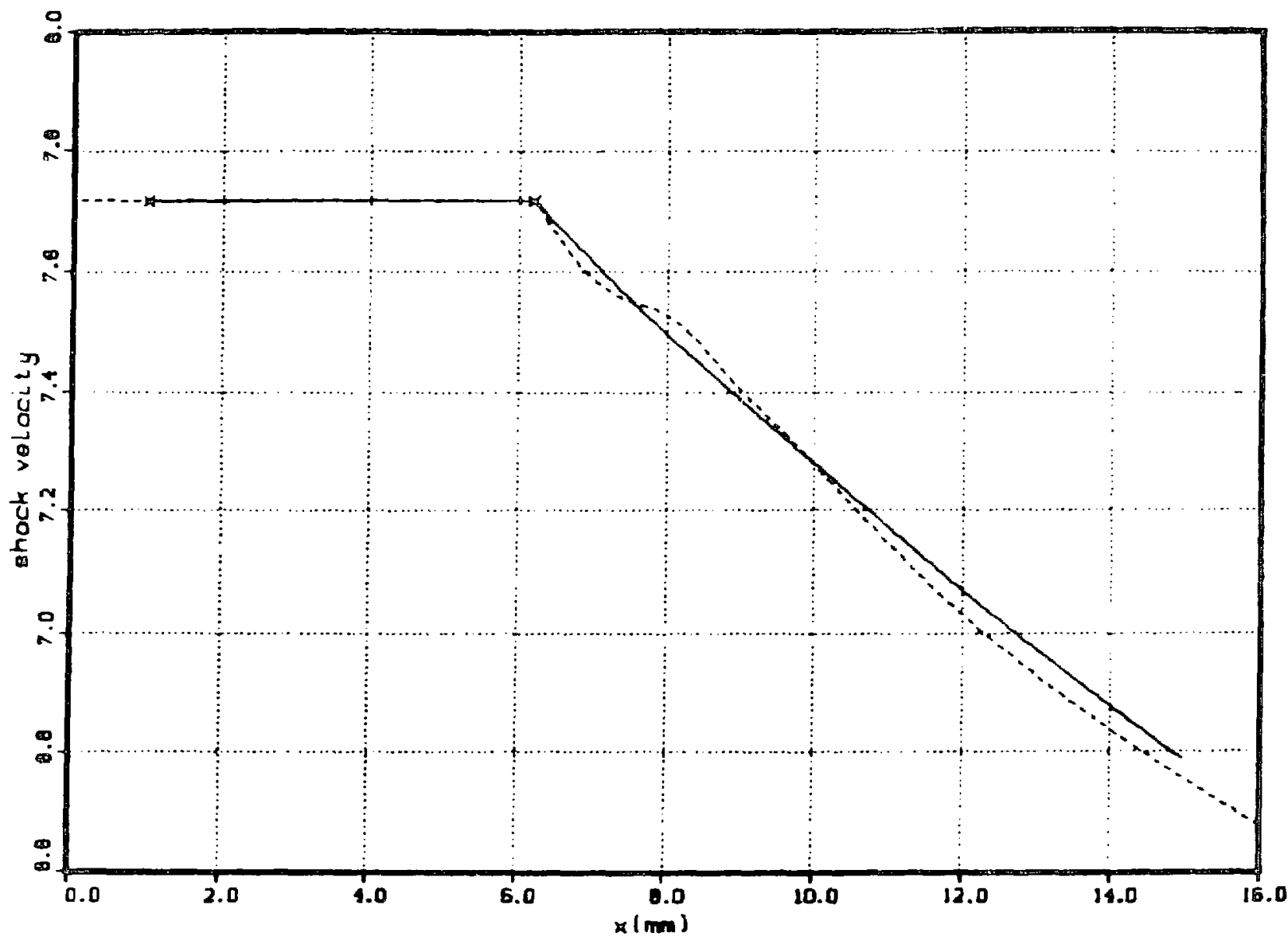

Figure 136: A comparion of the shock velocity from the code calculation and the shock velocity curve from a fit to the data for RS-2315. The solid line is the velocity curve taken from the fit to the data. The corresponding $\delta t$ plots are in figure 135 (see also table 54). 


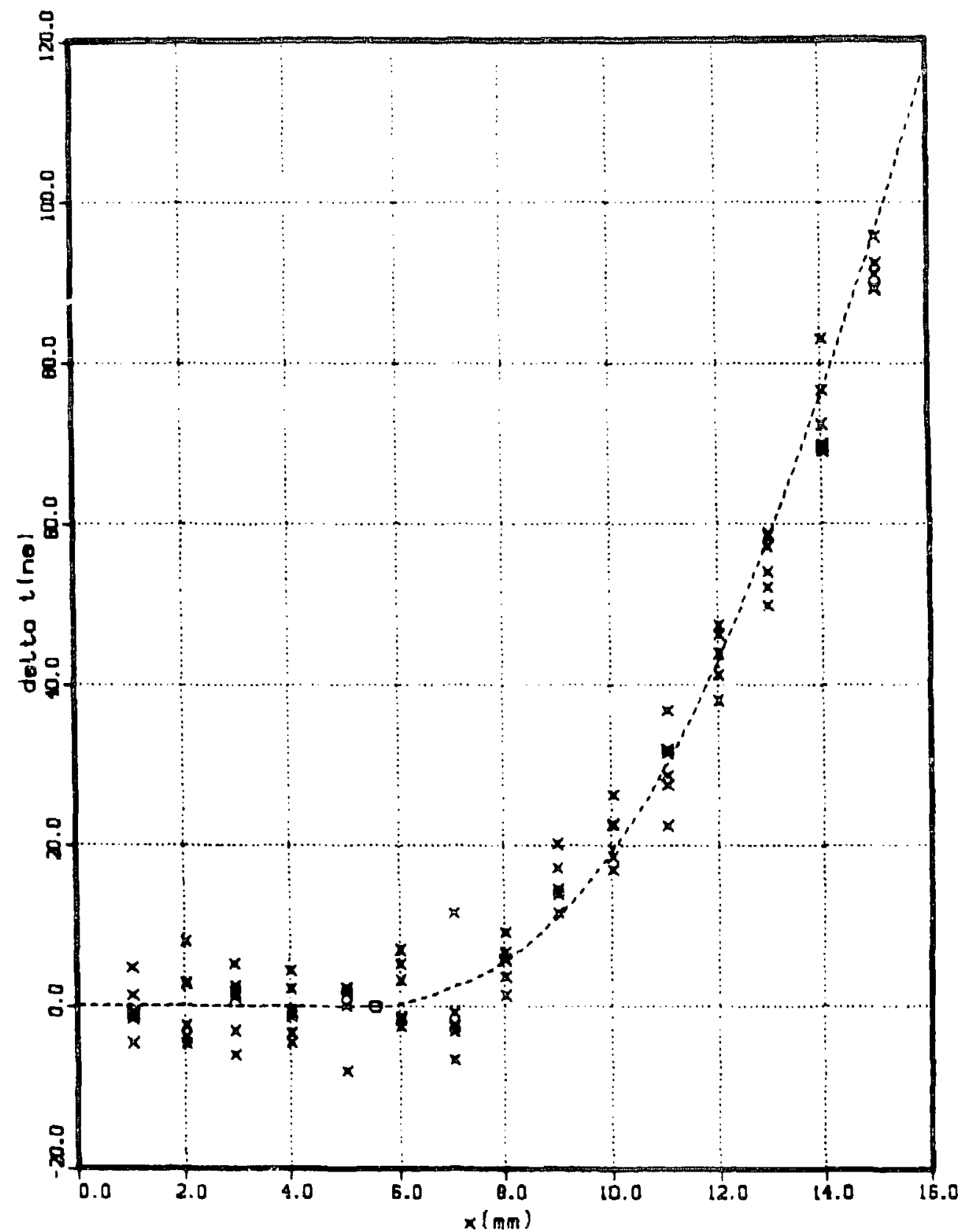

Figure 137: Comparison of the $\delta t$ data for RS-2309 and calculational results from the code SHKSJB. The dashed line is the $\delta t$ curve from the calculation. Parameterg for the calculational model and parameters for the data analysis are listed in table 55. 


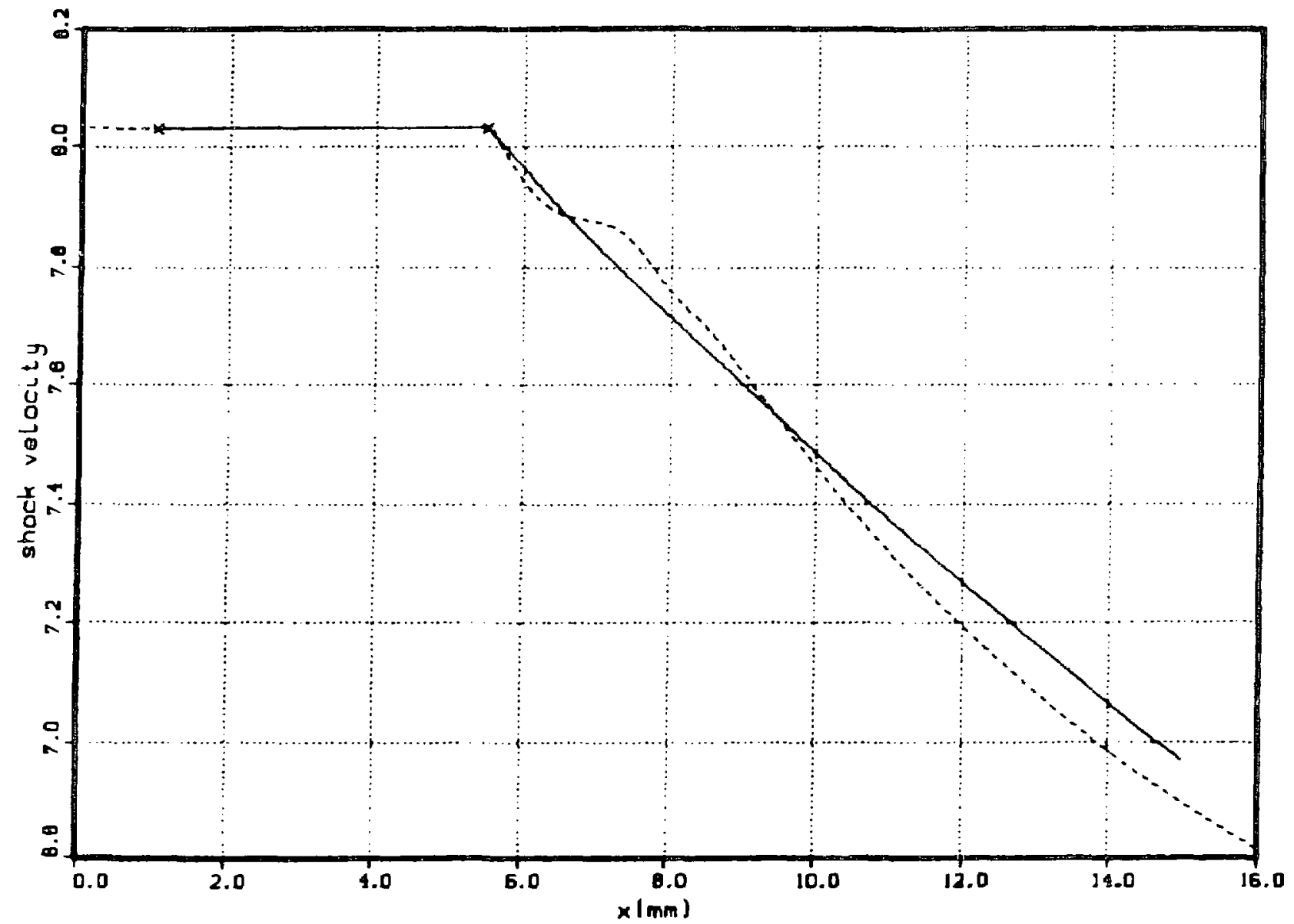

Figure 198: A comparison of the shock velocit: from the code calculation and the shock velocity curve from a fit to the data for RS-2309. The solid line is the velocity curve taken from the fit to the data. The corresponding $\delta t$ plots are in fgure 137 (see also table 55). 


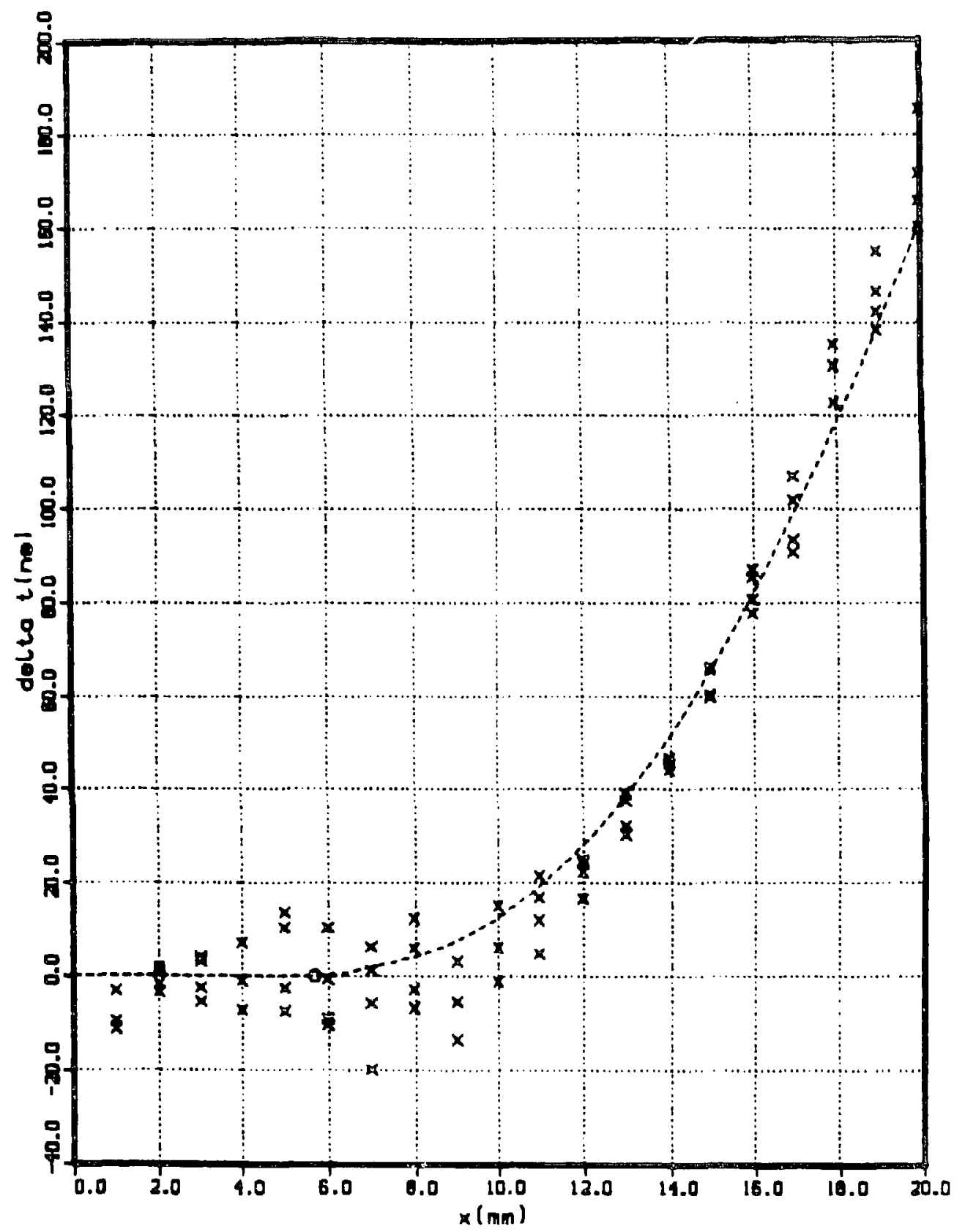

Figure 189: Comparison of the $\delta t$ data for RS-2302 and calculational regults from the code SHKSJB. The dashed line is the $\delta t$ curve from the code calculation. Pararneters for the calculational model and parameters for the data analysis are listed in table 56. 


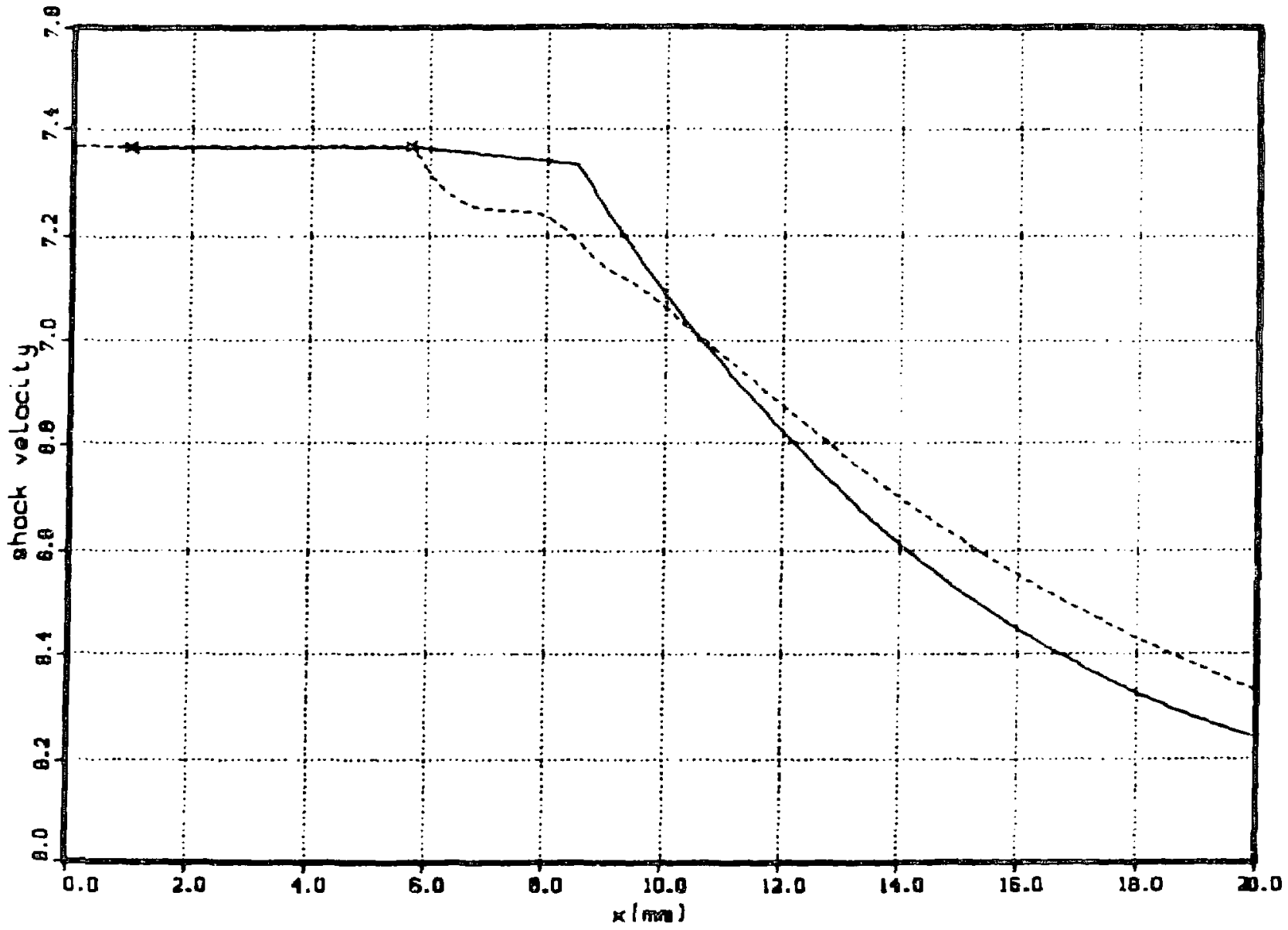

Figure 140: A comparison of the shock velocity from the code calculation and the shock velocity curve from a fit to the data for RS-2302. The solid line is the velocity curve taken from the fit to the data. The corresponding $\delta t$ plots are in figure 139 (see also table 56). 


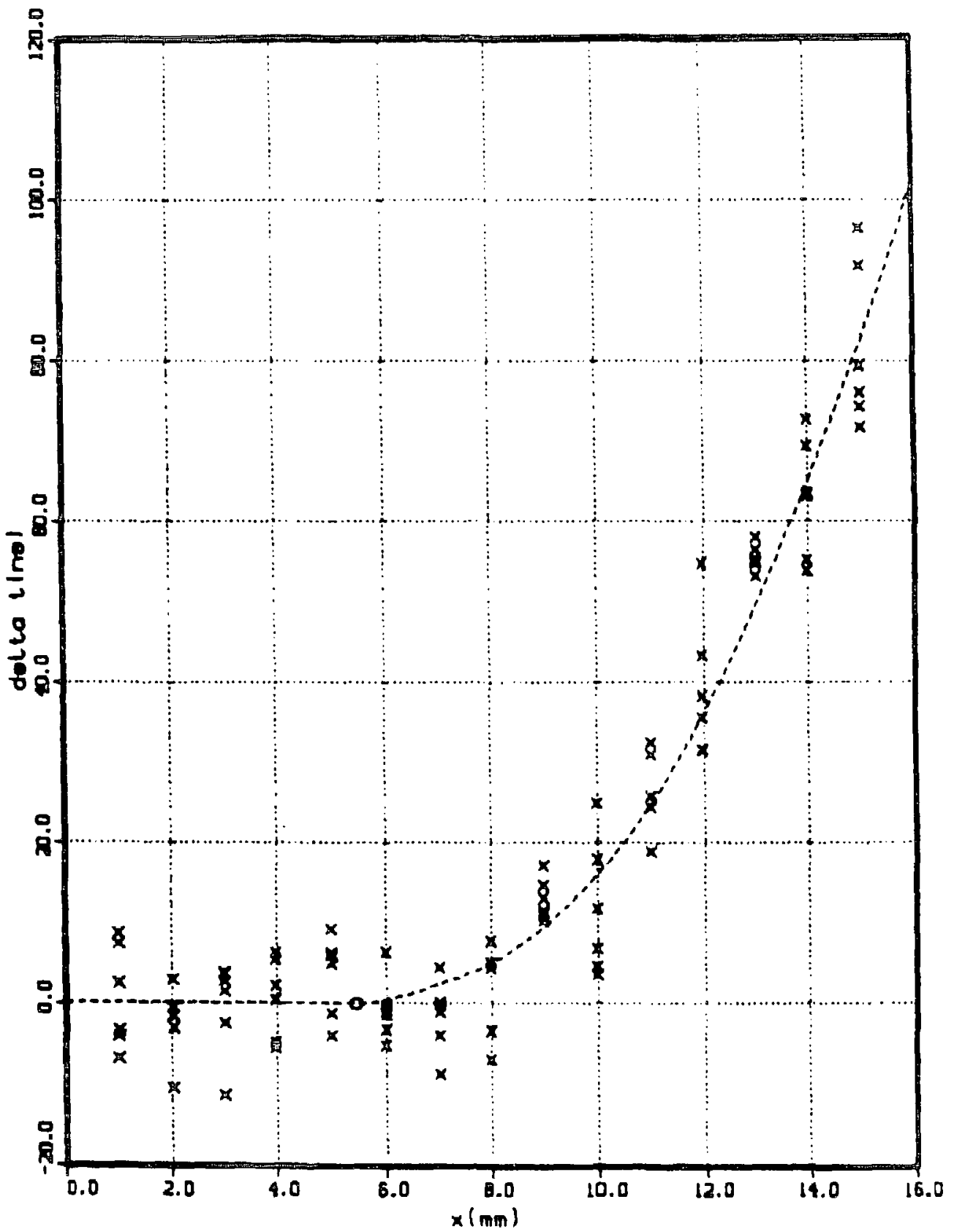

Figure 141: Comparison of the $\delta t$ data for RS-2315 and calculational results from the code SHKS JB. The dashed line is the $\delta t$ curve from the code calculation. Parameters for the calculational model and parameters for the data analysis are listed in table 57. 


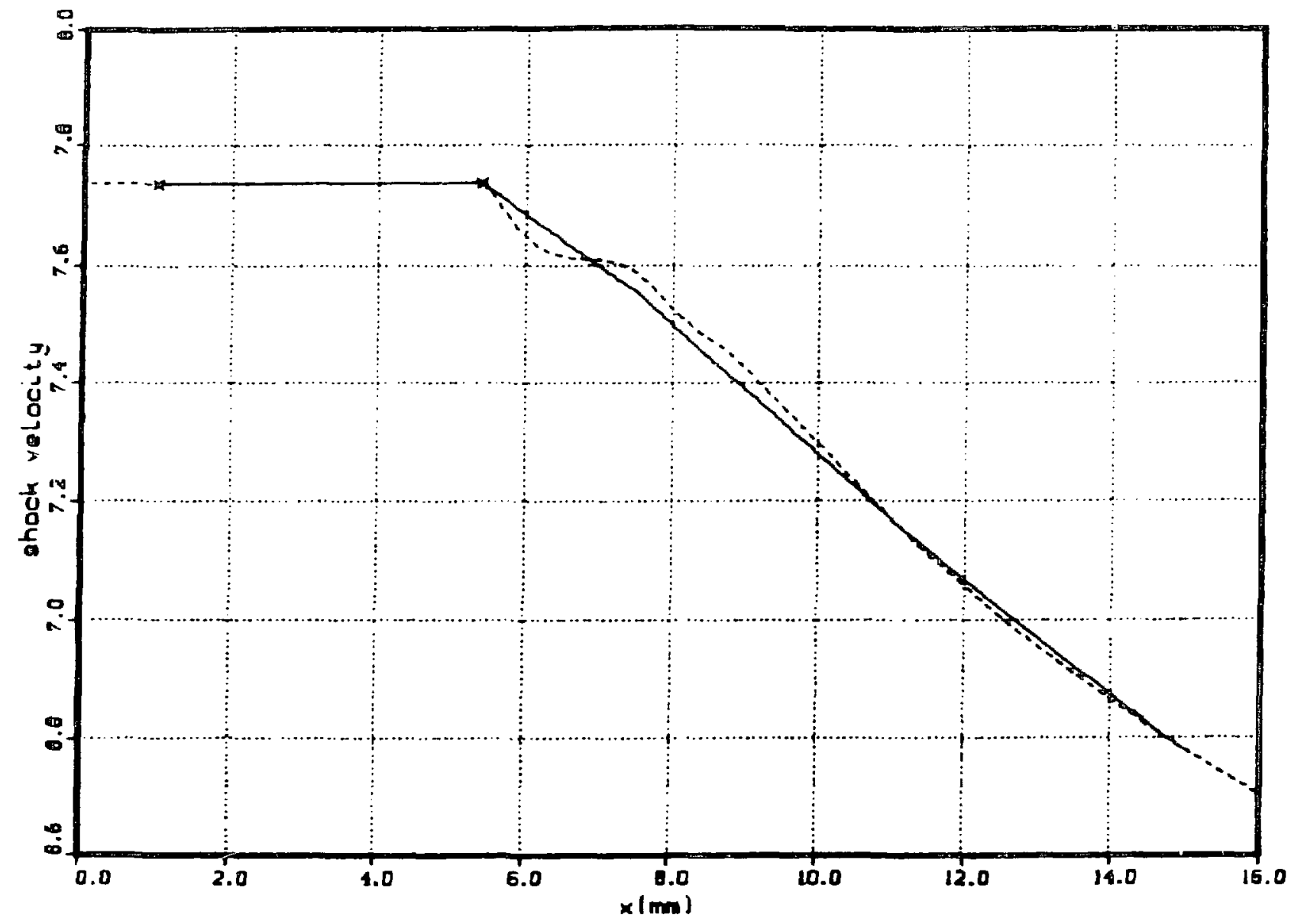

Figure 142: A comparison of the shock velocity from the code calculation and the shock velocity curve from a fit to the data for RS-2315. The solid line is the velocity curve taken from the fit to the data. The corresponding $\delta t$ plots aı 2 in figure 141 (see also table 57). 


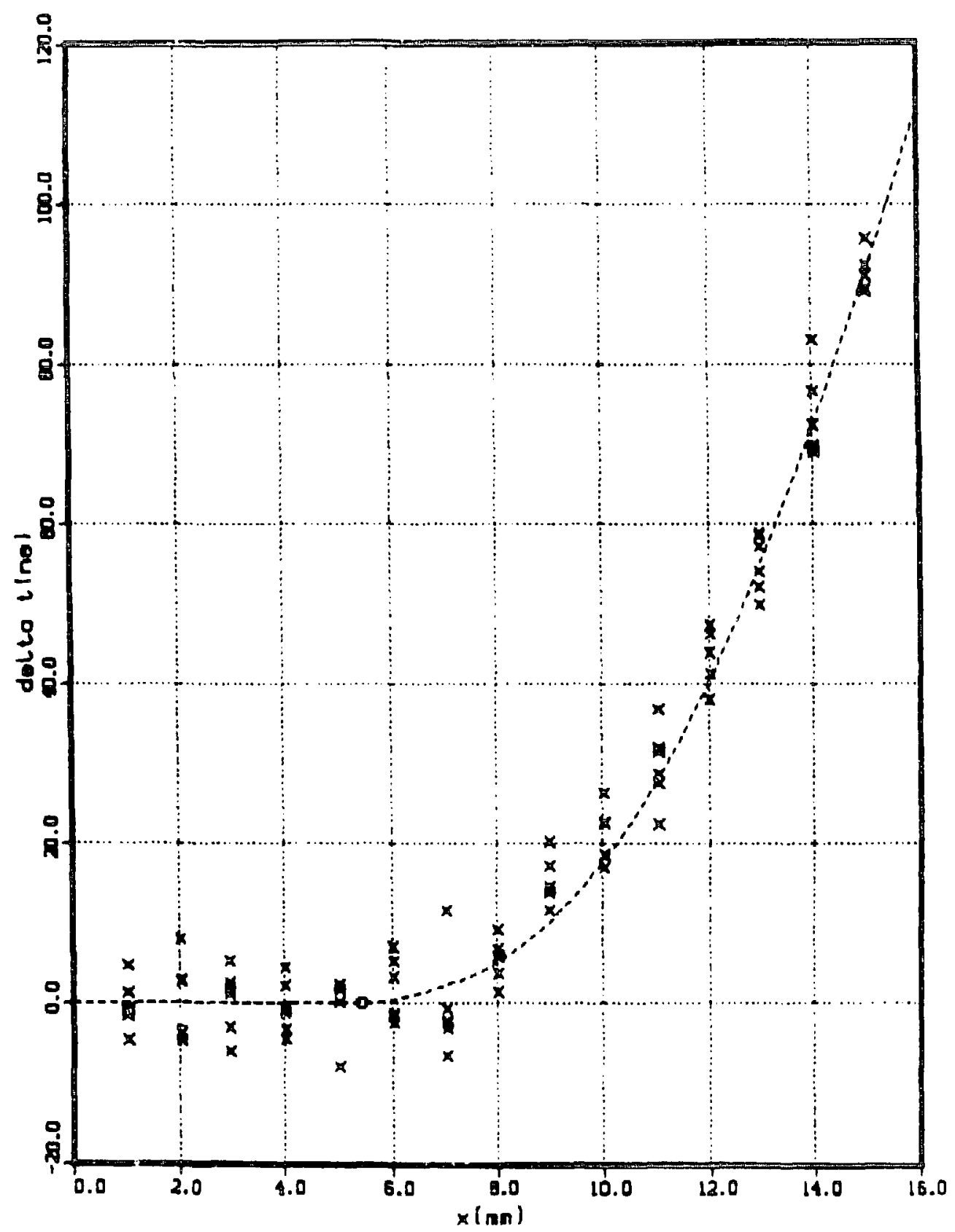

Figure 143: Comparion of the $\delta t$ data for RS-2309 and calculational results from the code SHKS JB. The dashed line is the $\delta t$ curve from the code calculation. Parameters for the calculational model and parameters for the data analysis are listed in table 58. 


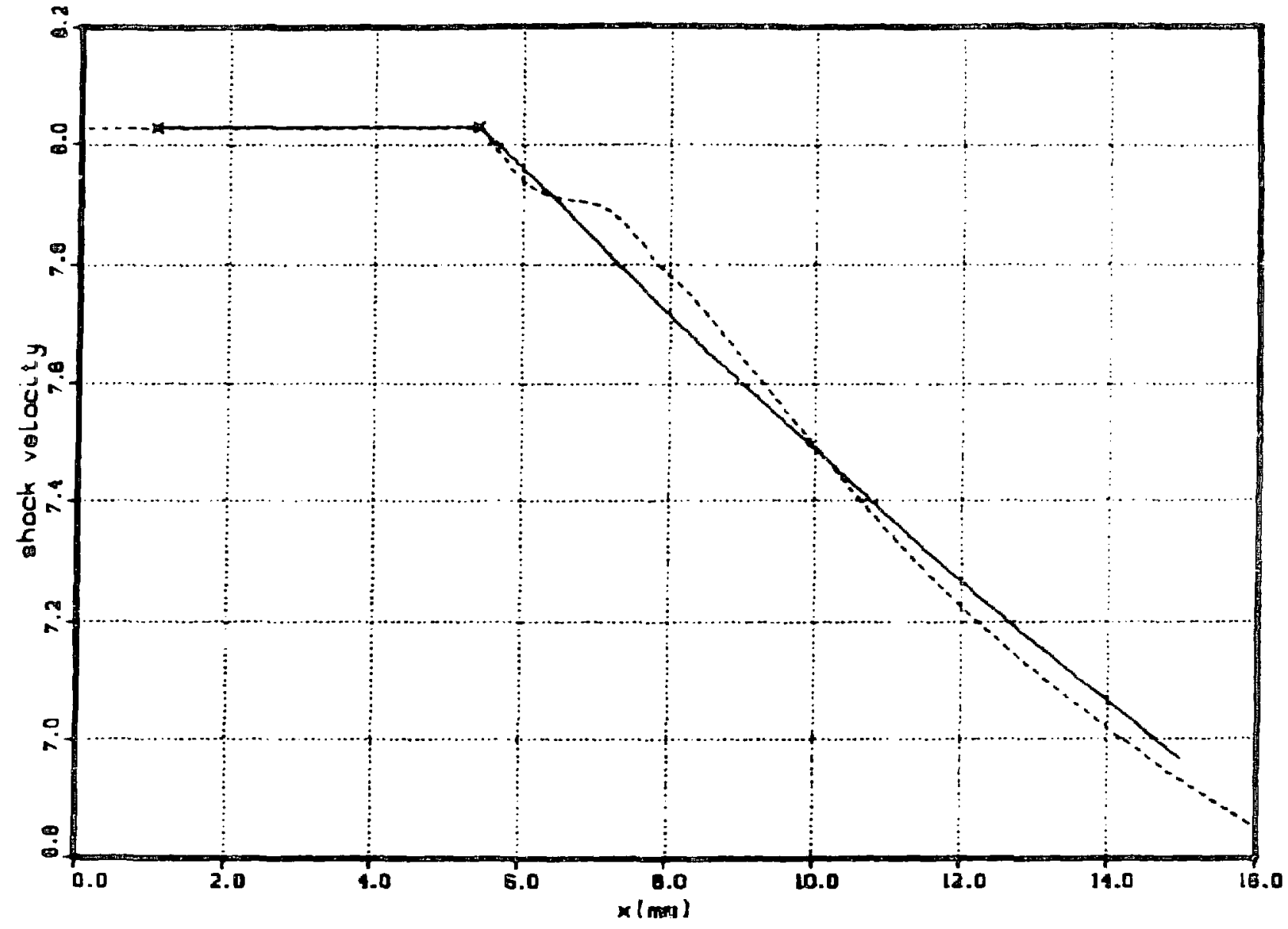

Figure 144: A comparison of the shock velocity from the code calculation and the shock velocity curve from a fit to the data for RS-2309. The solid line is the velocity curve taken frorn the fit to the data. The corresponding $\delta t$ plots are in figure 143 (see also table 58). 


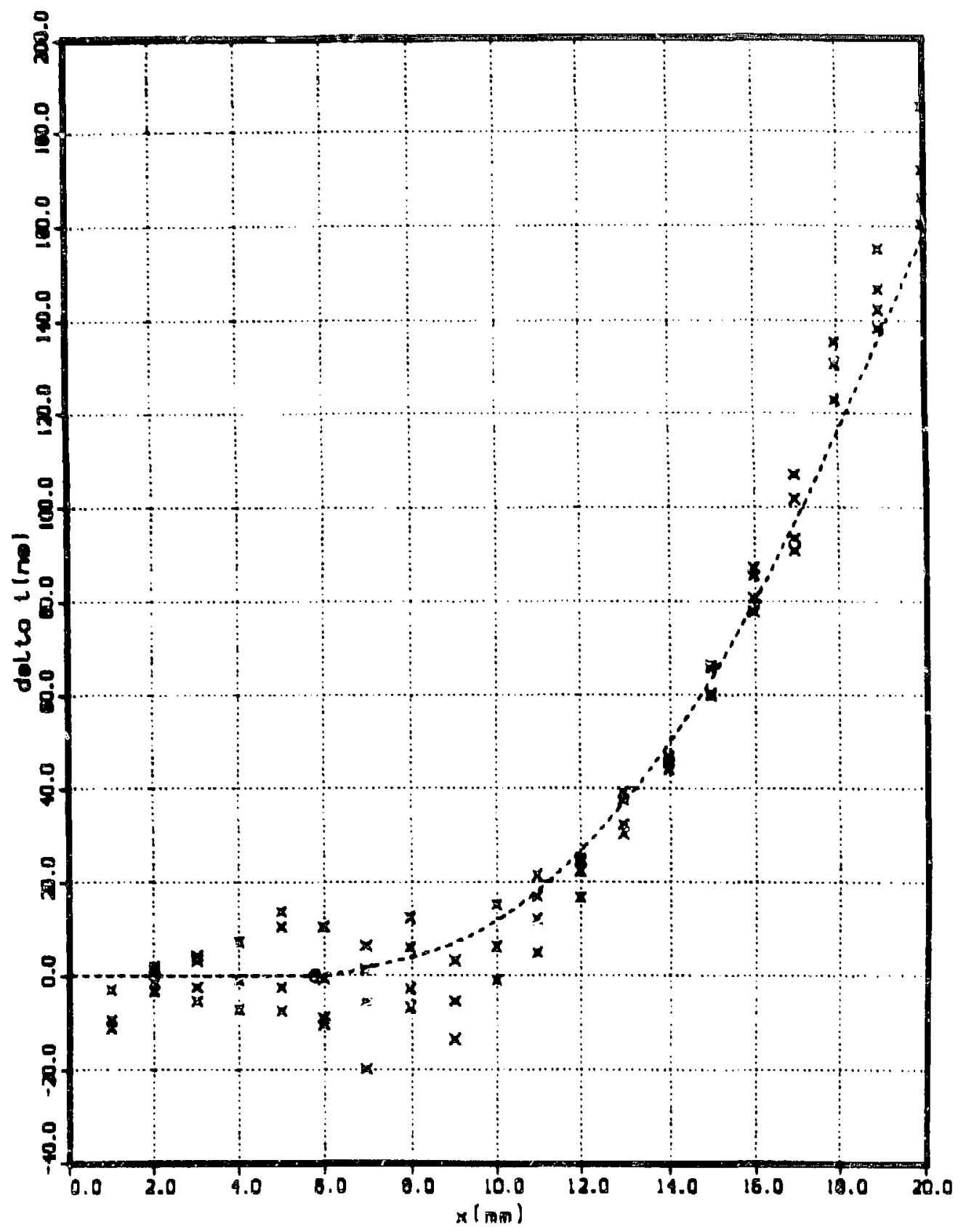

Figure 1A5: Comparison of the $\delta t$ data for RS-2302 and calculational results from the code SHKSJB. The dashed line is the $\delta t$ curve from the code calculation. Parameters for the calculational model and parameter for the data analysis are listed in table 59. 


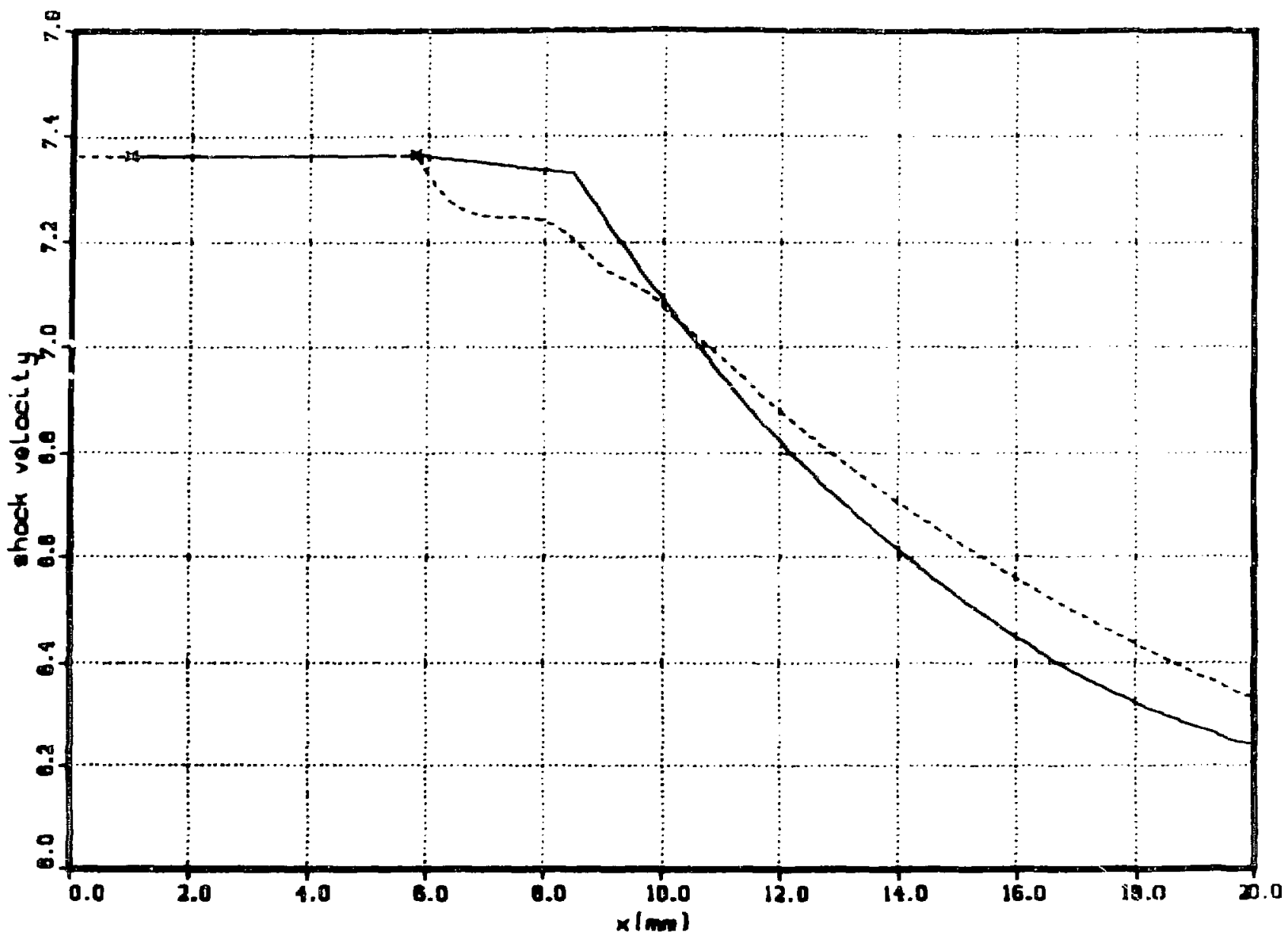

Figure 146: A comparison of the shock velocity from the code calculation and the shock velocity curve from a fit to the data for RS-2302. The solıd line is the velocity curve taken from the fit to the data. The corresponding $\delta t$ plots are in figure 145 (see also table 59). 


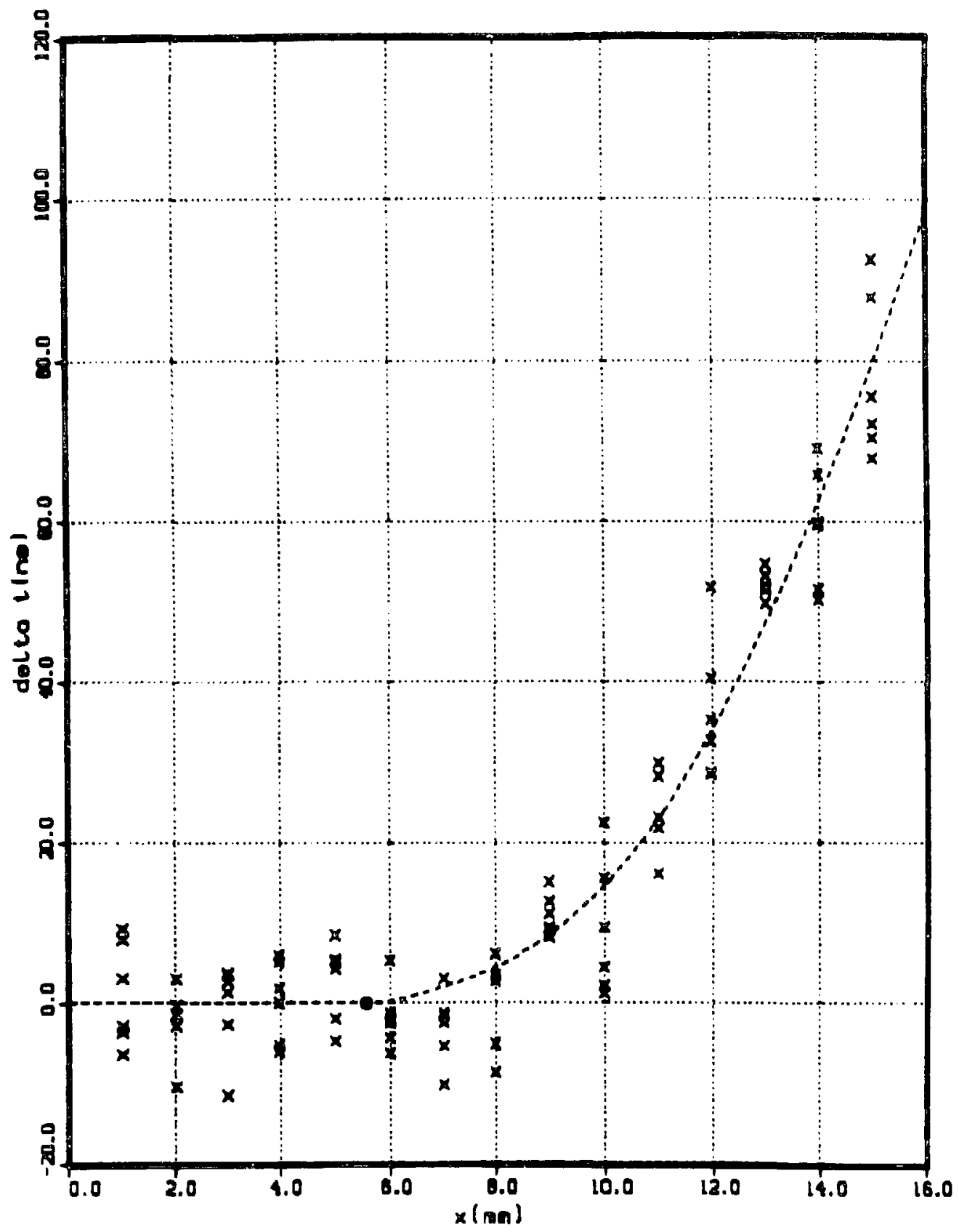

Figure 147: Comparison of the $\delta t$ data for RS-2315 and calculational results from the code SHKSJB. The dashed line is the ot curve from the code calculation. Parameters for the ca..ulational model and parameters for the data analysis are listed in table 60. 


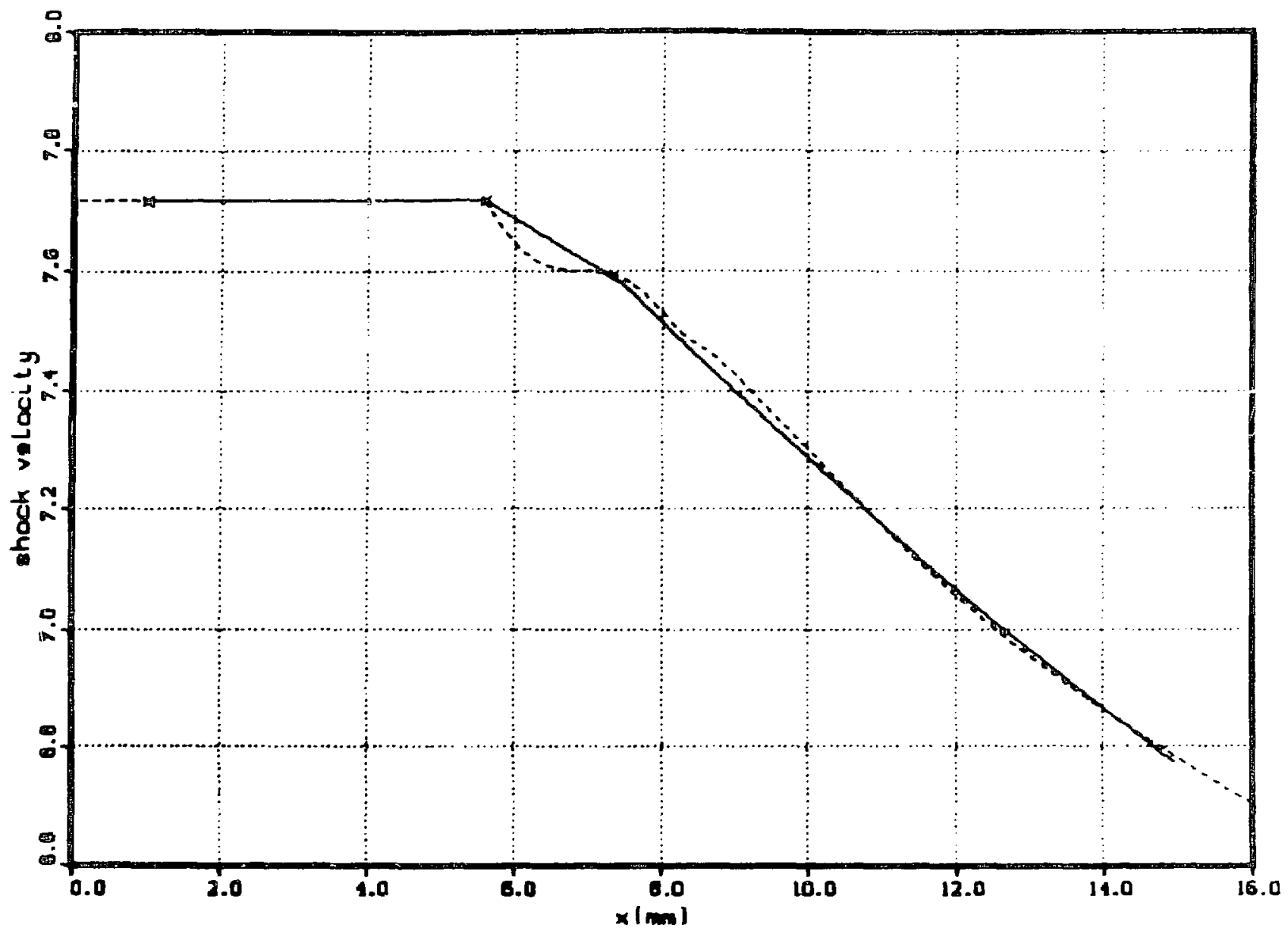

Figure 148: A comparison of the shock velocity from the code calculation and the shock velocity curve from a fit to the data for RS-2315. The solid line is the velocity curve taken from the fit to the data. The corresponding $\delta t$ plots are in figure 147 (see also table 60) 


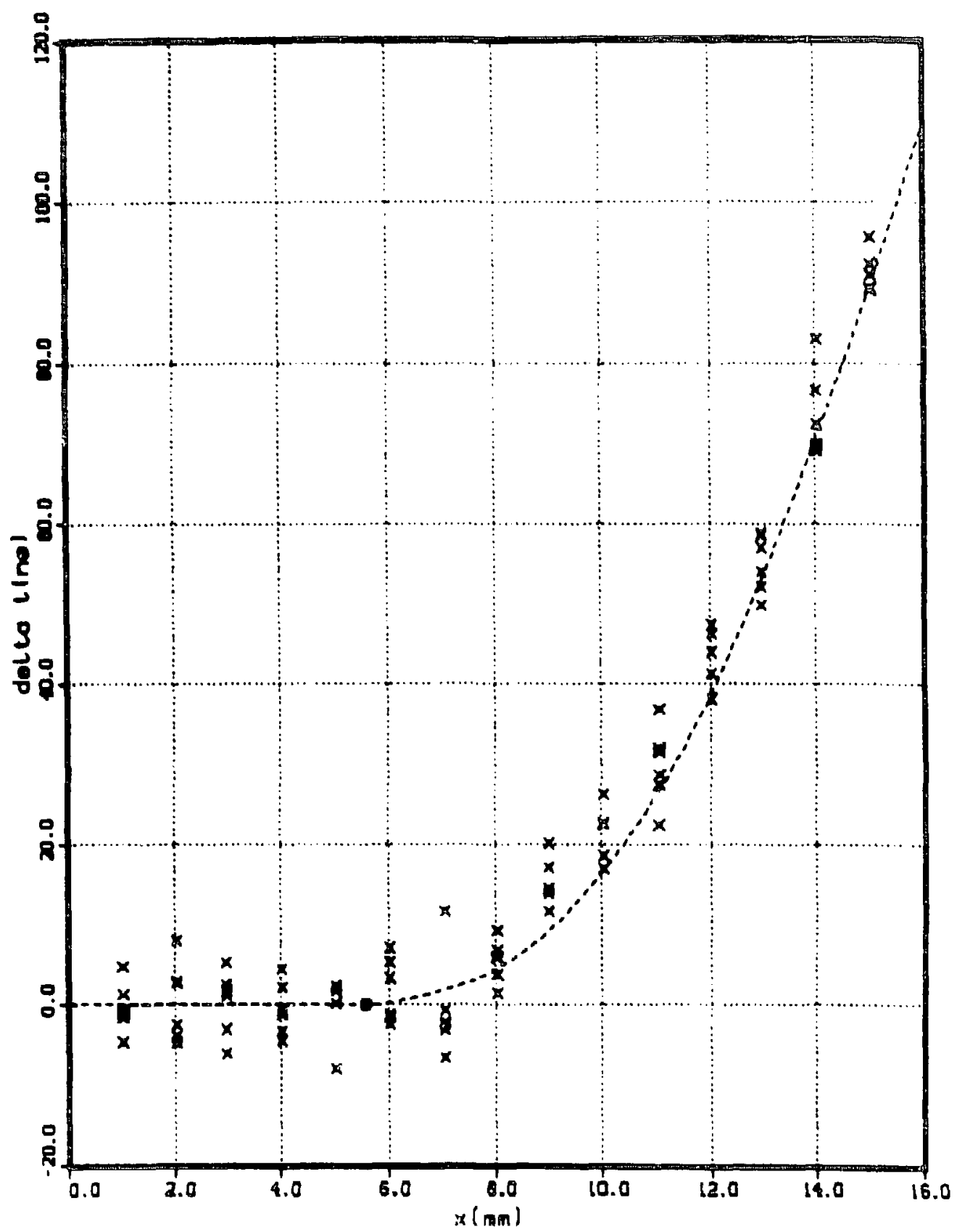

Figure 149: Comparison of the $\delta t$ data for RS-2309 and calculational results from the code SHKSJB. The dashed line is the $\delta$ t curve from the code calculation. Parameters for the calculational model and parameters for the data analysis are listed in table 61 . 


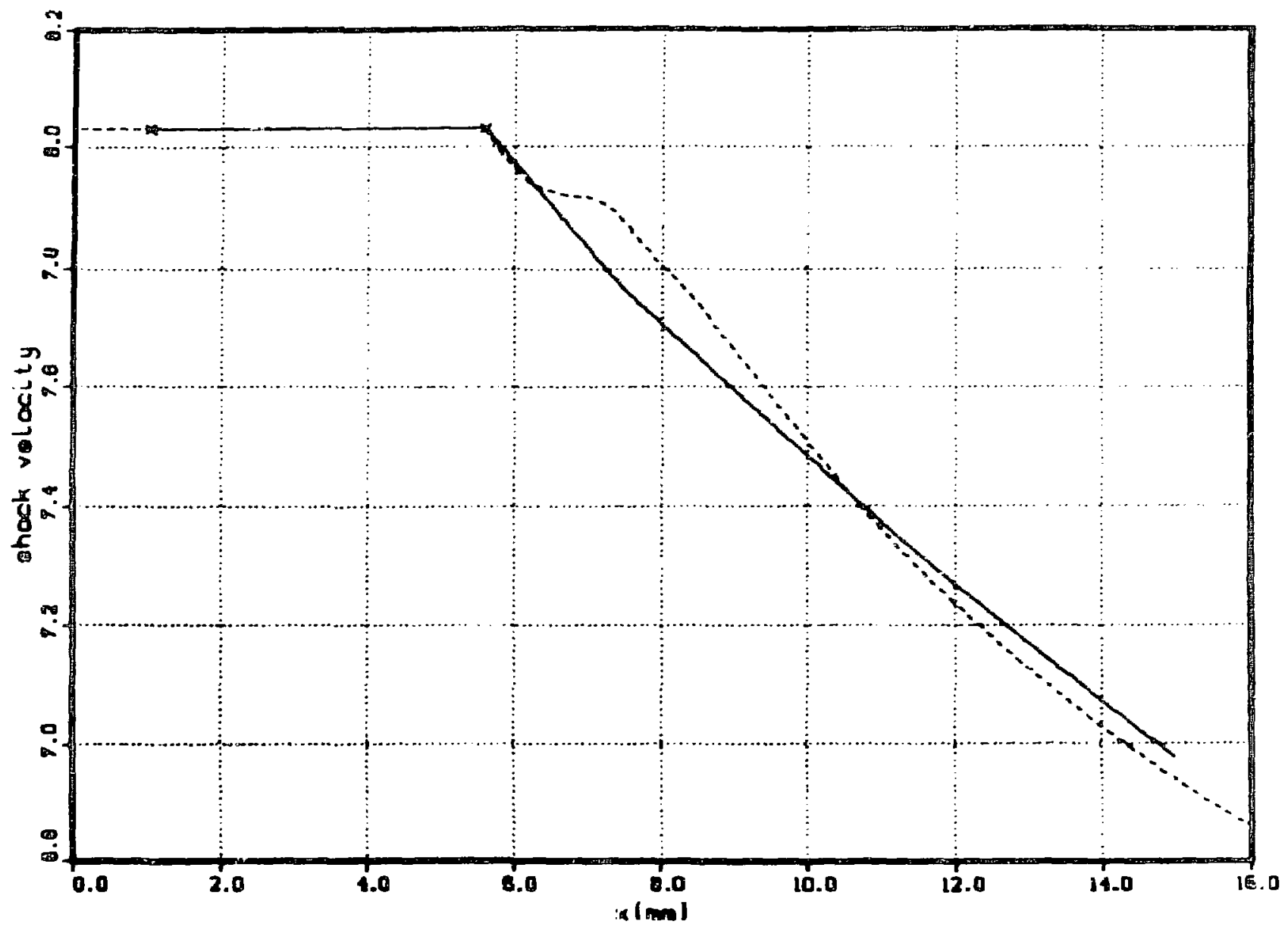

Figure 150: A comparison of the shock velocity from the code calculation and the shock velocity curve from a fit to the data for RS-2309. The solid line is the velocity curve taken from the fit to the data. The corresponding $6 t$ plots are in fgure 149 (see also table 61). 


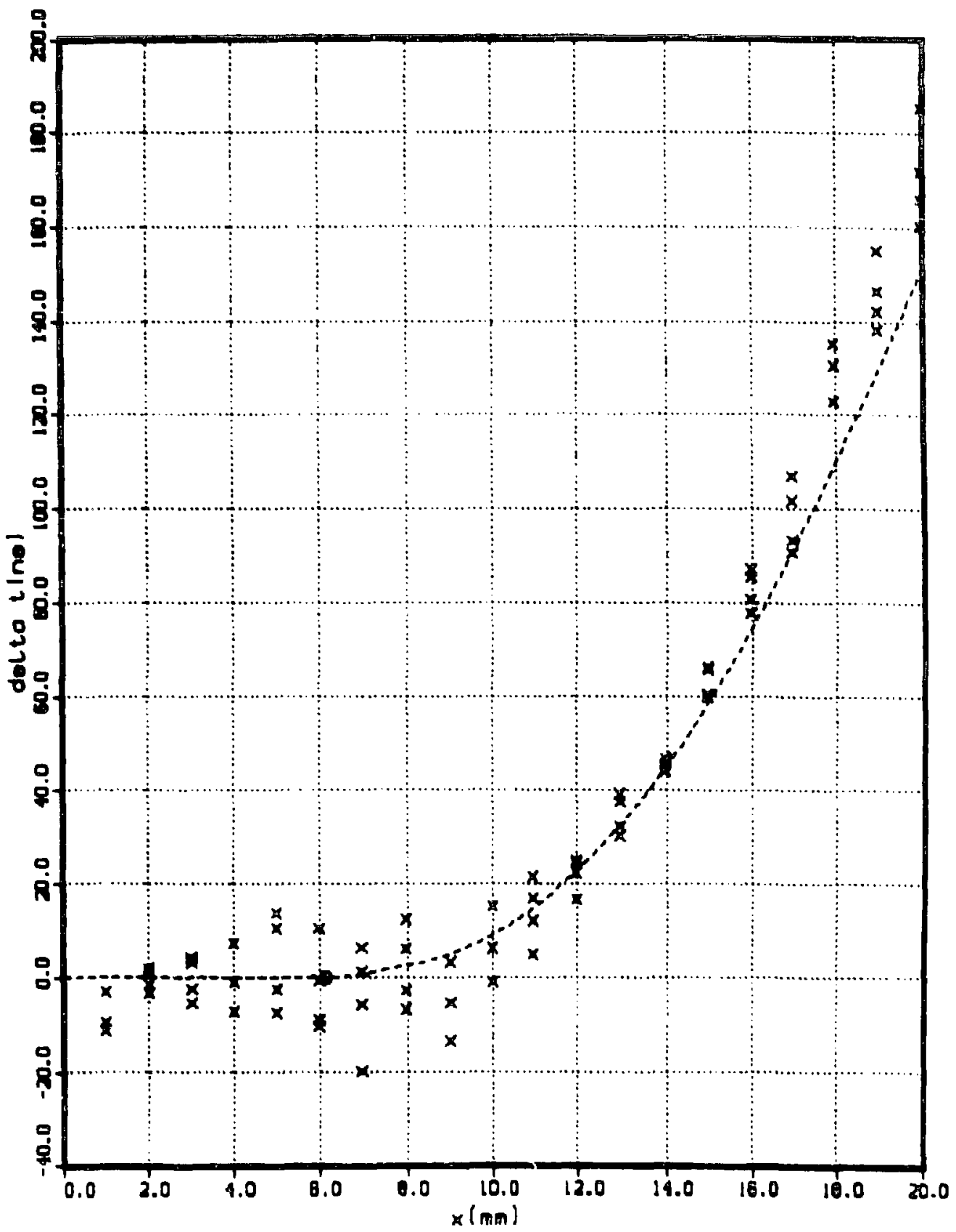

Figure 151: Comparien of the $6 t$ data for RS-2302 and calculational results from the code SHKSJB. The dashed line is the $\delta t$ curve from the code calculation. Parameters for the calculational model and parametors for the data analysis are listed in table $\mathbf{6 2}$. 


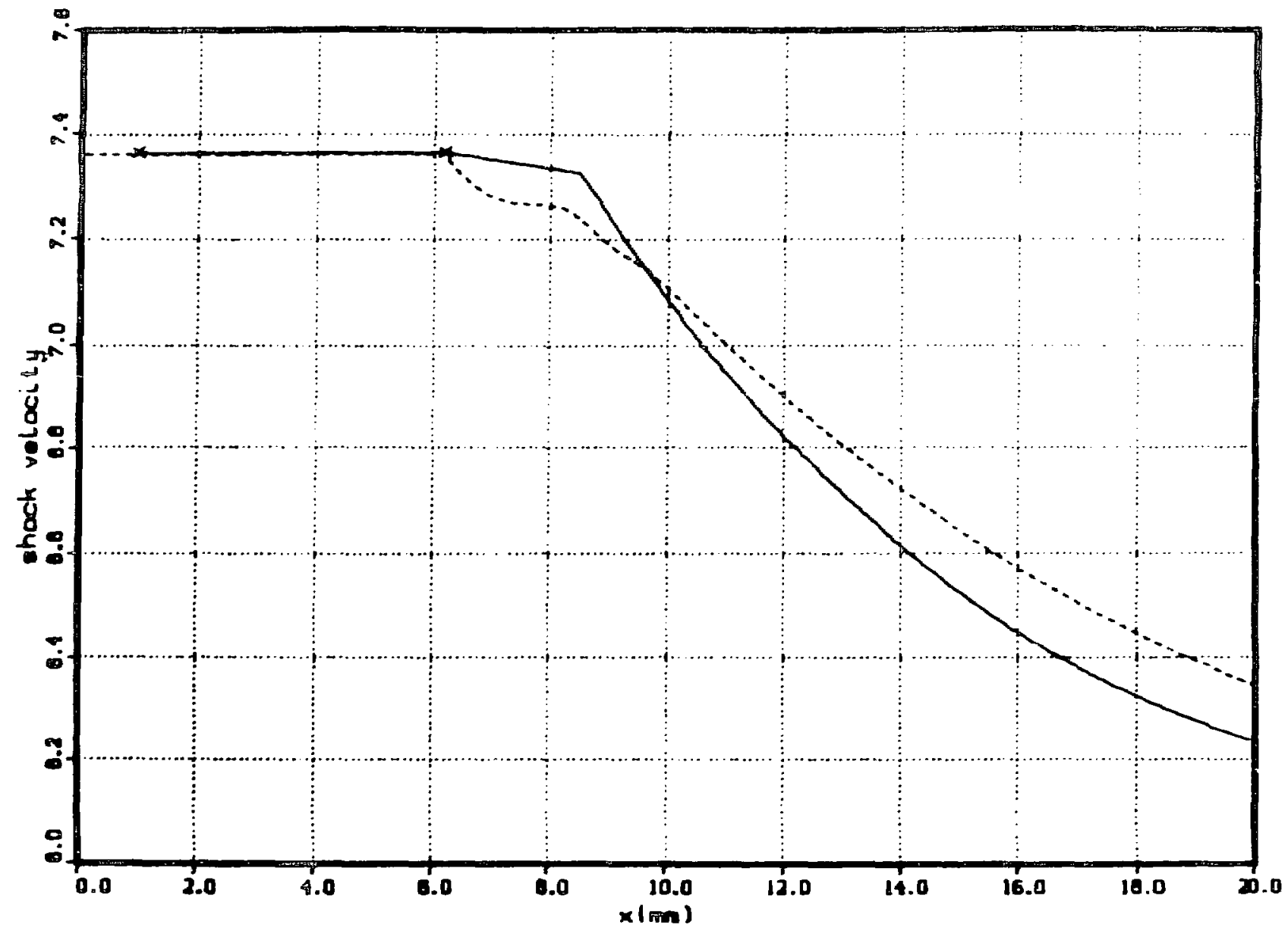

Figure 152: A comparion of the shock velocity from the code calculation and the slock velocity curve from a fit to the data for RS-2302. The solid line is the velocity curve taken from the fit to the data. The corresponding $\delta t$ plots are in tigure 151 (see also tail' 62 ). 


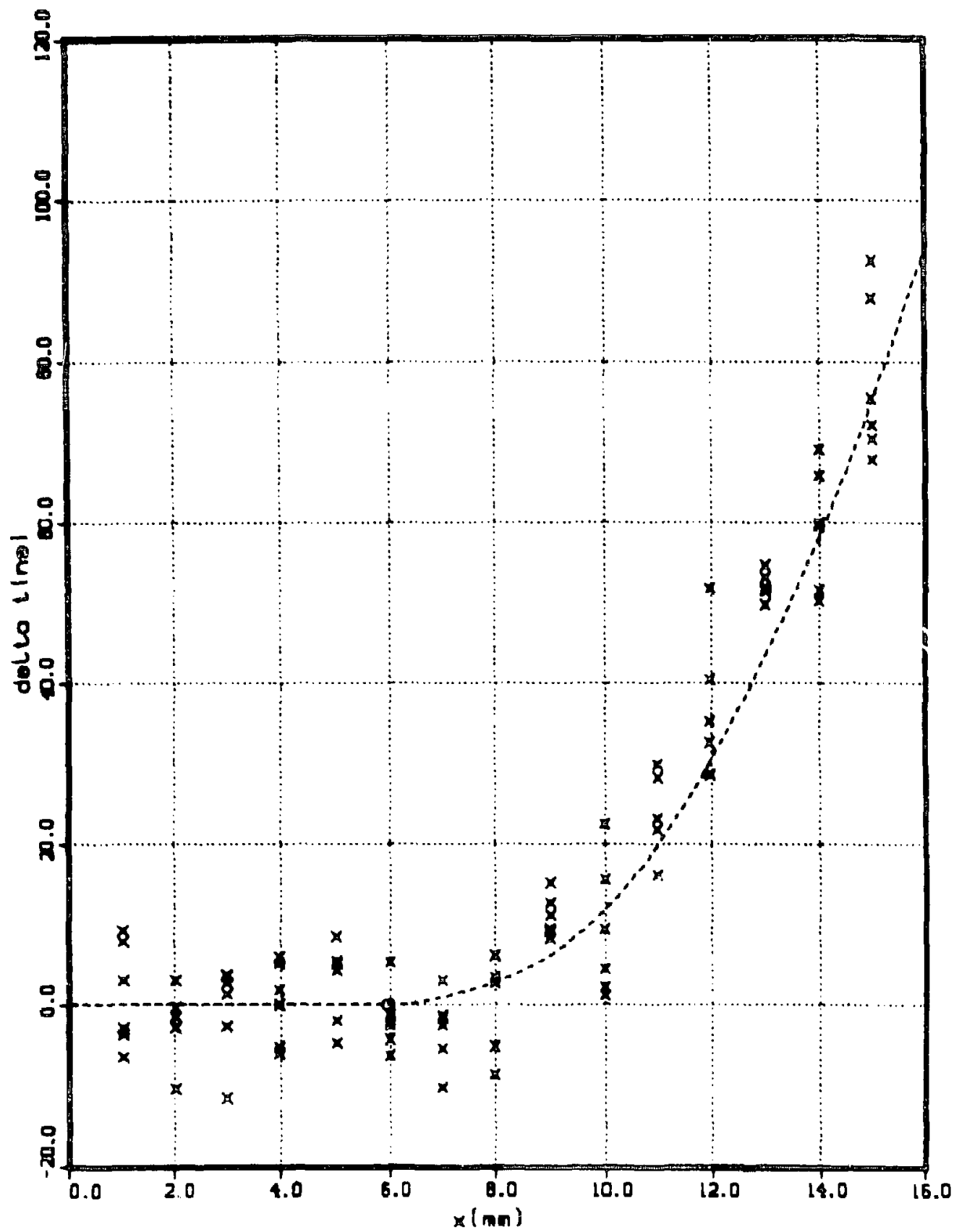

Figure 153: Comparison of the $\delta t$ data for RS-2315 and calculational results from the code SHKSJB. The daghed line is the $\delta t$ curve from the code calculation. Parameterg for the calculational model and parameters for the data analysis are listed in table 63. 


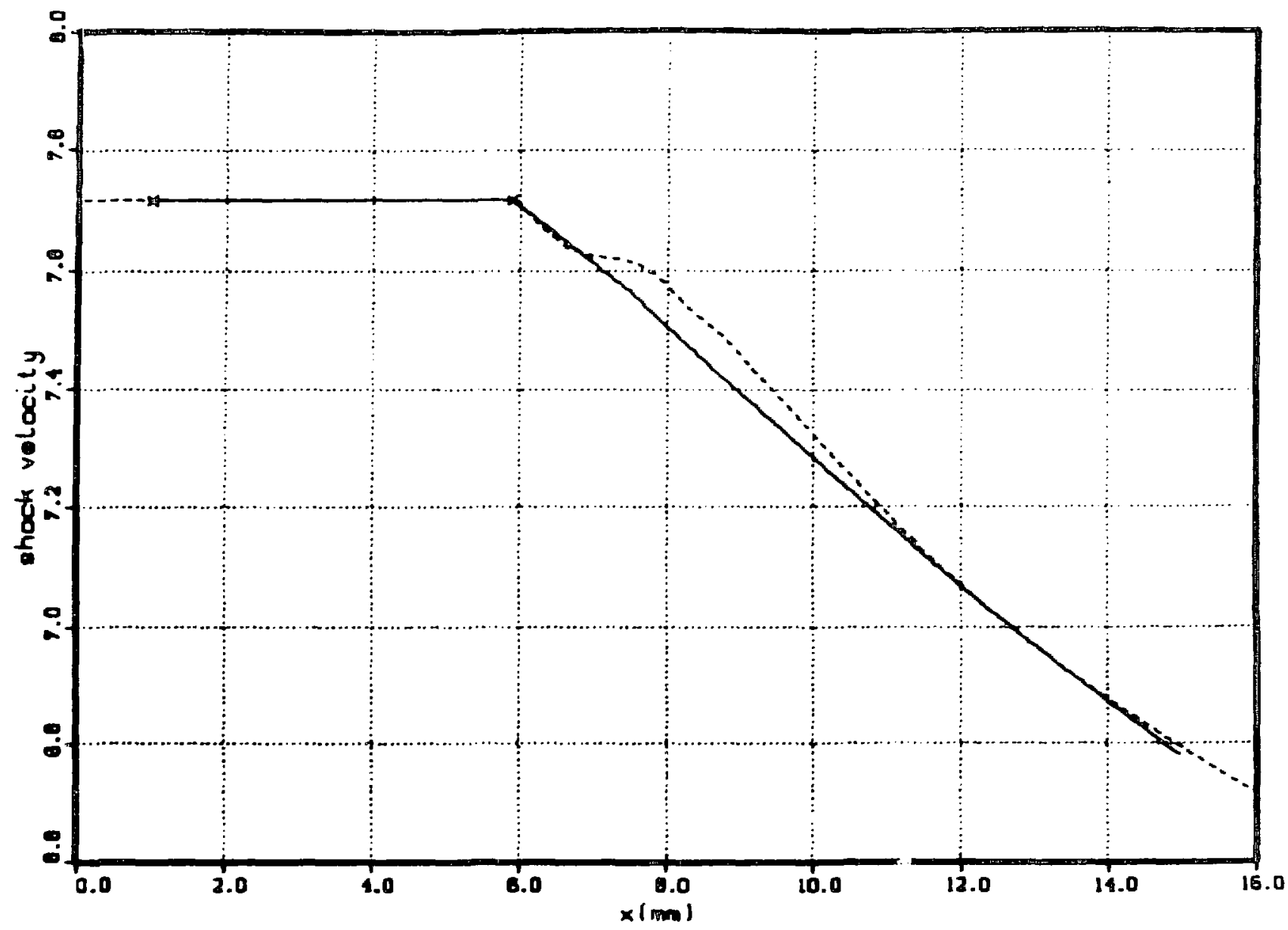

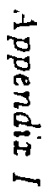

Figure 154: A comparison of the shock velocity from the code calculation and the shock velocity curve from a fit to the data for RS-2315. The solid line is the veiocity curve taken from the fit to the data. The corresponding $\delta t$ plots are in figure 153 (see also table 63). 


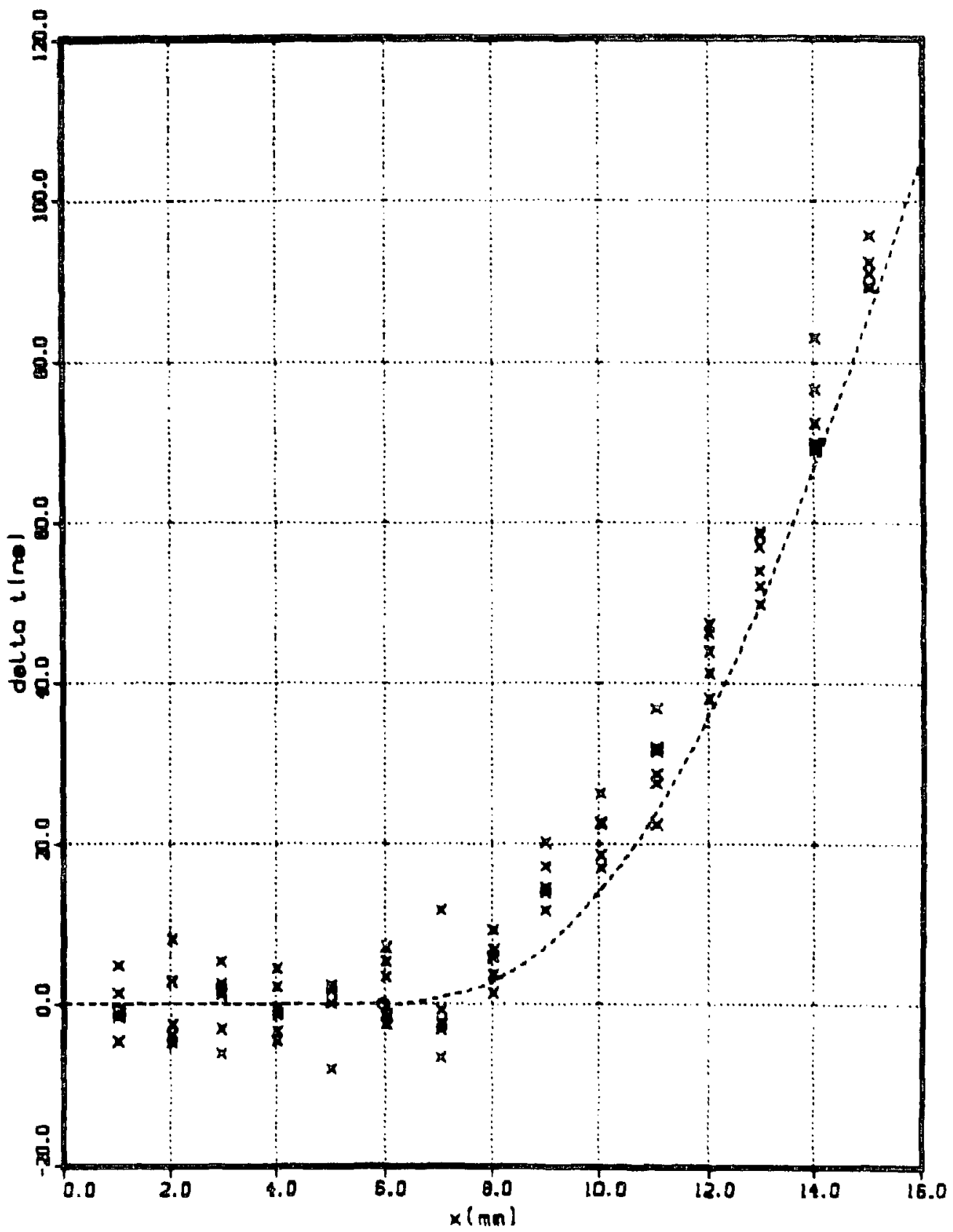

Figure 155: Comparison of the $\delta t$ data for RS-2309 and calculational results from the code SHKSJB. The dashed line is the $\delta t$ curve from the code calculation. Parameters for the calculational model and parameters for the data analysis are listed in table $\mathbf{6 4}$. 


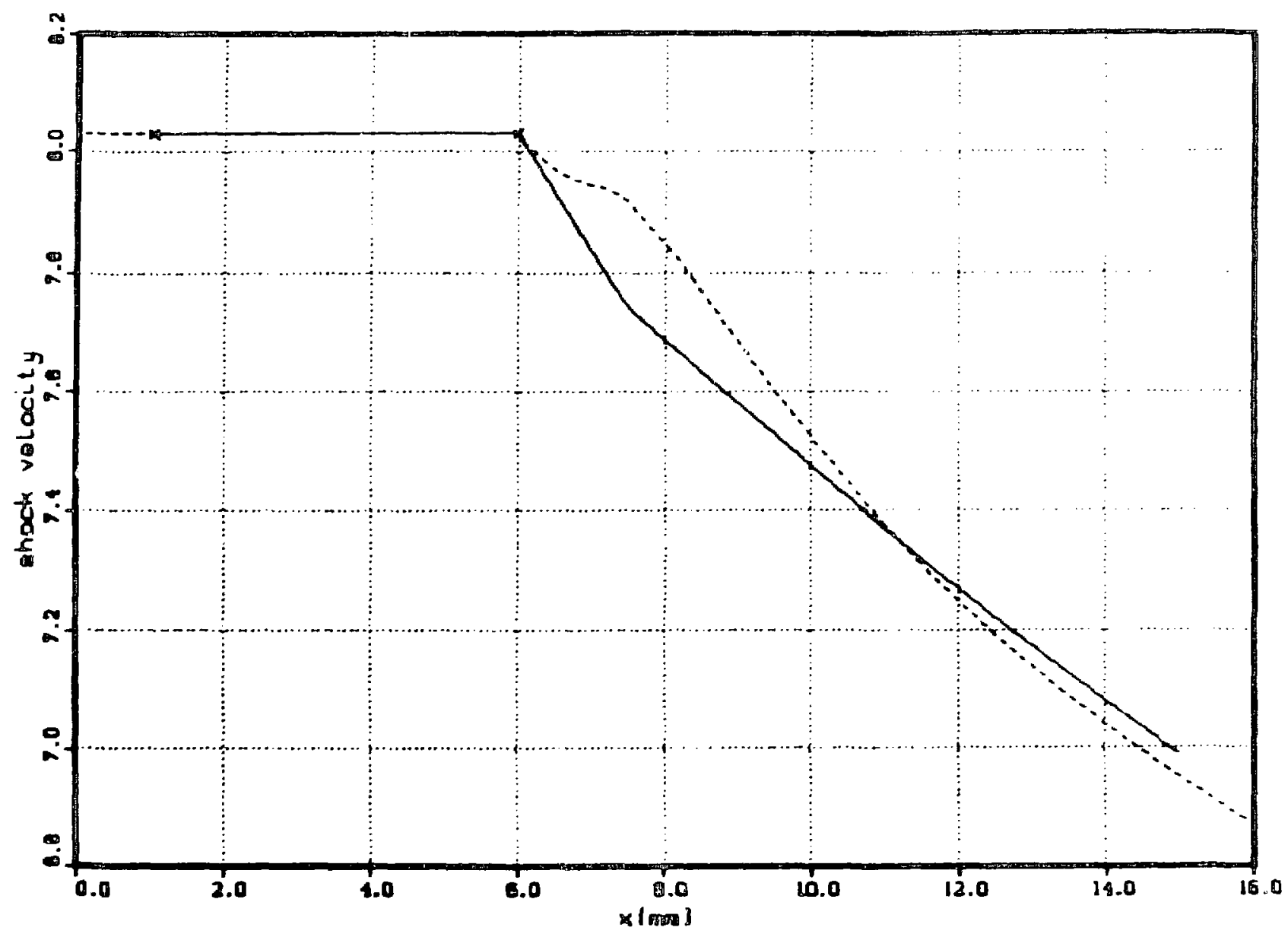

Figure 156: A comparison of the shock velocity from the code calculation and the shock velocity curve from a lit to the data for RS-2309. The solid line is the velocity curve taken from the fi to the data. The corresponding $\delta t$ plots are in Ggure 155 (see also table 64) 


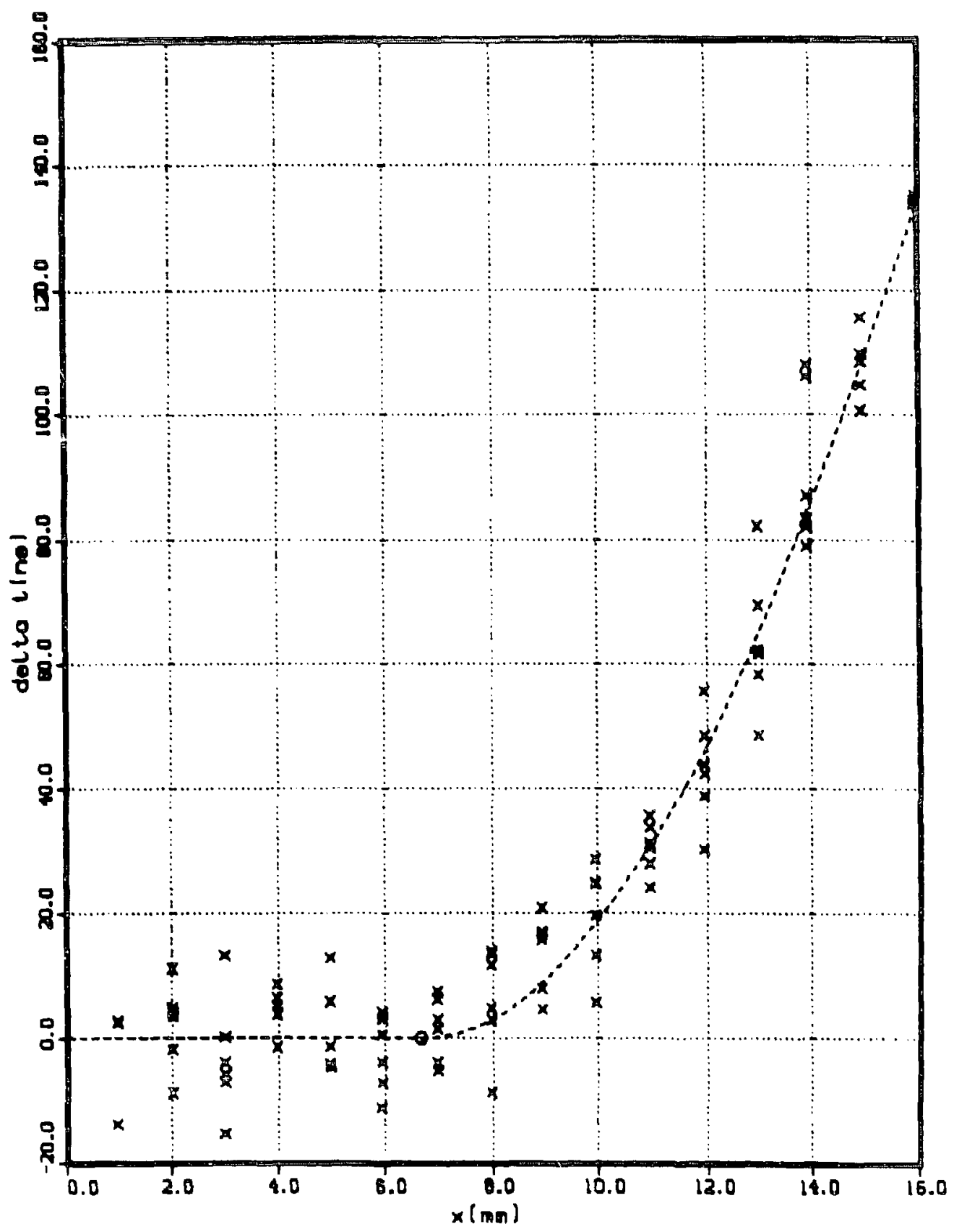

Figure 157: Comparison of the $\delta t$ data for RS-2306 and calculational results from the code SHKS. $\exists$. The dashed line is the $\delta t$ curve from the code calculation. Parameters for the calculational model and parameters for the data analysis are listed in table 65 . 


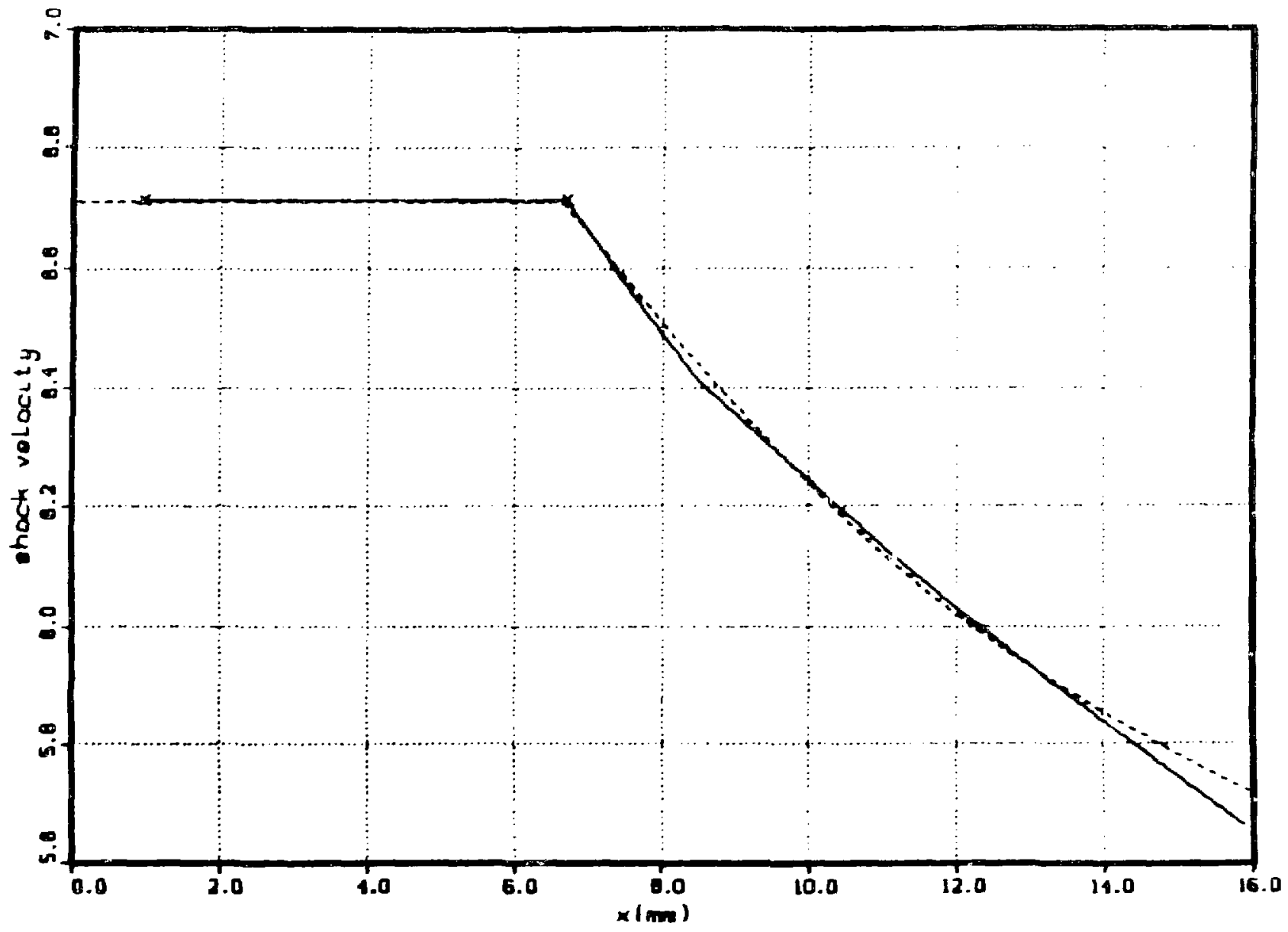

Figure 158: A compisrigun of the shock velocily fiom the code calculation and the shuck velucily curve frun a lit to the data for HS-2306. The solid line is the velocity curve baken from the fit tu the data. The corresponding of pluts are in tigure 157 (see also table b5). 


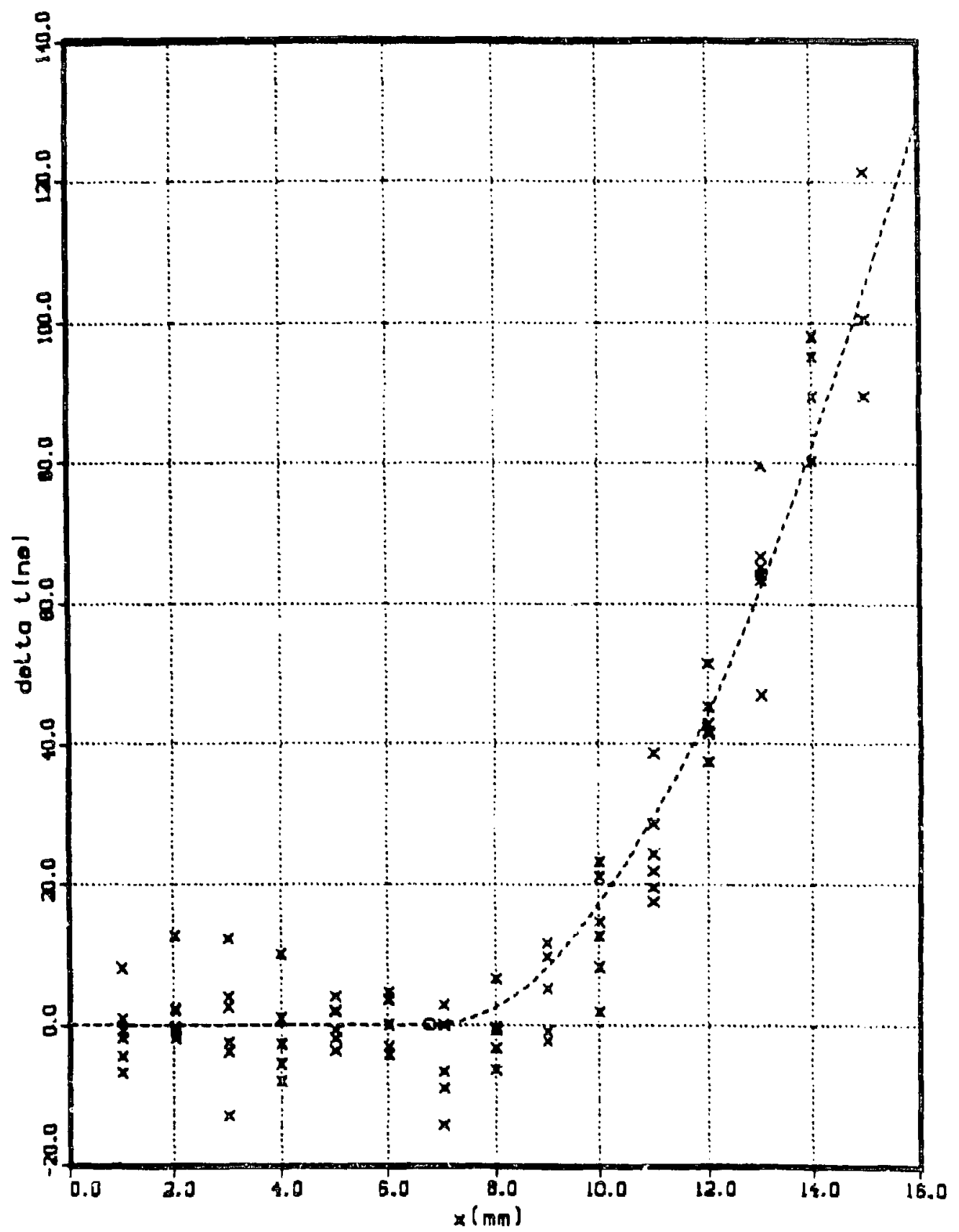

Figure 159: Comparison of the $\delta t$ data for RS-2314 and calculational resulte from the code SHKSJB. The dashed line is the $\delta t$ curve from the code calculation. Parameter for the caitulational model and parameters for the data analygis are listed in table 66. 


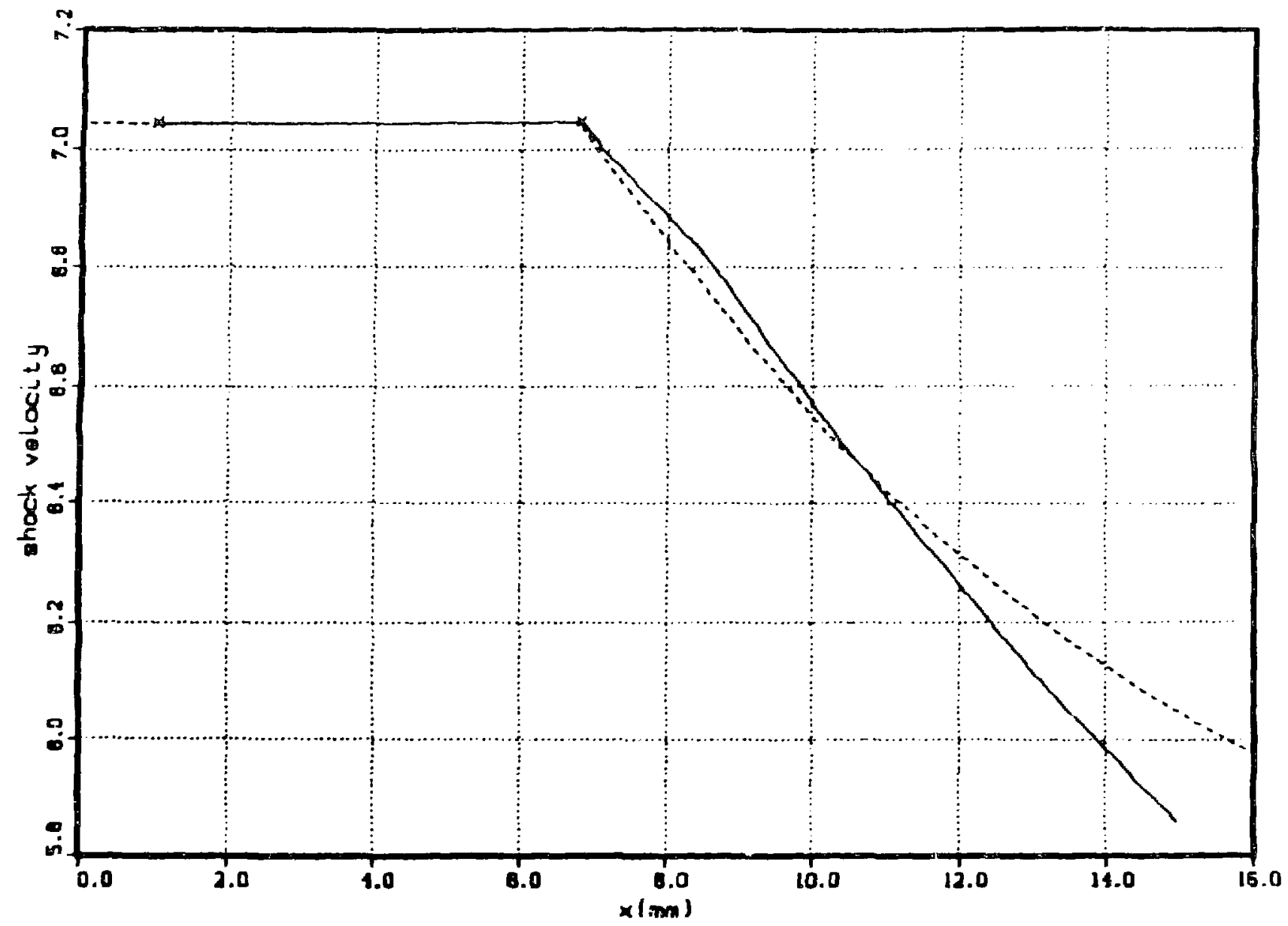

Figure 160: A comparison of the shock velocity from the cude calculation and the shock velocily curve from a fit to the data for RS-2314. The solid line is the velocity curve taken from the fit to the data. The corresponding $\delta t$ plots are in figure 159 (see also table 66). 


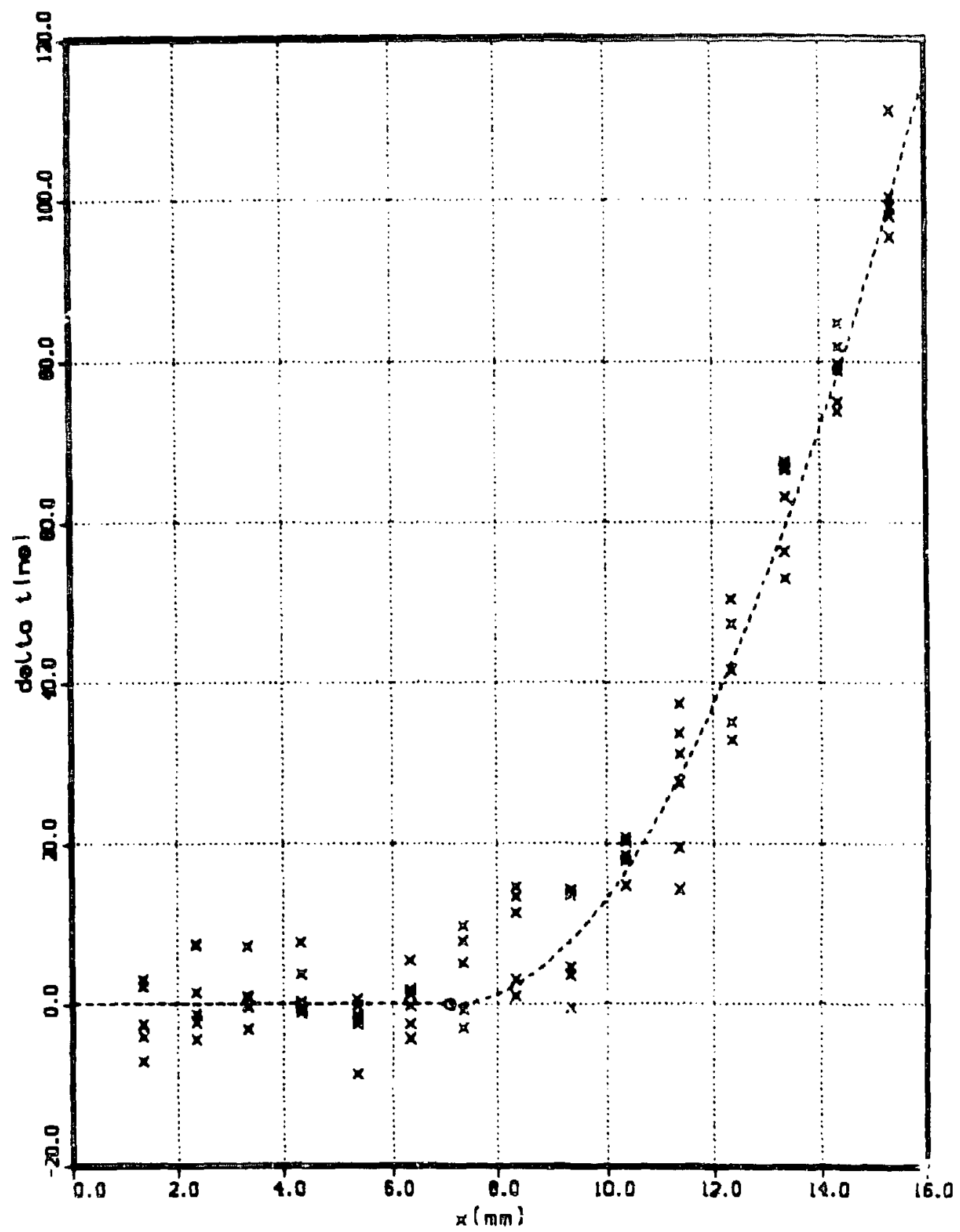

Figure 161: Comparison of the $\delta t$ data ior RS-2308 and calculational resulta from the code SHKSJB. The dashed line is the $\delta t$ curve from the code calculation. Parameters for the calculational model and pa:ameters for the data analygis are listed in table 67. 


$$
D
$$




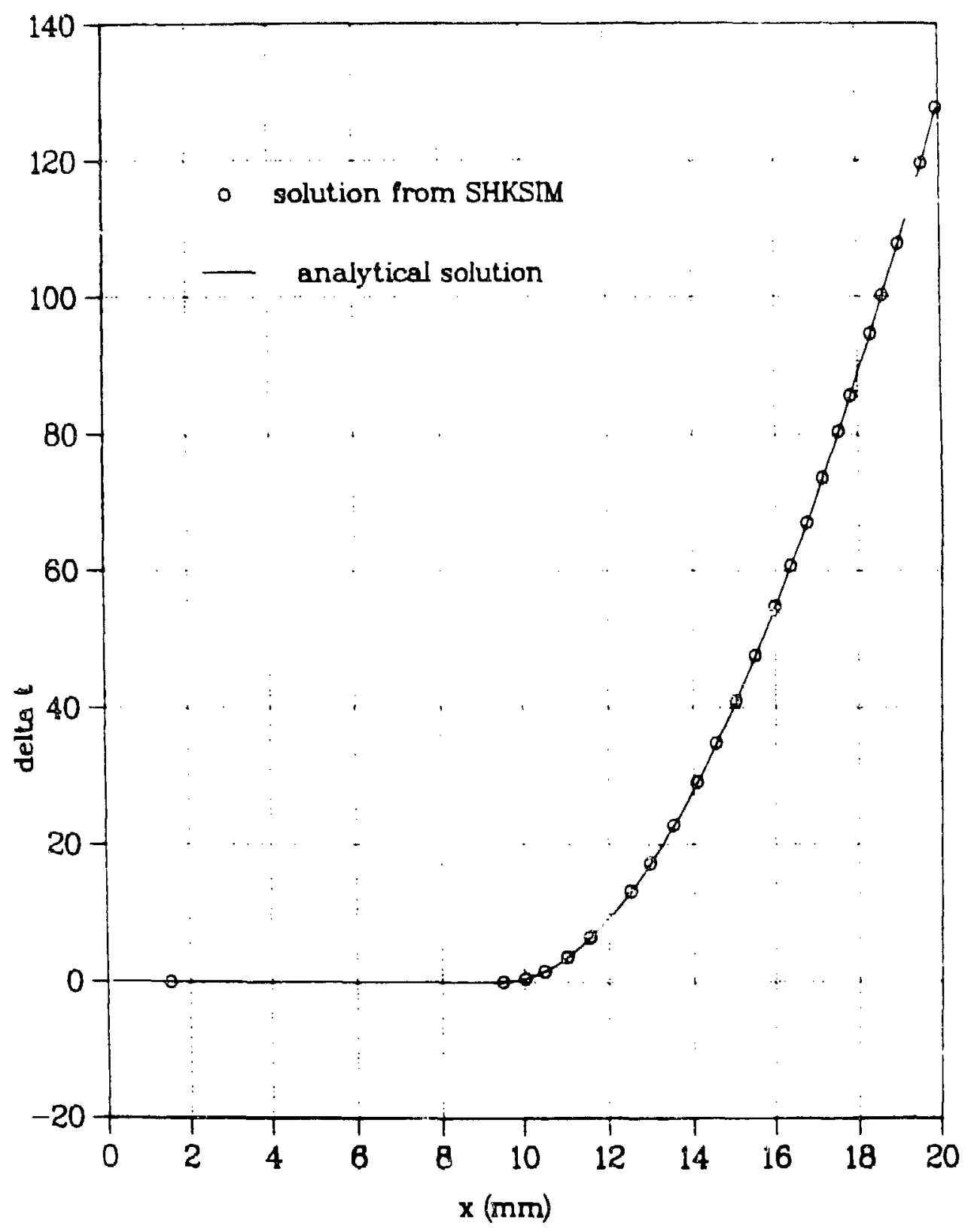

Figure 163. Comparieon of numerical resulta from SHKSIM and an analytical solution for shock attenuation. The initial oning for SHKSIM was $0.05 \mathrm{~mm}$. The initial flyer plate velocity in the calculation was $3.7 \mathrm{~km} / \mathrm{s}$. 


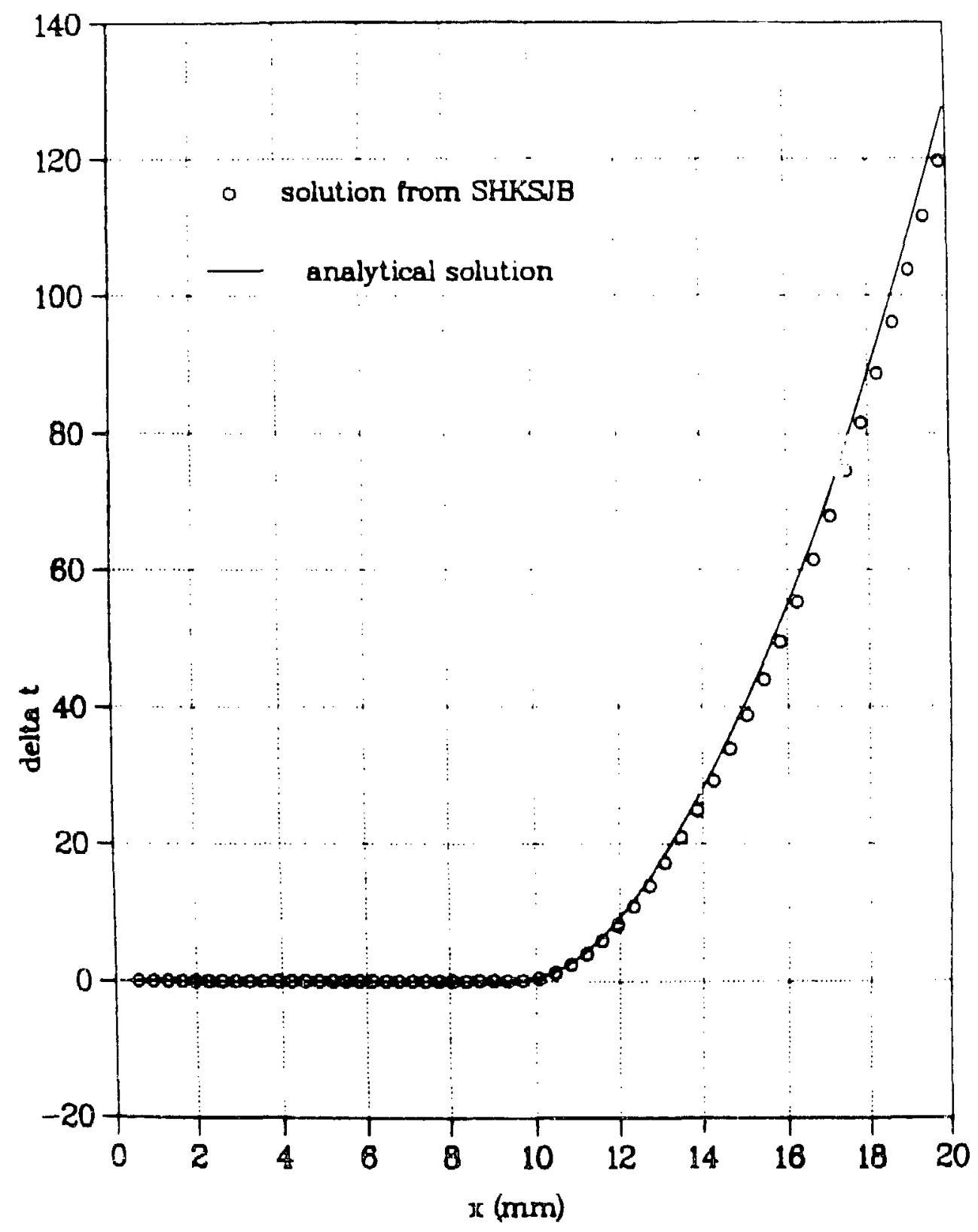

Figure 164. Comparison of numerical results from SHKSJB and an analytical solution for shock attenuation. The initial zoning for SHKSJB was $0.05 \mathrm{~mm}$. The initial tyer plate velocity in the calculation was $3.7 \mathrm{~km} / \mathrm{s}$. 


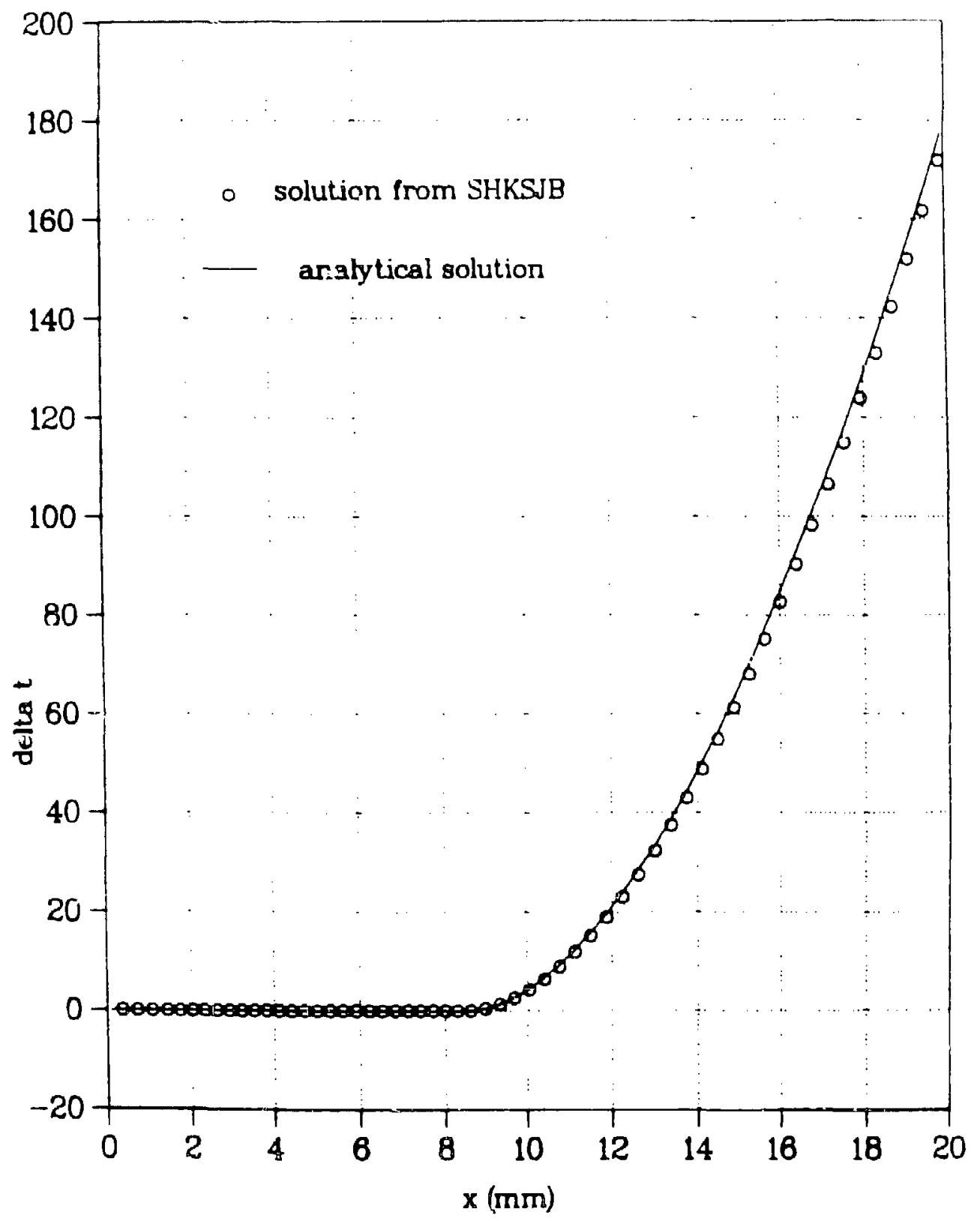

Figure 165. Comparison of numerical results from SHKSJB and an analytical solution for shock attenuation. The initial soning for SHKSJB was $0.025 \mathrm{~mm}$. The initial tyer plate velocity in the calculation was $4.65 \mathrm{~km}_{/}^{\prime} \mathrm{s}$. 


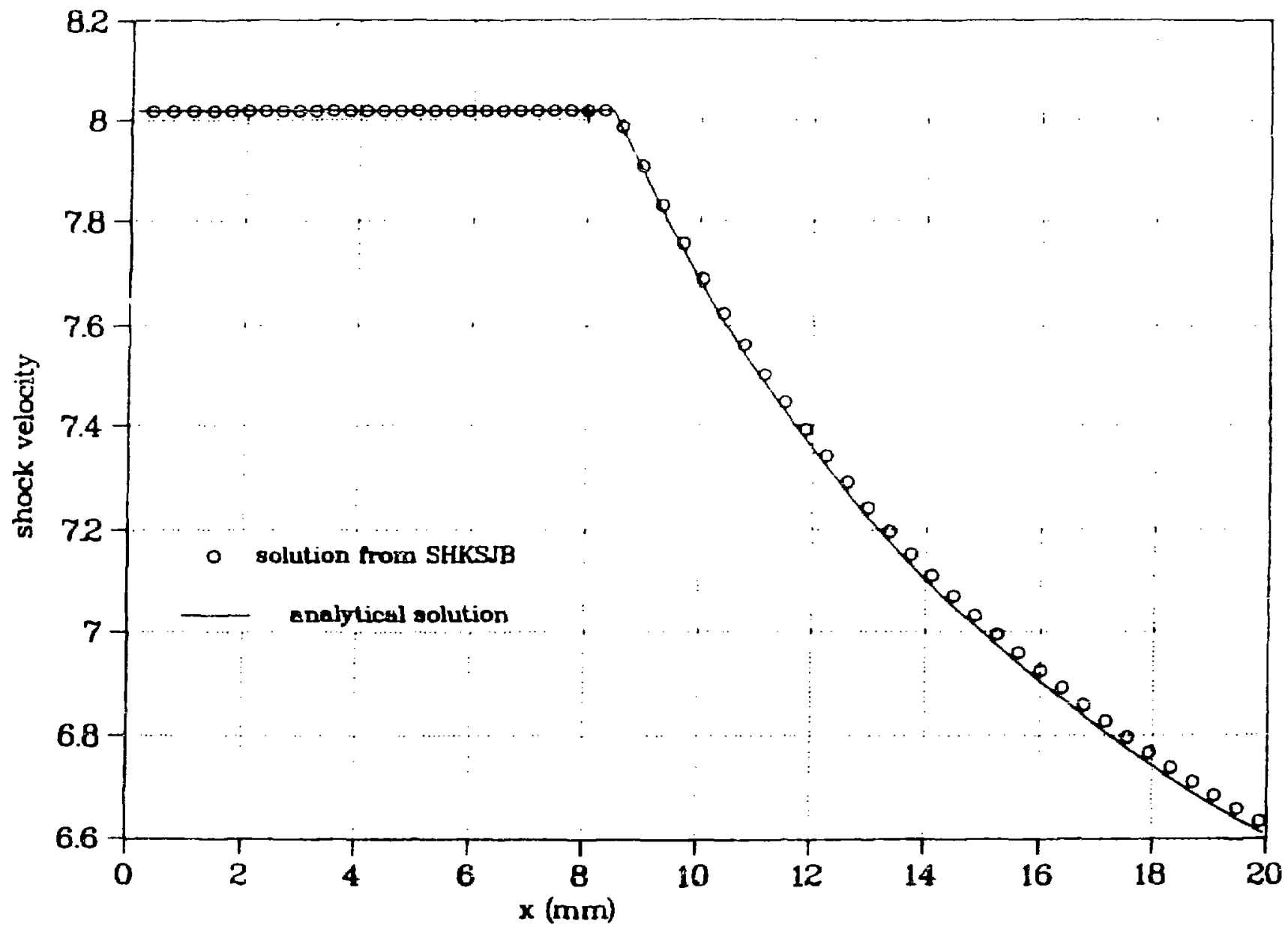

它

Figure 166. Comparion of the shock velocities from SHJSJB and an analytical solution plotted as a function of shock position. The corresponding $\delta t-x$ plots are shown in figure 165 . 


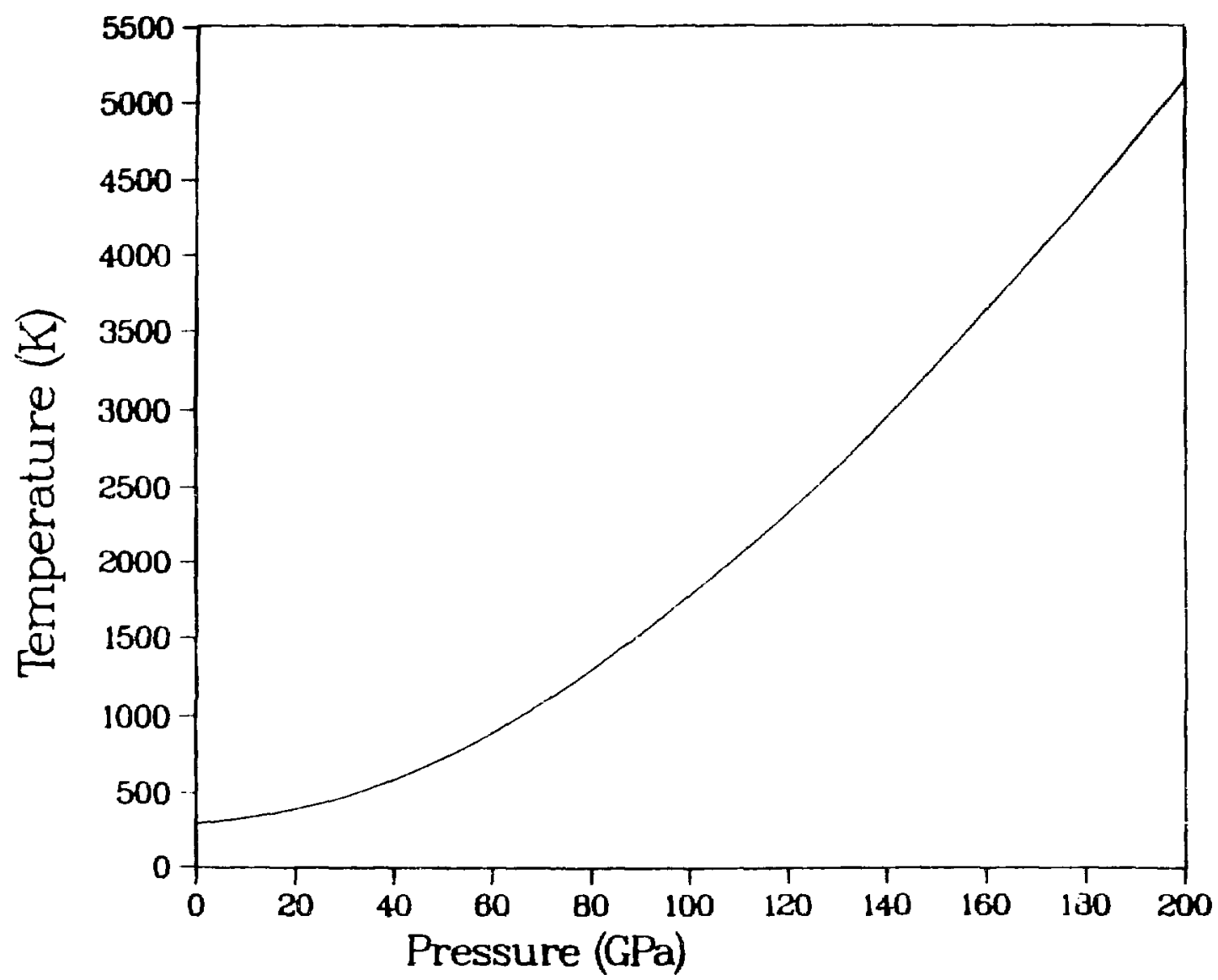

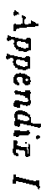

Figure 167. Temperature along the principal Hugoniot for copper taken from the integration of equations (d3)-(d5). Electronic effects are not explicitly taken into account.

$\stackrel{\vec{\omega}}{\omega}$ 


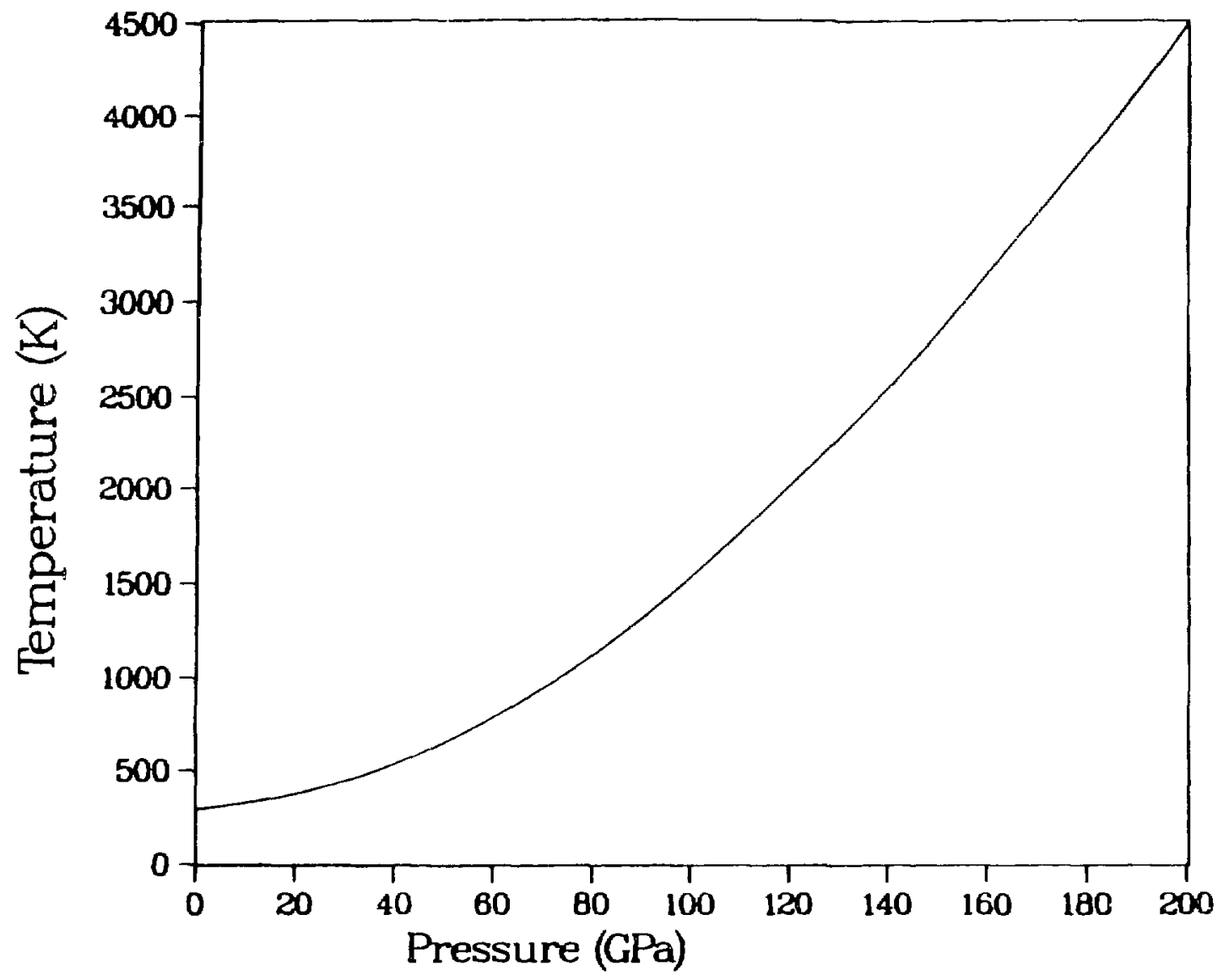

过

Fifure 168. Temperature along the principal Hugoniot for stainless-steel taken from the in:-siation of equations $(d 3)-(d 5)$. Electronic effects are not explicitly taken into account. 


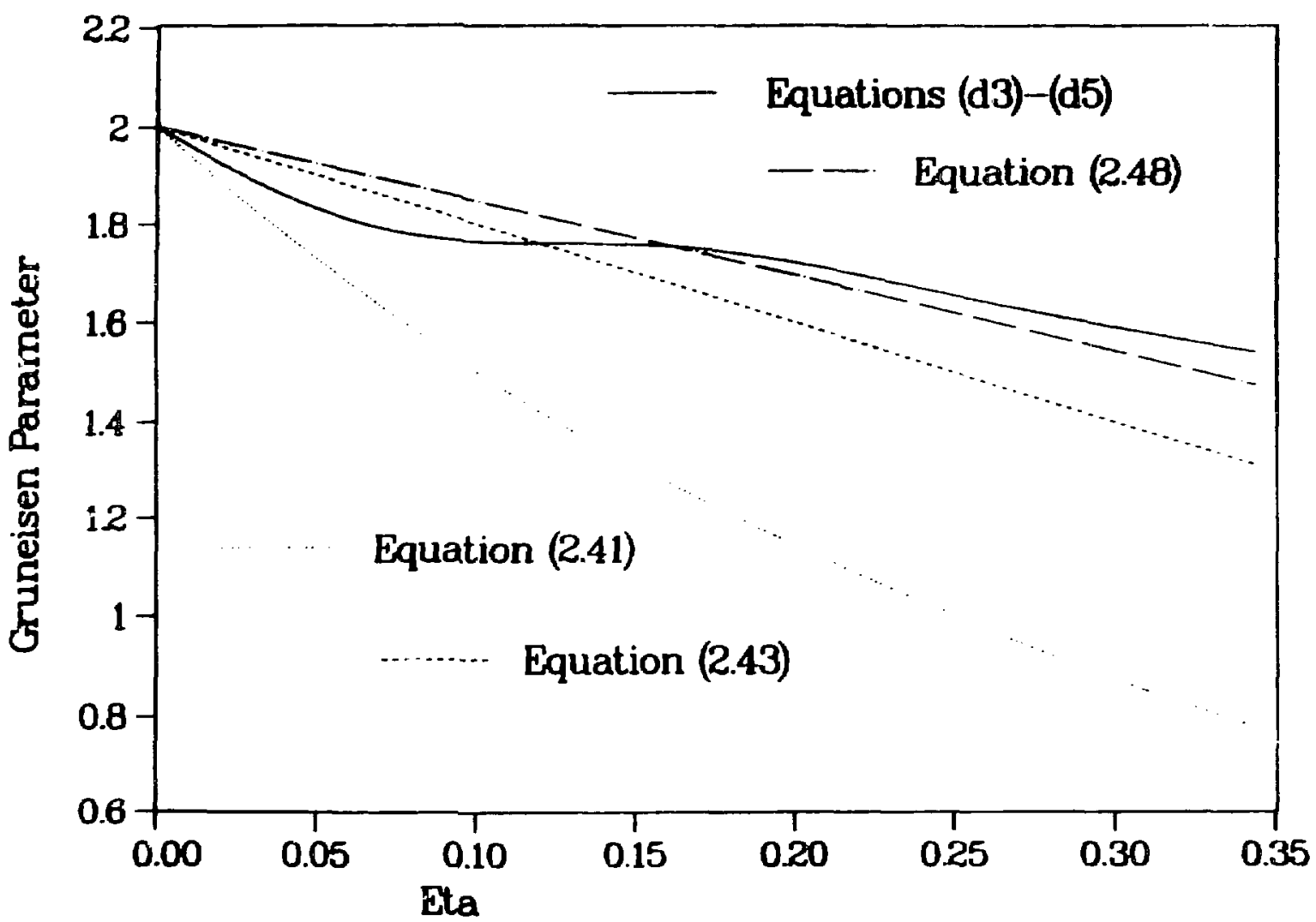

Figure 169. The Gruneisen parameter for copper plotted as a function of $\eta$ from different formulas. 


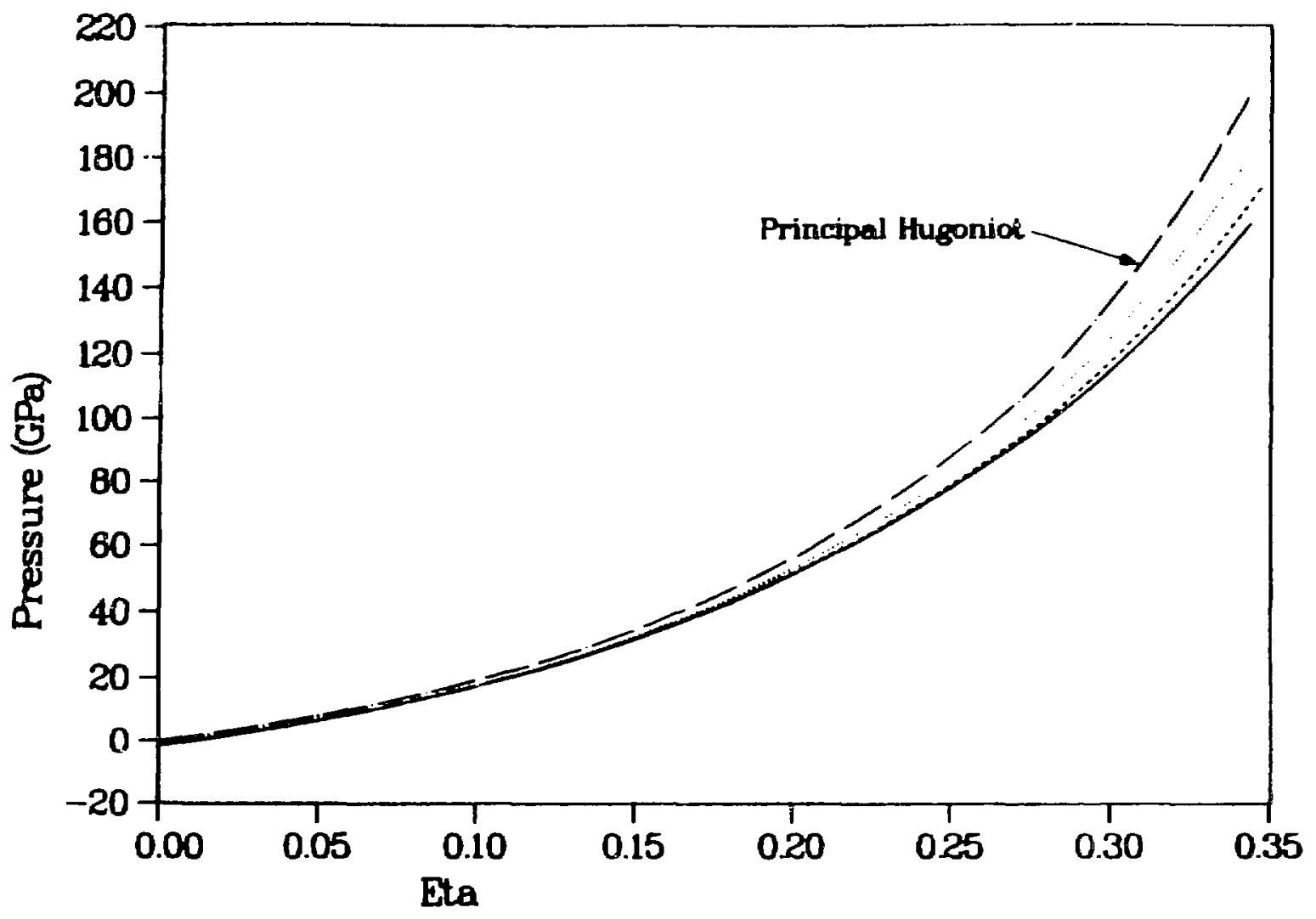

Figure 170. Pressure as a function of $\eta$ along sero-degree isotherms for copper. The dashed line is calculated using the Mie-Gruneisen equation of state. The dotted line is calculated u.sing the Rice equation of state. The solid line is from the integration of equaticns $(d 3)-(d 5)$. 


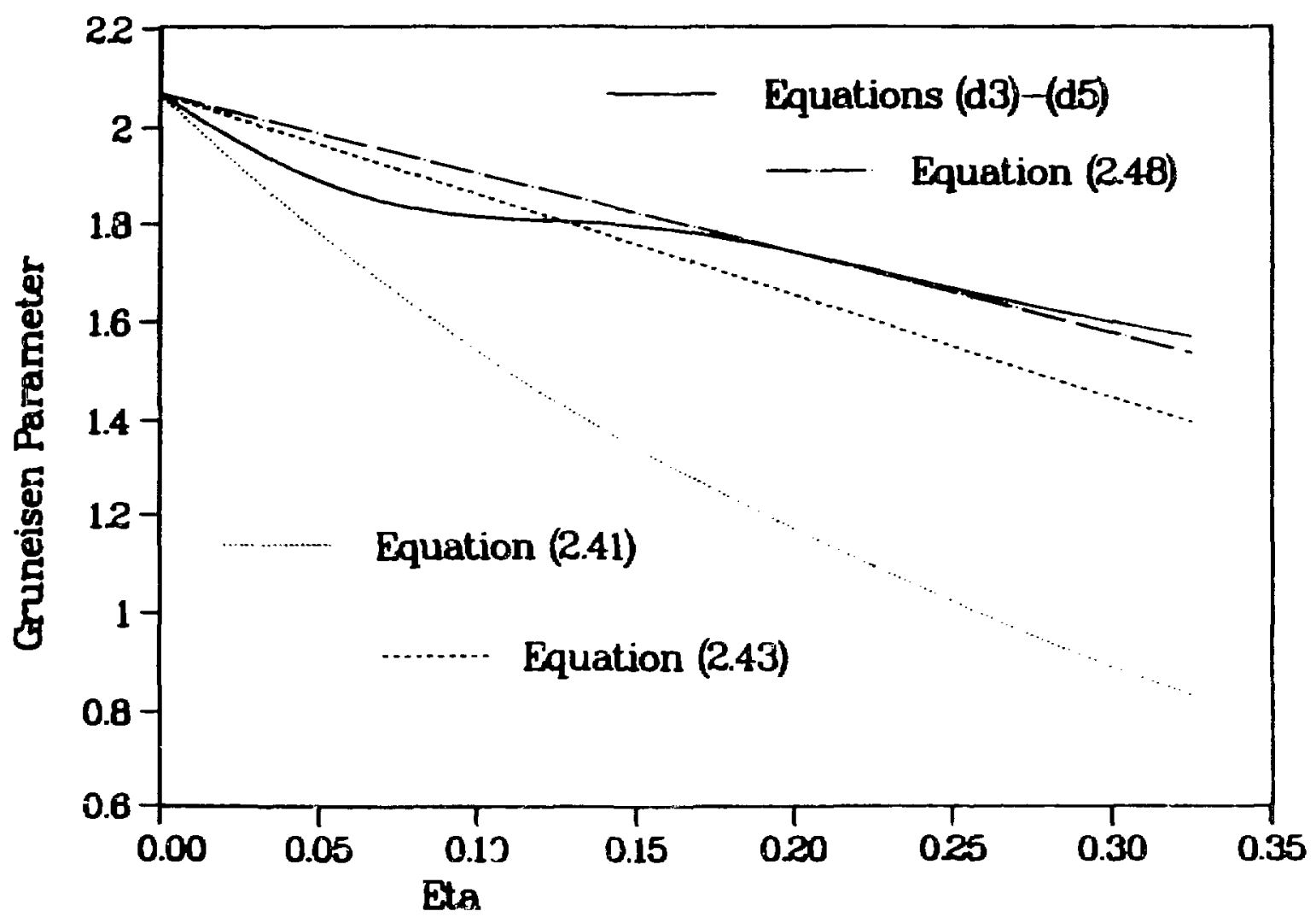

Figure 171. The Gruneisen parameter for stainless-steel plotted as a function of $\eta$ from different formulas. 


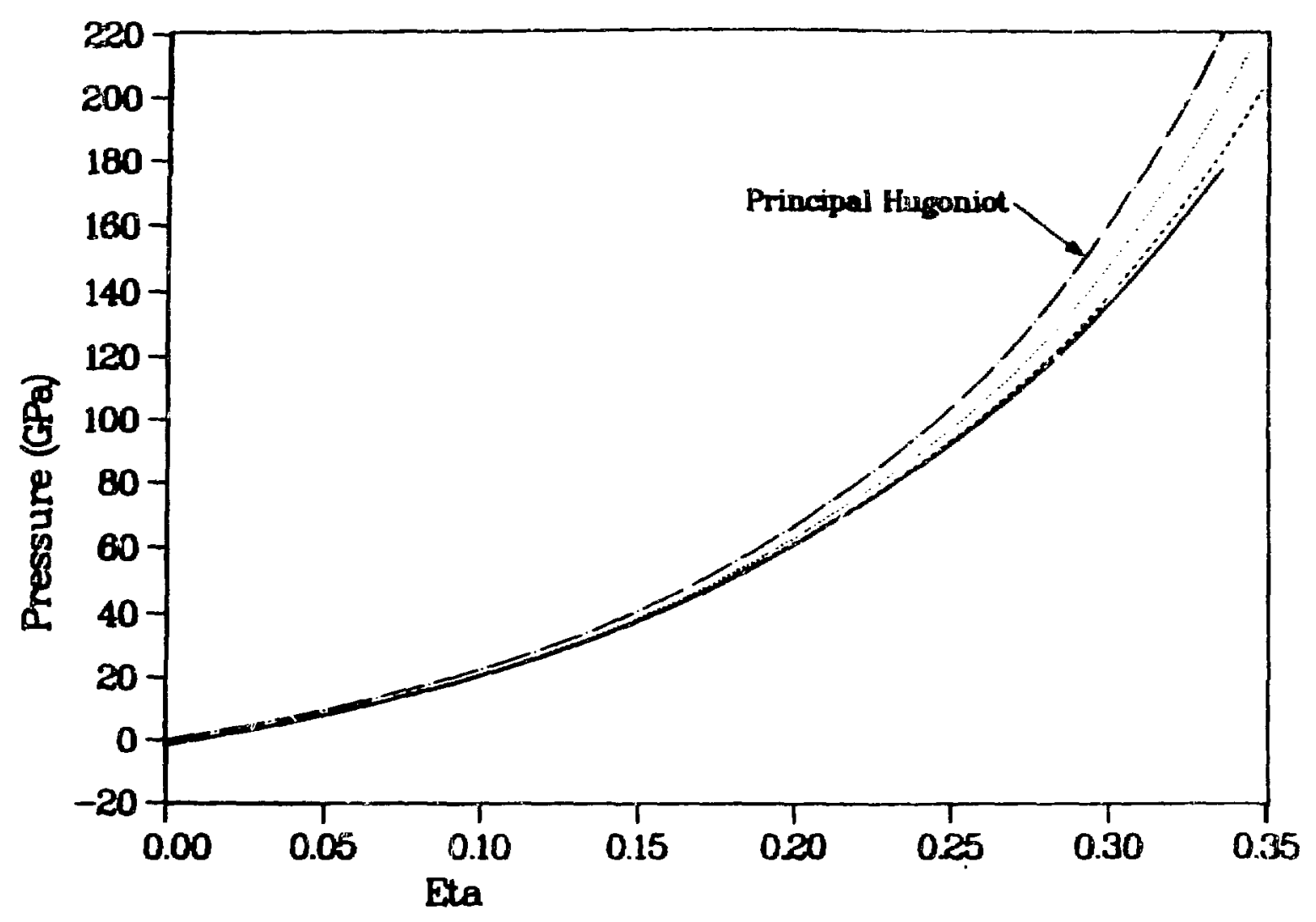

Figure 172. Pressure as a function of $\eta$ along sero-degree isotherms for stainless-steel. The dashed line is calculated using the Mie-Gruneisen equation of state. The dotted line is calculated using the Rice equation of state. The solid line is from the integration of equations $(d 3)-(d 5)$ 


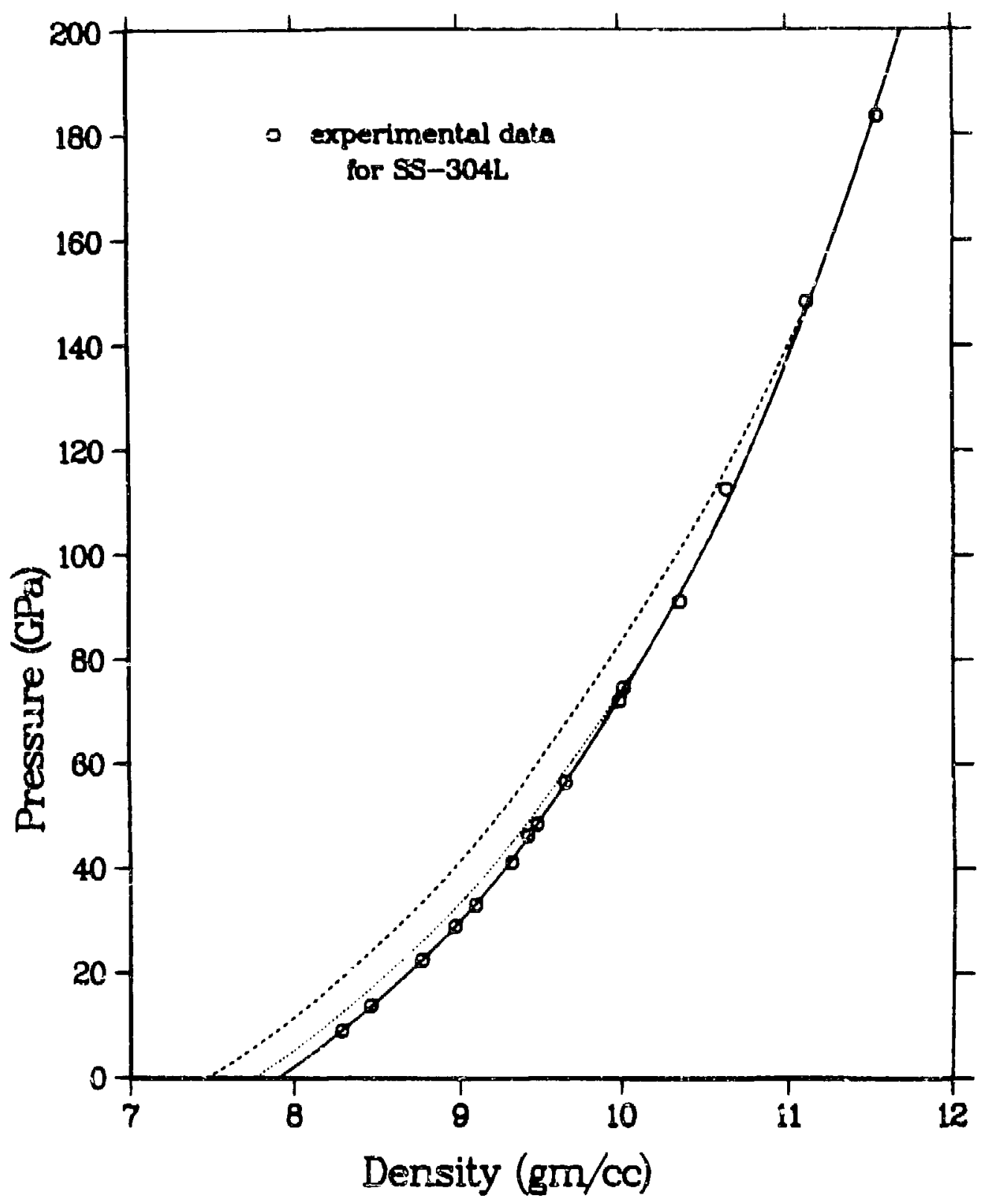

Figure 173. The Hugoniot for stainless-steel in the $P$ - $\rho$ plane as calculated from the MieGruneisen equation of state. The dashed line is the calculated release isentrope from $150 \mathrm{GPa}$. The dotted line is the isentrope from $80 \mathrm{GPa}$. 


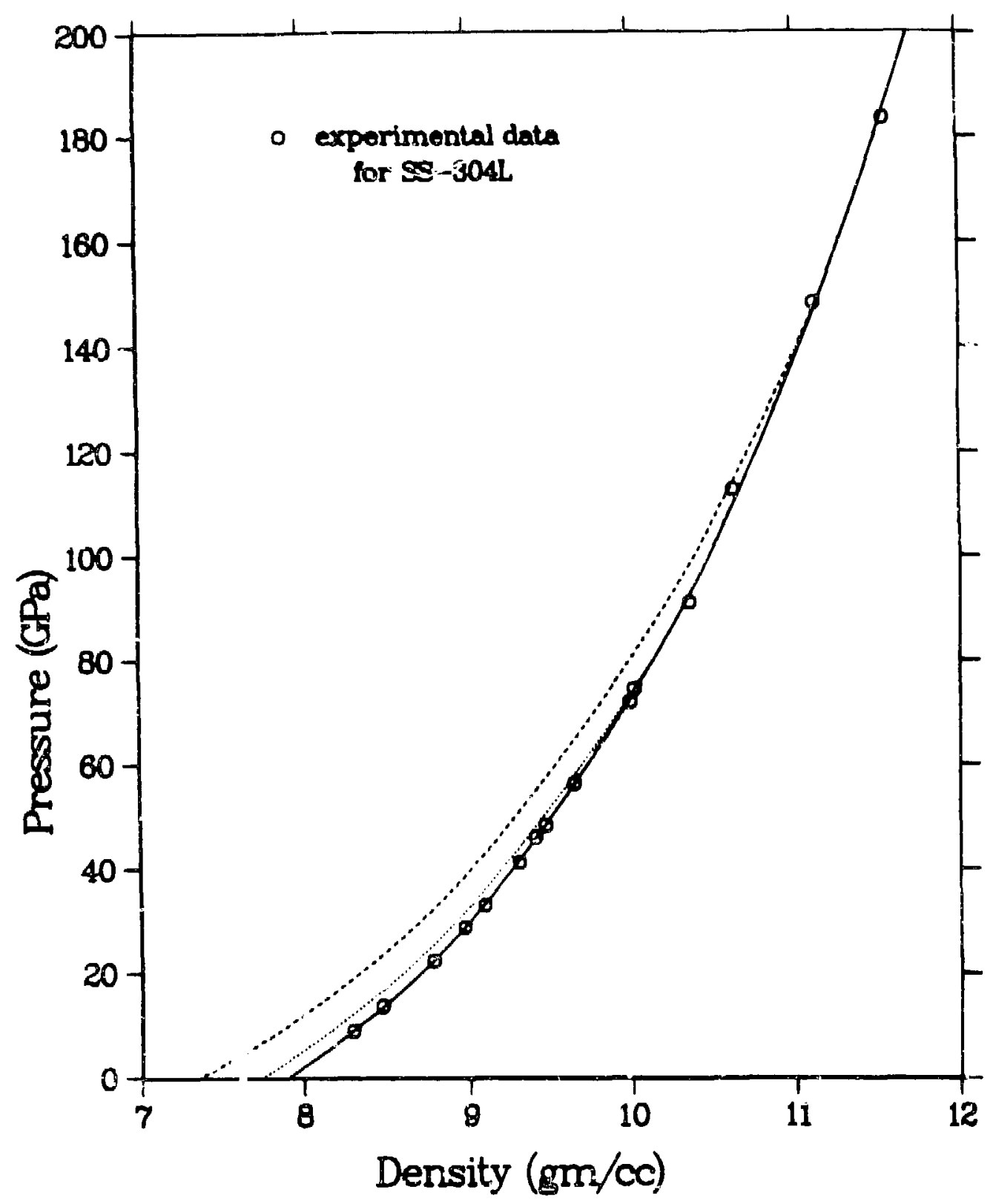

Figure 174. The Hugoniot for stainless-gteel in the $P$ - $\rho$ plane as calculaced from the Rice aquation of atate. The dashed line is the calculated release isentrope frum $150 \mathrm{GPA}$. The dotted ine is the isentrope from $80 \mathrm{GPa}$. 


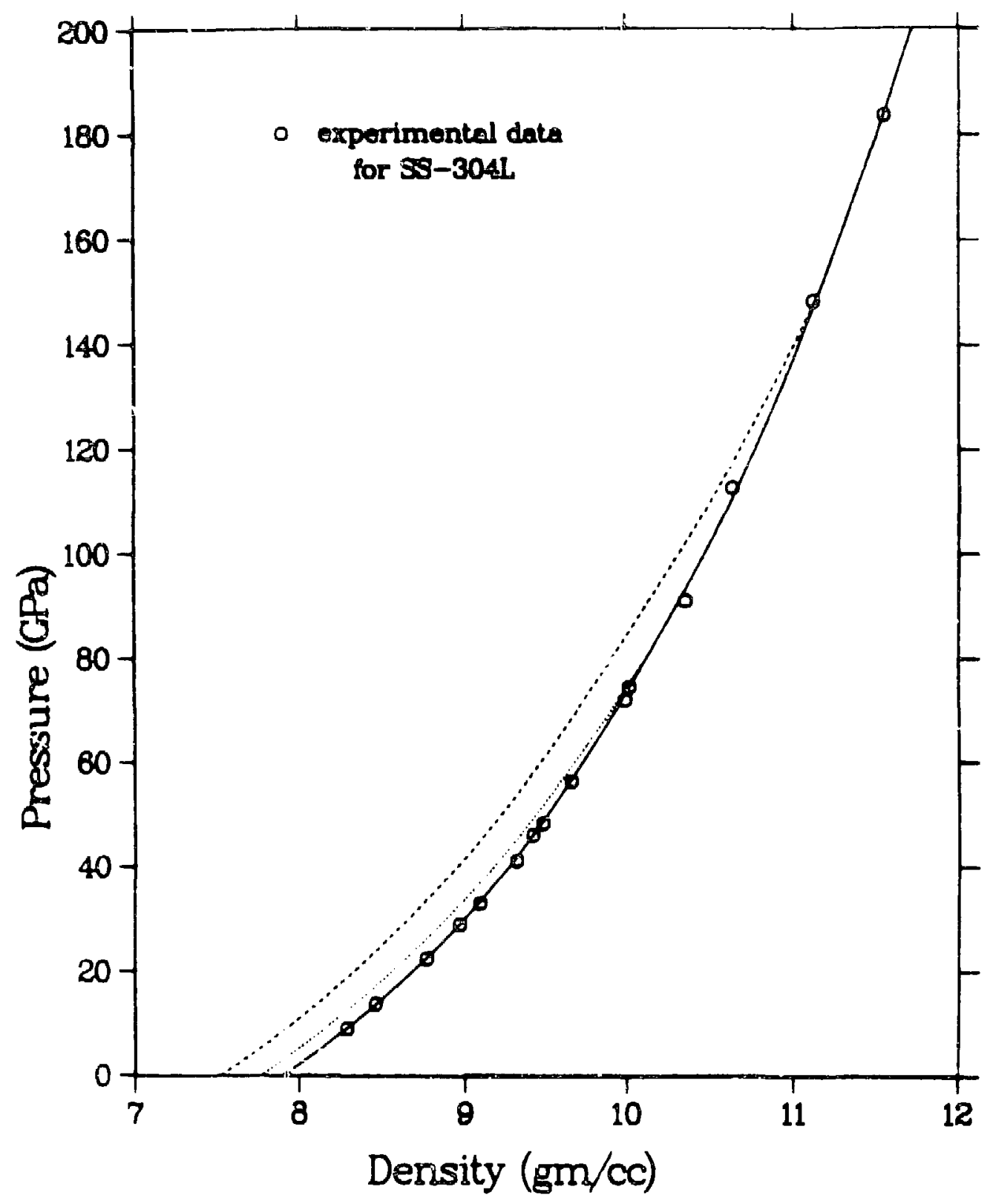

Figure 175. The Hugoniot for stainless-steel in the $P$ - $\rho$ pianc ag calculated from the Tillots $n$ equation of state. The dashed line is th, calculated release isentrope from $150 \mathrm{GPa}$. The dotted line is the isentrope from $80 \mathrm{GPa}$. 


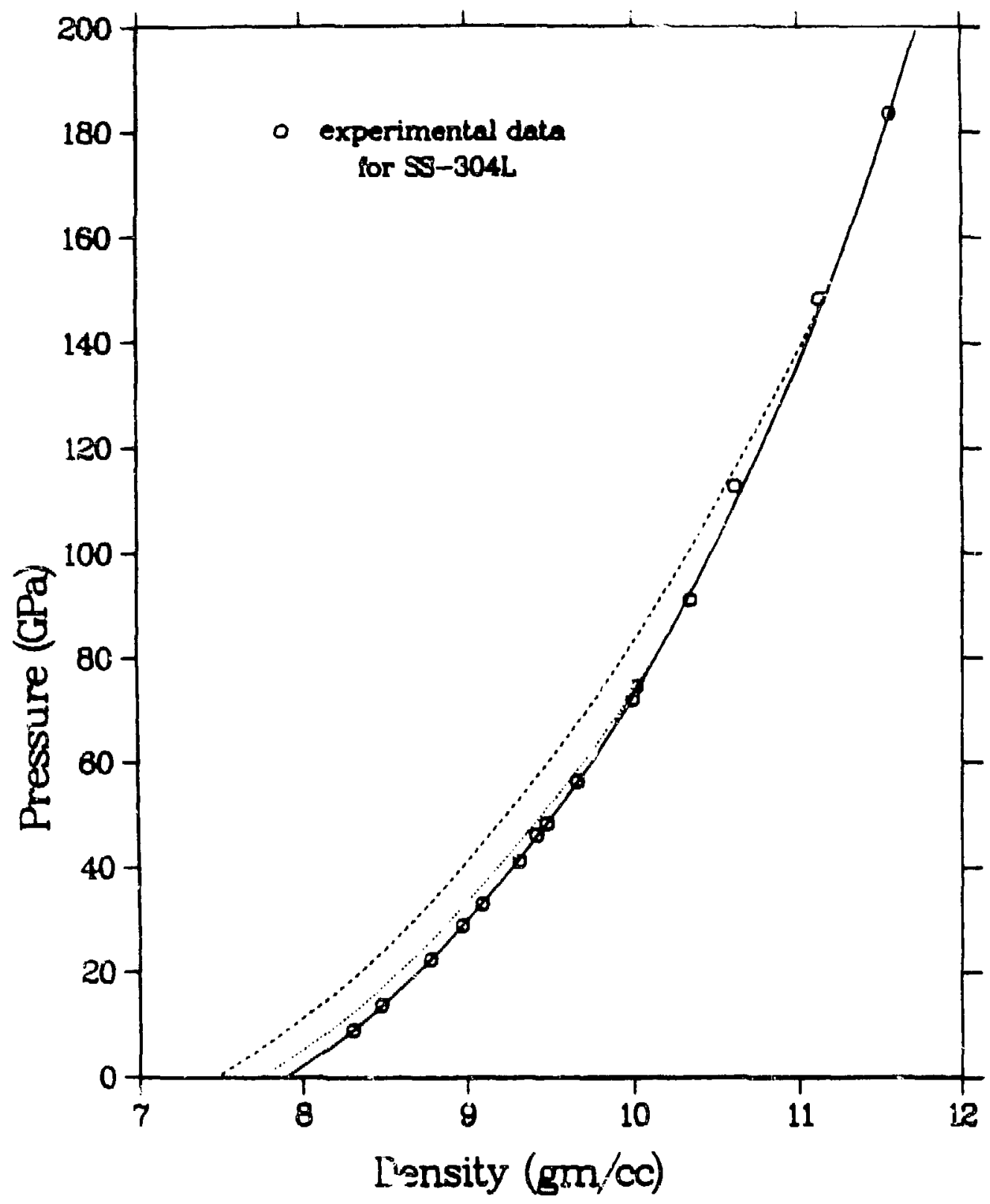

Figure 176. The Hugoniot for atainless-steel in the $P$ - $\rho$ plane as calculated from the Gray equation of state. The dasht $d$ line is the calculated release isentrope from $150 \mathrm{GPa}$. The dotted line is the isentrope from $80 \mathrm{GPa}$. 


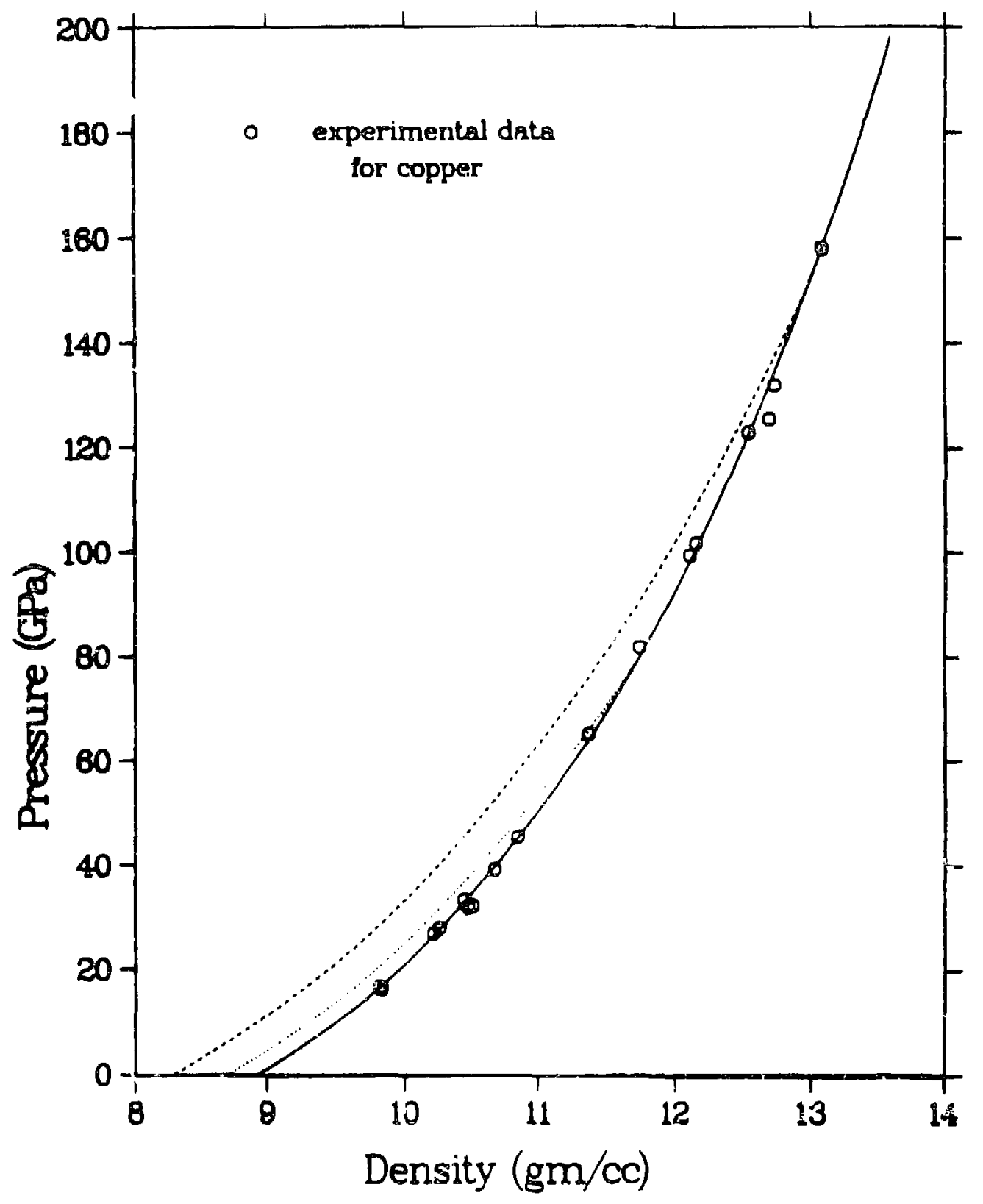

Figure 177. The Hugoniot for copper in the $P_{-\mu}$ plane as calculated from the Mie-Gruneisen equation of state. The 5 ind line is the cslculated release isentrope from $150 \mathrm{GPa}$. The dotted line is the igentrope from $\therefore, r a$. 


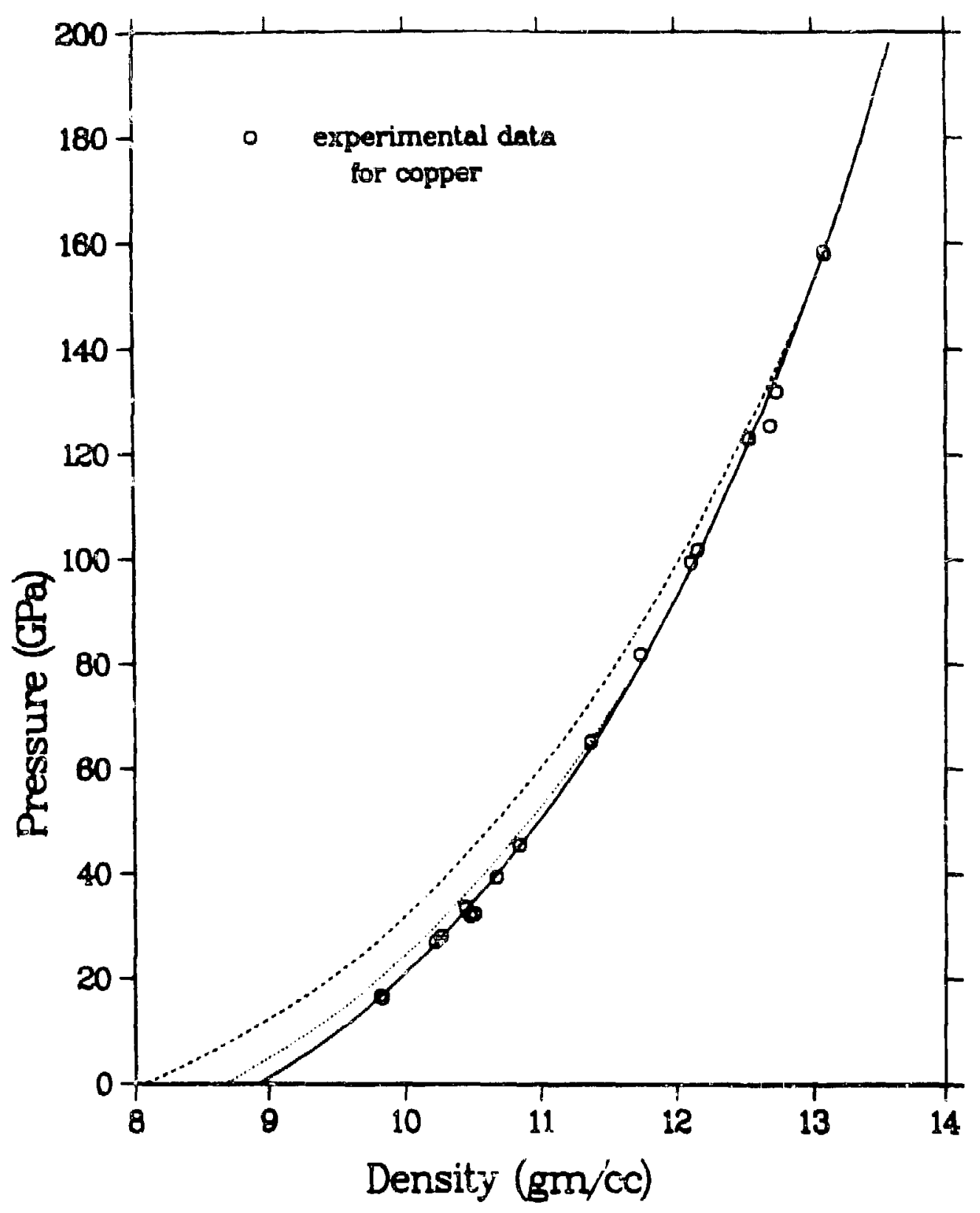

Figure 17a. The Yugoniot for copper in the $P$ - $\rho$ plane as calculated from the Rice equation of state. The dashed line is the calculated release isentrope from $150 \mathrm{GPa}$. The dotted line is the isentrope from $80 \mathrm{GPa}$. 


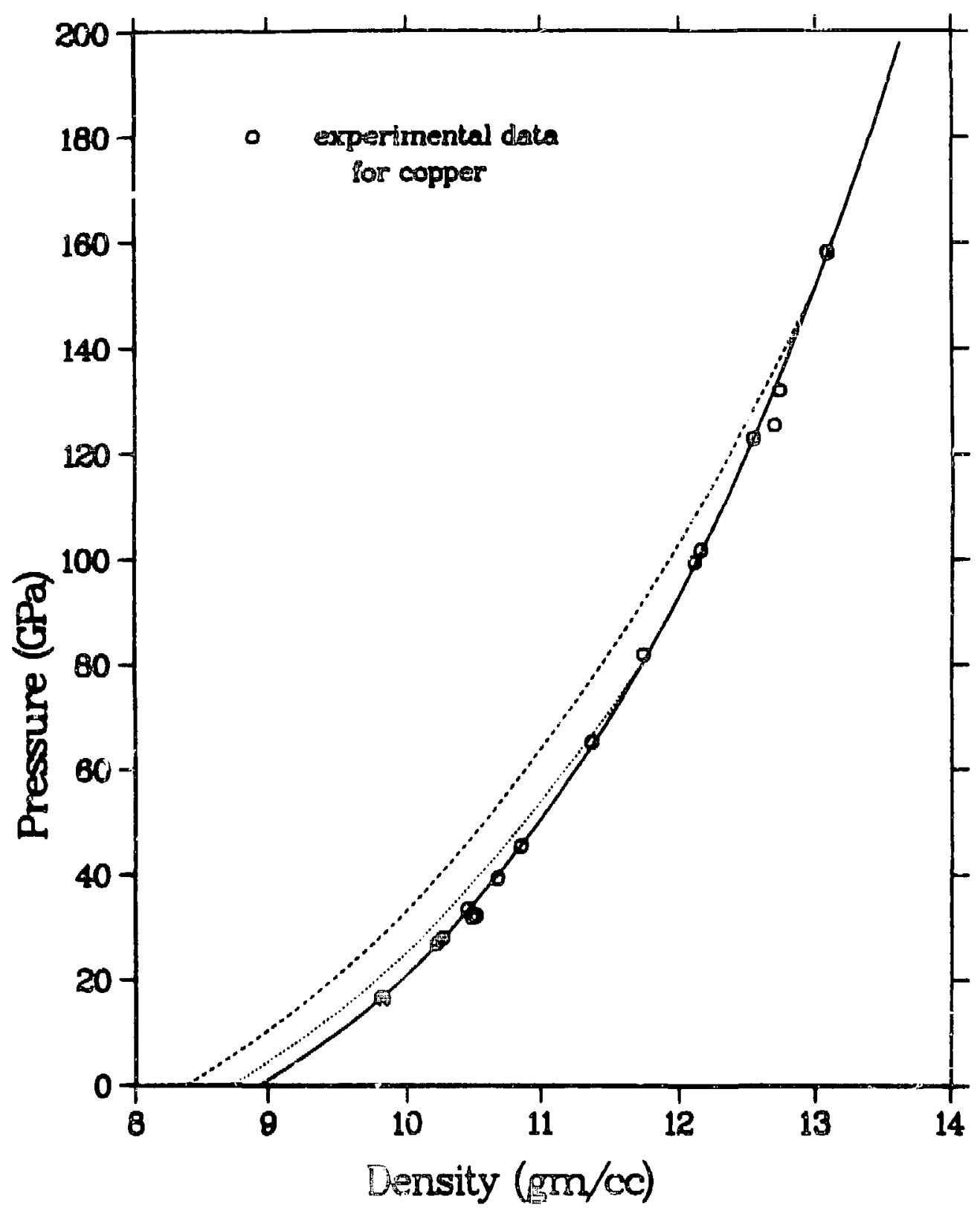

Figure 179. The Hugoniot for copper in is $P$ - $\rho$ plane as calculated from the Tillotson equation of atate. The dashed line is the calculated release isentrope from $150 \mathrm{GPa}$. The dotted line is the isentiope from $80 \mathrm{GPa}$. 


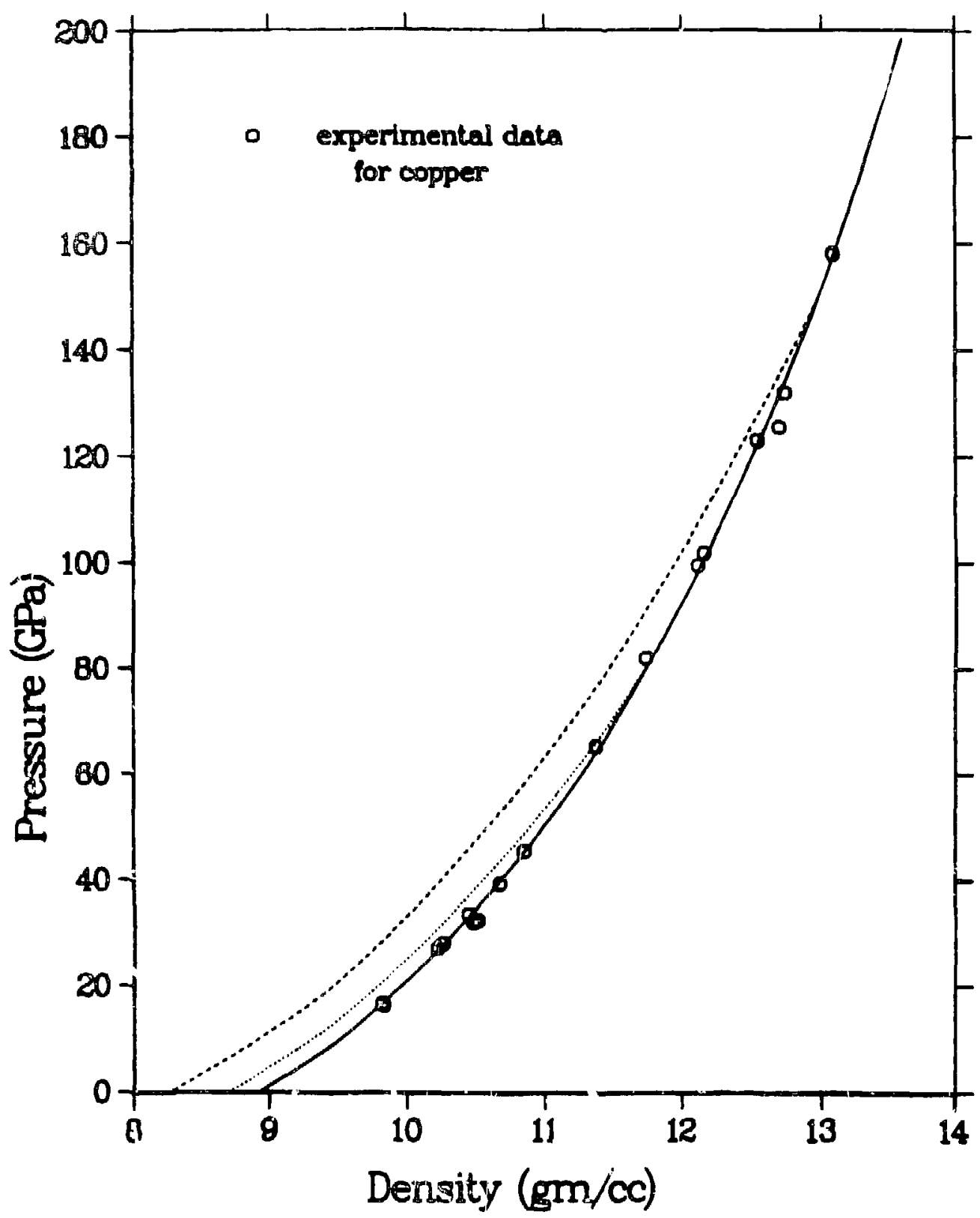

Flgure 180. The Hugoniot for copper in the $P$ - $\rho$ plane as calculated from the Gray equation of state. The dashed line is the ralculated release isentrope fron $150 \mathrm{GPa}$. The dotted line is the isentrope from $80 \mathrm{GPa}$. 


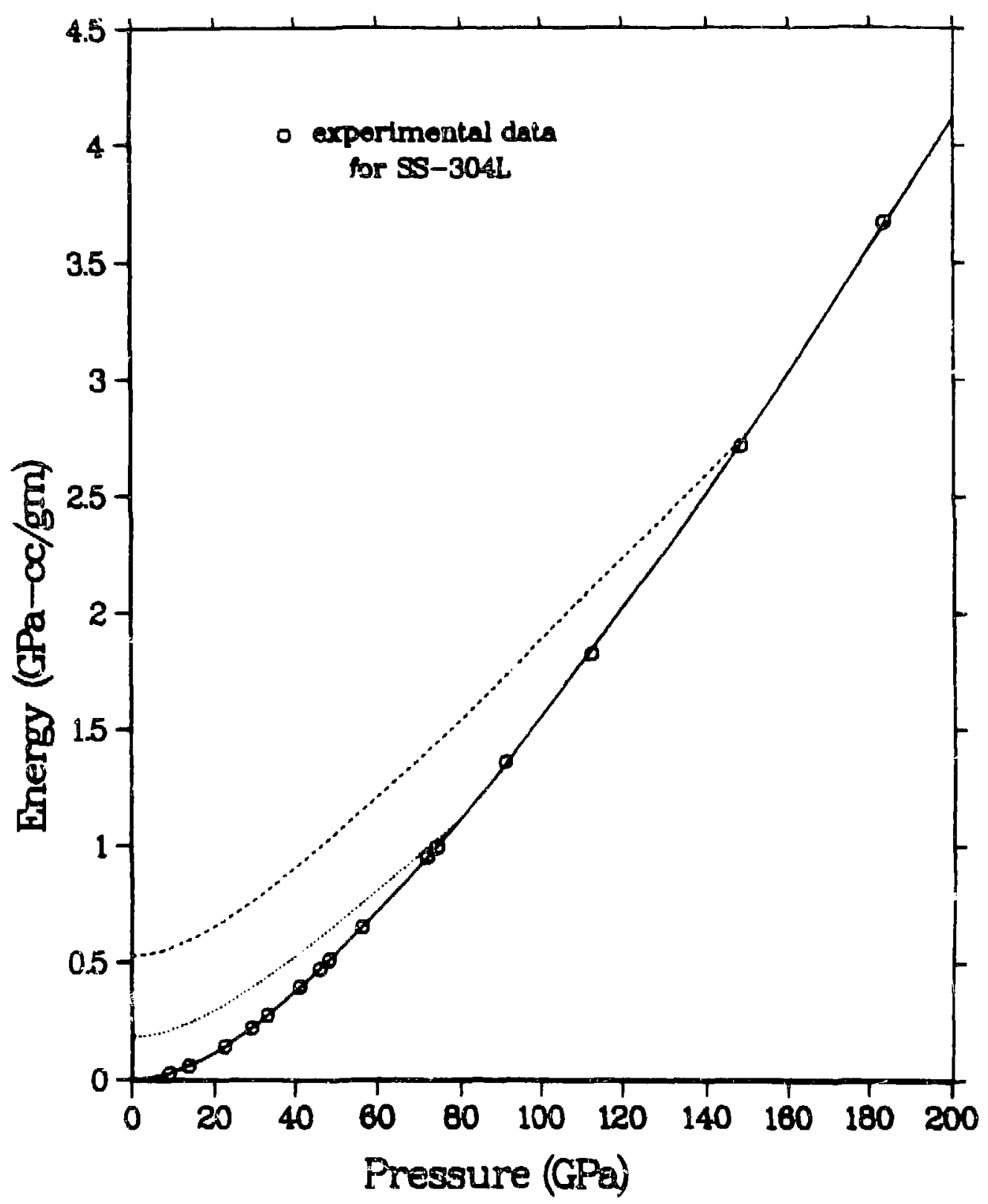

Figure 181. The Hugoniot for otainless-steel in the $E-P$ plane as calculated from the MieGrumeisen equation of state. The dashed line is the calculated release isentrope from $150 \mathrm{GPa}$. The dotted lise is the isentrope from $80 \mathrm{GPa}$. 


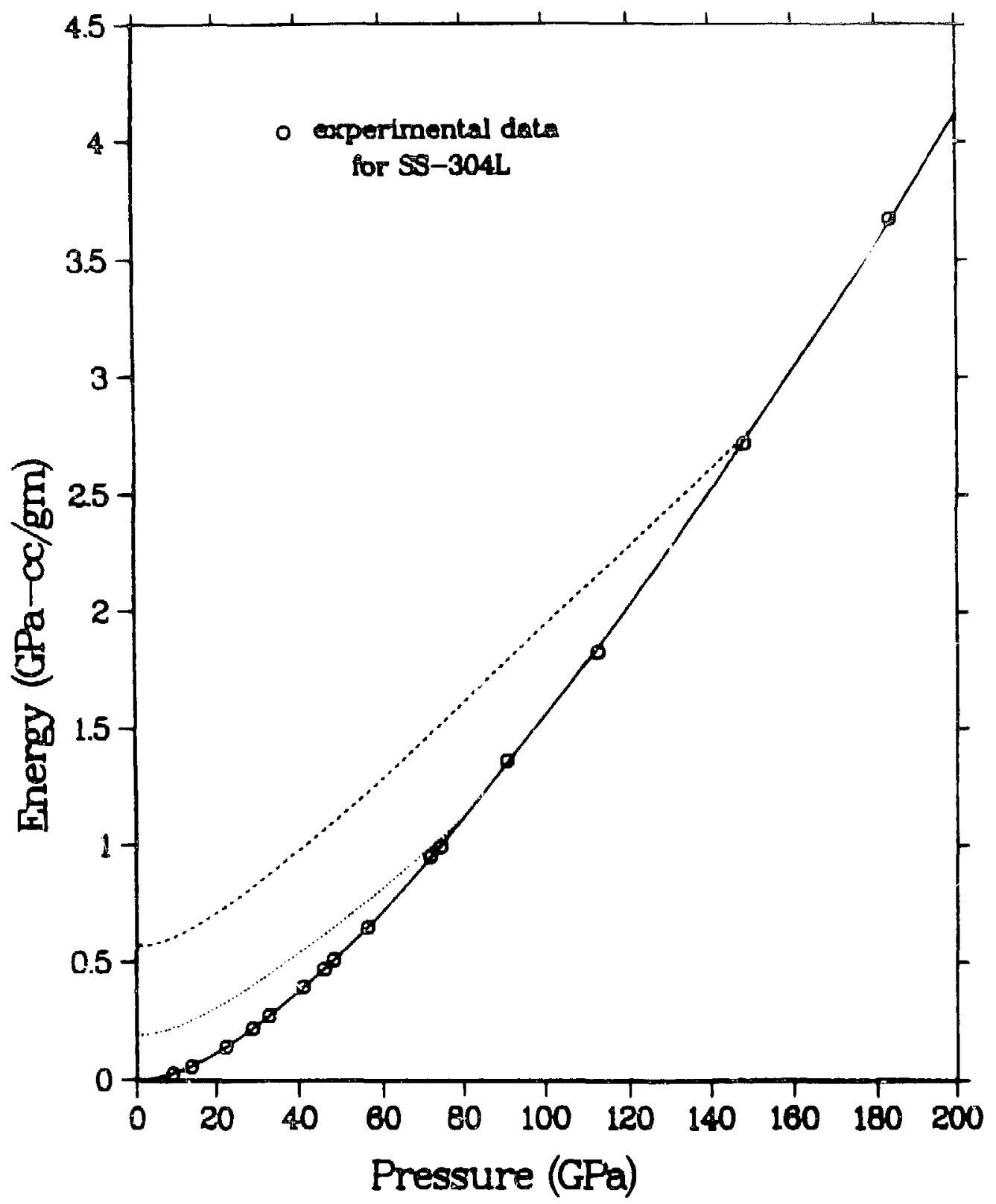

Figure 182. The Hugoniot for stainless-steel in the E-P plane as calculated from the Rice equation of state. The dashed line is the calculated release isentrope from $150 \mathrm{GPa}$. The dotted line is the isentrope from $80 \mathrm{GPa}$. 


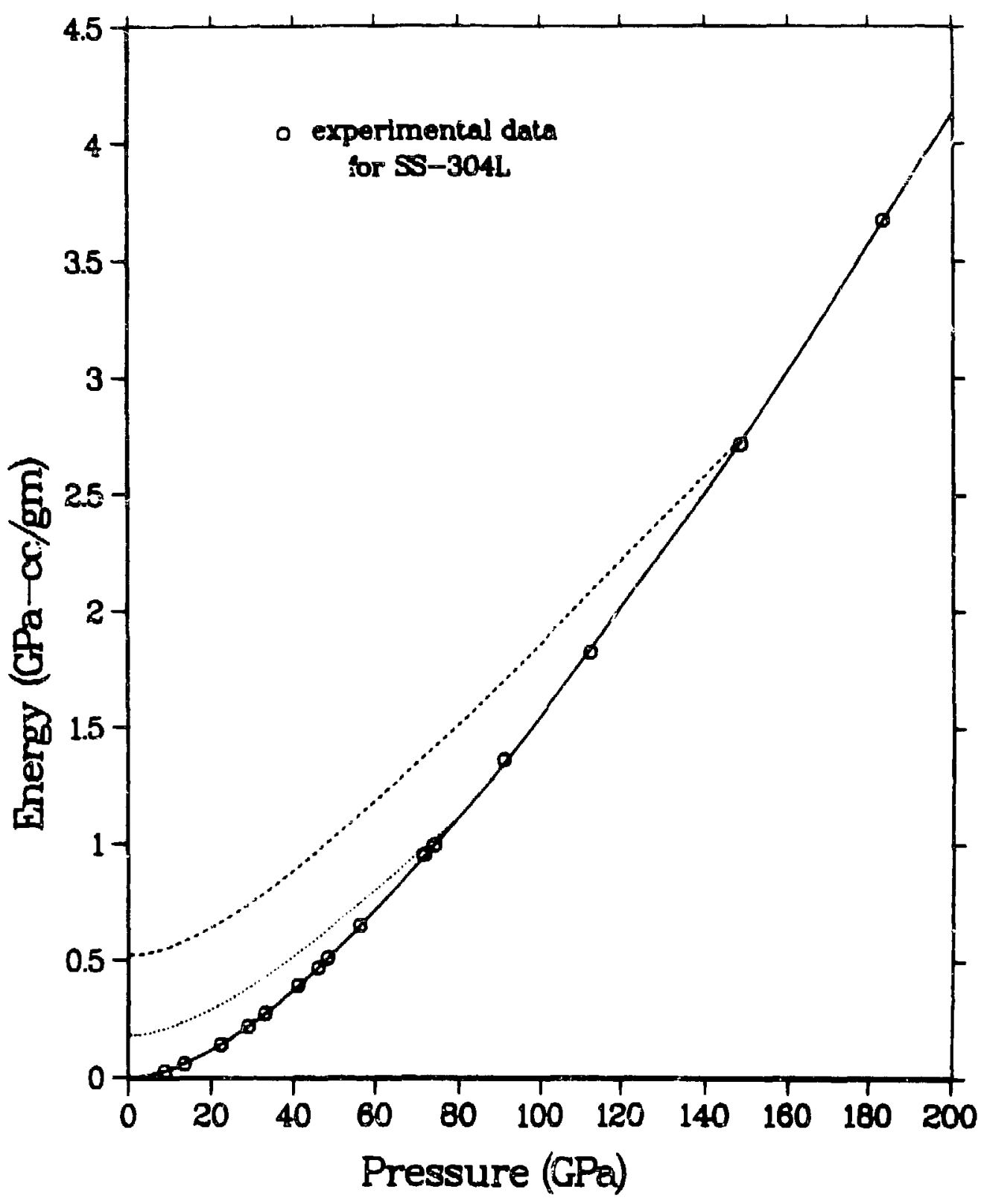

Figure 183. The Hugoniot for stainless-steel in the E-P plane as calculated from the Tillotson equation of atate. The dashed line is the calculated release isentrope from $150 \mathrm{GPa}$. The dotted line is the isentrope from $80 \mathrm{GPa}$. 


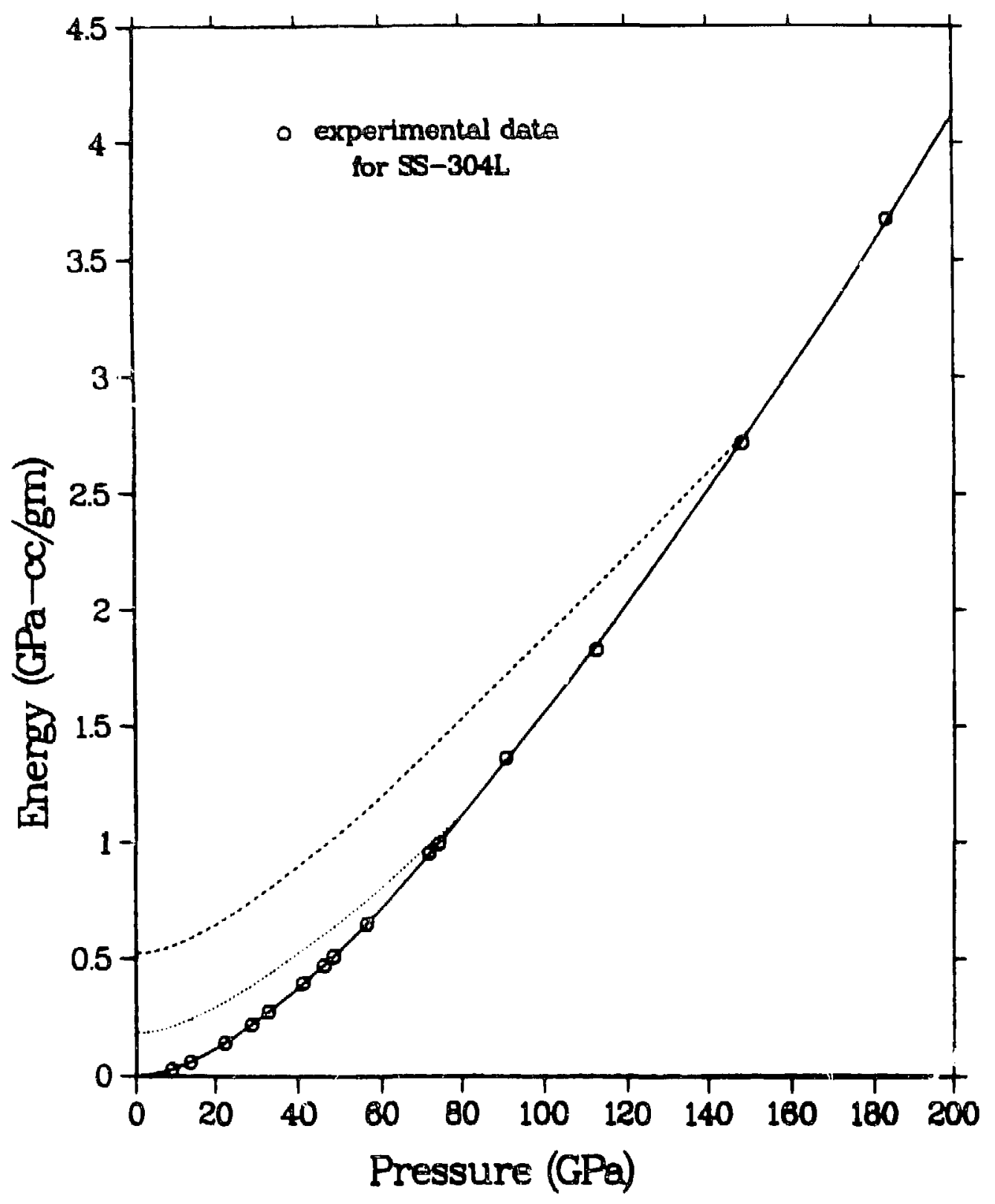

Figure 184. The Hugoniot for atainless-steel in the $E \cdot P$ plane as calculated from the Gray equation of state. The dashed line is the calcuiated release isentro, se from $150 \mathrm{GPa}$. The dotted line is the iseut:cipe from $80 \mathrm{GPa}$. 


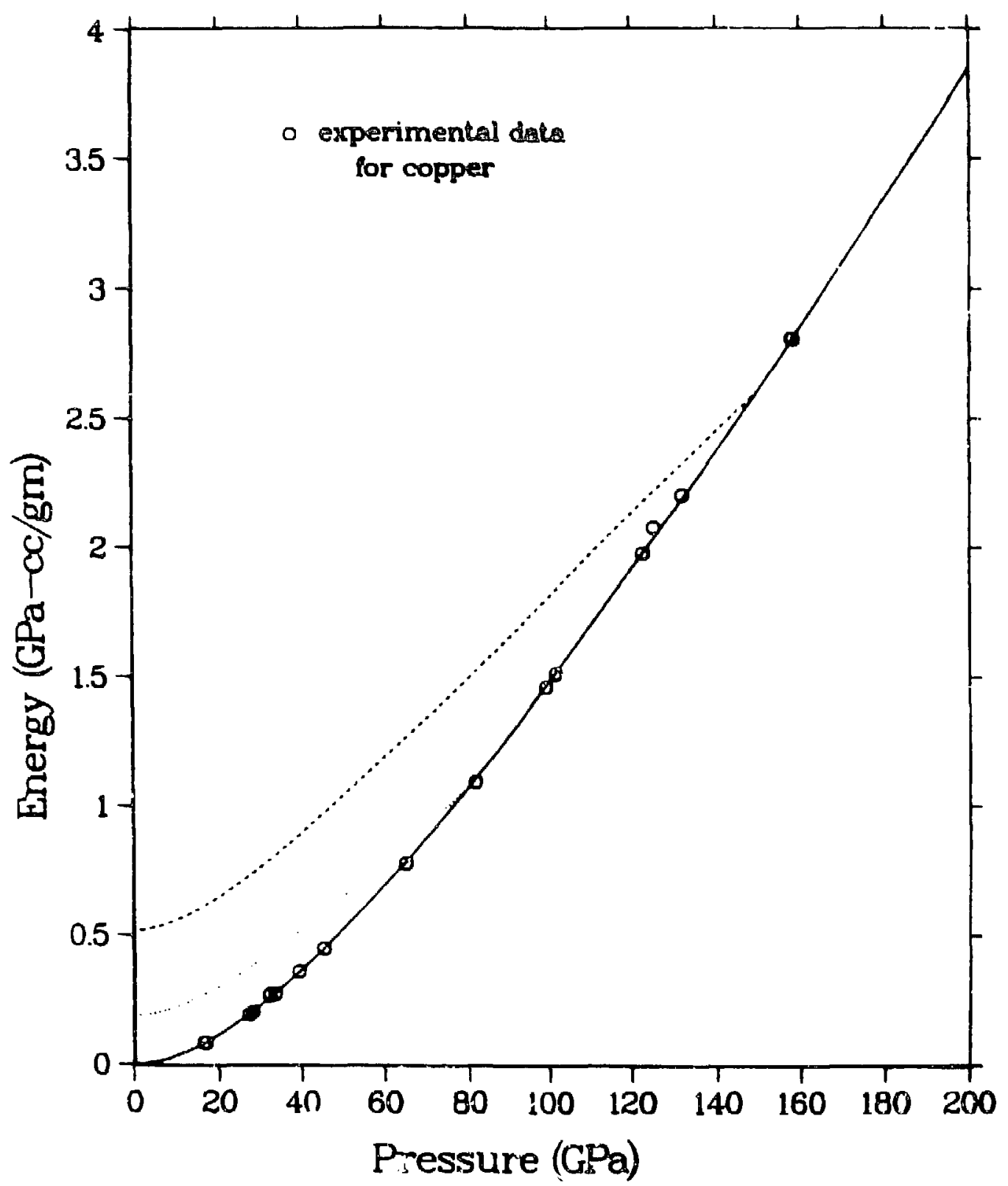

Figure 185. The Hugoniot for copper in the $E-P$ plane as calculated from the Mie-Gruneisen equatious of state. The dashed line is the calculated release isentrope from $150 \mathrm{GPa}$. The dotted line is the isentrope from $80 \mathrm{GPa}$. 


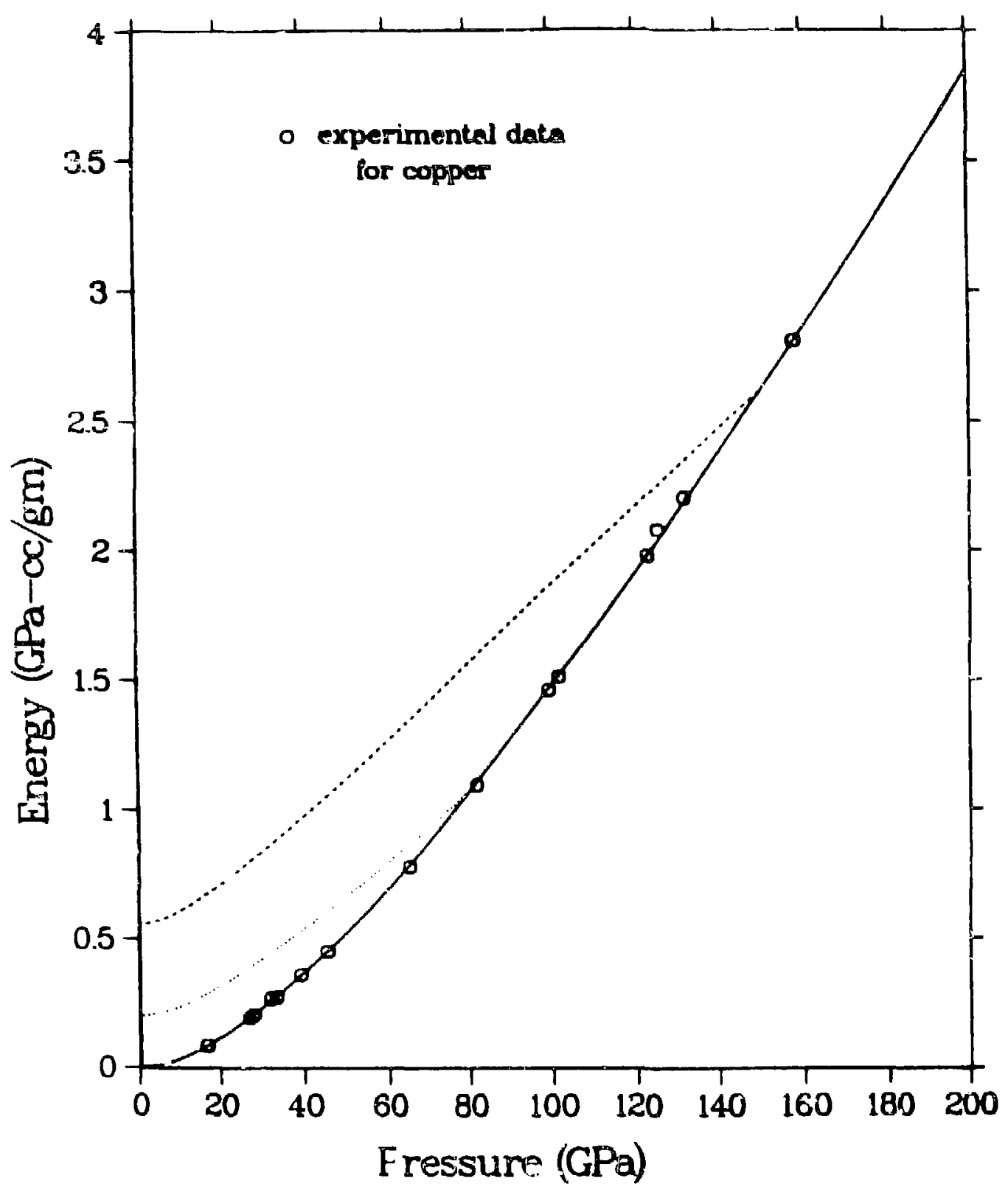

Figure 186. The Hugoniot for copper in the E-P plane as calculatea from the Rice equation of state. The dashed line is the calculated release isentrope from $150 \mathrm{GPa}$. The dotted line is the isentrope from $80 \mathrm{GPa}$. 


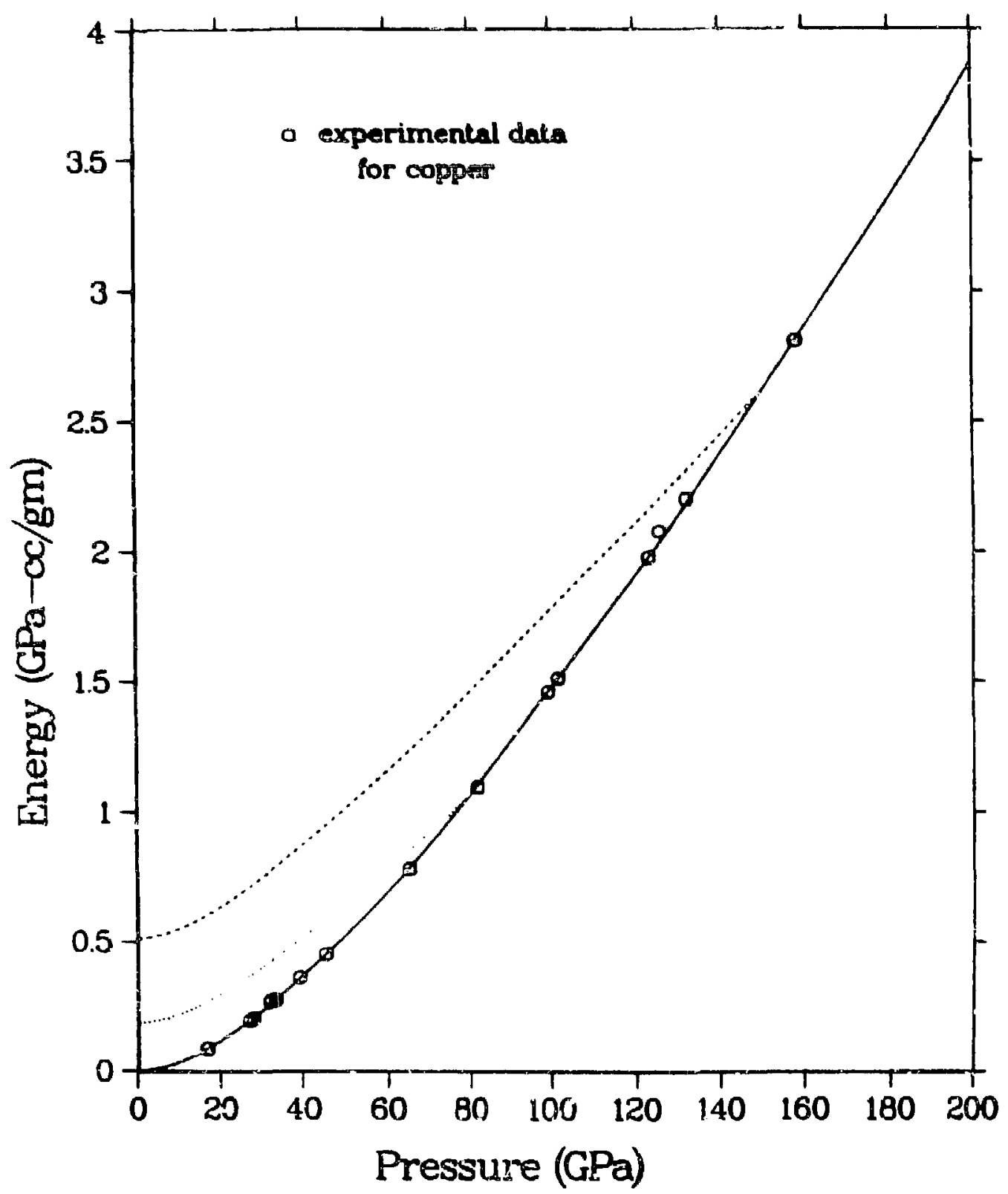

Figure 187. The Hugoniot for copper in the E.P plane as calculated from the Tillotson equation of state. The dached line is the calculaied release isentrope from $150 \mathrm{GPa}$. The dotted line is the isentrope f:om $80 \mathrm{GPa}$. 


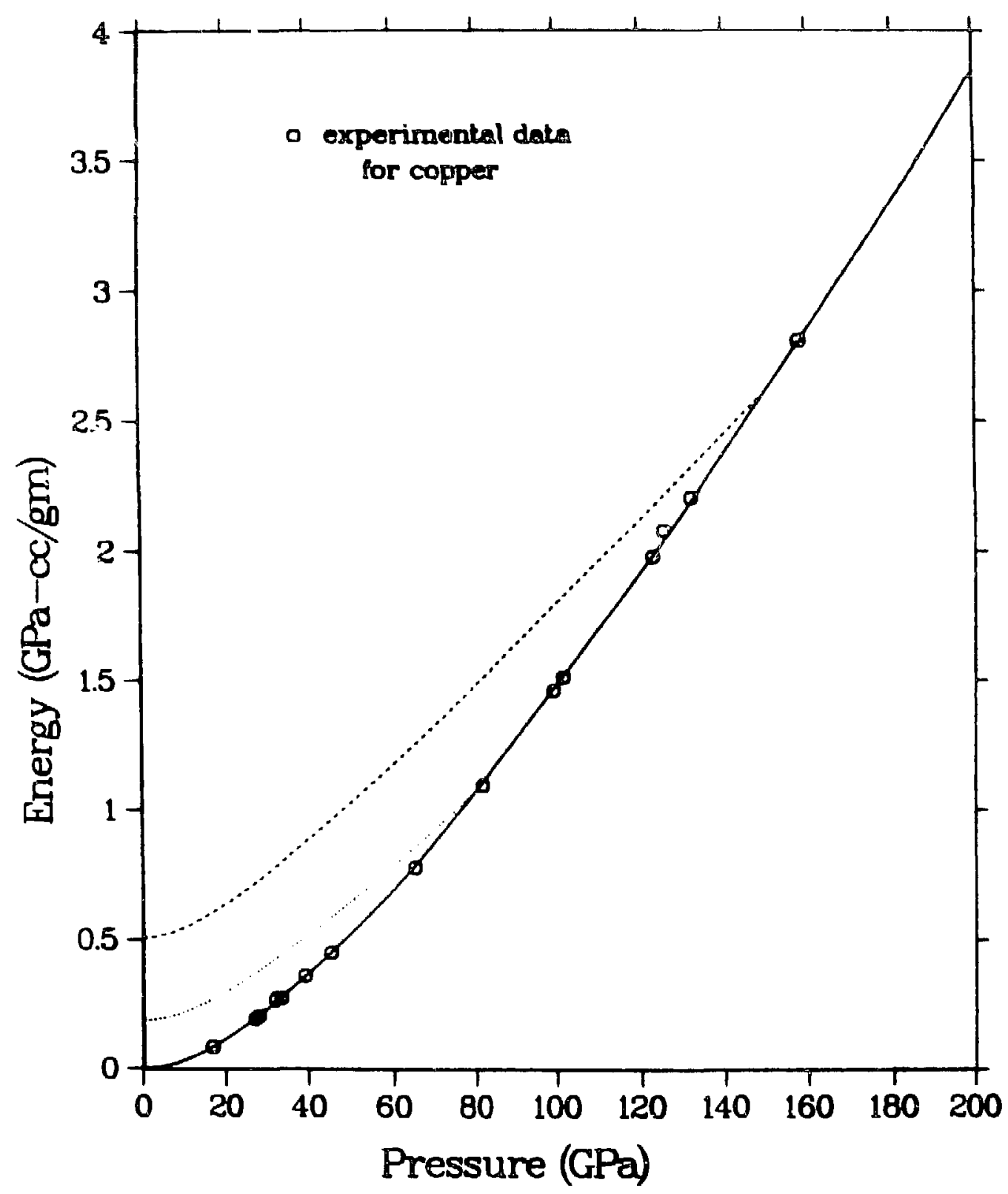

Figure 188. The Hugoniot for copper in the $E-P$ plane as calculated from the Gray equation of state. The dashed line is the calculated release isentrope from $150 \mathrm{GPa}$. The dotted line is the isentrope frum $80 \mathrm{GPa}$. 


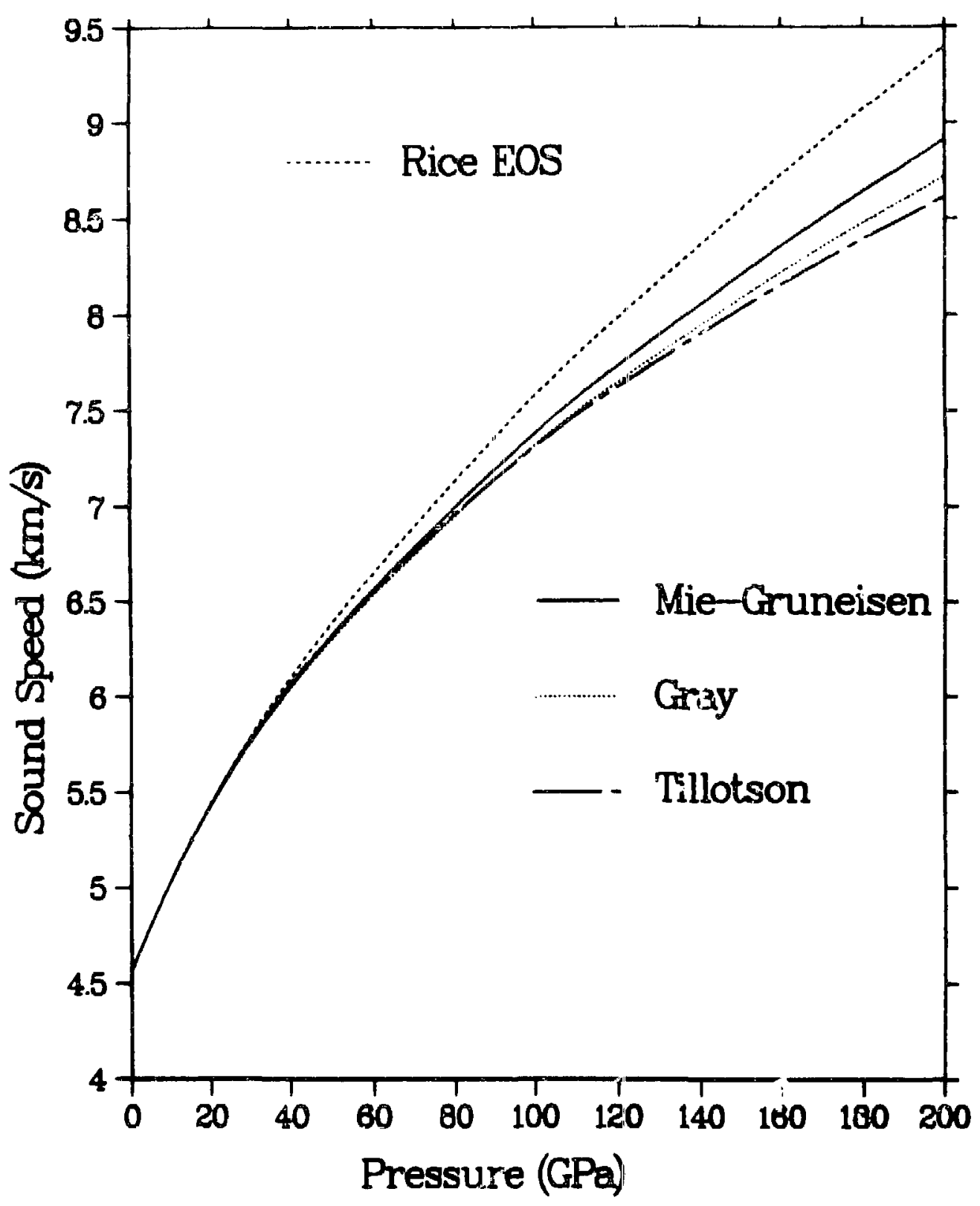

Plgure 189. The sound gpeeds for stainlesr-steel along the Hugoniot as calculated from the various equations of state. 


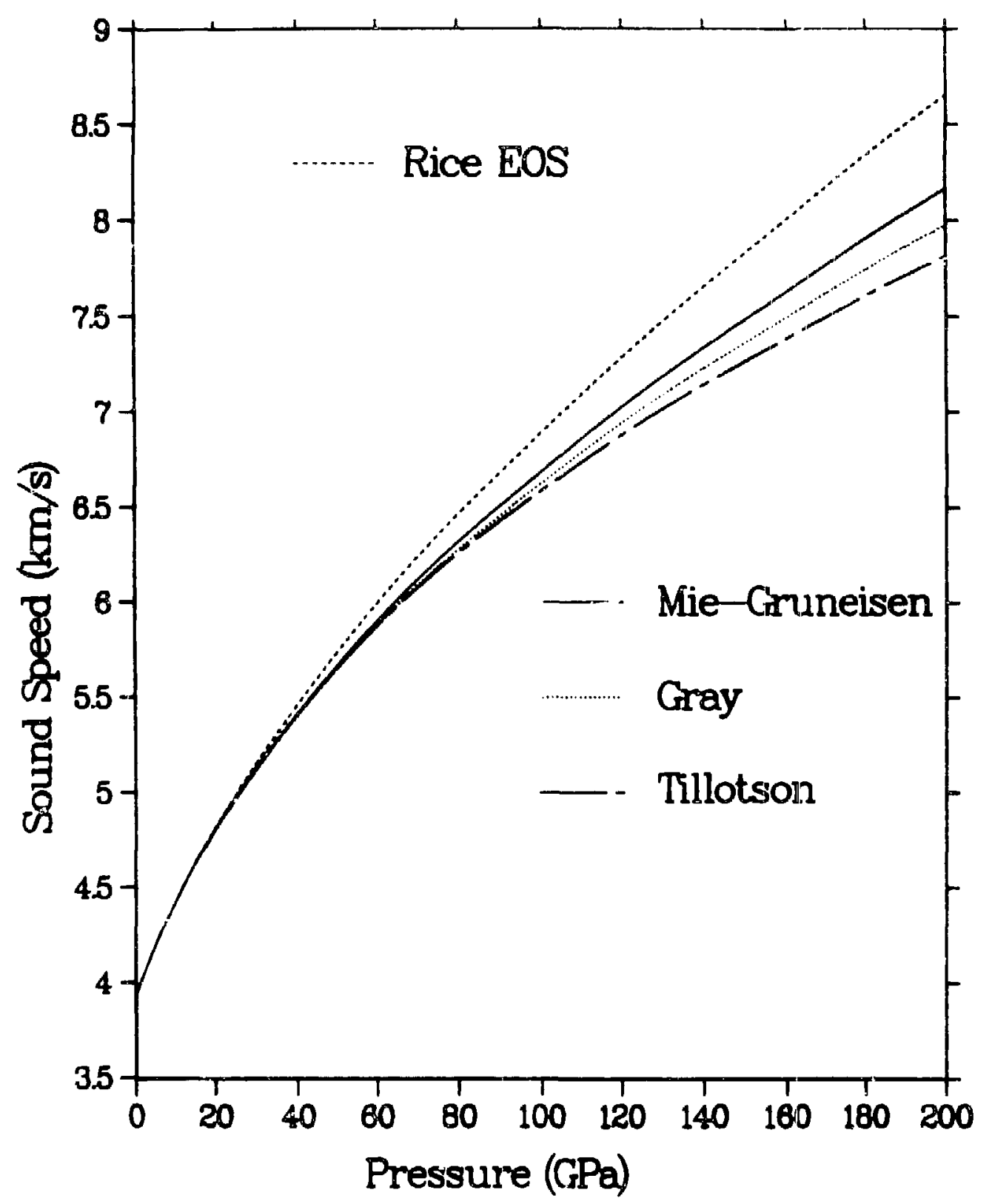

Figure 190. The sound speeds for copper along the Hugoniot as calculated from the various equations of state. 


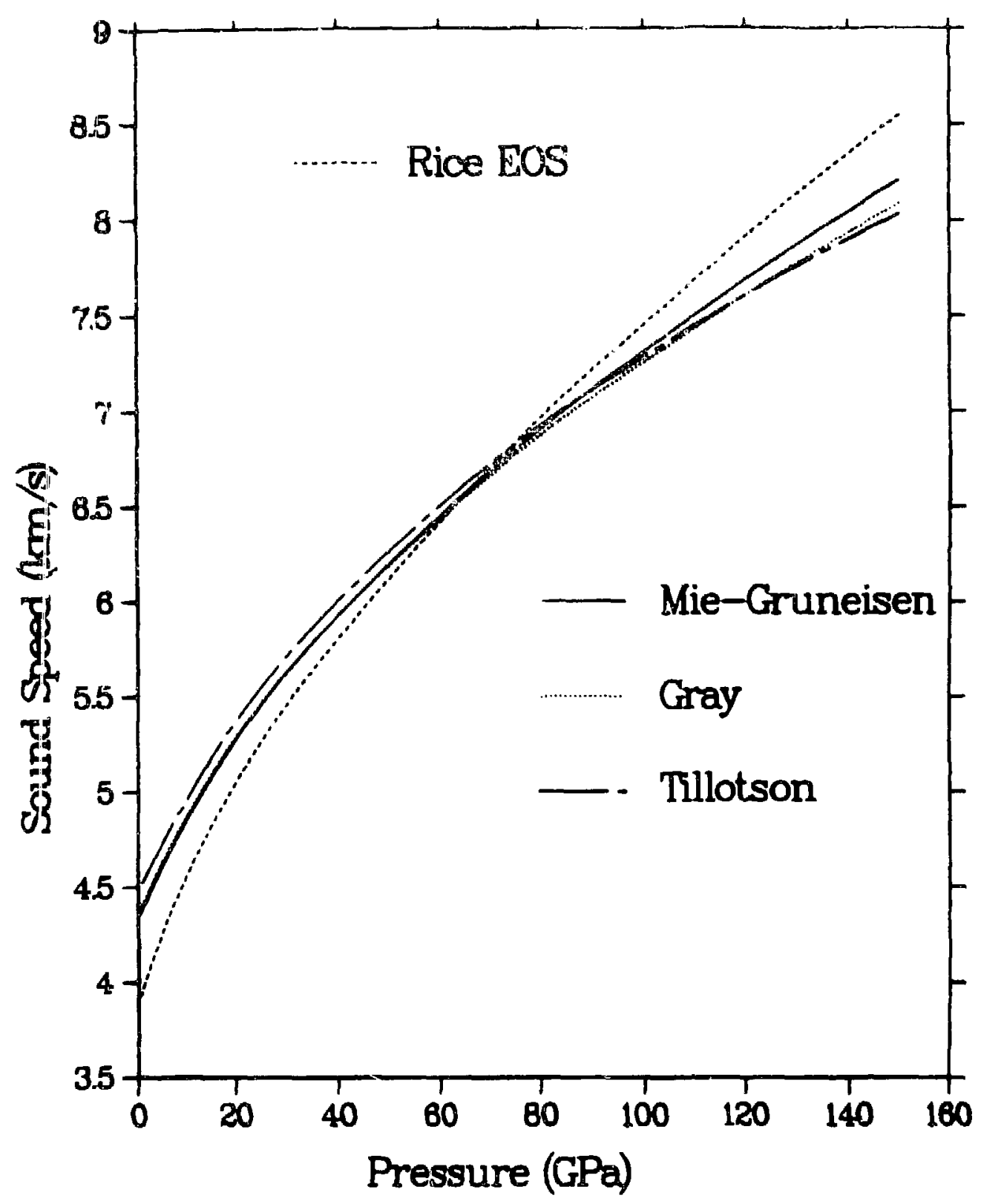

Figure 191. The sound speeds for stainless-steel along the 150-GPa isentrope as calculated from the varior s equations of atate. 


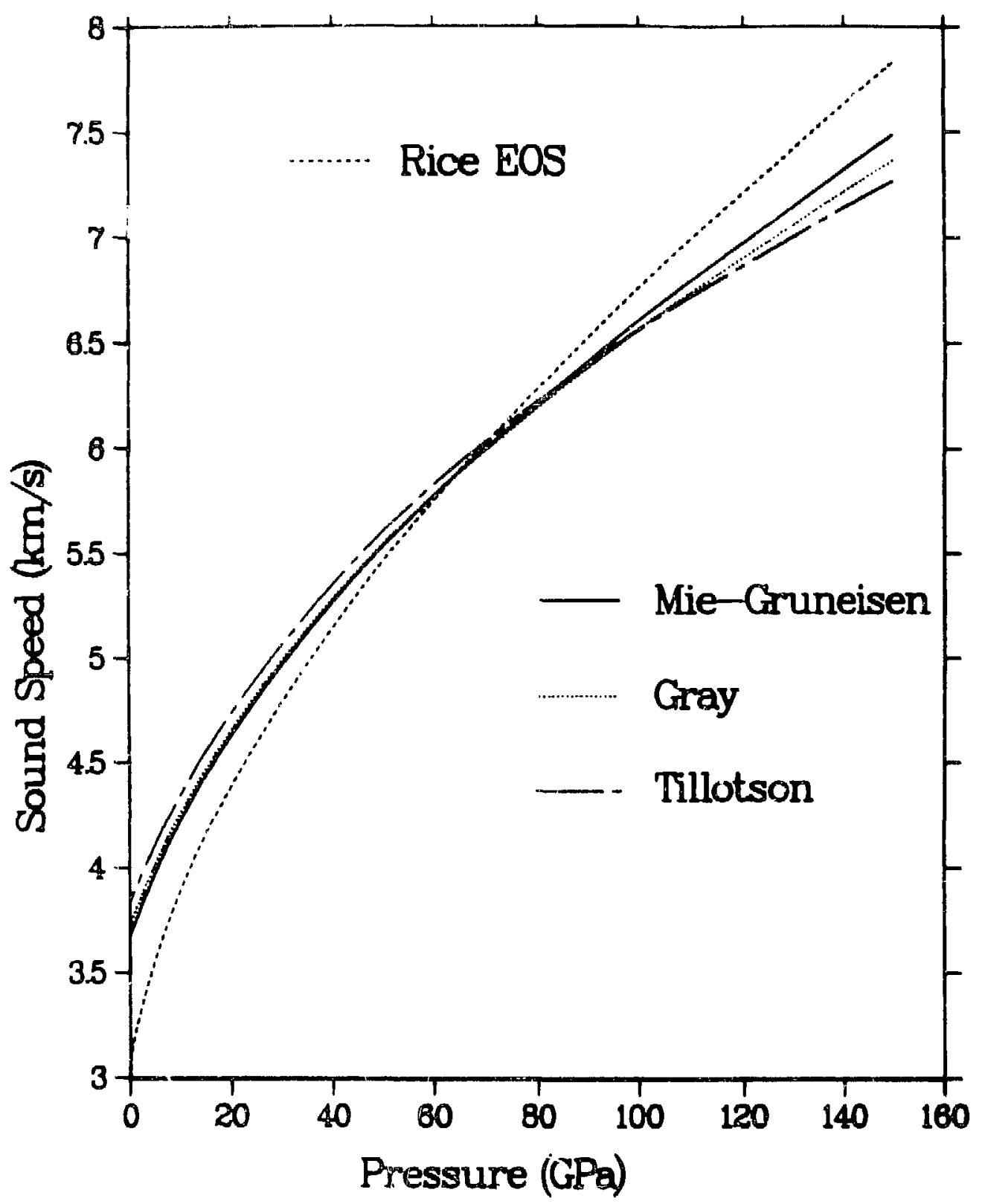

Figure 192. The sound apeeds for copper along the 150-GPa isentrope as calculated from the various equations of state. 


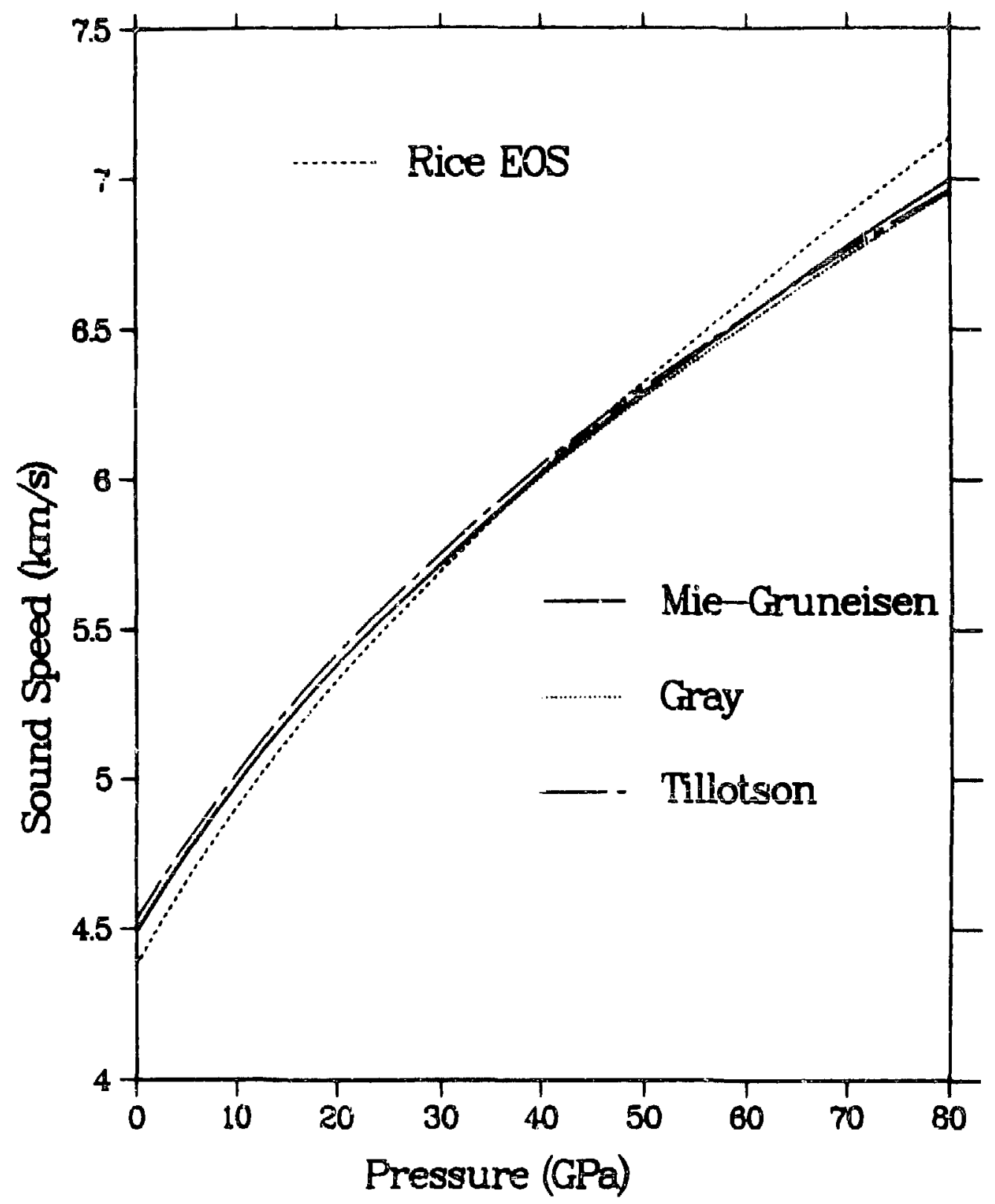

Figure 198. The sound speeds for stainless-steel along the 80-GPa isentrope as calculated from the various equations of atate. 


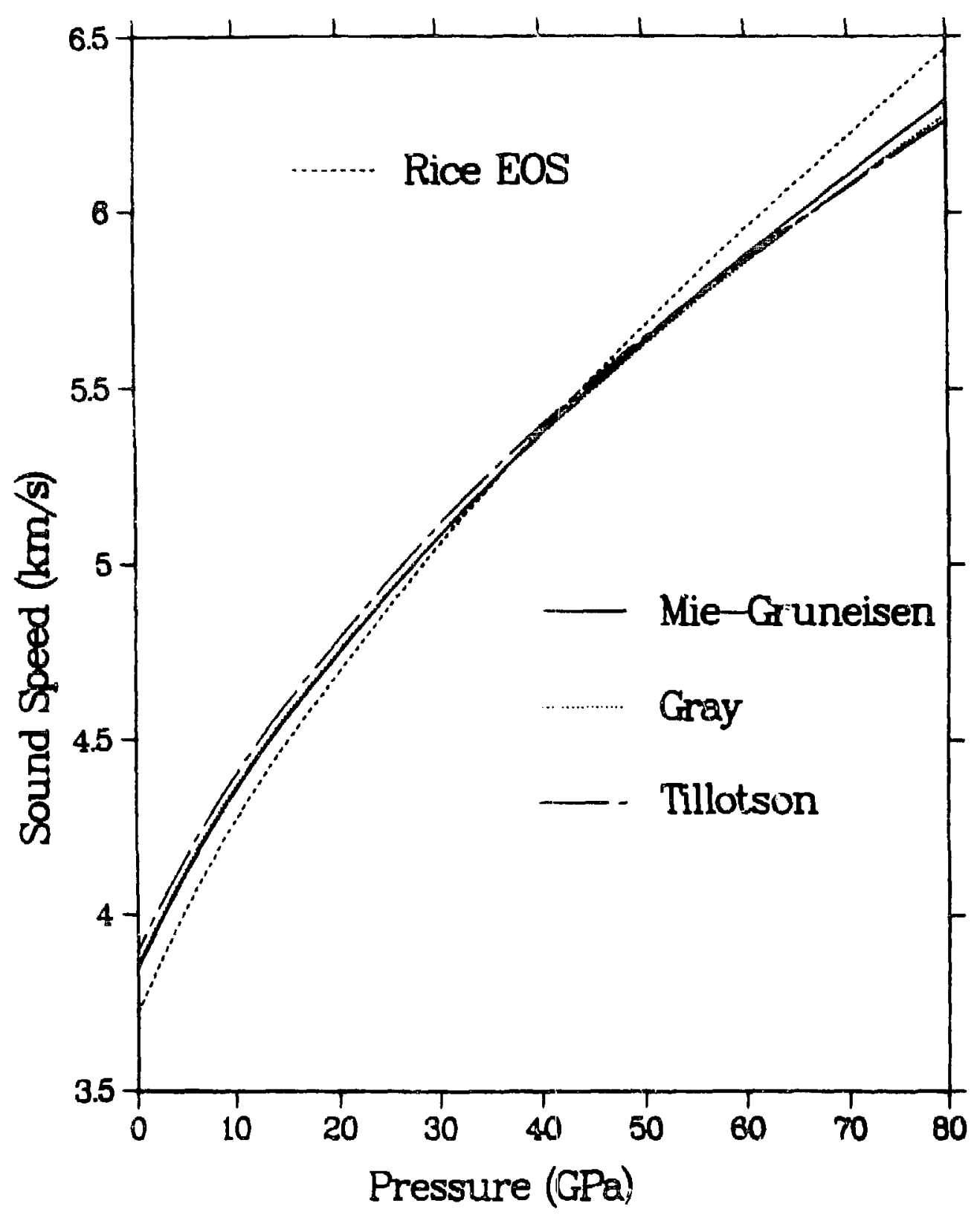

Figure 194. The sound speeds for copper along the 80-GPa isentrope as calculated from the various equations of atate. 


\section{ACKNOWLEDGEMENTS}

The research work reported in this dissertation would not have been possible without the help of many people. I thank Dr. J. M. Brown for supplying the sound speed data for tantalum. Dr. B. W. Olinger kindly supplied me with the longicudinal and transverse wave velocities for stainless-steel. I wish to thank Dr.

A. C. Juveland for many interesting and illuminating discussions on equations of state. I owe a debt of gratitude to the administration of the Los Alamos National Laboratory for allowing me the time to conduct this research. I am especially indebted to the many fine people at group M-4 of the laboratory. Ali the experiments were conducted at the firing sites of $\mathrm{M}-4$. I owe special thanks to four people in particular: Dr. T. R. Neal initially suggested this research work, Mr. R. E. Faudree and Mr. G. J. Martinez skillfully and carefully constructed most of the experiments, and Dr. J. M. Walsh who kindly served as my thesis advisor at Los Alamos. I am also very thankful to Dr. Alex Burr who agreed to be my thesis advisor at New Mexico State University.

D.O.M. 